\title{
Synthesis of Natural Products Containing Fully Functionalized
}

\section{Cyclopentanes}

\author{
Andrew J. Ferreira and Christopher M. Beaudry* \\ Department of Chemistry, Oregon State University, 153 Gilbert Hall, Corvallis, OR \\ 97331-4003, USA. \\ christopher.beaudry@oregonstate.edu
}

\begin{abstract}
Andrew J. Ferreira was born in San Francisco, CA. He received his A.B. degree in chemistry from the University of California, Davis in 2010. Currently, he is a Ph.D. candidate in the chemistry department of Oregon State University studying alkaloid synthesis with Prof. Christopher Beaudry.
\end{abstract}

Christopher M. Beaudry received his B.S. degree from the University of Wisconsin in 2000. As an undergraduate, he conducted research with Prof. Steven D. Burke. He obtained his Ph.D. at the University of California, Berkeley under the direction of Prof. Dirk Trauner. He was an NIH postdoctoral fellow in the lab of Prof. Larry Overman at the University of California, Irvine. He joined the chemistry department at Oregon State University in 2009.

\section{Introduction}

The five-membered carbocycle is a familiar and widespread structural motif in natural product architectures. As a result, the collection of chemical reactions that construct cyclopentanes represents an indispensible toolbox for synthetic chemists. Moreover, methods that build cyclopentanes with control of $\mathrm{sp}^{3}$-hybridized stereocenters are particularly valuable. The importance of creating substituted cyclopentanes with control of stereochemistry has been a driving force inspiring the creation of synthetic methods and strategies for building functionalized five-membered carbocycles. The pursuit of substituted cyclopentanes has showcased the wide scope, functional group tolerance, and elegance of many synthetic reactions. The pursuit has also exposed the limitations of various synthetic transformations, and it has inspired chemists to improve such processes. This review provides a comprehensive discussion on the methods that have been used in the synthesis of fully functionalized cyclopentanes in natural product synthesis. Previous reviews have appeared in this area; however, they are either not comprehensive for fully functionalized cyclopentanes, ${ }^{1}$ or they are a general review of annulation. ${ }^{2}$ We use these natural products as a lens to view the current state-of-the-art methods and strategies to form functionalized cyclopentanes.

\section{Figure 1.}

\footnotetext{
${ }^{1}$ Heasley, B. Eur. J. Org. Chem. 2009, 1477-1489.

2 Jung, M. E. Tetrahedron 1976, 32, 3-31.
} 


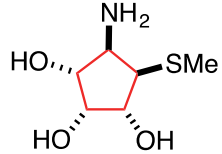

mannostatin A

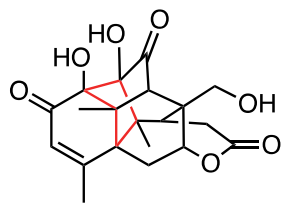

shinjulactone $\mathrm{C}$

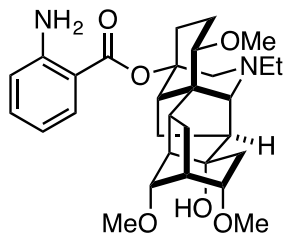

neofinaconitine
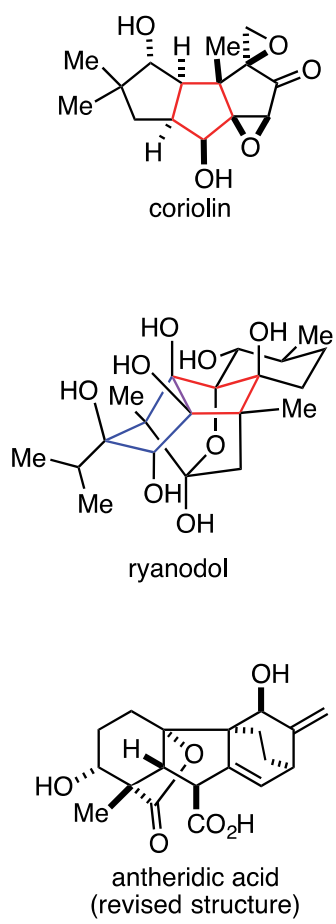

In this review, we define fully functionalized cyclopentanes as five membered carbocycles where each of the five $\mathrm{sp}^{3}$-hybridized carbons bears at least one non-hydrogen substituent. Such structures are exemplified by the monocyclic substituted cyclopentanoid mannostatin A and the polycyclic framework of coriolin (Figure 1). Of course, a cyclopentane may display up to ten non-hydrogen substituents. We include the examples of such decasubstituted cyclopentanes (shinjulactone $\mathrm{C}$ and ryanodol), but the discussion is not limited to these cases. Not included in this review are natural products that have methylene $\left(\mathrm{CH}_{2}\right)$ groups in the five membered ring (such as neofinaconitine), nor natural products with $\mathrm{sp}^{2}$-hybridized carbons in the five membered ring, such as antheridic acid.

Importantly, this review is organized by the reaction that assembles the 5-membered carbocycle, rather than an organizational system based on the target natural product. Particular emphasis is placed on the overall synthetic strategy used to assemble the stereochemically-rich cyclopentane. We organize this Report in 10 distinct sections based on the method by which the key five-membered carbocycle is forged. Within each main section we group mechanistically or topologically-related strategies into well-defined subsections. Within each subsection, reports are grouped according to the natural product target, and presented in chronological order of the earliest total synthesis.

Throughout the review, the carbon atoms that will constitute the cyclopentane have been color coded in red; if two fully functionalized cyclopentanes exist in the target, then the carbons are color-coded red and blue, as appropriate. This review focuses on the key steps in constructing and functionalizing the cyclopentane, and is not an exhaustive analysis of each total synthesis. Comparative reviews of total syntheses of target natural products in this Report appear elsewhere. ${ }^{3}$ Consequently, some of the noteworthy steps in the syntheses may be excluded

3 (a) Berecibar, A.; Grandjean, C.; Siriwardena, A. Chem. Rev. 1999, 99, 779-844. (b) Gelsemine: Lin, H.; Danishefsky, S. J. Angew. Chem. Int. Ed. 2003, 42, 36-51. (c) Basler, B.; Brandes, S.; Spiegel, A.; Bach, T. Topics in Current Chemistry; Springer-Verlag, 2005; pp 1-42. (d) Köck, M.; Grube, A.; Seiple, I. B.; Baran, P. S. Angew. Chem. Int. Ed. 2007, 46, 6586-6594. (e) Li, X. -W.; Ear A.; Nay, B. Nat. Prod. Rep. 2013, 30, 765-782. 
from the discussion if they do not contribute to the cyclopentane construction or functionalization. We show all steps that affect substitution or stereochemical changes to carbons of the eventual fully functionalized cyclopentane, and steps that do not result in changes to the cyclopentane structure or substitution are generally not shown nor discussed in detail.

Figure 2.

McMurry, 1969:
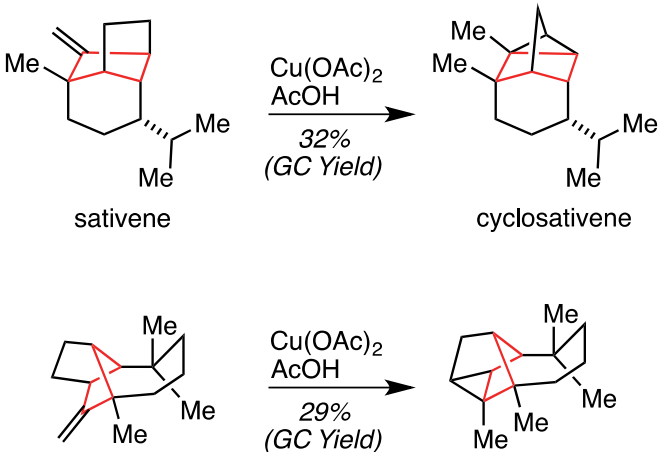

longifolene

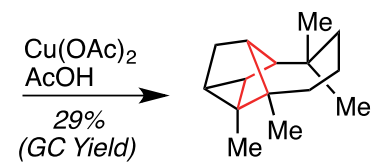

longicyclene

This review is limited to natural product total synthesis, and fully functionalized cyclopentanes that are not natural products have not been included. Also omitted are synthetic studies toward natural products that did not result in completion of the target. However, in the event that a partial synthesis bears close similarity to related total syntheses, we included those studies. Simple interconversions between natural products with fully functionalized five membered rings performed upon isolation are not covered. Similarly, studies of rearrangements between natural product congeners, such as those shown in Figure 2, are not covered. Of course, we recognize that the studies above render all previous and future syntheses of sativene and longifoline formal syntheses of cyclosativene and longicyclene, respectively; however, the syntheses of the former molecules do not address the complexities of forming fully functionalized cyclopentanes, and are not covered here. We cover those syntheses that have appeared through the first half of 2016. In the event that an example from the literature has been missed, the authors deeply regret the omission. 


\section{Table of Contents}

1. Introduction

2. Cycloadditions

$2.1[2+2]$ Cycloadditions

$2.2[3+2]$ Cycloadditions

2.2.1 Bimolecular Reactions of All-Carbon Dipoles

2.2.2 Intramolecular Reactions of Heteroatom Dipoles

2.3 [4+2] Cycloadditions

2.3.1 Bimolecular Diels-Alder Reactions of Cyclopentadienes

2.3.2 Intramolecular Diels-Alder Reactions of Skipped Trienes

$2.4[5+2]$ Cycloadditions

3. Electrocyclizations

3.1 Nazarov Reactions

3.2 Pyridinium Ring Contraction

4. Transition-Metal Mediated Cyclizations

4.1 Pauson-Khand Reactions

4.2 Ring-Closing Metathesis

4.3 Carbenoid Cyclizations

4.4 Intramolecular Kulinkovich Reaction

5. Rearrangement Reactions

5.1 Ring Contractions of Cyclohexane Derivatives

5.2 Oxa-di- $\pi$-methane Rearrangements

5.3 Ring Expansions of Cyclobutane Derivatives

5.4 Cope Rearrangement

6. Aldol and Related Reactions

6.1 1,4-Dicarbonyl Cyclizations

6.2 1,6-Dicarbonyl Cyclizations

6.3 Henry Reactions

7. Mannich Reactions

8. Conjugate Addition Reactions

9. Friedel-Crafts and Related Reactions of Nucleophilic Alkenes

10. Radical Reactions

10.1 Pinacol-type Reactions of Carbon-Heteroatom Double Bonds

$10.2 \sigma$-Bond Homolysis-5-exo-trig Cyclizations

10.3 Radical Cyclizations that Form Multiple Rings 
11. Functionalization of 5-Membered Carbocyclic Starting Materials

11.1 Cyclopentadiene Functionalizations

11.2 Cyclopentene Functionalizations

11.3 Cyclopentanone Functionalizations

11.4 Cyclopentenone Functionalizations

12. Conclusion 


\section{Cycloadditions}

Cycloadditions represent versatile and powerful transformations for the formation of ring structures, and it is not surprising that they are employed in preparing functionalized cyclopentane architectures. There are examples of $[2+2]^{4},[3+2]-,[4+2]-$ (the Diels-Alder reaction), and $[5+2]$-cycloadditions ${ }^{5}$ that are used in the formation of fully functionalized cyclopentane natural products.

\section{Scheme 1}

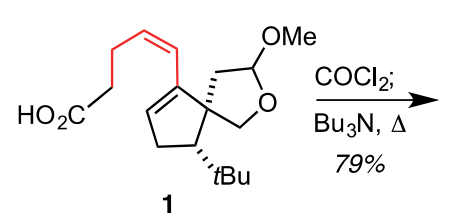

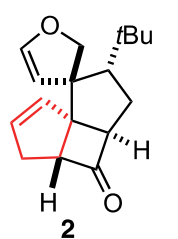

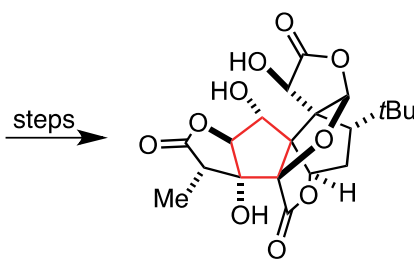

$( \pm$-ginkgolide B

The [2+2]-cycloaddition of 1,5-dienes leads to cis-fused bicyclo[3.2.0]heptane archtitectures, and these reactions have been used in the synthesis of targets with fully functionalized cyclopentanes (Scheme 1). The key fivemembered ring is formed from the carbons in the intramolecular tether connecting the alkene functional groups. Strategically, functional groups may be installed in the tether to enable subsequent transformations that create substitution in the target cyclopentane (such as in eq. 1). Alternatively, substitutents with appropriate stereochemistry may be established prior to the cycloaddition event (eq. 2). The stereochemistry at the bridgehead atoms favors the cis-fused diastereomer that delivers the substituents in sterically less-hindered positions. The synthesis of gingkolide exemplifies use of the [2+2]-cycloaddition where the reactivity of the cylcobutane is leveraged in the formation of additional rings, and the strategic use of the $[2+2]$-cycloaddition is non-obvious. The bicyclo[3.2.0]heptane moiety in the kelsoene target is more suggestive of the use of the [2+2]cycloaddition.

\section{Scheme 2.}

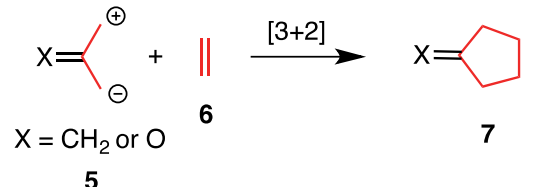

The intermolecular combination of an all-carbon 1,3-dipole (5) with an alkene (6) produces a 5-membered carbocycle (7) (Scheme 2). ${ }^{6}$ These reactions often proceed with high levels of substrate-controlled diastereoselectivity. Five natural products with fully functionalized cyclopentanes have been prepared using allcarbon [3+2]-cycloadditions to construct the five-membered ring. It is perhaps surprising that relatively few fully functionalized cyclopentanes are made with this reaction because it inherently constructs a five-membered

\footnotetext{
${ }^{4}$ For review, see: Crimmins, M. T.; Reinhold, T. L. Org. React. 1993, 44, $297-588$.

${ }^{5}$ For reviews, see: (a) Pellissier, H. Adv. Synth. Catal. 2011, 353, 189-218. (b) Ylijoki, K. E. O.; Stryker, J. M. Chem. Rev. 2013, 113, 2244-2266.

${ }^{6}$ Note that we organized all metal-mediated [3+2] cycloadditions in this section rather than removing the transition metal catalyzed examples to section 4.
} 
carbocycle, vis-à-vis the Diels-Alder reaction, which inherently forms a six-membered ring. However, methods available for the formation of all-carbon dipoles are relatively few in number. ${ }^{7}$

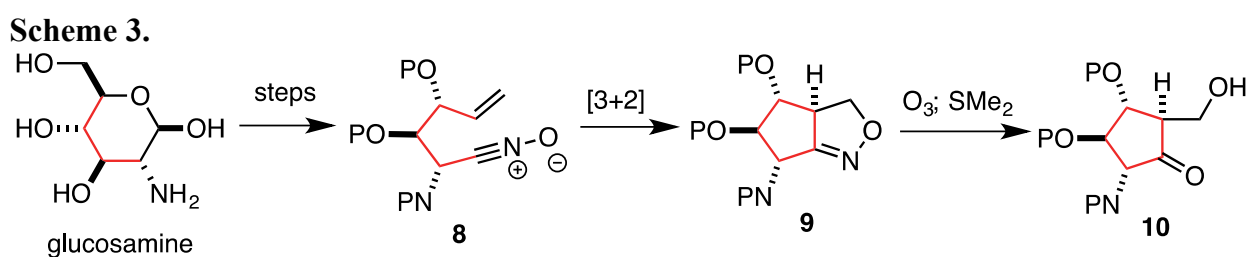

Intramolecular [3+2]-cycloadditions may also deliver functionalized cyclopentanes where the tether carbons become the eventual carbocycle (Scheme 3). In the context of this review, the stereogenic carbons in the tether are derived from carbohydrate feedstock molecules. The cycloaddition involves reaction of a nitrile oxide $\mathbf{8}$ or a nitrone dipole (not shown) to give an isoxazoline (9) or isoxazolidine, respectively. The stereochemistry of the bridgehead carbon is controlled by the adjacent stereocenter, leading to positioning of the substituent on the less hindered face of the bicycle. The heterocyclic five-membered ring is subsequently opened using standard conditions to give $\mathbf{1 0 .}$

\section{Scheme 4.}

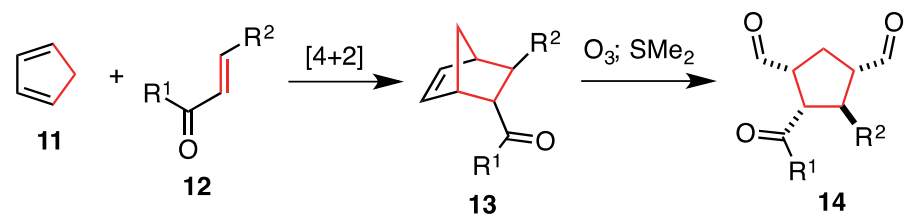

The intermolecular Diels-Alder reaction of cyclopentadienes with alkenes leads to the formation of substituted cyclopentanes that are part of bicyclo[2.2.1] heptenes (Scheme 4). Cases related to this review (with one exception: Zhai's biomimetic synthesis of absinthin) involve relatively simple and symmetrically-substituted cyclopentadienes (11) reacting with substituted alkene dienophiles 12. The cycloaddition proceeds with predictable endo diastereoselectivity, setting four carbon stereocenters in 13. Variation in the substitution pattern of the dienophile is widely tolerated, and appropriate substitution gives functional handles for further transformations. Invariably, subsequent transformations include the oxidative cleavage of the resident alkene to reveal intermediate 14 that undergoes further processing. A possible limitation of this synthetic strategy is the relatively few methods for the preparation of functionalized non-symmetric cyclopentadienes. ${ }^{8}$ Likely as a result of this limitation, this type of Diels-Alder reaction occurs early in the synthetic route to the natural product target.

\section{Scheme 5.}

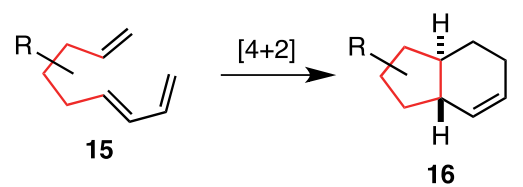

The intramolecular Diels-Alder reaction of skipped trienes $\mathbf{1 5}$ may also be used in the formation of substitituted cyclopentanes embedded in hydrindane architectures 16 (Scheme 5). In such cases, the functionalized cyclopentane is made from the molecular tether connecting the diene and dienophile. A key consideration of this strategy is the establishment of substitution on the tether with the desired stereochemical configuration. The intramolecuar Diels-Alder reaction is a well-studied and dependable transformation, even with quite complex substrates. ${ }^{9,10}$ The hydrindanes formed show trans ring fusions that position the tether substituents in the

\footnotetext{
${ }^{7}$ Little, R. D. [3+2] Cycloadditions-Thermal. In Comp. Org. Syn. Trost, B. M.; Fleming, I., Eds.; Pergamon Press: Oxford 1991, Vol 5; 239-270.

${ }^{8}$ Ivchenko, N. B.; Ivchenko, P. V.; Nifant'ev, I. E. Russ. J. Org. Chem. 2000, 36, 609-637.

${ }^{9}$ Roush, W. R. Intramolecular Diels-Alder Reations. In Comp. Org. Syn. Trost, B. M., Fleming, I., Paquette, L. A., Eds.; Pergamon Press: Oxford, 1991; Vol. 5; 513-550.
} 
sterically less hindered location. As a result of such dependability and predictable control elements, this type of Diels-Alder reaction has been used late in the synthetic route to complex natural product targets.

\section{$2.1[2+2]$ Cycloadditions}

In the synthesis of $( \pm)$-ginkgolide $\mathrm{B}$, Corey ${ }^{11}$ uses a [2+2] cycloaddition to construct a polycyclic ring system containing the five-membered ring of interest (Scheme 6). The synthesis begins with 1-morpholinocyclopentene (17), which was converted to cyclopentanone 18 by reaction with dimethoxyacetaldehyde followed by hydrolysis with $\mathrm{HCl}$. This cyclopentenone was advanced to spirocyclic intermediate $\mathbf{1 9}$ over two steps. Conversion to the enol triflate and coupling with alkyne 20 gave 21. Partial reduction of the alkyne furnished the desired $Z$-alkene. Acidic hydrolysis of the ortho-ester revealed carboxylic acid 1. Formation of the acid chloride with oxalyl chloride was followed by slow addition to tri- $n$-butylamine to give a ketene intermediate that underwent a [2+2] cycloaddition to give 2. Baeyer-Villiger oxidation with tert-butyl hydrogen peroxide produced $\gamma$-lactone 22 . This material was advanced over four steps to 23. Enolate formation and addition to the Davis oxaziridine gave an $\alpha$ hydroxy lactone. The ether bridge in $\mathbf{2 4}$ could then be constructed with catalytic camphorsulfonic acid in methanol. Oxidation of the cyclopentene to cyclopentenone $\mathbf{2 5}$ was completed by allylic bromination and subsequent oxidation with silver nitrate. An acid-promoted elimination of methanol gave a dihydrofuran. Stereoselective nucleophilic epoxidation yielded 26. Introduction of the final required substituent was accomplished by a diastereoselective aldol addition with tert-butyl propionate to give $\mathbf{2 7}$. Lactonization with camphorsulfonic acid delivered bislactone $\mathbf{2 8}$ and a fully functionalized cyclopentane with the desired substitution in ( \pm )-ginkgolide B.

Scheme 6. Total Synthesis of ( \pm )-Ginkgolide B (Corey, 1988)

\footnotetext{
${ }^{10}$ After this report was accepted, a synthesis of three denudatine natural products was reported using an intramolecular Diels-Alder reaction. See, Kou, K. G. M.; Li, B. X.; Lee, J. C.; Gallego, G. M.; Lebold, T. P.; DiPasquale, A. G.; Sarpong, R. J. Am. Chem. Soc. 2016, 138, 10830-10833.

${ }^{11}$ Corey, E. J.; Kang, M.-C.; Desai, M. C.; Ghosh, A. K.; Houpis, I. N. J. Am. Chem. Soc. 1988, 110, 649-651.
} 


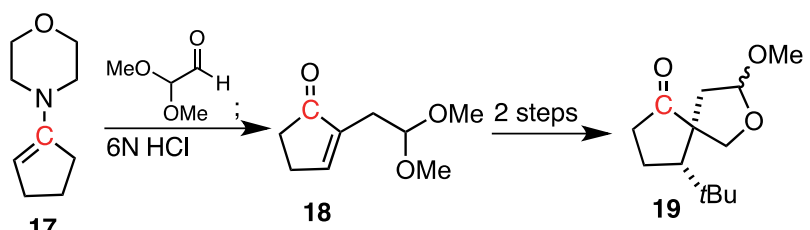

1) $\mathrm{LDA}, \mathrm{PhNTf}_{2}$

2) $\mathrm{Pd}\left(\mathrm{PPh}_{3}\right)_{4}$ cat.; $\mathrm{n} \mathrm{PrNH}_{2}$, Cul cat.

17

18

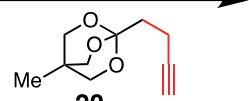

$64 \%$ two steps

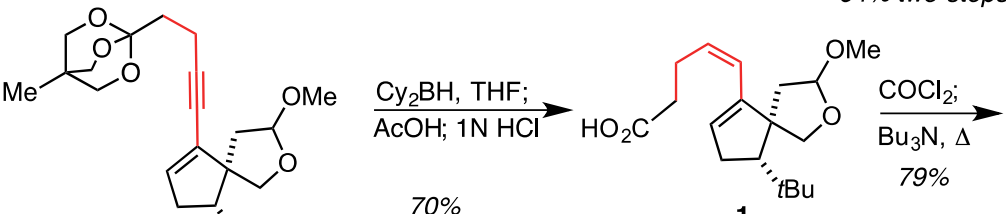

$70 \%$

1

$79 \%$

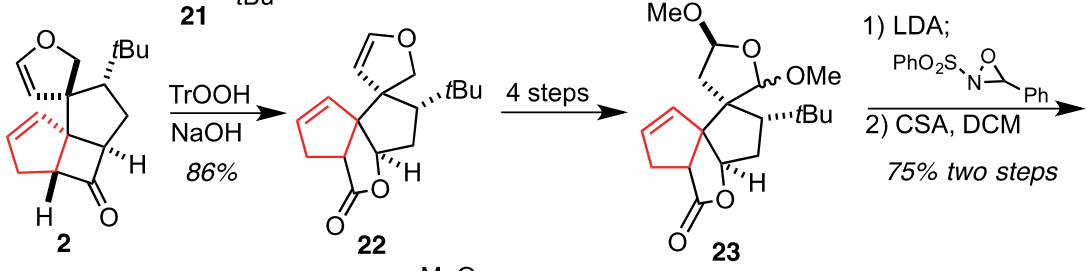

(50\%

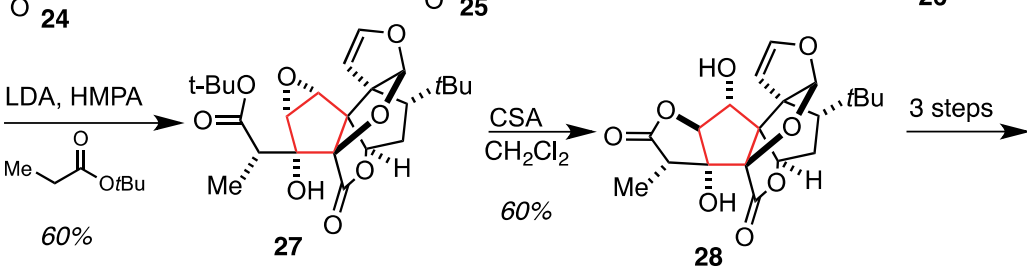

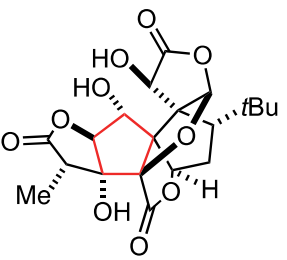

( \pm -ginkgolide B

The Bach ${ }^{12}$ synthesis of $( \pm)$-kelsoene utilizes a [2+2] photocycloaddition of a 1,6-diene to construct a fully functionalized cyclopentane (Scheme 7). The route begins with a Knovenagal condensation of citronellenedervied aldehyde $\mathbf{2 9}$ with dimethyl malonate to give 30. An Alder-ene reaction using conditions developed by Tietze ${ }^{13}$ delivered cyclopentane 31. This material was advanced over four steps to cycloaddition precursor $\mathbf{3}$. A $[2+2]$ photocycloaddition in the presence of CuOTf delivered a fully functionalized cyclopentane (4) as a mixture of diastereomers. Acetate cleavage, oxidation of the resulting alcohol and $\alpha$-bromination delivered $\mathbf{3 2}$ as a single diastereomer. Addition of methyllithium, oxidation of the resulting alcohol to the ketone, and elimination of bromide gave enone 33. Hydrogenation occurred preferentially from the face opposite the methyl groups. An acid-catalyzed epimerization of the resulting ketone delivered a fully functionalized cyclopentane with the desired stereochemistry for the target. A Wittig olefination completed the synthesis of (+)-kelsoene.

\section{Scheme 7. Total Synthesis of $( \pm)-$ Kelsoene (Bach, 2002)}

\footnotetext{
${ }^{12}$ Bach, T.; Spiegel, A. Synlett 2002, 8, 1305-1307.

${ }^{13}$ Tietze, L. F.; Beifuss, U.; Ruther, M.; Rühlman, A.; Antel, J.; Sheldrick, G. M. Angew. Chem. Int. Ed. Engl. 1988, 27, 1186-1187.
} 

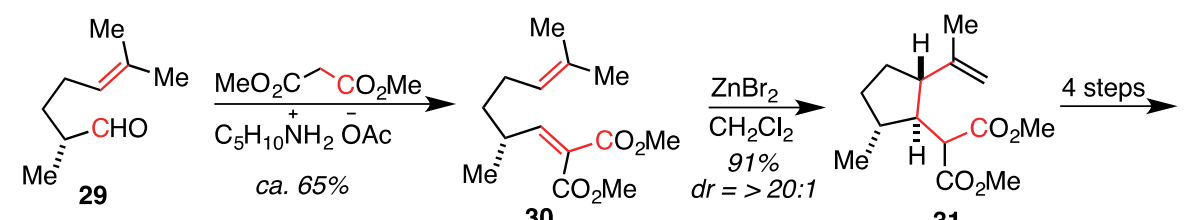<smiles>C=CC(COC(C)=O)C1C(C)CCC1C(C)=O</smiles>
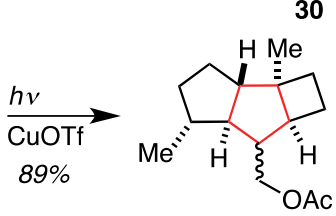

1) $\mathrm{K}_{2} \mathrm{CO}_{3} \mathrm{MeOH}$
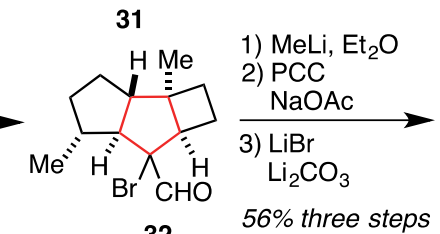

3<smiles>CC(=O)C1=C2[C@@H](C)CC[C@H]2[C@H]2CC[C@H]12</smiles>

2) $(\mathrm{COCl})_{2}$, DMSO

3) $\mathrm{py} \cdot \mathrm{HBr}_{3}$ $\mathrm{CHCl}_{3}$ $73 \%$ three steps

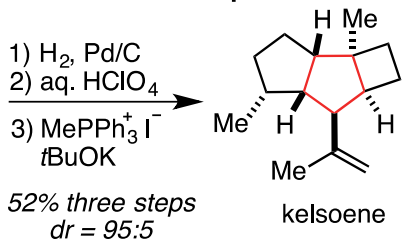

32

\section{$2.2[3+2]$ Cycloadditions}

\subsubsection{Bimolecular Reactions of All-Carbon Dipoles}

The Trost ${ }^{14}$ synthesis of (-)-rocaglamide begins with a palladium-catalyzed cycloaddition of the trimethylenemethane (TMM) ${ }^{15}$ precursor 35 and alkene 34 (Scheme 8 ). The chiral-auxiliary ${ }^{16}$ promotes addition of the TMM from the convex face of the boat-like confomer $\mathbf{3 6}$ and delivers $\mathbf{3 7}$ as a single diastereomer. Removal of the auxiliary, and oxidative cleavage produces cyclopentanone 39. Condensation with dimethyl phloroglucinol followed by selective transesterification gave $\mathbf{4 0}$ as a mixture of alkene regioisomers. Oxidative cyclization with DDQ delivers 41. Note the oxidative cyclization gives the undesired trans relationship between the phenyl and PMP groups, which must be corrected. In a preliminary study, the TMM [3+2] cyclization of model compounds lacking the auxiliary set the tertiary stereocenter with the desired cis configuration, albeit as a racemate. Dihydroxylation occurs from the top face of the cyclopentene promoting a cis fusion of the 5membered rings. Functional group manipulation (oxidation, silylation, and hydrogenolysis/decarboxylation) gave cyclopentanone 42. Reaction with phenylsulfenyl chloride $/ m$ CPBA delivers a cyclopentenone, which installs the necessary functionality to set the desired stereochemical configuration. Amide formation under Weinreb's conditions gives 43. Hydrogenation using Pearlman's catalyst sets the two tertiary stereocenters and delivers 44 as a 3:1 diastereomeric mixture. Presumably, the syn delivery of hydrogen is followed by epimerization of the methyl ester, which affords the trans relationship on the five-membered ring. Desilylation and stereoselective reduction with hydride directed by the vicinal hydroxide completes the synthesis of (-)rocaglamide.

\section{Scheme 8. Total Synthesis of (-)-Rocaglamide (Trost, 1990)}

\footnotetext{
${ }^{14}$ Trost, B. M.; Greenspan, P. D.; Yang, B. V.; Saulnier, M. G. J. Am. Chem. Soc. 1990, 112, 9022-9024.

15 Trost, B. M.; Chan, D. M. T. J. Am. Chem. Soc. 1983, 105, 2315-2325.

${ }^{16}$ Trost, B. M.; Yang, B.; Miller, M. L. J. Am. Chem. Soc. 1989, 111, 6482-6484.
} 

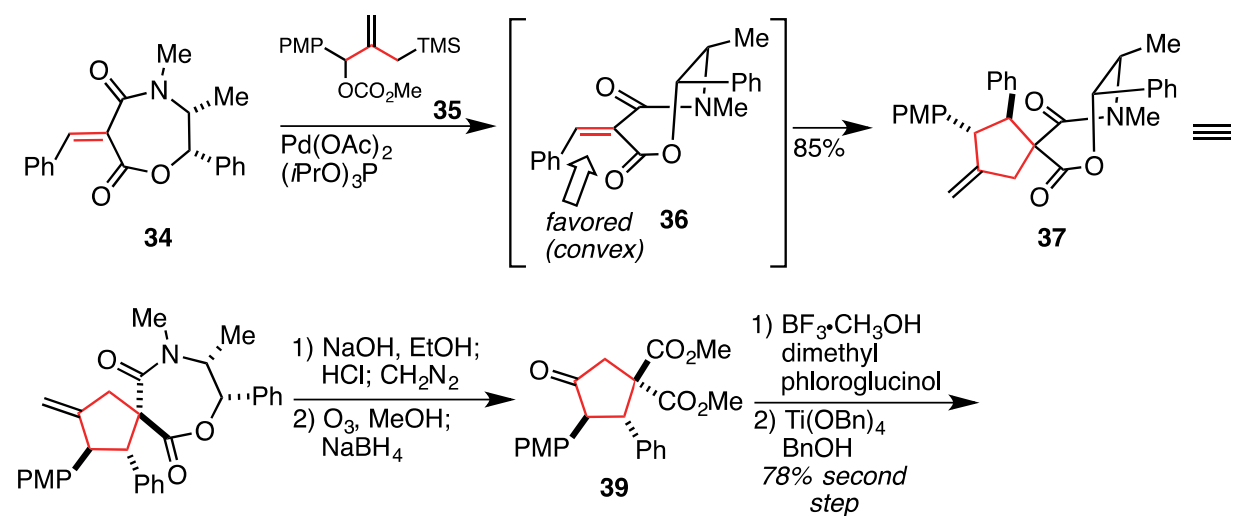

38
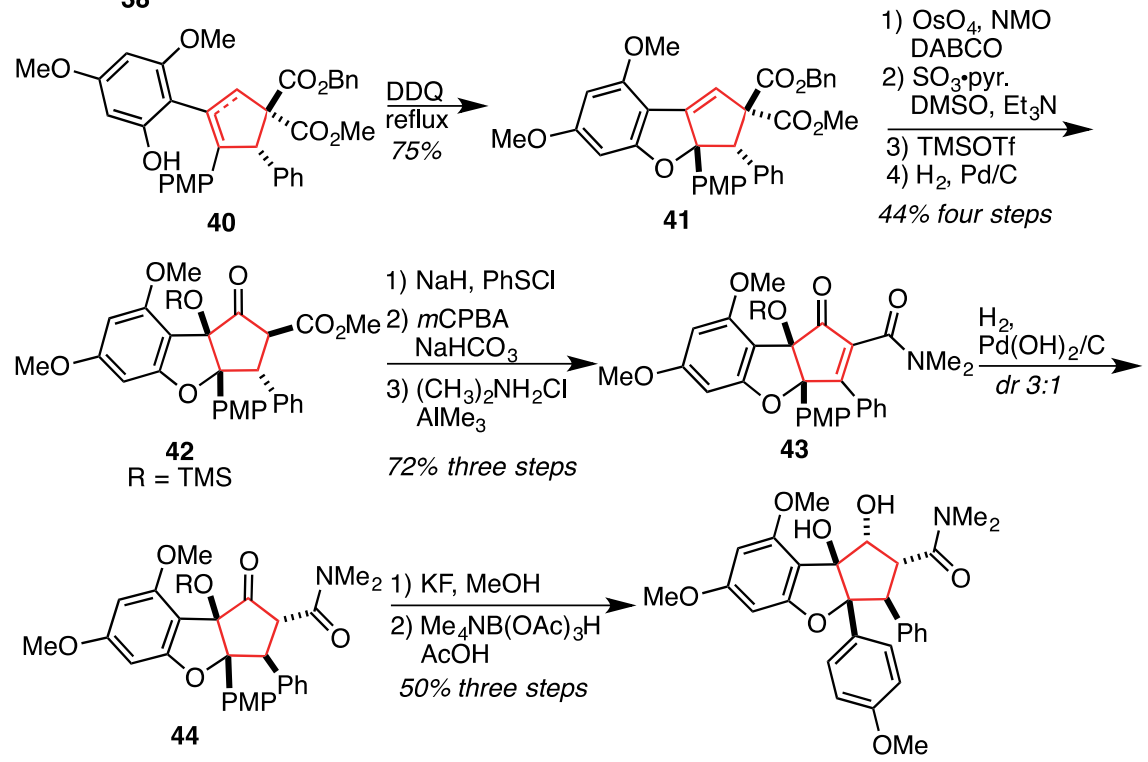

$(-)$-rocaglamide

Kuwajima's ${ }^{17}$ total synthesis of (-)-coriolin begins with a [3+2] cycloaddition to construct the cyclopentane that will eventually become fully functionalized (Scheme 9). This [3+2] reaction of vinyl sulfide $\mathbf{4 5}$ and the silyloxy allyl species $\mathbf{4 6}$ delivers two annulation products $(\mathbf{4 7}$ and $\mathbf{4 8})$ in a 1:1 ratio. This mixture was subjected to an additional three steps to deliver 46. The appropriate cis fusion of the cyclopentanes has been installed for coriolin. A second [3+2] cycloaddition with silyloxy allyl species $\mathbf{4 7}$ gave triquinane $\mathbf{4 8}$ and sets the quaternary stereocenter found in coriolin. With three stereocenters in place, functional group manipulation ( $\beta$-elimination with fluoride, desulfurization with phenyl thiolate, elimination, and silylation) delivered $\mathbf{4 9}$ with the necessary functionality to install the final two stereocenters. Deprotonation of the enone and silylation of the extended enolate was performed with potassium hydride and TIPSCl. An epoxide was formed on the less hindered top face of the molecule. Epoxide opening under acidic conditions delivered $\mathbf{5 0}$ and set the secondary alcohol stereocenter of coriolin. This material was advanced over seven steps to 51. Nucleophilic epoxidation of the enone set the final stereocenter on the fully functionalized cyclopentane and completed the synthesis of (-)-coriolin.

Scheme 9. Total Synthesis of (-)-Coriolin (Kuwajima, 1999)

\footnotetext{
${ }^{17}$ Mizuno, H.; Domon, K.; Masuya, K.; Tanino, K.; Kuwajima, I. J. Org. Chem. 1999, 64, 2648-2656.
} 

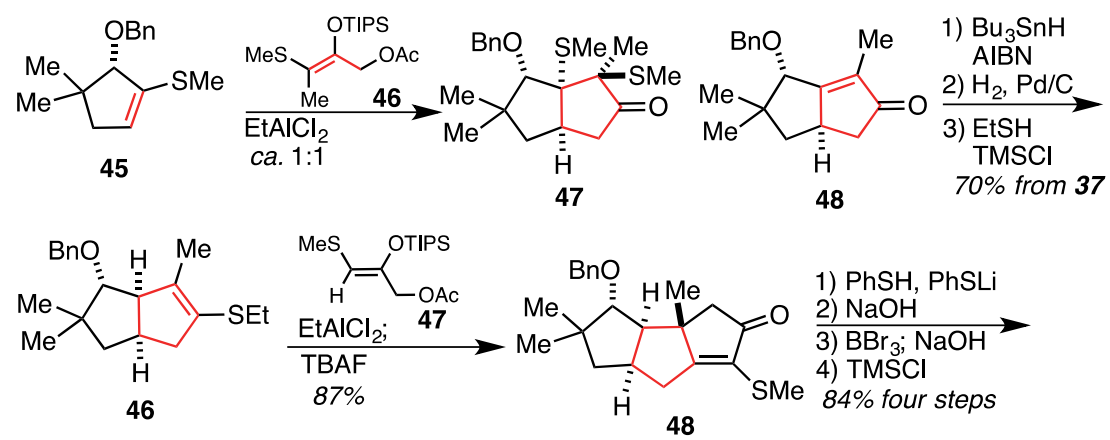

1) $\mathrm{PhSH}, \mathrm{PhSLi}$

3) $\mathrm{BBr}_{3} ; \mathrm{NaOH}$

4) $\mathrm{TMSCl}$

48
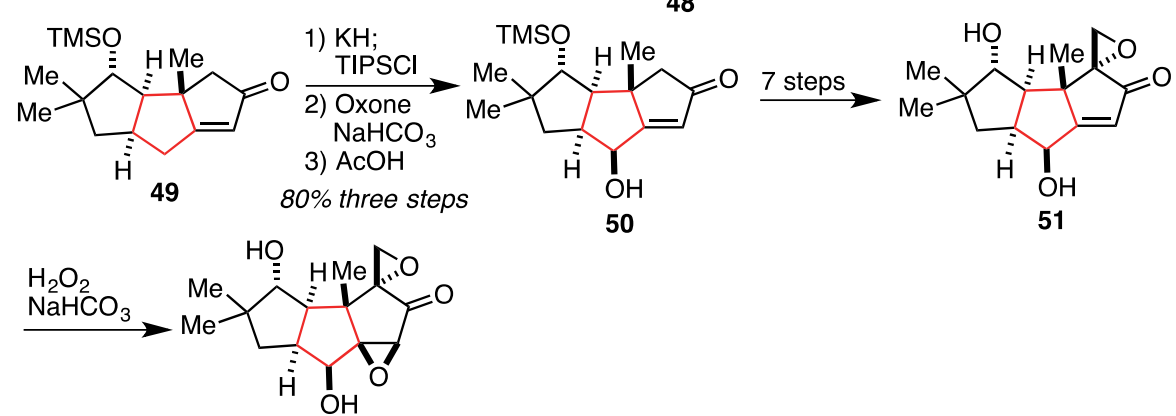

(-)-coriolin

The total synthesis of (-)-silvestrol by Porco ${ }^{18,19}$ features a [3+2] photocycloaddition between a 3-

hydroxyflavone and a cinnamate dipolarophile to assemble a functionalized cyclopentane (Scheme 10). The route commences with aryl ketone $\mathbf{5 3}$ (synthesized from phloroglucinol $\mathbf{5 2}$ in three steps). Deprotection of the MOM group (cat. $\mathrm{I}_{2} / \mathrm{MeOH}$ ) was followed by acylation with 4-methoxybenzoyl chloride to give 54. Treatment with LHMDS promoted a Baker-Venkataraman rearrangement ${ }^{20}$ yielding diketone 55. Sodium acetate in acetic acid promoted cyclization, dehydration and MOM deprotection. Reintroduction of the MOM group and hydrogenolysis of the benzyl ether delivers 56. The enantioselective [3+2] photocycloaddition was performed with methyl cinnamate (58) and chiral TADDOL derivative 57, leading to cycloadduct 60 in $71 \%$ ee. An $\alpha$-ketol (acyloin) rearrangement promoted by sodium methoxide was followed by a hydroxyl-directed reduction to complete to give the molecular architecture of (-)-silvestrol.

\section{Scheme 10. Total Synthesis of (-)-Silvestrol (Porco, 2007)}

\footnotetext{
${ }^{18}$ Porco used a similar synthetic strategy to prepare the related natural product rocaglamide, see: (a) Gerard, B.; Jones, G., II; Porco, J. A., Jr. J. Am. Chem. Soc. 2004, 126, 13620-13621. (b) Gerard, B.; Sangji, S.; O’Leary, D. J.; Porco, J. A., Jr. J. Am. Chem. Soc. 2006, 128, 7754-7755.

${ }^{19}$ Gerard, B.; Cencic, R.; Pelletier, J.; Porco Jr., J. A. Angew. Chem. Int. Ed. 2007, 46, 7831-7834.

${ }^{20}$ W. Baker, J. Chem. Soc. 1933, 1381-1389.
} 

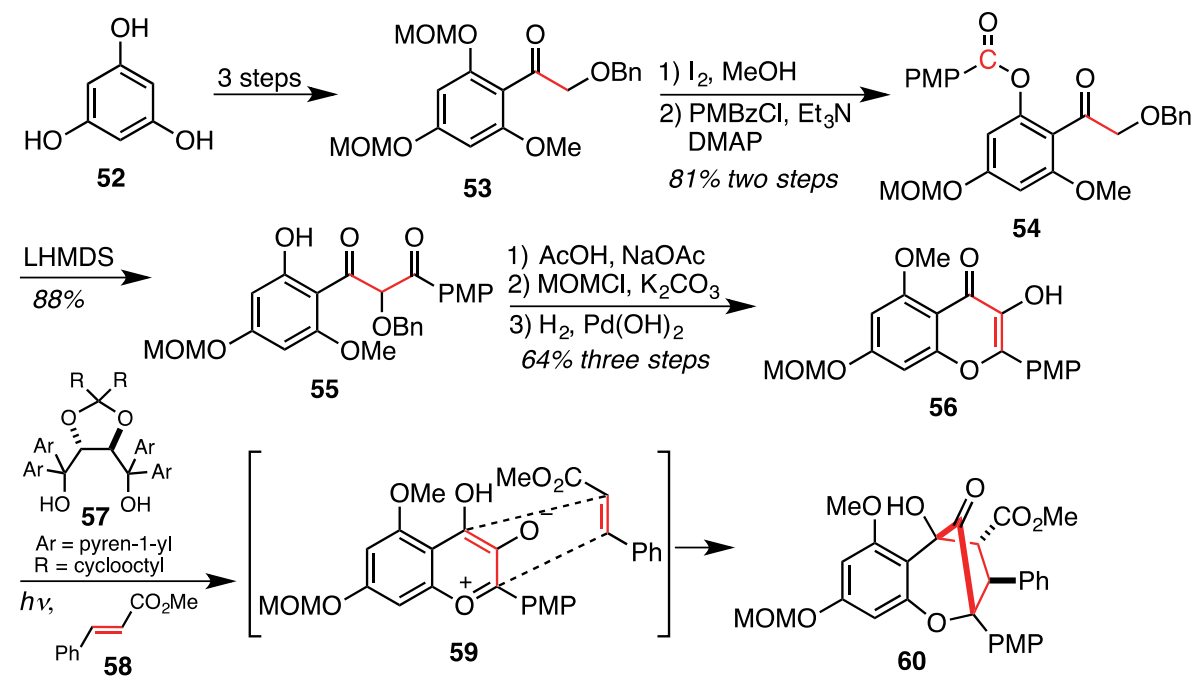

$66 \%, 71 \%$ ee

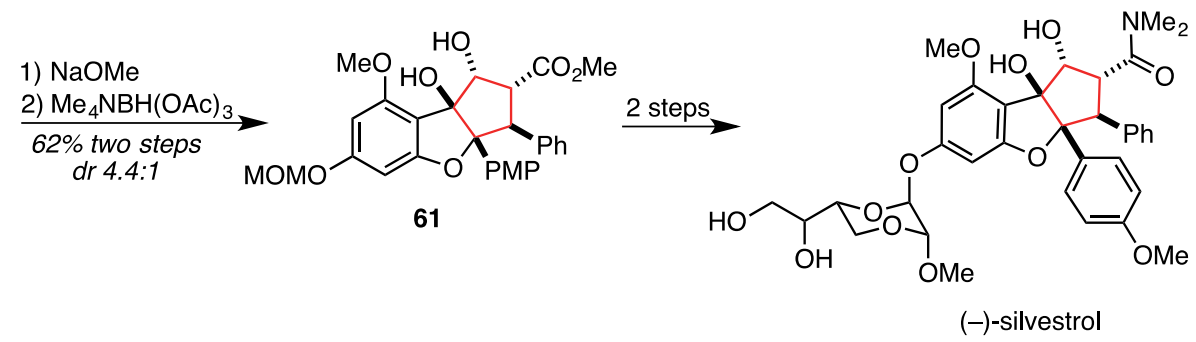

In a synthesis of (-)-silvestrol, Rizzacasa ${ }^{21}$ uses the photocycloaddition developed by Porco (see above) to assemble the fully functionalized cyclopentane (Scheme 11). The route to the 3-hydroxyflavone starting material began with the commercially available natural product naringenin. Selective benzylation delivered 62. An oxidation with iodine yielded a flavone 63 , which upon methylation furnished 64 . The desired 3-hydroxyflavone 65 could be obtained by deprotonation at $\mathrm{C} 3$ position followed by boration and oxidative workup. The photocycloaddition with methyl cinnamate (58) resulted in bicyclic intermediate $\mathbf{6 6}$ as a racemate. Treatment with base promoted an $\alpha$-ketol rearrangement to the $\beta$-ketoester $\mathbf{6 7}$. Hydride reduction from the convex face of the bicycle delivers the desired hydroxyl configuration in a 4.6:1 ratio. This advanced intermediate has a fully functionalized cyclopentane, and it was advanced to the natural product. Racemic intermediate $\mathbf{6 8}$ was advanced and subsequently conjugated with an enantioenriched chiral sugar moiety; separation of the diastereomers allowed for a synthesis of non-racemic (-)-silvestrol.

\section{Scheme 11. Total Synthesis of (-)-Silvestrol (Rizzacasa, 2007)}

\footnotetext{
${ }^{21}$ El Sous, M.; Khoo, M. L.; Holloway, G.; Owen, D.; Scammells, P. J.; Rizzacasa, M. A. Angew. Chem. Int. Ed. 2007, $46,7835-7838$
} 

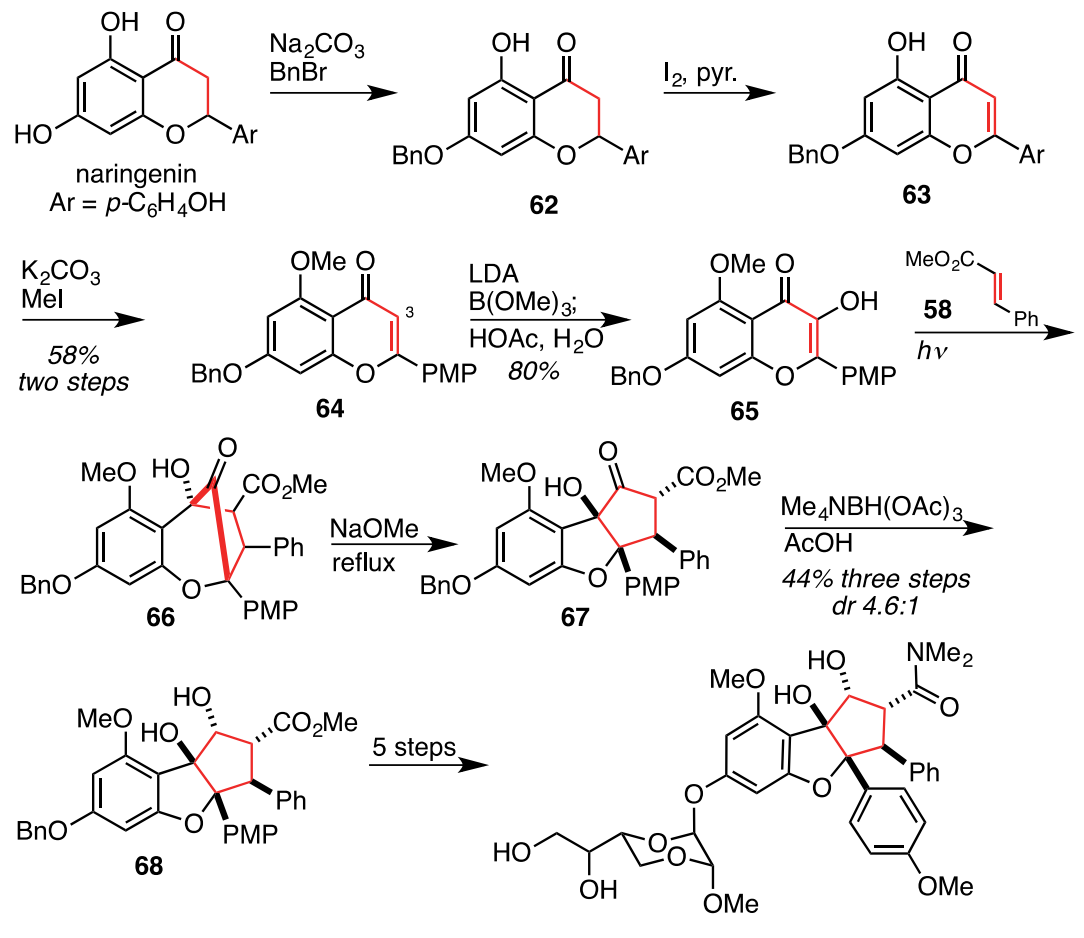

$(-)$-silvestrol

The synthesis of $(+)$-ponapensin and (+)-elliptifoline by Porco $^{22}$ (Scheme 12) uses a similar [3+2]

photocycloaddition strategy to that discussed above. The 3-hydroxyflavone $\mathbf{6 9}$ underwent a TADDOL (70) promoted [3+2] photocycloaddition to give cycloadduct $\mathbf{7 2}$ in good yield and good stereochemical control. Hydride reduction of the ketone hydrate delivered the fully functionalized cyclopentane $\mathbf{7 3}$ with the desired stereochemistry for $(+)$-ponapensin and $(+)$-elliptifoline, and it could be converted to either natural product in an additional two steps.

Scheme 12. Total Synthesis of (+)-Ponapensin and $(+)$-Eliptifoline (Porco, 2012)

\footnotetext{
${ }^{22}$ Lajkiewicz, N. J.; Roche, S. P.; Gerard, B.; Porco, J. A., Jr. J. Am. Chem. Soc. 2012, 134, 13108-13113.
} 

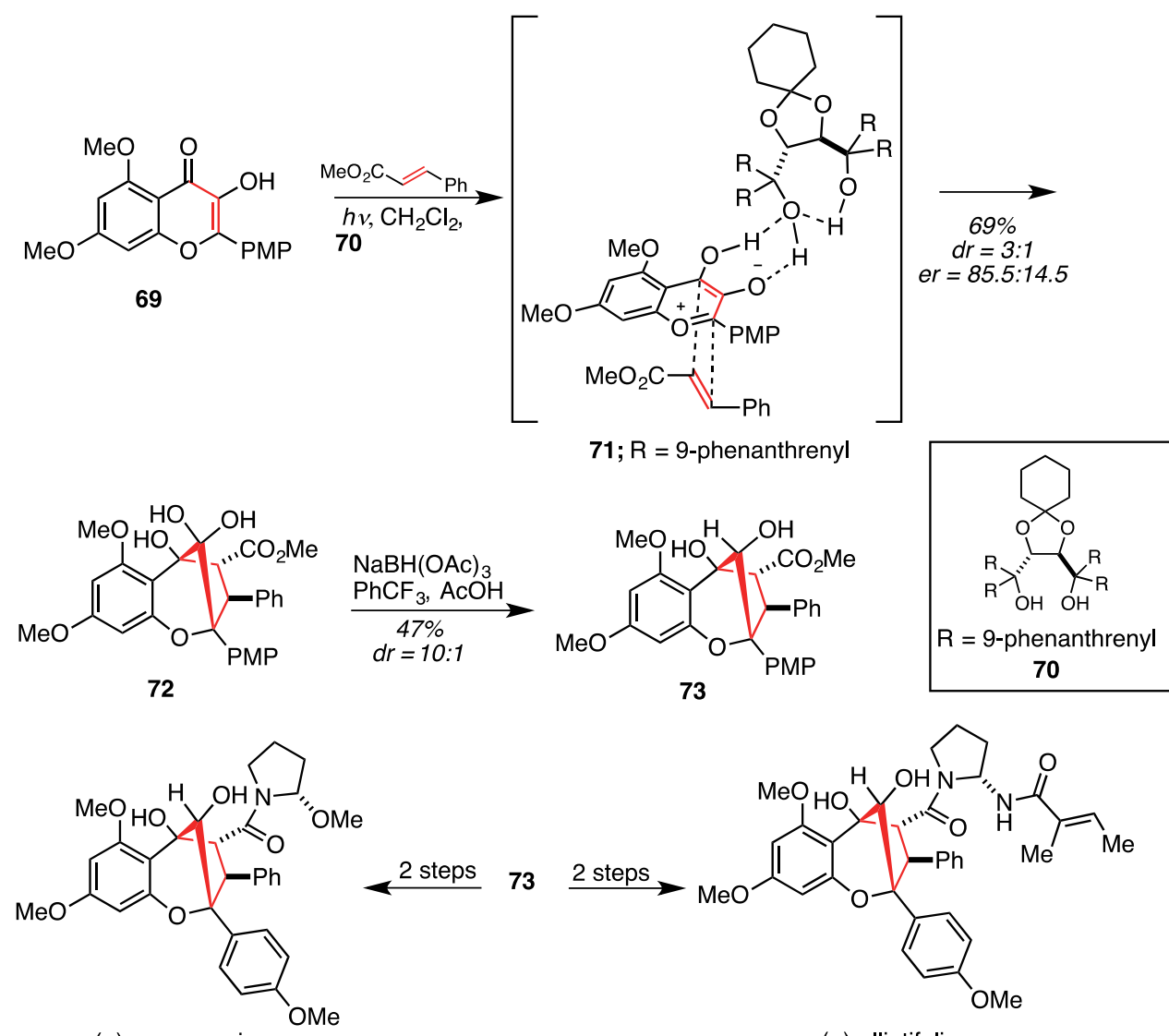

(+)-ponapensin

(+)-elliptifoline

\subsubsection{Intramolecular Reactions of Heteroatom Dipoles}

Tatsuta's $\mathrm{s}^{23}$ synthesis of (-)-allosamizoline begins with D-glucosamine, which contains the desired configuration at four of the five allosamizoline stereocenters (Scheme 13). The route begins with the conversion of Dglucosamine to furanose $\mathbf{7 4}$ over three steps. Treatment with ethanethiol and $\mathrm{HCl}$ delivered a dithioacetal, which underwent TBS protection of the secondary alcohols to give 75 . Desulfurization was performed with mercuric chloride and the resulting aldehyde was transformed into oxime 76. Oxidation to a nitrile oxide with sodium hypochlorite promoted an intramolecular 1,3-dipolar cycloaddition to deliver isoxazoline 77. Fragmentation of the isoxazoline with ozone yielded a $\beta$-hydroxy ketone. The final stereocenter of allosamizoline was set by hydroxyl-directed reduction of the ketone with zinc borohydride giving 78. This advanced interemediate contains a fully functionalized cyclopentane, and the natural product was completed in an additional four steps.

Scheme 13. Total Synthesis of (-)-Allosamizoline (Tatsuta, 1991)

${ }^{23}$ Nakata, M.; Akazawa, S.; Kitamura, S.; Tatsuta, K. Tetrahedron Lett. 1991, 32, 5363-5366. 


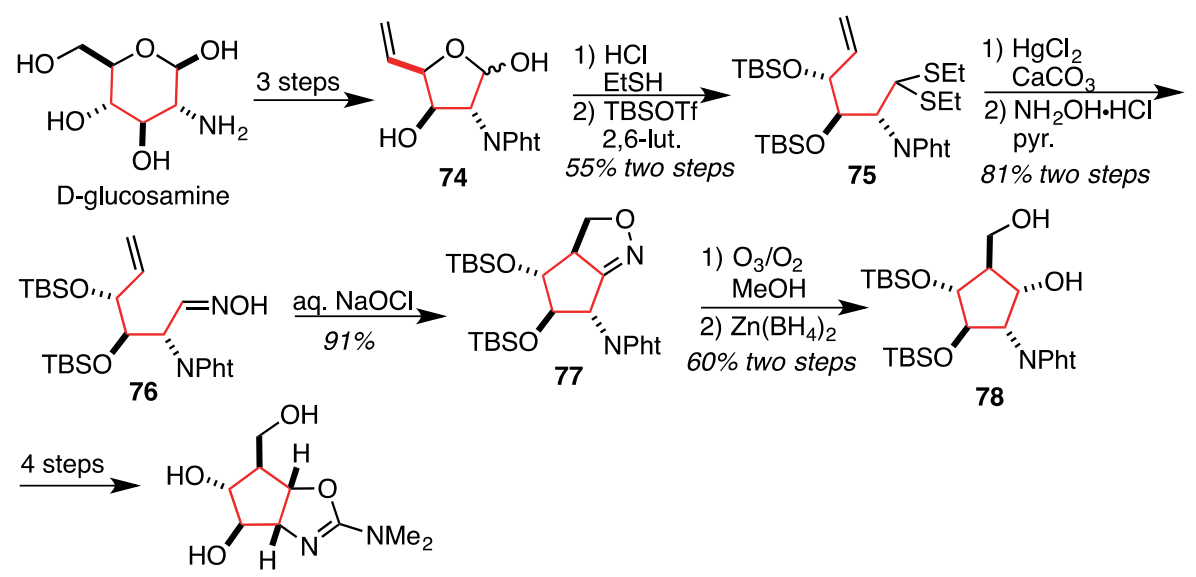

(-)-allosamizoline

In a synthesis of (-)-allosamizoline, Koseki ${ }^{24}$ uses a similar 1,3-dipolar cyclization of a nitrile oxide (Scheme 14). Beginning with the stereochemically rich glucosamine, intermediate 79 could be obtained over eight steps. Ring cleavage was achieved by reduction with activated zinc powder in wet THF delivering an aldehyde. The unpurified mixture was treated with hydroxylamine hydrochloride to afford oxime 80. A 1,3-dipolar cyclization was promoted by treatment with sodium hypochlorite to deliver isoxazoline 81. Protecting group exchange affords the TBS-protected intermediate 82. Oxidative cleavage with ozone was followed by hydroxyl-directed reduction to give $\mathbf{8 3}$, which installed the final stereocenter of the fully functionalized cyclopentane. This intermediate was converted to the natural product over an additional seven steps.

Scheme 14. Total Synthesis of (-)-Allosamizoline (Koseki, 1993)

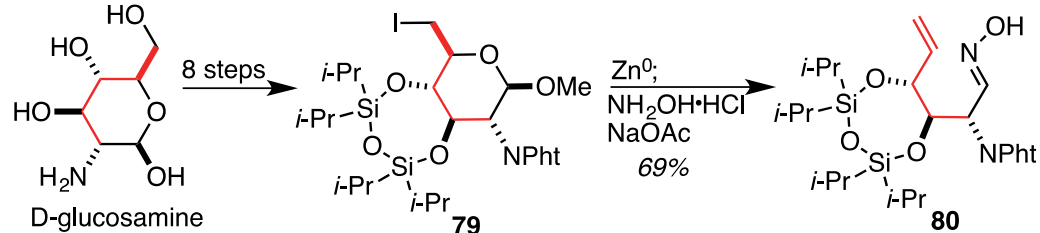

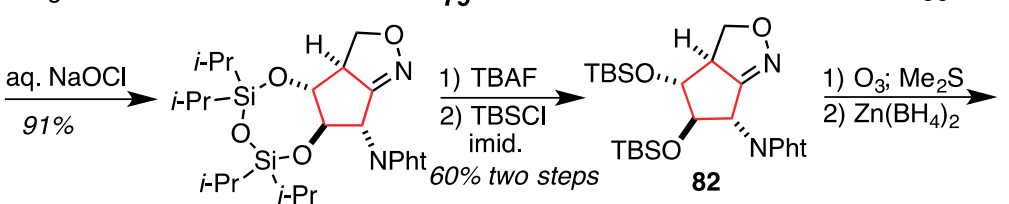<smiles>OC[C@H]1[C@@H](O)[C@@H](O)[C@@H](Nc2ccccc2)[C@@H]1O</smiles>

83

$(-)$-allosamizoline

The Ferrier ${ }^{25}$ synthesis of (-)-allosamizoline utilized a 1,3-dipolar cycloaddition to assemble a fully functionalized cyclopentane (Scheme 15). The route begins with D-glucose, which was converted to 84 over five steps. Reductive opening of the substituted pyranoside was accomplished by treatment with zinc powder to afford enal 85. This intermediate contains three of the required stereocenters for allosamizoline. Heating enal $\mathbf{8 5}$ with $\mathrm{N}$-methylhydroxylamine produced an intermediate nitrone that cyclized to form isoxazolidine 86. The [3+2] cycloaddition sets the eventual hydroxymethyl stereocenter, but the adjacent amino alcohol stereodiad requires adjustment. Reduction with hydrogen and Raney nickel delivered an aziridine. Peracid oxidation of the aziridine

${ }^{24}$ Kitahara, T.; Suzuki, N.; Koseki, K.; Mori, K. Biosci. Biotech. Biochem. 1993, 11, 1906-1909.

${ }^{25}$ (a) Blattner, R.; Furneaux, R. H.; Kemmit, T.; Tyler, P. C.; Ferrier, R. J.; Tidén, A.-K. J. Chem. Soc., Perkin Trans. 1 1994, 3411-3421. (b) Ferrier, R. J.; Ferneaux, R. H.; Prasit, P.; Tyler, P. C.; Brown, K. L.; Gainsford, G. J.; Diehl, J. W. J. Chem. Soc., Perkin Trans. 1 1983, 1621-1628. 
resulted in elimination to form cyclopentene 87. The final two stereocenters were installed by way of a modified Sharpless aminohydroxylation ${ }^{26}$ to give $\mathbf{8 8}$. This intermediate was advanced five steps to (-)-allosamizoline.

\section{Scheme 15. Total Synthesis of (-)-Allosamizoline (Ferrier, 1994)}<smiles>OCC1OC(O)[C@H](O)C(O)[C@@H]1O</smiles>

D-glucose

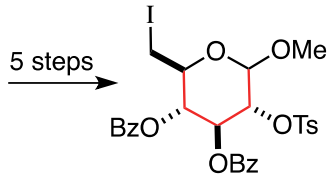

84

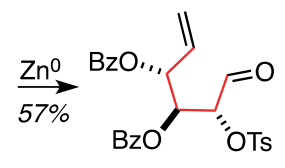

85

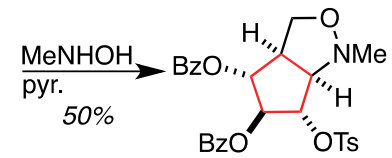

86

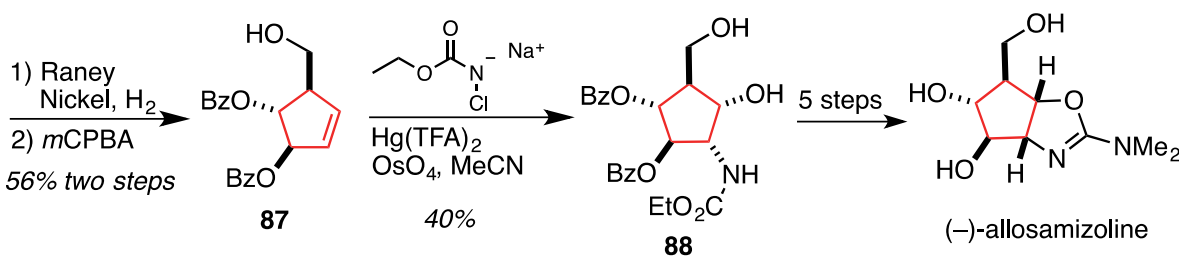

The Shiozaki ${ }^{27}$ synthesis of $(+)$-trehalamine featured a nitrile oxide [3+2] cycloaddition to construct the fivemembered ring (Scheme 16). The synthesis begins with D-glucose, which could be advanced over standard transformations to aldehyde 89. Oxime formation delivered cyclization precursor $\mathbf{9 0}$. The [3+2] cycloaddition was effected by treatment with sodium hypochlorite to form a nitrile oxide which cyclized to isoxazoline $\mathbf{9 1 .}$ Hydrogenolysis of the isoxazoline gave a hydroxymethyl ketone and led to $\beta$-elimination of the benzoyl group. Silyl protection of the primary alcohol and Luche reduction of the ketone gave desired diastereomer 92 with its hydroxyl epimer $(d r=2.5: 1)$. Benzyl protection of the secondary alcohol and fluoride-mediated removal of the silyl ether gives 93. Asymmetric epoxidation with Sharpless conditions gave 94 as a single isomer. Nucleophilic opening of the epoxide with azide delivers fully functionalized cyclopentane 95 with appropriate stereochemistry and substitution for $(+)$-trehalamine. This advanced intermediate underwent an additional eight steps to complete the natural product.

Scheme 16. Total Synthesis of (+)-Trehalamine (Shiozaki, 1994)

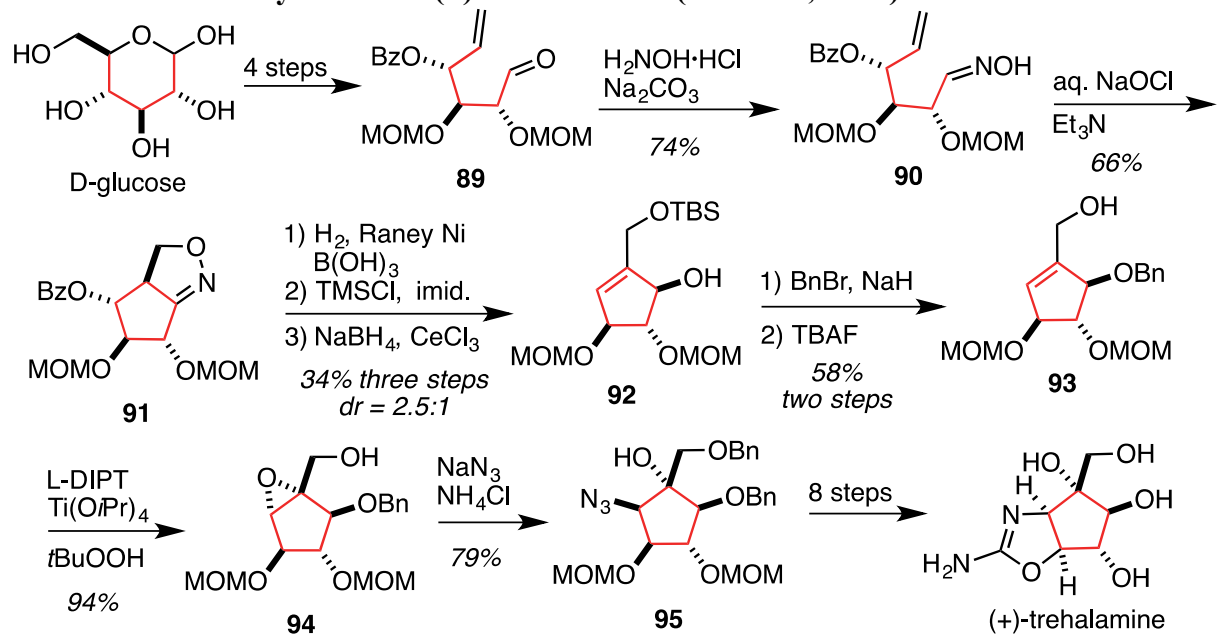

In the formal synthesis of $( \pm)$-coriolin by Funk ${ }^{28}$, a functionalized cyclopentane is formed in a nitrone [3+2] cyclization (Scheme 17). The route begins with a conjugate addition of allylsilane 96 to enone 97 delivering 98 as a mixture of diastereomers. The mixture was separated and individual diastereomers were converted to the corresponding nitrone and heated to give cyclization product 99. Methylation of the nitrogen atom was followed by reduction of the $\mathrm{N}-\mathrm{O}$ bond to give amino alcohol 100. Treatment with peracid formed an $N$-oxide and

${ }^{26}$ Herranz, E.; Biller, S. A.; Sharpless, K. B. J. Am. Chem. Soc. 1978, 100, 3596-3598.

${ }^{27}$ Kobayashi, Y.; Miyazaki, H.; Shiozaki, M. J. Org. Chem. 1994, 59, 813-822.

${ }^{28}$ Funk, R. L.; Bolton, G. L.; Daggett, J. U.; Hansen, M. M.; Horcher, L. H. M. Tetrahedron 1985, 41, 3479-3495. 
selective Cope elimination gave olefin 101. Hydroboration-oxidation of intermediate 101 was plagued by low regio- and stereoselectivity as a result of the resident hydroxyl group. Therefore, this functional group was removed by dehydration following the method of Gerlach $^{29}$ with chloridothioate 102 to give a diene. Hydroboration of this diene proceeded with complete control of chemo- and regioselectively to give an inconsequential mixture of diastereomers. A second hydroboration-oxidation proceeded to give alcohol 103 with control of alcohol stereochemistry. Selective oxidation with Fetizon's reagent ${ }^{30}$ delivered a hydroxyketone, and Saegusa oxidation gave enone 104, which is an intermediate previously taken to coriolin by Matsumoto. ${ }^{32}$

Scheme 17. Formal Synthesis of $( \pm)$-Coriolin (Funk, 1985)

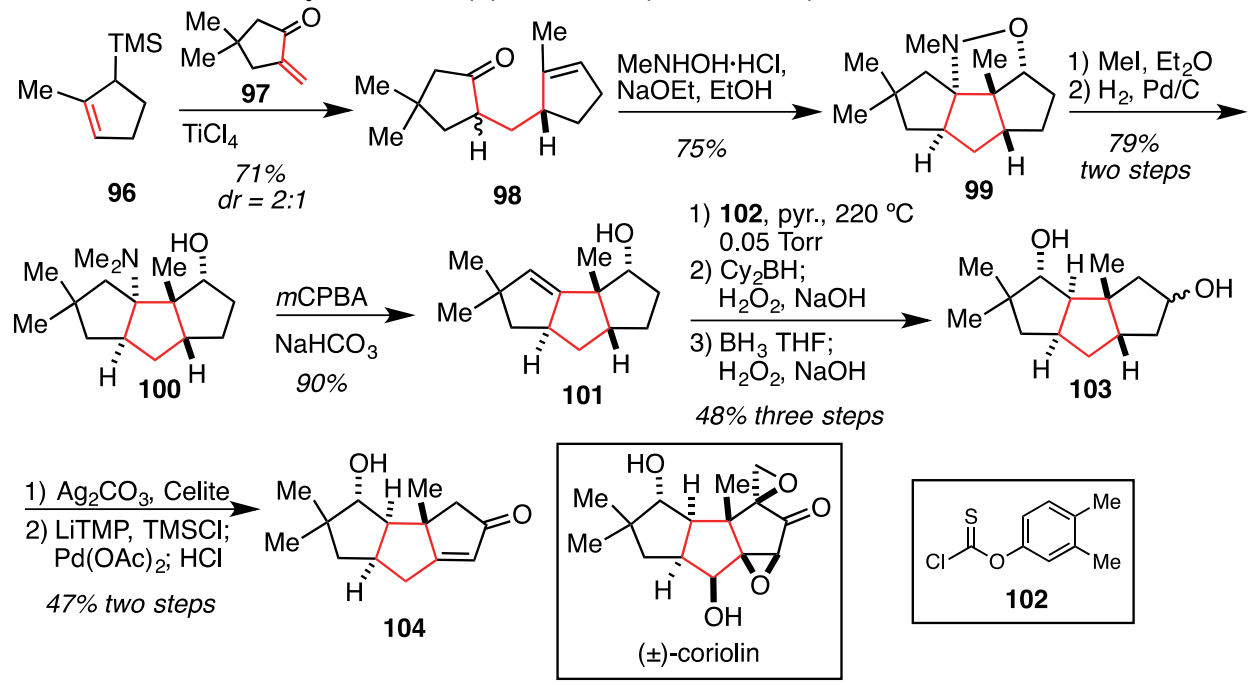

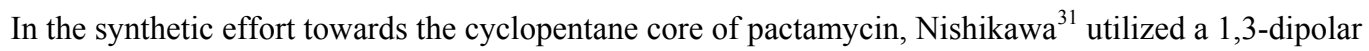
cycloaddition of a nitrone and an alkyne (Scheme 18). The synthesis begins glucose derivative 105. The unprotected hydroxyl undergoes an oxidation/Wittig olefination sequence with ylide $\mathbf{1 0 6}$ to obtain the desired Zalkene product $\mathbf{1 0 7}$ as the major isomer in a 5:1 ratio with its geometric isomer. Reduction to the allylic alcohol and subsequent Overman rearrangement afforded 108. This material was advanced over 11 steps to enal 109. Acetylide addition proceeded with moderate stereocontrol to give the desired $\mathbf{1 1 0}$ in a 3:1 diastereomer ratio. Desilylation of the alkyne and protection of the hydroxyl groups as benzyl ethers delivers 111. Chemoselective ozonolytic cleavage of the alkene was followed by condensation with $N$-hydroxyaniline to give nitrone 112. The $[3+2]$ cycloaddition was performed in refluxing toluene and a single product was isolated. The major diastereomer is the result of a favored transition state that positions the benzyl ethers in the pseudo-equatorial position (114). The expected cycloaddition product 115 was not obtained; however aziridine 116 was isolated. Presumably, the aziridine arises from rearrangement of the isoxazoline 115. The stereochemical assignment of the aziridine was ambiguous at this stage, but was later clarified following dihydroxylation (see below). The aldehyde was reduced, activated as the sulfonate and displaced with iodide to give 117. Treatment with TBAI and Lewis acid opened the aziridine to give allylic amine 118. Dihydroxylation of this intermediate delivered fully functionalized cyclopentane 119. While the functionalization and stereochemistry do not directly correspond to pactamycin, in principle this route could be used to obtain the target.

Scheme 18. Synthetic Studies Towards Pactamycin (Nishikawa, 2012)

\footnotetext{
${ }^{29}$ Gerlach, H.; Huong, T. T.; Müller, W. J. Chem. Soc., Chem. Commun. 1972, 1215-1216.

${ }^{30}$ Fétizon, M.; Golfier, M.; Louis, J.-M. J. Chem. Soc. D, Chem. Commun. 1969, 1102.

${ }^{31}$ Matsumoto, N.; Tsujimoto, T.; Nakazaki, A.; Isobe, M.; Nishikawa, T. RSC Adv. 2012, 2, 9448-9462.
} 


105

diacetone-D-glucose

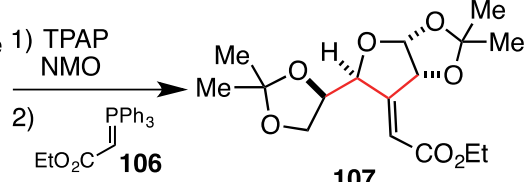

107

1) DIBAL

2) $\mathrm{Cl}_{3} \mathrm{CCN}, \mathrm{DBU}$

3) $\mathrm{K}_{2} \mathrm{CO}_{3}$

xylenes, $\Delta$

$84 \%$ three steps

$50 \%$ two steps<smiles>C=C[C@H](O)C1(C(O)C#CC#N)C(C)OC(=O)N1Cc1ccccc1</smiles>

109

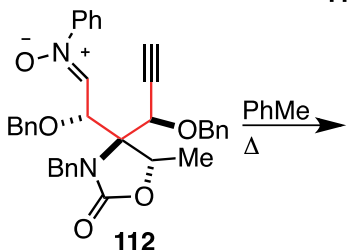

2) $\underset{\substack{\mathrm{NaH}, \mathrm{BnBr} \\ \mathrm{MeOH}}}{\substack{\mathrm{K}_{2} \mathrm{CO}_{3} \\ \text { two steps }}}$

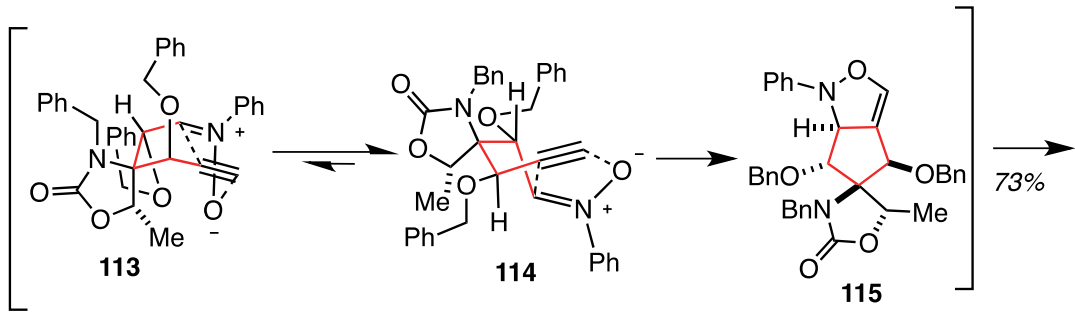<smiles>C=C1C(N)C(Cc2ccccc2)C(O)C12NC(=O)OC2[C@H](Br)c1ccccc1</smiles>

116

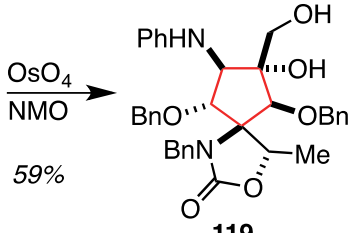

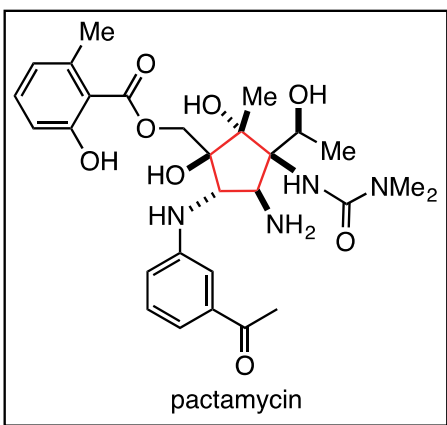

\section{3 [4+2] Cycloadditions}

\subsubsection{Bimolecular Diels-Alder Reactions of Cyclopentadienes}

In the formal synthesis of $( \pm)$-coriolin by Matsumoto ${ }^{32}$, dimerization of cyclopentadiene (11) assembles two of the three five-membered rings contained in the target architecture (Scheme 19). Dicyclopentadiene (120) was advanced five steps to intermediate 121. Ozonolytic cleavage of the alkene forms a dialdehyde, which exists as hemiacetal 122. Oxidation followed by decarboxylation leads to cyclopentene 123. Standard functional group

${ }^{32}$ Ito, T.; Tomiyoshi, N.; Nakamura, K.; Azuma, S.; Izawa, M.; Maruyama, F.; Yanagiya, M.; Shirahama, H.; Matsumoto, T. Tetrahedron Lett. 1982, 23, 1721-1724. 
manipulations converted this material to substituted cyclopentene 124 over seven steps. Hydroboration-oxidation followed by Jones oxidation furnishes a ketone. Formation of the thermodynamic enolate is followed by alkylation with 2,3-dichloropropene (125) on the convex face of the bicyclic ring system to deliver $\mathbf{1 2 6}$ with good control of stereochemistry. Oxymercuration of the vinyl chloride following Matsumoto's method ${ }^{33}$ gave $^{2}$ methyl ketone 127. An aldol condensation delivered the triquinane ring system (104) of ( \pm )-coriolin. Treatment with isopropenyl acetate converted cyclopentenone 104 to linear dienol acetate 128 under acidic conditions. Epoxidation was followed by an elimination to give enone 128. In this case, epoxidation on the hindered face of the polycycle is driven by the preferential formation of a cis-fused diquinane substructure. This material was advanced over five steps to an intermediate published by Danishefsky and Tatsuta (130) in their coriolin syntheses. $^{34,35}$

\section{Scheme 19. Formal Synthesis of $( \pm)$-Coriolin (Matsumoto, 1982)}
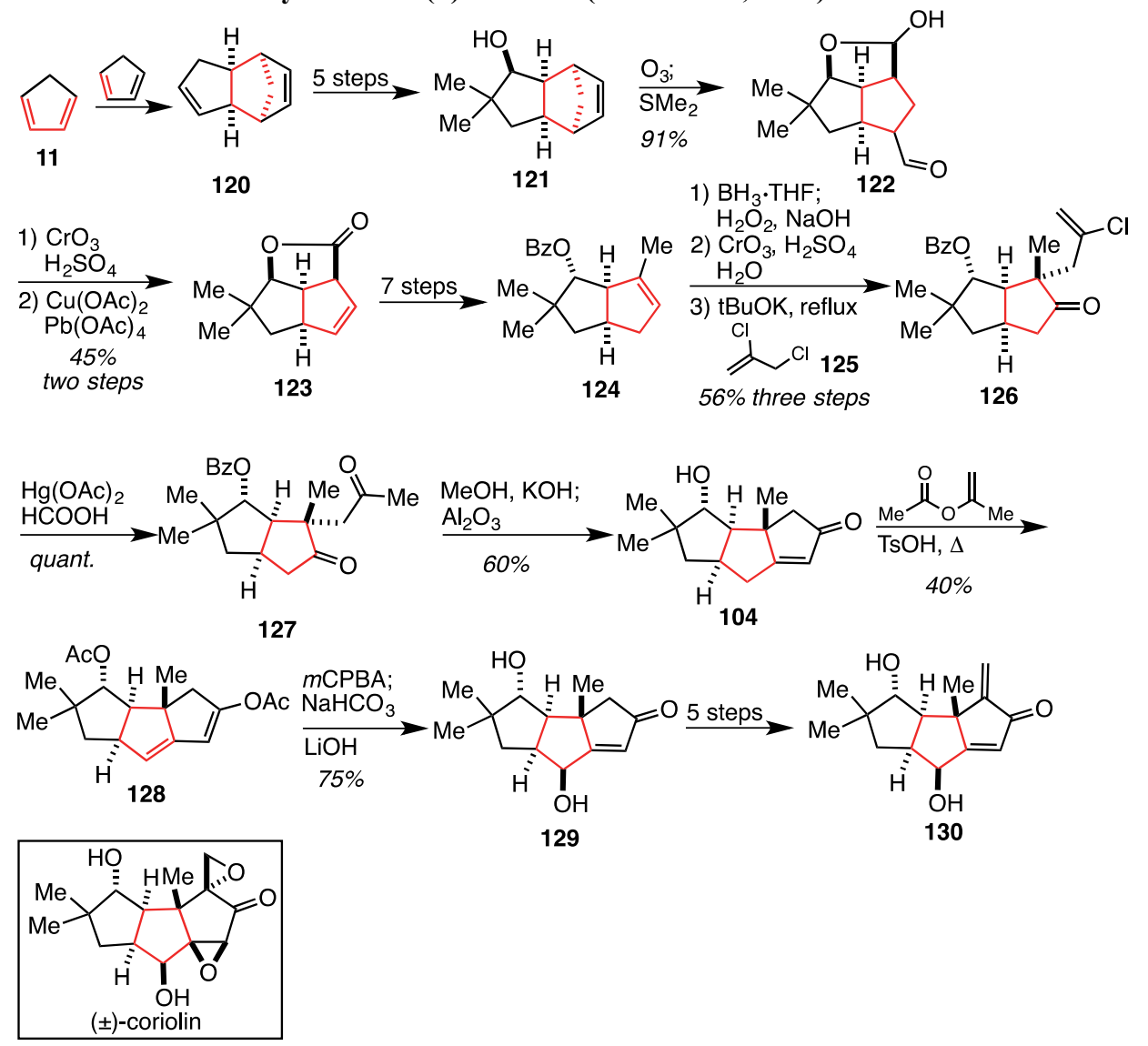

129

The formal synthesis of $( \pm)$-coriolin by Schuda ${ }^{36}$ begins with dibromo ketal 131, which could be obtained from cyclopentanone in two steps (Scheme 20). Double elimination of bromide gave a cyclopentadiene (132) that dimerized via the Diels-Alder reaction to give the bicyclo[2.2.1] heptane substructure in 134 after hydrolysis. This material was functionalized over six steps to give 135. Oxidative cleavage of $\mathbf{1 3 5}$ was followed by reduction giving a diol. Selective protection of the hydroxyl at C8 delivers 136. It is proposed that an intramolecular hydrogen bond between the $\mathrm{C} 1$ oxygen and the $\mathrm{C} 3$ carbinol renders the $\mathrm{C} 3$ alcohol less reactive. A one-pot threestep sequence (triflic anhydride, tert-butyl ammonium iodide, zinc metal) reduces the primary alcohol to a methyl group yielding 137. This intermediate is functionalized to cyclopentanone 138 over three steps: ester hydrolysis, oxidation of the alcohol and benzyl ether, and acid-promoted decarboxylation and simultaneous ketal hydrolysis. The acidic conditions led to epimerization of the methyl group to give an inconsequential mixture of

${ }^{33}$ Yoshioka, H.; Takasaki, K.; Kobayashi, M.; Matsumoto, T. Tetrahedron Lett. 1979, 20, 3489-3492.

${ }^{34}$ Danishefsky, S.; Zamboni, R.; Kahn, M.; Etheredge, S. J. J. Am. Chem. Soc. 1980, 102, 2097-2098.

35 (a) Tatsuta, K.; Akimoto, K.; Kinoshita, M. J. Antibiot. 1980, 33, 100-102. (b) Tatsuta, K.; Akimoto, K.; Kinoshita, M. Tetrahedron 1981, 37, 4365-4369.

${ }^{36}$ Schuda, P. F.; Heimann, M. R. Tetrahedron 1984, 40, 2365-2380. 
diastereomers. Treatment of ketone 138 with alcohol 139 induces enol ether formation and subsequent Claisen rearrangement on the convex face of the ring system to give 140. Saponification of the benzoate gave an intermediate alcohol. Treatment with mercuric acetate induced hydrolysis of the vinyl chloride and elimination of the thiopropyl group to give desired enone 141. Aldol condensation of 141 was promoted by potassium tertbutoxide to deliver the triquinane ring system (142) of coriolin. Deconjugation of enone 142 gave a $\beta$, $\gamma$ unsaturated ketone. Epoxidation with peracid occurs from the top face, forming a cis-fused diquinane substructure. Epoxide opening and subsequent elimination with DBU delivers $\mathbf{1 3 0}$ and intersects a published intermediate used in the Danishefsky and Tatsuta syntheses of coriolin. ${ }^{34,35}$

Scheme 20. Formal Synthesis of ( \pm )-Coriolin (Schuda, 1984)

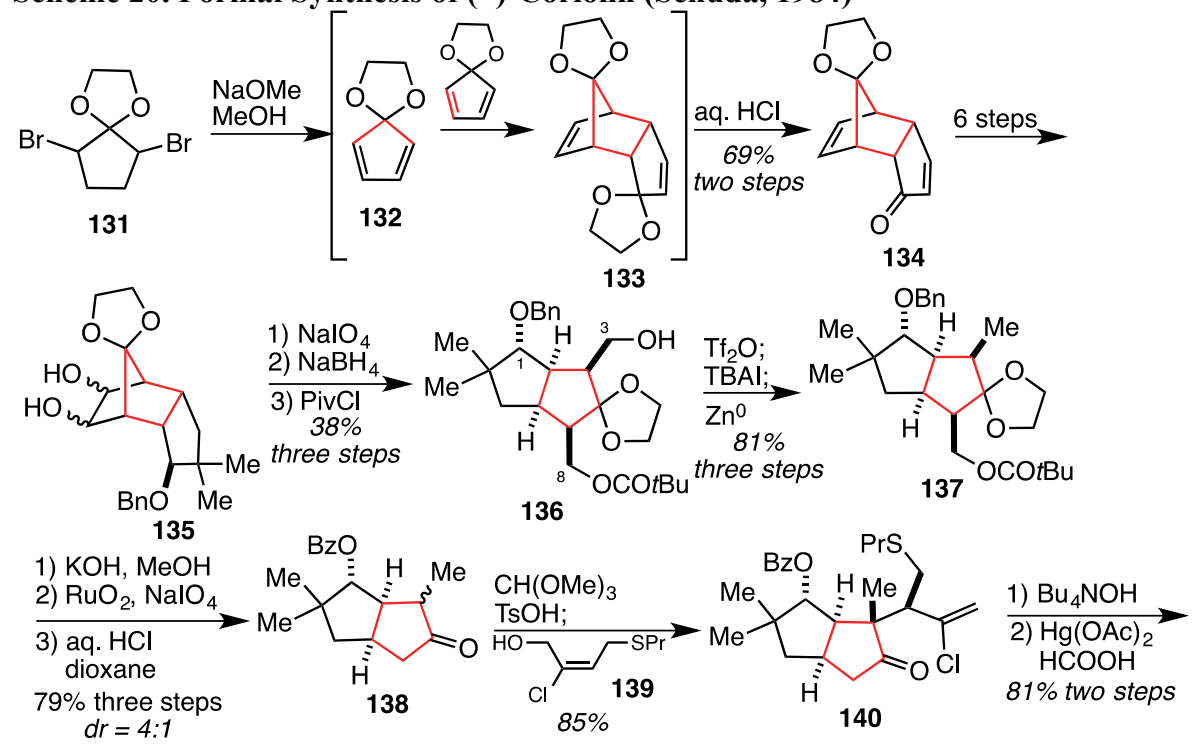

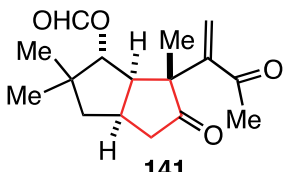

141

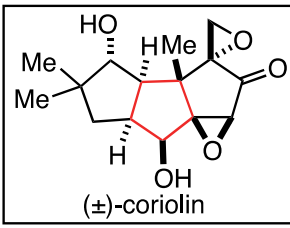

1) $t B u O K, D M E$ $\mathrm{AcOH}$ 2) $m \mathrm{CPBA}$ 3) DBU $13 \%$ three steps $47 \%$ recovered 142

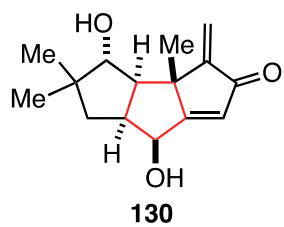

In the formal synthesis of $( \pm)$-coriolin, Mehta $^{37}$ functionalizes cyclopentadiene through a Diels-Alder cycloaddition and a photo-induced rearrangement (Scheme 21). The route commences with the endo selective Diels-Alder of quinone 143 and cyclopentadiene (11) to give 144. Upon irradiation, a [2+2] cycloaddition delivers pentacyclic dione 145. A [2+2] cycloreversion reaction occurred under flash vacuum pyrolysis conditions to yield triquinane 146. The desired cis, anti,cis diastereomer (in 148) was obtained by establishing a thermal equilibrium of enone isomers by refluxing in benzyl benzoate. The resulting mixture contained a 1:3:3.5 ratio of starting material 146 and isomers 147 and 148 . While the isolated yield of 148 was $35-40 \%$, starting material 146 and enone 147 could be recycled by reestablishing the thermal equilibrium. This material was advanced seven steps to intermediate 149. A Saegusa oxidation gave enone 150. Enolate addition to phenylselenyl bromide followed by oxidation and elimination provided advanced intermediate 142. This material was prepared in the syntheses of $( \pm)$-coriolin by Ikegami ${ }^{38}$ and Schuda. ${ }^{36}$

\section{Scheme 21. Formal Synthesis of ( \pm )-Coriolin (Mehta, 1986)}

${ }^{37}$ Mehta, G.; Murthy, A. N.; Reddy, D. S.; Reddy, A. V. J. Am. Chem. Soc. 1986, 108, 3443-3452.

${ }^{38}$ Iseki, K.; Yamazaki, M.; Shibasaki, M.; Ikegami, S. Tetrahedron 1981, 37, 4411-4418. 

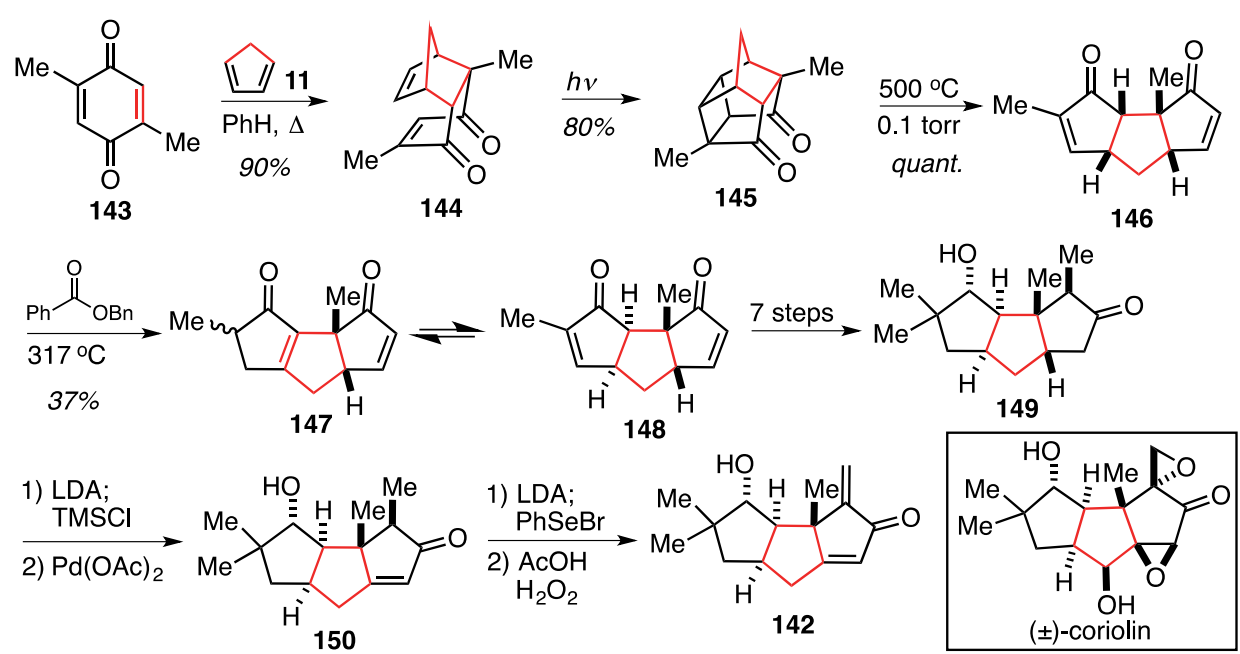

In Danishefsky's second generation ${ }^{39}$ approach to $( \pm)$-merrilactone $A^{40}$ the cyclopentane is constructed in the first step using a Diels-Alder reaction to form a bicyclo[2.2.1] heptane (Scheme 22). Specifically, the cycloaddition between the chlorinated cyclopentadiene 151 and 2-methylmaleic anhydride proceeds with endo selectivity and sets the quaternary stereocenter. Methanolysis of the anhydride and subsequent esterification of the free acid delivered 152. Installation of the second required methyl group for merrilactone A was accomplished by standard alkylation. This two-step sequence successfully produced 153, where as Diels-Alder cycloaddition of 150 with dimethylmaleic anhydride was unsuccessful. Reduction of the esters and global halide reduction occurs over two steps with concomitant ketal hydrolysis to give $\mathbf{1 5 4}$. Ketone $\mathbf{1 5 4}$ was advanced to ester $\mathbf{1 5 6}$ over three steps featuring a Horner-Wadsworth-Emmons olefination with 155 and subsequent alkene reduction. This material was advanced four steps to intermediate 157. The carboxylic acid was converted to alcohol 158 over three steps through carboxy inversion. ${ }^{41}$ Lewis acid opening of the acetal induced lactonization with the tethered methyl ester, and the aldehyde was concomitantly protected as the dithiane 159. Removal of the dithiane and reduction of the corresponding aldehyde gave primary alcohol 160. Elimination using the method developed by Grieco ${ }^{42}$ delivered the exocyclic cyclopentene 161. Iodolactonization furnishes tricyclic compound 162, which is an intermediate that intersects the first route to $( \pm)$-merrilactone A published by Danishefsky. ${ }^{43}$

Scheme 22. Formal Synthesis of ( \pm )-Merrilactone A (Danishefsky, 2005)

\footnotetext{
${ }^{39}$ For the first generation synthesis see Scheme 100, reference Error! Bookmark not defined.

${ }^{40}$ Meng, Z.; Danishefsky, S. J. Angew. Chem. Int. Ed. 2005, 44, 1511-1513.

${ }^{41}$ (a) Denney, D. B.; Sherman, N. J. Org. Chem. 1965, 30, 3760-3761. (b) Danishefsky, S. J.; Tsuzuki, K. J. Am. Chem. Soc. 1980, 102, 6891-6893.

${ }^{42}$ Grieco, P. A.; Gilman, S.; Nishizawa, M. J. Org. Chem. 1976, 41, 1485-1486.

${ }^{43}$ Birman, V. B.; Danishefsky, S. J. J. Am. Chem. Soc. 2002, 124, 2080-2081.
} 

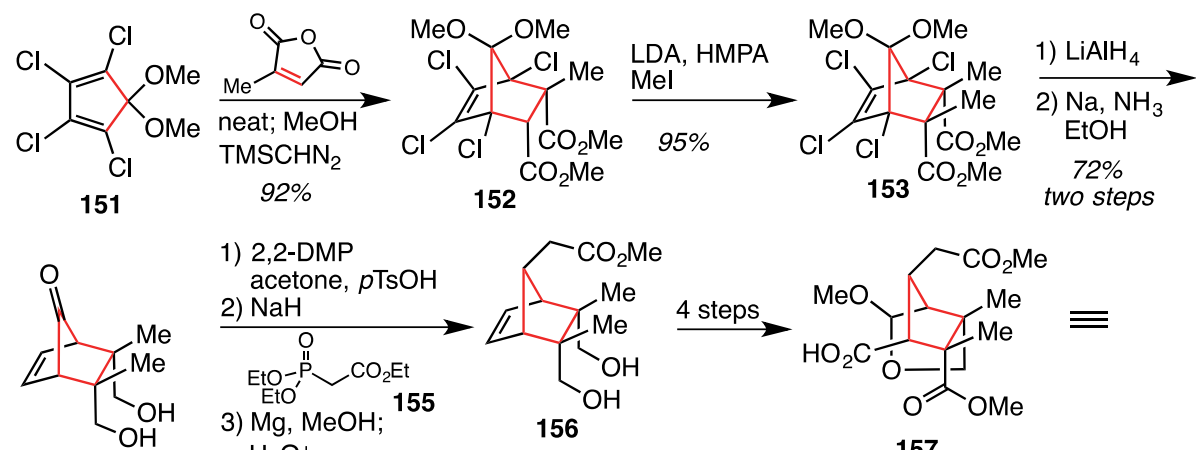

1) 2,2-DMP

acetone, $p \mathrm{TsOH}$

2) $\mathrm{NaH}$

152 $153 \mathrm{CO}_{2} \mathrm{Me} \quad \begin{gathered}72 \% \\ \text { two step }\end{gathered}$

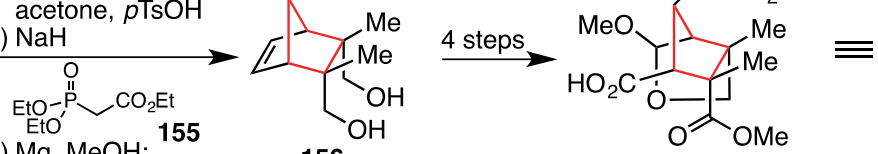

3) $\mathrm{Mg}, \mathrm{MeOH} ;$ $\mathrm{H}_{3} \mathrm{O}^{+}$

157

$66 \%$ three steps<smiles>COC(=O)C[C@@H]1[C@H]2C(OC)OC[C@@]2(C)[C@](C)(C(=O)O)[C@@H]1C(=O)O</smiles>
1) $\mathrm{DCC}, m \mathrm{CPBA}$ 2) $\mathrm{PhH}$, 3) $\mathrm{K}_{2} \mathrm{CO}_{3}, \mathrm{MeOH}$ $58 \%$ three steps<smiles>COC(=O)C[C@H]1[C@H](O)[C@](C)(C(=O)OC)[C@@]2(C)COC(OC)[C@H]12</smiles><smiles>CCO[SbH3]</smiles>
157<smiles>C=C1OC[C@]2(C)[C@H](C3SCCCS3)[C@H](O)[C@@H](CCOC)[C@]12C</smiles>

159

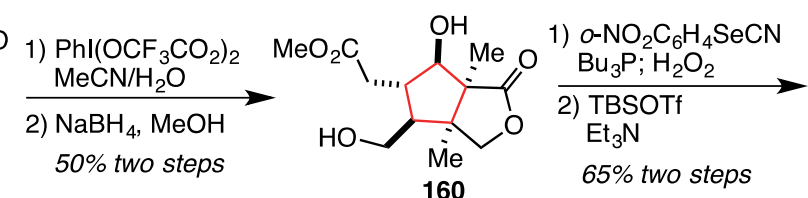

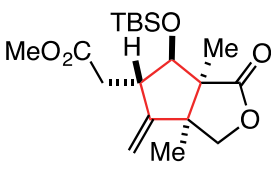

161

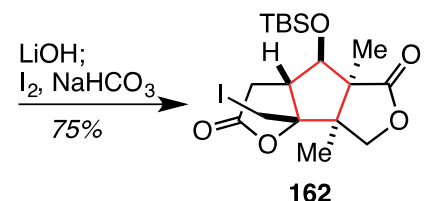

162

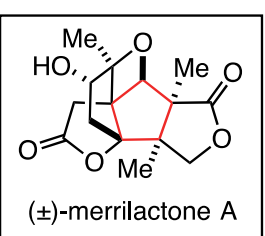

In the synthetic effort towards the axinellamines by Carreira ${ }^{44}$, a Diels-Alder reaction of the spirocyclopentadiene $\mathbf{1 6 5}$ is used to synthesize a bicyclo[2.2.1] heptane (Scheme 23). The synthesis begins with lithiocyclopentadienide (163), which is converted to spirocyclic intermediate 165 by addition to epichlorohydrin (164) and silylation. The cycloaddition of $\mathbf{1 6 5}$ with $N$-phenylmaleimide (166) delivers a 1:1 mixure of diastereomers. The desired stereoisomer 168 could be obtained preferentially through recrystallization from cyclohexane. Resubjection of the undesired $\mathbf{1 6 7}$ in hot chlorobenzene gave the 1:1 mixture, which could then be crystallized as before. Desired intermediate 168 is functionalized over six steps to give cyclic anhydride 169. A desymmetrization sequence using the method developed by Bolm, produced acid-ester $170{ }^{45}$ Epimerization of the methyl ester with LDA gave the trans configuration of the acid-ester 171. This material was advanced six steps to acid chloride 172. Conversion of $\mathbf{1 7 2}$ to the corresponding Cbz carbamate $\mathbf{1 7 3}$ was accomplished using the Curtius procedure, and it elegantly establishes the $\alpha$-tertiary amine with the desired stereochemistry for the axinellamines. Ozonolysis of the cyclopentane and subsequent treatment with base resulted in dialdehyde 174. The base selectively epimerizes the aldehyde with the more accessible $\alpha$-position. Note that this transformation converts the $\alpha$-tertiary amine stereocenter into a non-stereogenic chirotopic center. Interestingly, a selective mono-protection of the dialdehyde was possible, which results in desymmetrization of the pseudo- $C_{2}$ symmetry in the molecule. The factors responsible for the difference in reactivity of the two aldehydes are unknown; however, it may be attributable to either the steric difference or the proximity of the Cbz-carbamate versus the silyloxymethyl group. Oxidation of the remaining aldehyde to the corresponding carboxylic acid was performed with Masamune's conditions to give $175{ }^{46}$ The hindered chloride was installed by radical decarboxylation in the presence of carbon tetrachloride. This advanced intermediate (176) displays a fully functionalized cyclopentane with the required substitution and stereochemistry found in the axinellamines.

\footnotetext{
${ }^{44}$ Starr, J. T.; Koch, G.; Carreira, E. M. J. Am. Chem. Soc. 2000, 122, 8793-8794.

${ }^{45}$ Bolm, C.; Gerlach, A.; Dinter, C. L. Synlett 1999, 195-196.

${ }^{46}$ Abiko, A.; Roberts, J. C.; Takemasa, T.; Masamune, S. Tetrahedron Lett. 1986, 27, 4537-4540.
} 
Scheme 23. Synthetic Studies Towards Axinellamines (Carreira, 2000)
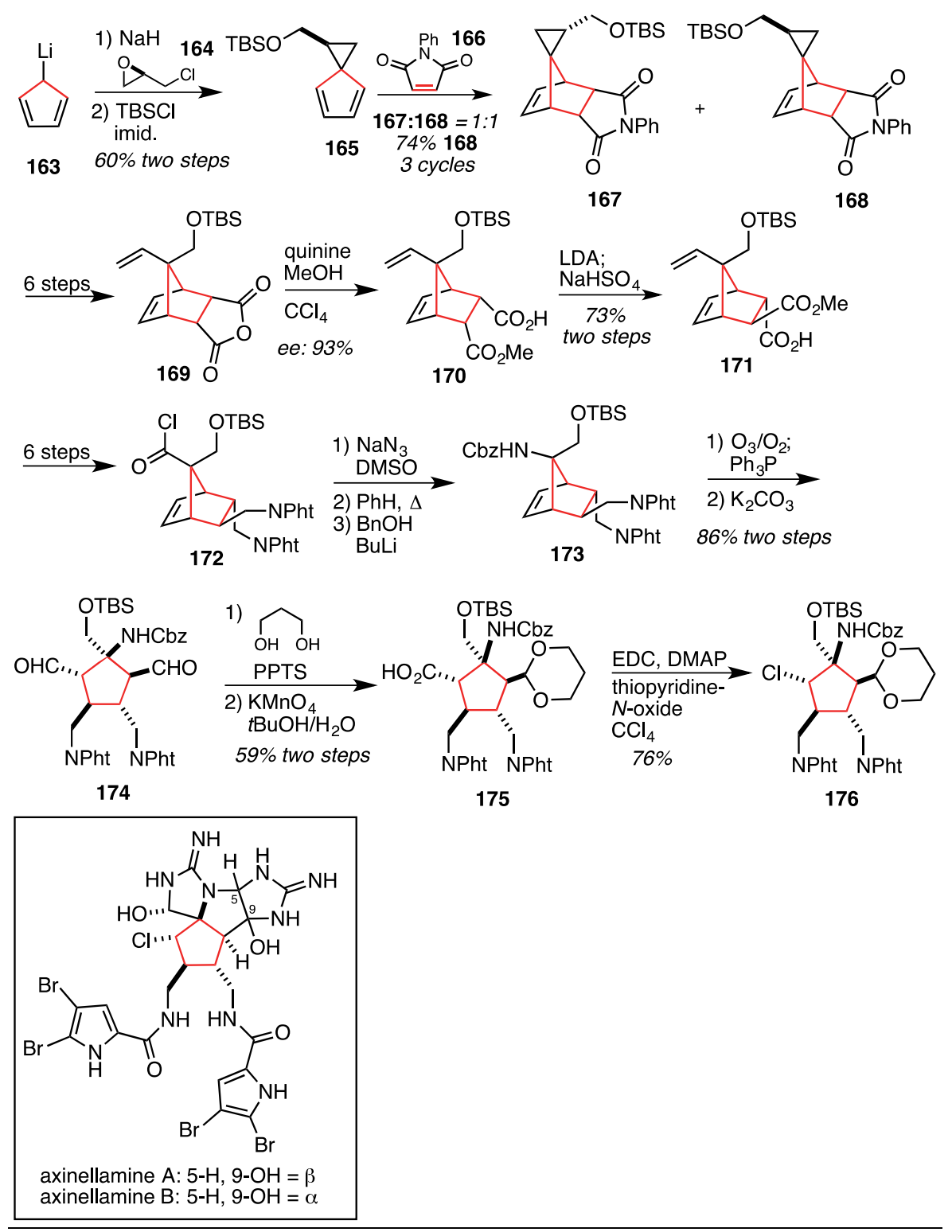

\subsubsection{Intramolecular Diels-Alder Reactions of Skipped Trienes}

The De Clerq ${ }^{47}$ synthesis of the gibberellin natural product $( \pm)-\mathrm{GA}_{5}$ uses an intramolecular Diels-Alder reaction, where the molecular tether connecting the diene and dienophile forms the five-membered ring (Scheme 24). The route begins with conjugate addition of a furan-derived Grignard reagent to 177, followed by intermolecular enolate trapping with 2,3-dibromopropene (178). The alkylation occurs on the face opposite the furan substituent setting the first two stereocenters of the eventual fully functionalized cyclopentane. Enol ether hydrolysis gives a ketone. Lithium-halogen exchange of the vinyl bromide induces ring closure to give 179. Reduction of the ester and oxidation of the resulting alcohol gives an aldehyde. Addition of the lithiated propiolate gave $\mathbf{1 8 0}$ as the major product in 4:1 diastereomeric ratio, presumably as a result of Felkin-Anh selectivity. Intramolecular Diels-Alder reaction of $\mathbf{1 8 0}$ in the presence of $\beta$-cyclodextrin gave cycloadduct $\mathbf{1 8 1}$ in high yield and high dr; however, there was no explanation given for the diastereoselectivity. Selective hydrogenation of the strained disubstituted alkene with $\mathrm{Pd} / \mathrm{BaSO}_{4}$ was followed by hydride reduction of the unsaturated ester to give the

${ }^{47}$ (a) Grootaert, W. M.; De Clercq, P. J. Tetrahedron Lett. 1982, 23, 3291-3294. (b) Grootaert, W. M.; De Clercq, P. J. Tetrahedron Lett. 1986, 27, 1731-1734. 
thermodynamically favored trans-ring fusion in 182. At this stage, the cyclopentane is fully functionalized with five stereocenters; however, in order to complete the synthesis of $\mathrm{GA}_{5}$, the secondary alcohol must be converted to a carboxy substituent. Oxidation of the secondary alcohol, protection of the tertiary alcohol and olefination gave a methyl vinyl ether. An ester methylation and transesterification sequence delivers $\mathbf{1 8 3}$. Hydrolysis of the vinyl ether proceeded with protonation on the less hindered face to set the final stereocenter on the fully functionalized cyclopentane. Pinnick ${ }^{48}$ oxidation completed the synthesis of $( \pm)-\mathrm{GA}_{5}$.

Scheme 24. Total Synthesis of ( \pm )-GA (De Clercq, 1986)
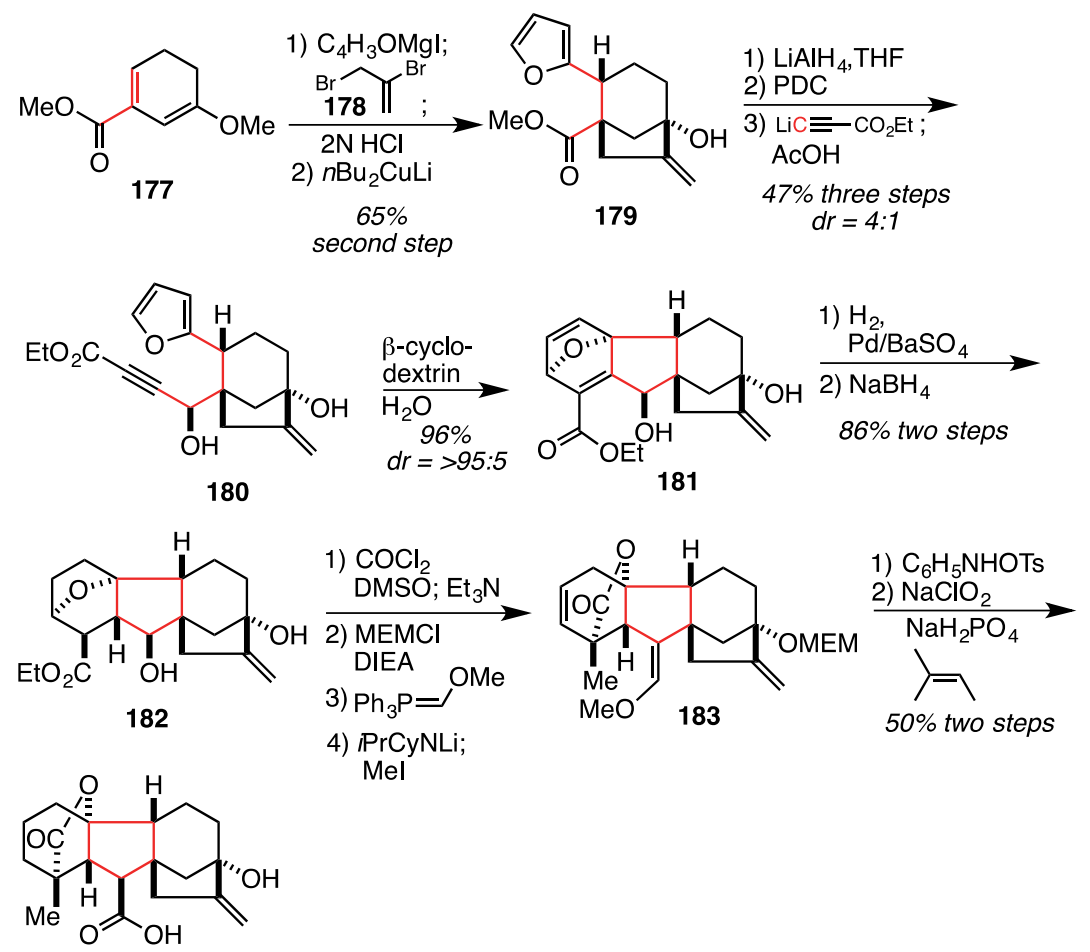

$( \pm)-\mathrm{GA}_{5}$

Sorensen's ${ }^{49}$ biogenetic synthesis of (+)-FR182877 employs a double transannular Diels-Alder reaction to construct five rings, including a fully functionalized cyclopentane (Scheme 25). This remarkable transformation forms seven stereocenters with complete stereocontrol. The route commences with the assembly of coupling fragment 190. An Evans syn-aldol reaction between 185 and aldehyde 186 sets two stereocenters for the eventual fully functionalized cyclopentane. The chiral auxiliary was then cleaved with conditions delivering a Weinreb amide. Silylation of the secondary alcohol and addition of dimethyl lithiomethylphosphonate gives ketophosphonate 187. Olefination with $(E)$-3-iodo-2-methacrolein (188) and subsequent cleavage of the silyl ethers delivers diol 189. A chelation-controlled reduction of the ketone gave a syn-1,3-diol. Silylation and stannylation delivers coupling fragment 190. Cross-coupling of the dienylstannane and allyl acetate 191 delivered 192. This intermediate was functionalized over four steps to give 193. Exposure to $\mathrm{Pd}_{2} \mathrm{dba}_{3}$ promoted macrocyclic ring closure. To install the necessary unsaturation for the Diels-Alder reaction, the stabilized enolate was formed and reacted with phenylselenyl bromide to afford an inseparable mixture of organoselenide diastereomers $(d r=3: 1)$. When this mixture was treated with $m \mathrm{CPBA}$ and warmed to $40{ }^{\circ} \mathrm{C}$ in chloroform, Diels-Alder substrate 194 was formed and the cyclization cascade occurred spontaneously to furnish 195. The cycloaddition leads to the establishment of seven stereogenic centers including the final two stereocenters for the fully functionalized cyclopentane. After this spectacular transformation, an additional three steps were required to complete the synthesis of the natural product.

Scheme 25. Total Synthesis of (+)-FR182877 (Sorensen, 2002)

${ }^{48}$ Bal, B. S.; Childers, W. E.; Pinnick, H. W. Tetrahedron 1981, 37, 2091-2096.

${ }^{49}$ Vosburg, D. A.; Vanderwal, C. D.; Sorensen, E. J. J. Am. Chem. Soc. 2002, 124, 4552-4553. 

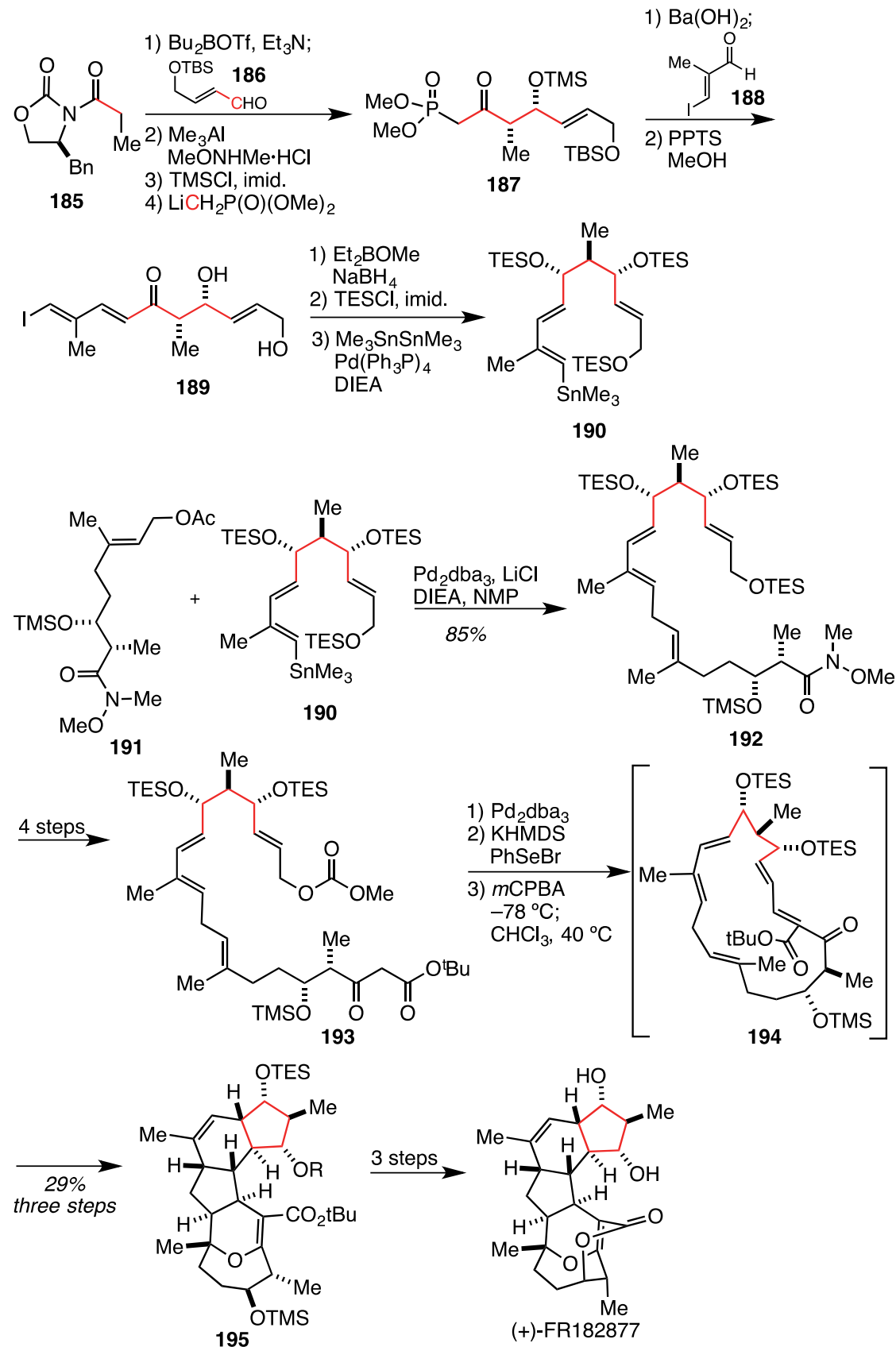

In the Evans synthesis of (-)-FR182877 ${ }^{50}$, a similar cascade of transannular [4+2] cycloadditions construct much of the molecular architecture of the natural product, including the fully functionalized cyclopentane (Scheme 26). The route begins with the assembly of coupling fragment 199. An Evans syn-aldol reaction between 196 and aldehyde $\mathbf{1 8 6}$ sets two stereocenters for the eventual fully functionalized cyclopentane. Cleavage of the auxiliary and formation of a Weinreb amide was followed by addition of magnesium acetylide. A stereoselective DIBAL reduction delivered $s y n-1,3-$ diol 198. ${ }^{51}$ Silylation and hydroboration-oxidation of the alkyne delivered boronic acid coupling partner 199. The Suzuki coupling of fragments 199 and 200 gave bromodiene 201 as the desired geometrical diene isomer. Three additional steps including installation of the $\beta$-keto ester gave 202. Iodination delivered an allyl iodide that cyclized upon exposure to cesium carbonate furnishing macrocycle $\mathbf{2 0 3}$ as an inconsequential 1:1 mixture of diastereomers (epimeric at C2). Oxidation produced a diene (204) and initiated

${ }^{50}$ Evans, D. A.; Starr, J. T. J. Am. Chem. Soc. 2003, 125, 13531-13540.

${ }^{51}$ Kiyooka, S. -i.; Kuroda, H.; Shimasaki, Y. Tetrahedron Lett. 1986, 27, 3009-3012. 
the first transannular Diels-Alder reaction. The initial cycloadduct 205 proceeded through the cyclization cascade resulting in $\mathbf{2 0 6}$ as the only isolable product. The result of the cascade is a fully functionalized cyclopentane bearing all the required stereochemistry for (-)-FR182877. The target was completed in four additional steps.

Scheme 26. Total Synthesis of (-)-FR182877 (Evans, 2003)
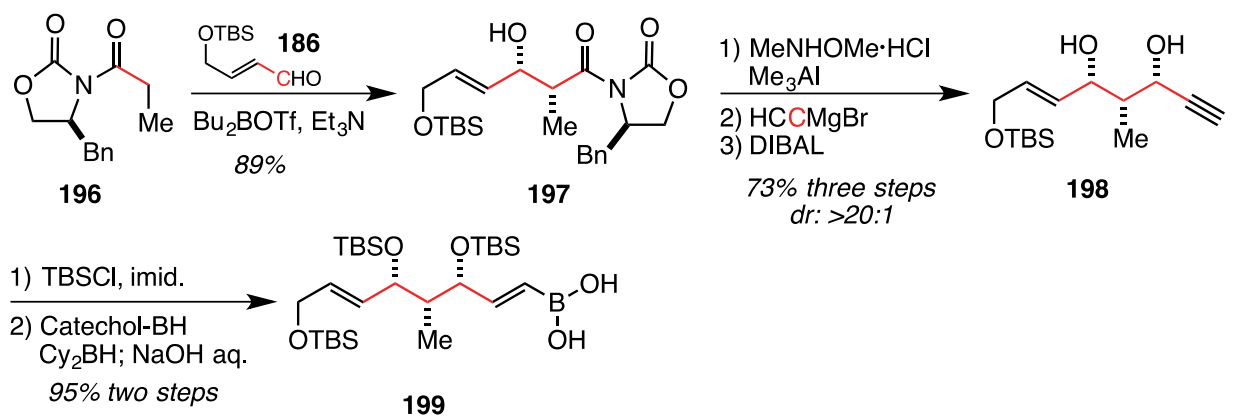

$$
d r:>20: 1
$$
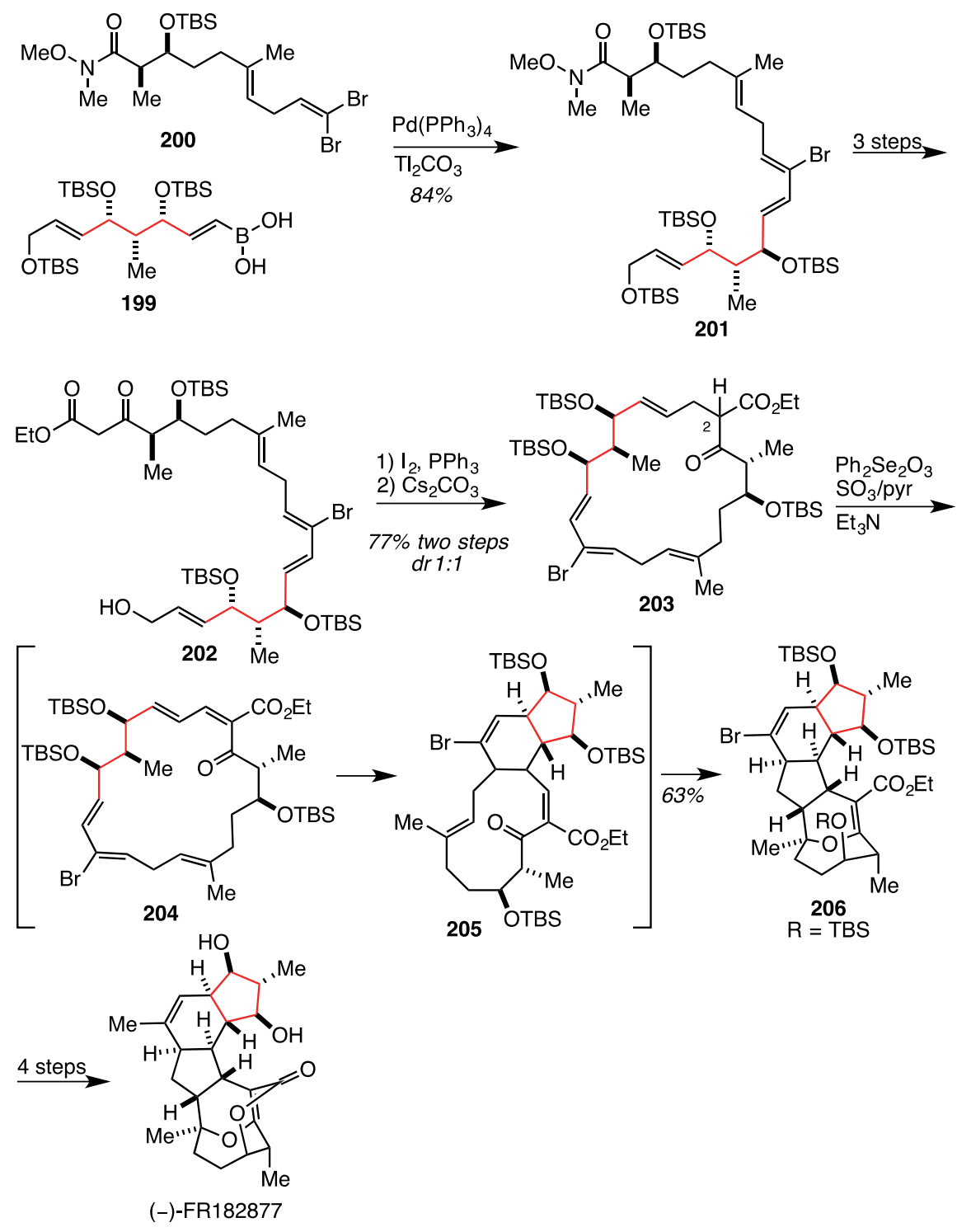
In the synthesis of (-)-FR182877 by Nakada ${ }^{52}$ an intramolecular Diels-Alder reaction cascade constructs the polycyclic ring system (Scheme 27). The route begins with an Evans aldol between 207 and aldehyde 208. The chiral auxiliary was cleaved to give a Weinreb amide, which was advanced to intermediate $\mathbf{2 1 1}$ over four steps. Addition of dimethyl lithiomethylphosphonate installs the final carbon atom of the eventual cyclopentane to give a ketophosphonate. Olefination with aldehyde $\mathbf{2 1 2}$ gives intermediate 213. Reduction of the ketone gave the undesired anti-1,3-diol as the major product and subsequent TES protection delivered 214. The allylic alcohol was oxidized to give 215, which spontaneously underwent the intramolecular Diels-Alder (IMDA) reaction cascade to give 216. While a fully functionalized cyclopentane has been assembled, the stereochemistry of the TES-protected hydroxyl required stereochemical inversion. Desilylation with PPTS and oxidation with DMP gave cyclopentanone 217. A stereoselective reduction from the less hindered face gave alcohol 218 bearing the desired stereochemistry for the natural product. The completion of (-)-FR182877 was completed after an additional 11 steps.

Scheme 27. Total Synthesis of (-)-FR182877 (Nakada, 2009)
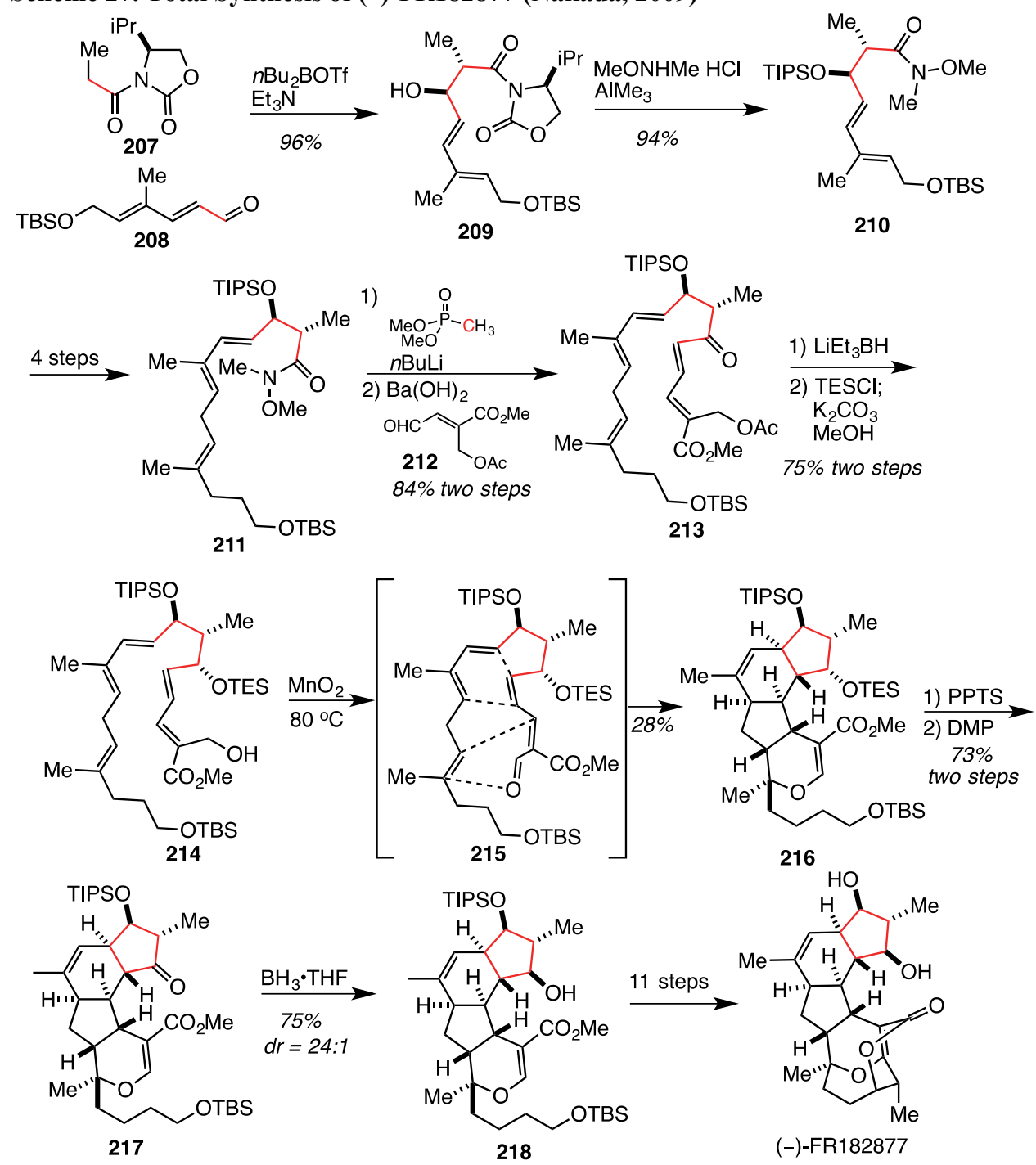

\footnotetext{
52 Tanaka, N.; Suzuki, T.; Matsumura, T.; Hosoya, Y.; Nakada, M. Angew. Chem. Int. Ed. 2009, 48, 2580-2583.
} 
In the synthesis of the C20-diterpenoid alkaloid (+)-nominine, $\mathrm{Gin}^{53}$ uses a pyrrolidine-induced dienamine isomerization/Diels-Alder cascade to construct the fully functionalized cyclopentane (Scheme 28). Synthetic fragments 219 and 220 were condensed via a Staudinger-aza-Wittig reaction, and a subsequent hydride reduction of the resultant imine gave amine 221. This mixture of four diastereomers converged through a TFA-catalyzed $\mathrm{MeOH}$ extrusion and isomerization to provide the aza-1,3-dipole 222. The 1,3-dipolar cycloaddition between the betaine and unsaturated nitrile groups yields desired isomer $\mathbf{2 2 4}$ as a minor product with its constitutional isomer 223. The mixture is a result of thermodynamic selection, and resubjection of pure $\mathbf{2 2 3}$ to the reaction conditions allowed for the undesired material to be recycled. This transformation builds a substantial portion of the natural product architecture including two stereocenters of the fully functionalized cyclopentane. Exhaustive reduction of the ketone carbon to a methylene delivers 225. Conversion of the nitrile to alkene $\mathbf{2 2 6}$ was completed through DIBAL reduction followed by a Wittig olefination that unveiled the dienophile functionality. A Birch reduction of the aromatic ring furnished the $\beta, \gamma$-unsaturated cyclohexanone 227. Treatment with $\mathrm{MeOH}$ and pyrrolidine promoted the formation of a dienamine (228) that participated in the IMDA to deliver fully functionalized cyclopentane 229. The synthesis of (+)-nominine was completed in two additional steps.

\section{Scheme 28. Total Synthesis of (+)-Nominine (Gin, 2008)}
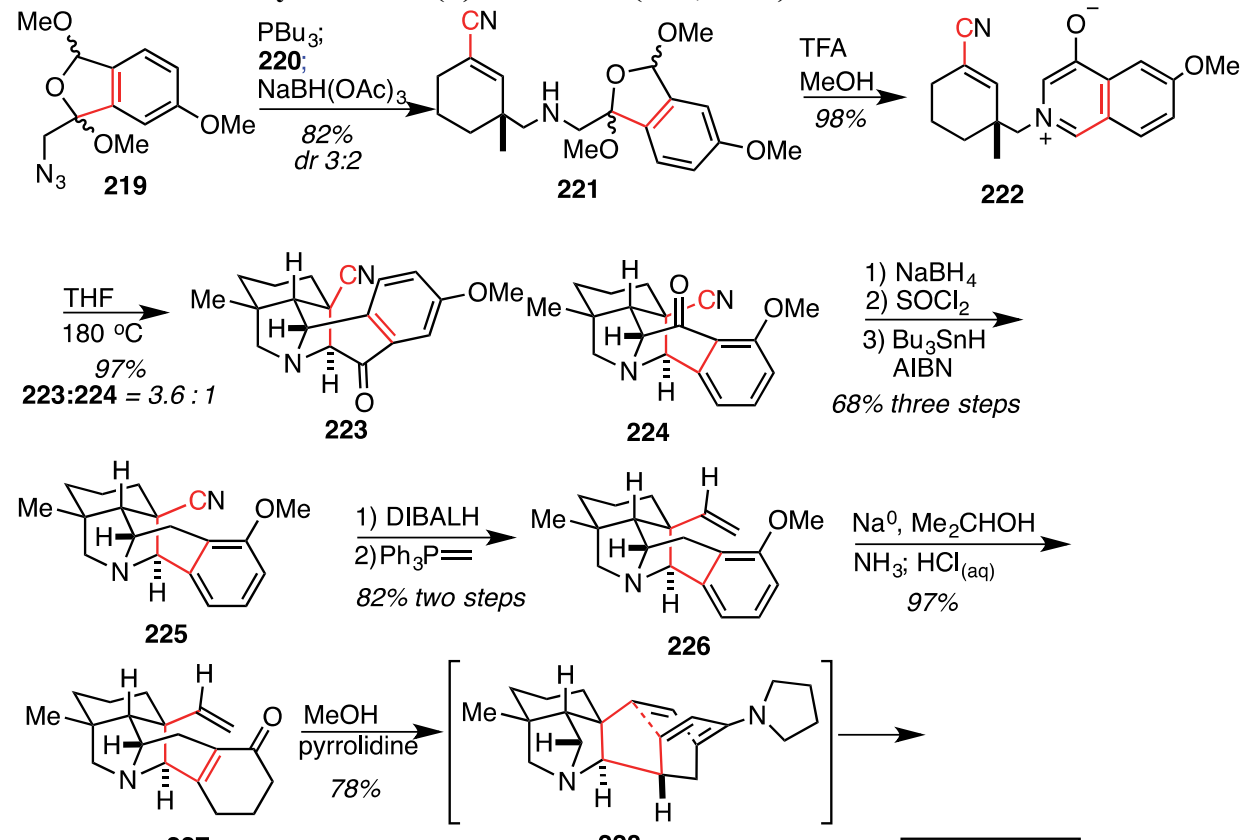

227

228
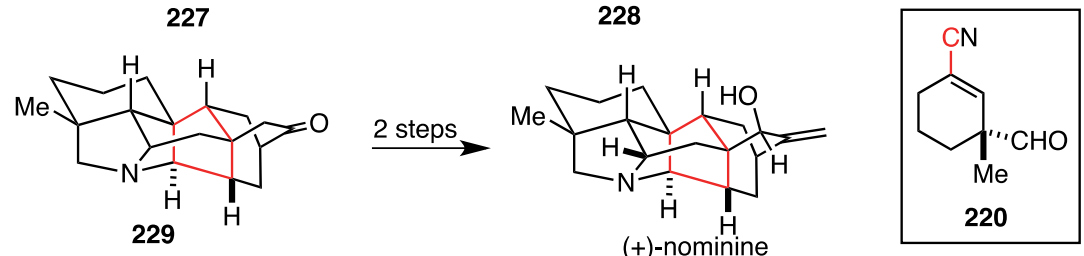

The Nicolau ${ }^{54}$ synthesis of (+)-hirsutellone B employs an epoxide opening/Diels-Alder reaction sequence to construct a fully functionalized cyclopentane (Scheme 29). The route begins with a Stork-Zhao olefination of $(R)-(+)$-citronellal followed by oxidative cleavage to give iodo aldehyde 230. An olefination reaction of aldehyde 230 gives an $\alpha, \beta$-unsaturated aldehyde. A Jørgensen asymmetric epoxidation ${ }^{55}$ with proline-derived catalyst 231 yielded an epoxyaldehyde. This was immediately exposed to $\mathrm{Ph}_{3} \mathrm{PCHCO}_{2} \mathrm{Me}$ to furnish intermediate 232 with the expected $E$-geometry. A coupling reaction with stannane 233 yielded annulation precursor 234. The epoxide opening/Diels-Alder cascade was promoted with $\mathrm{Et}_{2} \mathrm{AlCl}$ to give the tricyclic core of hirsutellone $\mathrm{B}$. The stereochemical outcome of the epoxide opening was postulated to result from the preferred conformation $\mathbf{2 3 5}$, and the subsequent Diels-Alder reaction delivered a single diastereomer (237) that resulted from endo-transition

\footnotetext{
${ }^{53}$ Peese, K. M.; Gin, D. Y. Chem. Eur. J. 2008, 14, 1654-1665.

${ }^{54}$ Nicolaou, K. C.; Sarlah, D. Wu, T. R.; Zhan, W. Angew. Chem. Int. Ed. 2009, 48, 6870-6874.

${ }^{55}$ Marigo, M.; Franzén, J.; Poulsen, T. B.; Zhuang, W.; Jørgensen, K. A. J. Am. Chem. Soc. 2005, 127, $6964-6965$.
} 
state 236. The resulting fully functionalized cyclopentane contains the desired stereochemistry for $(+)$ hirsutellone B. The natural product was completed in 17 additional steps.

\section{Scheme 29. Total Synthesis of (+)-Hirsutellone B (Nicolaou, 2009)}

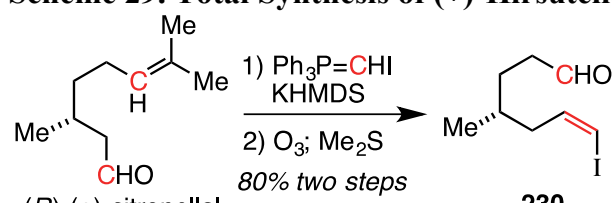

(R)-(+)-citronellal 230

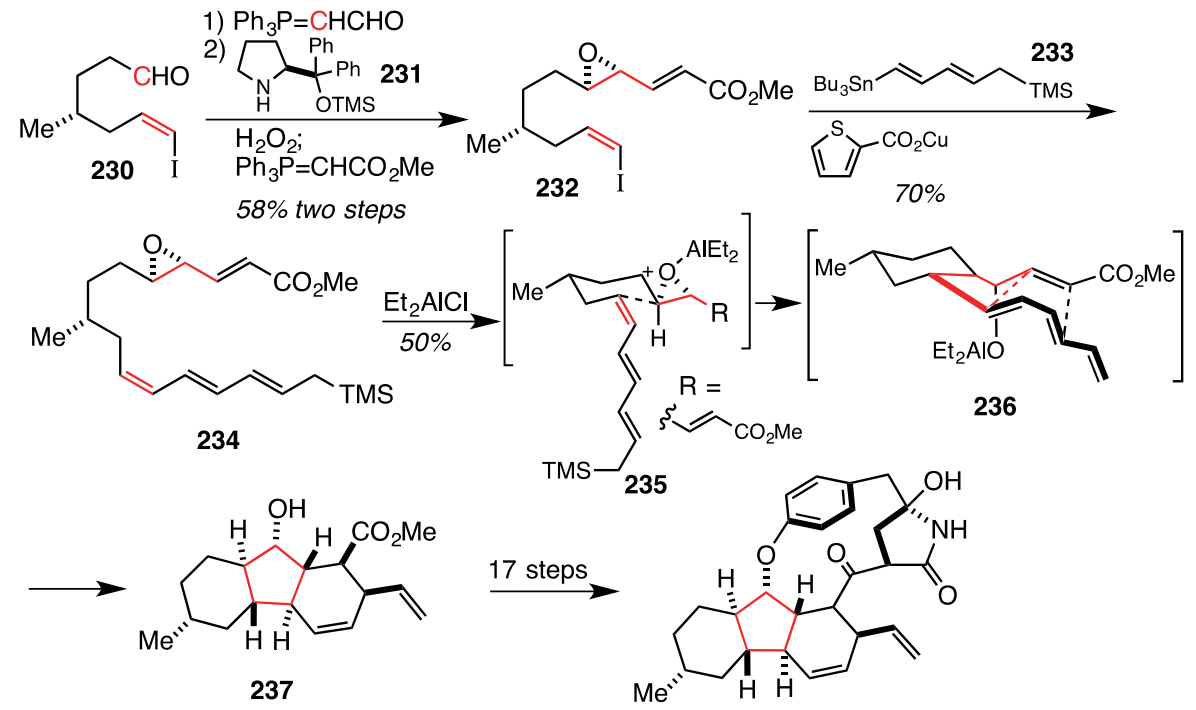

(+)-hirsutellone B

The Uchiro ${ }^{56}$ synthesis of (+)-hirsutellone B features a cascade Diels-Alder reaction sequence to form a functionalized cyclopentanone (Scheme 30). The route begins with an oxidative cleavage of the tertiary alkene in $(R)-(-)$-citronellene. Reductive workup and silylation gives TBS ether 238. Ozonolysis of the remaining alkene and Wittig homologation delivers 239. Ester reduction delivered an allylic alcohol, which underwent reagentcontrolled epoxidation using the Sharpless ${ }^{57}$ method to furnish 240. A Swern oxidation of the primary alcohol gave an aldehyde that was homologated using the Ohira-Bestmann reagent (241). ${ }^{58}$ Fluoride-mediated removal of the silyl ether delivers alcohol 242. A Swern oxidation gave the corresponding aldehyde, which was olefinated with 243 to give diene 244 as the desired geometrical isomer $(E / Z=10: 1)$. Treatment with TMSOTf promoted an intramolecular cyclization, presumably through a chair-like intermediate, yielding $\mathbf{2 4 5}$ as a single diastereomer. A Diels-Alder reaction of the alkene and 1,2,3,4,5-pentamethylcyclopentadiene (246) served to protect the eventual alkene dienophile in 247. Conversion of the alkyne group to the olefinic iodide was achieved via hydrostannylation followed by iodination to deliver 248. A Stille cross-coupling reaction with 249 gave trienyl stannane, which was followed by silylation of the secondary alcohol yielding $\mathbf{2 5 0}$. Warming to reflux in the presence of BHT promoted a retro-DA-IMDA reaction sequence and the cycloadduct $\mathbf{2 5 1}$ was obtained. In this particular IMDA no hypothesis was given for the diastereoselectivity; however, a rationale similar to that discussed above $(\mathbf{2 3 4} \rightarrow \mathbf{2 3 7})$ could be used to explain the selectivity. Reduction with $\mathrm{NaBH}_{4}$ installs the final stereocenter of the fully functionalized cyclopentane. The reduction gave the desired product 252 in a 1:1.5 ratio with the undesired alcohol epimer. The undesired diastereomer could be converted to 252 using standard transformations. The completion of (+)-hirsutellone B was completed with an additional 16 steps (longest linear sequence).

\section{Scheme 30. Total Synthesis of (+)-Hirsutellone B (Uchiro, 2011)}

${ }^{56}$ Uchiro, H.; Kato, R.; Arai, Y.; Hasegawa, M.; Kobayakawa, Y. Org Lett. 2011, 13, 6268-6271.

${ }^{57}$ Gao, Y.; Hanson, R. M.; Klunder, J. M.; Ko, S. Y.; Masamune, H.; Sharpless, K. B. J. Am. Chem. Soc. 1987, 109, 5765-5780.

${ }^{58}$ (a) Ohira, S. Synth. Commun. 1989, 19, 561-564. (b) Müller, S.; Liepold, B.; Roth, G. J.; Bestmann, H. J. Synlett 1996, 521-522. 

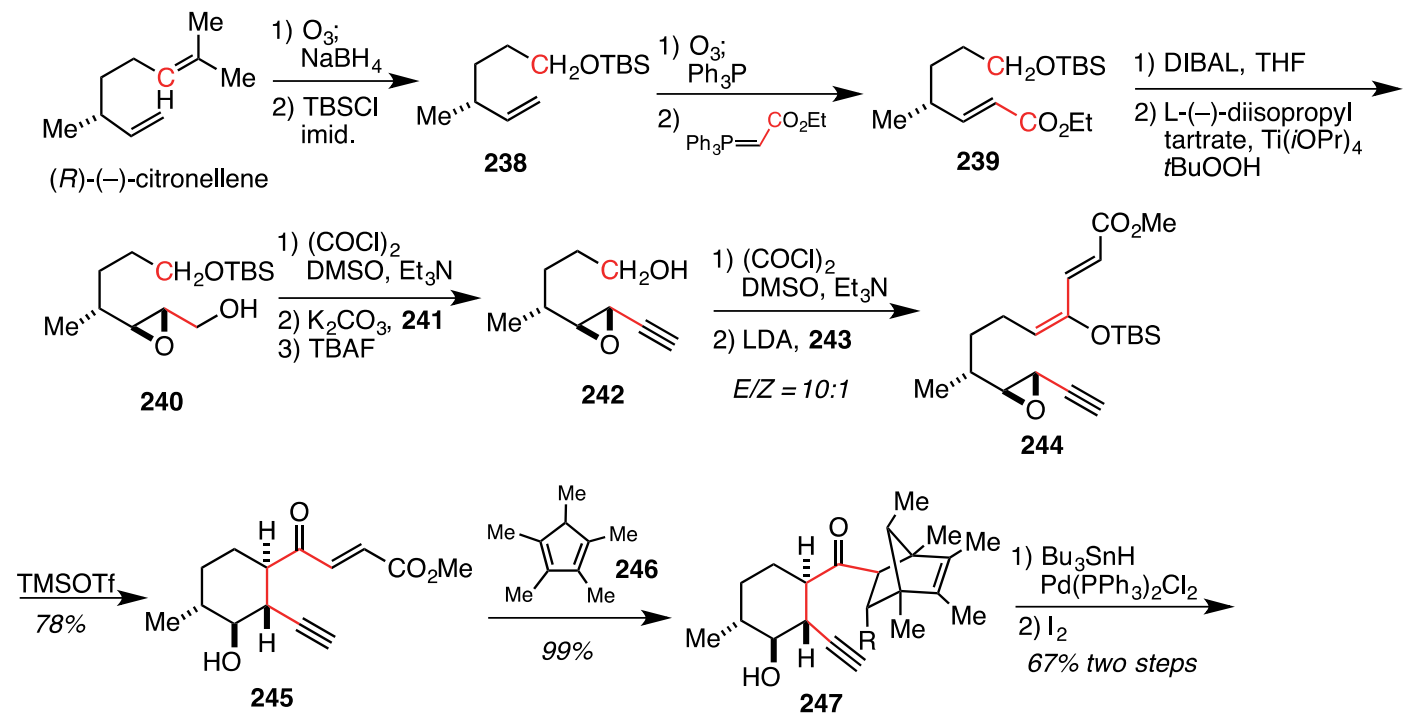

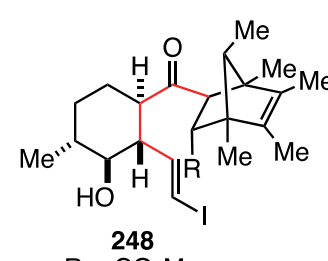
$\mathrm{R}=\mathrm{CO}_{2} \mathrm{Me}$

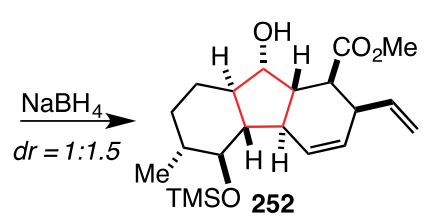

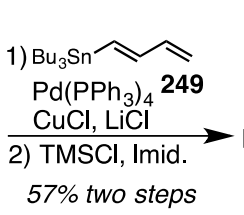
$57 \%$ two steps

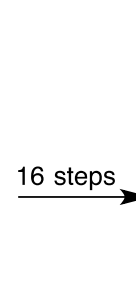

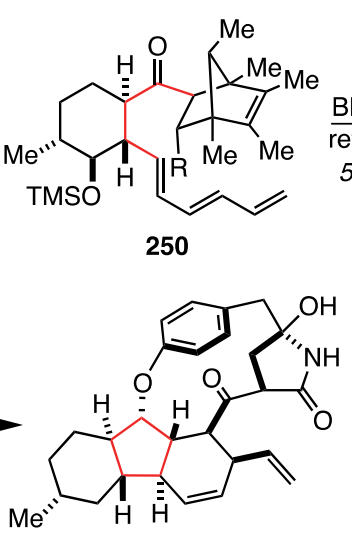

(+)-hirsutellone B

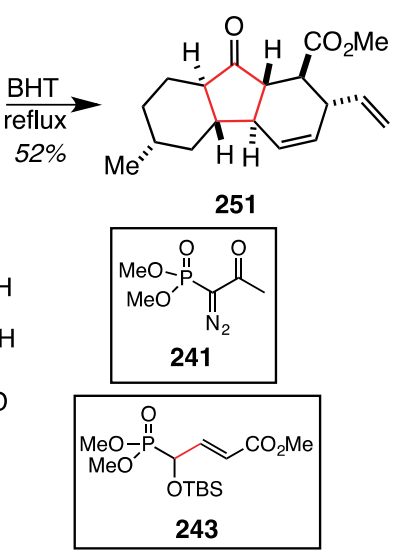

Roush $^{59}$ reported synthetic studies toward hirsutellone B where the cyclopentane core was constructed with an IMDA reaction of a siloxacyclopentene (Scheme 31). The route begins with an enantioselective Diels-Alder reaction of the dienol 253 with methyl acrylate (254) in the presence of the reagent derived from $(R)$-BINOL, $\mathrm{Me}_{2} \mathrm{Zn}$, and $\mathrm{MeMgBr} .{ }^{60}$ Cycloadduct 255 was converted to the trienyl aldehyde 256 over five steps. Exposure to the lithium acetylide derived from 257 afforded alcohol 258 with Felkin-Anh selectivity $(d r>95: 5)$. This propargyl alcohol was hydrosilylated with tetramethyldisilizane. Addition of $t \mathrm{BuOK}$ gave siloxacyclopentene 259. Treatment with TMSOTf promoted the Diels-Alder cycloaddition and a subsequent protodesilylation of the crude cycloadduct delivered decahydrofluorene 261. The Diels-Alder proceeds through the endo conformation 260 and delivers the product with good diastereoselectivity. Advanced intermediate $\mathbf{2 6 1}$ possesses the required substitution and stereochemical configuration present in hirsutellone B.

Scheme 31. Synthetic Studies Towards Hirsutellone B (Roush, 2011)

\footnotetext{
${ }^{59}$ Halvorsen, G. T.; Roush, W. R. Tetrahedron Lett. 2011, 52, 2072-2075.
}

${ }^{60}$ Ward, D. E.; Souweha, M. S. Org. Lett. 2005, 7, 3533-3536 

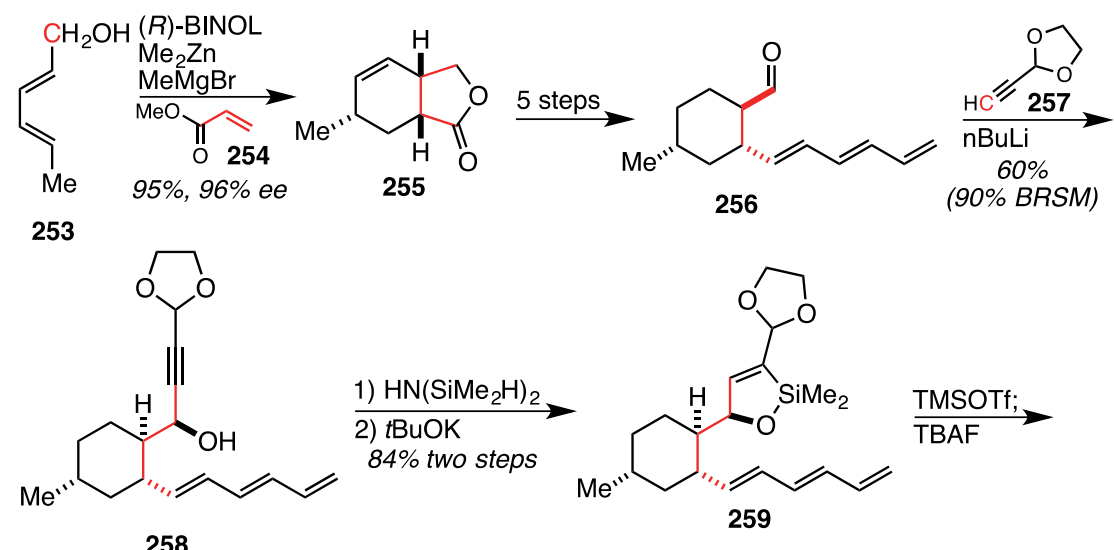

258

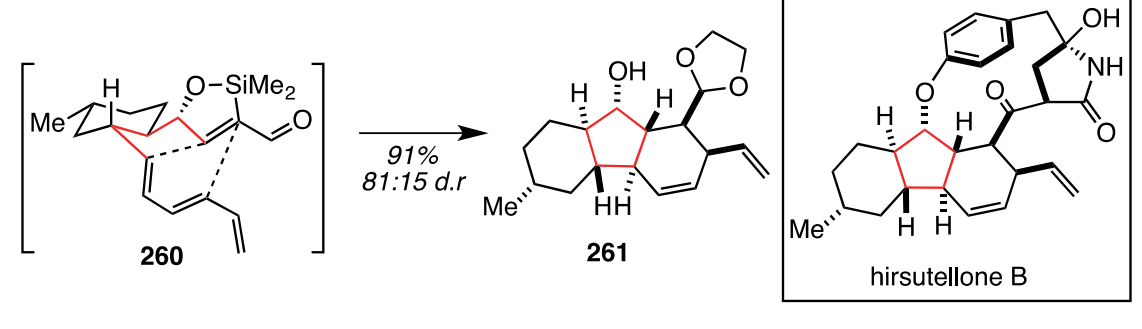

In the synthesis of the decahydrofluorene core of the hirsutellones, Sorensen ${ }^{61}$ employs a tandem ketenetrapping/Diels-Alder cyclization sequence (Scheme 32). The synthesis commences with an diastereoselective Diels-Alder reaction of olefin 262 and diene 263 to form cyclohexene 264 as a single diastereomer. This intermediate was carried forward to the aldehyde 265 over four steps. Homologation with phosphonium 266 following Kluge's method ${ }^{62}$ gave enol ether $\mathbf{2 6 7}$ as an inconsequential mixture of alkene isomers $(E / Z=6: 1)$. This mixture of alkenes was subjected to dihydroxylation conditions giving $\alpha$-hydroxylated aldehyde 268. Direct treatment with ylide $\mathbf{2 6 9}$ gave alkene $\mathbf{2 7 0}$ with complete control of alkene stereochemistry. This material was converted to the primary alcohol $\mathbf{2 7 1}$ over four steps. Oxidation and Wittig olefination with $\mathbf{2 7 2}$ gave cyclization substrate 273. Upon heating in toluene, elimination of acetone reveals both an electron withdrawing goup conjugated to the dienophile and an electrophilic ketene (274). Trapping of the ketene with the tethered amine forms the macrocyclic substructure of hirsutellone B (275). The Diels-Alder reaction then spontaneously occurs to complete the molecular architecture of the natural product in 276. This advanced intermediate contains a fully functionalized cyclopentane with the desired substitution and stereochemistry for hirsutellone B.

\section{Scheme 32. Synthetic Studies Towards the Hirsutellones (Sorensen, 2013)}

${ }^{61}$ Reber, K. P.; Tilley, S. D.; Carson, C. A.; Sorensen, E. J. J. Org. Chem. 2013, 78, 9584-9607.

${ }^{62}$ Kluge, A. F. Tetrahedron Lett. 1978, 39, 3629-3632. 

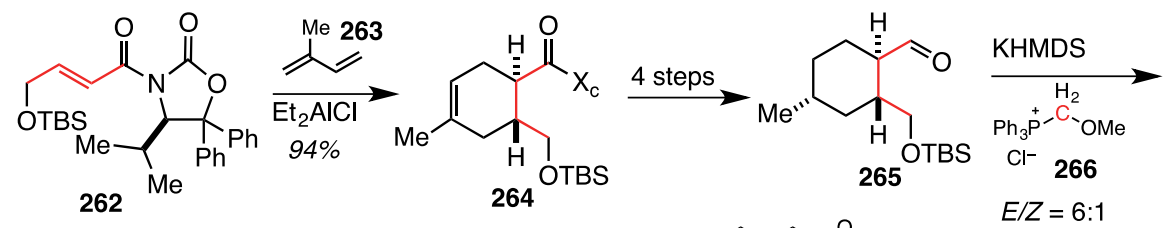

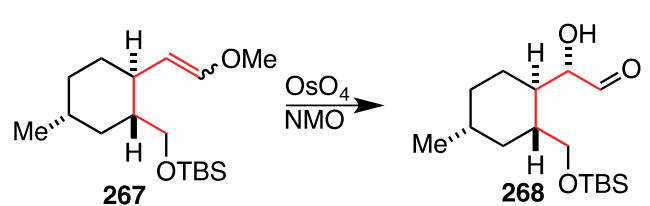<smiles>O=C1C=C(C=[PbH])OCO1</smiles>

$E / Z=6: 1$

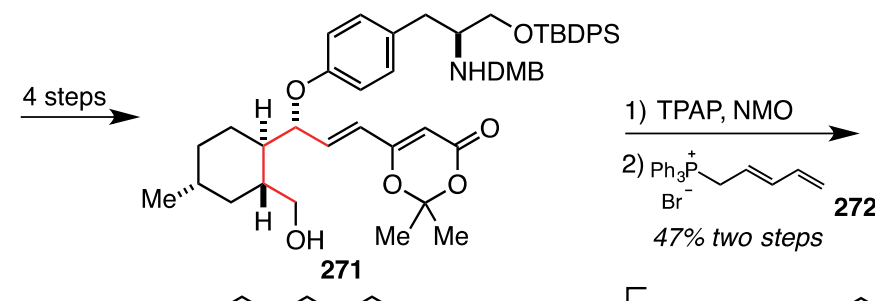

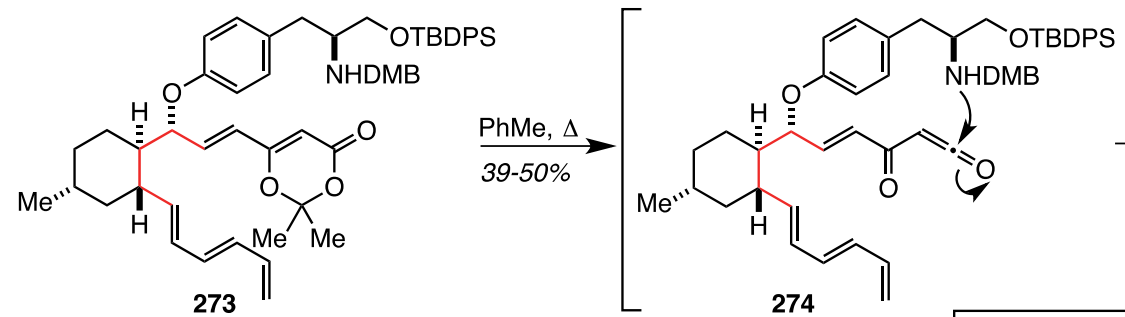

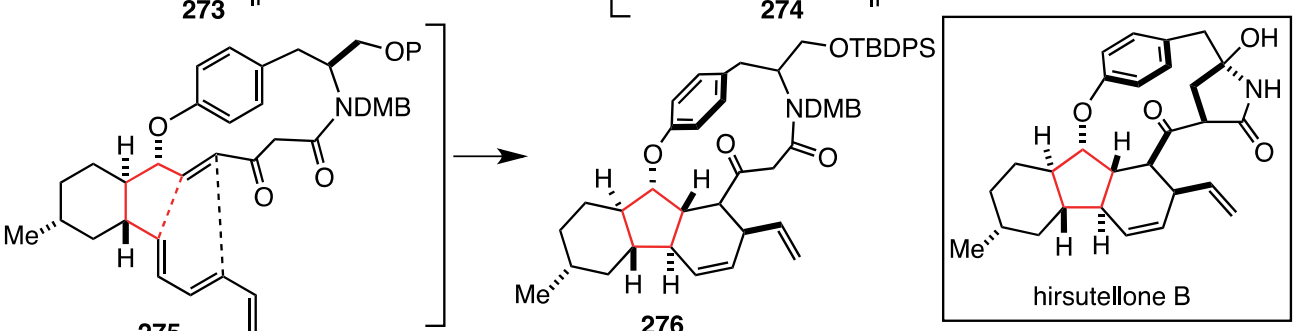

In the synthesis of the dimeric guaianolide $(+)$-absinthin by Zhai ${ }^{63}$, the construction of the five-membered carbocycle arises from a dimerization reation of $\mathbf{2 7 8}$ via a Diels-Alder cycloaddition (Scheme 33). The route commences with natural product (-)-santonin, which was photolyzed to give cyclopentenone 277. A three-step sequence (hydride reduction, selenylation, and oxidation/elimination) leads to cyclopentadiene 278. When stored neat under an inert atmosphere, the slow dimerization of 278 via a Diels-Alder reaction proceeded to deliver fully functionalized cyclopentane $\mathbf{2 7 9}$. This intermediate was advanced to the natural product over an additional five steps.

Scheme 33. Total Synthesis of (+)-Absinthin (Zhai, 2005)

\footnotetext{
${ }^{63}$ Zhang, W.; Luo, S.; Fang, F.; Chen, Q.; Hu, H.; Jia, X.; Zhai, H. J. Am. Chem. Soc. 2005, 127, $18-19$.
} 
(-)

$(-)$-santonin

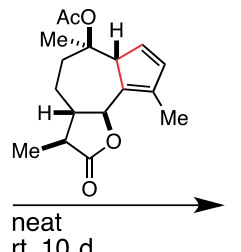

$\mathrm{rt}, 10 \mathrm{~d}$

$72 \%$

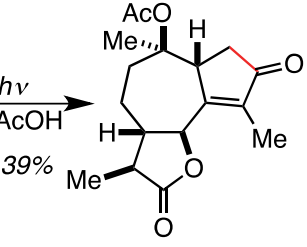

277
1) $\mathrm{NaBH}_{4}$

2) $\mathrm{O}-\mathrm{NO}_{2} \mathrm{C}_{6} \mathrm{H}_{4} \mathrm{SeCN}$ $n \mathrm{Bu}_{3} \mathrm{P}$

3) $\mathrm{NaIO}_{4}$

$47 \%$ two steps

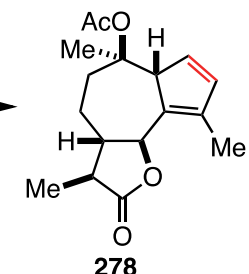

278

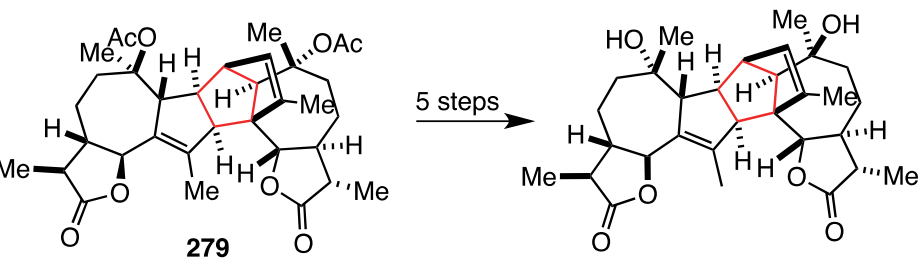

In the total synthesis of $( \pm)-\mathrm{GA}_{111}$ methyl ester, Ihara ${ }^{64}$ employs a Diels-Alder reaction of a skipped triene to construct the hydrindane substructure of the target (Scheme 34). The synthesis begins with a palladium-catalyzed cycloalkenylation of $\mathbf{2 8 0}$ to form cyclohexenone 281. Conjugate addition of the vinyl cuprate occurred from the less hindered face to give 282. This material was advanced to intermediate $\mathbf{2 8 3}$ over six steps. Ester reduction and alcohol oxidation gave an aldehyde. Olefination with $\mathbf{2 8 4}$ delivered the desired $E$-alkene $\mathbf{2 8 5}$ in a 1.3:1 ratio. A similar sequence was repeated (reduction, oxidation, olefination) furnishing the skipped triene 286. Lithiumhalogen exchange of the vinyl bromide and addition to ethyl chloroformate gave 287. An inverse electron demand Diels-Alder reaction formed the hydrindane core of the gibberellins (289) and delivered the product as a single diastereomer. The authors hypothesize that the high facial selectivity may be attributed to the avoidance of steric interactions between the carboethoxy group and the $\mathrm{C} 7$ methylene protons in the transition state $\mathbf{2 8 8}$. This reaction delivers a fully functionalized cyclopentane intermediate that was converted to the $( \pm)-\mathrm{GA}_{111}$ methyl ester over seven steps.

Scheme 34. Total Synthesis of ( \pm )-GA $\mathbf{A}_{11}$ Methyl Ester (Ihara, 2000)

\footnotetext{
${ }^{64}$ Toyota, M.; Odashima, T.; Wada, T.; Ihara, M. J. Am. Chem. Soc. 2000, 122, 9036-9037.
} 

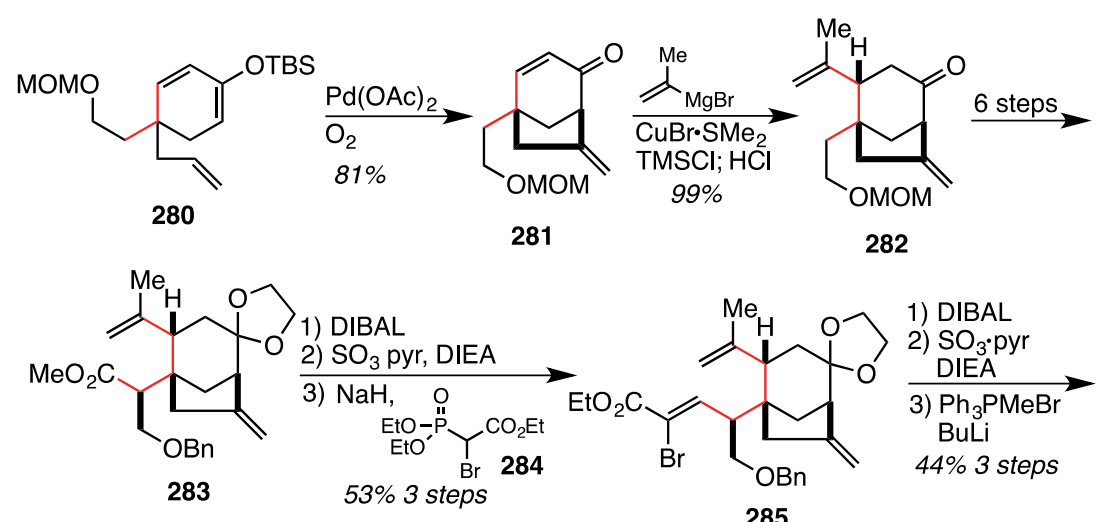

$E / Z: 1.3: 1$

285
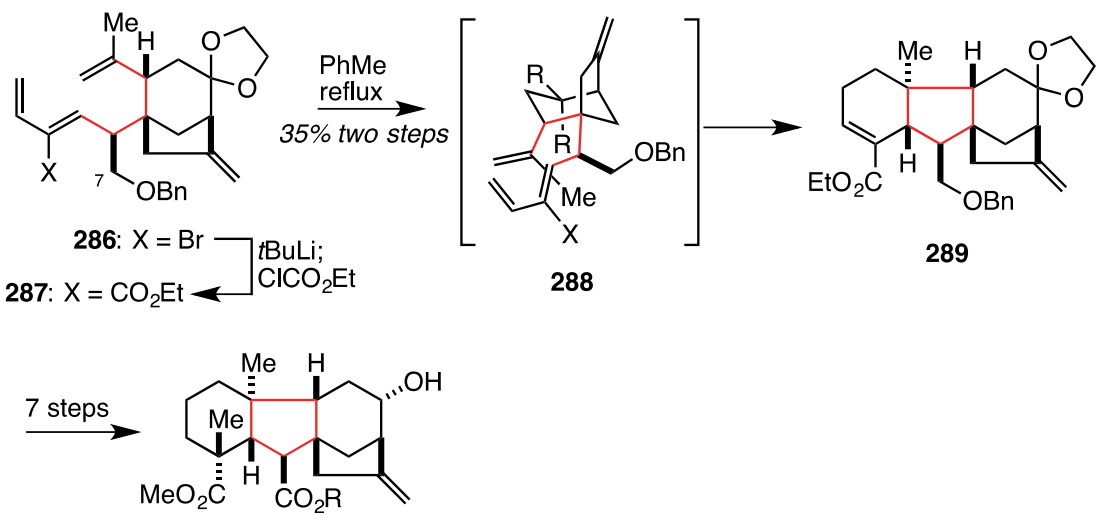

288

$( \pm)-\mathrm{GA}_{111}$ methyl ester: $\mathrm{R}=\mathrm{Me}$

$( \pm)-G A_{111}: R=H$

In the synthetic effort towards hexacyclinic acid, Kalesse ${ }^{65}$ constructs a fully functionalized cyclopentane with an intramolecular Diels-Alder reaction (Scheme 35). The route begins with the olefination of aldehyde 290, followed by a two-step oxidation state adjustment to give unsaturated aldehyde 292. An Evans aldol reaction of the aldehyde with 293 and subsequent conversion to the Weinreb amide provided 294. The aldol reaction appropriately sets two of the stereocenters for the fully functionalized cyclopentane. Following silylation of the secondary alcohol, the Weinreb amide was reduced to the aldehyde. Acetylide addition and oxidation of the resulting alcohol provided ynone 295. The IMDA reaction proceeded to furnish the hydrindane core in 297 with complete diastereocontrol. The selectivity can be attributed to the pseudo-equatorial positioning of the silyl ether in the boat-like transition state 296. Addition of vinyl cuprate occurs trans to the adjacent substitutent, and the resulting enolate protonates to give the $c i s$-fused hydrindane structure. Hydride reduction occurs on the concave face presumably to avoid interactions with the TBS ether delivering 298. This advanced intermediate contains appropriate substitution and stereochemical configuration for hexacyclinic acid.

Scheme 35. Synthetic Studies Towards Hexacyclinic Acid (Kalesse, 2004)

${ }^{65}$ Stellfeld, T.; Bhatt, U.; Kalesse, M. Org. Lett. 2004, 6, 3889-3892. 

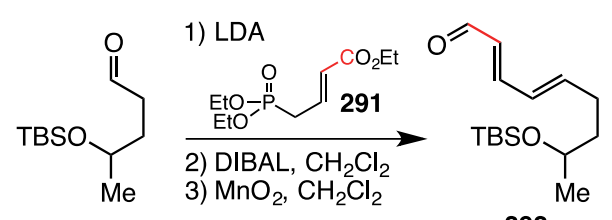

290

292
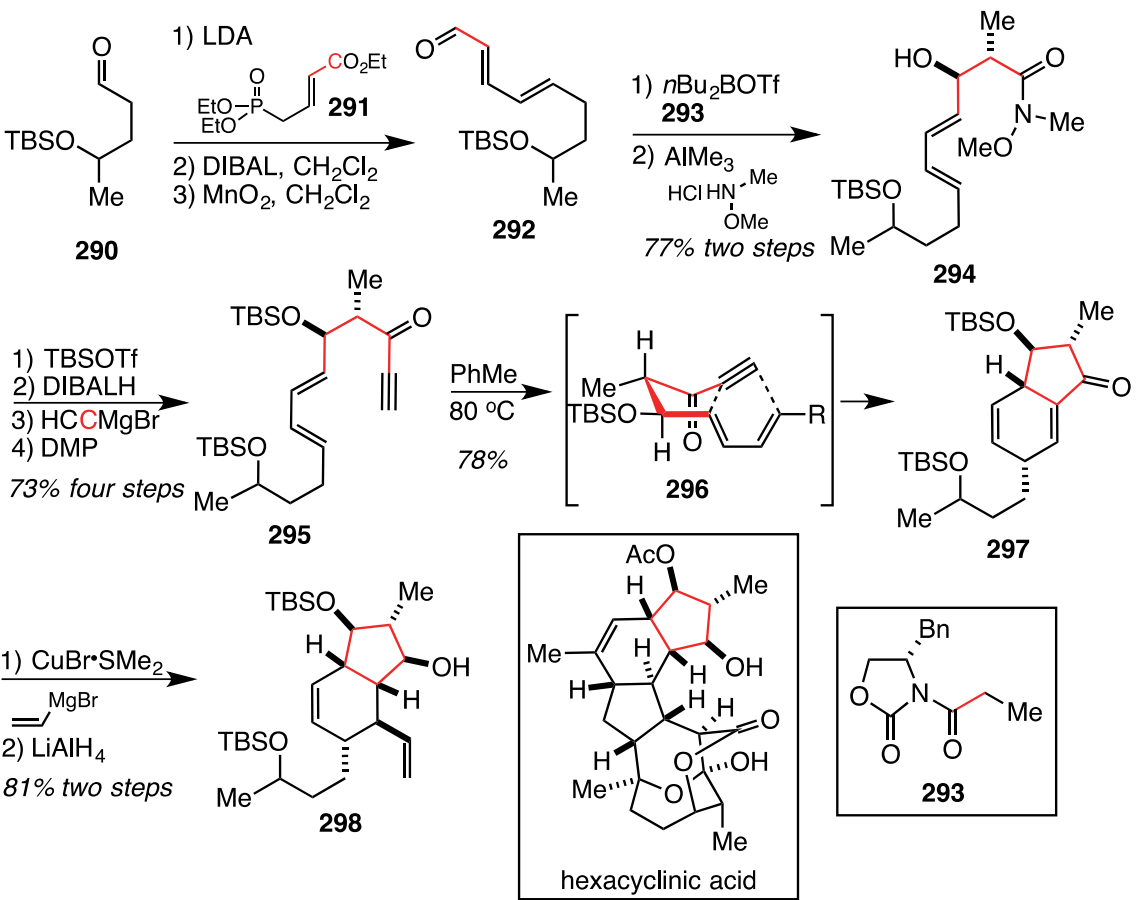

297

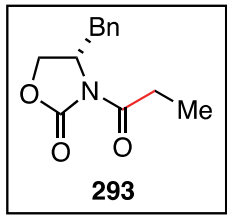

\section{$2.4[5+2]$ Cycloadditions}

The synthesis of $( \pm)$-epicolactone by Trauner used a biomimetic cycloaddition of two oxygenated arenes to construct a fully functionalized cyclopentane (Scheme 36). The route commences with vanillyl alcohol, which could be converted to cycloaddition partner 299 over six steps. The known metabolite epicoccine was synthesized in five steps from eudesmic acid. Addition of two equivalents of formalin to eudesemic acid in the presence of $\mathrm{HCl}$ resulted in chlorinated isobenzofuranone 300. Dechlorination with zinc delivered 301. The lactone was reduced in two steps with DIBAL followed by TFA and triethylsilane. Demethylation with boron tribromide furnished epicoccine. The key cycloaddition takes place in the presence of oxidant potassium ferricyanide to give epicolactone methyl ether $\mathbf{3 0 2}$ and the regioisomeric heterodimer $\mathbf{3 0 3}$. The cycloaddition sequence results in a pentacyclic cage-like structure with three contiguous quaternary stereocenters. Demethylation yields $( \pm)$-epicolactone.

Scheme 36. Total Synthesis of ( \pm )-Epicolactone (Trauner, 2015) 


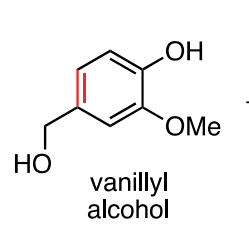

s steps<smiles>COc1cc(C(=O)O)cc(OC)c1OC</smiles>

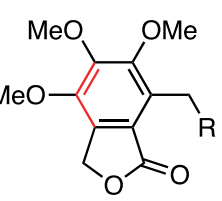<smiles>Cc1c(O)c(O)c(O)c2c1COC2</smiles>

eudesmic acid

300: $\mathrm{R}=\mathrm{Cl} \longrightarrow \mathrm{Zn}, \mathrm{THF}$

301: $\mathrm{R}=\mathrm{H} \longleftarrow$ aq. $\mathrm{KH}_{2} \mathrm{PO}_{4}$<smiles>COc1c(CO)cc(O)c(O)c1O</smiles>

299

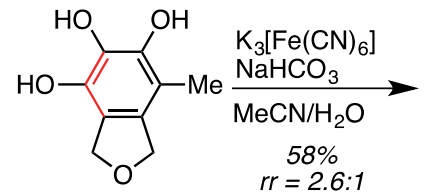

epicoccine

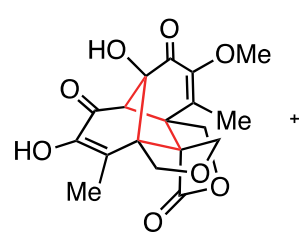

302

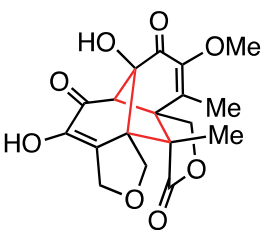

303

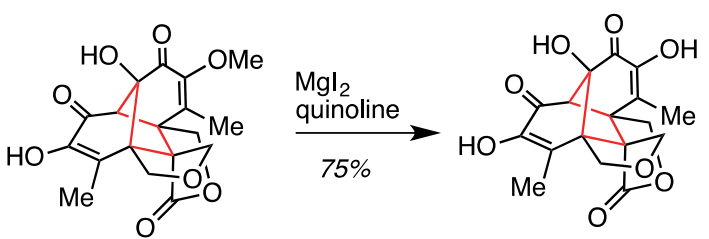

304

(士)-epicolactone

The formal synthesis of $( \pm)$-coriolin was completed by Wender ${ }^{66}$ and employs an arene-olefin photocyclization to construct a highly congested ring system containing a fully functionalized cyclopentane (Scheme 37). The synthesis begins with an addition of the Grignard derived from bromide 305 to aldehyde 306 . An in situ acetylation of the alkoxide delivers 307. Oxidative cleavage and Wittig olefination gave acetal 309. This material underwent photocyclization to give adduct $\mathbf{3 1 0}$ as the major product. Upon cleavage of the cyclopropane ring and reductive desulfuration, the triquinane structure is revealed in 311. Oxidation with peracid in the presence of water stereoselectively epoxidizes the cyclopentene and performs a Baeyer-Villiger oxidation of a transient aldehyde to give a formate (312). Semi-pinacol rearrangement of the epoxide gave cyclopentanone 313 .

Oxidation to the cyclopentenone proceeded with concomitant transformation of the formate to the corresponding silyl ether. The $\alpha$-sulfenyl group was installed providing compound 314. Acidic removal of the TMS group delivers intermediate 315, which was previously taken forward to $( \pm)$-coriolin by Danishefsky. ${ }^{34}$

\section{Scheme 37. Formal Synthesis of $( \pm)$-Coriolin (Wender, 1983)}

${ }^{66}$ (a) Wender, P. A.; Howbert, J. J. Tetrahedron Lett. 1982, 23, 3983-3986. (b) Wender, P. A.; Howbert, J. J. Tetrahedron Lett. 1983, 24, 5325-5328. 


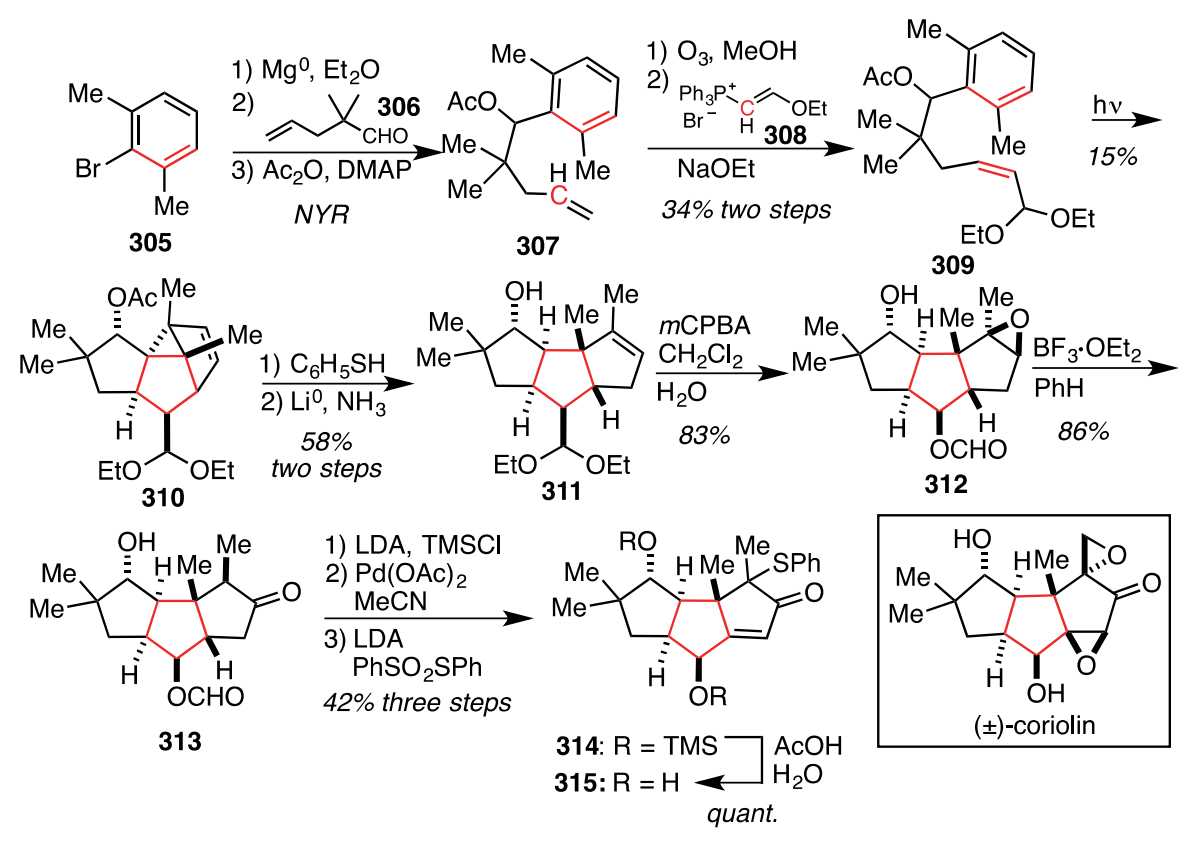

\section{Electrocyclizations}

Electrocyclizations ${ }^{67}$ are powerful ring-closing reactions that predictably follow the Woodward-Hoffman rules ${ }^{68}$ for conservation of orbital symmetry. As reactions that tolerate diverse substrates and predictably deliver cyclic products as single diasteromers, it is not surprising that they have been used to create fully functionalized cyclopentanes.

The Nazarov ${ }^{69}$ mechanism (Scheme 38) involves a pentadienyl cation (317) derived from Lewis (or Brønsted) acid complexation of a divinyl ketone (316), which undergoes a thermally promoted conrotatory $4 \pi$ electrocyclization to give an oxyallyl cation (318). Loss of a proton gives dienolate 319, which may be protonated to give the final cyclopentenone product 320. A main concern with the Nazarov cyclization of substituted non-symmetric substrates is the deprotonation of the locally symmetric oxyallyl cation $\mathbf{3 1 8}$. There are two regioisomeric possibilities for the deprotonation, and regioisomeric mixtures of products can be observed.

\section{Scheme 38 .}

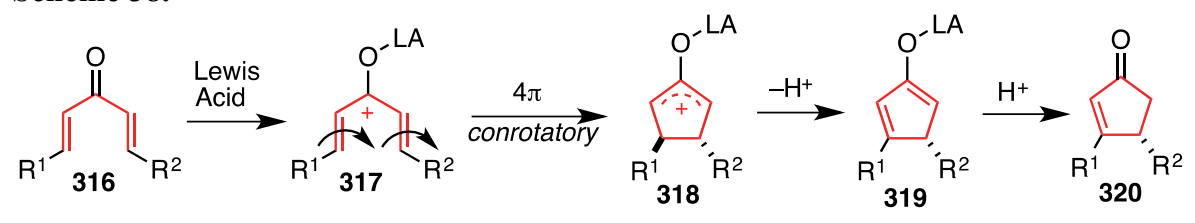

Modern advances in controlling the Nazarov reaction rely on substrates with coordinating groups, electronic polarization, or cation-stabilizing atoms to control the fate of the oxyallyl cation. ${ }^{70}$ The Nazarov reactions in this review all utilize electronically polarized divinyl ketones that contain a furan (Scheme 39, eq. 1), benzofuran (eq.

\footnotetext{
${ }^{67}$ For a review of biomimetic electrocyclizations in synthesis, see: Beaudry, C. M.; Malerich, J. P.; Trauner, D. Chem. Rev. 2005, 105, 4757-4778.

68 (a) Woodward, R. B.; Hoffmann, R. Angew. Chem. Int. Ed. 1969, 8, 781-853. (b) Hoffmann, R.; Woodward, R. B. Acc. Chem. Res. 1968, 1, 17-22.

${ }^{69}$ (a) Nazarov, I. N.; Zaretskaya, I. I. Zh. Obshch. Khim. 1960, 30, 746-754. (b) Habermas, K. L.; Denmark, S. E.; Jones, T. K. Org. React. 1994, 45, 1-158.

${ }^{70}$ (a) Denmark, S. E.; Jones, T. K. J. Am. Chem. Soc. 1982, 104, 2642-2645. (b) He, W.; Sun, X.; Frontier, A. J. J. Am. Chem. Soc. 2003, 125, 14278-14279. (c) Liang, G.; Gradl, S. N.; Trauner, D. Org. Lett. 2003, 5, 4931-4934. (d) Bee, C.; Leclerc, E.; Tius, M. A. Org. Lett. 2003, 5, 4927-4930. (e) Aggarwal, V. K.; Beffield, A. J. Org. Lett. 2003, 5, 5075-5078. (e) Occhiato, E. G.; Prandi, C.; Ferrali, A.; Guarna, A.; Venturello, P. J. Org. Chem. 2003, 68, $9728-9741$.
} 
2-3), or tricarbonyl (eq. 4) motif to give a single regioisomeric product. The reaction shown in eq. 1 is a particularly elegant example where silyl transfer arrests the Nazarov reaction at the dienolsilane 323, preserving both fully-substituted stereocenters created in the conrotatory electrocyclization. Two distinct Nazarov reactions of benzofuran-containing substrates have been used in the stereoselective synthesis of functionalized cyclopentenones related to rocaglamide (eq. 2-3).

\section{Scheme 39.}
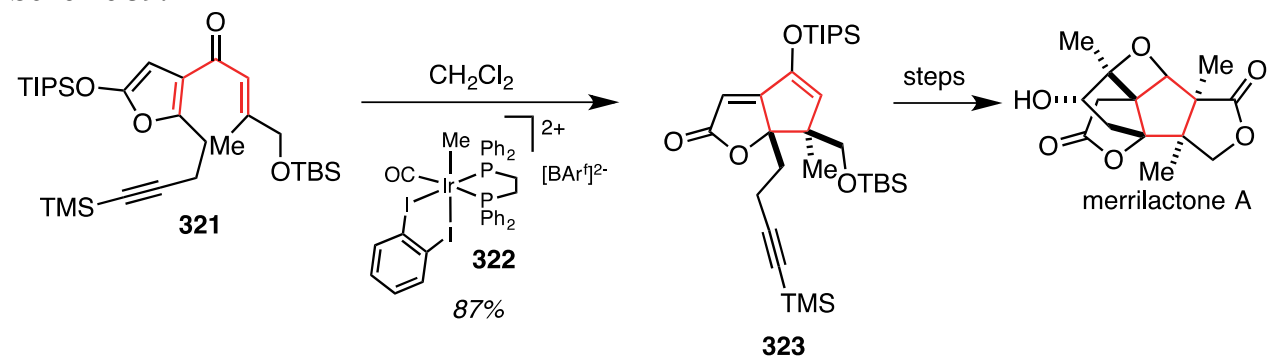

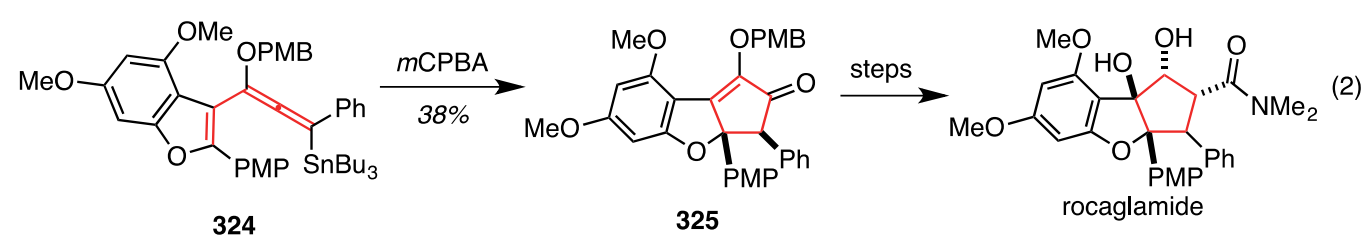<smiles>COc1cc(OC)c2c(C(=O)/C(C)=C/P)c([Pb])oc2c1</smiles>

326

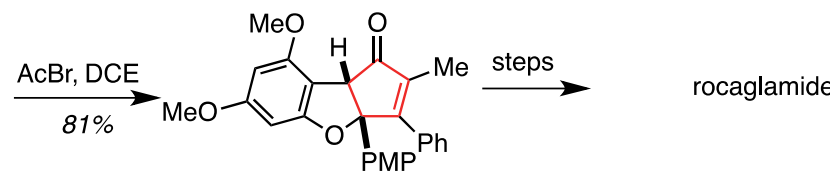

327

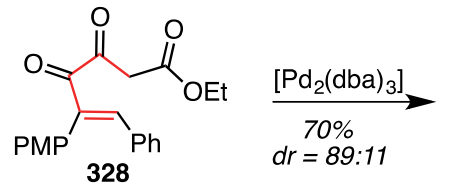

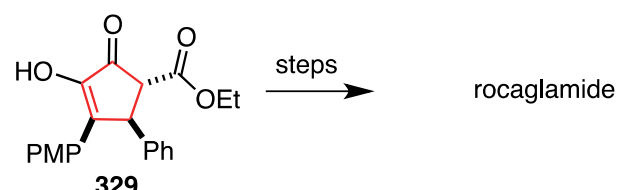

329

There has been one electrocyclization of a pyridine used in the synthesis of mannostatin A (Scheme 40). Photochemical cyclization of pyridinium chlorate (328) in the presence of water gave symmetric cyclopentene 329, which contained an amino-diol stereotriad. Subsequent steps, including an enzymatic desymmetrization, were used to prepare the natural product.

\section{Scheme 40.}

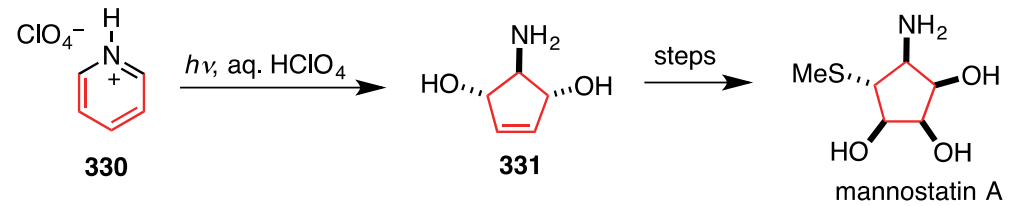

\subsection{Nazarov Reactions}

In the synthesis of $( \pm)$-merrilactone A, Frontier ${ }^{71}$ employs a Nazarov cyclization with a siloxyfuran to construct a functionally rich five-membered carbocycle (Scheme 41). Beginning with furan 332, lithiation and addition to Weinreb amide 333 furnishes divinyl ketone 321. Treatment with the dicationic iridium catalyst $\mathbf{3 2 2}$ promotes

${ }^{71}$ (a) He, W.; Huang, J.; Sun, X.; Frontier, A. J. J. Am. Chem. Soc. 2007, 129, 498-499. (b) He, W.; Huang, J.; Sun, X.; Frontier, A. J. J. Am. Chem. Soc. 2008, 130, 300-308 
the $4 \pi$-electrocyclic ring closure to deliver $\mathbf{3 2 3}$ as a single diastereomer. As anticipated, the stereospecific nature of the conrotatory electrocyclization ensures that the alkene stereochemistry is translated to the desired diastereomeric product. This is an elegant application of the Nazarov reaction that leads to vicinal fully substituted carbons, and the use of the siloxyfuran functional group allows for regioselective trapping of the oxyallyl cationic intermediate to form the dienoate. Chemoselective removal of the trimethyl silyl group was accomplished to give 1,6-enyne 334. Radical cyclization of the enyne gave a vinyl stannane intermediate, which upon protonolysis delivered an exocyclic alkene. Desilylation with fluoride provides the functionalized cyclopentanone 335. Conversion of the primary alcohol to the ethyl carbonate was followed by treatment with sodium hydride leading to a Diekmann cyclization yielding 337. This product was obtained in a 1:1 ratio with the uncyclized $\beta$-ketoester 336, which could be converted to the desired lactone by treatment with $\mathrm{TsOH}$.

Stereoselective methylation occurs to give the more stable cis-fused ring system in $\mathbf{3 3 8}$. Hydride reduction gave fully functionalized cyclopentane $\mathbf{3 3 9}$ as a 1.2:1 mixture with the undesired alcohol epimer. The undesired material could, however, be recycled by near quantitative oxidation with DMP and resubjection to the reduction conditions. An additional three steps were required to convert advanced intermediate $\mathbf{3 3 9}$ to the natural product.

Scheme 41. Total Synthesis of ( \pm )-Merrilactone A (Frontier, 2007)
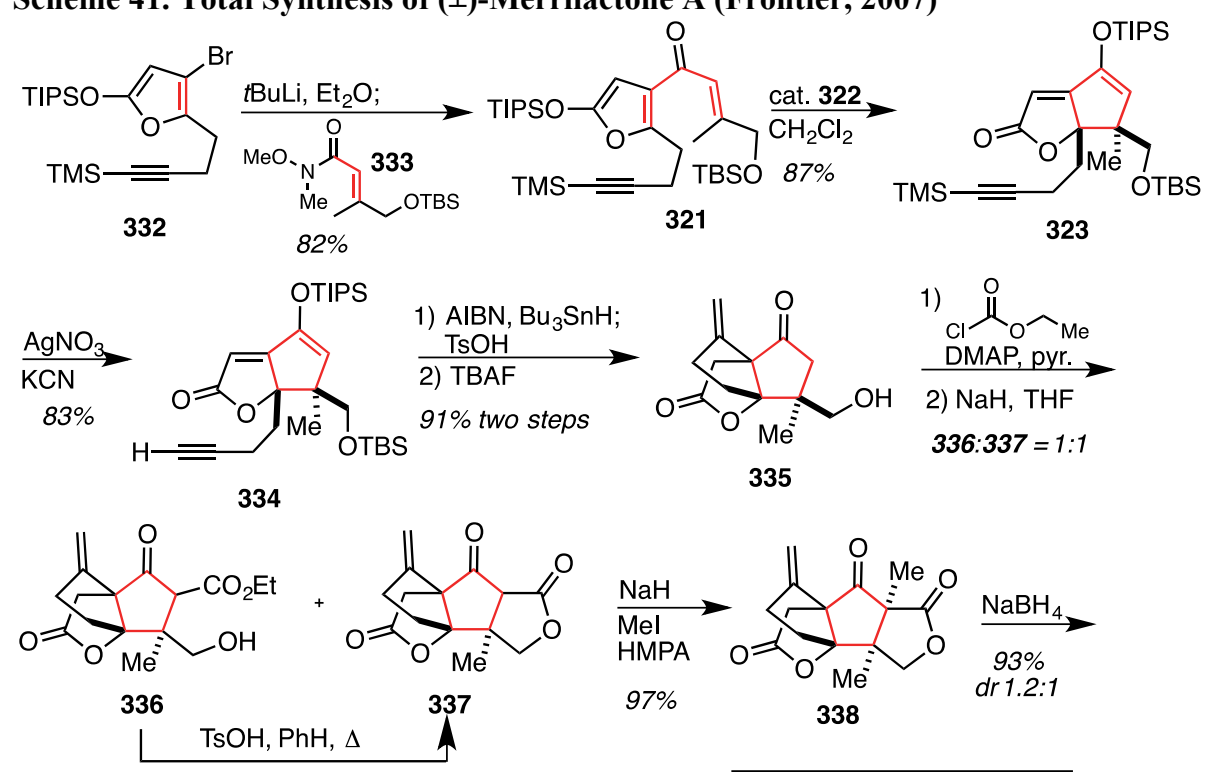

$90 \%$ two steps
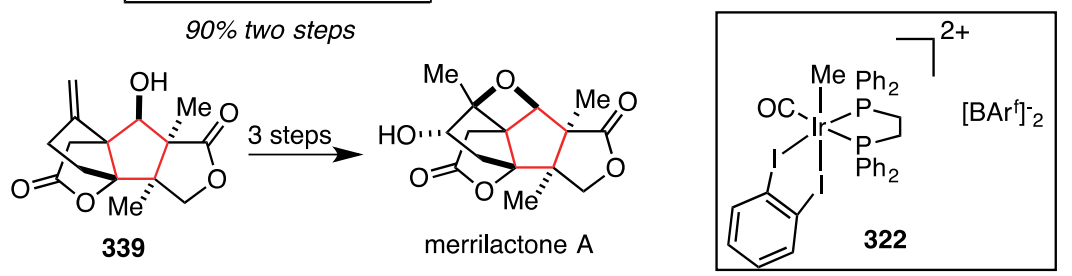

The synthesis of $( \pm)$-rocaglamide by Frontier ${ }^{72}$ utilizes a Nazarov cyclization of a benzofuran and a stannyl alkoxyallene to form a cyclopentenone (Scheme 42). The synthesis begins with addition of vinylmagnesium bromide to the known benzofuranone 340, dehydration and subsequent oxidative cleavage gave aldehyde 341 . Addition of phenyl acetylide and subsequent protection of the resulting alcohol as the PMB ether delivered 342 . Deprotonation of the propargylic position with tert-butylithium and trapping gave stannyl alkoxyallene 324 . Reaction with excess $m$ CPBA promoted the oxidation/Nazarov cyclization cascade to give cyclopentenone 325 as a single diastereomer. The reaction is thought to proceed by oxidation of the central allene carbon to give pentadienyl cation 343. Protodestannylation and conrotatory electrocyclization delivers cyclopentenone 325. Treatment with DDQ resulted in both removal of the PMB-ether and oxygenation of the bridgehead carbon. Triflation of the enolizable ketone delivers 345. A palladium-mediated carbonylation installs the final carbon substituent on the five-membered ring to give ester 346. Hydrogenation over platinum oxide delivers hydrogen from the concave face, and epimerization of the ester yields a single diastereomer. Finally, ketone reduction of

72 (a) Malona, J. A.; Cariou, K.; Frontier, A. J. J. Am. Chem. Soc. 2009, 131, 7560-7561. (b) Malona, J. A.; Cariou, K.; Spencer, W. T.; Frontier, A. J. J. Org. Chem. 2012, 77, 1891-1908. 
the cyclopentenone proceeds with substrate-directed delivery of hydride to afford a fully functionalized cyclopentane 347 . The synthesis of $( \pm$ )-rocaglamide was completed in two additional steps.

Scheme 42. Total Synthesis of ( \pm )-Rocaglamide (Frontier, 2009)
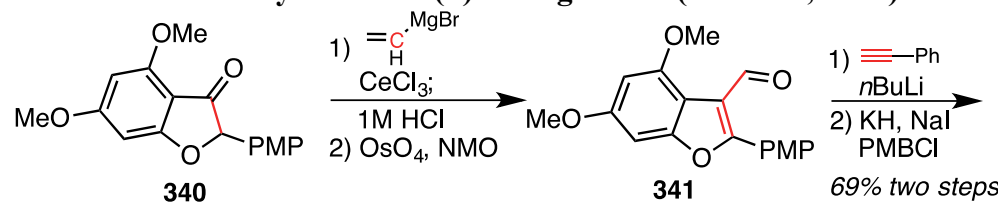

340

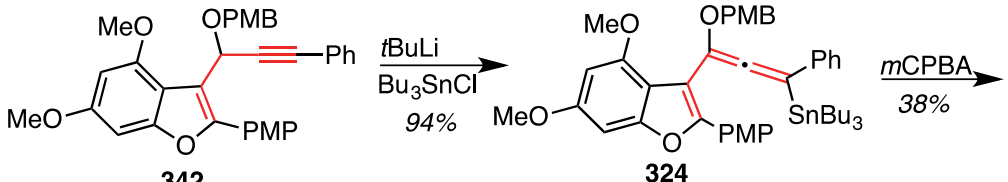

342

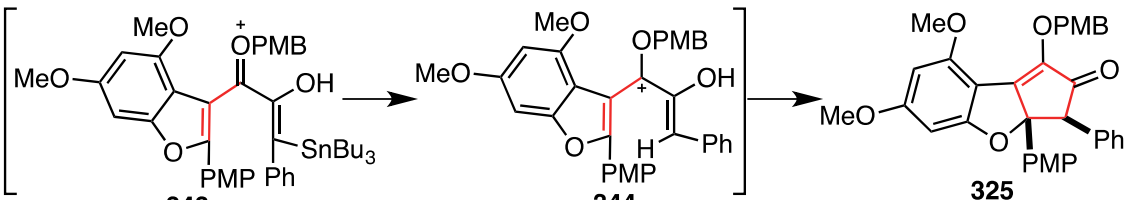

343

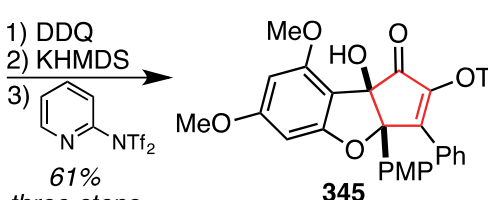

344

325

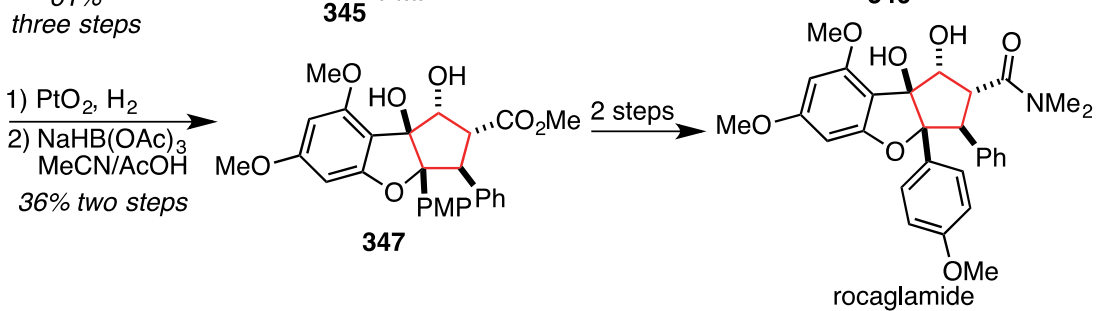

In the formal synthesis of $( \pm)$-rocaglamide by Magnus ${ }^{73}$, the five-membered ring of interest is formed through a Nazarov cyclization reaction (Scheme 43). The synthesis begins with an alkynylation of iodophenol 348 under Kumada conditions to give 349. Palladium-catalyzed carbonylative cyclization delivered benzofuran 350. Addition of diethyl ethylphosphonate gave a ketophosphonate. Olefination with benzaldehyde was performed under Masamune-Roush ${ }^{74}$ conditions to deliver $(E)$-configured 326. After experimentation with several Lewis acids, it was discovered that acetyl bromide gave the desired Nazarov cyclization product 327. It is postulated that acetylation of $\mathbf{3 2 6}$ gives an oxonium ion (351) which proceeds through the Nazarov cyclization to give $\mathbf{3 2 7}$ after aqueous workup. Oxidation with ceric ammonium nitrate installed the tertiary alcohol in 352. Four additional steps advanced intermediate 352 to functionalized cyclopentenone 346, which has been taken to ( \pm )rocaglamide by Frontier. ${ }^{72}$

Scheme 43. Formal Synthesis of ( \pm )-Rocaglamide (Magnus, 2012)

\footnotetext{
${ }^{73}$ Magnus, P.; Freund, W. A.; Moorhead, E. J.; Rainey, T. J. Am. Chem. Soc. 2012, 134, 6140-6142.

${ }^{74}$ Blanchette, M. A.; Choy, W.; Davis, J. T.; Essenfeld, A. P.; Masamune, S.; Roush, W. R.; Sakai, T. Tetrahedron Lett. 1984, 25, 2183-2186.
} 

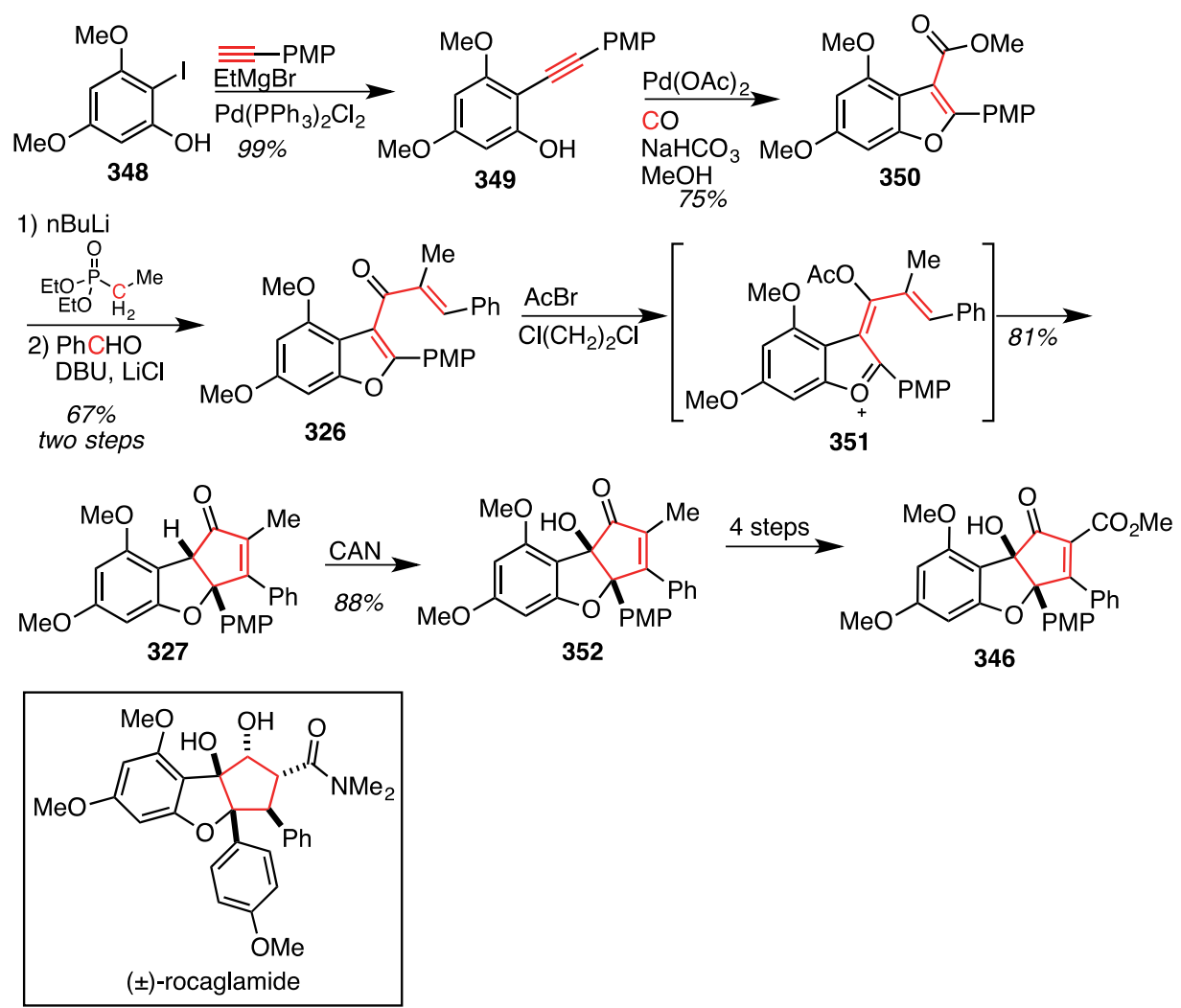

The total synthesis of (-)-rocaglamide by Tius ${ }^{75}$ used an enantioselective Nazarov-type cyclization to construct a functionalized cyclopentenone (Scheme 44). The route begins with reaction of vinyl carbonate 353 with cinnamate derivative 354 to deliver dienone $355 .{ }^{76}$ Oxidative cleavage of the DMB protecting group gave tricarbonyl 328. Palladium-catalyzed Nazarov electrocyclization proceeded in the presence of ligand $\mathbf{3 5 6}$ derived from D-(-)-tartrate, and gave desired product 329 with the appropriate $(S)$-C3 stereochemistry. Oxidative etherification and enol ether formation gave 357. Hydrolysis of the ethyl ester and conversion of the resulting carboxylate to the dimethyl amide delivered 358. The aryl lithium derived from 1-fluoro-3,5-dimethoxybenzene (359) was transmetallated with $\mathrm{LaCl}_{3}$ and added to ketone 360. Oxidative cleavage of the allyl protecting group furnished diol 361. Treatment with base led to formation of the core dihydroxybenzofuran substructure of rocaglamide via a nucleophilic aromatic substitution. Cleavage of the enol ether delivered a cyclopentanone, which was stereoselectively reduced following the method of Qin ${ }^{77}$ completing the synthesis of $(-)$-rocaglamide.

Scheme 44. Total Synthesis of (-)-Rocaglamide (Tius, 2015)

\footnotetext{
${ }^{75}$ Zhou, Z.; Tius, M, A. Angew. Chem. Int. Ed. 2015, 54, 6037-6040.

${ }^{76}$ Bresser, T.; Knochel, P. Angew. Chem. Int. Ed. 2011, 50, $1914-1917$.

${ }^{77}$ Li, H.; Fu, B.; Wang, M. A.; Li, N.; Liu, W. J.; Xie, Z. Q.; Ma, Y. Q.; Qin, Z. Eur. J. Org. Chem. 2008, $1753-1758$.
} 


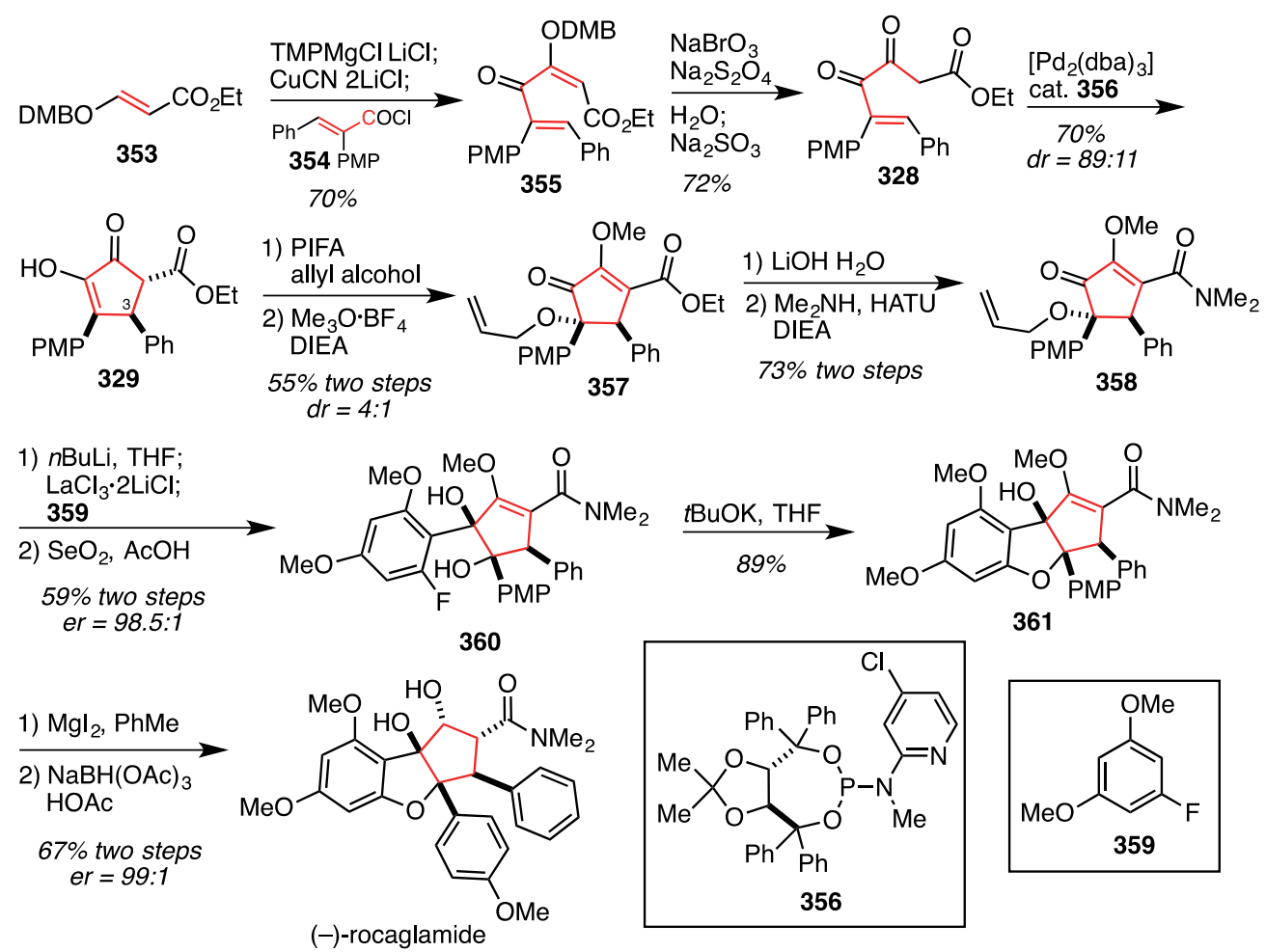

\subsection{Pyridinium Ring Contraction}

In the synthetic approach to members of the aminocyclopentitol family, Mariano ${ }^{78}$ developed a ring contraction of a pyridine to give a symmetric cyclopentene (Scheme 45). Specifically, photolysis of pyridinium perchlorate (330) induces a disrotatory $4 \pi$ electrocyclization to give cation 362, which is intercepted by water. Treatment with acetic anhydride and DMAP leads to the isolated product 363. Enzymatic desymmetrization delivers chiral cyclopentene 364. Protecting group manipulation over three steps provides orthogonally protected diol $\mathbf{3 6 5}$. A Pd-catalyzed addition of methylthiol delivers 366 with retention of stereochemistry. ${ }^{79}$ Removal of the silyl protecting group was performed with hydrofluoric acid. Inversion of the secondary alcohol following the conditions developed by Wipf ${ }^{80}$ delivers cis-amido alcohol 367. Directed dihydroxylation delivers a triol and completes the fully functionalized cyclopentane. Amide hydrolysis yields (+)-mannostatin A as its hydrochloride salt.

Scheme 45. Total Synthesis of (+)-Mannostatin A (Mariano, 1998)

\footnotetext{
${ }^{78}$ Ling, R.; Mariano, P. S. J. Org. Chem. 1998, 63, 6072-6076.

${ }^{79}$ Trost, B. M.; Scanlan, T. S.; Tetrahedron Lett. 1986, 27, 4141-4144.

${ }^{80}$ Wipf, P.; Miller, C. P. J. Org. Chem. 1993, 58, 1575-1578.
} 


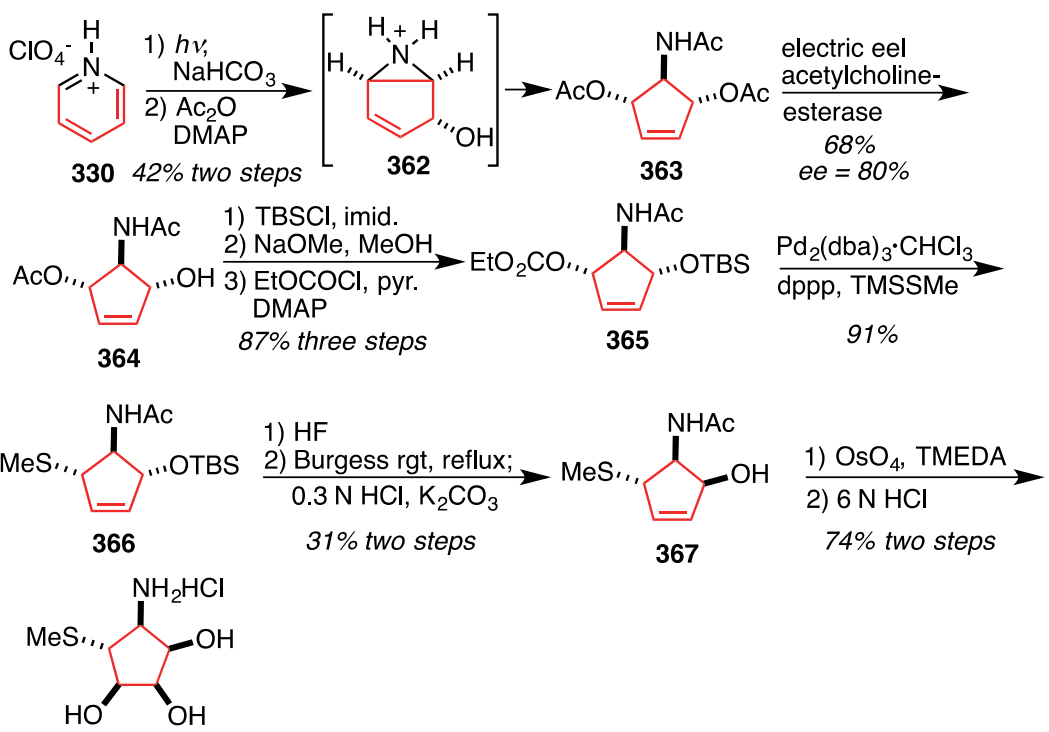

(+)-mannostatin $\mathrm{A} \cdot \mathrm{HCl}$

\section{Transition-Metal Mediated Cyclizations}

Transition metal complexes mediate a diverse variety of ring-forming reactions. ${ }^{81}$ Control of regio- and stereoselectivity can be quite high in these transformations, and as a result, it is not surprising that transition metal-mediated reactions have been used in the assembly of fully functionalized cyclopentane natural products.

The Pauson-Khand ${ }^{82,83}$ reaction is a well-established method for the construction of five-membered rings. In principle, up to four substituents and two stereocenters can be installed on a five-membered carbocycle in a single transformation (Scheme 46, eq. 1). The regiochemistry and stereochemistry are key concerns in the strategic use of this reaction, and tethering the reactive components is a common strategy that limits the regiochemical possibilities. The Pauson-Khand has been used in three distinct strategies directed toward the synthesis of natural products with fully functionalized cyclopentanes. The intermolecular Pauson-Khand has been used to combine an alkene (e.g. 368), an alkyne (e.g. 369), and a molecule of CO to construct a 5membered carbocycle (370, eq. 1). The intramolecular Pauson-Khand of a skipped enyne (371) has been used, which converts the molecular tether into the functionalized carbocycle (372) as part of a fused bicycle (eq. 2).

Finally, there is an example of a Mo-promoted intramolecular Pauson-Khand reaction where an aldehyde (373) serves as a reactive component in the cyclization to form furanone 374. In this case, the five-membered carbocycle is also formed from the atoms in the intramoleclar tether (eq. 3). The latter two strategies are particularly well-suited for the synthesis of polyquinanes and related structure types with fused five-membered rings.

Scheme 46.

${ }^{81}$ Note that the Pd-catalyzed [3+2]-cycloaddition appears in Section 2.2.

${ }^{82}$ Khand, I. U.; Knox, G. R.; Pauson, P. L.; Watts, W. E.; Foreman, M. I. J. Chem. Soc., Perkin Trans. 1 1973, $977-$ 981.

${ }^{83}$ For reviews, see: (a) Schore, N. E. Org. React. 1991, 40, 1-90. (b) Brummond, K. M.; Kent, J. L. Tetrahedron 2000, $56,3263-3283$. 


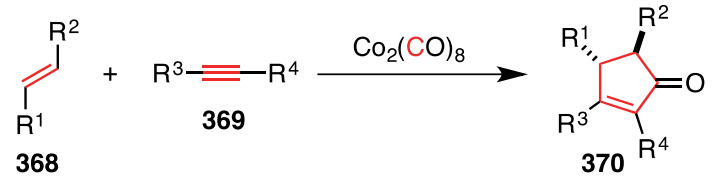<smiles>[R]C#CC[R7]([H])([H])CCC=C</smiles>

371<smiles>[R]C#CCCCC[R]</smiles>

373

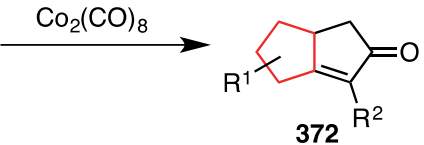

$372^{R^{2}}$

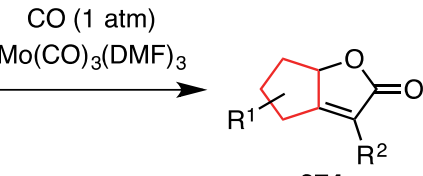

An attractive strategic advantage of the Pauson-Khand reaction is the production of an enone product, which can be subsequently manipulated with countless alkene or carbonyl transformations. In the context of the synthesis of cyclopentane targets, this advantage is accompanied by the requirement that the $\mathrm{sp}^{2}$-hybridized carbons must be processed into substituted $\mathrm{sp}^{3}$-hybridized carbons. Perhaps for this reason, many examples of the Pauson-Khand in this review occur early in the synthetic route. However, judicious use of reactions that simultaneously leverage the reactivity of the enone and build complexity can be quite powerful. For a particularly elegant example, the Pauson-Khand reaction to form $\mathbf{3 7 6}$ establishes two fused five-membered carbocycles, and just seven subsequent transformations established the required susbstitution and stereochemistry on the adjacent fully functionalized cyclopentanes of ryanodol (Scheme 47).

Scheme 47.

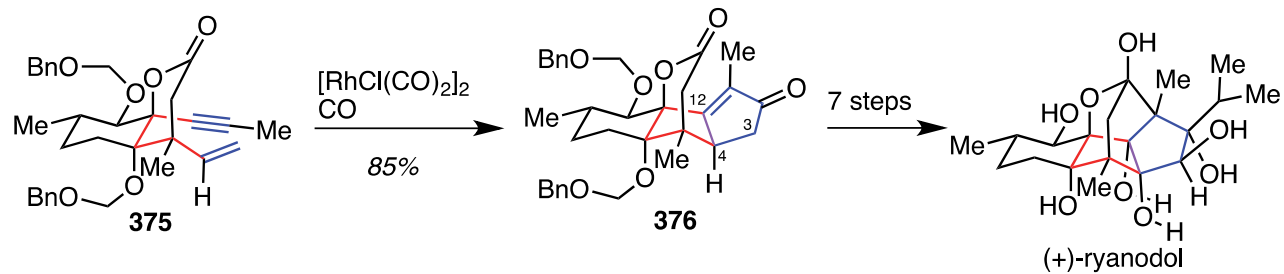

Ring-closing metathesis ${ }^{84}$ is another venerable reaction that performs reliably to construct five-membered rings from 1,6-diene starting materials (Scheme 48). An auspicious feature of alkene metathesis using Grubbs' catalyst is the high functional-group tolerance of the reaction, and this aspect of the reaction allows cyclization of $\mathbf{3 7 7}$ to cyclopentene $\mathbf{3 7 8}$ to take place in the presence of many resident functional groups. In order to form a fully functionalized cyclopentane, the alkene product must undergo further transformations. As with other cyclization strategies where the tether becomes the eventual cyclopentane, establishing the stereocenters on the tether is a key strategic consideration.

\section{Scheme 48.}

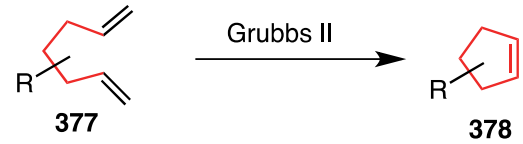

$\alpha$-Diazocarbonyls (e.g. 379, 381) react with metals to form metal carbenoid intermediates that may undergo cyclization reactions to form cyclopentanones (e.g. 380, 382) (Scheme 49). In the context of fully functionalized cyclopentane synthesis, a $\mathrm{C}-\mathrm{H}$ insertion reaction and a cyclopropanation and have been performed. Notably, both reactions form all-carbon quaternary stereocenters on the functionalized cyclopentane.

\section{Scheme 49.}

\footnotetext{
${ }^{84}$ For reviews, see: (a) Grubbs, R. H.; Miller, S. J.; Ru, G. C. Acc. Chem. Res. 1995, 28, 446-452. (b) Yet, L. Org. React. 2016, 89, 1-1304.
} 

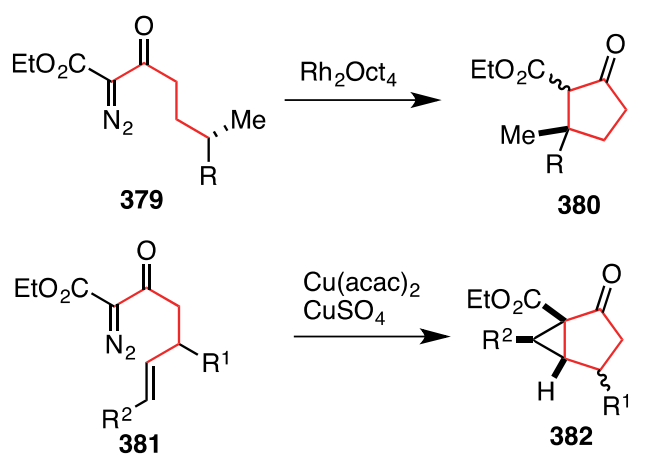


\subsection{Pauson-Khand Reactions}

In the synthesis of $( \pm)$-axinellamines $A$ and $B, \operatorname{Baran}^{85}$ utilizes a Pauson-Khand reaction of a symmetric unactivated alkene to construct a functionalized cyclopentenone (Scheme 50). Initial efforts towards cyclization of alkene 383 and propargyl amine 384 led to a decomposition of the alkyne cobalt complex. Conditions were developed using NMO and ethylene glycol as an additive to dramatically improve the yield of the desired cyclopentenone 385. Luche reduction of the ketone proceeded with concomitant desilylation. The resulting crude mixture was chlorinated to give trichloride $\mathbf{3 8 6}$ as an inconsequential mixture of epimers. A desymmetrizing Barbier reaction was performed with zinc and indium metals to give addition product $\mathbf{3 8 9}$. In the addition step, the aldehyde $\mathbf{3 8 8}$ approaches opposite the chloromethyl group of $\mathbf{3 8 7}$ to set the sterocenter on the cyclopentene. Displacement with azide, Boc-deprotection and installation of the guanidine (with 390) yielded 391. A chlorination-induced cyclization was achieved with $t$-butyl hypochlorite with $\mathrm{TfNH}_{2}$ as an essential required additive delivering 392 as a single diastereomer. Presumably, stereoselective formation of a bridged-chloronium species forms opposite the adjacent alkyl substituents. Opening of the chloronium ion with the tethered guanidine furnishes the spirocycle with complete stereocontrol. This transformation completes the fully functionalized cyclopentane in $( \pm)$-axinellamines A and B, and the natural product was completed in an additional five steps.

Scheme 50. Total Synthesis of ( \pm )-Axinellamines A and B (Baran, 2011)
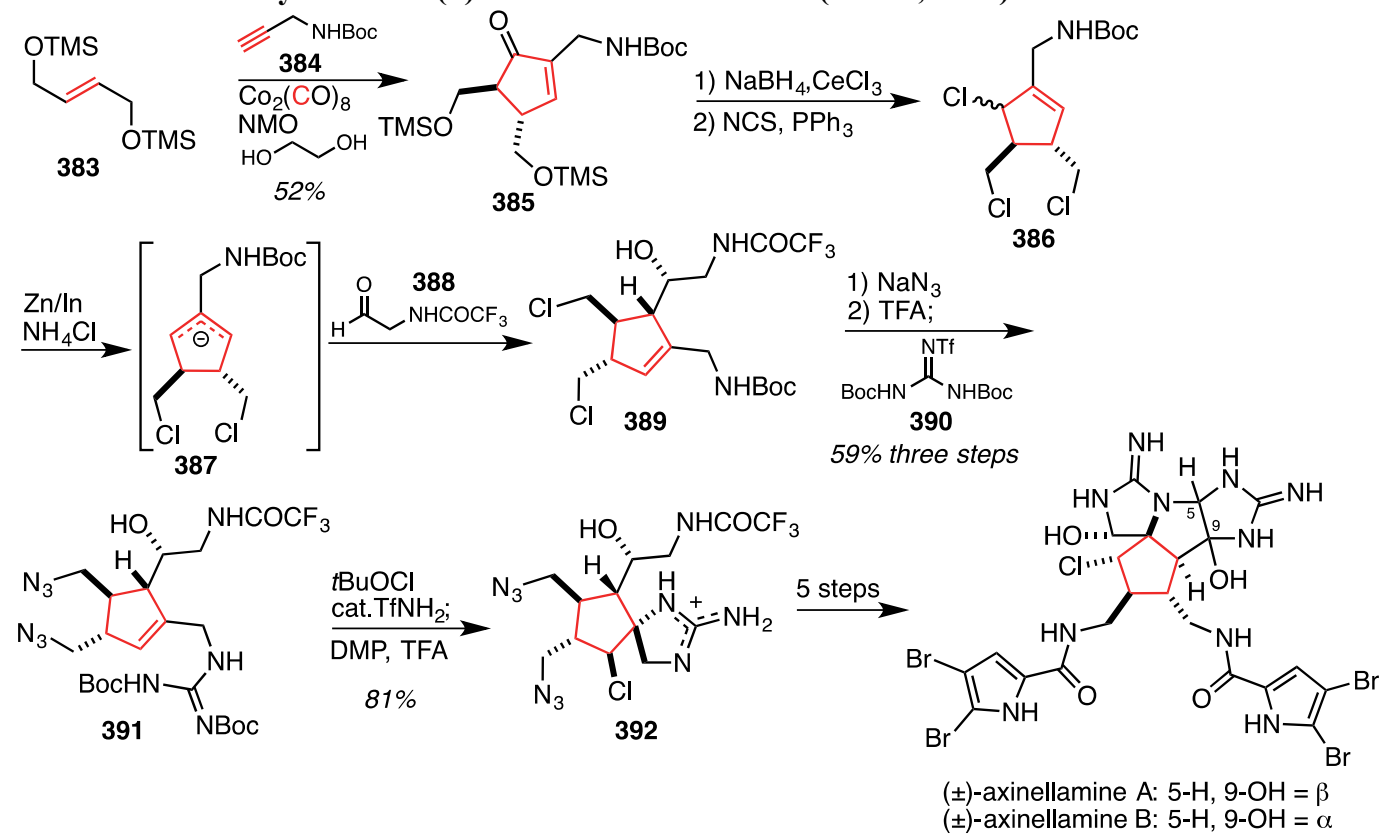

The pentacyclic sesquiterpene $( \pm)$-merrilactone A was synthesized by Zhai ${ }^{86}$, and utilizes a hetero-Pauson-Khand reaction to construct a $\gamma$-lactone fused to a functionalized cyclopentane (Scheme 51). The route begins with a Johnson-Claisen rearrangement of alcohol 393. The rearrangement occurs through the anticipated chair-like transition state and proceeds with a $d r$ of 3.8:1 favoring the desired product 394. Simultaneous desilylation and lactonization yields substituted $\gamma$-lactone $\mathbf{3 9 5}$ as an inconsequential mixture of racemic diastereomers. Deprotonation of lactone 395 gave a single racemic $(E)$-enolate that added to 3-trimethylsilylpropynal (396) to deliver 397 and $\mathbf{3 9 8}$ as a 1:1 mixture of racemic distereomers. The aldol reaction proceeds with near complete Zimmerman-Traxler control but with no facial selectivity with respect to the chiral racemic enolate. This mixture of distereomers was advanced 3 steps to to give aldehyde 399, which was purified from the undesired diastereomer. Note that the undesired diastereomer could be recycled to 397 over three steps. The key heteroPKR was accomplished by stirring aldehyde 399 with $\left[\mathrm{Mo}(\mathrm{CO})_{3}(\mathrm{DMF})_{3}\right]$ first in an atmosphere of argon and then under $1 \mathrm{~atm}$ of carbon monoxide to give tricycle intermediate 400. The unsaturated lactone was converted to siloxyfuran $\mathbf{4 0 1}$ by treatment with TBSOTf. Conjugate addition to methyl vinyl ketone (MVK) delivers adduct 402. The good facial selectivity $(d r=7.2: 1)$ is presumably because of the addition of MVK on the face opposite the large TBS-ether. A radical cyclization gives a tetracyclic product in good (albeit inconsequential) selectivity

${ }^{85}$ Su, S.; Rodriguez, R. A; Baran, P. S. J. Am. Chem. Soc. 2011, 133, 13922-13925.

${ }^{86}$ Chen, J.; Gao, P.; Yu, F.; Yang, Y.; Zhu, S.; Zhai, H. Angew. Chem. Int. Ed. 2012, 51, 5897-5899. 
yielding a tertiary alcohol. Simulateneous desilylation and dehydration occurred with acid in refluxing benzene to yield alkene 403. The alcohol stereocenter could be inverted by oxidation to a ketone and hydride reduction, which gave a mixture of the desired alcohol $\mathbf{4 0 4}$ with its alcohol epimer, which could be recycled. This intermediate was advanced two steps to the natural product.

\section{Scheme 51. Total Synthesis of ( \pm )-Merrilactone A (Zhai, 2012)}
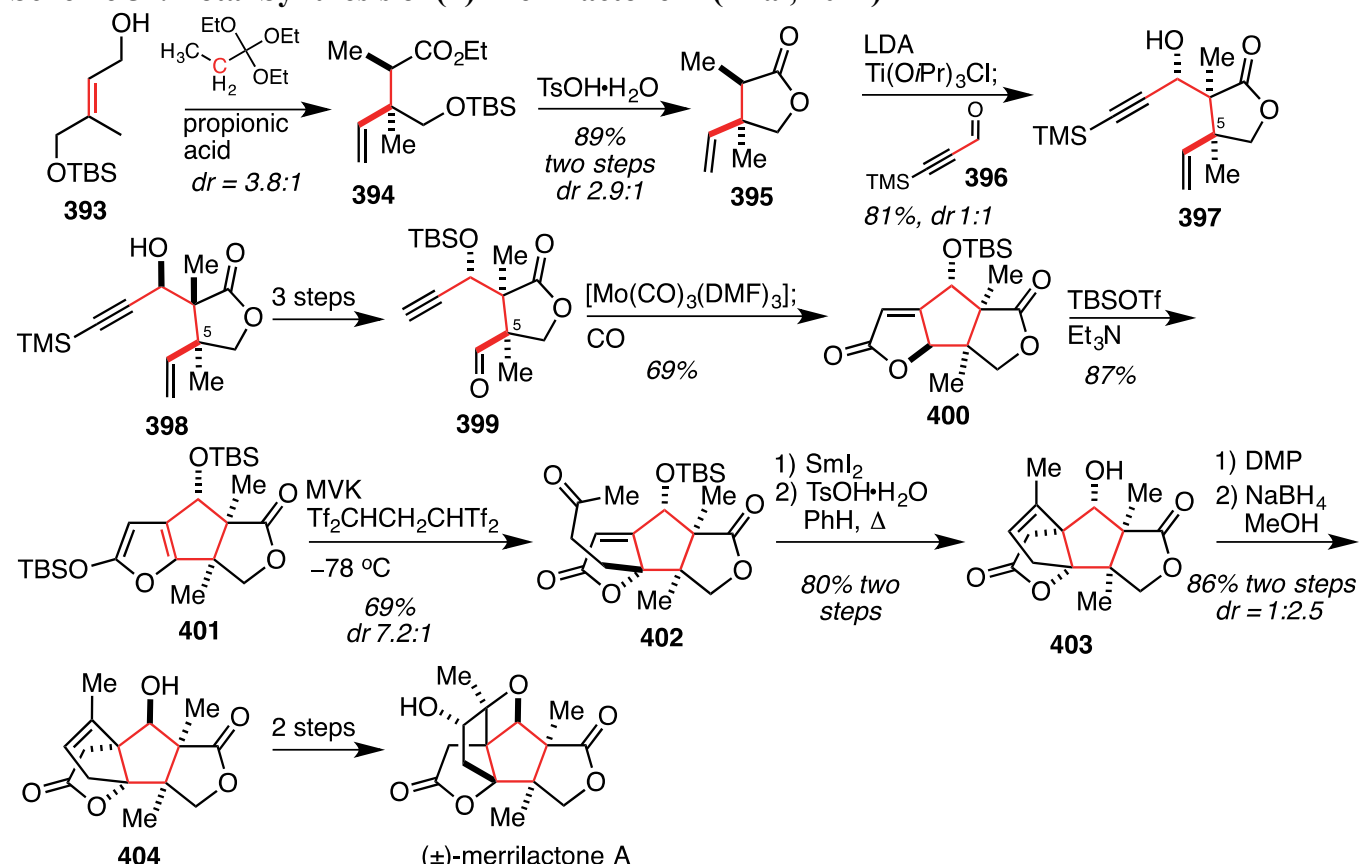

In the Reisman ${ }^{87}$ synthesis of (+)-ryanodol, a Pauson-Khand reaction constructs two five-membered rings that eventually become fully functionalized (Scheme 52). The route begins with a stereoselective oxidation of $(S)$ pulegone to give diol 406. The hydroxyl groups were protected as benzyloxymethyl ethers to give 407. Addition of the magnesium acetylide occurred preferentially with equatorial approach $(d r=5: 1)$. Chemoselective ozonolysis of the alkene gave methyl ketone 408. The unsaturated lactone of $\mathbf{4 0 9}$ was installed by a 1,2-addition of ethoxyethynylmagnesium bromide followed by a silver-catalyzed cyclization and elimination. Conjugate addition of divinyl cuprate occurred stereoselectively to construct the all quaternary stereocenter and deliver enyne 375. The Pauson-Khand cyclization occurred with high yield and good $d r$ when using $\left[\mathrm{RhCl}(\mathrm{CO})_{2}\right]_{2}$ to furnish 376. This spectacular transformation creates two fused five-membered rings with substitution and stereochemistry appropriate for the congested cis-diquinane embedded in the ryanodol architecture. Heating of the enone with selenium dioxide and molecular sieves enabled the stereoselective installation of the $\mathrm{C} 4, \mathrm{C} 12$ synvicinal diol and oxidation of $\mathrm{C} 3$ in a single step producing the first fully functionlized cyclopentane in $\mathbf{4 1 0}$. Enol triflate formation was followed by a Pd-catalyzed cross-coupling with stannane 411 to install the final carbons of the target in 412. Hydride reduction occurred from the less hindered face of the ring system, and hydrogenation of the disubstituted alkene with simultaneous hydroxyl deprotection delivered $(+)$-anhydroryanodol. Epoxidation with trifluoroperacetic acid and reductive cyclization onto the lactone delivered $(+)$-ryanodol.

Scheme 52. Total Synthesis of (+)-Ryanodol (Reisman, 2016)

${ }^{87}$ Chuang, K. V.; Xu, C.; Reisman, S. E. Science 2016, 353, 912-915. 

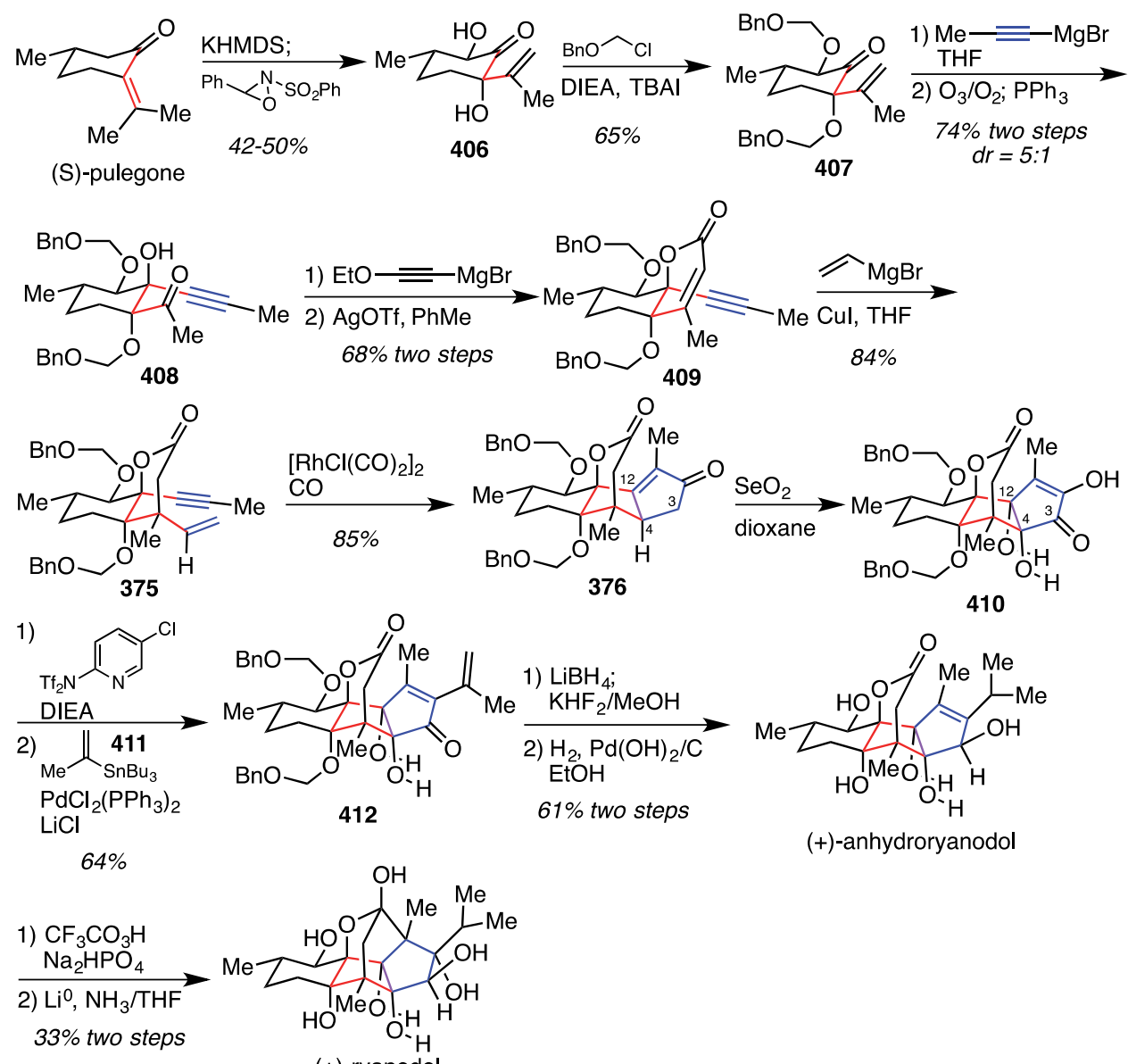

(+)-anhydroryanodol

(+)-ryanodol

In the formal synthesis of $( \pm)$-coriolin, Magnus ${ }^{88}$ employs a Pauson-Khand reaction to construct a functionalized cyclopentanone (Scheme 53). The synthesis begins with addition of lithium-TMS acetylide to aldehyde 413, and TBS protection of the resulting alcohol delivers 414. Removal of the TMS ether and methylation gave cyclization precursor 415 . Heating enyne 415 with $\mathrm{Co}_{2}(\mathrm{CO})_{8}$ in a solution saturated with carbon monoxide yielded cyclopentenone $\mathbf{4 1 6}$ as the major diastereomer. The origin of the diastereoselectivity is not known; however, the silyl acetylene analog gave a much higher diastereomer ratio $(d r=26: 1)$. Hydrogenation of the enone gave a cis-bicyclo[3.3.0]octane which could be allylated on the convex face. A Wacker oxidation delivers diketone 417. Treatment with $t \mathrm{BuOK}$ induced an aldol condensation yielding tricyclic enone 418. Deprotonation with $t \mathrm{BuOK}$ followed by acidic workup gave deconjugated $\beta, \gamma$-enone 419. Epoxidation with $m \mathrm{CPBA}$ proceeds to give the cis-fused dihydroquinane substructure. Basic workup induces elimination to form the enone. Deprotection of the silyl group delivers $\mathbf{1 2 9}$ which is an intermediate in a synthesis of coriolin by Matsumoto. ${ }^{32}$

\section{Scheme 53. Formal Synthesis of ( \pm )-Coriolin (Magnus, 1983)}

${ }^{88}$ (a) Exon, C.; Magnus, P. J. Am. Chem. Soc. 1983, 105, 2477-2478. (b) Magnus, P.; Exon, C.; Albaugh-Robertson, P. Tetrahedron 1985, 41, 5861-5869. 

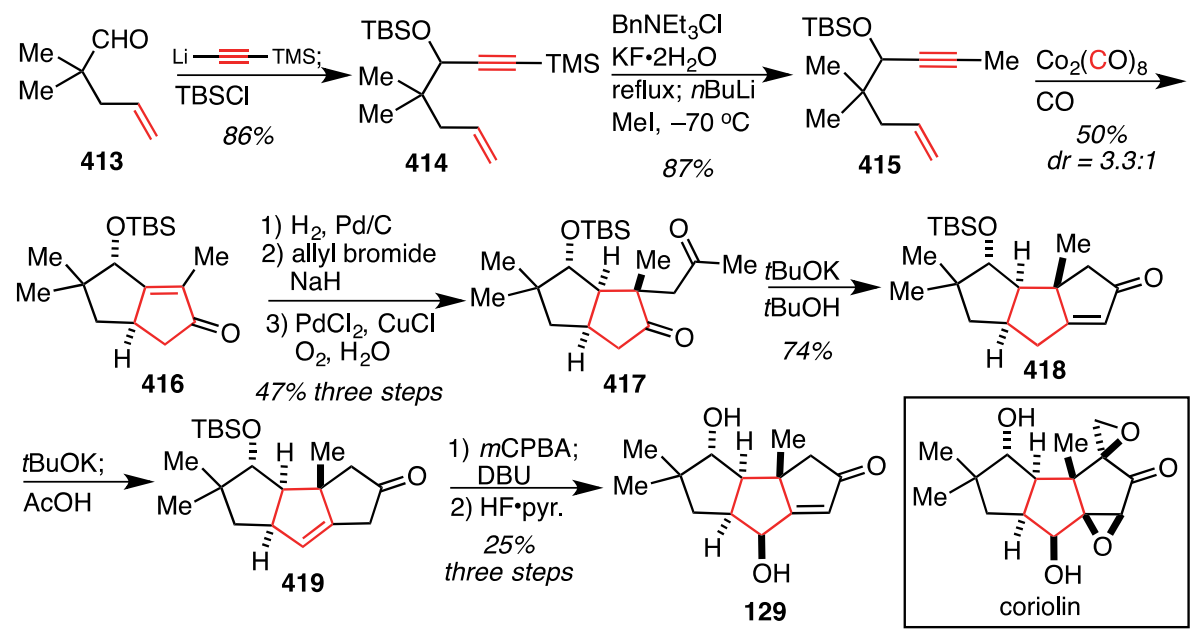

In the formal synthesis of $( \pm)$-coriolin, Oppolzer ${ }^{89}$ employs a nickel-catalyzed metallo-ene-type cyclization to construct a cyclopentanone (Scheme 54). Beginning with aldehyde 413, addition of a lithium acetylide 420 delivers a 1,5-enyne. The resulting alcohol was silylated and the THP group was removed to give 421. Transselective reduction of the alkyne was followed by two-step iodination of the alcohol to give allylic iodide $\mathbf{4 0 2}$. The key cyclization reaction was performed by stirring diene $\mathbf{4 2 2}$ with $\mathrm{Ni}(\mathrm{COD})_{2}$ under an atmosphere of carbon monoxide to deliver two isolable products. The desired ketoester $\mathbf{4 2 4}$ was obtained as a 3:2 ratio with the isomeric lactone 423. The authors did not comment on the origin of diastereoselectivity; however, it is plausible the favored diastereomer positions the ether and alkyl groups on the less hindered convex face of the cis-fused bicycle. Saponification of this mixture with lithium hydroxide in aqueous methanol provided ketoacid 425 . The unpurified material was taken forward to a Barton-type decarboxylation ${ }^{90}$ yielding methylcyclopentanone 426. Stereoselective allylation occurs from the convex face of the ring system to furnish 427, which is an intermediate synthesized by Magnus ${ }^{88}$ in a synthesis of coriolin.

Scheme 54. Formal Synthesis of $( \pm)$-Coriolin (Oppolzer, 1992)
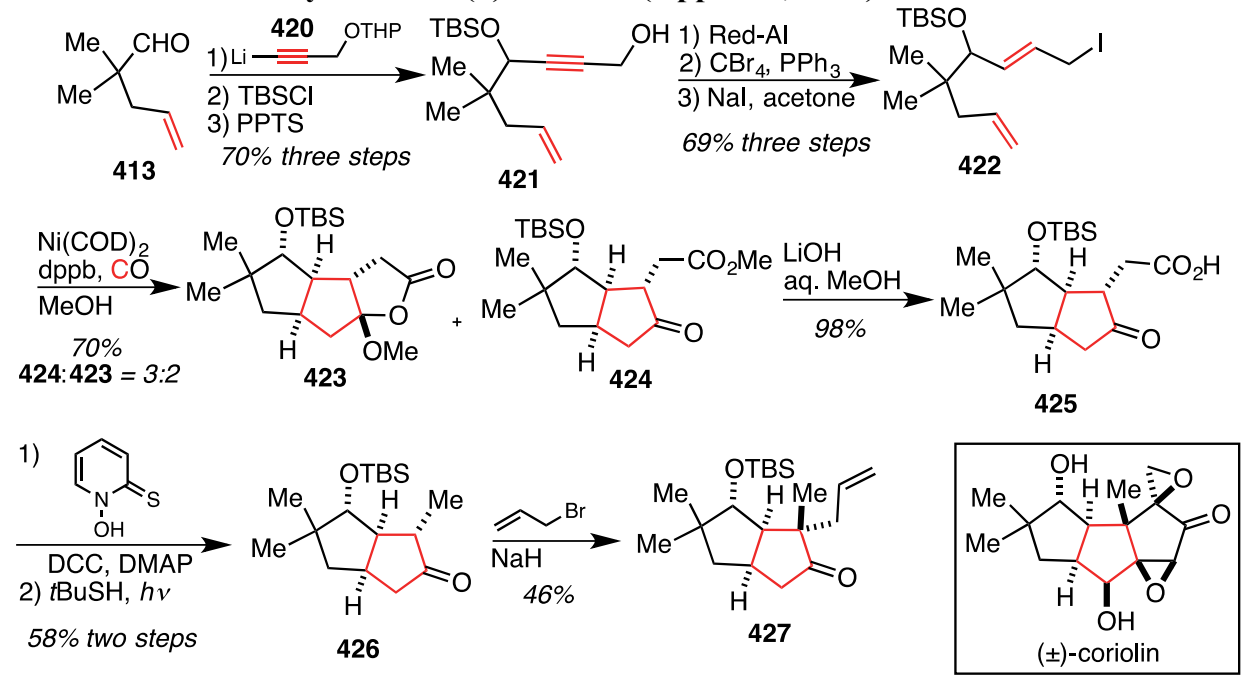

\subsection{Ring-Closing Metathesis}

\footnotetext{
${ }^{89}$ Oppolzer, W.; Ando, A. Chimia 1992, 46, 122-125.

90 (a) Barton, D. H. R.; Dowlatshahi, H. A.; Motherwell, W. B.; Villemin, D. A. J. Chem. Soc., Chem. Commun. 1980, 732-733; (b) Barton, D. H. R.; Crich, D.; Motherwell, W. B. Tetrahedron Lett. 1983, 24, 4979-4982; (c) Barton, D. H. R.; Crich, D.; Motherwell, W. B. J. Chem. Soc., Chem. Commun. 1983, 939-941.
} 
In the synthesis of (-)-allosamizoline, Donohoe ${ }^{91}$ employs a ring-closing metathesis to construct a functionalized cyclopentene (Scheme 55). The synthesis begins with methyl pyranoside 428, which was synthesized from Dglucosamine over four steps. Reductive cleavage of the ring gives an aldehyde that was directly subjected to Wittig olefination delivering diene 429. Ring closing metathesis with Grubbs 2nd generation catalyst furnishes substituted cyclopentene 430. Conversion of the methyl carbamate to the urea $\mathbf{4 3 1}$ was accomplished using the procedure developed by Basha. ${ }^{92}$ Iodocyclization of $\mathbf{4 3 1}$ gave an oxazoline. Reaction of the intermediate iodide using conditions developed by $\mathrm{Keck}^{93}$ led to carbon-carbon bond formation on the less hindered face of the cyclopentane affording $\mathbf{4 3 2}$ as a single diastereomer. Isomerization of the terminal alkene with Grubbs II catalyst gave an inconsequential mixture of alkene isomers. Ozonolysis with reductive workup and a global MOM deprotection yields (-)-allosamizoline.

Scheme 55. Total Synthesis of (-)-Allosamizoline (Donohoe, 2007)

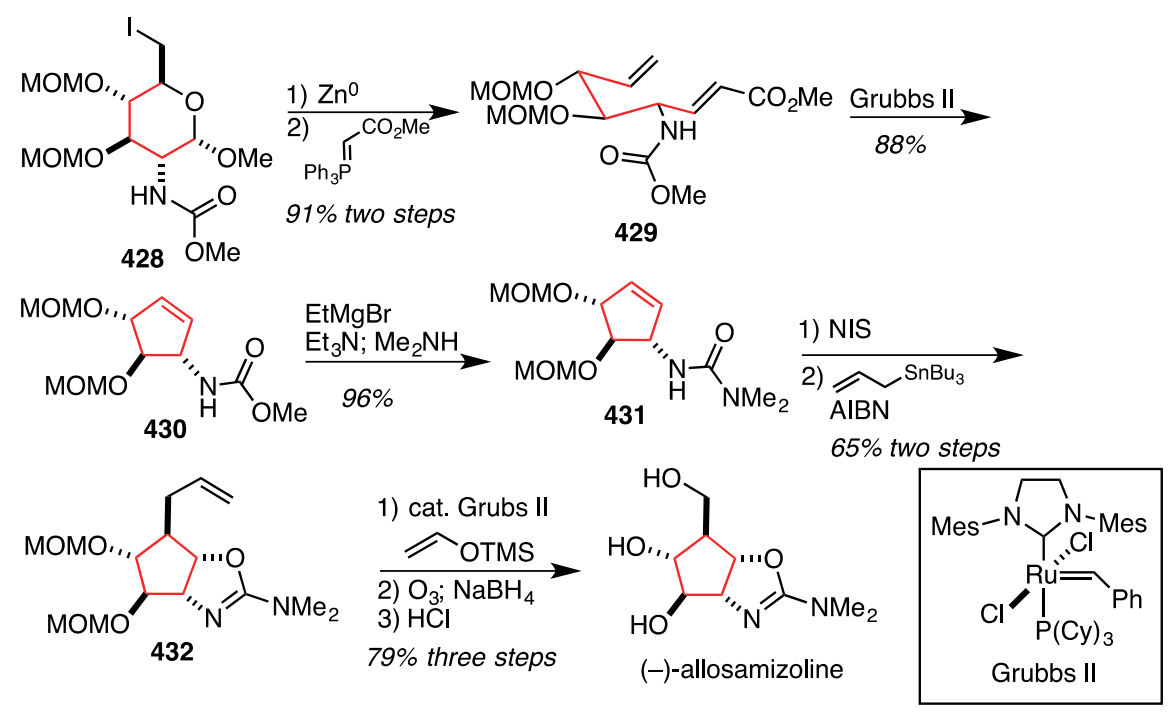

In the synthetic effort towards hexacyclinic acid, Prunet ${ }^{94}$ uses ring-closing metathesis to construct a functionalized cyclopentenone (Scheme 56). The route commences with the asymmetric syn-aldol of 433 with acrolein to give 434. Cleavage of the chiral auxiliary delivered an aldehyde, and a subsequent tin-mediated addition of ethyl diazoacetate gave $\beta$-ketoester 435. A Lewis acid promoted condensation with acetaldehyde gives enone $\mathbf{4 3 6}$ as a 1:1 mixture of inconsequential alkene isomers. Ring-closing metathesis proceeded smoothly to deliver functionalized cyclopentenone 437. Mukaiyama Michael addition of enol silane 438 to 437 led to the formation of three stereocenters and highly functionalized cyclopentanone 439. In this key Michael reaction the forming stereocenters are the result of the stereochemical preference of each of the reactive coupling partners. It was hypothesized that conducting the reaction in a polar solvent reduces complexation of the reactants and allows addition of each reactive component on their respective least hindered diastereoface. ${ }^{95}$ A radical cyclization of $\beta$-ketoester 439 furnishes the fused carbocyclic ring system 440. Finally, Luche reduction proceeds with hydride delivery from the convex face of the ring system to deliver fully functionalized cyclopentane 441.

\footnotetext{
${ }^{91}$ Donohoe, T. J.; Rosa, C. P. Org. Lett. 2007, 9, 5509-5511.

${ }^{92}$ Basha, A. Tetrahedron Lett. 1988, 19, 2525-2526.

${ }^{93}$ Keck, G. E.; Enholm, E. J.; Yates, J. B.; Wiley, M. R. Tetrahedron 1985, 41, 4079-4094.

94 Toueg, J.; Prunet, J. Org. Lett. 2008, 10, 45-48.

${ }^{95}$ For related examples, see (a) Kishi, Y.; Wang, W.; Org. Lett. 1999, 7, 1129-1132. (b) Lebsack, A. D.; Overman, L. E.; Valentekovich, R. J.; J. Am. Chem. Soc. 2001, 123, 4851-4852.
} 
This advanced intermediate contains much of the hexacyclinic acid structure including the fully functionalized cyclopentane. $^{96}$

Scheme 56. Synthetic Studies Towards Hexacyclinic Acid (Prunet, 2008)

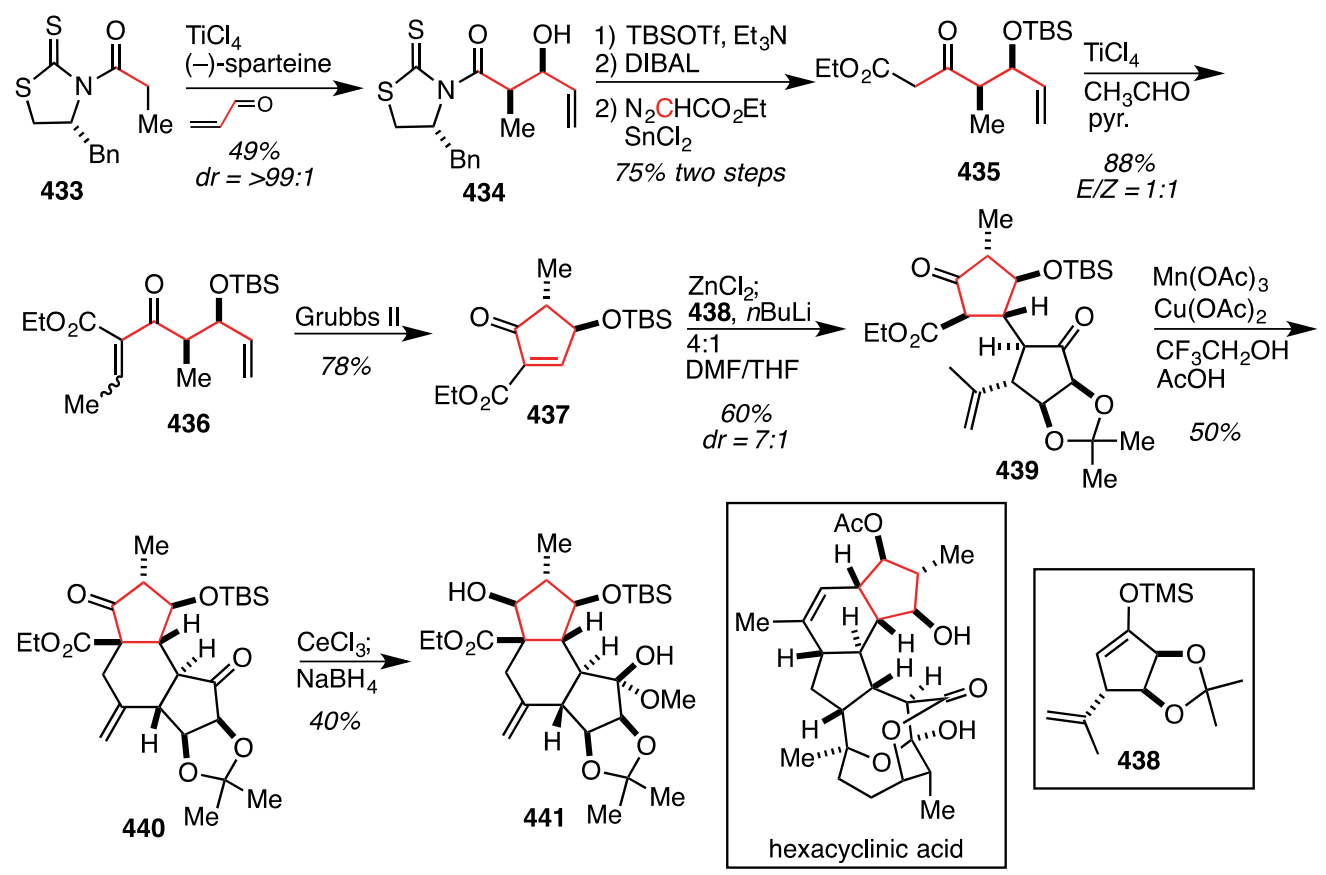

\subsection{Carbenoid Cyclizations}

In Fukuyama's ${ }^{97}$ first total synthesis of $( \pm)$-gelsemine, a copper-mediated cyclopropanation results in the formation of a functionalized cyclopentanone (Scheme 57). ${ }^{98}$ Beginning with a protocol first published by Kondo $^{99}$, addition of the dianion derived from methyl acetoacetate (442) to sorbic aldehyde was followed by protection with ethyl vinyl ether to deliver intermediate 443. Treatment with tosyl azide and triethylamine results in diazo transfer to give the $\beta$-ketoester 444 . The copper-mediated cyclopropanation was successful in constructing cyclopentanone $\mathbf{4 4 5}$ as an inconsequential mixture of diastereomers. Hyride reduction of the ketone occurs from the convex face of the bicycle to deliver 446, although the diastereoselectivity is inconsequential. This intermediate was advanced over four standard transformations to give 447. A Pfitzner-Moffatt ${ }^{100}$ oxidation followed by elimination of acetic acid produced enone 448. Refluxing intermediate $\mathbf{4 4 8}$ in a toluene/acetonitrile mixture promotes a divinylcyclopropane Cope rearrangement to furnish the bicyclo[3.2.1] octadienone system 449. This material was converted to unsaturated ester $\mathbf{4 5 0}$ by radical deiodination, a Horner-Wadsworth-Emmons

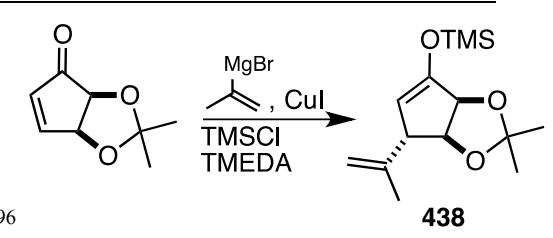

96

Interestingly, advanced intermediate $\mathbf{4 4 1}$ contains two fully functionalized cyclopentanes; however, the target hexacyclinic acid requires only one fully functionalized cyclopentane. The uncolored fully functionalized cyclopentane of $\mathbf{4 4 1}$ was constructed beginning with the cyclopentenone building block starting material shown above, by way of intermediate 438. See section 11 for similar functionalizations of related enones.

${ }^{97}$ Fukuyama, T.; Liu, G. J. Am. Chem. Soc. 1996, 118, 7426-7427.

${ }^{98}$ Here we discuss Fukuyama's 1996 report of the synthesis of $( \pm)$-gelsemine. An enantioselective synthesis of $(+)-$ gelsemine was published by Fukuyama in which intermediate 449 was prepared as a single enantiomer, see:

Yokoshima, S.; Tokuyama, H.; Fukuyama, T. Angew. Chem. Int. Ed. 2000, 39, 4073-4075.

${ }^{99}$ Kondo, K.; Umemoto, T.; Yako, K.; Tunemoto, D. Tetrahedron Lett. 1978, 19, 3927-3930.

${ }^{100}$ Pfitzner, K. E.; Moffatt, J. G. J. Am. Chem. Soc. 1965, 87, 5661-5670. 
reaction of the cyclopentenone, and protection of the indole nitrogen as an $\mathrm{N}, \mathrm{O}$-acetal. Conjugate addition of dimethylamine occurred exclusively from the less hindered exo-face yielding trans-amino ester $\mathbf{4 5 1}$. This material was advanced four steps through functional group manipulations to deliver intermediate 452. Upon exposure to silver triflate and silver carbonate an unusual lactam formation occurred to provide fully functionalized cyclopentane $\mathbf{4 5 3}$. This advanced intermediate contains the fully functionalized cyclopentane core of $( \pm)$-gelsemine, and it was advanced to the natural product over an additional five steps.

\section{Scheme 57. Total Synthesis of ( \pm )-Gelsemine (Fukuyama, 1996)}
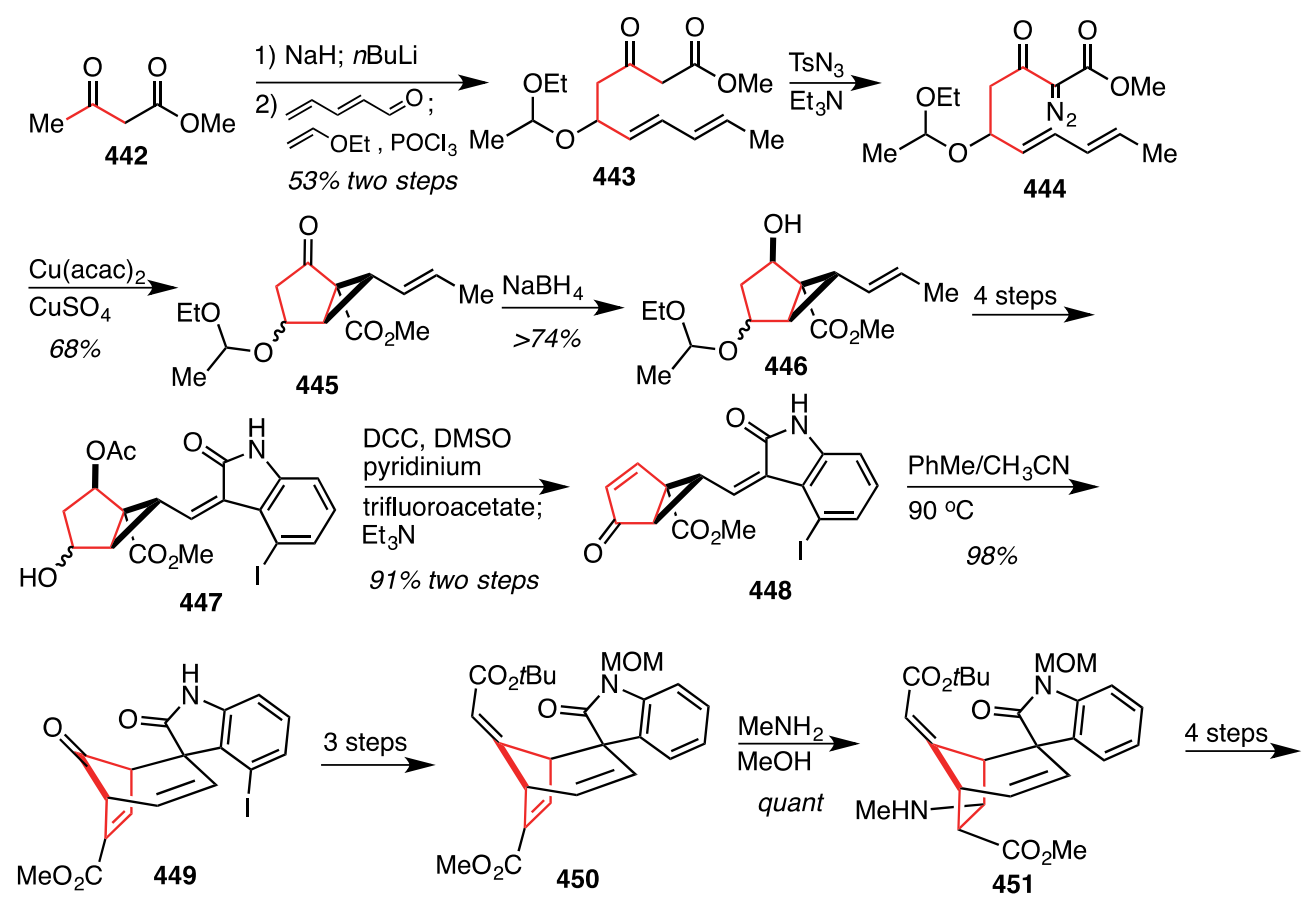

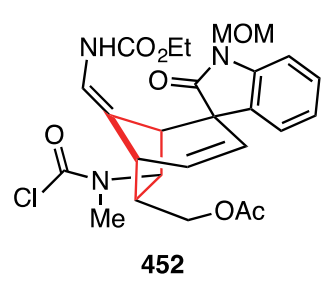

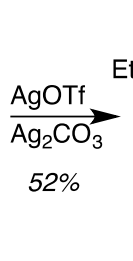

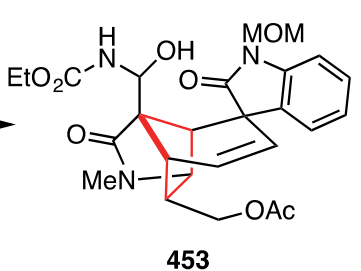<smiles>C=CC12CC3CC(CC(C3)C13C(=O)Nc1ccccc13)N(C)C2</smiles>

$( \pm)$-gelsemine

453

The synthesis of $(+)$-sulcatine $\mathrm{G}$ by Taber ${ }^{101}$ employs a rhodium catalyzed intramolecular $\mathrm{C}-\mathrm{H}$ insertion for the construction of the cyclopentane ring (Scheme 58). The route begins with commercial $(S)$ - $(+)$-citronellyl bromide 454. This was subjected to an ozonolysis/diazocoupling sequence ${ }^{102}$ to deliver a $\beta$-ketoester. Diazotransfer using methanesulfonyl azide furnished cyclization substrate 455. Rhodium catalyzed intramolecular $\mathrm{C}-\mathrm{H}$ insertion gave cyclopentane $\mathbf{4 5 6}$ without erosion of enantiomeric excess, although the product was isolated as an inconsequential mixture of ethyl ester epimers. Treatment of $\mathbf{4 5 6}$ with base under Finkelstein conditions induced intramolecular alkylation to give the cis-bicyclo[3.2.0]heptane (457). Oxidation of the cyclopentenone was accomplished by monobromination of the ketone followed by dehydrobromination with calcium carbonate yielding 458. A Trost annulation ${ }^{103}$ occurs from the convex face of the bicyclic ring system and formed the tricyclic intermediate $\mathbf{4 5 9}$ with good diastereocontrol. Hydride reduction occurs to give the desired diastereomer of the secondary alcohol (460) in good yield, which represents delivery of hydride on the diastereoface of the

\footnotetext{
${ }^{101}$ Taber, D. F.; Frankowski, K. J. J. Org. Chem. 2005, 70, 6417-6421.

${ }^{102}$ Holmquist, C. R.; Roskamp, E. J. J. Org. Chem. 1989, 54, 3258-3260.

${ }^{103}$ Trost, B. M.; Chan, D. M. T. J. Am. Chem. Soc. 1983, 105, 2315-2325.
} 
cyclopentanone with no less than four substituents. No explanation was given for the origin of this

diastereoselectivity. ${ }^{104}$ This advanced intermediate contains a fully functionalized cyclopentane, and (+)-sulcatine $\mathrm{G}$ was completed in an additional five steps.

Scheme 58. Total Synthesis of Sulcatine G (Taber, 2005)

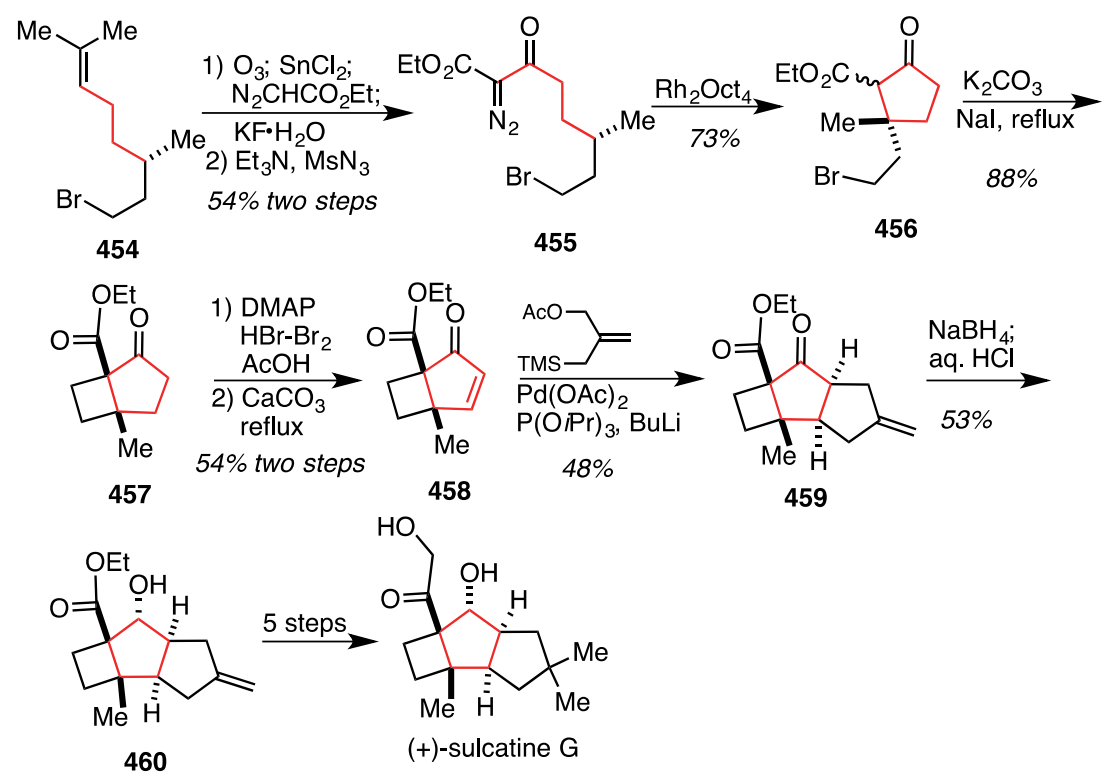

\subsection{Intramolecular Kulinkovich Reaction}

In the efforts towards the gelsemine skeleton, $\mathrm{Cha}^{105}$ utilizes a titanium-mediated cyclization of an $\omega$-vinylamide to construct the tricyclic core the natural product (Scheme 59). A Diels-Alder reaction between $N$ phenylmaleimide (461) and (E)-4,6-heptadienoic acid (462) provided cycloadduct 463 with good endo selectivity. Hydrogenation of the cyclohexene was followed by oxidative decarboxylation to yield $\mathbf{4 6 4}$. Treatment of $\mathbf{4 6 4}$ with modified Kulinkovic conditions led to intramolecular addition of an organotitanium intermediate to the imide carbonyl. Oxidative workup with oxygen gave the primary alcohol $\mathbf{4 6 5}$. This intermediate contains a fully functionalized cyclopentane with substitution and stereochemistry that matches many of the structural features found in gelsemine.

Scheme 59. Synthetic Studies Towards Gelsemine (Cha, 1999)
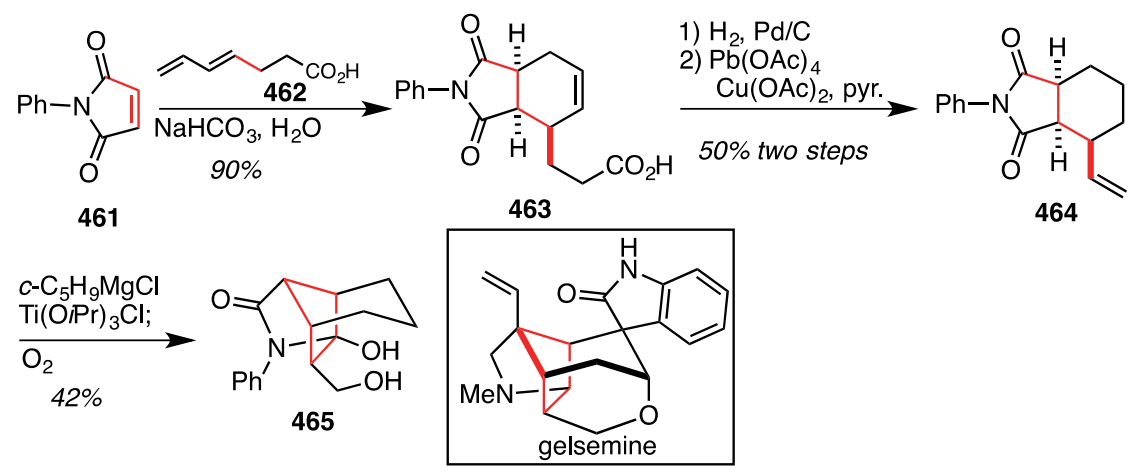

\section{Rearrangements}

104 The ester may direct the hydride to the more hindered ketone face.

105 (a) Sung, M. J.; Lee, C. -W.; Cha, J. K. Synlett 1999, 561-562. (b) For the Kulinkovich reaction, see: Kulinkovich, O. G.; de Meijere, A. Chem. Rev. 2000, 100, 2789-2834. 
Several natural products contianing fully functionalized cyclopentanes have been prepared using a skeletal rearrangement to build the five-membered ring. In such cases, stereogenic carbon atoms are installed on a larger or smaller ring; a subsequent transformation either contracts or expands, respectively, the carbocyle size to arrive at the cyclopentane. All examples of rearrangements in the context of this review fall into four well-defined categories: ring contraction of six-membered rings, oxa-di- $\pi$-methane rearrangements, ring expansion of cyclobutanes, and a Cope rearrangement.

Ring contraction of six-membered rings represents a common strategy for the formation of cyclopentane architectures. The value of this strategy stems from the myriad of sources of six-membered rings; suitably substituted benzenes, cyclohexenes prepared in Diels-Alder reactions, $\delta$-valerolactone derivatives, or other cyclohexane derivatives could all conceivably undergo contraction to a substituted cyclopentane. A common strategy for ring-contraction features a 1,2-disubstituted cyclohexane starting material (466) that undergoes the semipinacol $^{106}$ (Scheme 60), Wolff ${ }^{107}$, or acyloin-type rearrangements (less common regarrangements have also been used) to give cyclopentanes (e.g. 467). A useful feature of these reactions is that migrating carbon retains its stereochemical configuration during the rearrangement.

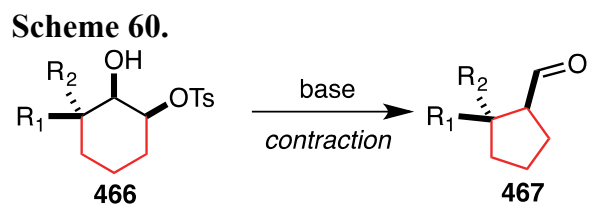

The oxa-di- $\pi$-methane rearrangement ${ }^{108}$ is a process by which six-membered rings can be contracted into suitably functionalized cyclopentanes with predictable control of resident stereochemical configuration (Scheme 61). The bicyclic starting materials (468) are produced using standard Diels-Alder cycloaddition reactions. Exposure to UV light induces the rearrangement to give the products 469. A subsequent cleavage of the embedded cyclopropane gives a cis-fused bicyclo[3.3.0]heptanone (470). In the context of this review, this strategy has been used in three separate examples, all in the context of a coriolin synthesis. Depending on the substitution pattern of the rearrangement substrate 468 , the reaction can deliver a fused-diquinane or fused-triquinane molecular architecture.

\section{Scheme 61.}

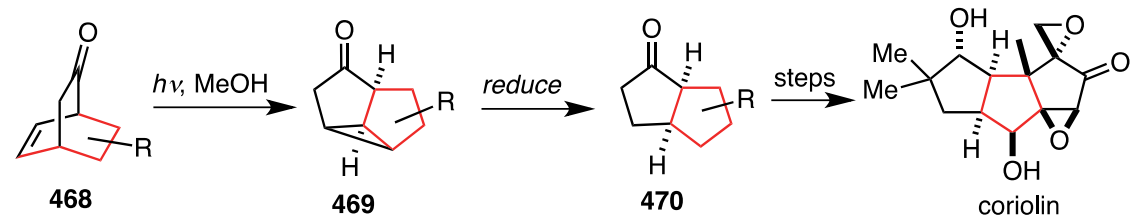

Substituted cyclobutanes may undergo ring expansion to give cyclopentanes (Scheme 62). In the context of this review, all such cyclobutanes are prepared using photochemical [2+2]-cycloadditions between alkenes (471) and substituted unsaturated carbonyls (472). The stereospecificity of the cycloaddition allows for the controlled production of several stereogenic carbons. The ring strain in the cyclobutane provides a potent driving force for the subsequent cyclopentane formation. A potential limitation of this strategy is that regioselectivity in the photochemical event requires careful selection of alkene substitution; as a result, several steps are required to transform the cycloaddition product (473) to the substrate for ring enlargement (474). The reaction used to expand the four-membered ring to the cyclopentane varies widely. A Tiffeneau-Demjanov-type rearrangement has been used (e.g. $\mathbf{4 7 4} \rightarrow \mathbf{4 7 5}$ ), but more unusual rearrangements also appear in this review.

\section{Scheme 62 .}

${ }^{106}$ For review of semipinacol rearrangements in synthesis, see: Song, Z. -L.; Fan, C. -A.; Tu, Y. -Q . Chem. Rev. 2011, 111, 7523-7556.

${ }^{107}$ For a thorough microreview of the Wolff rearrangement, see: Kirmse, W. Eur. J. Org. Chem. 2002, 2193-2256.

${ }^{108}$ For review of the di- $\pi$-methane and oxa-di- $\pi$-methane rearragnements, including a mechanistic analysis, see: Hixson, S. S.; Mariano, P. S.; Zimmerman, H. E. Chem. Rev. 1973, 73, 531-551. 


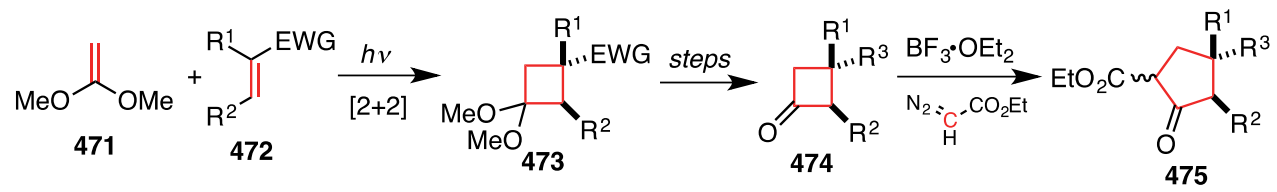

\subsection{Ring Contractions of Cyclohexane Derivatives}

In the synthesis of $( \pm)$-gibberellin $\mathrm{A}_{1}$ and gibberellic acid, Mander ${ }^{109}$ employs a photochemical ring contraction of an $\alpha$-diazo cyclohexenone to produce a cyclopentene (Scheme 63). Cyclohexadienone 477 was obtained after a five step conversion from starting material 476. Conjugate reduction of the enone was accomplished using standard conditions, and the unpurified material was transformed into diazoketone 479 using sulfonyl azide 478. Irradiation of the diazo species initiated a Wolff rearrangement to furnish $\mathbf{4 8 0}$ as a mixture of carboxylic acids $(d r=3.6: 1)$ that were methylated with diazomethane. A three-step oxidation sequence furnished cyclopentenone 481. Organometallic addition followed by esterification gave intermediate $\mathbf{4 8 2}$. Finally, conjugate addition of the propionate ester to the enone delivered fully functionalized cyclopentane 483 as a mixture of $\mathrm{C} 4$ epimers. This advanced intermediate contains the substituents and stereochemistry suitable for the synthesis of the target gibberellins, and completion of $( \pm)$-gibberellin $A_{1}$ required an additional seven steps.

\section{Scheme 63. Total Synthesis of $\left( \pm\right.$ )-Gibberellin $A_{1}$ (Mander, 1980)}<smiles>COc1cccc2c1CC(O)(C(=O)O)CC2</smiles>

476

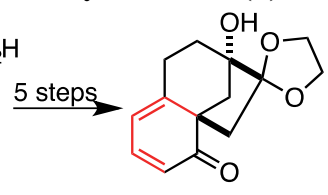

477

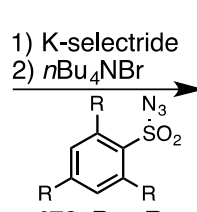

478; $\mathrm{R}=\mathbb{P r}$

$80 \%$ two steps

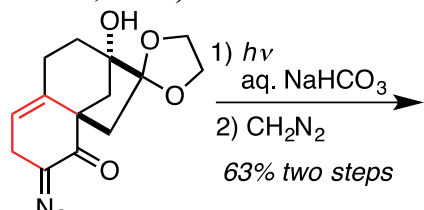

$\mathrm{N}_{2} 479$

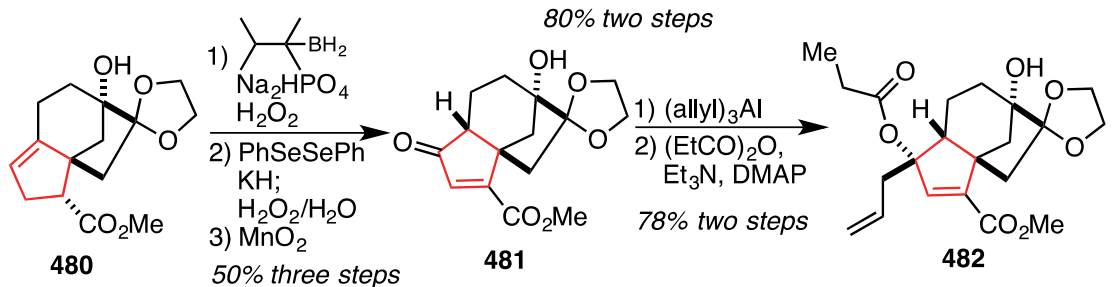

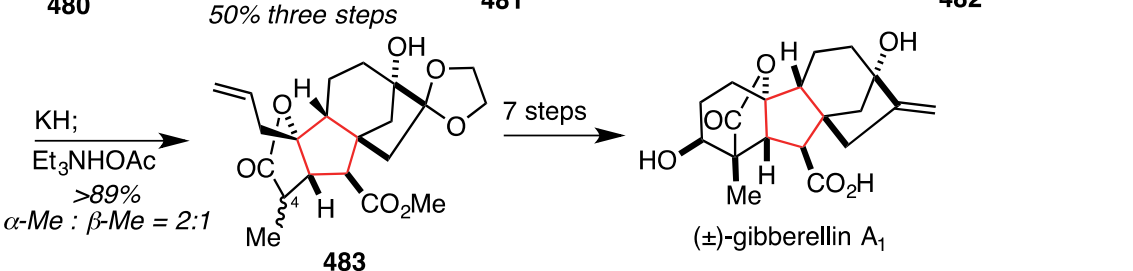

In the synthesis of (-)-allosamizoline, Kuzuhara ${ }^{110}$ performs a ring contraction of a six-membered sugar derivative to a fully functionalized cyclopentane (Scheme 64). The synthetic intermediate 484 was obtained from D-glucosamine over seven steps. A modified Ferrier reaction was performed using mercuric sulfate to give a $\beta$ hydroxy ketone, which underwent dehydration to give enone 485. A Luche reduction was followed by activation of the resulting allylic alcohol as the mesylate. Displacement with the pendant urea delivered the dimethylamino oxazoline ring 487. Dihydroxylation of the cyclohexene with $\mathrm{OsO}_{4}$ proceeded from the less hindered convex face giving a syn-diol. Monosulfonylation of the hydroxyl group was accomplished using tosyl chloride in pyridine. Treatment with L-Selectride initiated semi-pinacol rearrangement to give transient intermediate aldehyde $\mathbf{4 8 8}$, which was reduced under the conditions to give fully functionalized cyclopentane 489. This advanced intermediate was advanced to (-)-allosamizoline over two additional transformations.

Scheme 64. Total Synthesis of (-)-Allosamizoline (Kuzuhara, 1991)

\footnotetext{
${ }^{109}$ Lombardo, L.; Mander, L. N.; Turner, J. V. J. Am. Chem. Soc. 1980, 102, 6626-6628.
}

${ }^{110}$ Takahashi, S.; Terayama, H.; Kuzuhara, H. Tetrahedron Lett. 1991, 32, 5123-5126. 

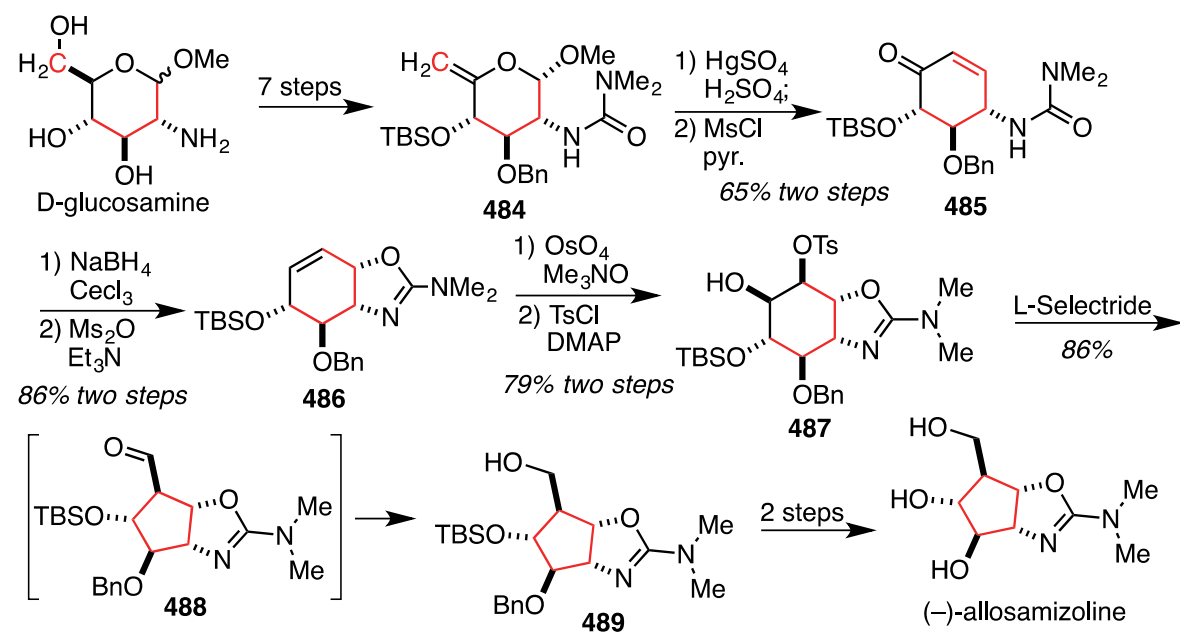

In the synthesis of $( \pm)$-kelsoene by Koreeda ${ }^{111}$, the key step is a homo-Favorskii rearrangement which effects a ring contraction from a six-membered carbocycle to a bicyclo[3.2.2] heptane and delivers a fully functionalized cyclopentane (Scheme 65). The synthesis begins with methoxycyclohexa-1,4-diene (490) which is hydrolyzed, protected as the ketal and epoxidized to give 491. Regioselective epoxide opening with selenium occurred away from the ketal and upon elimination gave allylic alcohol 492. Chlorination and addition of Grignard 493 gave enyne 494. A Pd-catalyzed cyclization of enyne 494 delivers annulation product $\mathbf{4 9 5}$. This material was carried forward over four steps to cyclohexenone 496. Conjugate addition occurs under conditions developed by $\mathrm{Kharasch}^{112}$ on the less hindered face of the enone to give intermediate 497. Exposure of the $\beta$-keto tosylate 497 to base resulted in a mixture of two cyclobutanones. Minor product $\mathbf{5 0 2}$ results from the desired homo-Favorskii rearrangement producing a fully functionalized cyclopentane (mechanism shown), and $\mathbf{5 0 1}$ is the product of intramolecular enolate alkylation with the tethered tosylate. The crude ketone mixture was treated with acid, which induced the isomerization of the undesired $\mathbf{5 0 1}$ to an inconsequential mixture of cyclobutanones containing the desired $\mathbf{5 0 2}$. The isomerization of $\mathbf{5 0 1}$ to $\mathbf{5 0 2 / 5 0 3}$ may proceed via intermediates $498-\mathbf{5 0 0}$ The separable cyclobutanones were converted to their respective tosylhydrazone derivatives, and they were subsequently reduced to $( \pm)$-kelsoene.

Scheme 65. Total Synthesis of $( \pm)$-Kelsoene (Koreeda, 2002)

${ }_{111}$ Zhang, L.; Koreeda, M. Org. Lett. 2002, 4, 3755-3758.

${ }^{112}$ Reetz, M. T.; Kindler, A. J. Organomet. Chem. 1995, 502, C5-C7. 

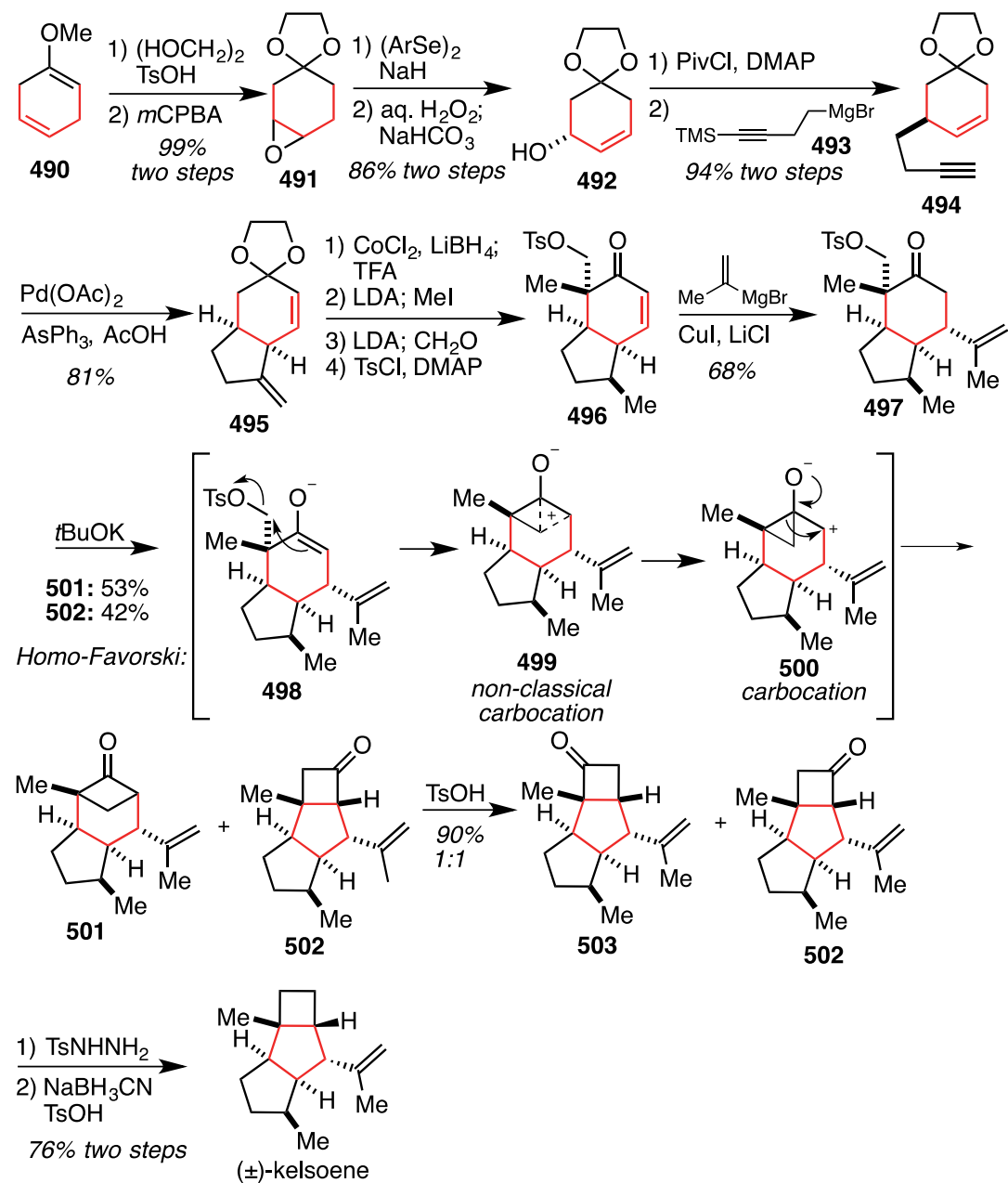

In the synthesis of the $\alpha$-mannosidase inhibitor, $(+)$-mannostatin $\mathrm{A}$, Vasella ${ }^{113}$ employs a ring contraction from an $\mathrm{N}$-aminolactam (504) to form a fully functionalized cyclopentane (Scheme 66). Mesylate $\mathbf{5 0 3}$ (derived from D-ribonolactone) was converted to $N$-aminolactam 504 over two steps. Oxidation with lead (IV) acetate provided fully functionalized cyclopentane 505. This oxidative ring contraction was developed by Vasella and has multiple proposed mechanistic pathways. ${ }^{114}$ This material was converted over four steps to the orthogonally protected triol 506. Substitution with methanethiolate installed the thioether. Fluoride-mediated removal of the silyl protecting group furnished 507. The final stereocenter of the cyclopentane was installed by imidate formation with spontaneous displacement of the mesylate to give fully functionalized cyclopentane $\mathbf{5 0 8}$. Hydrolysis of the oxazoline delivers (+)-mannostain A as the hydrochloride salt.

\section{Scheme 66. Total Synthesis of (+)-Mannostatin A (Vasella, 2003)}

\footnotetext{
${ }^{113} \mathrm{Hu}, \mathrm{G} . ;$ Zimmerman, M.; Ramana, C. V.; Vasella, A. Chem. Comm. 2003, 952-953.

${ }^{114}$ Hu, G.; Vasella, A. Helv. Chim. Acta 2004, 87, 2434-2446.
} 

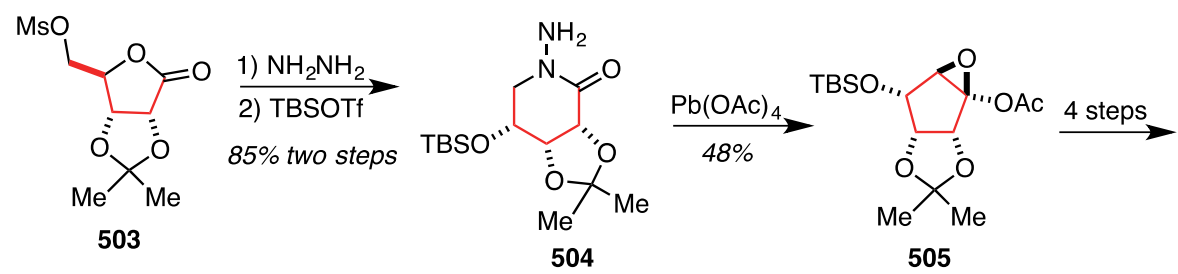

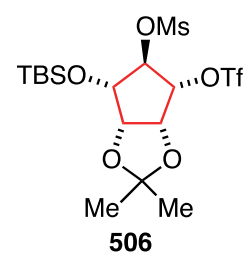
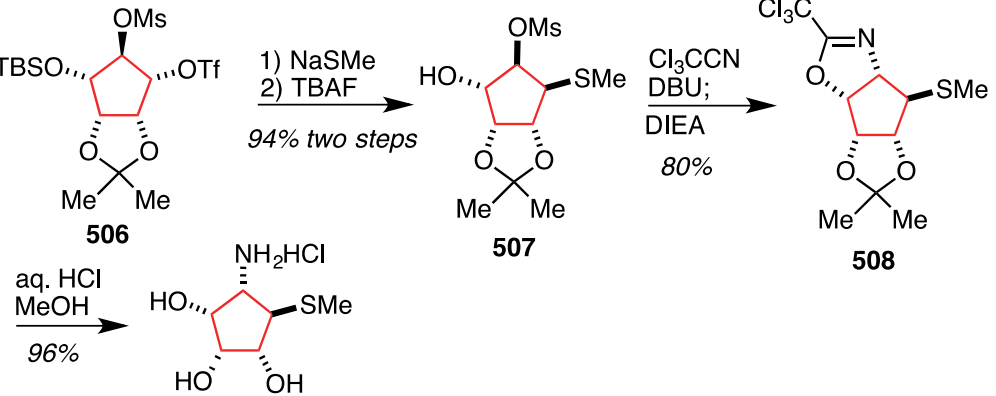

(+)-mannostatin $\mathrm{A} \cdot \mathrm{HCl}$

The total synthesis of (-)-massadine was completed by Baran and Chen utilizing a ring contraction (Scheurer rearrangement) to give a fully functionalized cyclopentane (Scheme 67). ${ }^{115}$ The route begins with $(S)$-Garner's aldehyde (509), which was olefinated and reduced to give allylic alcohol 510. ${ }^{116}$ Carbodiimide coupling with carboxylic acid 511 delivers ester 512. Enolate formation and addition to aldehyde 513 was followed by oxidation of the resulting alcohol giving cyclization precursor 514. Treatment with $\mathrm{Mn}(\mathrm{OAc})_{3}$ induced a radical cyclization resulting in the formation of three stereocenters in 515. Hydrolysis of the lactone delivered 516, which was advanced nine steps to intermediate 517. Epimerization of the C9' (massadine numbering) stereocenter was performed under acidic conditions to deliver 518. Reduction of the ketone proceeded with axial attack by the hydride reagent to deliver alcohol 519. Treatment of 519 with titanium isopropoxide and TBHP induced the bioinspired skeletal rearrangement proposed by Scheuer ${ }^{117,118}$ to give fully functionalized cyclopentane 520. An additional six steps were required to complete the synthesis of (-)-massadine.

\section{Scheme 67. Total Synthesis of Massadine (Chen, 2014)}

\footnotetext{
${ }^{115}$ Ma, Z.; Wang, X.; Wang, X.; Rodriguez, R. A.; Moore, C. E.; Gao, S.; Tan, X.; Ma, Y.; Rheingold, A. L.; Baran, P. S.; Chen, C. Science 2014, 346, 219-224.

${ }^{116}$ Early publications in this area by Chen depict $(R)$-Garner's aldehyde and related intermediates that were transformed to diastereomers of 516. Investigations of the stereostructure of massadine, palau'amine and related alkaloids led to correction of the stereochemistry of $\mathbf{5 1 5}$ and 516. We depict the corrected structures based on personal communication with Prof. Chuo Chen.

${ }^{117}$ Kinnel, R. B.; Gehrken, H. -P.; Swali, R.; Skoropowski, G.; Scheuer, P. J. J. Org. Chem. 1998, 63, 3281-3286.

118 The Scheuer hypothesis has been investigated and experimentally validated by several groups. See: Romo, reference 123; Sivappa, R.; Hernandez, N. M.; He, Y.; Lovely, C. J. Org. Lett. 2007, 9, 3861-3864, and references therin.
} 

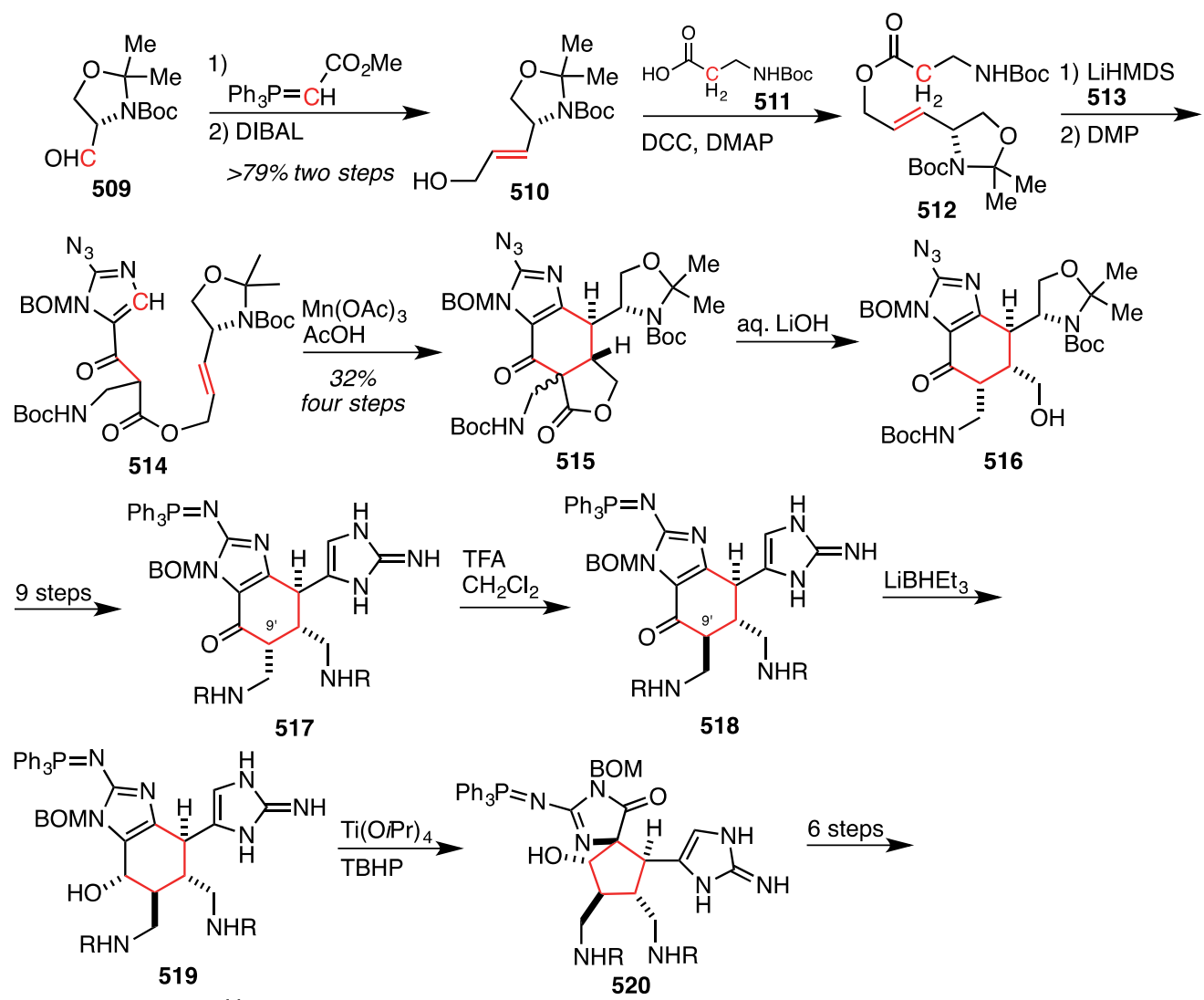<smiles>N=C1N[C@H]2O[C@@H]3NC(=N)N4[C@H]([C@H](CNC(=O)c5cc(Br)c(Br)[nH]5)[C@H](CNC(=O)c5cc(Br)c(Br)[nH]5)[C@@]34O)[C@]2(O)N1</smiles>
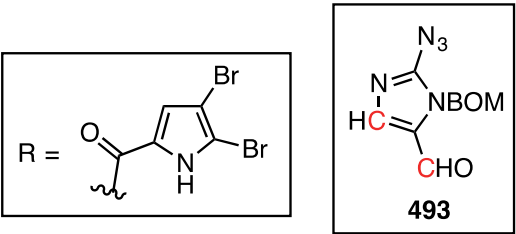

$(-)$-massadine

The Chen ${ }^{119}$ synthesis of (-)-axinellamines A and B takes advantage of a similar ring-contraction (Scheuer rearrangement) to give the fully functionalized cyclopentane core (Scheme 68). The route begins with 516, which was prepared as shown above. This intermediate was advanced six steps to $\mathbf{5 2 1}$. Basic epimerization of the $\mathrm{C} 12$ (axinellamine numbering) stereocenter delivered 522. Ketone reduction proceeded with axial attack of hydride to give 523. The oxidative cyclization delivered 524 and the spiro-cyclic core of the axinellamines. Installation of the chlorine atom with retention of configuration was accomplished by activation of the alcohol as the sulfonate and treatment with nucleophilic chloride to give 525. Mechanistically, the reaction proceeds through intermediate aziridine 525. Advanced intermediate $\mathbf{5 2 6}$ has the appropriate substitution and stereochemistry for the axinellamines, and the synthesis was completed in an additional 13 steps.

Scheme 68. Total Synthesis of Axinellamines A and B (Chen, 2016)

119 (a) Ma, Z.; Lu, J.; Wang, X.; Chen, C. Chem. Commun. 2011, 47, 427-429. (b) Wang, X.; Ma, Z.; Lu, J.; Tan, X.; Chen, C. J. Am. Chem. Soc. 2011, 133, 15350-15353. (c) Ma, Z.; Wang, X.; Ma, Y.; Chen, C. Angew. Chem. Int. Ed. 2016, 55, 4763-4766. 


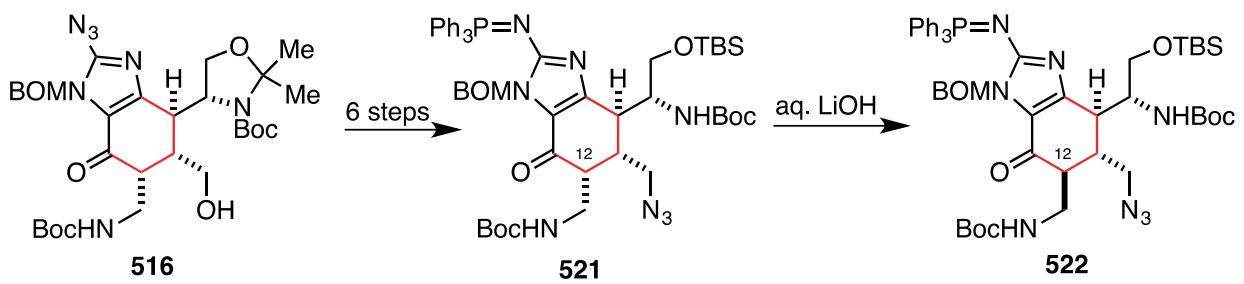

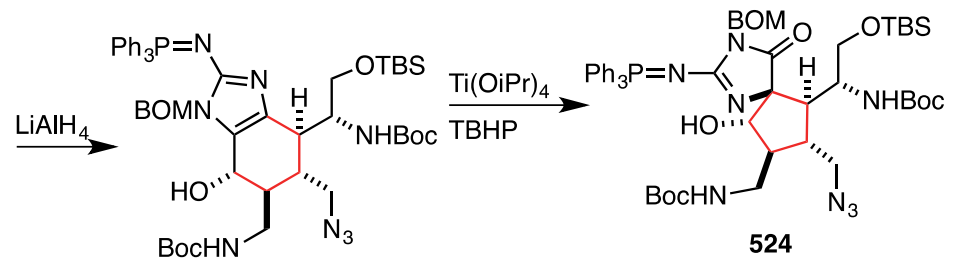

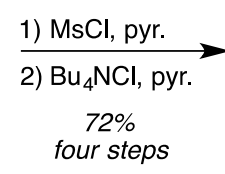

523

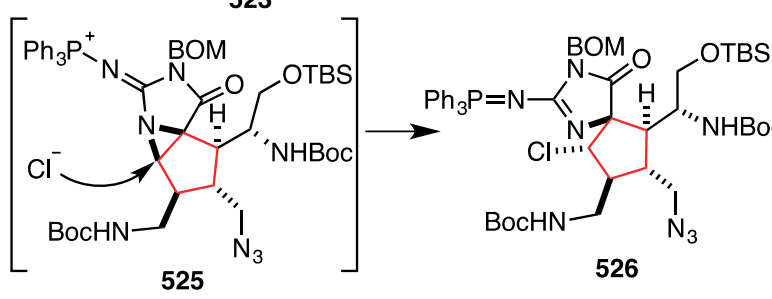

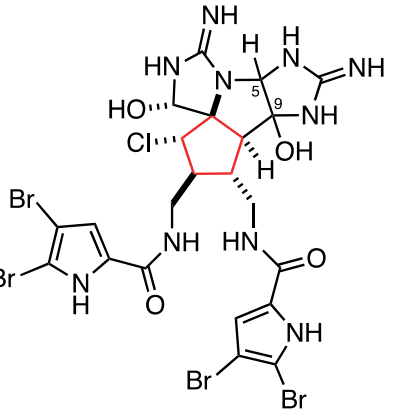

$(-)$-axinellamine $\mathrm{A}: 5-\mathrm{H}, 9-\mathrm{OH}=\beta$ $(-)$-axinellamine $\mathrm{B}: 5-\mathrm{H}, 9-\mathrm{OH}=\alpha$

The formal synthesis of $( \pm)$-gymnomitrol by Itô ${ }^{120}$ employs a skeletal rearrangement of a bicyclo[2.2.2] octane derivative (Scheme 69). The synthesis commences with an alkene isomerization/Diels-Alder cycloaddition between diene 527 and cyclopentene-1,2-dicarboxylic anhydride (528). Refluxing in toluene with TsOH delivered endo-adduct $\mathbf{5 2 9}$ as the only product. Four functional group manipulations were used to convert cycloaddition product 509 to intermediate 530. Desulfurization gave intermediate 531. Epoxidation with $m$ CPBA gave epoxy alcohol 532. Upon alumina chromatography a skeletal rearrangement took place to deliver ketoalcohol 533. Finally, Jones oxidation yielded diketone 534, which intersected a synthetic intermediate previously taken to $( \pm)$-gymnomitrol by Coates. ${ }^{121}$

\section{Scheme 69. Formal Synthesis of ( \pm )-Gymnomitrol (Itô, 1979)}

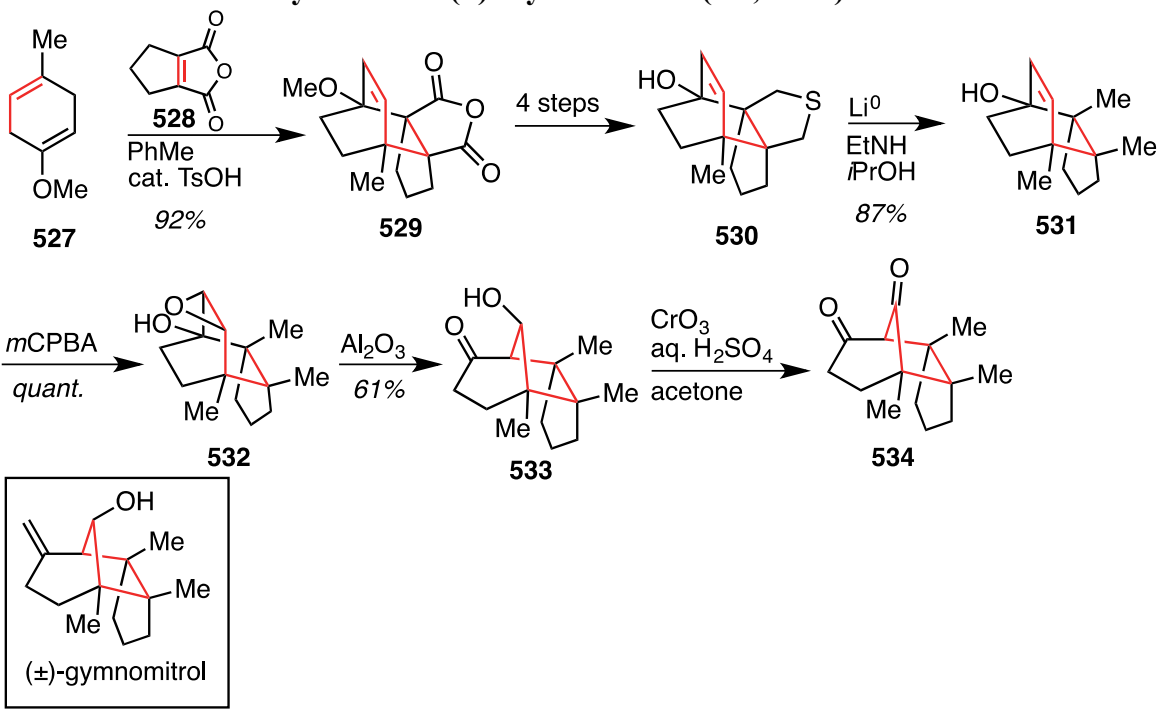

\footnotetext{
${ }^{120}$ Kodama, M.; Kurihara, T.; Sasaki, J.; Itô, S. Can. J. Chem. 1979, 57, 3343-3345.

${ }^{121}$ Coates, R. M.; Shah, S. K.; Mason, R. W. J. Am. Chem. Soc. 1982, 104, 2198-2208.
} 
In an approach toward gelsemine, Fleming ${ }^{122}$ assembles a fully functionalized cyclopentane using a rearrangement from a bicyclo[2.2.2] octane into a bicyclo[3.2.1] octane (Scheme 70). The route commences with a Diels-Alder reaction of diene $\mathbf{5 3 5}$ and $\beta$-nitroacrylate to give desired bicycle $\mathbf{5 3 6}$ in a 1:1 diastereomer ratio. Functional group manipulation was performed over five steps to deliver 537. Epoxidation with perbenzoic acid 538 occurred unexpectedly on the more hindered face to give 539. Opening of the epoxide with magnesium bromide gave a bromohydrin (540), which underwent a skeletal rearrangement to give $\mathbf{5 4 1}$. This material was advanced to tricyclic intermediate $\mathbf{5 4 2}$ over four steps. While a fully functionalized cyclopentane was formed in 543, the substitution pattern needed to be adjusted for gelsemine. Oxidation of the secondary alcohol and addition of vinylmagnesium bromide gave allylic alcohol 543. Chloride 544 was obtained by reaction with thionyl chloride. Presumably, chloride substitution of the alcohol was followed by an $\mathrm{S}_{\mathrm{N}} 2$ ' process resulting in the trisubstituted alkene. Substitution of the allylic chloride gave an allyl silane, and treatment with trioxane in formic acid induced an allyl silane addition to an acyl iminium ion. This reaction gives advanced intermediate 545, which contains a fully functionalized cyclopentane and the core molecular architecture of gelsemine.

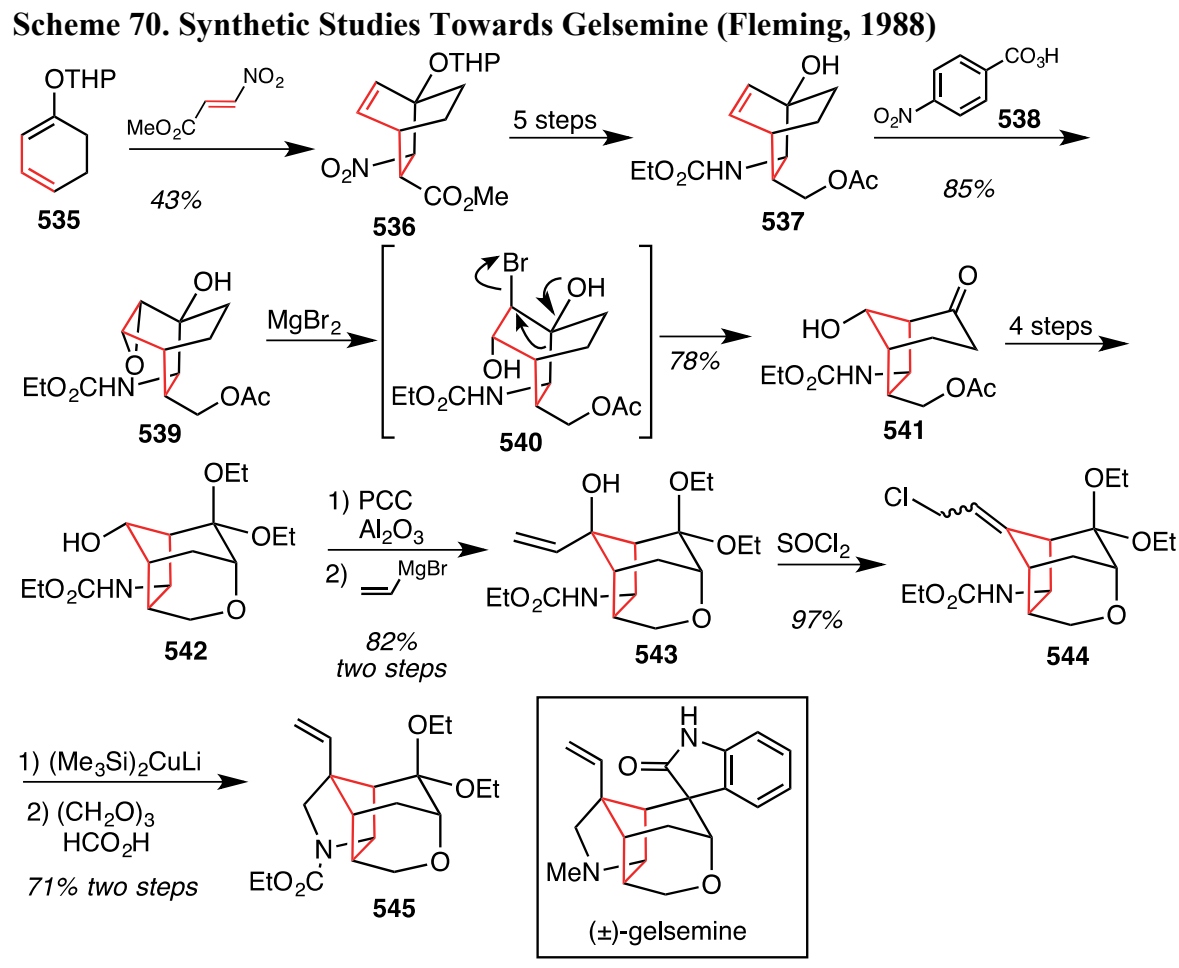

Romo ${ }^{123,124}$ developed an enantioselective strategy to the spirocyclic core of palau'amine in which a chlorination/ring contraction sequence delivers a fully functionalized cyclopentane (Scheme 71). A Diels-Alder reaction of vinyl imidazolone 546 with the chiral lactam 547 provides the tricyclic product with good control of regioselectivity. Under the conditions of the cycloaddition the double bond migrates regenerating the imidazolone ring. Silyl protection of the primary alcohol delivers 548. An oxidation/elimination sequence performed with dimethyldioxirane provides carbinolurea 549. Ring contraction with $N$-chlorosuccinimide furnished the chlorinated spirocyclic hydantoin and fully functionalized cyclopentane $\mathbf{5 5 0}$.

Scheme 71. Synthetic Studies Towards Palau'amine (Romo, 2001)

\footnotetext{
${ }^{122}$ Clarke, C.; Fleming, I.; Fortunak, J. M. D.; Gallagher, P. T.; Honan, M. C.; Mann, A.; Nübling, C. O.; Raithby, P. R.; Wolff, J. J. Tetrahedron, 1988, 44, 3931-3944.

${ }^{123}$ Dilley, A. S.; Romo, D. Org. Lett. 2001, 3, 1535-1538.

${ }^{124}$ A second approach to a palau'amine-type intermediate was later published by Romo using a similar synthetic strategy. See: (a) Dransfield, P. J.; Wang, S.; Dilley, A.; Romo, D. Org. Lett. 2005, 7, 1679-1682. (b) Dransfield, P. J.; Dilley, A. S.; Wang, S.; Romo, D. Tetrahedron 2006, 62, 5223-5247. (c) Wang, S.; Dilley, A. S.; Poullennec, K. G.; Romo, D. Tetrahedron 2006, 62, 7155-7161. (d) Zancanella, M. A.; Romo, D. Org. Lett. 2008, 10, 3685-3688.
} 


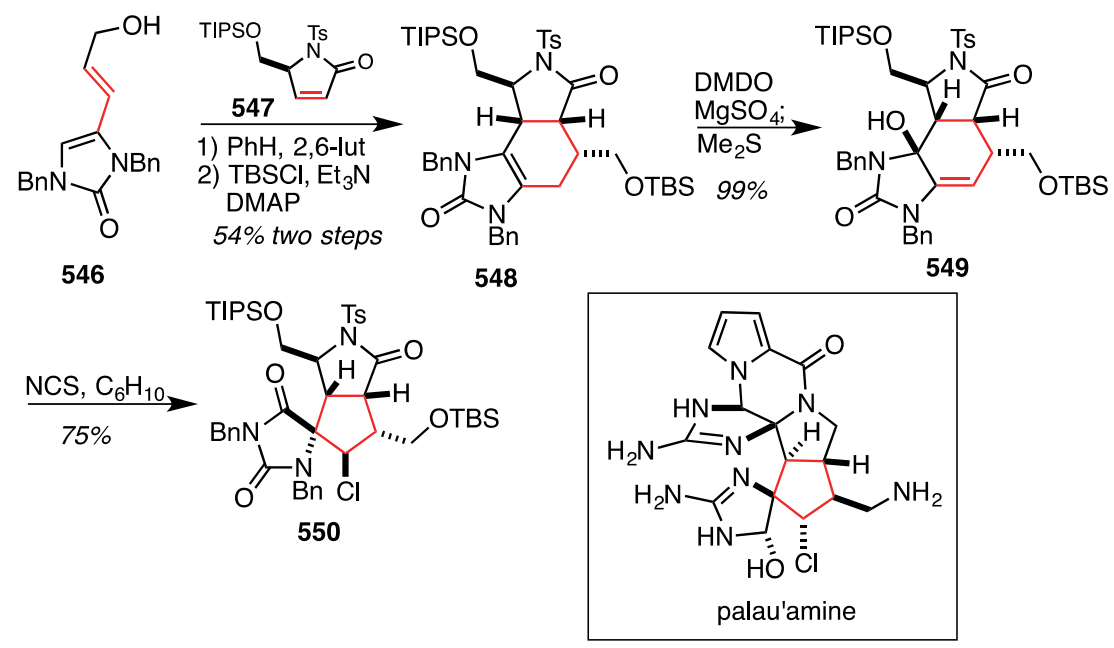

In the synthetic effort towards massadine, Carreira ${ }^{125}$ reports a silver-promoted norbornyl skeletal rearrangement to form the cyclopentane of massadine (Scheme 72). The route begins with a cycloaddition of cyclopentadiene 551 with dimethyl fumarate delivering norbornylsilane 552. Bromolactonziation provided tricyclic intermediate 553. The skeletal rearrangement occurs upon treatment with silver nitrate wherein desilylation and bond migration occurs with elimination of bromide giving intermediate lactone 555. Cleavage with methanol furnishes alcohol 556. A Swern oxidation gave ketone 557, which was advanced to $\mathbf{5 5 8}$ over four steps. Imine formation with 2,4-dimethoxybenzylamine was followed by addition of cyanide in the presence of zinc chloride to deliver iminonitrile $\mathbf{5 5 9}$ with good diastereoselectivity ( $>95: 5)$. The selectivity can be attributed to addition of TMScyanide to the ketone face opposite the benzyloxymethylene groups. This material was advanced over six steps to intermediate 560. Ozonolysis fragmented the norbornene skeleton. Workup with acetic anhydride and triethylamine resulted in regioselective ester formation ${ }^{126}$, which successfully differentiated the carbonyls to give 561 and completed the core of massadine.

Scheme 72. Synthetic Studies Towards Massadine (Carreira, 2008)

\footnotetext{
${ }^{125}$ (a) Breder, A.; Chinigo, G. M.; Waltman, A. W.; Carreira, E. M. Angew. Chem. Int. Ed. 2008, 47, 8514-8517. (b) Breder, A.; Chinigo, G. M.; Waltman, A. W.; Carreira, E. M. Chem. Eur. J. 2011, 17, 12405-12416.

${ }^{126}$ Schreiber, S. L.; Claus, R. E.; Reagan, J. Tetrahedron Lett. 1982, 23, 3867-3870.
} 

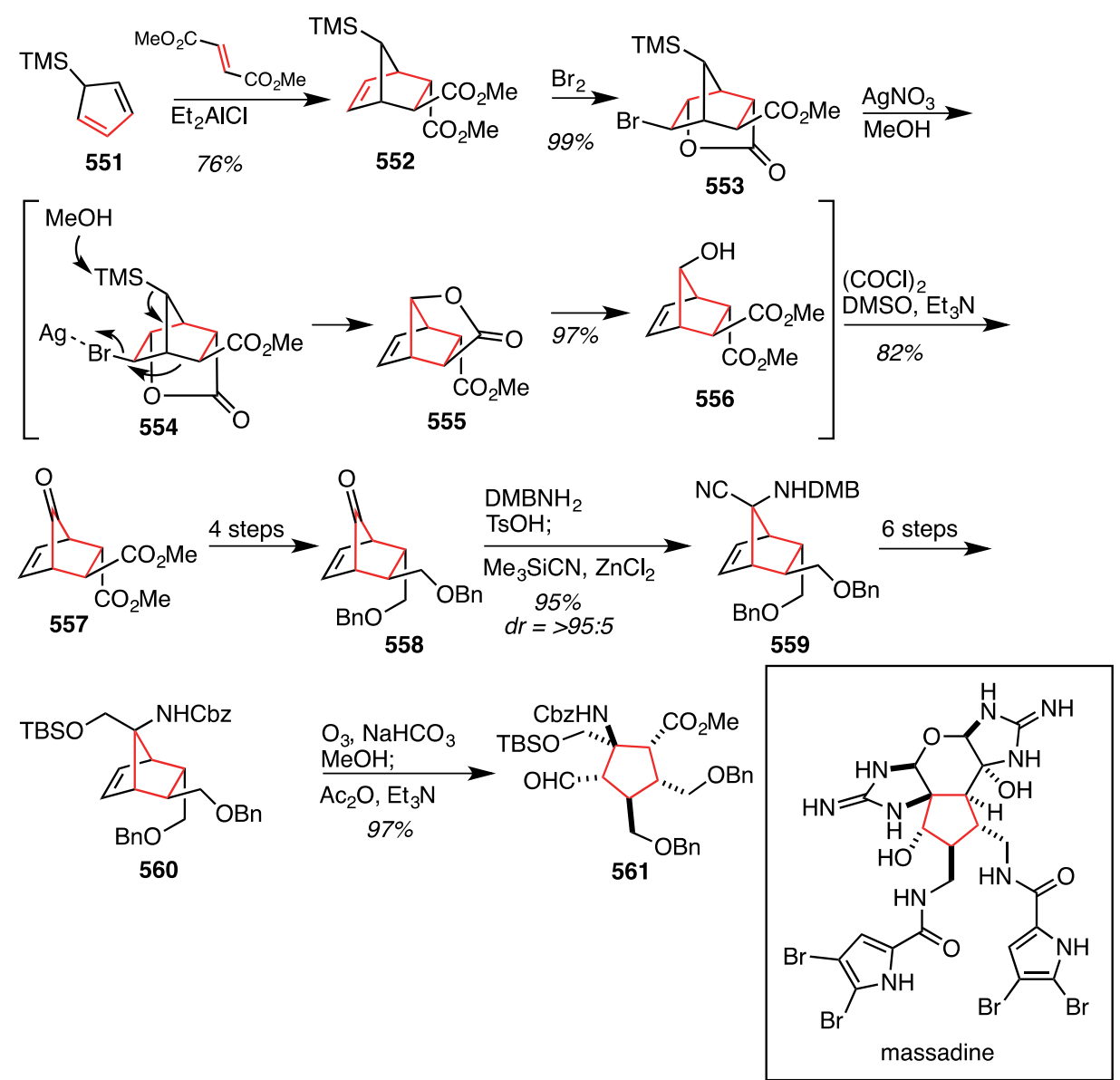

In an alternative route towards, Carreira ${ }^{127}$ uses the same skeletal rearrangement to construct the cyclopentane but functionalizes the core using a 4-component-Ugi-reaction (Scheme 73). Ketone 557 underwent an Ugireaction with 2,2-dimethoxybenzylamine and 2-nitrophenylisonitrile (563) to give amide 564. As above, the major diastereomer results from nucleophilic attack on the less hindered imine face. Over the next seven steps this material was advanced to intermediate 565. Ozonolytic cleavage and workup according to Schreiber's method differentiates the resulting carbonyl groups and gives 566. ${ }^{126} \mathrm{~A}$ Barton decarboxylation/oxygenation ${ }^{90}$ was used to convert the aldehyde to secondary alcohol of $\mathbf{5 6 7}$, which proceeded with retention of configuration. To complete the core of massadine, the alcohol was silylated and the ester stereocenter was epimerized yielding 568. This advanced intermediate contains a fully functionalized cyclopentane with appropriate substitution for a massadine synthesis.

Scheme 73. Synthetic Studies Towards Massadine (Carreira, 2011)

${ }^{127}$ Chinigo, G. M.; Breder, A.; Carreira, E. M. Org. Lett. 2011, 13, 78-81. 

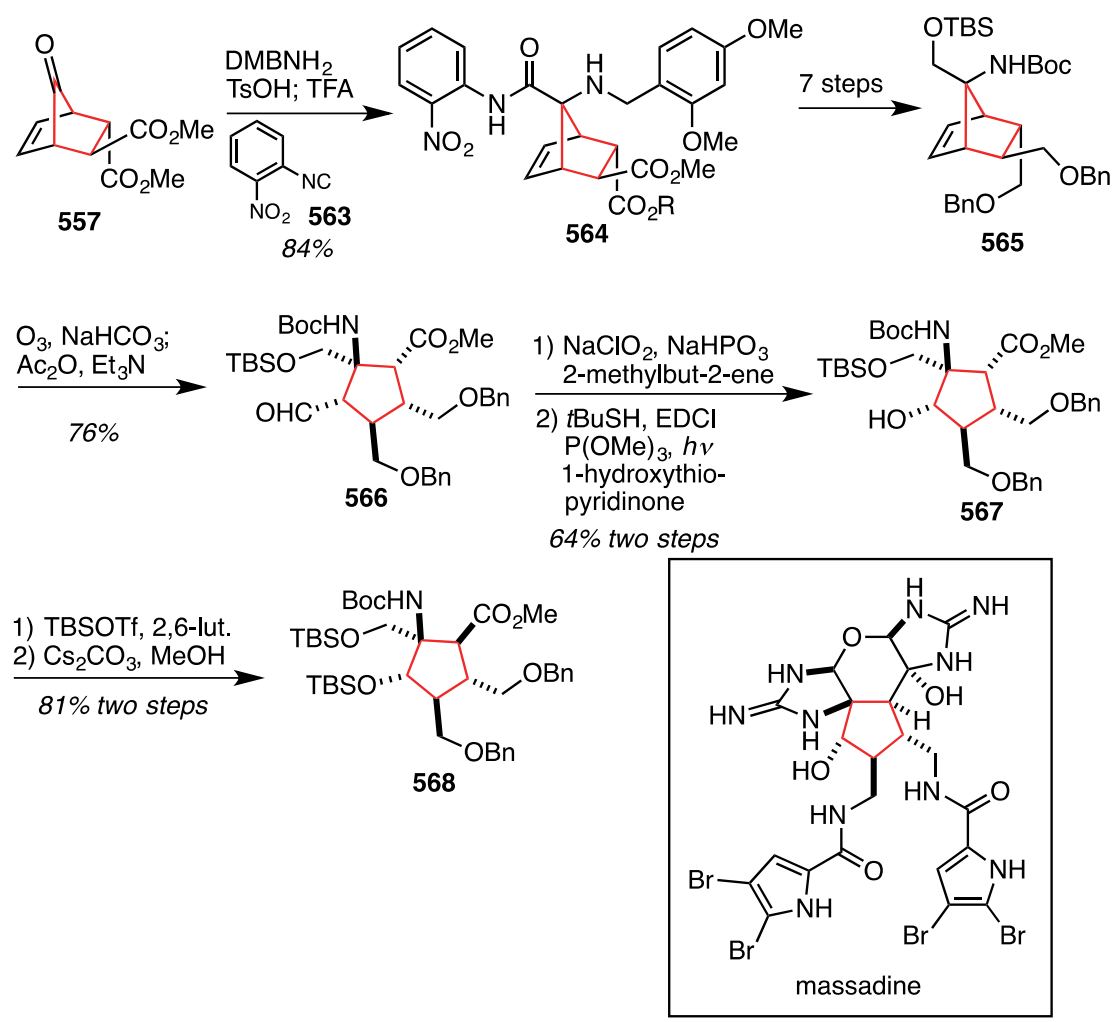

\subsection{Oxa-di- $\pi$-methane Rearrangements}

In the first enantioselective synthesis of (-)-coriolin, Demuth ${ }^{128}$ utilizes an oxa-di- $\pi$-methane rearrangement to assemble the cyclopentane (Scheme 74). The synthesis begins with chiral resolution of racemic diketone ( \pm )-569 to deliver (-)-569 over three steps. Methylation of enantiopure $\mathbf{5 6 9}$ was carried out with two equivalents of sodium hydride and methyl iodide; subsequently, an additional equivalent of sodium hydride and 18-crown-6 with excess methyl iodide gave tri-methyl diketone 570. When this mixture was irradiated in acetone, the oxa-di$\pi$-methane rearrangement occurred to give cyclopentanone $\mathbf{5 7 1}$ with it's $\alpha$-methyl diastereomer. The unpurified product mixture was alkylated with chloride $\mathbf{5 7 2}$ under standard conditions. Dissolving metal conditions resulted in cyclopropane fragmentation and selective ketone reduction. Finally, oxidative cleavage of the alkene furnished 573. A base-catalyzed aldol condensation formed the triquinane core. Direct reaction with isopropenyl acetate formed the dienol acetate, which upon epoxidation and elimination delivered alcohol 129. The synthesis was completed in an additional three steps following the protocol of Trost. ${ }^{129}$

\section{Scheme 74. Total Synthesis of (-)-Coriolin (Demuth, 1986)}

${ }^{128}$ (a) Demuth, M.; Ritterskamp, P.; Weigt, E.; Schaffner, K. J. Am. Chem. Soc. 1986, 108, 4149-4154. (b) Demuth, M.; Ritterskamp, P.; Schaffner, K. Helv. Chim. Acta 1984, 67, 2023-2027.

${ }^{129}$ Trost, B. M.; Curran, D. P. J. Am. Chem. Soc. 1981, 103, 7380-7381. 

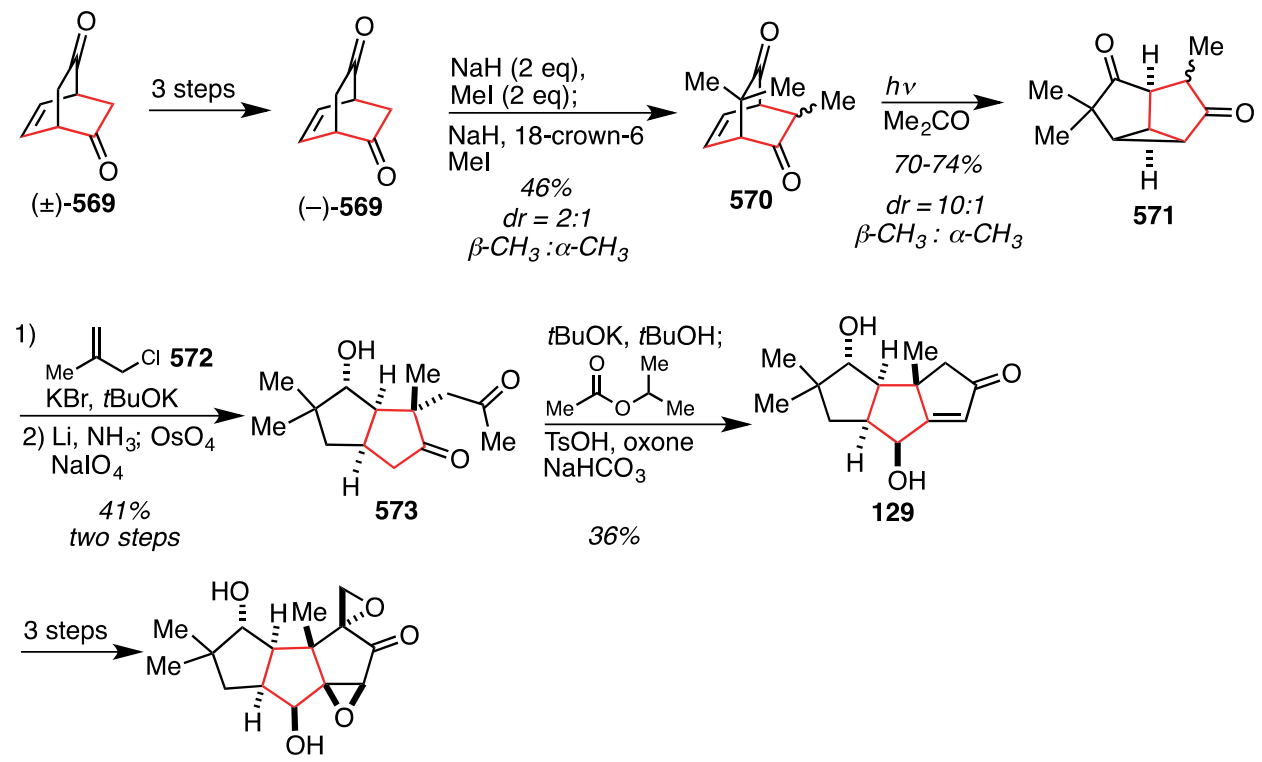

(-)-coriolin

A photo-induced skeletal rearrangement was employed by $\operatorname{Singh}^{130}$ to assemble the triquinane core of $( \pm)$ coriolin (Scheme 75). The synthesis begins with aryl aldehyde 575, which underwent organometallic addition of $\mathbf{5 7 6}$ to give intermediate 577. This material was advanced to $\beta, \gamma$-enone $\mathbf{5 7 8}$ over three steps. Aryl oxidation, under conditons developed by Adler, ${ }^{131}$ results in an oxaspirodienone (579), which undergoes an IMDA reaction to give the functionalized bicyclo[2.2.2] octenone 580. This material was advanced to intermediate $\mathbf{5 6 1}$ over six steps. Photochemical rearrangement of $\mathbf{5 8 1}$ in acetone delivered triquinane $\mathbf{5 8 2}$. Radical cleavage of the cyclopropane delivered $\mathbf{5 8 3}$, which is an intermediate in coriolin syntheses by both Little ${ }^{132}$ and Funk. $^{28}$

Scheme 75. Formal Synthesis of $( \pm)$-Coriolin (Singh, 1999)
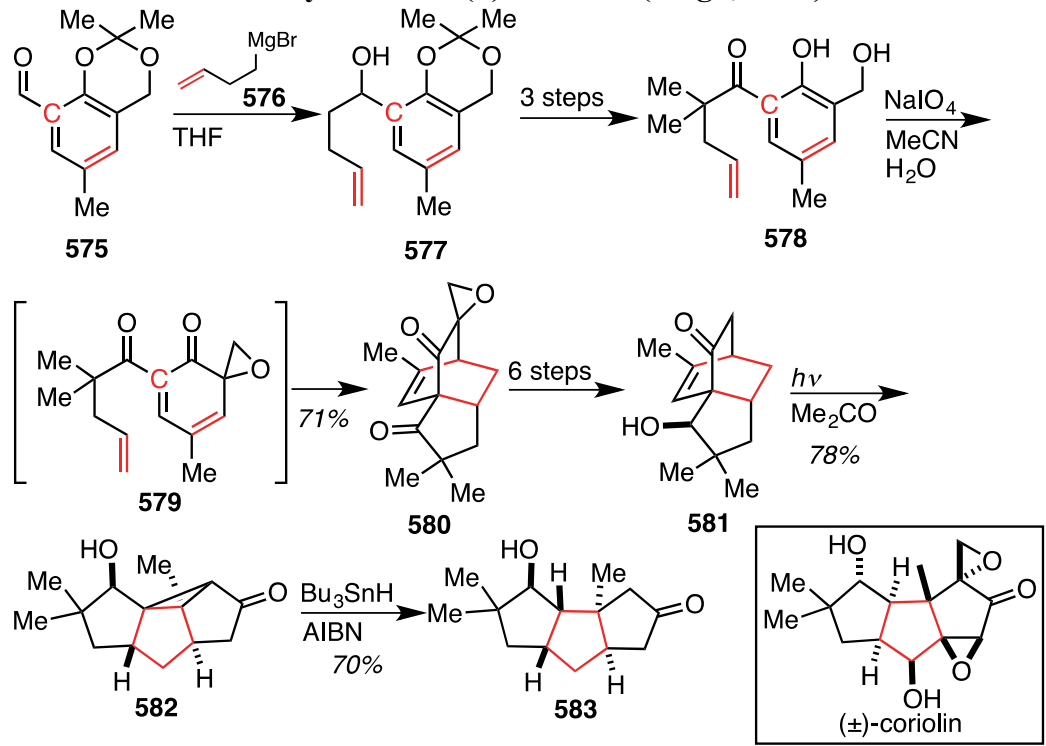

\footnotetext{
${ }^{130}$ Singh, V.; Alam, S. Q. Chem. Commun. 1999, 2519-2520.

${ }^{131}$ Adler, E.; Holmberg, K. Acta. Chem. Scand. B 1974, 28, 465-472.

${ }^{132}$ Van Hijfte, L.; Little, R. D.; Peterson, J. L.; Moeller, K. D. J. Org. Chem. 1987, 52, 4647-4661.
} 
In the formal synthesis of (-)-coriolin by Banwell ${ }^{133}$, a key oxa-di- $\pi$-methane rearrangement was used to construct the triquinane scaffold (Scheme 76). The route commences with a Diels-Alder cycloaddition between diene $\mathbf{5 8 4}$ and cyclopentenone to deliver 585. This cycloadduct was functionalized over seven steps to give photo-rearrangement precursor 586. Irradiation promoted the oxa-di- $\pi$-methane rearrangement to give an inconsequential epimeric mixture of benzoyl esters (587). Reductive cleavage of the benzoyloxy group with samarium iodide delivered 588. Radical cleavage of the cyclopropane ring gave a cyclopentanone that was oxidized with IBX to the corresponding cyclopentenone 589. Acetate cleavage delivers 104, which is an intermediate prepared by Trost in a synthesis of coriolin. ${ }^{129}$

Scheme 76. Formal Synthesis of (-)-Coriolin (Banwell, 2013)
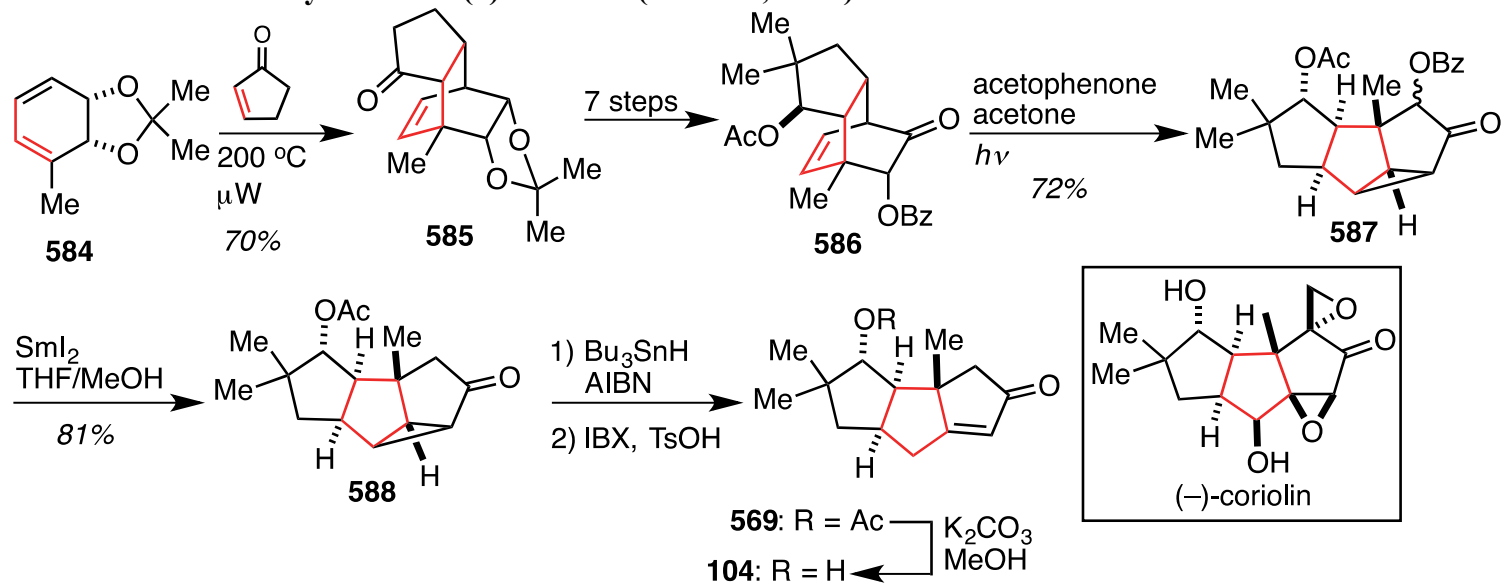

\subsection{Ring Expansions of Cyclobutane Derivatives}

In the total synthesis of $( \pm)$ - $\alpha$-caryophyllene alcohol, Corey ${ }^{134}$ uses a skeletal rearrangement to construct a cyclopentane (Scheme 77). The route begins with a [2+2] photocycloaddition of cyclohexenone 591 with 4,4dimethylcyclopentene (592) to give fully functionalized cyclobutane $\mathbf{5 9 3}$ as the major stereoisomer. Addition of methyllithium gave the tertiary alcohol 594. Treatment of 594 with aqueous sulfuric acid induced fragmentation of the cyclobutane ring and skeletal rearrangement to give the natural product. The mechanism is hypothesized to proceed through intermediate carbocation 595, which results from loss of water. Fragmentation of the cyclobutane gives a carbocation (596) that can be intercepted by water to give $\alpha$-caryophyllene alcohol.

Scheme 77. Total Synthesis of ( \pm )- $\alpha$-Caryophyllene Alcohol (Corey, 1965)

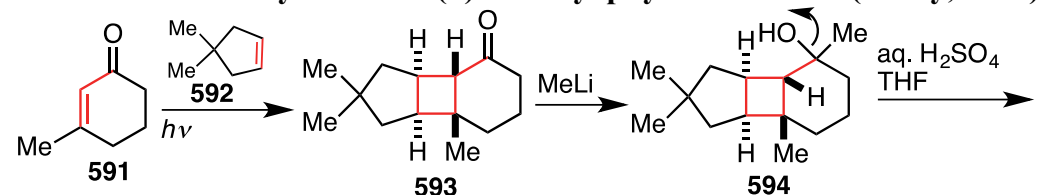

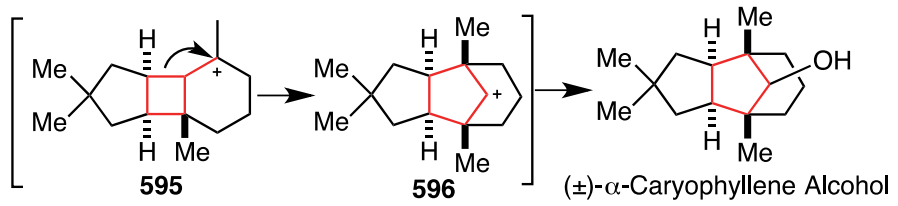

In the synthesis of $( \pm)$-coriolin, Tatsuta ${ }^{35}$ constructs the triquinane ring system using a ring expansion (Scheme 78). A stereocontrolled [2+2] photocycloaddition of cyclohexene 597 with 3-acetoxy-2-methyl-cyclopent-2enone (598) gives a fully functionalized cyclobutane. This first step sets four of the required five stereocenters for the fully functionalized cyclopentane. Ketone reduction furnishes 599. Protecting group manipulations and activation of the secondary hydroxyl as a tosylate gave $\mathbf{6 0 0}$. Treatment of intermediate $\mathbf{6 0 0}$ with base induced a

133 (a) Bon, D. J. -Y. D.; Banwell, M. G.; Ward, J. S.; Willis, A. C. Tetrahedron 2013, 69, 1363-1368. (b) Reekie, T. A.; Austin, K. A. B.; Banwell, M. G.; Willis, A. C. Aust. J. Chem. 2008, 61, 94-106. (c) Bon, D. J. -Y. D.; Banwell, M. G.; Willis, A. C. Tetrahedron 2010, 66, 7807-7814.

${ }^{134}$ Corey, E. J.; Nozoe, S. J. Am. Chem. Soc. 1965, 87, 5733-5735. 
unique skeletal rearrangement to give fully functionalized cyclopentane 602. Reductive deoxygenation gave a cyclopentene, and the MOM ether and acetonide were hydrolyzed under acidic conditions. Dihydroxylation occurred stereoselectively from the concave face of the bicycle to form the energetically favored cis-fused diquinane substructure in $\mathbf{6 0 3}$. This material was carried forward eight steps to intermediate 604. Elimination of the two tertiary alcohols gave a cross-conjugated dienone and subsequent removal of the acetates gave intermediate 130. A non-stereoselective epoxidation gave, among other diastereomers, $( \pm)$-coriolin.

\section{Scheme 78. Total Synthesis $( \pm)$-Coriolin (Tatsuta, 1980)}
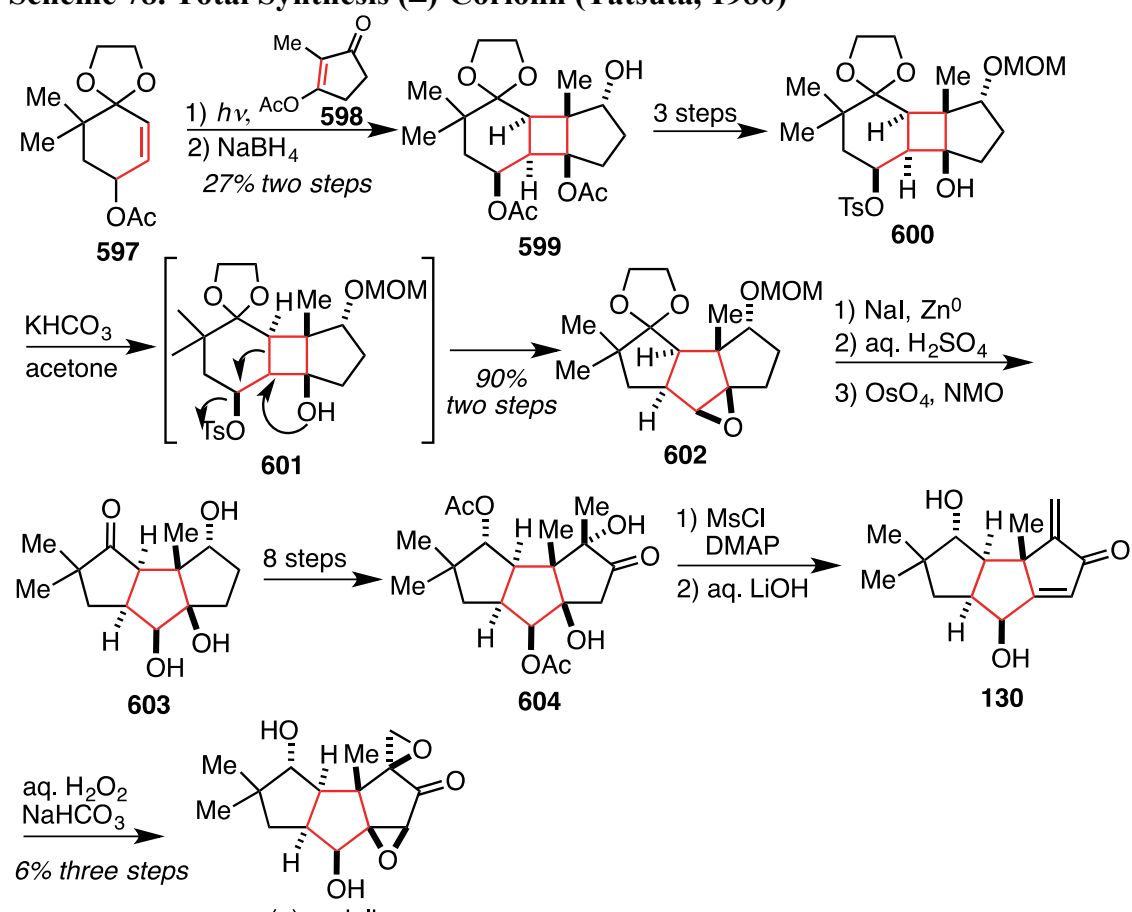

$( \pm)$-coriolin

The Greaney ${ }^{135}$ synthesis of $( \pm)$-merrilactone A and $( \pm)$-anislactone A employs a Tiffeneau-Demjanov-type ring expansion to construct a cyclopentanone (Scheme 79). The synthesis begins with a [2+2] photocycloaddition of 4,5-dimethylmaleic anhydride $\mathbf{6 0 5}$ and dimethylketene acetal to deliver cyclobutane 606. Reductive opening of the anhydride gives a diol, which is benzyl protected. Treatment with sulfuric acid delivers cyclobutanone 607. Reaction with ethyl diazoacetate (608) in the presence of a Lewis acid effected a Tiffeneau-Demjanov-type reaction yielding cyclopentanone 609. Transesterification with allyl alcohol was followed by $C$-alkylation of the $\beta$-keto ester with allyl bromide. Upon reaction with $\mathrm{Pd}(\mathrm{OAc})_{2}$, a Tsuji-Trost decarboxylation-dehydrogenation sequence proceeded to deliver cyclopentenone 610. A three-step functional group manipulation sequence gave alcohol 611. Nucleophilic epoxidation gave a diastereomeric mixture favoring the undesired $\beta$-epoxide diastereomer (612) with the desired $\alpha$-epoxide 613. The $\beta$-diastereomer could, however, be recycled through reductive deoxygenation to regenerate enone 611. Silyl protection of 613 was followed by a stereoselective addition of acetylide $\mathbf{6 1 4}$ on the convex face of the 6-oxa-bicyclo[3.1.0]hexane to deliver $\mathbf{6 1 5}$ as a single stereoisomer. Basic methanolysis of the silyl group afforded 616. Cleavage of the epoxide with titanium was followed by a 5-exo-dig cyclization onto the alkyne affording a single stereoisomer $617 .{ }^{136} \mathrm{~A}$ fully functionalized cyclopentane has been synthesized and was elaborated to $( \pm)$-anislactone A in six steps. Additionally, advanced intermediate 617 could be advanced over eight steps to complete a synthesis of ( \pm )-merrilactone A.

Scheme 79. Total Synthesis of ( \pm -Merrilactone A and ( \pm )-Anislactone A (Greaney, 2010)

\footnotetext{
${ }^{135}$ Shi, L.; Meyer, K.; Greaney, M. F. Angew. Chem. Int. Ed. 2010, 49, 9250-9253.
}

${ }^{136}$ RajanBabu, T. V.; Nugent, W. A. J. Am. Chem. Soc. 1994, 116, 986-997. 

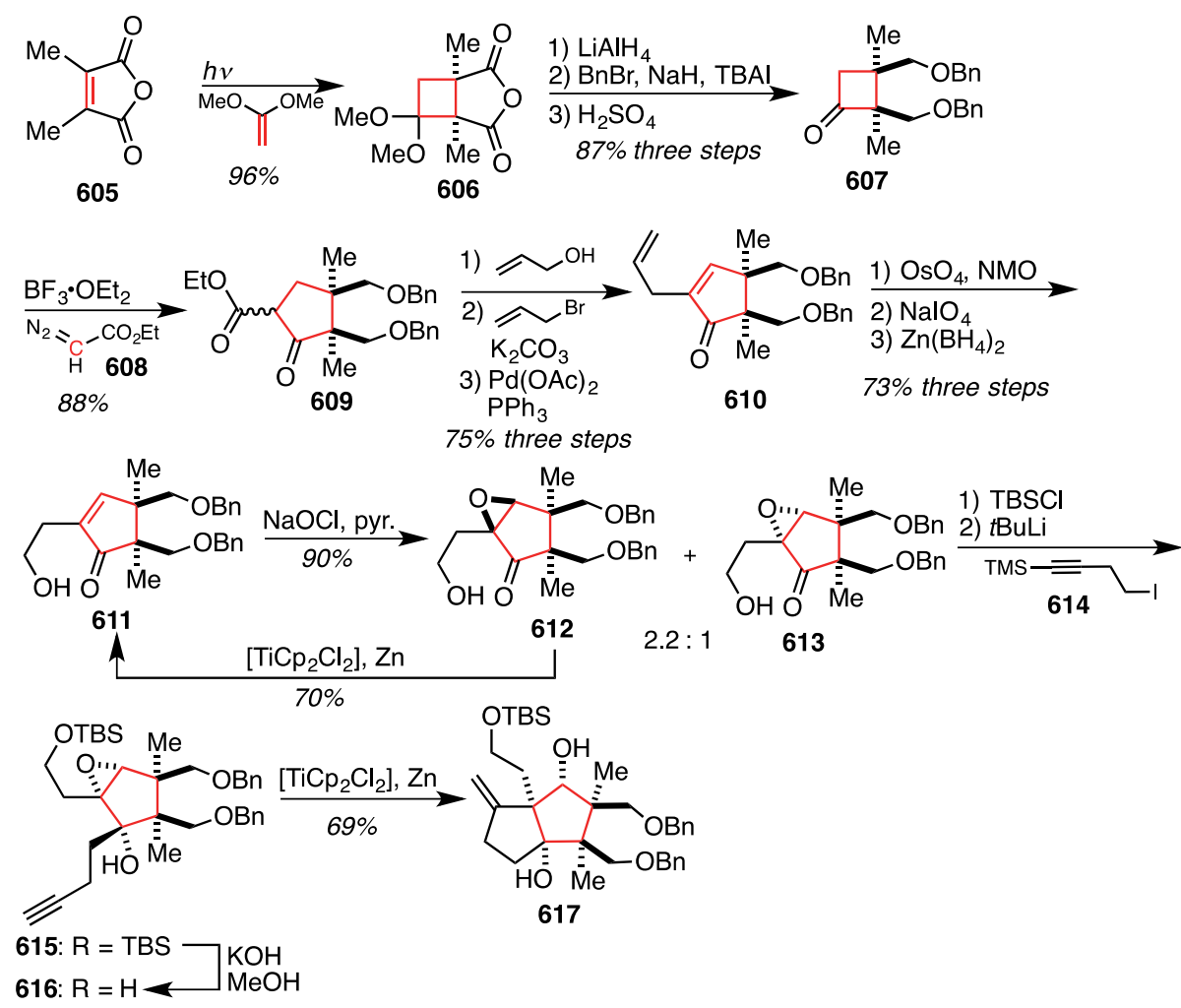

$90 \%$ three steps

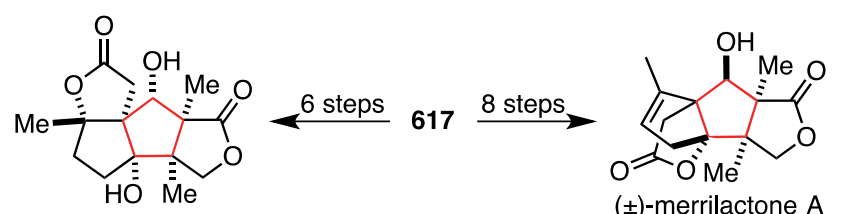

anislactone $\mathrm{A}$

$( \pm)$-merrilactone A

\subsection{Cope Rearrangement}

In the synthetic effort toward palau'amine, $\operatorname{Gin}^{137}$ constructs a fully functionalized cyclopentane through a $[3,3]$ sigmatropic rearrangement of a bridged tricyclodecadiene (Scheme 80). The synthesis begins with the DielsAlder reaction of 1,4-benzoquinone and cyclopentadiene (11) to give dihydroquinone 618. Epoxidation of the enone was followed by a Favorskii-like rearrangement of the keto-epoxide to deliver keto-ester 619. This intermediate underwent enolate alkylation to give 620, which has a 1,5-diene substructure. This intermediate underwent spontaneous [3,3]-sigmatropic rearrangement to give a dynamic mixture of enone $\mathbf{6 2 0}$ and cyclopentanone 621. This dynamic mixture was subjected to Meerwein-Pondorf-Verley reduction conditions which selectively consume the more reactive cyclopentanone and converts all the reactive mixture to secondary alcohol 622. Chloride displacement proceeded with retention of configuration to deliver $\mathbf{6 2 3}$. ${ }^{138}$ This material was advanced three steps to bridged ketone 624. Oxime formation followed by treatment with thionyl chloride induced a regioselective Beckmann rearrangement to form the lactam, which was subsequently protected as the Boc-carbamate (625). Oxidative cleavage of the alkene and reductive workup gave a diol that spontaneously cyclized to a lactone upon treatment with TsOH. Silylation of the primary alcohol delivered $\mathbf{6 2 6}$. The benzyl ether was removed, and cyclization of the free alcohol with the carbamate and dimethoxymethyl benzene delivered 627. Hydrolysis of the lactone was followed by methylation of the resulting carboxylic acid. Oxidation of the primary alcohol gave an aldehyde that was epimerized to the more stable trans configuration in $\mathbf{6 2 8}$. The fully functionalized cyclopentane contains the appropriate functionalization and stereochemistry for palau'amine.

${ }^{137}$ Bultman, M, S.; Ma, J.; Gin, D. Y. Angew. Chem. Int. Ed. 2008, 47, 6821-6824.

${ }^{138}$ Story, P. R. J. Org. Chem. 1961, 26, 287-290. 
Scheme 80. Synthetic Studies Towards Palau'amine (Gin, 2008)
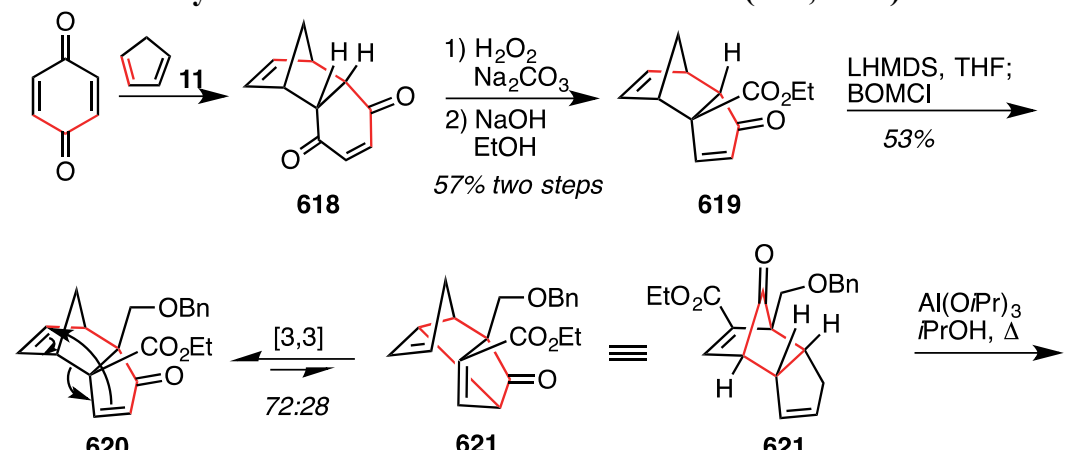

620

621

621

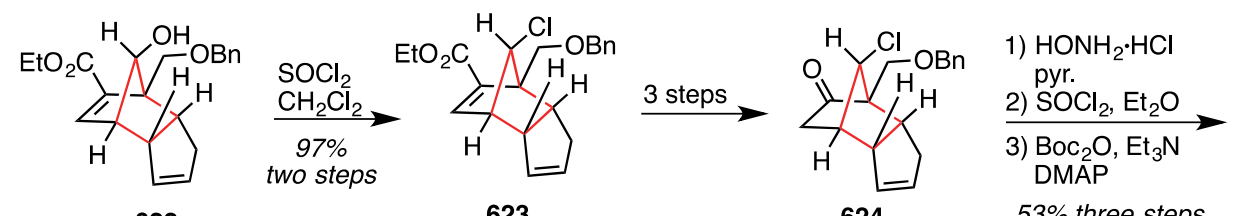

622

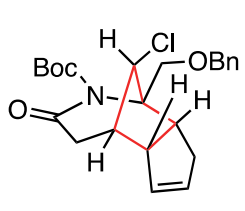

625

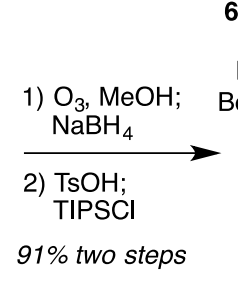

91\% two steps

623

624

$53 \%$ three steps

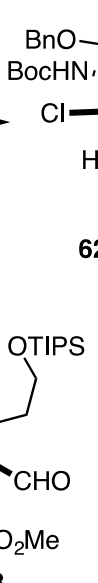

OTIPS
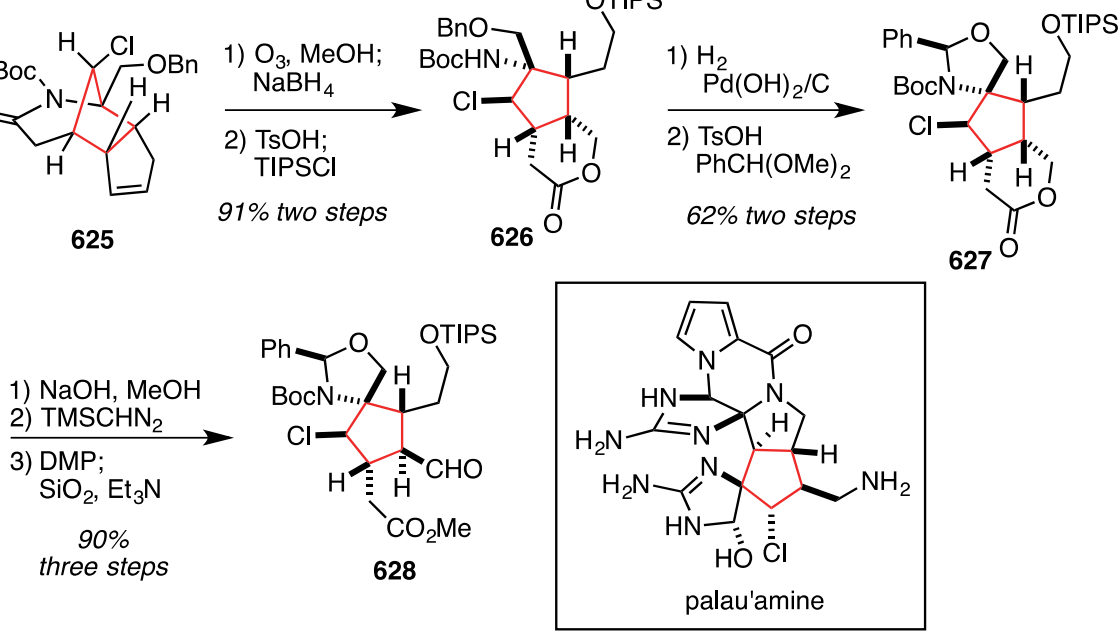

\section{Aldol and Related Reactions}

The aldol reaction and related reactions of enolate-type nucleophiles with carbonyl electrophiles are versatile processes that have been used in many syntheses of natural products with fully functionalized cyclopentanes. Such reactions have performed well in complex substrates decorated with many functional groups. The diversity in target molecules made with these reactions attests to their value as bond-forming processes.

The intramolecular aldol reaction forms a five membered carbocycle when the substrate is either a 1,4- or 1,6dicarbonyl (Scheme 81). The former synthetic strategy begins with a ketone starting material 629 (eq. 1), which undergoes functionalization (e.g. alkylation) at the $\alpha$-position to reveal a 1,4-dicarbonyl (630). Cyclization gives the five-membered carbocycle (631).

\section{Scheme 81.}


1,4-dicarbonyl aldol:

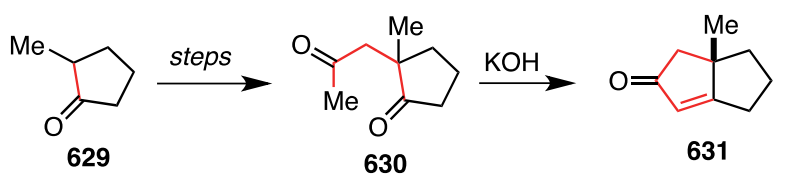

1,6-dicarbonyl aldol:

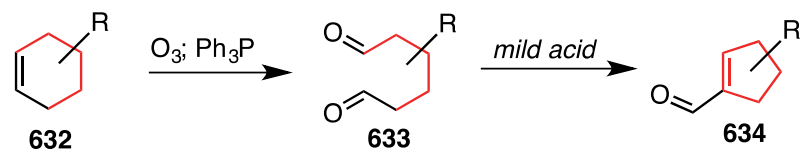

The latter strategy (1,6-dicarbonyl aldol) commonly begins with cyclohexenes of the type 632 (eq. 2). Such molecules are often prepared with good control of diastereoselectivity using the Diels-Alder reaction. A subsequent oxidative cleavage reveals the 1,6-dicarbonyl adorned with stereocenters at the intervening carbons (633). Aldol condensation with mild buffered-acid conditions gives the five-membered ring architecture (634). Typically, the more hindered carbonyl acts as the electrophile.

A particularly striking strategic use of aldol reactions appears in a ryanodol synthesis where no less than three intramolecular aldol reactions are used to prepare two fused fully functionalized cyclopentanes (Scheme 82). The substrate for the final intramolecular aldol is a 1,6-dicarbonyl that is revealed from oxidative cleavage of a cyclohexene prepared by a Diels-Alder reaction.

\section{Scheme 82 .}

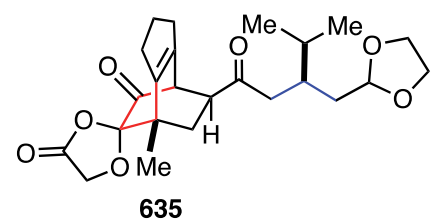

635

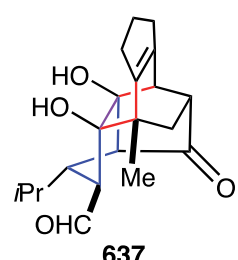

637

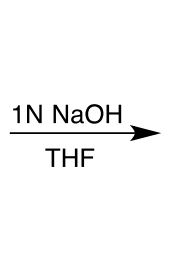

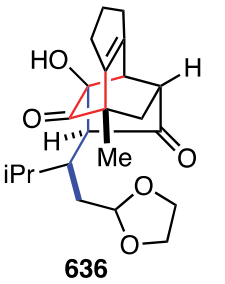

aq. $\mathrm{AcOH}$;

$1 \mathrm{~N} \mathrm{NaOH}$

636

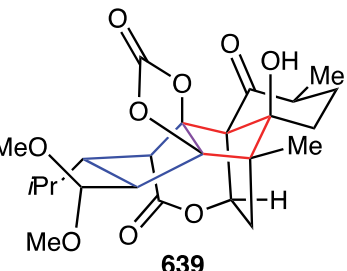




\subsection{1,4-Dicarbonyl Cyclizations}

In the total synthesis of $(+)$-cyclosativene by Piers ${ }^{139}$ performs an aldol reaction of a 1,4-ketoester to construct a five-membered ring (Scheme 83). The route begins with known enone 640, which oxidation to furnish 641. Conjugate addition of methyl cuprate and acylation of the resulting enolate delivers enol acetate 642. Ozonolysis, oxidative workup, and methylation of the resulting carboxylic acid gave aldol cyclization precursor 643. Enolate formation and Diekmann-type condensation delivered a functionalized five-membered ring (644). The ketone bearing an acidic $\alpha$-hydrogen was deprotonated and protected as the isopropyl enol ether. A Wittig olefination was performed on the remaining ketone and hydrolysis of the two enol ethers delivered keto-aldehyde 645. Wittig olefination of the aldehyde gave alkene 646. Ketone reduction and dehydration gave a functionalized cyclopentene. Hydroboration-oxidation of the less hindered alkene delivered 647. Oxidation and hydrazone formation furnished 648. Treatment with butyllithium formed the lithium salt, which upon heating induced a 1,3dipolar cycloaddition to deliver pyrazoline 649. Photolysis of this compound gave $(+)$-cycosativene displaying a fully functionalized cyclopentane.

\section{Scheme 83. Total Synthesis of (+)-Cyclosativene (Piers, 1973)}

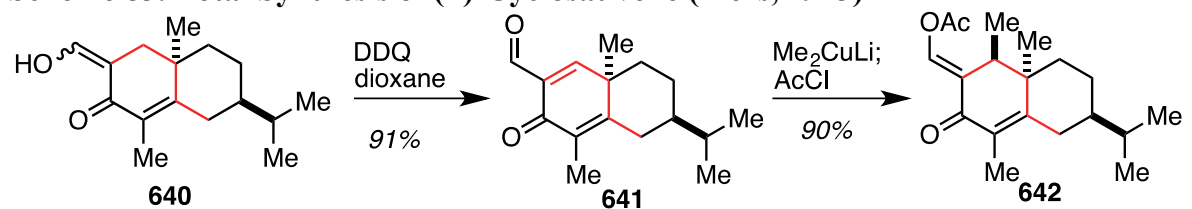
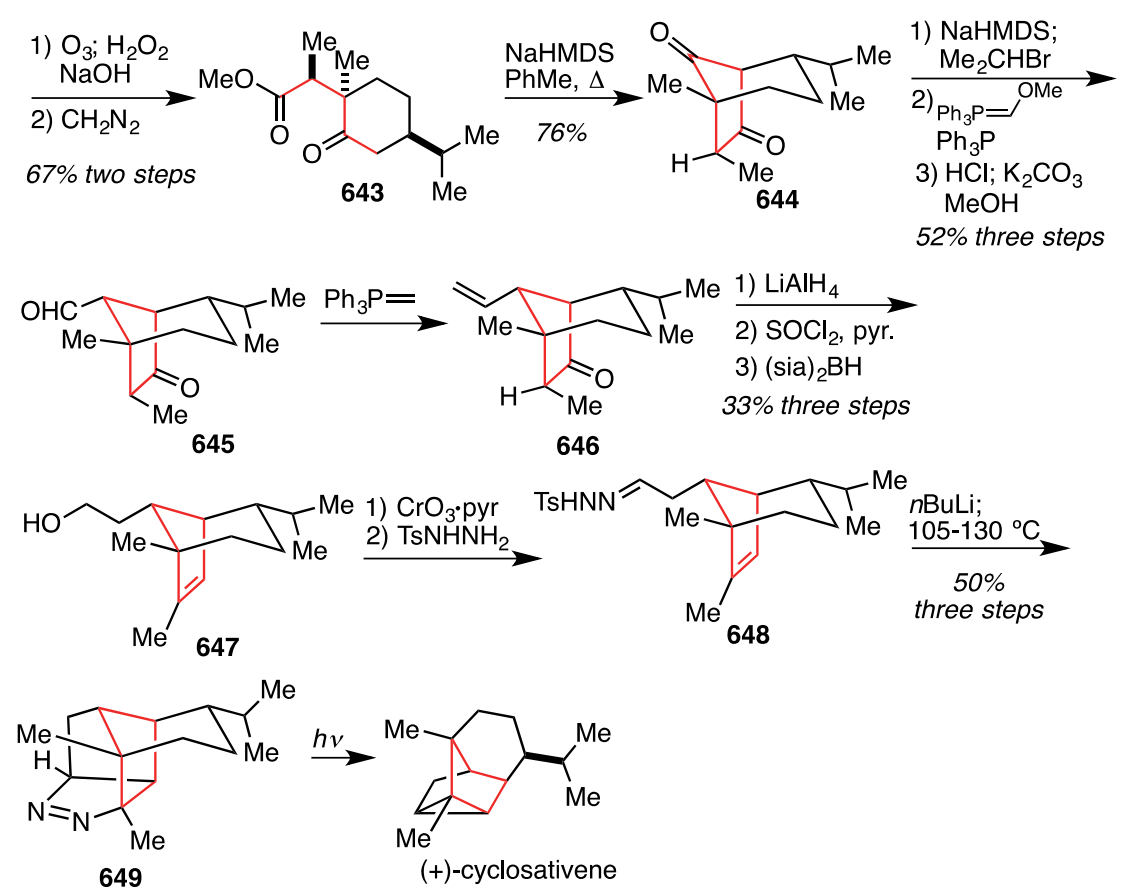

In the formal synthesis of (+)-cyclosativene by Yoshikoshi ${ }^{140}$, an intramolecular aldol of a 1,4-dicarbonyl constructs the cyclopentane (Scheme 84). The synthesis begins with intermediate $\mathbf{6 5 0}$ available from $(-)$-carvone. Consecutive enolate additions to dimethyl carbonate and allyl bromide deliver 651. Demethoxycarbonylation was performed by heating in DMSO with sodium cyanide. An oxidative cleavage of the allyl group gives ketoaldehyde 652. Treatment with base induces an aldol reaction of the 1,4-dicarbonyl to form cyclopentane 653 as a mixture of alcohol epimers. Oxidation state adjustment required six steps and gave cyclopentanone $\mathbf{6 5 5}$. Methylation of the ketone and subsequent alcohol oxidation gave a cyclopentenone, which was dehydrated to a mixture of exo- and endo-cyclic alkenes. This mixture could be resolved to give the desired endo-cyclic alkene

139 (a) Piers, E.; Geraghty, M. B.; Kido, F.; Soucy, M. Synth. Commun. 1973, 3, 39-43. (b) Piers, E.; Geraghty, M. B.; Soucy, M. Synth. Commun. 1973, 3, 401-405.

${ }^{140}$ Hagiwara, H.; Miyashita, M.; Uda, H.; Yoshikoshi, A. Bull. Chem. Soc. Jpn. 1975, 48, 3723-3726. 
by heating in the presence of iodine delivering $\mathbf{6 5 6}$ as the sole product. A Wittig reaction/hydrolysis sequence provided a mixture of aldehydes, which was epimerized with base to the more stable 657 . A second homologation sequence was performed, and the resulting aldehyde was reduced to give primary alcohol 627. This highly functionalized cyclopentene is an intermediate taken to $(+)$-cyclosativene by Piers. ${ }^{139}$

Scheme 84. Formal Synthesis of $( \pm)$-Cyclosativene (Yoshikoshi, 1975)<smiles>CC1CCC(C(C)C)CC1=O</smiles>

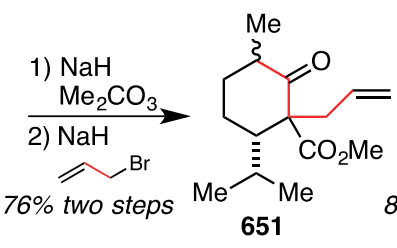

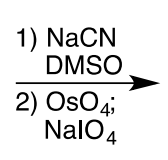
2) $\mathrm{OsO}_{4}$; $84 \%$ two steps

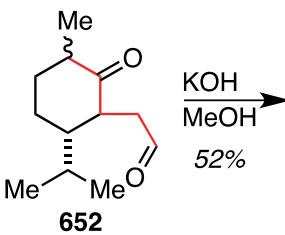<smiles>CC1CC2CC(C1=O)C(C)CC2(O)C(C)C</smiles>

653

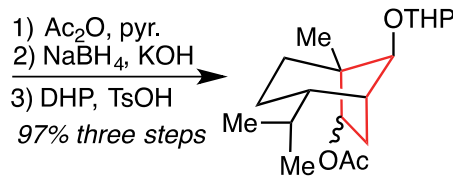

654

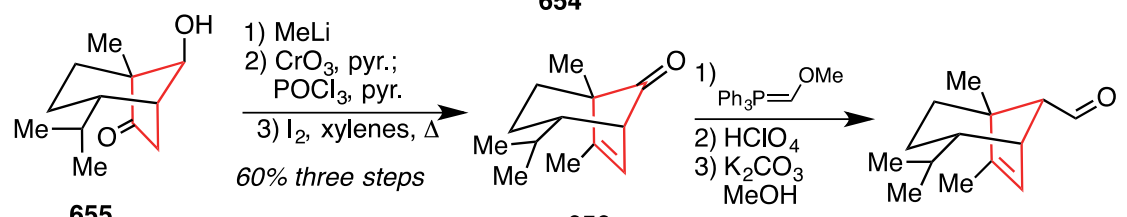

655

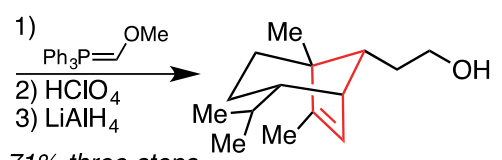

$71 \%$ three steps
1) $\mathrm{LiAlH}_{4}, \mathrm{Et}_{2} \mathrm{O}$

2) $\mathrm{CrO}_{3}$, pyr.

3) $\mathrm{TsOH}, \mathrm{H}_{2} \mathrm{O}$

dioxane

$60 \%$ three steps

In the synthesis of sesquiterpene natural products $( \pm)$-longicyclene and $( \pm)$-longiborneol, Welch ${ }^{141}$ constructs the cyclopentane through a reductive aldol cyclization (Scheme 85 and 86 ). The synthesis begins with (-)-carvone, which can be transformed into tetrahydroeucarvone $\mathbf{6 5 9}$ over three steps. Enolate formation under thermodynamic conditions and alkylation with chloride 660 delivers keto-olefin 661 as an inconsequential mixture of diastereomers. Oxidative cleavage of the alkene produced keto-acid 662. Acid-catalyzed lactonization delivered enol lactone 663. Reductive cleavage of the lactone and aldol cyclization gave cyclopentanone 664. This compound was discovered to be prone to fragmentation, so the material was immediately subjected to mesylation/elimination to give $\beta, \gamma$-unsaturated ketone 665. A Wittig reaction installed the methyl enol ether 666 , and hydrolysis gave a mixture of aldehydes that underwent epimerization to the more stable configuration in 667 . This aldehyde served as a key intermediate in the synthesis of both longicyclene and longiborneol. Towards the synthesis of longicyclene, Jones oxidation provided carboxylic acid $\mathbf{6 6 8}$. Conversion to the acid chloride and addition of diazomethane delivered a diazoketone that underwent copper-mediated cyclization yielding cyclopropyl ketone 669. This advanced intermediate contains a fully functionalized cyclopentane with the appropriate substitution and stereochemistry for $( \pm)$-longicyclene, and an additional three steps completed the natural product.

Scheme 85. Total Synthesis of ( \pm )-Longicyclene (Welch, 1974)

${ }^{141}$ Welch, S. C.; Walters, R. L. J. Org. Chem. 1974, 39, 2665-2673. 

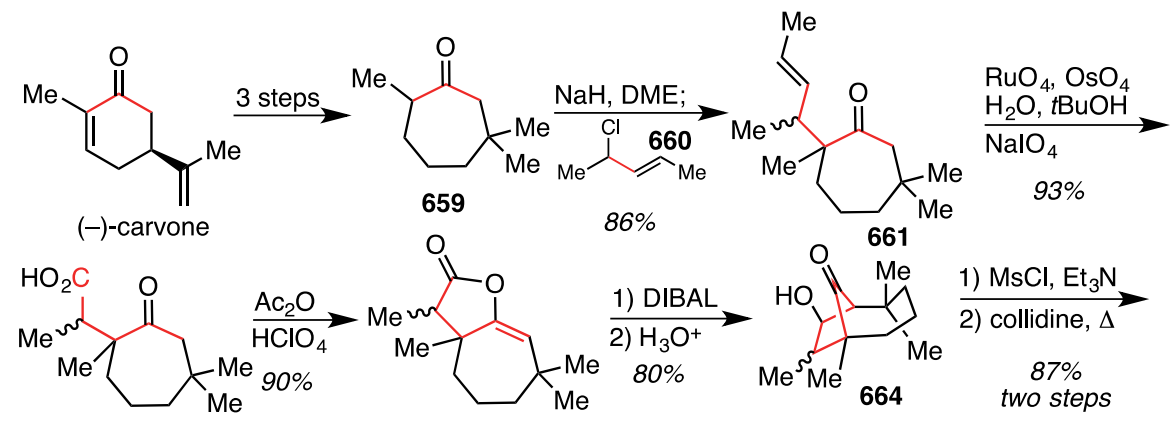

662

663
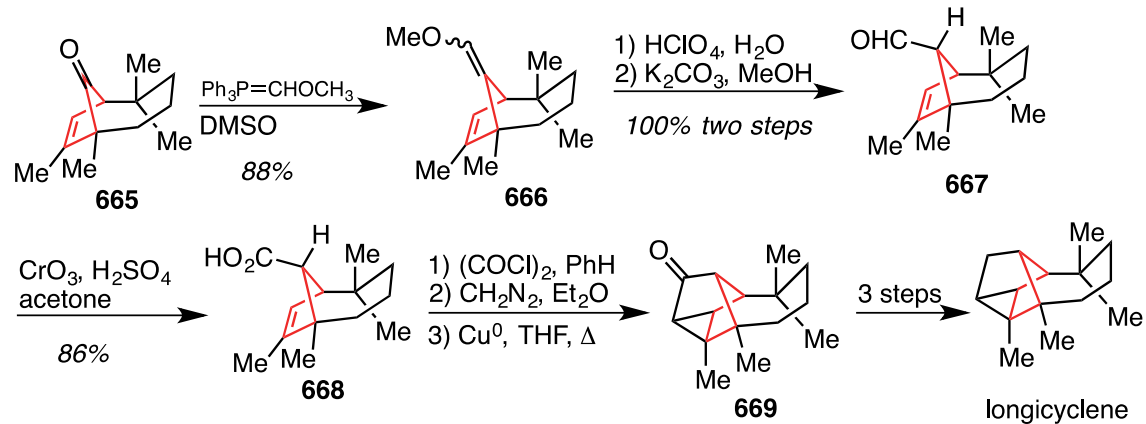

The synthesis of ( \pm )-longiborneol from intermediate aldehyde 667 continued with a Wittig reaction to give diene 670 (Scheme 86). Hydroboration-oxidation of the diene produced diol 671. Sulfonylation of the primary alcohol and oxidation of the secondary alcohol delivered ketone 672. An intramolecular enolate alkylation delivered a tetracyclic intermediate, which is the natural product longicamphor. Finally, a dissolving metal reduction with calcium delivered the fully functionalized cyclopentane of longiborneol.

Scheme 86. Total Synthesis of $( \pm)$-Longiborneol (Welch, 1974)
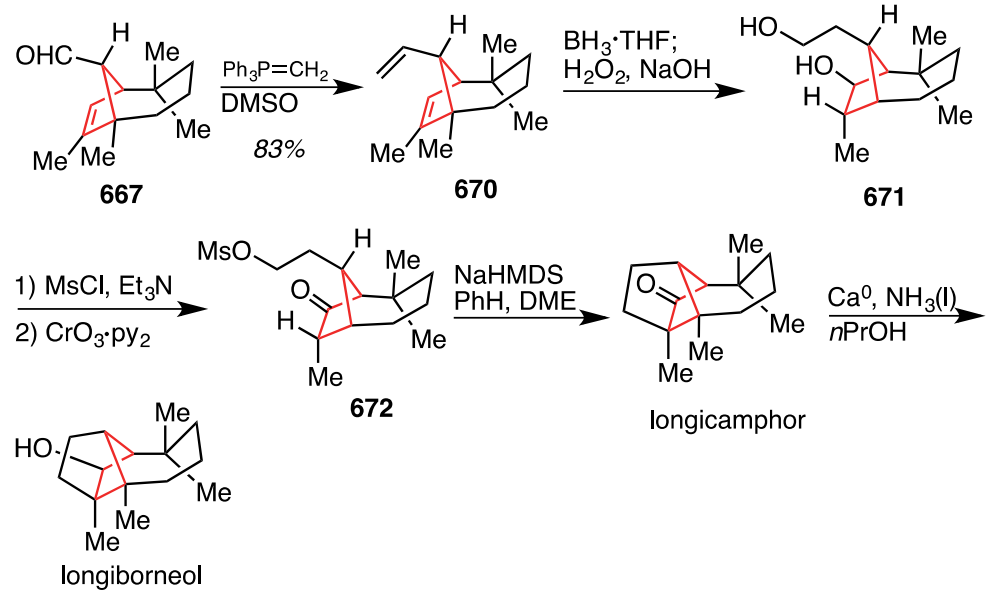

672

longicamphor

In the synthesis of tricyclic sesquiterpenoid $\left( \pm\right.$ )-gymnomitrol, Welch ${ }^{142}$ utilizes an aldol condensation to construct a cyclopentenone (Scheme 87). Cyclopentanone 629 was converted to 1,4-diketone 630 by alkylation with dibromide 673 and oxidation. A base-catalyzed aldol condensation furnished bicyclic cyclopentenone 631 Conjugate addition of methyl cuprate gives the cis-fused diquinane, and trapping of the enolate with allyl chloride gives 674 as an inconsequential mixture of diastereomers. Formation of the thermodynamic enolate was followed by alkylation on the convex face of the bicycle to yield 675 as a single diastereomer. Hydroborationoxidation of the alkene occurs with concomitant ketone reduction to deliver diol 676. Jones oxidation gave a carboxylic acid, which was methylated to yield ester 677. A Dieckmann-type condensation occurs with strong base, and silyl trapping of the enolate provides tricyclic ketone 678. Stereoselective reduction of the ketone from

${ }^{142}$ Welch, S. C.; Chayabunjonglerd, S. J. Am. Chem. Soc. 1979, 101, 6768-6769. 
the less hindered face furnishes a fully functionalized cyclopentane (679) bearing the necessary stereochemistry for gymnomitrol. Completion of the synthesis required an additional five steps.

\section{Scheme 87. Total Synthesis of ( \pm )-Gymnomitrol (Welch, 1979)}

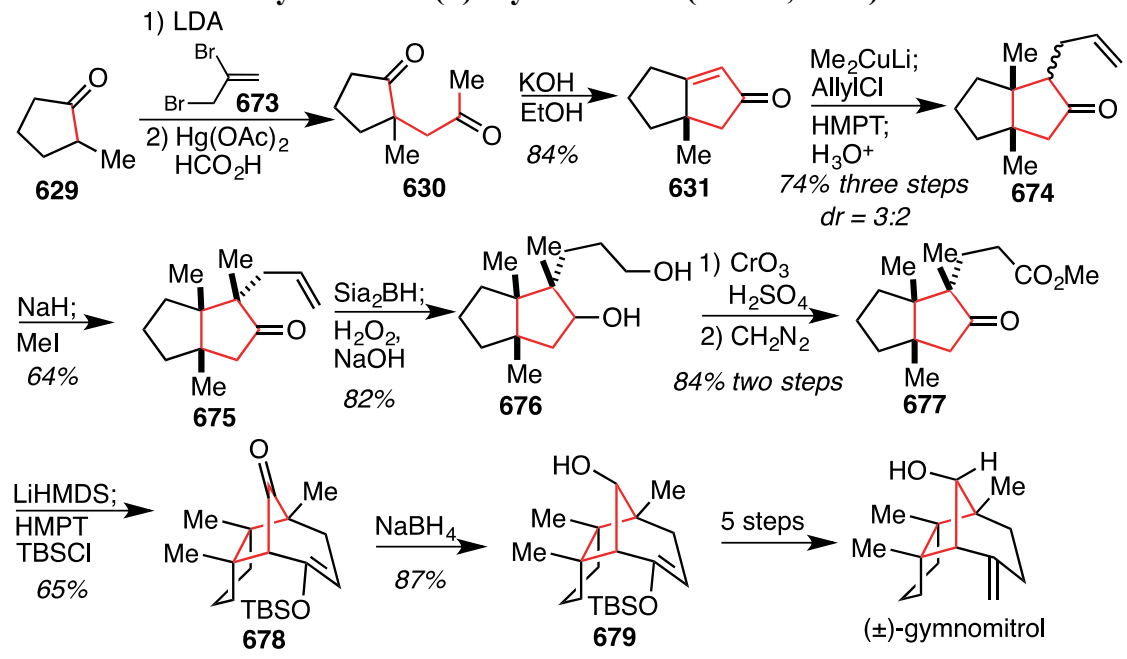

In the synthesis of the tricothecene mycotoxin T-2 tetraol tetra-acetate, Colvin ${ }^{143}$ builds the cyclopentane ring using an aldol addition of a 1,4-ketoaldehyde (Scheme 88). The synthetic route begins with lactone $\mathbf{6 8 0}$, which can be advanced to bicyclic intermediate $\mathbf{6 8 1}$ over two steps. Conjugate addition of methyl cuprate occurs from the convex face of the ring system. Epoxidation and a semi-pinacol rearrangement in the presence of ethylene glycol gives 682. Two successive oxidations gave the keto lactone 683 which existed exclusively in the enolized form. Allylation delivered enol ether $\mathbf{6 8 4}$. Reduction of the lactone with DIBAL produced an unstable lactol, which could be further reduced with triethylsilane to give deoxygenated $\mathbf{6 8 5}$. Hydride reduction of the ester was followed by heating in toluene to promote a Claisen rearrangement to give a ketone. Silylation of the primary alcohol gave 686. Dihydroxylation and oxidative cleavage delivered keto aldehyde 687 . The aldol addition took place with sodium methoxide to give a 4:1 mixture of diastereomers favoring the desired $\mathbf{6 8 8}$ (the configuration of the secondary alcohol is inconsequential). The alcohol was subsequently protected as the pyranyl ether, and olefination of the ketone gave 689. The pyranyl ether was cleaved and the resulting alcohol oxidized to ketone 690. The ketone was $\alpha$-hydroxylated with oxaziridine 691 and then reduced with hydride to give the trans-1,2diol 692; both additions having occurred from the less substituted exo face of the bicycle. Treatment with PPTS selectively deprotected the ketal, and epoxidation with peracid gave fully functionalized cyclopentane 693. This advanced intermediate was taken to $\mathbf{6 9 4}$, a relay intermediate in the synthesis of T-2 tetraol, which was completed in an additional four steps.

Scheme 88. Total Synthesis of T-2 Tetraol Tetraacetate (Colvin, 1990)

${ }^{143}$ Colvin, E. W.; Egan, M. J.; Kerr, F. W. J. Chem. Soc., Chem. Commun. 1990, 1200-1202. 
<smiles>COC(=O)c1ccc(=O)oc1</smiles>

1) $\mathrm{LiCuMe}_{2}, \mathrm{TMSCl}$

2) $m$ CPBA

3) ethylene glycol

$\mathrm{BF}_{3} \cdot \mathrm{OEt}_{2}$

681

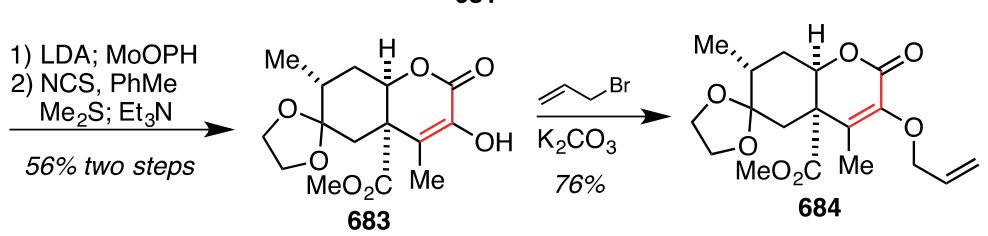<smiles>COC(=O)[C@@]12CC3(OCCO3)[C@@H](C)C[C@H]1OC(=O)C[C@H]2C</smiles>

682
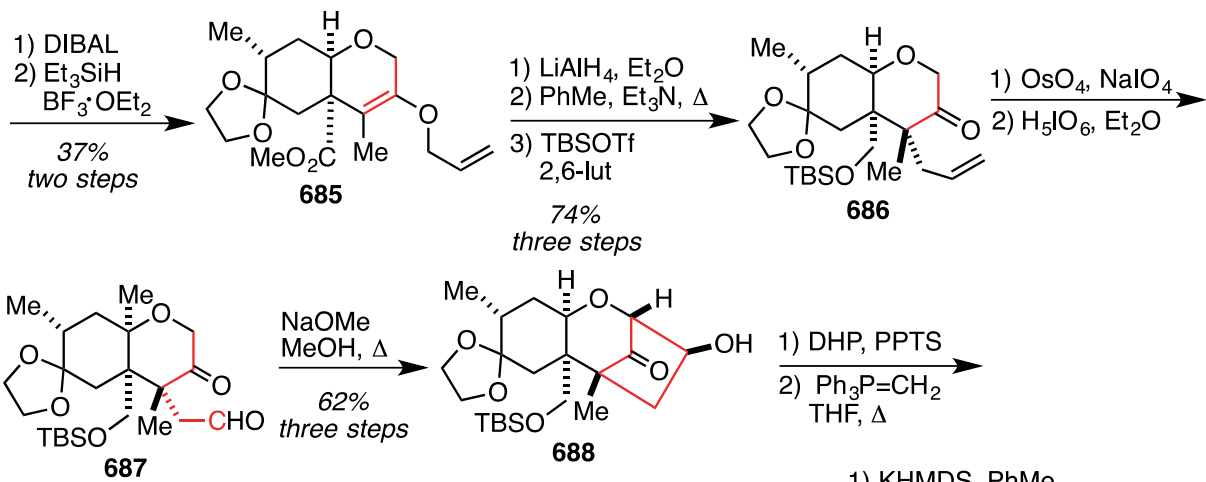

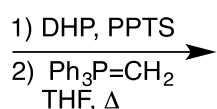

$\mathrm{THF}, \Delta$

1) KHMDS, PhMe

2)

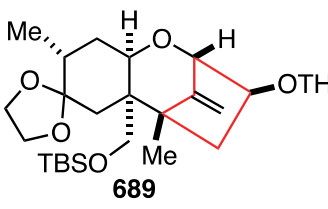

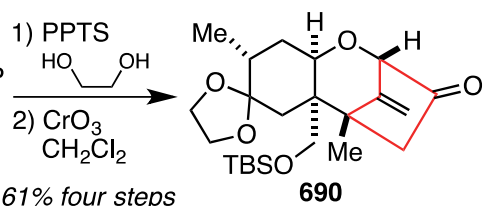

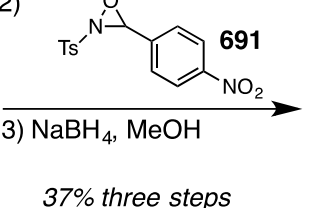

$61 \%$ four steps

$37 \%$ three steps
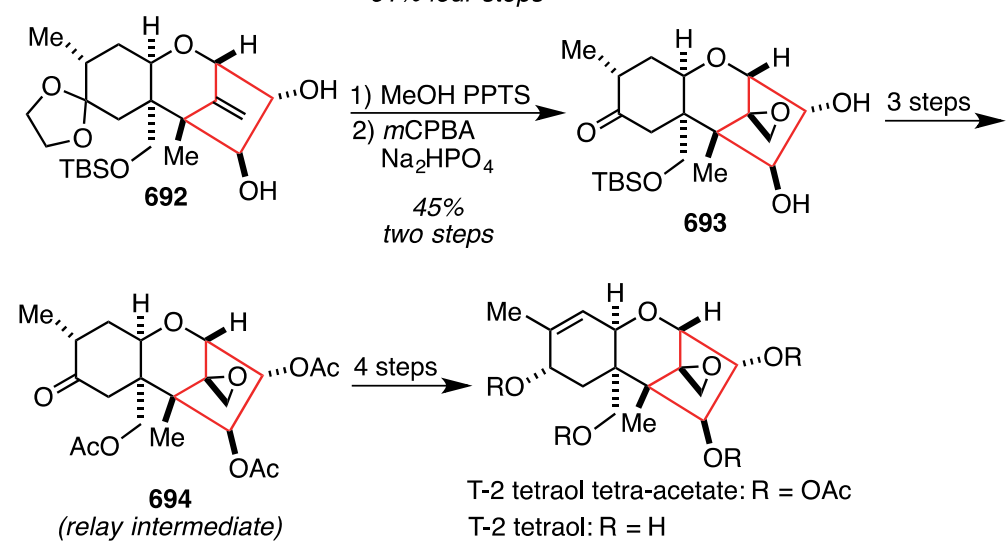

The sesquiterpenoid (-)-merrilactone A was synthesized by Inoue ${ }^{144}$ and utilized a transannular aldol reaction of a cyclooctenedione (Scheme 89). Beginning with the unsaturated $\gamma$-lactone 695, a [2+2]-photocycloaddition with trans-1,2-dichloroethylene (696) successfully establishes the two methyl stereocenters with the desired synrelationship. Dechlorination with zinc delivers bicyclic intermediate 697. This material was advanced to cyclobutene 698 over six steps. Dihydroxylation, alcohol oxidation, and Grignard addition gives fully functionalized cyclobutane 699. Ring closing metathesis was followed by oxidative cleavage of the diol yielding a cyclooctenedione (701). With carefully optimized conditions, the transannular aldol reaction gave diastereoselective carbon-carbon bond formation to yield the cis-fused diquinane 704. Deprotonation occurs at the most sterically accessible position (distal from the BTB group) to give a favored $(E)$-configured enolate, which exists in conformation $\mathbf{7 0 3}$ that minimizes diaxial interactions of the substituents. Transannular cyclization of $\mathbf{7 0 3}$ leads to the observed product $\mathbf{7 0 4}$ with excellent stereoselectivity. This material was advanced over three

${ }^{144}$ Inoue, M.; Sato, T.; Hirama, M. Angew. Chem. Int. Ed. 2006, 45, 4843-4848. 
steps to $\alpha$-bromoacetal 705. Treatment of $\mathbf{7 0 5}$ with radical conditions led to 5-exo-trig cyclization in good yield producing a mixture of diastereomers. The mixture could be simplified to isomer $\mathbf{7 0 7}$ by treatment of 706 with $\mathrm{EtOSiMe}_{3}$ and Lewis acid. This material was advanced over six steps to trisubstituted alkene 708. Reduction of the ketone with sodium in ammonia resulted in a stereoselective reduction to the desired $\beta$-hydroxy compound. This sets the final stereocenter in the fully functionalized cyclopentane, giving 710. This advanced intermediate has appropriate substitution and stereochemistry for (-)-merrilactone A, and the synthesis was completed in an additional two steps.

\section{Scheme 89. Total Synthesis of (-)-Merrilactone A (Inoue, 2006)}

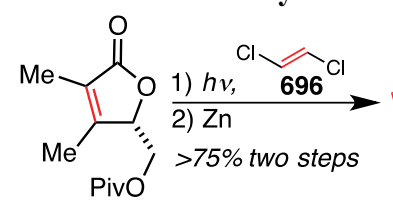

695

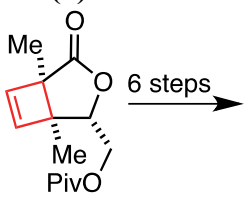

697

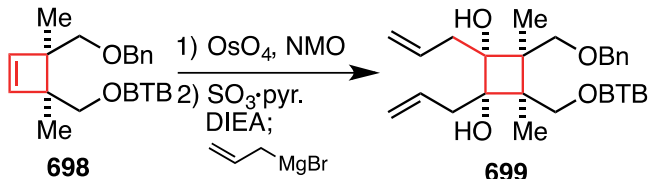

$73 \%$ two steps

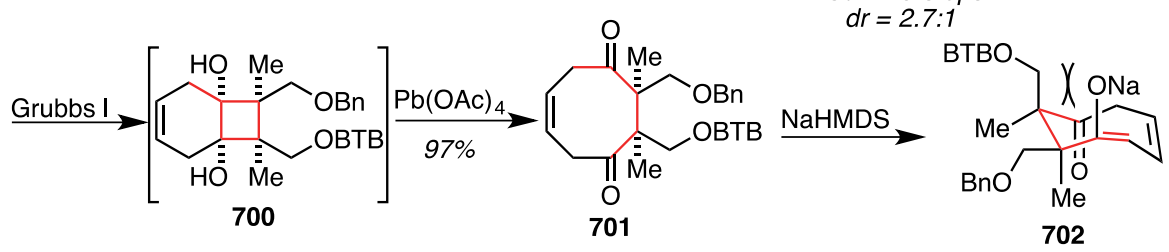<smiles>CC(CO)(COCc1ccccc1)C(C)(COCc1ccccc1)C(=O)OCc1ccccc1</smiles>

703

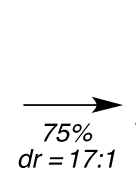<smiles>CC[C@]1(CBr)C(=O)[C@@H]2C=CC[C@]2(O)[C@]1(C)CBr</smiles>

704
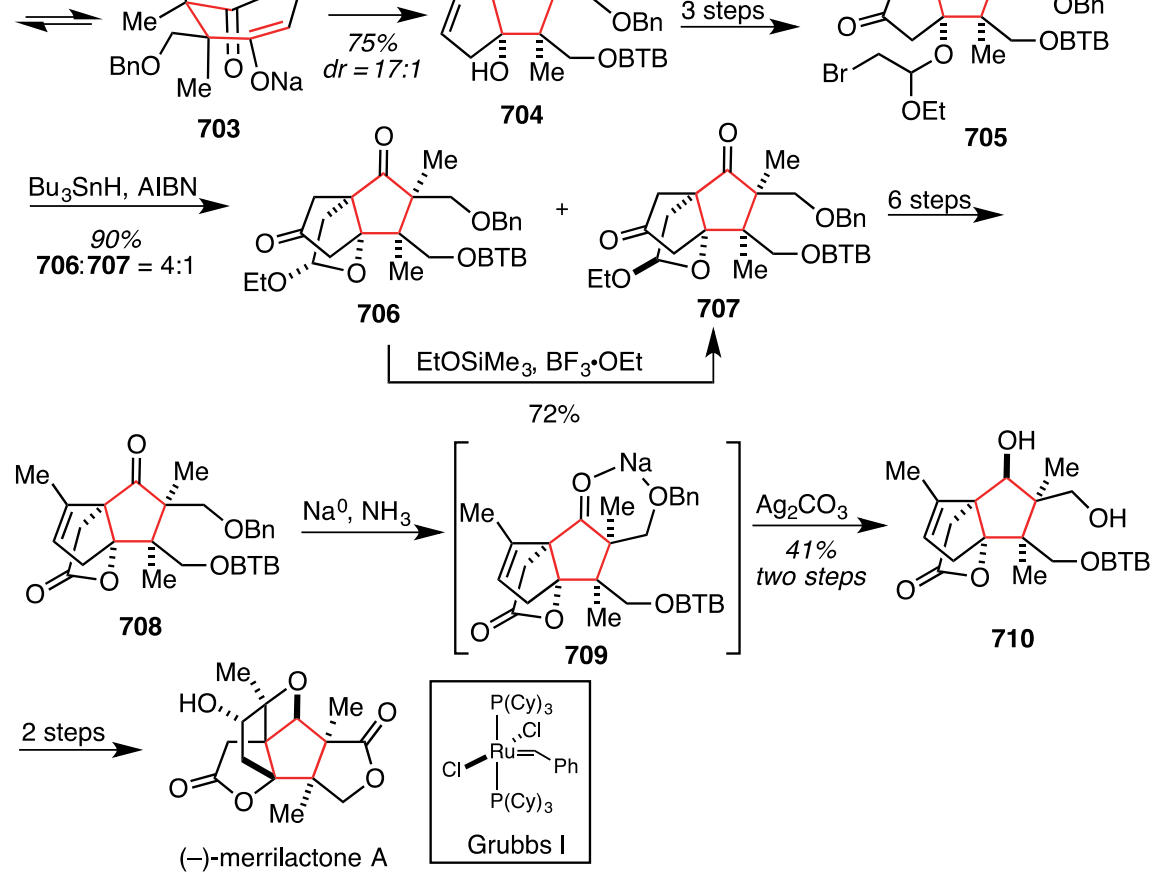

The first synthesis of (+)-pactamycin was accomplished by Hannesian (Scheme 90). ${ }^{145}$ Pactamycin has a densely functionalized cyclopentane core with three contiguous fully substituted stereocenters. The cyclopentane was constructed using an aldol condensation. Commercially available L-threonine was used to synthesize intermediate 711 over three steps. Condensation with unsaturated aldehyde $\mathbf{7 1 2}$ and silylation of the resulting secondary alcohol delivers 714. The stereochemical outcome can be rationalized by the favored ZimmermanTraxler transition state model 713. Intermediate $\mathbf{7 1 4}$ was advanced three steps to ketone $\mathbf{7 1 5}$. Ozonolysis of the alkene provides the desired 1,4-dicarbonyl relationship. A Mukaiyama-type aldol condensation proceeded in the presence of $\mathrm{TiCl}_{4}$ to yield cyclopentanone 696. Alcohol elimination yields cyclopentenone 717. Unexpectedly,

${ }^{145}$ Hanessian, S.; Vakiti, R. R.; Dorich, S.; Banerjee, S.; Deschnês-Simard, B. J. Org. Chem. 2012, 77, 9458-9472. 
nucleophilic epoxidation occurred from the undesired bottom face of the carbocycle. Luche reduction delivered epoxy alcohol 718. At this stage, it was known that an inversion of the epoxide would be necessary to complete the synthesis of pactamycin. An invertive displacement of the secondary alcohol generated azide $\mathbf{7 1 9}$ with the required nitrogen stereochemistry. A three-step sequence (TES removal, oxidation, Grignard addition) delivered tertiary alcohol $\mathbf{7 2 0}$ as a single diastereomer. The epoxide inversion began with liberation of the primary alcohol with fluoride. Treatment of the epoxy alcohol with zinc triflate induces a Payne rearrangement to give an intermediate spiro-oxirane, which is subsequently opened by acetic acid giving 721. The acetate-protected alcohol was converted to the corresponding TBDPS ether. Treatment of the diol with triflic anhydride led to Williamson-type epoxide formation yielding 722. Finally, opening of the epoxide with aniline $\mathbf{7 2 3}$ gave advanced intermediate $\mathbf{7 2 4}$, which has the desired substitution and stereochemistry on the five-membered ring of pactamycin. Completion of the synthesis required an additional ten steps.

\section{Scheme 90. Total Synthesis of (+)-Pactamycin (Hanessian, 2012)}<smiles>CC(O)C(N)C(=O)O</smiles>

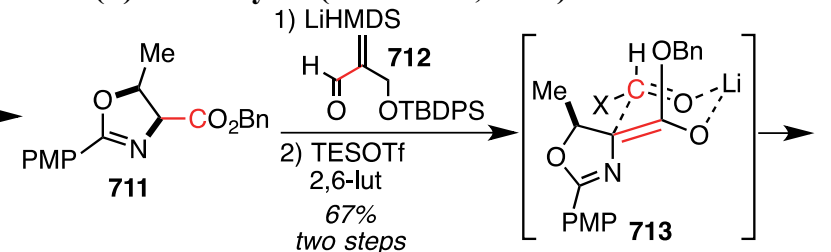

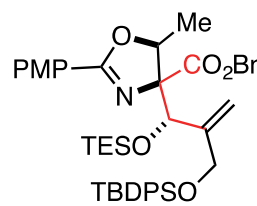

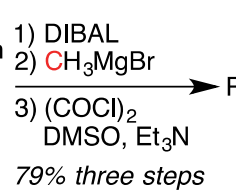

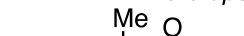
two step<smiles>C=C(C)C(=O)O[Mg]</smiles>

TBDPSO DIPEA

$715 \quad 71 \%$ two steps

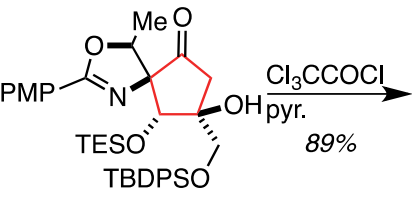

714

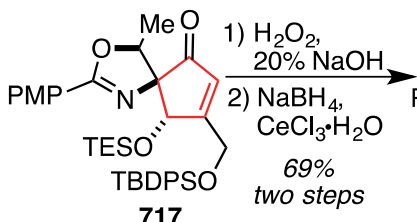

717

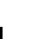

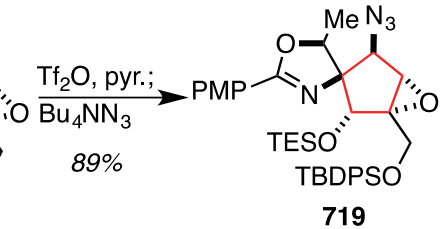

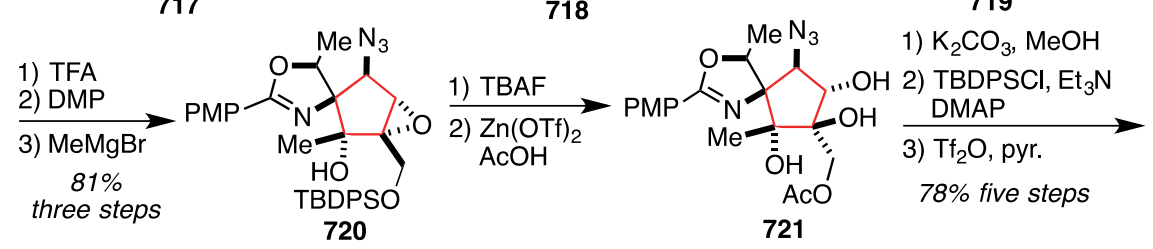<smiles>C/C(=C\Cc1ccccc1)c1cccc(N)c1</smiles><smiles>CC(=O)OC[C@@H](C)[C@H](C)OC(C)C</smiles>

(+)-pactamycin

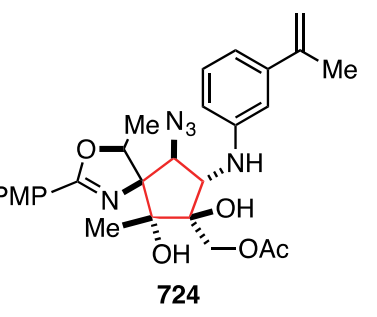


In the total synthesis of pactamycin, Johnson ${ }^{146}$ used an aldol condensation to form a highly functionalized cyclopentenone (Scheme 91). The synthesis begins with a cinchonidine catalyzed Mannich reaction of diketone 725 and imine $\mathbf{7 2 6}$. When catalyst 727 was employed, product 728 could be obtained with good enantioselectivity. Desymmetrization of the Mannich product was accomplished using a hydride reduction where addition of hydride to a lithium chelate of $\mathbf{7 2 8}$ occurs away from the sterically large urea group. TBS protection gives 729. An intermolecular aldol reaction with formaldehyde homologated the ketone. Oxidative cleavage of the alkene delivers the 1,4 dicarbonyl relationship in $\mathbf{7 3 0}$ necessary to deliver a cyclopentenone. Reaction with sodium methoxide promotes an aldol condensation delivering 731. Nucleophilic epoxidation on the same face as the nitrogen groups yields $\mathbf{7 3 2}$ in high diastereoselectivity. Silylation of the primary alcohol with the sterically demanding TBDPS group shielded one reactive diastereoface of the ketone, and addition of methyl Grignard led to the desired tertiary alcohol stereocenter on 733. Finally, nucleophilic opening of the epoxide with 3acetylaniline (734) yielded the fully functionalized cyclopentane 735, which contains the desired molecular architecture of pactamycin. An additional three steps was required to complete the natural product.

Scheme 91. Total Synthesis of (+)-Pactamycin (Johnson, 2013)
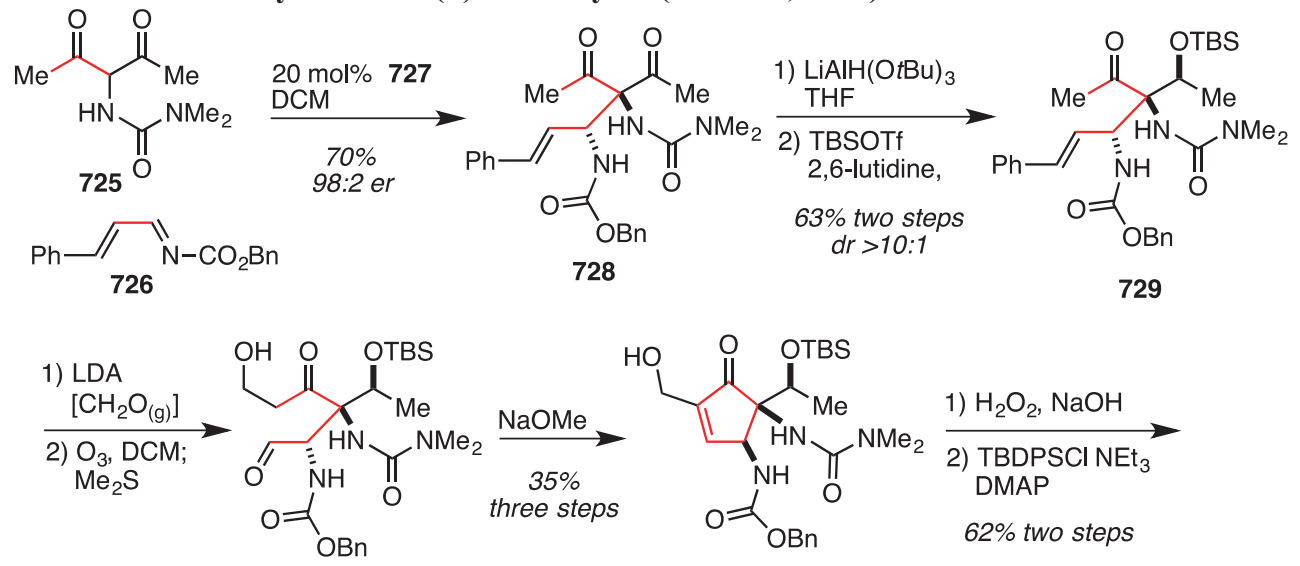

730
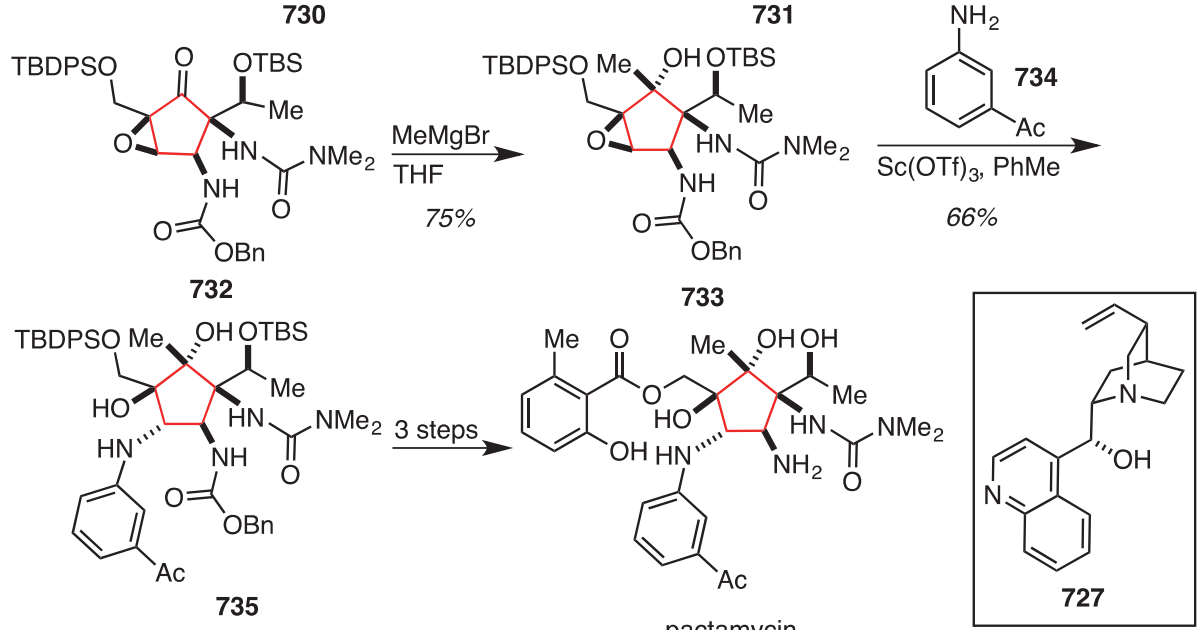

Corey ${ }^{147}$ reported an approach to gibberellic acid using an aldol of a 1,4-ketoaldehyde that was an improvement over their previously published route ${ }^{151}$, which used the aldol of a 1,6-dicarbonyl (Scheme 92). As such, this effort represents a formal (or second generation) synthesis of gibberellic acid. This route begins with the HornerWadsworth-Emmons olefination of ketone $\mathbf{7 3 6}$ with ylide $\mathbf{7 3 7}$. Vinyl cuprate conjugate addition occurs to give an inconsequential mixture of diastereomers, and the product mixture undergoes oxidative cleavage to give 1,4ketoaldehyde 738. An aldol condensation in ethanolic sodium hydroxide furnishes spiro enone 739. This material was advanced to the ketal $\mathbf{7 4 0}$ over six steps. Treatment with potassium tert-butoxide induced intramolecular alkylation to give $\mathbf{7 4 1}$. Intermediate $\mathbf{7 4 1}$ was converted to ketone $\mathbf{7 4 2}$ over 10 steps. Selective formylation at the

\footnotetext{
${ }^{146}$ Malinowski, J. T.; Sharpe, R. J.; Johnson, J. S. Science, 2013, 340, $180 \square 182$.
}

${ }^{147}$ Corey, E. J.; Smith, J. G. J. Am. Chem. Soc. 1979, 101, 1038-1039. 
least hindered $\alpha$-position provided 743. Methylation of the enol tautomer $\mathbf{7 4 3}$ gave $(E)$-enol ether $\mathbf{7 4 4}$ in a 6:1 ratio with its geometrical isomer. This mixture was formylated and the aldehyde was reduced to give $\mathbf{7 4 5}$. Subsequent ketone reduction delivered diol $\mathbf{7 4 6}$ as a mixture of alcohol epimers. Treatment with oxalic acid promoted formation of enal 747. Finally, standard Wittig olefination completed the second generation synthesis of 748 .

Scheme 92. Formal Syntheis of Gibberellic Acid (Corey, 1979)
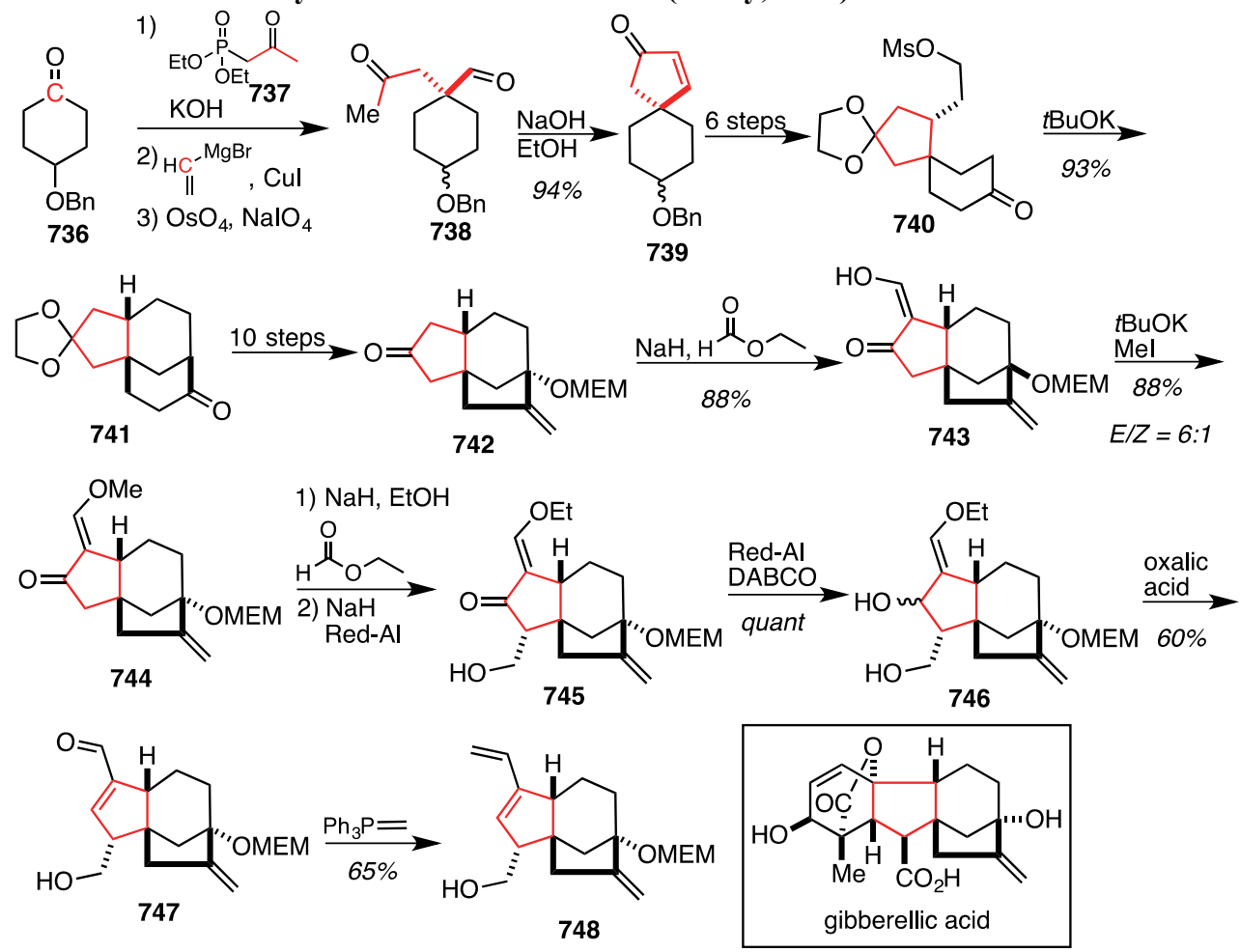

In the synthetic effort towards delphinine, Wiesner ${ }^{148}$ used an intramolecular aldol addition to create the central five-membered carbocycle of the target (Scheme 93). ${ }^{149}$ The methoxy tetralone starting material (749) underwent consectutive with allyl bromide and $\mathrm{BOMCl}$ to give 750. Subsequent oxidative cleavage gave aldol substrate 751. A base-catalyzed aldol reaction constructed the five membered carbocycle (752). This tricyclic intermediate was functionalized over 12 steps to 753. Amination with Raney nickel in methanolic ammonia proceeded to deliver the desired amine $\mathbf{7 5 4}$ with $d r=10: 1$. The unpurified amine was acetylated, the benzyl groups were removed by hydrogenolysis, and the diol was oxidized to the diketone $\mathbf{7 5 5}$. Heating the diketone with potassium cyanide promoted a sequence of reactions that resulted in the fully functionalized cyclopentane 759. First, aldol condensation delivered an $\alpha, \beta$-unsaturated ketone, which was followed by a stereoselective conjugate addition of cyanide. Subsequent hydrolysis of the nitrile provided an amide, which cyclized to the lactamol 759. Treatment of this intermediate with acidic methanol opened the lactamol to give ketone $\mathbf{7 6 0}$. Hydride reduction gave a separable mixture of diastereomers containing 761. This advanced intermediate contains the central fivemembered ring of the delphinine-type alkaloids with appropriate substitution and stereochemistry for delphinine.

\section{Scheme 93. Synthetic Studies Towards Delphinine (Wiesner, 1972)}

\footnotetext{
148 (a) Wiesner, K.; Jay, E. W. K.; Tsai, T. Y. R.; Demerson, C.; Jay, L.; Kanno, T.; Křepinský, J.; Vilím, A.; Wu, C. S. Can. J. Chem. 1972, 50, 1925-1943. (b) Wiesner, K. Pure \& Appl. Chem., 1979, 51, 689-703.

${ }^{149}$ For related work on this class of alkaloids by Wiesner, see : (a) Wiesner, K.; Bickelhaupt, F.; Babin, D. R.; Götz, M. Tetrahedron Lett. 1959, 3, 11-14. (b) Wiesner, K. Pure \& App. Chem. 1975, 41, 93-112. (c) Wiesner, K.; Tsai, T. Y. R.; Huber, K.; Bolton, S. E. J. Am. Chem. Soc. 1974, 96, 4990-4992.
} 

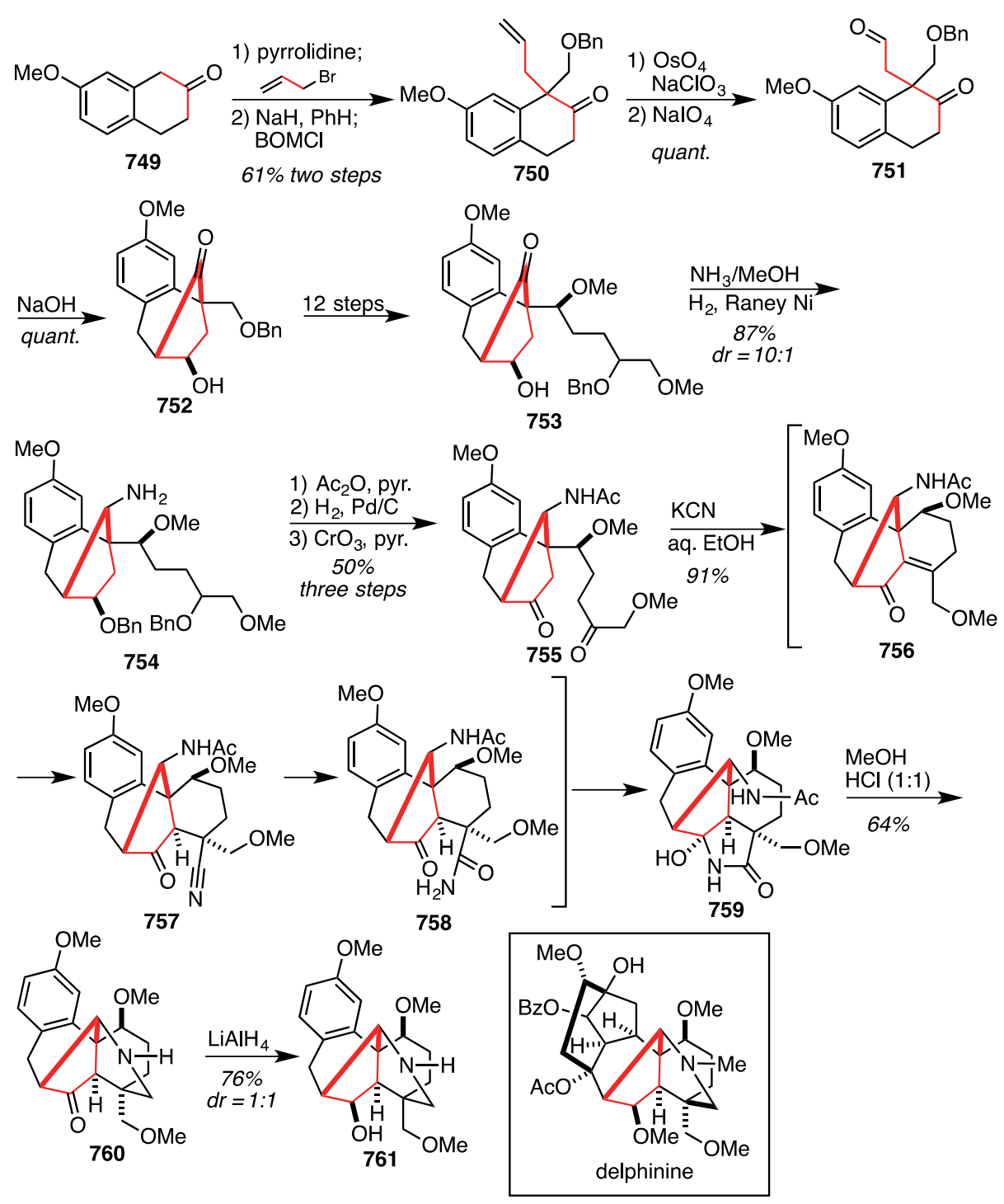

\subsection{1,6-Dicarbonyl Cyclizations}

The synthesis of ( \pm )-gibberellin $\mathrm{A}_{15}$ was completed by Nagata ${ }^{150}$ and built a fully functionalized cyclopentane using an aldol cyclization of a 1,6-ketoaldehyde (Scheme 94). The synthesis begins with tricyclic ketone $\mathbf{7 6 2}$ which underwent hydrocyanation to give a mixture of cis- and trans-cyano ketones. Recrystallization of the mixture in the presence of acid caused epimerization to the more stable trans isomer, which was isolated as a single product (763). This product was advanced to amine 764 over four steps. Birch reduction gave a dienol ether, and the amine was activated as the sulfonamide. Upon acidic workup, the dienol ether isomerized to cyclcohexenone 765. To prepare the ketoaldehyde for the aldol cyclization, the cyclohexenone was converted to the dienol and reduced to give a mixture of hydroxyl-olefin isomers. Oxidative cleavage of the alkene gave the 1,6-ketoaldehyde (766) that cyclized to cyclopentane $\mathbf{7 6 7}$ upon contact with neutral alumina. This material was advanced four steps to intermediate $\mathbf{7 6 8}$. The final substituent on the fully functionalized cyclopentane was installed by elimination of the tertiary alcohol to give enone $\mathbf{7 6 9}$ followed by hydrocyanation to give the ciscyano ketone 770. This advanced intermediate contains the appropriate functionality and stereochemistry for gibberellin $\mathrm{A}_{15}$ and the synthesis was completed in an additional 20 steps.

\section{Scheme 94. Total Synthesis of ( \pm )-Gibberellin $A_{15}$ (Nagata, 1971)}

${ }^{150}$ (a) Nagata, W.; Sugasawa, T.; Narisada, M.; Wakabayashi, T.; Hayase, Y. J. Am. Chem. Soc. 1963, 85, $2342-2343$. (b) Nagata, W.; Wakabayashi, T.; Narisada, M.; Hayase, Y. Kamata, S. J. Am. Chem. Soc. 1971, 93, 5740-5758. 

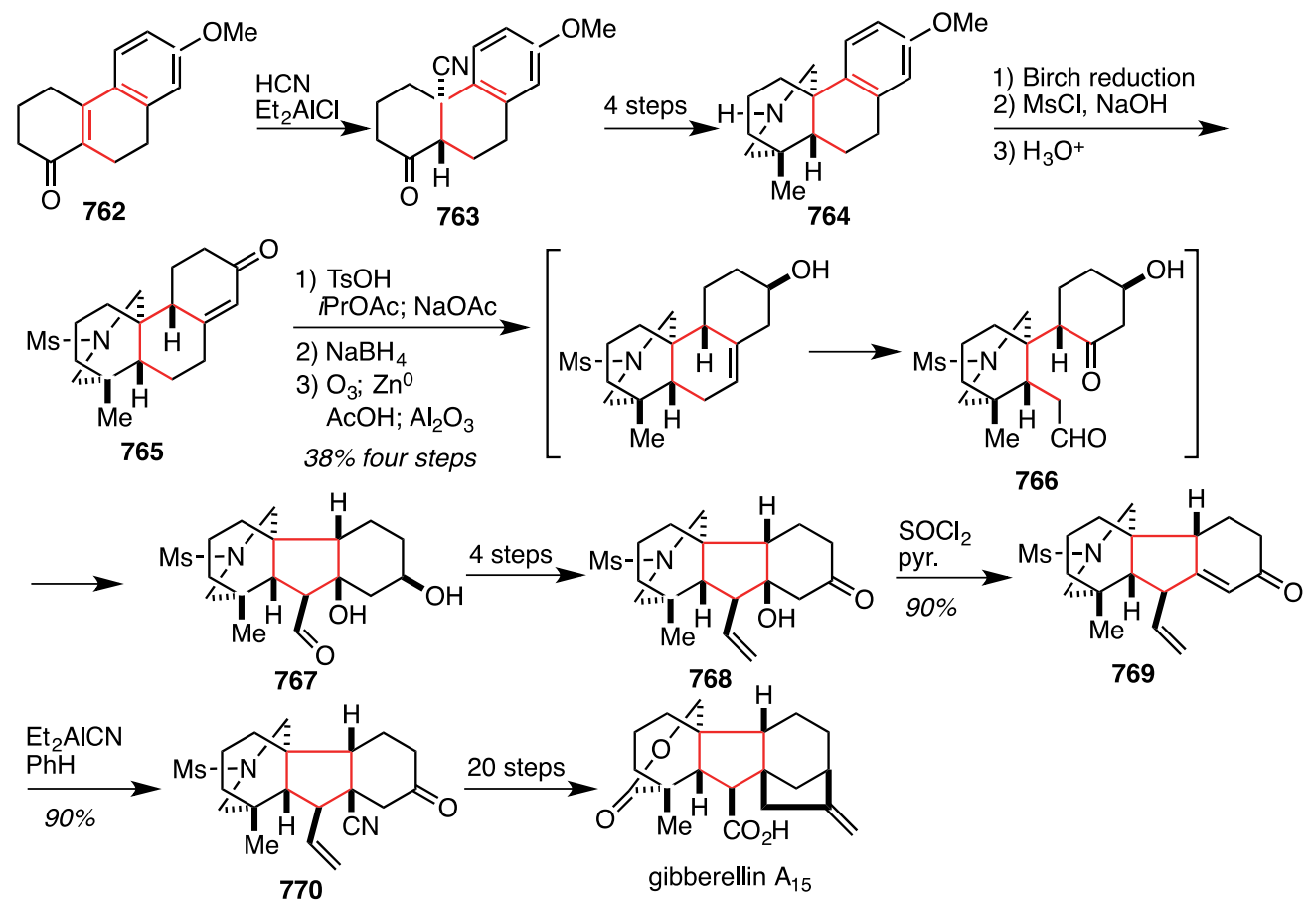

The Corey ${ }^{151}$ synthesis of (+)-gibberellic acid commences with a Diels-Alder reaction between quinone $\mathbf{7 5 1}$ and trans-2,4-pentadiene-1-ol to give 772 (Scheme 95). This material was carried forward to tricyclic intermediate 773 over 10 steps. Dihydroxylation followed by oxidative cleavage furnishes 1,6-dialdehyde 774 . When treated with dibenzylammonium trifluoroacetate an aldol condensation gave the desired $\alpha, \beta$-unsaturated aldehyde $\mathbf{7 7 5}$ and constructed the five-membered carbocycle. Olefination with methylenetriphenylphosphorane provided a diene, and acidic removal of the THP ether furnished 748. The alcohol functional group was deprotonated and acylated with trans-2-chloroacrylyl chloride (776) to give an unsaturated ester 777 . When heated to $160{ }^{\circ} \mathrm{C}$, an endo-selective Diels-Alder cycloaddition proceeded to give lactone $\mathbf{7 7 8}$ as the sole stereoisomer. Treatment of 778 with base and methyl iodide led to chloride elimination and enolate alkylation. Removal of the MEM protecting group with anhydrous zinc bromide yields $( \pm)$-779. This racemic intermediate was resolved by formation of two diastereomeric carbamates with (-)- $\alpha$-phenethylamine, separation by chromatography, and hydrolysis to give $(+)$-779. Hydrolysis of the lactone was followed by oxidation to the diacid with sodium ruthenate in sodium hydroxide. During the oxidation the intermediate acid aldehyde undergoes base-catalyzed epimerization to the more stable $\beta$-formyl derivative before being oxidized to the diacid. Chemoselective esterification gave 780. Hydroxylactonization was achieved by treatment with $m$ CPBA. Saponification of the intermediate lactone, performed under basic conditions, was followed by iodolactonization to give 781. This synthetic intermediate contains a fully functionalized cyclopentane with all the required stereochemistry for $(+)-$ gibberellic acid. The synthesis was completed in an additional three steps.

Scheme 95. Total Synthesis of (+)-Gibberellic Acid (Corey, 1978)

${ }^{151}$ (a) Corey, E. J.; Danheiser, R. L.; Chandrasekaran, S.; Siret, P.; Keck, G. E.; Gras, J. -L. J. Am. Chem. Soc. 1978, 100, 8031-8034. (b) Corey, E. J.; Danheiser, R. L.; Chandrasekaran, S.; Keck, G. E.; Gopalan, B.; Larsen, S. D.; Siret, P.; Gras, J. -L. J. Am. Chem. Soc. 1978, 100, 8034-8036. 

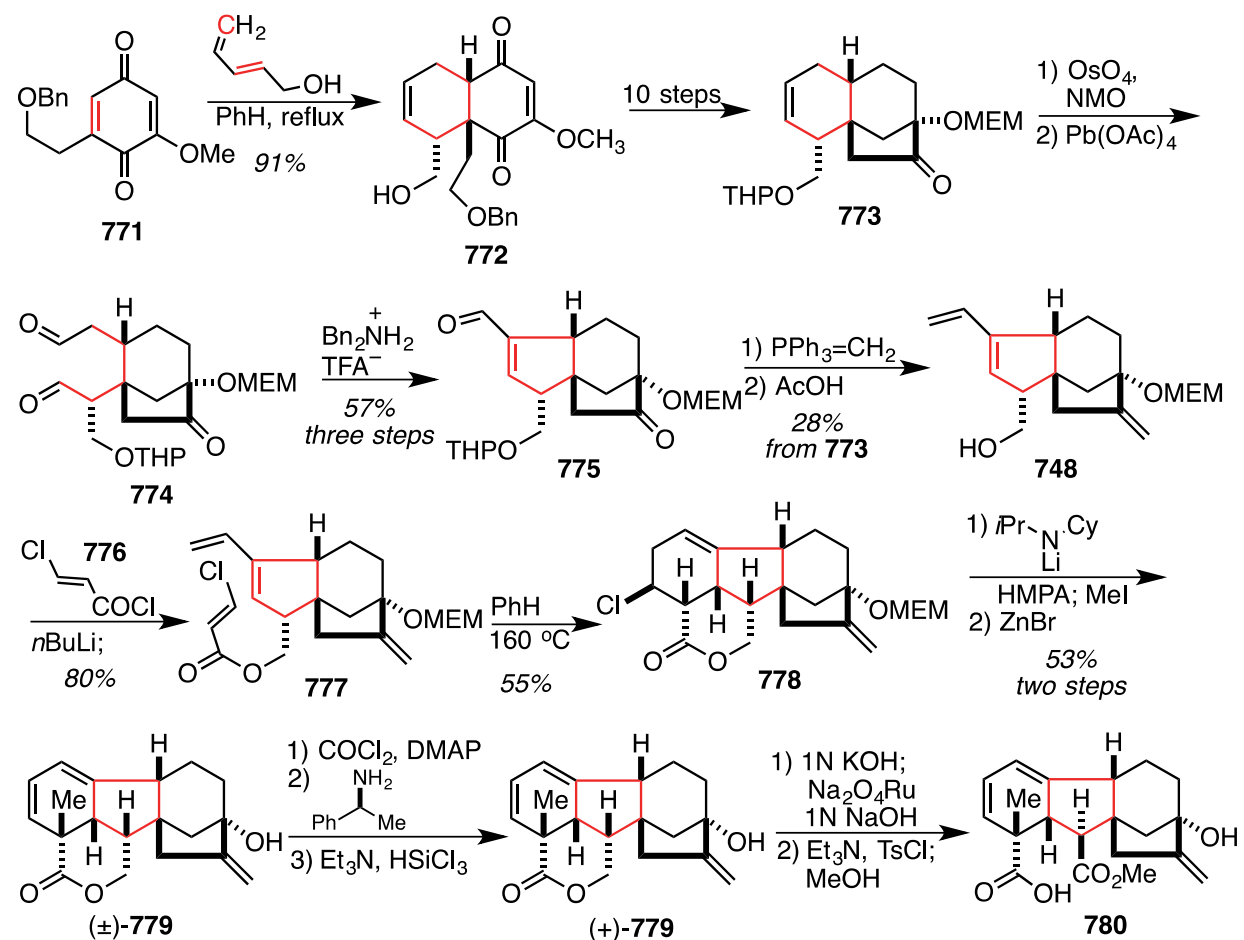

$( \pm)-779$

$(+)-779$

780

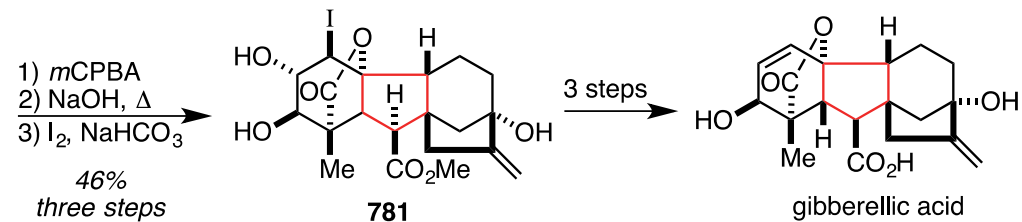

The first total synthesis of (+)-ryanodol was completed by Deslongchamps ${ }^{152}$ in 1979 (Scheme 96). The construction of the two fully functionalized cyclopentanes was performed in separate steps each using an aldol reaction. The synthetic route commences with 5,6-dimethoxyindane (782). Formylation of the indane with dichloromethoxy methane (783) gave $\mathbf{7 8 4}$, which was then advanced five steps to give aromatic lactone $\mathbf{7 8 5}$. Oxidative dearomatization/rearrangement of $\mathbf{7 8 5}$ gave racemic dienone 786. A Diels-Alder reaction occurred between racemic dienone 786 and enantiopure dienophile 787 (prepared in five steps from (+)-carvone) proceeded to give equimolar amounts of 788, 789 and 790, 791. Intermediates 788 and 789 arise from DielsAlder cycloaddition from the desired diastereoface of the diene, and they are inconsequential acetal epimers. Diels-Alder products $\mathbf{7 9 0}$ and $\mathbf{7 9 1}$ represent cycloadducts arising from addition to the undesired diene diastereoface. Nevertheless, the entire mixture was subjected to aqueous base, which induced acetal hydrolysis, ketone epimerization, enolization, and intramolecular aldol addition to give $\mathbf{7 9 2}$ and 793 as an exo/endo pair and 794 and 795 as an exo/endo pair. In this mixture, the endo diastereomers were favored $(\sim 3: 1)$ over the exo diastereomers. Treatment of this unpurified material with aqueous acetic acid followed by $1 \mathrm{~N} \mathrm{NaOH}$ induced an aldol reaction and delivered the first fully functionalized cyclopentane (796). ${ }^{153}$ The diol was advanced four steps to intermediate 797. Oxidative cleavage of the olefin in the presence of acid revealed a 1,6-diketone, which underwent transannular aldol addition to give 798. This material was advanced six steps to mesylate 799.

Treatment with base induced a Grob fragmentation to give an alkene. Hydrolysis of the orthocarbonate gave $\mathbf{8 0 0}$.

${ }^{152}$ Bélanger, A.; Berney, D. J. F.; Borschberg, H. -J.; Brousseau, R.; Doutheau, A.; Durand, R.; Katayama, H.; Lapalme, R.; Leturc, D. M.; Liao, C. -C.; MacLachlan, F. N.; Maffrand, J. -P.; Marazza, F.; Martino, R.; Moreau, C.; Saint-Laurent, L.; Saintonge, R.; Soucy, P.; Ruest, L.; Deslongchamps, P. Can. J. Chem. 1979, 57, 3348-3354.

${ }^{153}$ Control experiments indicated that purified 792 underwent quantitative cyclization to 796; however, purified 794 did not undergo productive aldol addition under these conditions. Furthermore, it was not reported whether purified 793 underwent a retro-aldol-aldol addition with epimerization to give $\mathbf{7 9 6}$. 
Epoxidation of the alkene was performed with trifluoroperacetic acid to give $\beta$-epoxide $\mathbf{8 0 1}{ }^{154}$ Basic hydrolysis of the lactone occurred with concomitant nucleophilic opening of the epoxide yielding lactone triol $\mathbf{8 0 2}$. This material was advanced four steps to intermediate 803. Treatment of $\mathbf{8 0 3}$ with acid led to enol ether $\mathbf{8 0 4}$ as a mixture of stereoisomers. Ozonolysis delivered a ketone, which was transformed to the enol acetate 805. This material was converted to the corresponding enone $\mathbf{8 0 6}$ by an elimination reaction with DBN. Hydride reduction of the ketone gave the endo allylic alcohol. Hydrolysis of the acetonide with aqueous sodium hydroxide delivered anhydroryanodol $(\mathbf{8 0 7})$ in a 3:1 ratio with the isomerized lactone epianhydroryanodol $(\mathbf{8 0 8})$. This mixture could be separated and each alkene was epoxidized to give $\mathbf{8 0 9}$; note that $\mathbf{8 0 7}$ undergoes oxidation with concomitant transesterification to give 809. Finally, a reductive cyclization under dissolving metal conditions furnished (+)-ryanodol.

Scheme 96. Total Synthesis of (+)-Ryanodol (Deslongchamps, 1979)

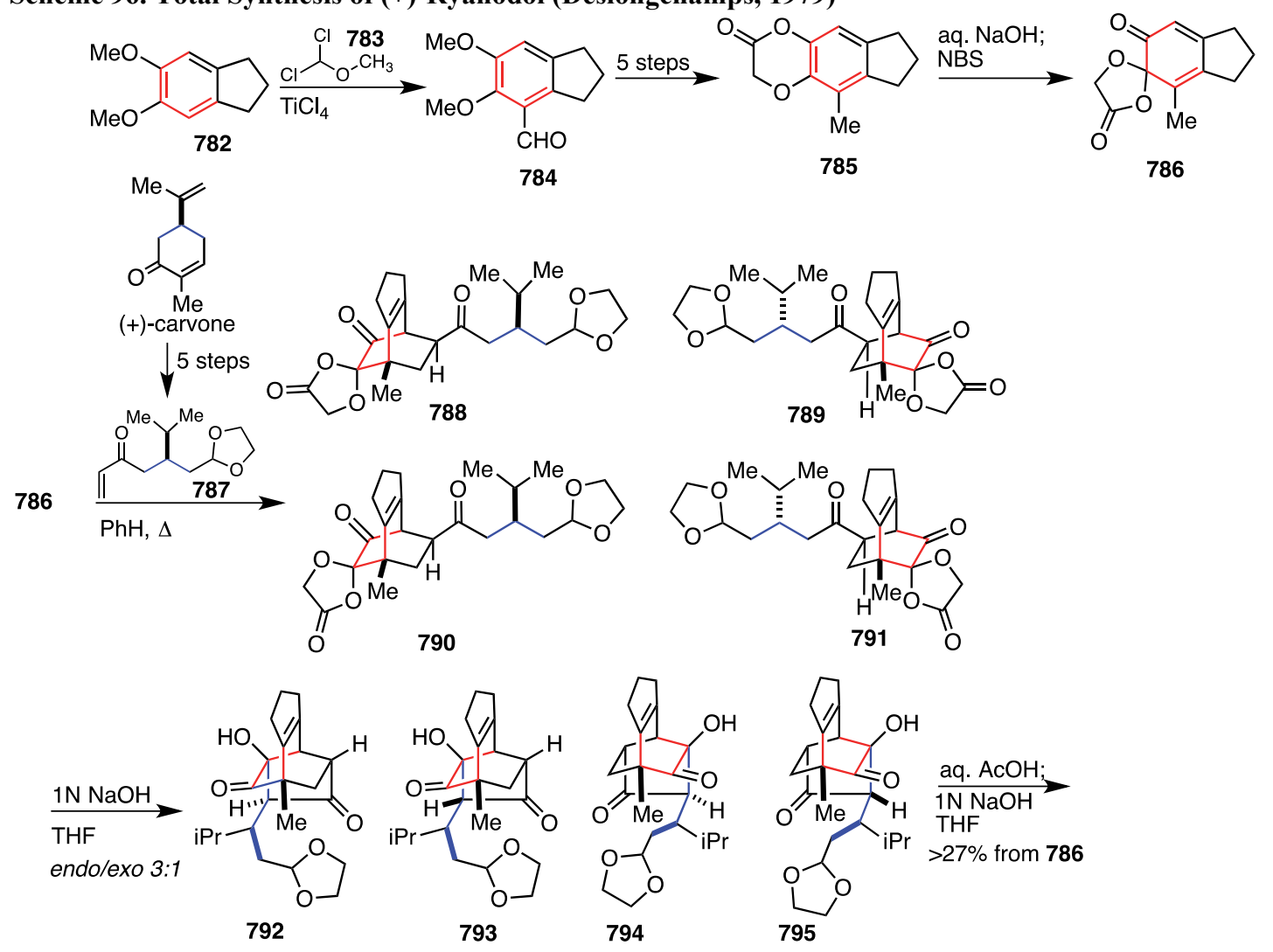

\footnotetext{
${ }^{154}$ No rationale was given to explain the origin of diastereoselectivity in the epoxidation; however, it may arise from steric considerations or the Henbest effect.
} 

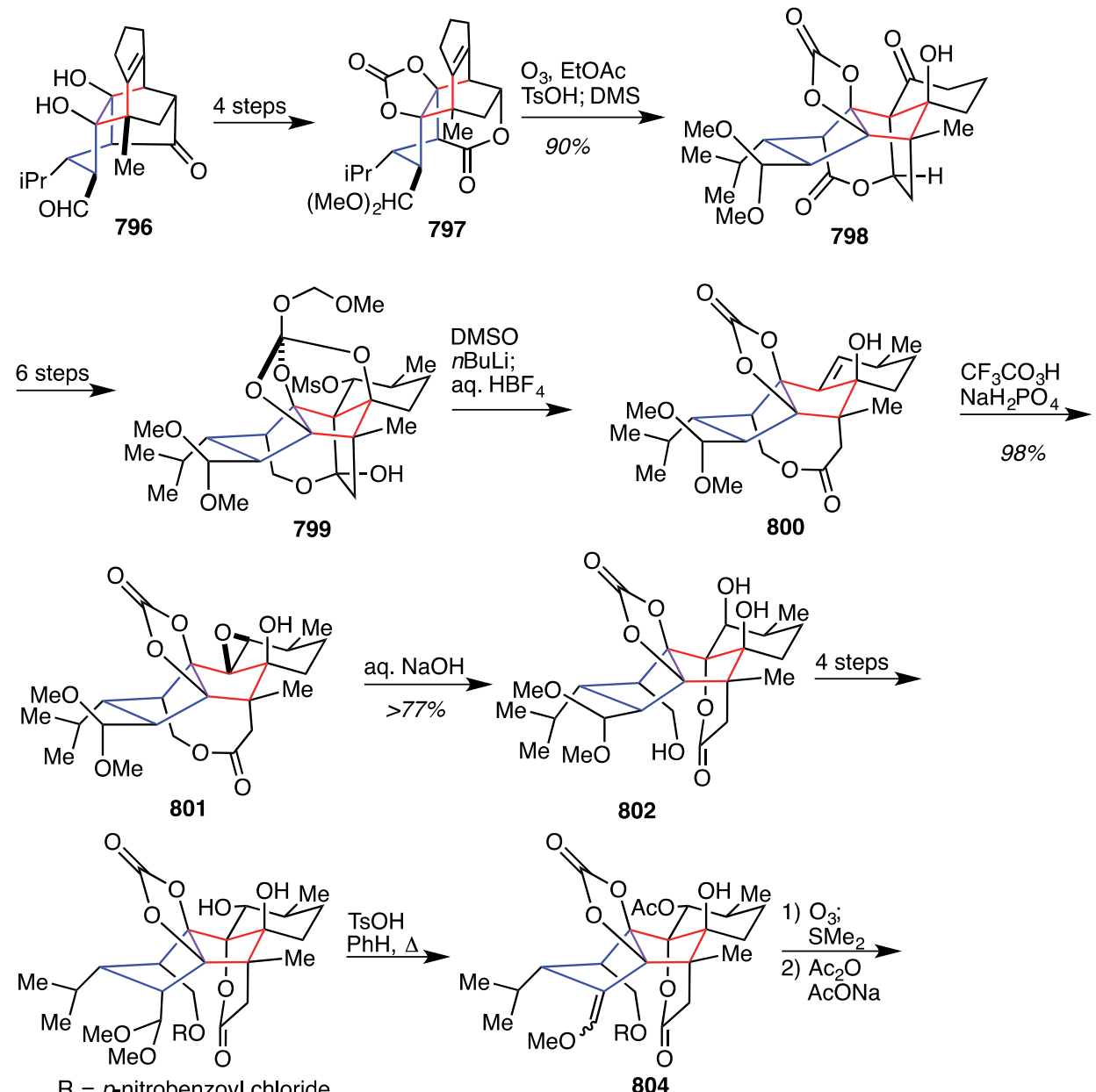

$\mathrm{R}=p$-nitrobenzoyl chloride

804
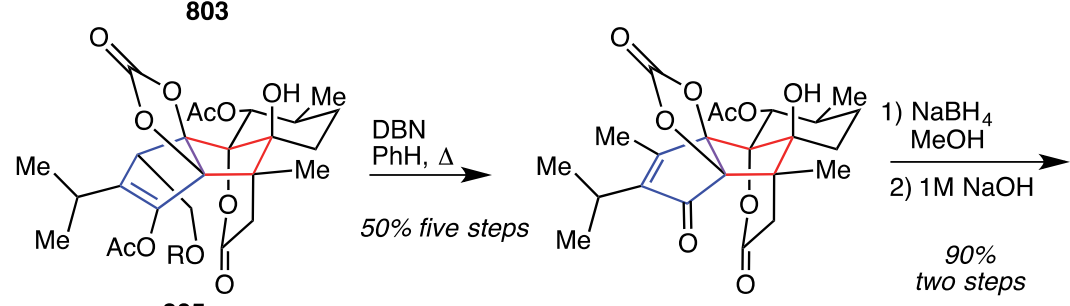

805

806
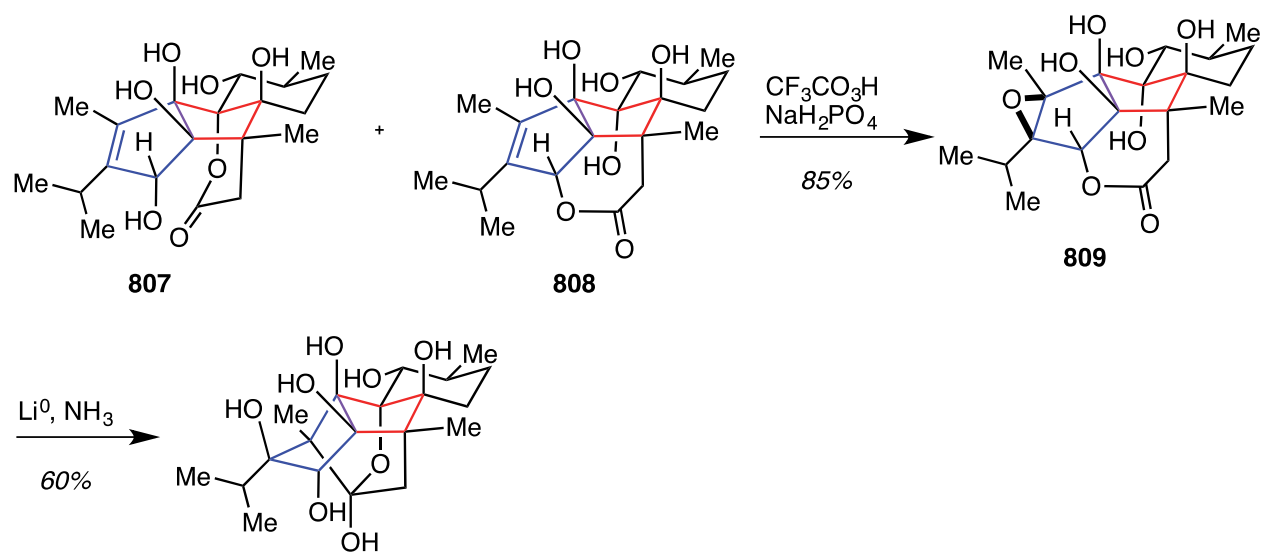

(+)-ryanodol 
The complex natural product $( \pm$ )-ryanodol bears two fully functionalized cyclopentanes (Scheme 97). In the Inoue $^{155}$ total synthesis both functionalized cyclopentanes are formed in a transannular aldol reaction of an eightmembered ring. The synthesis begins with a Diels-Alder reaction between 2,5-dimethylbenzene-1,4-diol (810) and maleic anhydride (811) to give $\mathbf{8 1 2}$ which creates two quaternary stereocenters. The anhydride could be hydrolyzed to the dicarboxylic acid, which underwent electrolysis with a platinum electrode to give the $C_{2^{-}}$ symmetric intermediate $\mathbf{8 1 3}$. To obtain the fused functionalized five membered rings a two-directional functionalization with an intramolecular aldol cyclization was performed. ${ }^{156}$ A stereoselective CoreyChaykovsky epoxidation was followed by regioselective ring-opening with ammonia to produce 814. Treatment with sodium nitrite in acetic acid results in two regioselective ring-expansions to give the 1,5-cyclooctanedione eight-membered ring of $\mathbf{8 1 5}$. Formation of the silyl enol ethers was followed by double epoxidation with DMDO from the bottom face to give 816. Desilylation with triflic acid resulted in diketone 817. The transannular aldol was found to proceed in the presence of Brønsted acid, and the optimized conditions of $3 \mathrm{~mol} \%$ triflic acid in methylene chloride delivered the fused five-membered ring system of $\mathbf{8 1 9}$. This transformation is thought to proceed through intermediate $\mathbf{8 1 8}$, which minimizes ring strain and transannular interactions and leads to the observed aldol diastereomer. The resulting syn-diol was protected as the acetonide, and the remaining hydroxyl group was oxidized to give ketone $\mathbf{8 2 0}$. Ketone $\mathbf{8 2 0}$ is a $C_{2}$-symmetric molecule, and it enabled resumption of the two-directional synthetic strategy toward ryanodol. Two-fold oxidation of the diketone $\mathbf{8 2 0}$ gave $\mathbf{8 2 1}$. The resulting hydroxyl groups were protected as the methoxymethyl ethers. Oxidation of the alkene using catalytic cobalt and molecular oxygen gave an inconsequential mixture of stereoisomers 822. ${ }^{157}$ Silyl protected peroxide 822 was transformed to the corresponding $C_{2}$-symmetric ketone, which underwent hydration upon chromatography to give 823. Differential protection of the two hydroxyl groups was required at this stage. To this end, benzyl ether formation at the less hindered C15 position was followed by conversion of the remaining hydroxyl to the corresponding thiocarbonate $\mathbf{( 8 2 5 )}$. The $C_{2}$-symmetry of previous intermediates has now been broken and the five-membered carbocycles are further functionalized independently. Homolytic cleavage of the $\mathrm{C}-\mathrm{O}$ bond of the thiocarbonate gave a bridgehead radical which successfully formed $\mathbf{8 2 6}$ upon reaction with allyltributyltin. This material was advanced over four steps to intermediate 827. Organolithium addition to the more reactive ketone gave intermediate 829, which has the first fully functionalized cyclopentane installed. The equatorial approach of the organolithium was attributed to sterically less hindered approach of the nucleophile. This material was advanced five steps to intermediate 830. Oxidation of the secondary alcohol was followed by addition of isopropenyl lithium, which occurred away from the acetonide to give 831. In the final stages of the synthesis, the silyl ethers and acetonide were cleaved. The ketone was then reduced by hydride, which is thought to be directed by the revealed tetraol to give the second fully functionalized cyclopentane. Hydrogenolysis of the benzyl ether delivered $( \pm$ )-ryanodol.

Scheme 97. Total Synthesis of ( \pm )-Ryanodol (Inoue, 2014)

\footnotetext{
155 (a) Hagiwara, K.; Himuro, M.; Hirama, M.; Inoue, M. Tetrahedron Lett. 2009, 50, 1035-1037. (b) Urabe, D.; Nagamoto, M.; Hagiwara, K.; Masuda, K.; Inoue, M. Chem. Sci. 2013, 4, 1615-1619. (c) Nagamoto, M.; Koshimizu, M.; Masuda, K.; Tabuchi, T.; Urabe, D.; Inoue, M. J. Am. Chem. Soc. 2014, 136, 5916-5919.

${ }^{156}$ For examples of two-directional strategies used in total synthesis, see: (a) Poss, C. S.; Schreiber, S. L.; Acc. Chem. Res. 1994, 27, 9-17.

${ }^{157}$ Note that the two alkene carbons are symmetry related.
} 
<smiles>O=[N+]([O-])c1cc(O)c(O)cc1O</smiles>

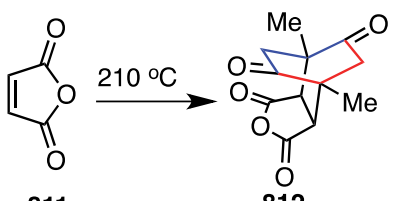
$\mathrm{H}_{2} \mathrm{O}, 80^{\circ} \mathrm{C} ;$
pyr. $\mathrm{Et}_{3} \mathrm{~N}$
$\mathrm{Pt}(+)-\mathrm{Pt}(-), 0.8 \mathrm{~A}$

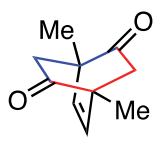
1) $\mathrm{Me}_{3} \mathrm{~S}(\mathrm{O}) \mathrm{I}$ 2) $28 \%$ aq. $\mathrm{NH}_{3}$

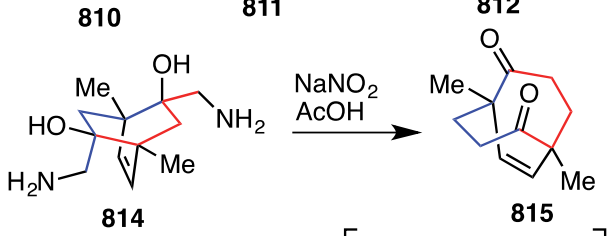
813

1) TMSOTf $\frac{\mathrm{Et}_{3} \mathrm{~N}}{\text { 2) } \mathrm{DMDO}}$

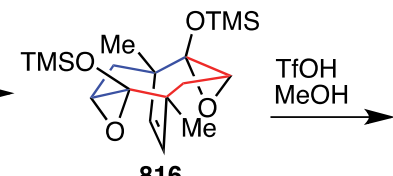
816

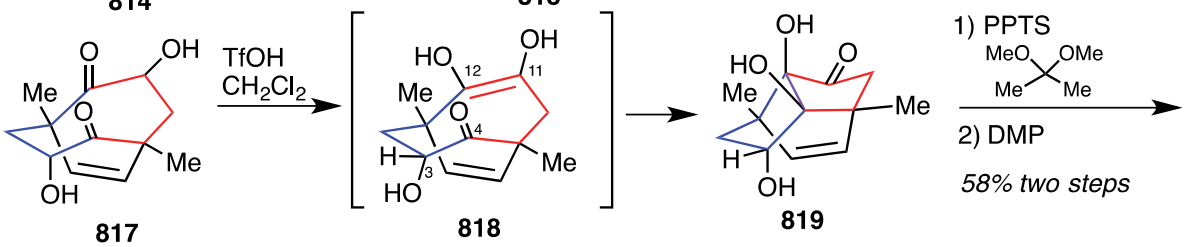

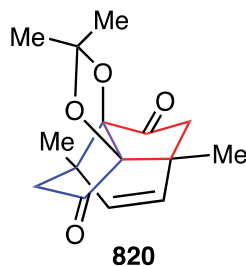

819
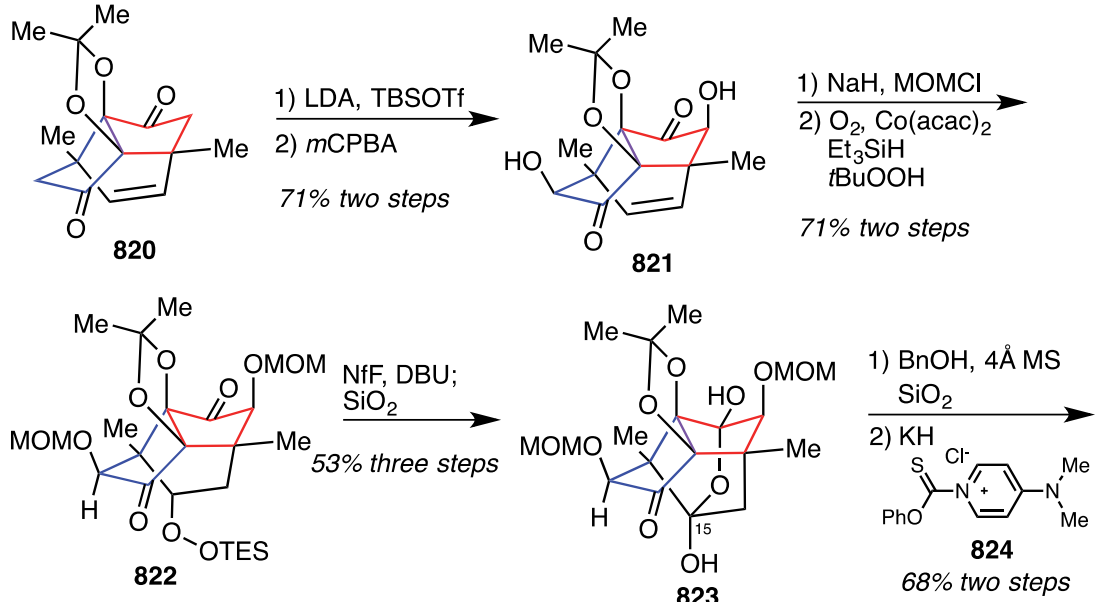


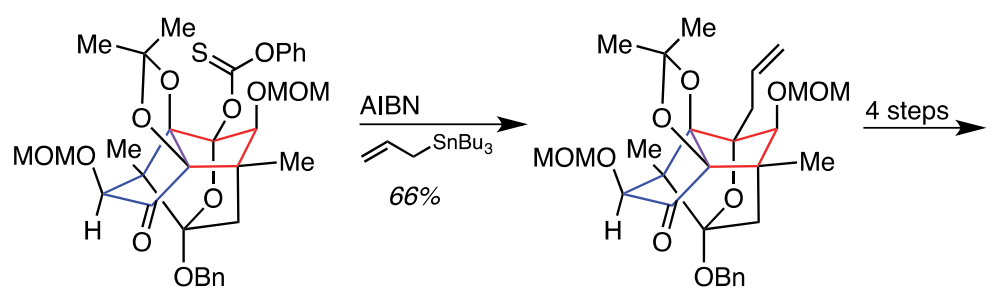

825

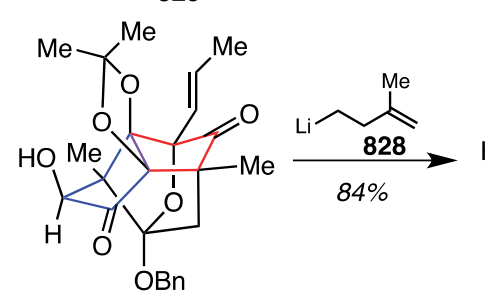

827
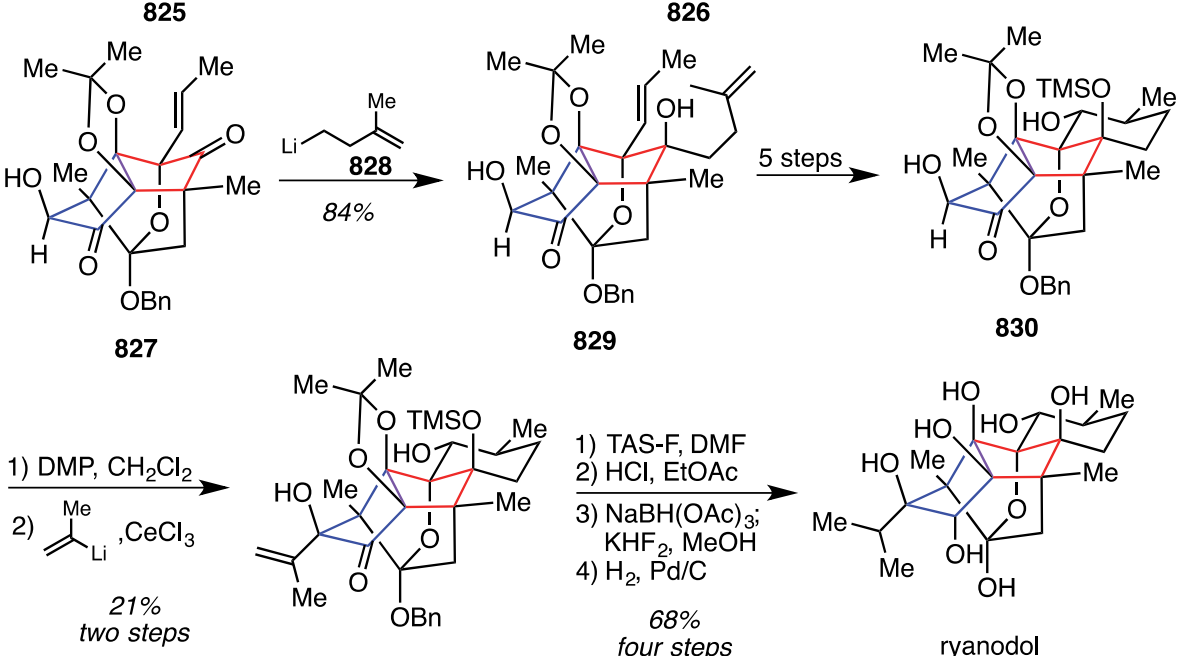

829

830

831

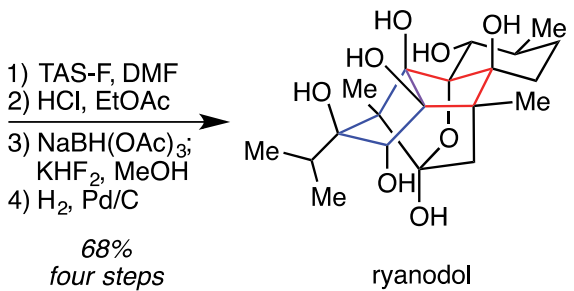

The first stereoselective synthesis of $\left( \pm\right.$ )-orostanal, completed by Zhou ${ }^{158}$, begins with commercially available hyodeoxycholic acid, which could be advanced to intermediate $\mathbf{8 3 2}$ following the two-step method of Ziegler (Scheme 98). ${ }^{159}$ Ozonolysis of the cyclohexene followed by reductive workup afforded keto-aldehyde 833. The aldol reaction was promoted with exposure to neutral alumina and resulted in a fully functionalized cyclopentane. Axial attack on the ketone leads to the cis-fused hydrindane substructure with the aldehyde positioned on the convex face of the polycyclic molecular architecture in $\mathbf{8 3 4}$. This intermediate contains a fully functionalized cyclopentane and was converted to the natural product over an additional ten steps.

Scheme 98. Total Synthesis of Orostanal (Zhou, 2002)

${ }^{158}$ Liu, B.; Zhou, B. Tetrahedron Lett. 2002, 43, 4187-4189.

${ }^{159}$ Bharucha, K. R.; Buckley, G. C.; Cross, C. K.; Rubin, L. J.; Ziegler, P. Can. J. Chem. 1956, 34, 982-990. 


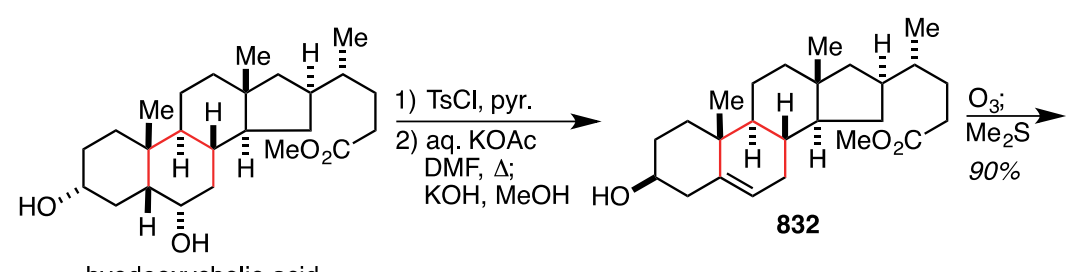

hyodeoxycholic acid

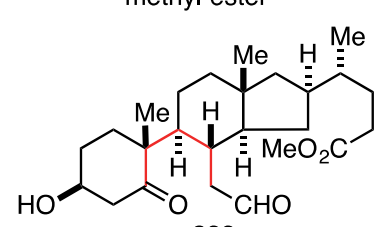

833
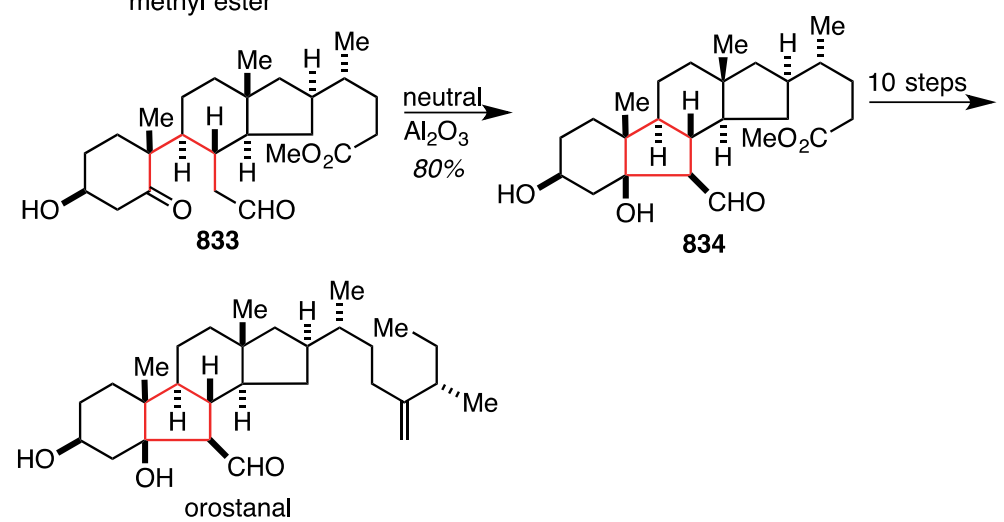

Baran's ${ }^{160}$ synthesized the fully functionalized cyclopentane $\mathbf{8 5 2}$, which served as a common intermediate for the synthesis of the (-)-axinellamines, (-)-palau'amine, and (-)-massadine (Scheme 99). The route commences with an enantioselective Diels-Alder reaction of a 1-siloxydiene 841 and 842 to give cyclohexene 844. This material was carried forward to the diazide $\mathbf{8 4 5}$ over six steps. Ozonolytic cleavage of the alkene gave a 1,6-diketone (846). Dibromination followed by exposure to dry silica promoted an intramolecular aldol reaction to form a fully functionalized cyclopentane (847). ${ }^{161}$ Chlorination of the more reactive bromide and cleavage of the PMBether furnished diol 848. Although a fully functionalized cyclopentane has been synthesized at this stage, it contains neither the correct substitution or desired stereochemistry for the natural targets. Tandem invertive chlorination and elimination were promoted with sulfuryl chloride to give enone 849. A Luche reduction of the chloroketone and displacement of the bromide with $N, N^{\prime}$-bis(Boc) guanidine provided 850. Re-oxidation of the allylic alcohol with IBX induced spirocyclization to give $\mathbf{8 5 1}$ as a mixture of diastereomers favoring the desired stereoisomer. Treatment with sodium diformylamide installed the primary ammonium group and Boc deprotection with TFA delivered $\mathbf{8 5 2}$. Advanced intermediate $\mathbf{8 5 2}$ was a versatile synthetic intermediate that was used in the synthesis of the (-)-axinellamines, (-)-palau'amine, and (-)-massadine.

Scheme 99. Total Synthesis of (-)-Palau'amine, (-)-Massadine, (-)-Axinellamines A and B (Baran, 2008)

${ }^{160}$ (a) Yamaguchi, J.; Seiple, I. B.; Young, I. S.; O’Malley, D. P.; Maue, M.; Baran, P. S. Angew. Chem. Int. Ed. 2008, 47, 3578-3580. (b) O’Malley, D. P; Yamaguchi, J.; Young, I. S.; Seiple, I. B.; Baran, P. S. Angew. Chem. Int. Ed. 2008, 47, 3581-3583. (c) Su, S.; Seiple, I. B.; Young, I. S.; Baran, P. S. J. Am. Chem. Soc. 2008, 130, $16490-16491$.

${ }^{161}$ Lee reported a somewhat similar oxidative cleavage/1,6-dicarbonyl aldol cyclization in a synthesis of the carbocyclic core of massadine. See: Sun, C.; Lee, H.; Lee, D. Org. Lett. 2015, 17, 5348-5351. 


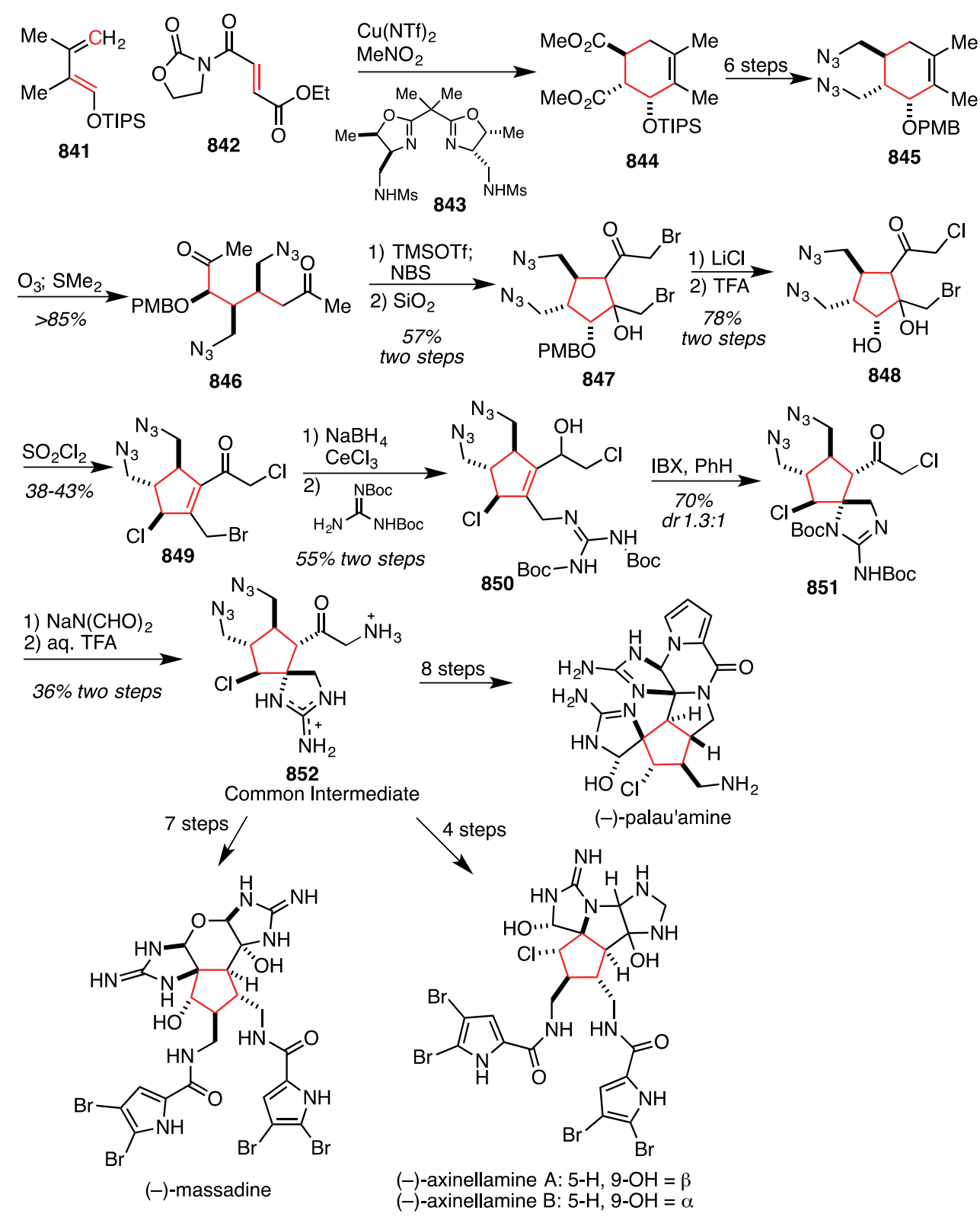

The first synthesis of $( \pm)$-pallambins $C$ and D by Wong ${ }^{162}$ employs a Grob fragmentation-intramolecular aldol cyclization sequence to construct a fully functionalized cyclopentane (Scheme 100). The synthesis commences with a reduction of the Wieland-Miescher ketone $(\mathbf{8 5 3})$ to diol 854. Benzyl protection of the diol was followed by epoxidation. Nucleophilic opening of the oxirane with methyl Grignard occurs at the less substituted carbon to give 855. Displacement of the hydroxyl with chloride and subsequent elimination delivers 856. Hydroborationoxidation proceeds stereoselectively to give the trans-decalin. Acylation of the resulting hydroxyl group and hydrogenolysis of the benzyl ethers provides 857. Oxidation to a diketone using Jones reagent was followed by selective conversion of the less hindered ketone to the diethyl ketal $\mathbf{8 5 8}$. The acetyl protecting group was removed and a Bamford-Stevens-type iodination gave the vinyl iodide 859. Benzyl protection of the secondary alcohol and transketalization was followed by a palladium-catalyzed alkoxycarbonylation to deliver ester $\mathbf{8 6 0}$. Ester reduction and protection of the resulting alcohol as the MOM ether gives $\mathbf{8 6 1}$. Hydroboration-oxidation on the less hindered alkene face, and acylation of the resulting alcohol delivered $\mathbf{8 6 2}$. This material was advanced over five steps to intermediate 863. Upon treatment with potassium tert-butoxide, a Grob fragmentation gave 1,6-ketoaldehyde 864 as an intermediate. A subsequent aldol cyclization occurred to give $\mathbf{8 6 5}$. The constraints of

${ }^{162}$ Xu, X. -S.; Li, Z. -W.; Zhang, Y. -J.; Peng, X. -S.; Wong, H. N. C. Chem. Commun. 2012, 48, 8517-8519. 
the intramolecular tether ensure the aldehyde approaches on one diastereoface of the enolate, and the secondary alcohol stereocenter is inconsequential. At this stage, a fully functionalized cyclopentane has been synthesized, but the secondary alcohol must be converted to a tertiary alcohol. Introduction of the methyl group on the more hindered face of the molecular architecture required multiple steps. ${ }^{163}$ Intermediate $\mathbf{8 6 5}$ was advanced five steps to homoallylic alcohol 866. Substrate directed epoxidation was accomplished using the method of Sharpless, and fragmentation of the $\mathrm{C}-\mathrm{O}$ bond was performed with $\mathrm{LiAlH}_{4}$. Advanced intermediate 867 contains a fully functionalized cyclopentane with appropriate functional groups for the synthesis of $( \pm)$-pallambins $\mathrm{C}$ and $\mathrm{D}$. The synthesis was completed in an additional six steps.

Scheme 100. Total Synthesis of ( \pm )-Pallambins C and D (Wong, 2012)
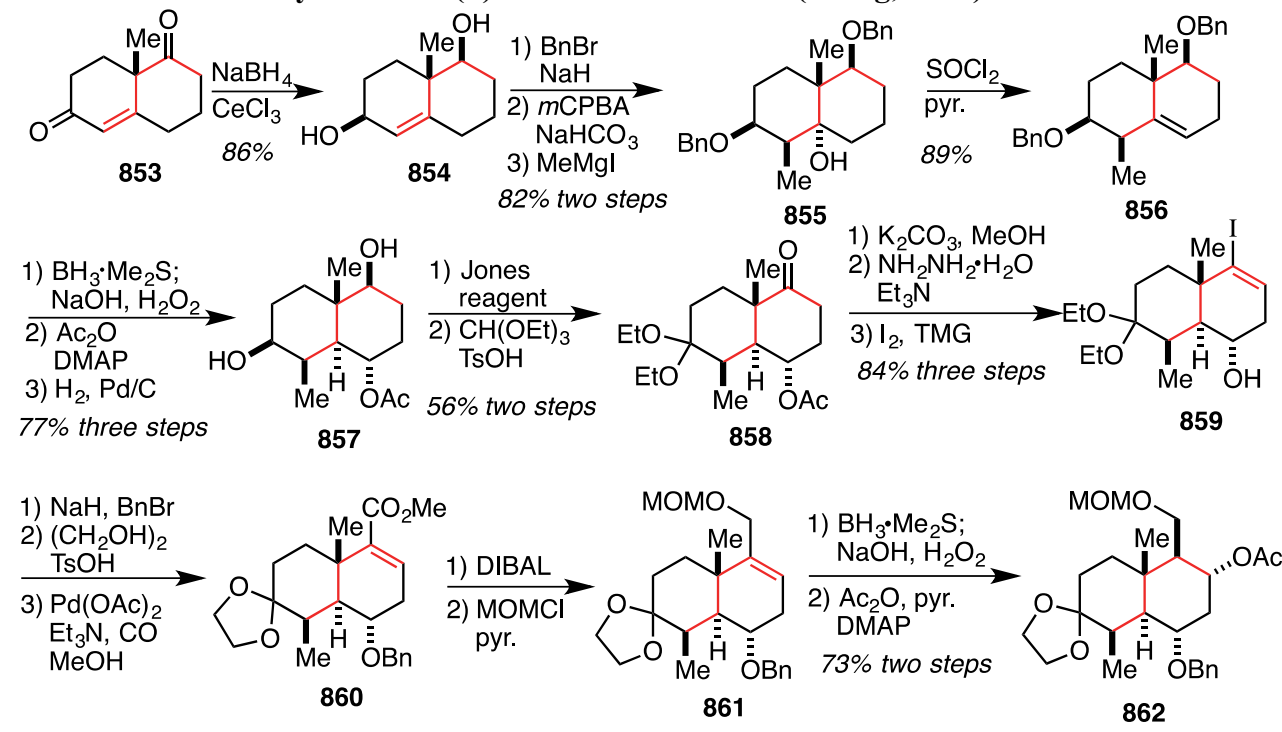

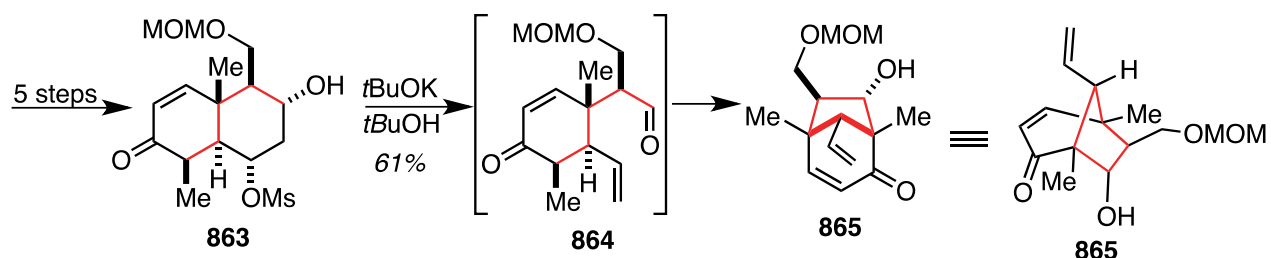

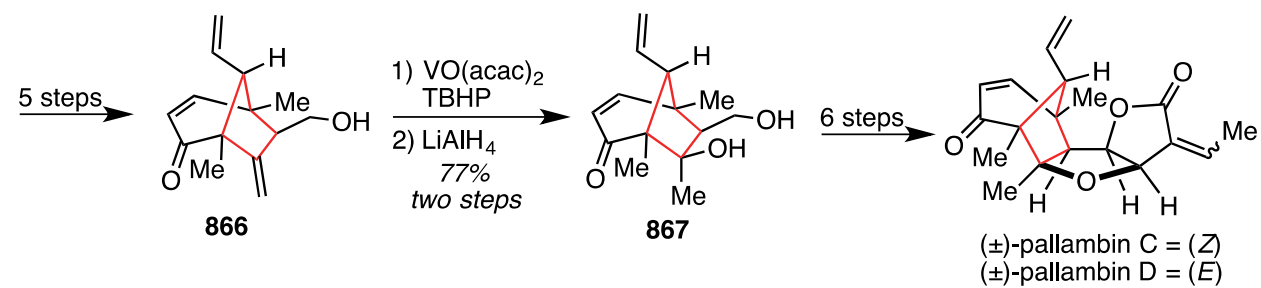

The total synthesis of ( \pm )-pallambins $C$ and $D$ was completed by Baran ${ }^{164}$ in eleven steps (Scheme 101). The cyclopentane was constructed with an intramolecular Mukaiyama aldol. The route commences with furfuryl alcohol (868). An Eschenmoser-Claisen rearrangement with $N, N$-dimethylpropionamide dimethyl acetal (869) and subsequent silyl hydride reduction of the resulting amide produces aldehyde 870. A Robinson annulation with ethylvinyl ketone (871) yielded cyclohexenone 872. Conjugate addition of vinyl cuprate and enolate trapping with TMS chloride delivers silyl enol ether 873. Oxidative ring-opening of the furan gave keto-aldehyde 874. This sensitive material was carried forward without chromatography into the aldol reaction. Lewis acid promoted Mukaiyama aldol reaction assembles the bicyclo[3.2.1] octane containing the functionalized fivemembered ring. The reaction proceeds with complete stereocontrol to give $\mathbf{8 7 5}$ as a single stereoisomer. The stereochemistry of the tertiary alcohol is the result of bidendate chelation of the Lewis acid with the ketone and aldehyde carbonyls and suppression of any retro-aldol, aldol processes. The tetrahydrofuran could be assembled

${ }^{163}$ Organometallic addition of methyl to the ketone derived from $\mathbf{8 6 5}$ led to the undesired tertiary alcohol diastereomer.

${ }^{164}$ Martinez, L. P.; Umemiya, S.; Wengryniuk, S. E.; Baran, P. S. J. Am. Chem. Soc. 2016, 138, 7536-7539. 
by treatment with trifluoroboron etherate and trimethylorthoformate. The reaction is thought to proceed through an acetal, which eliminates to an oxocarbenium ion. Nucleophilic conjugate addition of methanol occurring from the top face results in an unproductive intermediate as cyclization would deliver the higher energy trans-fused bicycle. Upon conjugate addition of methanol to the bottom face, cyclization with the hydroxyl group delivers the cis-fused tetrahydrofuran ring 876. This material was directly subjected to bromination with acetyl bromide to furnish 877. Reduction of the bromide using Stille conditions gave 878, which contains a fully functionalized cyclopentane with the required substitution and stereochemistry for pallambins $\mathrm{C}$ and $\mathrm{D}$. Completion of the synthesis required an additional four steps.

Scheme 101. Total Synthesis of ( \pm )-Pallambins C and D (Baran, 2016)

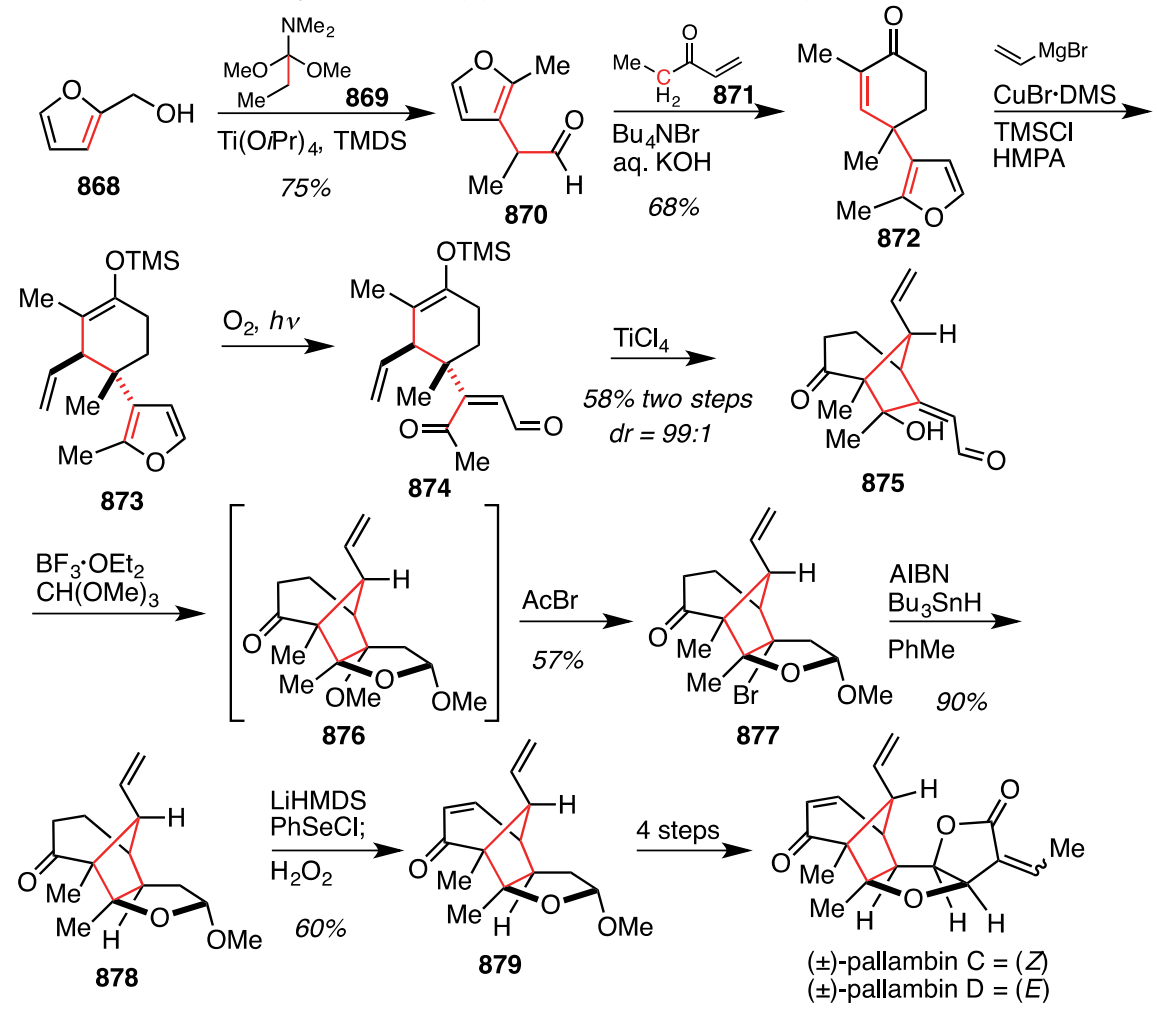

In the total synthesis of $( \pm)$-rocaglamide by Dobler ${ }^{165}$, addition of a cyanohydrin anion to a benzofuranone assembles the key five-membered ring (Scheme 102). The synthesis commences with an addition of benzofuranone $\mathbf{8 8 0}$ to $(E)$-cinnamaldehyde $(\mathbf{8 8 1})$ to give desired diastereomer of $\mathbf{8 8 2}$ with modest selectivity. Aldehyde $\mathbf{8 8 2}$ was transformed to the corresponding cyanohydrin $\mathbf{8 8 3}$. The cyanohydrin could be deprotonated with LDA, and an aldol-like addition to the ketone occurred and an acyloin (884) was isolated upon elimination of the nitrile. The diastereoselectivty is the result of preferential formation of the cis-fused bicycle. Following earlier precedent, carboxylation with Stiles ${ }^{166}$ reagent occurred on the concave face of the ring system opposite the two aryl substituents. Hydrolysis to the $\beta$-keto-acid, and amide formation delivered dimethylamide $\mathbf{8 8 5}$. Substrate directed hydride reduction completed the synthesis of $( \pm)$-rocaglamide.

Scheme 102. Total Synthesis of $( \pm)$-Rocaglamide (Dobler, 2001)

\footnotetext{
${ }^{165}$ Dobler, M. R.; Bruce, I.; Cederbaum, F.; Cooke, N. G.; Diorazio, L. J.; Hall, R. G.; Irving, E. Tetrahedron Lett. 2001, 42, 8281-8284.

${ }^{166}$ Stiles, M. J. Am. Chem. Soc. 1959, 81, 2598-2599.
} 

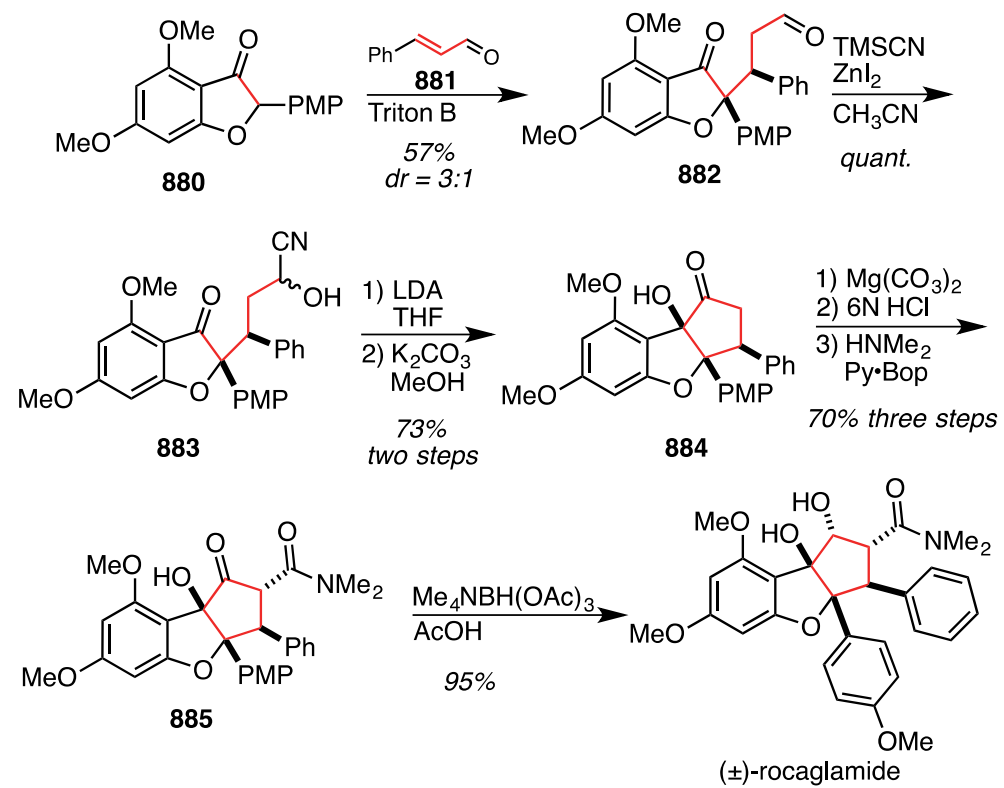

The asymmetric synthesis of $(+)$-gelsemine was completed by Zhai and Qiu, ${ }^{167}$ and the fully functionalized cyclopentane is constructed with an aldol addition of a 1,6-ketoaldehyde (Scheme 103). The route commences with an enantioselective organocatalytic Diels-Alder reaction of dihydropyridine $\mathbf{8 8 7}$ and dienophile $\mathbf{8 8 8}$ to give desired endo product $\mathbf{8 9 1}$ after reduction. ${ }^{168,169}$ Surprisingly, intermediate $\mathbf{8 9 0}$ was also isolated from the reaction mixture, which may suggest dienophile double bond isomerization under the reaction conditions. This material could be converted to the desired cycloadduct $\mathbf{8 9 1}$ by heating with DBU. Reduction with DIBAL gave a lactol, which upon Wittig reaction delivered a methyl enol ether that was converted to acetal 892. Ozonolysis of the alkene gave a 1,6-ketoaldehyde that undwerent aldol addition upon treatment with base to give fully functionalized cyclopentane 893. This material was advanced over six steps to intermediate 894. The final carbon substituent of the cyclopentane was installed with an enolate alkylation of the pendant oxindole moiety to give 895. Removal of the MOM ether delivered (+)-gelsemine.

Scheme 103. Total Synthesis of (+)-Gelsemine (Zhai \& Qiu, 2015)

${ }^{167}$ Chen, X.; Duan, S.; Tao, C.; Zhai, H.; Qiu, F. G. Nat. Commun. 2015, 6, 7204.

${ }^{168}$ Ahrendt, K. A.; Borths, C. J.; MacMillan, D. W. C. J. Am. Chem. Soc. 2000, 122, 4243-4244.

${ }^{169}$ Nakano, H.; Osone, K.; Takeshita, M.; Kwon, E.; Seki, C.; Matsuyama, H.; Takano, N.; Kohari, Y. Chem. Commun. 2010, 46, 4827-4829. 

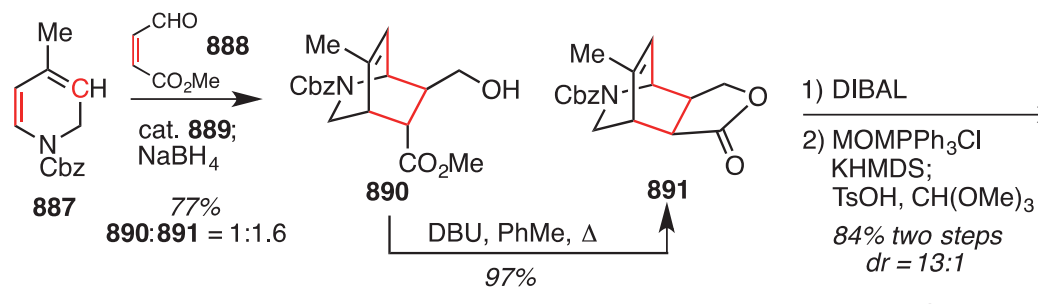

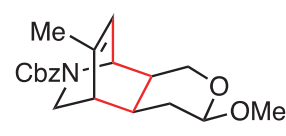

892

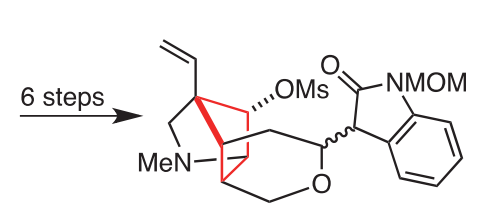

894

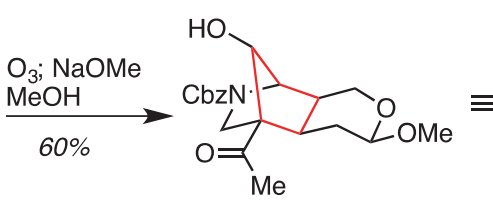

893
(Obz)

893

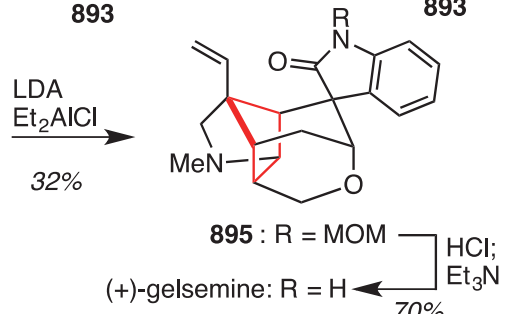

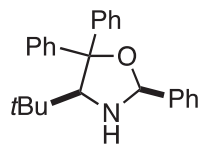

cat. $\mathbf{8 8 9}$

The squarate ester cascade is a reaction sequence developed by Paquette ${ }^{170}$, and it was used to efficiently assemble the triquinane framework of ( \pm )-coriolin (Scheme 104). Lithiated cyclopentene 897 was added to squarate ester 896, to which excess vinyl lithium was added. The two possible stereochemical outcomes of the organometallic additions are inconsequential due to independent pathways to the desired triquinane 904. The product of syn-addition (898) undergoes a di-anionic oxy-Cope rearrangement to give 871. Anti-addition induces a $4 \pi$-electrocyclic ring opening of the cyclobutane (899) to give $\mathbf{9 0 0}$, which is followed by an $8 \pi$ -

electrocyclization to give 901. After regeneration of the ketone (902), an intramolecular aldol furnishes the tricyclic intermediate 904. Treatment with acid leads to ketone 905. Deoxygenation of the tertiary alcohol occurred over two steps to give 906. A dehydrative 1,3-carbonyl transposition was effected with $\mathrm{LiAlH}_{4}$ and MOM protection of the secondary alcohol gave intermediate $\mathbf{9 0 7}$. Enolate alkylation stereoselectively installed the quaternary stereocenter forming the energetically favorable cis-ring fusion in $\mathbf{9 0 8}$. Nucleophilic addition of methyllithium and dehydration formed the exocyclic alkene. A Saegusa oxidation installed the final unsaturation and delivered 142, which is an intermediate previously synthesized by Ikegami ${ }^{38}$ in a synthesis of coriolin.

Scheme 104. Formal Synthesis of $( \pm)$-Coriolin (Paquette, 2002)

\footnotetext{
${ }^{170}$ Paquette, L. A.; Geng, F. J. Am. Chem. Soc. 2002, 124, $9199 \square 9203$.
} 


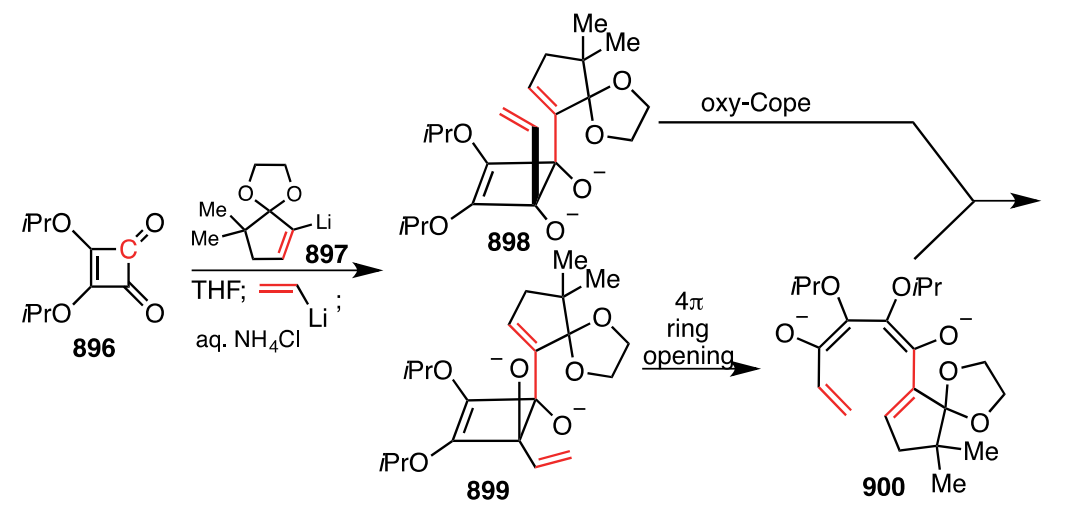

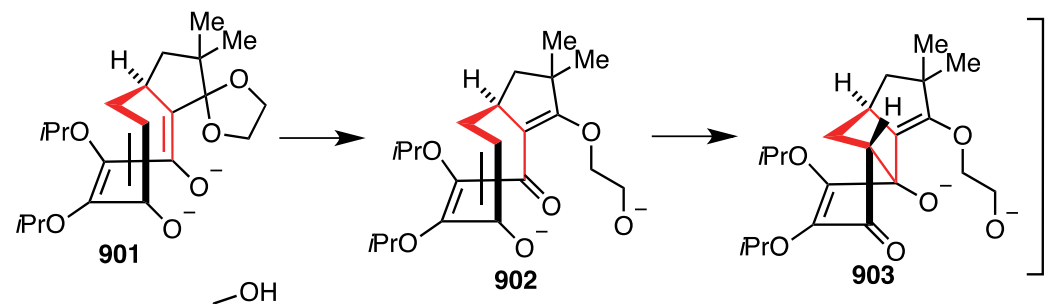

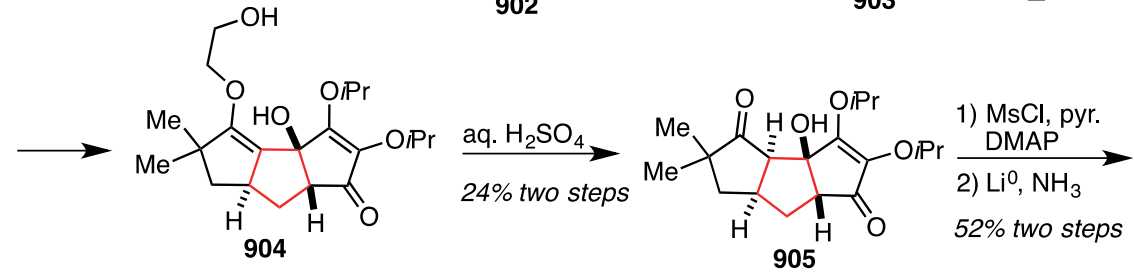

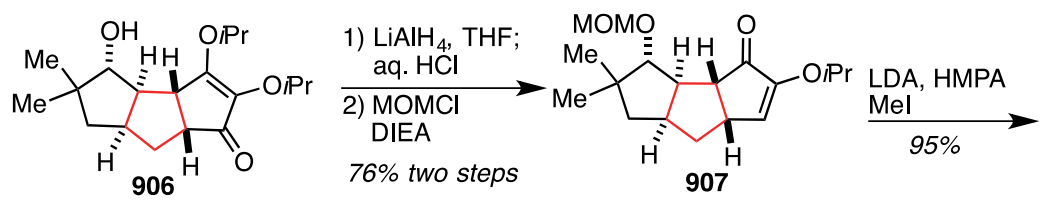

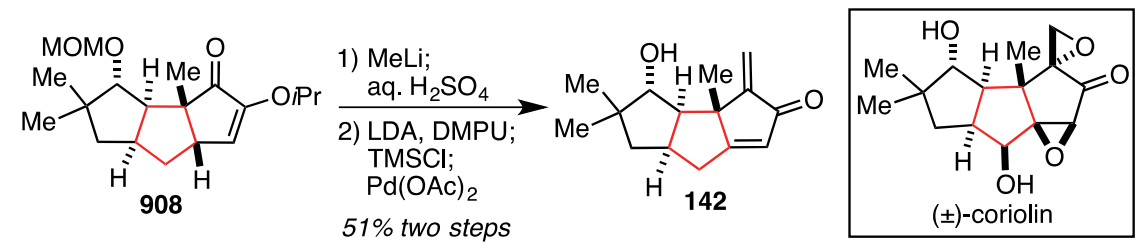

The first Danishefsky ${ }^{43}$ synthesis of the sesquiterpene $( \pm)$-merrilactone A begins with a Diels-Alder reaction between 2,3-dimethylmaleic anhydride and diene 909 to afford cyclohexene 910 (Scheme 105). The endo selectivity of the cycloaddition gives the desired relative stereochemistry for the target molecule. This material was carried forward to lactone $\mathbf{9 1 1}$ over five steps. The alkene underwent ozonolysis to a 1,6-dialdehyde that cyclized upon treatment with mild acid to give enal 912. Following reduction of the aldehyde, addition of triethyl orthoacetate and acid induced a Johnson-Claisen rearrangement to give ester 913 as a mixture of diastereomers favoring the desired stereoisomer. The ester was then hydrolyzed, and iodolactonization proceeded to give 914, which contains a fully functionalized cyclopentane complete with the substituents and stereochemistry required for merrilactone $\mathrm{A}$. The natural product was completed over an additional eight steps.

Scheme 105. Total Synthesis of ( \pm )-Merrilactone A (Danishefsky, 2002) 

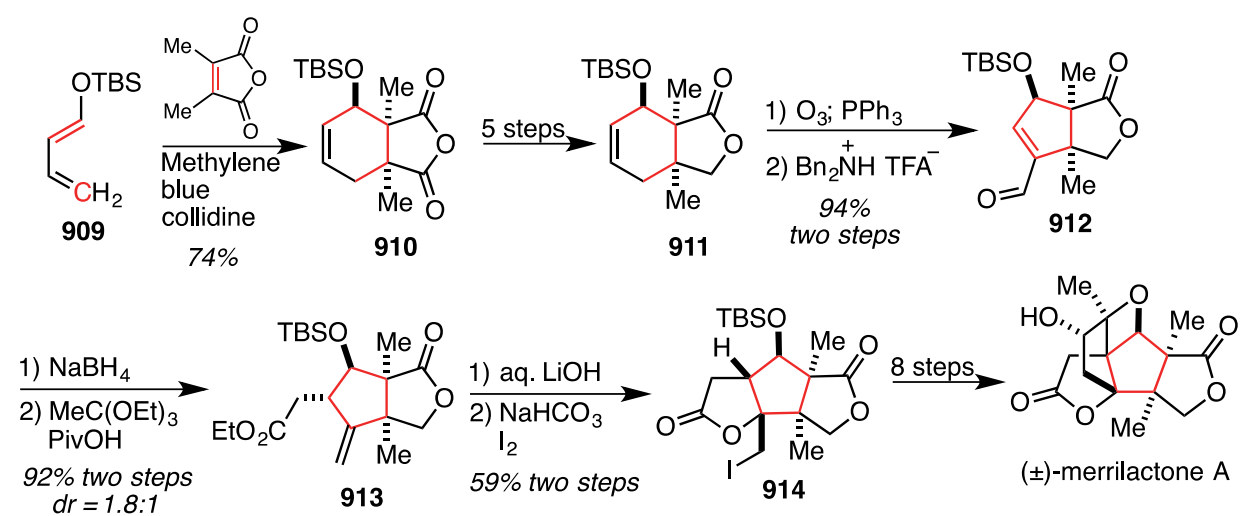

\subsection{Henry Reactions}

In the synthesis of $( \pm)$-mannostatin $\mathrm{A}$, Ogawa ${ }^{171}$ constructs the cyclopentane by double addition of nitromethane to a dialdehyde (Scheme 106). The synthesis begins with an oxidative cleavage of myo-inositol derivative 915 to give a dialdehyde intermediate (916). Base-catalyzed double addition of nitromethane gives diol $\mathbf{9 1 7}$ as a complex mixture of diastereomers. This mixture was advanced to intermediate 918 and separated, with the major diastereomer having the desired configuration. This material was converted to 919 by selective $O$-acetate removal and sulfonate formation. ${ }^{172,173}$ Heating in sodium acetate inverted the $\mathrm{C} 1$ and $\mathrm{C} 4$ configurations and resulted in a diol. Treatment of the diol with $\mathrm{MsCl}$ delivered racemic monomesylate 920. Nucleophilic substitution with potassium thioacetate proceeded with inversion to set the final stereocenter for mannostatin A (921). This material was converted to mannostatin A over an additional three steps.

Scheme 106. Total Synthesis of ( \pm )-Mannostatin A (Ogawa, 1994)

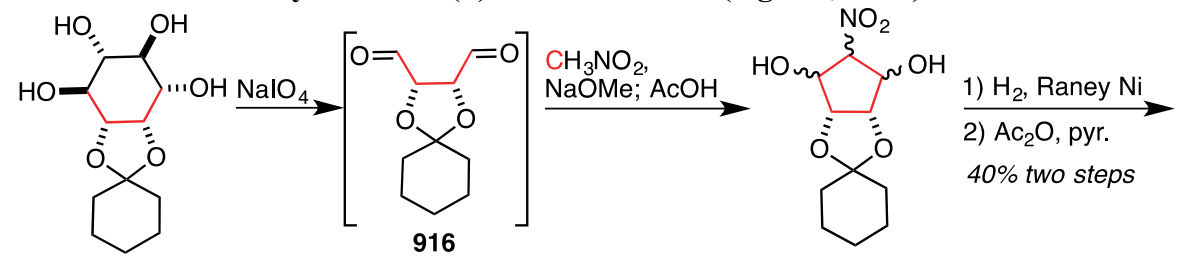

915

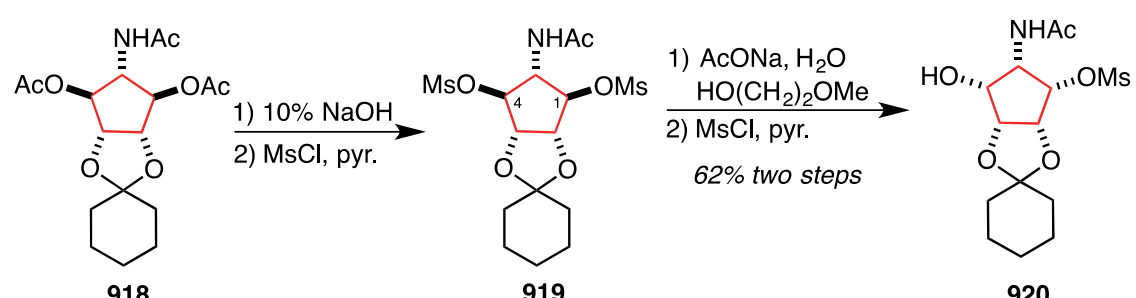

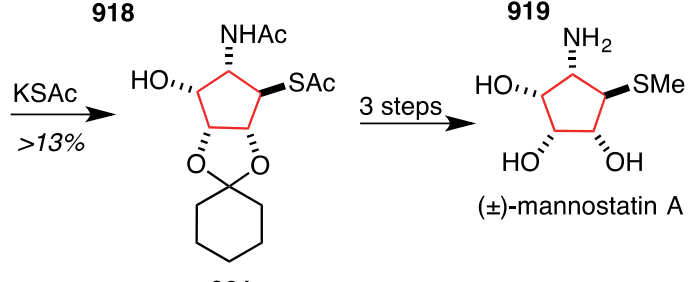

921

171 (a) Ogawa, S.; Yuming, Y. J. Chem. Soc., Chem. Commun. 1991, 890-891. (b) Ogawa, S.; Kimura, H.; Uchida, C.; Ohashi, T. J. Chem. Soc., Perkin Trans. 1 1995, 1695-1705. (c) Ogawa, S.; Yuming, Y. Bioorg. Med. Chem. 1995, 3, 939-943.

${ }^{172}$ Suami, T.; Tadano, K.; Nishiyama, S.; Lichtenthaler, F. W. J. Org. Chem. 1973, 38, 3691-3696.

${ }^{173}$ Ahluwalia, R.; Angyal, S. J.; Luttrell, B. M. Aust. J. Chem. 1970, 23, 1819-1829. 
In the synthesis of $(+)$-trehazolin, Ogawa ${ }^{174}$ constructs the cyclopentane by double addition of nitromethane to a dialdehyde (Scheme 107). The synthesis begins with intermediate 918 that was previously synthesized (see Scheme 106 above) in the route to mannostatin A. Conversion to the desired stereoisomer 922 was performed over five steps according to the protocol developed by Lichtenthaler. ${ }^{172}$ Selective $O$-deacetylation with sodium methoxide in methanol was followed by formation of the $\mathrm{N}, \mathrm{O}$-isopropylidene to give $\mathbf{9 2 3}$. A resolution of this racemic mixture was achieved by formation of a chromatographically separable diastereomeric mixture of $(S)$ acetylmandelates 925 and $\mathbf{9 2 6}$. Ester cleavage of $\mathbf{9 2 6}$ and oxidation of the resulting secondary alcohol yields ketone (+)-927. Conversion to the exo-olefin was accomplished by conversion to a mixture of spiro-epoxides with diazomethane/DMSO followed by reduction with trimethylphosphite to give $\mathbf{9 2 8}$. To complete the synthesis of trehazolin, stereochemical inversion of C4' was required. Hydrolysis of the ketal was followed by selective sulfonylation of the allylic alcohol. Acetate protection of the secondary alcohol delivered 929. Substitution with sodium acetate successfully inverted the C4' stereocenter and dihydroxylation from the less hindered face delivered 930. This advanced intermediate contains a fully functionalized cyclopentane with the desired substitution and stereochemistry for (+)-trehazolin, which was completed in an additional four steps.

\section{Scheme 107. Total Synthesis of (+)-Trehazolin (Ogawa, 1994)}

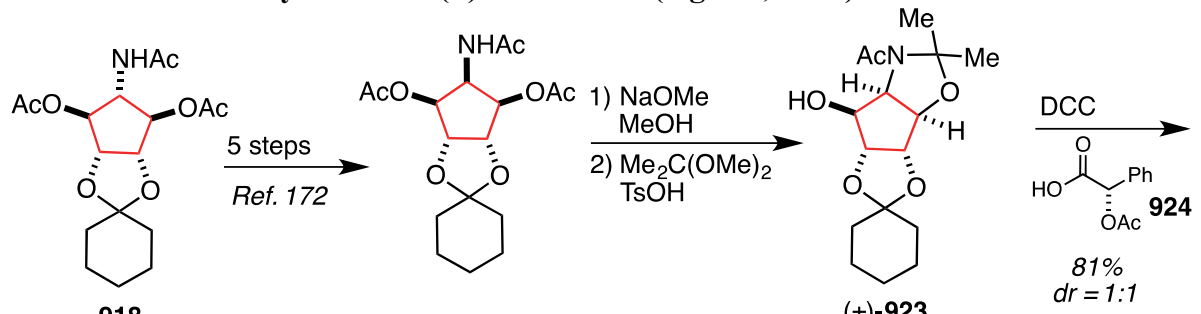

918

922

$( \pm)-923$

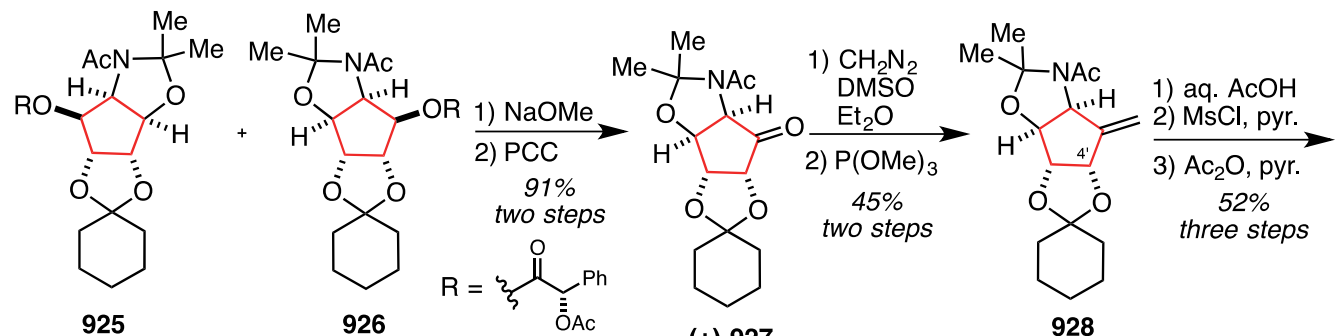

$(+)-927$

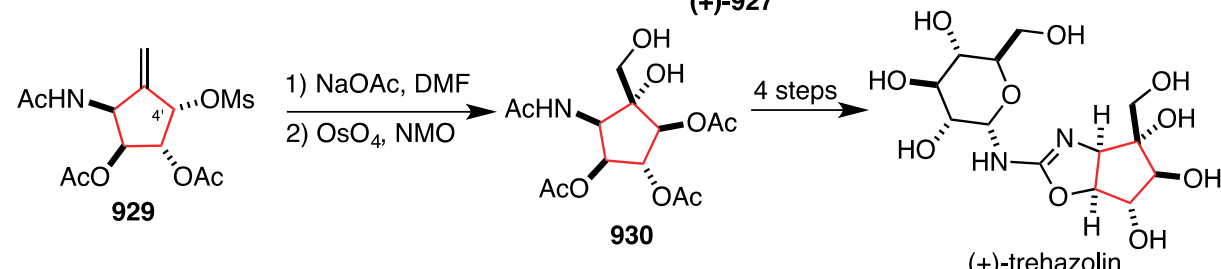

(+)-trehazolin

\section{Mannich Reactions}

The Mannich reaction is a powerful $\mathrm{C}-\mathrm{C}$ bond-forming reaction that is a mainstay of alkaloid total synthesis. The reaction is dependable in complex substrates. As a result, it is not surprising that the Mannich reaction has been employed in the creation of carbocycles that are part of fully functionalized cyclopentane natural products. Mannich reactions that form a five-membered ring require the substrate to contain a carbonyl and an iminium ion in a 1,6-relationship; however, the method by which the nucleophilic enol and the iminium are prepared vary widely.

The Mannich reaction gives products that display a 1,3-N,O-relationship (e.g. 3-amino ketone), and the Mannich reaction is well suited to targets with this functional group pattern. However, if such a relationship can be

${ }^{174}$ (a) Ogawa, S.; Uchida, C.; Yuming, Y. J. Chem. Soc., Chem. Commun. 1992, 886-888. (b) Uchida, C.; Yamagishi, T.; Ogawa, S. J. Chem. Soc., Perkin Trans. 1 1994, 589-602. (c) Ogawa, S.; Uchida, C. J. Chem. Soc., Perkin Trans. 1 1992, 1939-1942. 
revealed through retrosynthetic simplification, then other $\mathrm{C}-\mathrm{C}$ bonds may be targeted for the Mannich disconnection. For example, the central fully functionalized cyclopentane in gelsemine has been prepared using a Mannich reaction of 931 (eq. 1) to forge the $\mathrm{C} 5-\mathrm{C} 16$ bond of 932, which is part of a 1,3-N,O-relationship in the natural product (Scheme 108). However, the C5-C6 bond becomes another Mannich disconnection if the target is simplified to a C7 carbonyl containing intermediate $(\mathbf{9 3 3} \rightarrow \mathbf{9 3 4})$.

\section{Scheme 108.}
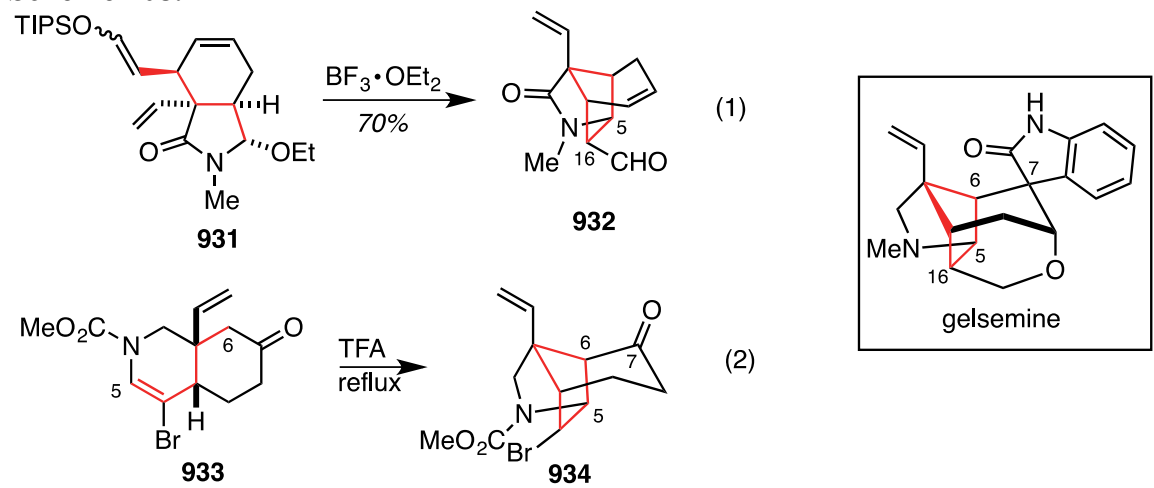

In the synthesis of $( \pm)$-gelsemine, Johnson ${ }^{175}$ employs an intramolecular Mannich reaction to form the polycyclic molecular architecture (Scheme 109). The synthesis commences with a conjugate addition/chloride elimination of alcohol 935 to the vinyl diester 936 to deliver 937. An intramolecular photochemical [2+2] cycloaddition provided tricyclic intermediate $\mathbf{9 3 8}$. The stereochemistry of the product is a result of the suprafacial addition of both alkene components and the constraints of the intramolecular tether. This material was carried forward nine steps to cyclobutanone 939. A retro-Claisen reaction was induced by addition of methylamine providing amido ester 940 as a single diastereomer. Conversion to the aldehyde was performed by an exhaustive reduction of the ester followed by oxidation to the corresponding aldehyde. Treatment with potassium carbonate epimerized the aldehyde and promoted cyclization to yield 941. This material was carried forward five steps to the Mannich reaction precursor $\mathbf{9 4 2}$. Treatment of 942 with refluxing TFA generated the acyliminium ion, and the desired intramolecular Mannich reaction occurred to deliver fully functionalized cyclopentane 943, and the natural product was completed in an additional nine steps.

\section{Scheme 109. Total Synthesis of $( \pm)$-Gelsemine (Johnson, 1994)}

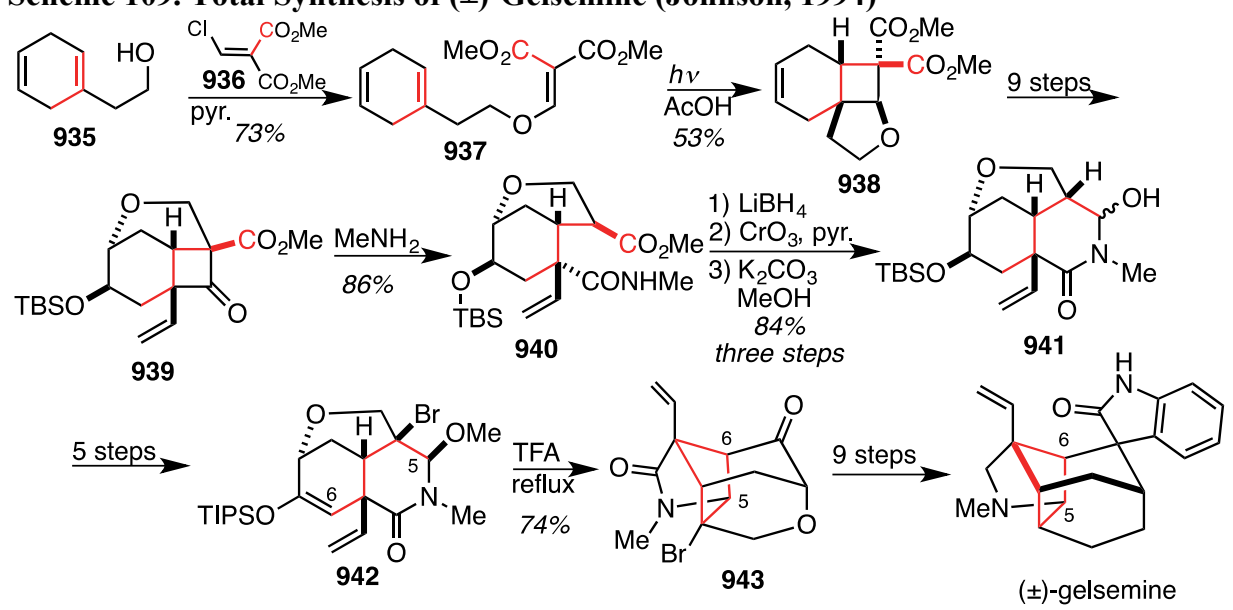

The Speckamp ${ }^{176}$ synthesis of $( \pm)$-gelsemine begins with a Diels-Alder reaction of $N$-methylmaleimide (944) and alcohol 945 (Scheme 110). The less hindered carbonyl of intermediate 946 was reduced and converted to the

175 (a) Sheikh, Z.; Steel, R.; Tasker, A. S.; Johnson, A. P. J. Chem. Soc., Chem. Commun. 1994, 763-764. (b) Dutton, J. K.; Steel, R. W.; Tasker, A. S.; Popsavin, V.; Johnson, A. P. J. Chem. Soc., Chem. Commun. 1994, 765-766.

${ }^{176}$ Newcombe, N. J.; Ya, F.; Vijn, R. J.; Hiemstra, H.; Speckamp, W. N. J. Chem. Soc., Chem. Commun. 1994, 767768. 
corresponding $\mathrm{N}, \mathrm{O}$-acetal 947. Two additional transformations gave intermediate $948 .{ }^{177}$ Enolate formation and addition to 2-(phenylseleno)-ethanal gave a mixture of aldol products, which were advanced to the desired vinyl compound 931 as a mixture of silyl enol ether geometrical isomers. Exposure to Lewis acidic conditions promoted formation of an $N$-acyliminium ion, which cyclized to give aldehyde $\mathbf{9 3 2}$ as a 3:1 mixture with its $\mathrm{C} 16$ epimer. This establishes the fully functionalized cyclopentane with the desired functionality and stereochemistry for gelsemine. An additional 11 steps were used to complete the synthesis of $( \pm)$-gelsemine.

\section{Scheme 110. Total Synthesis of $( \pm)$-Gelsemine (Speckamp, 1994)}

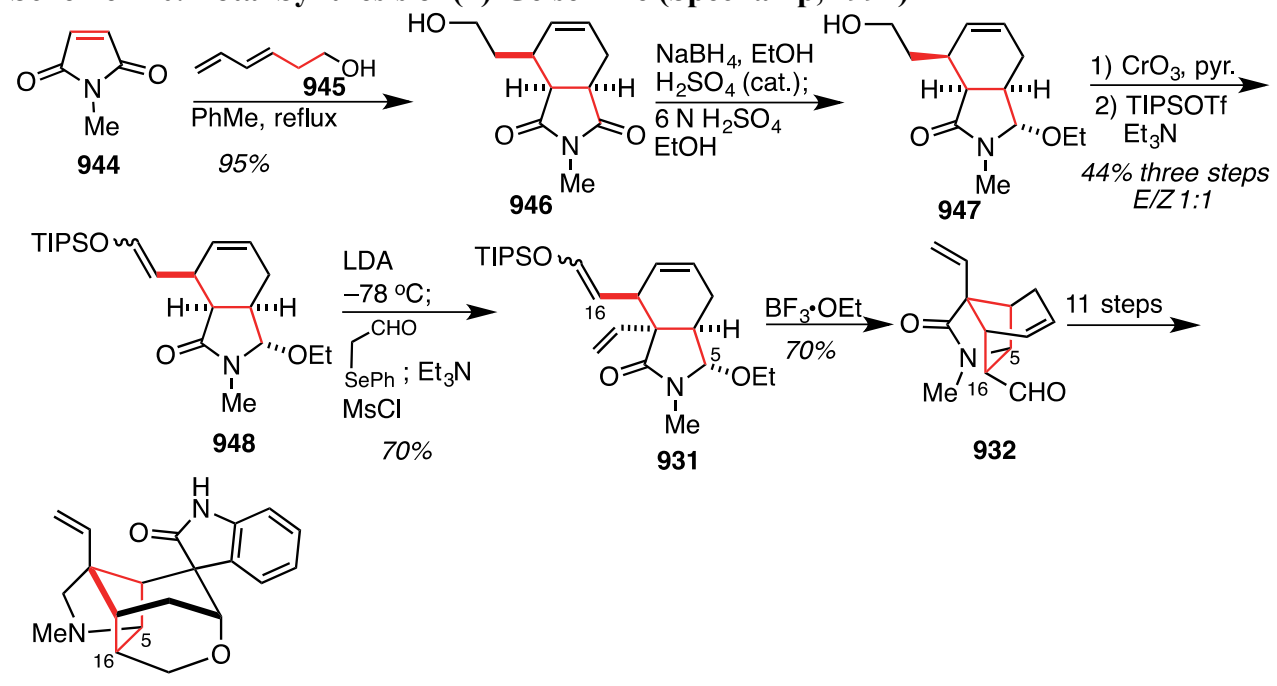

$( \pm)$-gelsemine

In the synthesis of $( \pm)$-gelsemine by Overman, ${ }^{178}$ the azatricyclodecane ring system was constructed with an intramolecular Mannich reaction (Scheme 111). The synthesis commenced with the Diels-Alder reaction of siloxy cyclohexadiene 949 and methyl acrylate to give bicyclo[2.2.2]octene 950. This material was functionalized over three steps to give 951. A Curtius rearrangement sequence converted the carboxylic acid to primary amine 952. Alkylation and TIPS removal delivered 953. Treatment with potassium hydride and 18crown-6 generated a formaldimine alkoxide (954), which participated in an anionic aza-Cope rearrangement. Quenching the reaction with methyl chloroformate delivered intermediate 956. Selective removal of the carbonate group delivered cis-hexahydroisoquinolinone 957. Bromination was envisioned as a means of installing a functional handle for the eventual hydroxymethyl group, and treatment of the enecarbamate with molecular bromine delivered monobrominated compound 933. Exposure to TFA generated the acyliminium ion and promoted the intramolecular Mannich reaction to give azatricyclodecane 934. In this cyclization, the tetrahydropyridine ring must exist in a boat conformation (as seen in 958) in order to obtain the necessary overlap with the enol $\pi$-system. The thermodynamically favored $\mathrm{C} 16$ epimer delivers the tricyclic product with the bromine substituent on the exo face. At this stage a fully functionalized cyclopentane has been achieved, but the substitution does not match gelsemine. Seven steps were used to install the spirooxindole moiety of gelsemine (959). Aziridine formation was achieved by heating the bromide in refluxing cyanide to give 960 . The aziridine nitrogen was methylated, the aziridine was opened with cyanide at the less hindered position, and the ethoxy ethyl protecting group was removed to give 961, which represents a fully functionalized cyclopentane with appropriate stereochemistry for gelsemine. Completion of the synthesis required an additional three steps.

\section{Scheme 111. Total Synthesis of $( \pm)$-Gelsemine (Overman, 1999)}

${ }_{177}^{177}$ Hiemstra, H.; Vijn, R. J.; Speckamp, W. N. J. Org. Chem. 1988, 53, 3882-3884.

178 (a) Madin, A.; O’Donnell, C. J.; Oh, T.; Old, D. W.; Overman, L. E.; Sharp, M. J. Angew. Chem. Int. Ed. 1999, 38, 2934-2936. (b) Earley, W. G.; Jacobsen, E. J.; Meier, G. P.; Oh, T.; Overman, L. E. Tetrahedron Lett. 1988, 29, 37813784. 

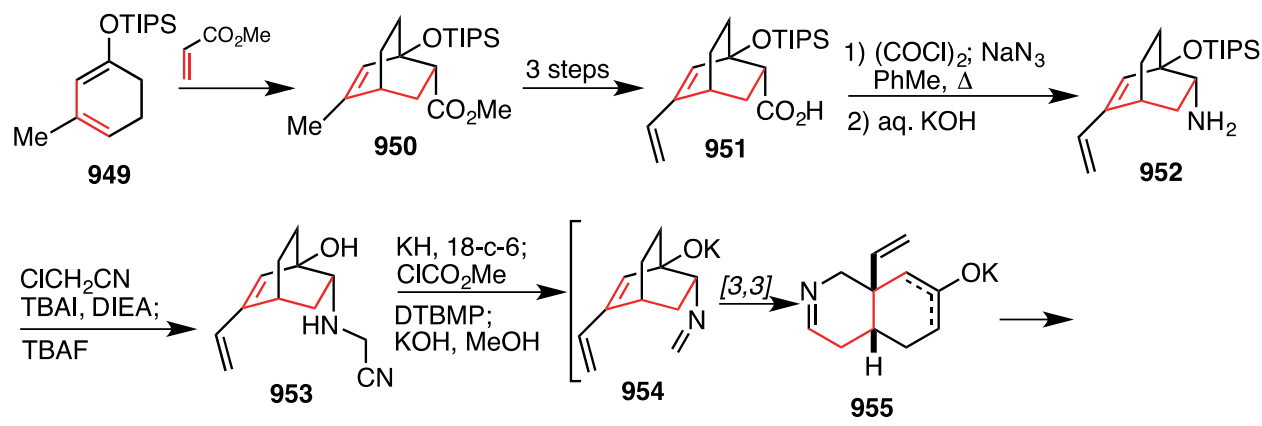

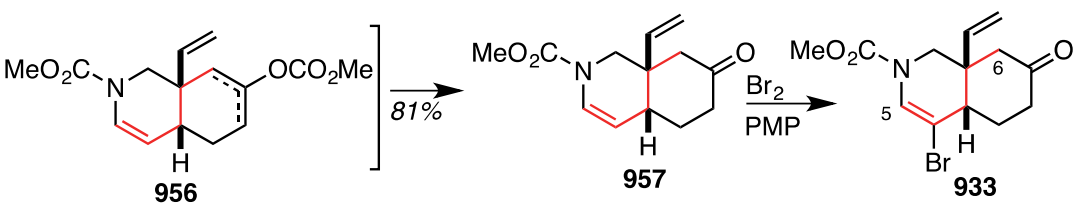
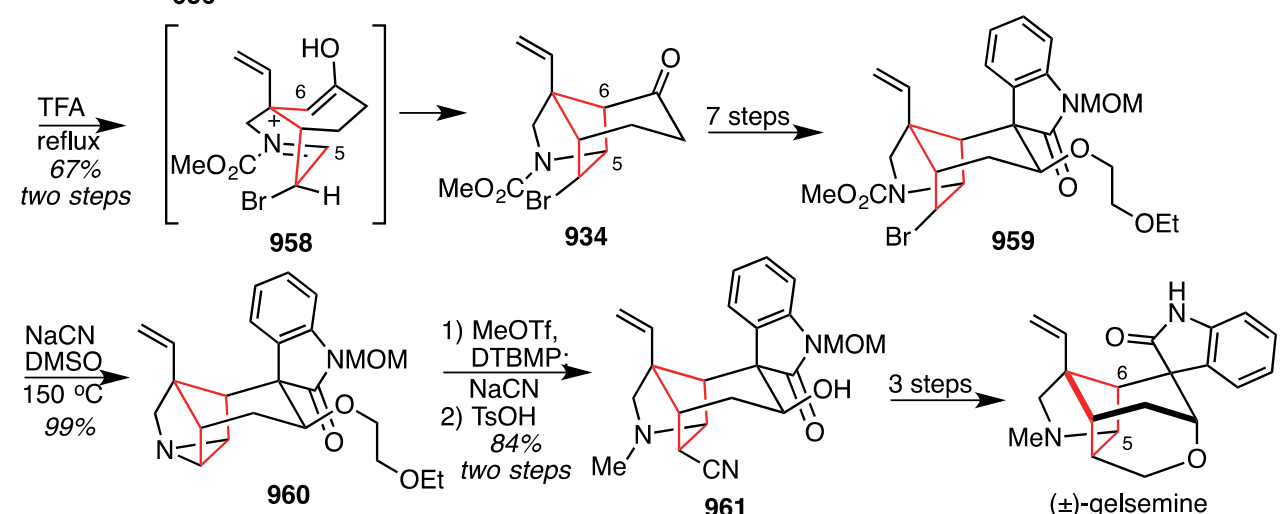

The synthesis of (-)-lepenine by Fukuyama ${ }^{179}$ features an intramolecular Mannich reaction to form the key fivemembered ring (Scheme 112). The synthesis begins with a Mitsunobu reaction with enantiopure lactic acid derivative (963) and guaiacol (962) to deliver 964 with complete inversion of stereochemistry. Reduction with DIBAL and addition of vinylmagnesium chloride provides 965 as a mixture of alcohol epimers. Heating the allylic alcohol in $p$-nitrophenol with triethyl orthoacetate promoted the Johnson-Claisen rearrangement to give the $\gamma, \delta$-unsaturated ester $\mathbf{9 6 6}$. This intermediate ester is also a Claisen substrate, and it undergoes a subsequent rearrangement to yield phenol 967. The Claisen rearrangement cascade proceeds through the usual chair-like transition state and transfer of stereochemical information gives the expected stereoisomer in 967. This material was advanced three steps to $\mathbf{9 6 8}$. Saponification of the ethyl ester and Friedel-Crafts acylation delivers 969. Addition of vinylmagnesium chloride and elimination of the resulting alcohol gave diene 970. Cleavage of the pivaloyl ester, esterification with methacrylic acid furnished cycloaddition substrate $\mathbf{9 7 1}$. Heating in benzonitrile promoted the Diels-Alder cycloaddition furnishing tetracyclic intermediate $\mathbf{9 7 2}$ in good yield. Hydroborationoxidation occurred on the convex face of the cis-6,6 ring system which gave complete diastereoselectivity to give alcohol 973. The Mannich precursor 974 was prepared over three steps. Treatment of key substrate 974 with $\operatorname{Pd}(0)$ in acetic acid led to loss of the alloc protecting group. Intramolecular cyclization gave an iminium ion that underwent Mannich cyclization to give 975. This material was advanced over three steps to $o$-quinone monoketal 976. Heating in an ethylene atmosphere, a Diels-Alder reaction occurred with complete diastereoselectivity on the less hindered face of the diene to give cycloadduct 977. This material was advanced three steps to 978 . The final stereocenter of the fully functionalized cyclopentane was set by hydroboration-oxidation of cyclopentene on the less hindered face to give $\mathbf{9 7 9}$. Advanced intermediate $\mathbf{9 7 9}$ contains a fully functionalized cyclopentane and could be advanced to (-)-lepenine in four additional steps.

Scheme 112. Total Synthesis of (-)-Lepinine (Fukuyama, 2014)

${ }^{179}$ Nishiyama, Y.; Han-ya, Y.; Yokoshima, S.; Fukuyama, T. J. Am. Chem. Soc. 2014, 136, 6598-6601. 

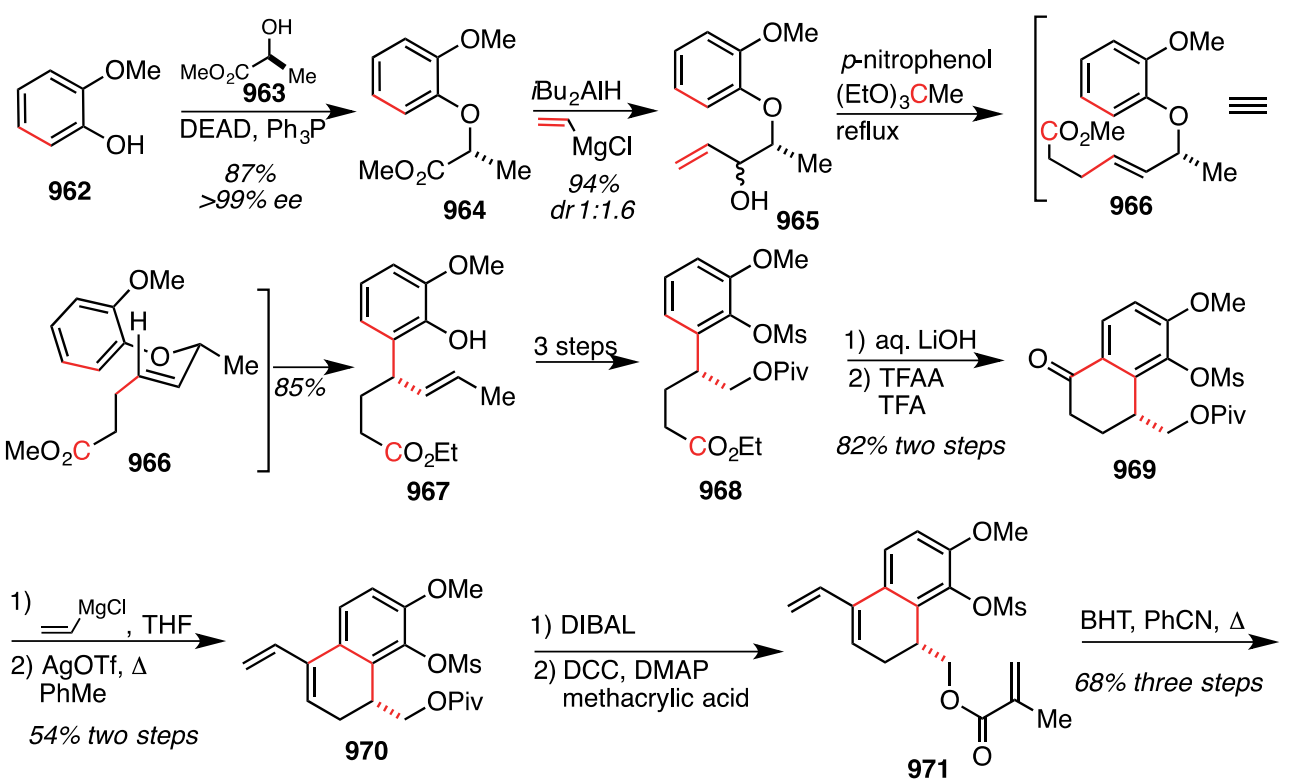

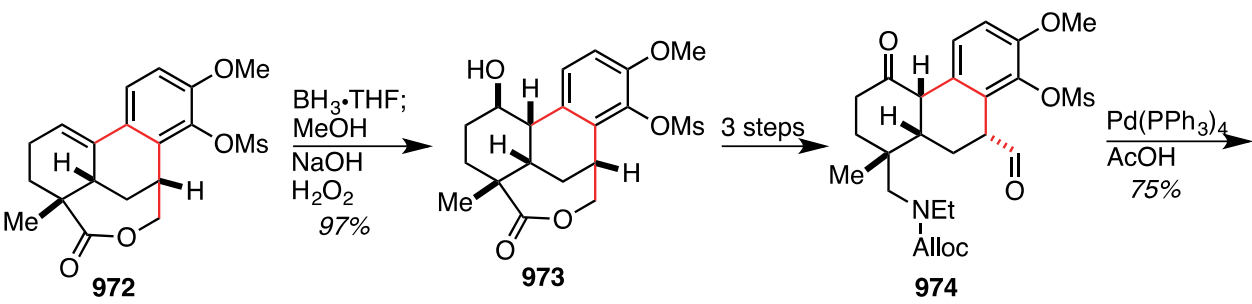

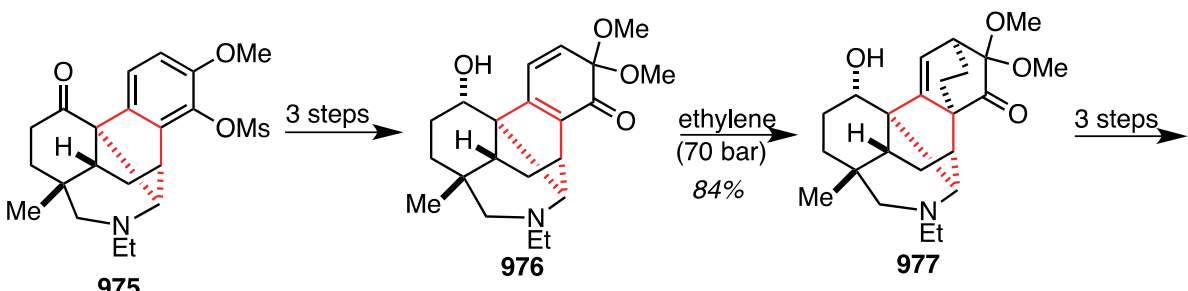

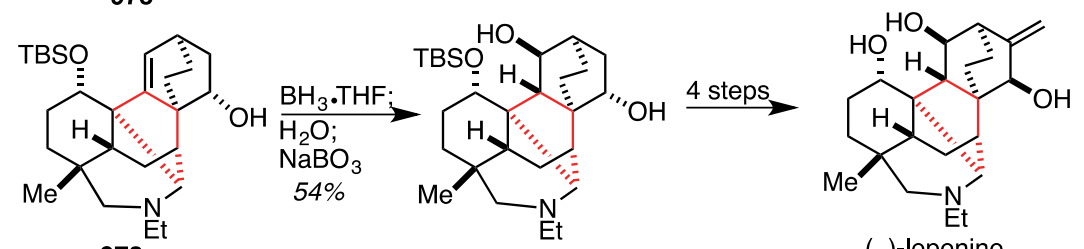

978

979

(-)-lepenine

In Baran's ${ }^{180}$ unified approach to the ent-atisane, atisine, and hetidine alkaloids, a fully functionalized five membered ring is synthesized via an azomethine ylide isomerization followed by a Mannich cyclization (Scheme 113). Beginning with the ent-kaurane (-)-steviol, much of the required stereochemistry is present for the hetidine alkaloids. Amide formation introduced the requisite nitrogen for the Mannich reaction. Reduction to the amine and reaction with diethyl chlorophosphate provided phosphoramidate $\mathbf{9 8 0}$. A three step sequence to form bicyclo[2.2.2] octane intermediate $\mathbf{9 8 1}$ begins with oxidative cleavage of the alkene with cobalt acetoacetate to form a 1,5 dicarbonyl. Aldol cyclization delivers the bicyclo[2.2.2] octane and the resulting tertiary alcohol is protected as the acetate (981). Following the Suárez conditions ${ }^{181}, \mathrm{C}-\mathrm{H}$ activation occurred selectively at C20. During the iodination, the phosphoramidate was also oxidized to the corresponding imine 982. Treatment with allyl amine in methanol led to condensation and alkylation to give imminium ion 983. Subsequent deprotonation to an azomethine imine, and re-protonation isomerized the imminium ion and triggered the Mannich cyclization

${ }^{180}$ Cherney, E. C.; Lopchuk, J. M.; Green, J. C.; Baran, P. S. J. Am. Chem. Soc. 2014, 136, 12592-12595.

${ }^{181}$ Francisco, C. G.; Herrera, A. J.; Suárez, E. J. J. Org. Chem. 2003, 68, 1012-1017. 
to give hetidine skeleton 984. While this structure is not a natural product, it contains the fully functionalized cyclopentane, all of the carbons, and the stereochemistry found in (-)-spirafine III and other structurally related natural products.

\section{Scheme 113. Synthetic Studies Toward ent-Atisane, Atisine, and Hetidine Diterpenes (Baran, 2014)}

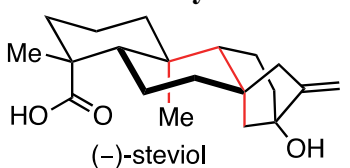

1) $\mathrm{EDCl}, \mathrm{HOBt}$; $\mathrm{NH}_{4} \mathrm{OH}$

2) $\mathrm{LiAlH}_{4}$

3) $(\mathrm{EtO})_{2} \mathrm{POCl}$

$70 \%$ three
steps

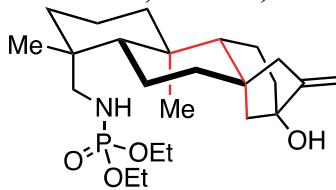

980
1) $\mathrm{Co}(\mathrm{acac})_{2}$

$\mathrm{Et}_{3} \mathrm{SiH}, \mathrm{O}_{2}$

2) Amberlyst 15

3) $\mathrm{Ac}_{2} \mathrm{O}$, DMAP

$46 \%$ three steps dr 1:1

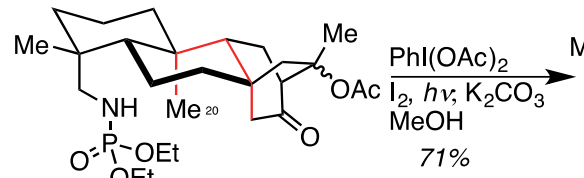

981

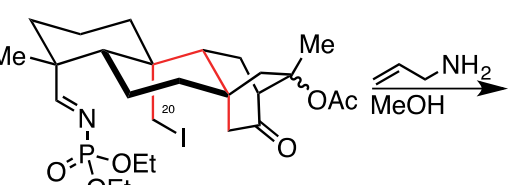

982
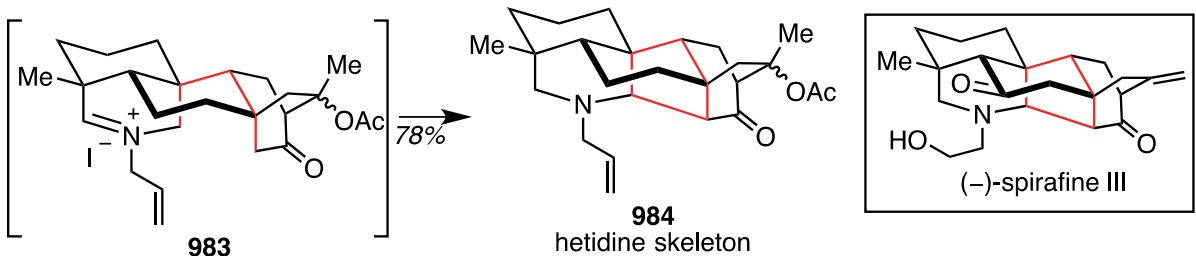

hetidine skeleton

The synthetic effort towards palau'amine by Harran ${ }^{182}$ seeks to take advantage of the symmetry present in the natural product (Scheme 114). The fully functionalized cyclopentane is constructed by a halogenative enamine Mannich-type reaction of $\mathbf{9 6 0}$. The synthesis begins with tetrahydropyridazine 954 which could be advanced four steps to give key dimerization precursor 955. Dienolate formation and subsequent oxidation gave meso-dimer 956 as the major product in a 3:1 ratio with its trans diastereomer. A rhodium-catalyzed reduction provided 959 as a complex mixture of stereoisomers. This inconsequential mixture of diastereomers was treated with $\mathrm{Cy}_{2} \mathrm{BOTf}$ and base, which induced a double fragmentation double tautomerization sequence to give $\mathbf{9 6 0}$. The halogenative desymmetrization reaction proceeds with $t \mathrm{BuOCl}$ and magnesium chloride to give $\mathbf{9 6 1}$ as a mixture of $\mathrm{C} 10$ epimers thus completing the fully functionalized cyclopentane. This advanced intermediate contains many of the structural features found in palau'amine.

Scheme 114. Synthetic Studies Toward Palau'amine (Harran, 2009)

${ }^{182}$ Li, Q.; Hurley, P.; Ding, H.; Roberts, A. G.; Akella, R.; Harran, P. G. J. Org. Chem. 2009, 74, 5909-5919. 


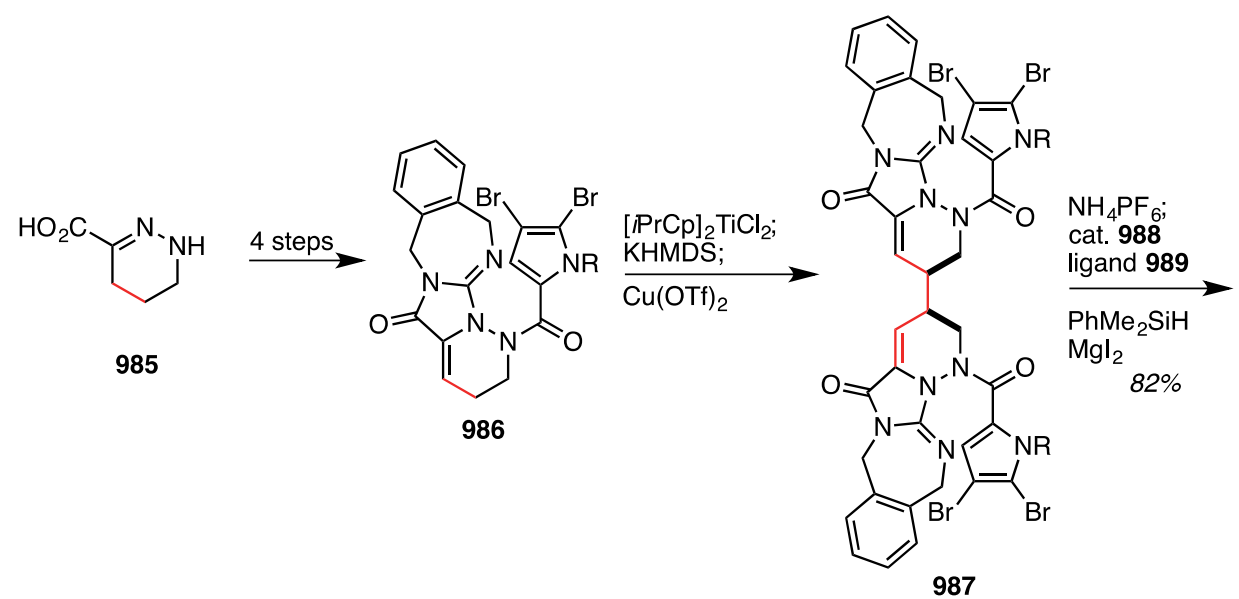<smiles>CCOCCNC(=O)c1cc(Br)c(Br)[nH]1</smiles>

990
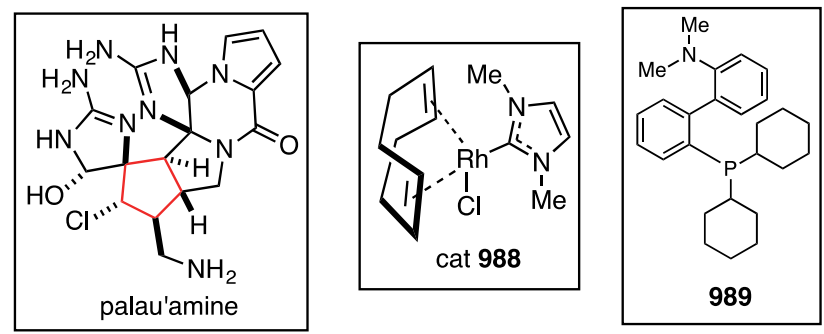

\section{Conjugate Addition Reactions}

This section describes syntheses where the fully funtionalized cyclopentane is created using a conjugate addition of an enolate (or related nucleophile) to an unsaturated carbonyl. Conjugate addition reactions, including the Michael reaction, inherently produce enolates; such enolates can be captured in a variety of creative ways. As a result, the reactions featured in this section are highly varied, and they contain many domino or cascade reactions of carbanions (e.g. tandem Michael-aldol reactions). We collect all relevant conjugate addition reactions and all cascade or tandem reactions involving conjugate additions in this section.

As mentioned above, the reactions in this section are particularly diverse. For example, there are two distinct syntheses of the complex alkaloid gelsemine (Scheme 115) that use enolate conjugate additions in markedly different strategies. The first (eq. 1) uses tandem nitronate and ester enolate (993) conjugate additions to divinyl ketone 994 to give a bicyclic product 995. This reaction builds the key cyclopentane while creating the C5-C6 and $\mathrm{C} 15-\mathrm{C} 16$ bonds of the target molecule. In another gelsemine synthesis (eq. 2) an intramolecular reaction of a nitrile enolate and an unsaturated oxindole (996) creates the fully functionalized cyclopentane and the C6-C20 bond in 997. The conjugate addition reactions shown were used to build a fully functionalized cyclopentane with 
good control of product stereochemistry; however, the reactions have little else in common. A feature of this section is the wide variety of creative uses and tandem tranformations that have been based on the conjugate addition reaction.

\section{Scheme 115.}

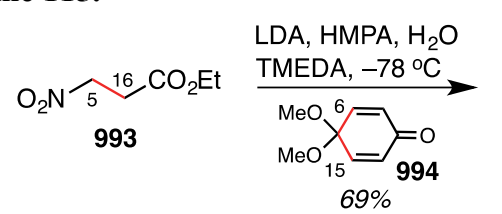

$69 \%$

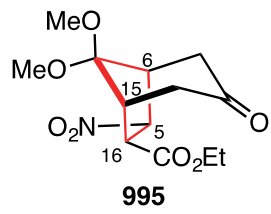<smiles>COC[C@H]1[C@@H](CC(OC)OC)[C@@H](C)CN(C)[C@@H]1/C=C1/C(=O)N(OC)c2ccccc21</smiles>

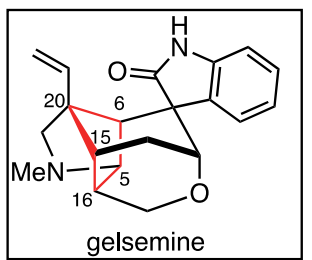

In the synthesis of $( \pm)$-gymnomitrol by Büchi ${ }^{183}$, a conjugate addition is followed by an intramolecular cyclization to construct the key five-membered ring (Scheme 116). The synthesis begins with quinone ketal 998 , which is obtained from 4,5-dimethoxy-2-methylbenzaldehyde in three steps. Condensation with 1,2-

dimethylcyclopentene delivered $\mathbf{1 0 0 1}$ as the major diastereomer. Mechanistically, the sequence begins with Lewis acid activation to give quinone derivative 999. Conjugate addition of 1,2-dimethylcyclopentene is followed by trapping of the resulting carbocation by the tethered enol ether in $\mathbf{1 0 0 0}$. This cyclization sets four of the five stereocenters in gymnomitrol favoring the desired stereoisomer. The unpurified material was reduced with hydride to set the final stereocenter of gymnomitrol giving intermediate 1002. Completion of the synthesis of $( \pm$-gymnomitrol required five additional steps for the deletion of the enol ether functionality and olefination of the ketone.

Scheme 116. Total Synthesis of ( \pm )-Gymnomitrol (Buchi, 1979)

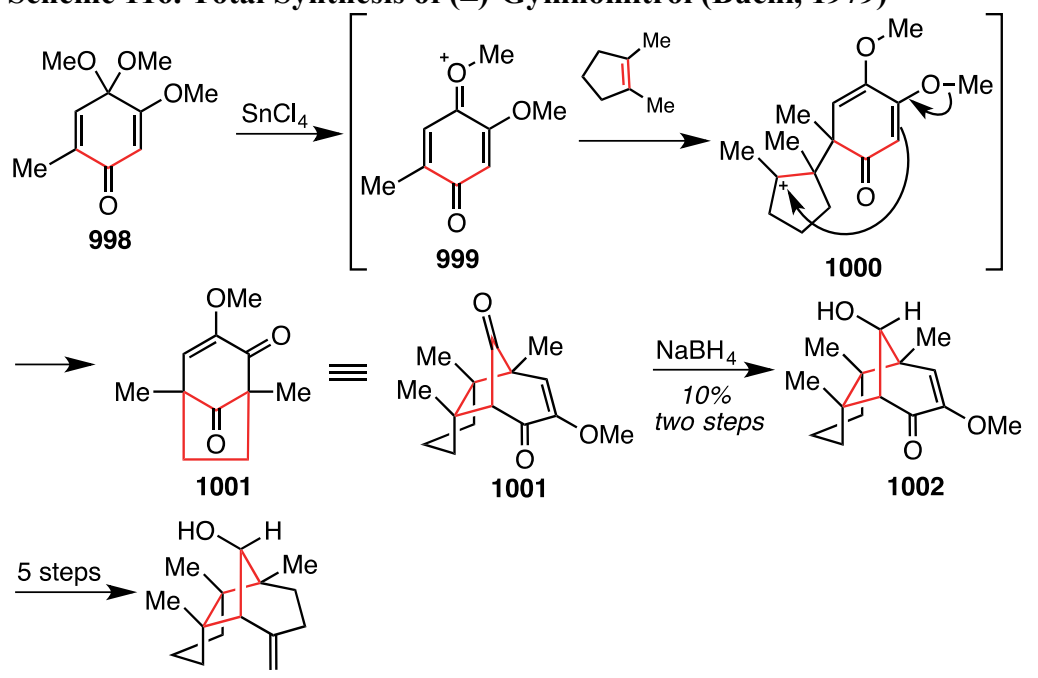

( \pm -gymnomitrol

In the synthesis of coriolin by Danishefsky ${ }^{34}$, the key five-membered ring in the target was constructed in a cascade process featuring a Michael addition and an aldol condensation (Scheme 117). An addition of the enolate derived from 1003 to the Michael acceptor 5,5-dimethyl cyclopentenone (1004) was followed by refluxing in acid to deliver 1005. A Diels-Alder reaction with diene $\mathbf{1 0 0 6}$ gives the tricyclic $\mathbf{1 0 0 7}$ as a single diastereomer. Addition from the bottom face of the cyclopentenone was controlled by formation of the energetically preferred

${ }^{183}$ Büchi, G.; Chu, P. -S. J. Am. Chem. Soc. 1979, 101, 6767-6768. 
cis fusion of the cyclopentanes. The enone functionality was installed by addition to phenylselenyl chloride and subsequent oxidation and elimination with $\mathrm{NaIO}_{4}$ to give 1008. Regiospecific addition of methyl to the enone carbonyl gave allylic alcohol 1009. Diketone 1010 was accessed over the following steps: ozonolysis, Jones oxidation, decarboxylation with barium hydroxide, and lead acetate cleavage of the hydroxy acid. An aldol condensation of the 1,4-dicarbonyl delivered tricyclic product 1011. Deconjugation of the $\alpha, \beta$-enone to the $\beta, \gamma-$ unsaturated ketone 1012 was completed with potassium tert-butoxide and acetic acid. Treatment with DIBAL resulted in reduction of the less-hindered ketone, and the more substituted ketone required dissolving metal reduction. These two steps resulted in the formation of diol 1013. Epoxidation with $m \mathrm{CPBA}$ occurred stereoselectively by forming the favorable cis ring fusion of the cyclopentanes. Chromium oxidation provides ketone 1014. Treatment of $\mathbf{1 0 1 4}$ with LDA resulted in $\beta$-elimination of the epoxide and subsequent formation of an enolate that underwent sulfenylation to yield 1015. The resulting thioether was oxidized with peracid to a sulfoxide that was eliminated upon reflux to give exo-methylene 130. Double epoxidation of the divinyl ketone with peroxide completed the synthesis of $( \pm)$-coriolin. ${ }^{184}$

Scheme 117. Total Synthesis of $( \pm$ )-Coriolin (Danishefsky, 1980)
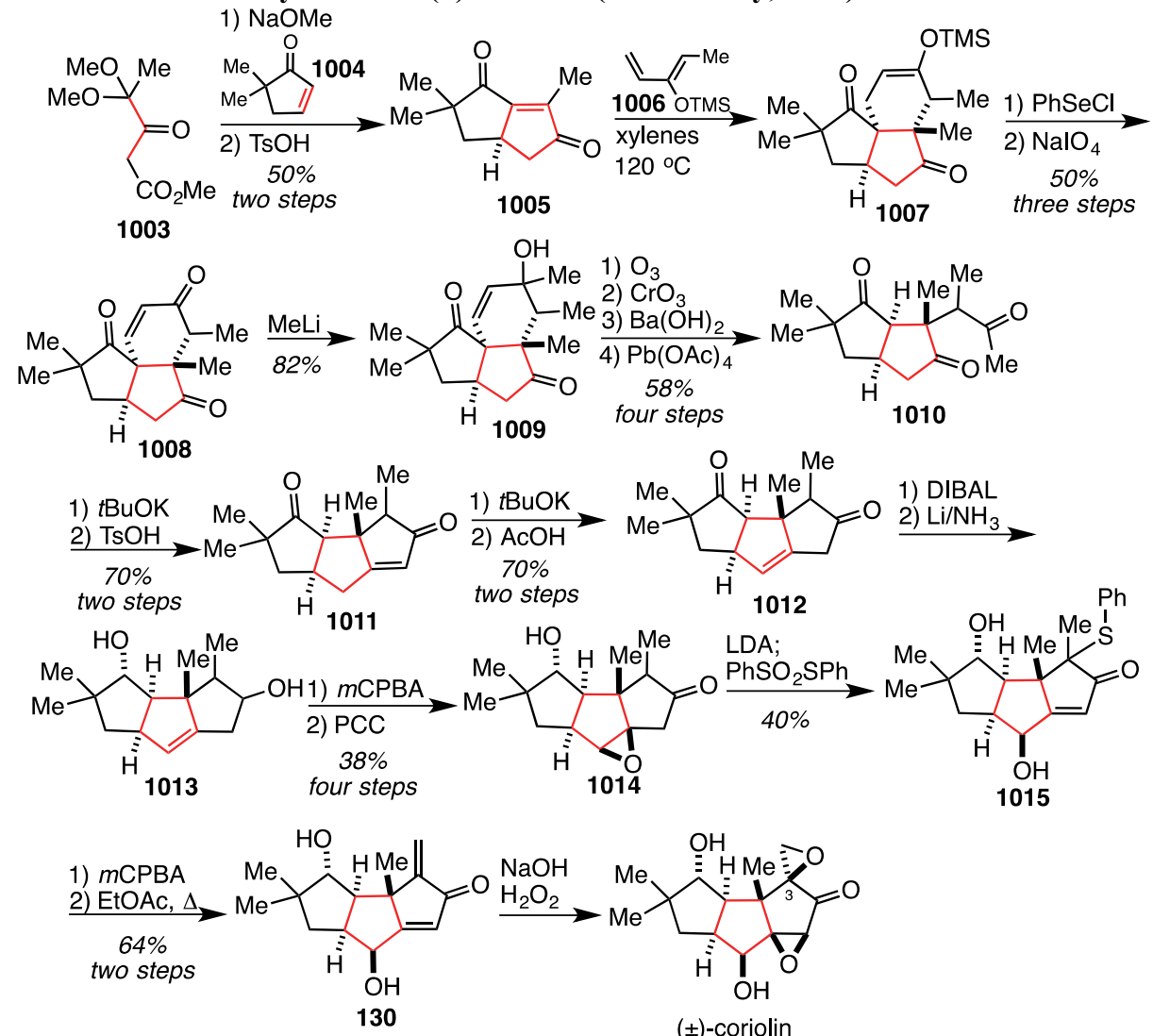

Considerations of the biosynthesis of shinjulactone $\mathrm{C}$ led Takahashi ${ }^{185}$ to hypothesize that this natural product was biosynthetically related to the structurally similar compound ailanthone (Scheme 118). It was found that ailanthone could be converted into shinjulactone $\mathrm{C}$ in low yield by prolonged reflux in pyridine. This spectacular reaction creates a decasubstituted cyclopentane embedded in the shinjulactone $\mathrm{C}$ architecture. Of the five contiguous fully substituted carbons, two of the quaternary carbons and both tertiary alcohols are forged in this transformation. With this discovery in hand additional effort was committed to a mechanistic investigation of the reaction. It was hypothesized that ailanthone undergoes oxidation at $\mathrm{C} 1$, hemiacetal fragmentation, isomerization of the exo-methylene, and epimerization at C9 to give presumed reactive intermediate 1017. This intermediate would be deprotonated at C5 to form a dienolate nucleophile, which would undergo conjugate addition to the

${ }^{184}$ The final transformation delivers a 7:5 ratio of the desired $( \pm)$-coriolin and its spiroepoxide epimer. A four-step stereoselective route was designed for the final transformation to $( \pm)$-coriolin, see: Danishefsky, S.; Zamboni, R.; Kahn, M.; Etheredge, S. J. J. Am. Chem. Soc. 1981, 103, 3460-3467.

${ }^{185}$ Ishibashi, M.; Tsuyuki, T.; Takahashi, T. Tetrahedron Lett. 1983, 24, 4843-4846. 
enone at $\mathrm{C} 12$. The conjugate addition presumably resulted in an enolate, nucleophilic at $\mathrm{C} 13$, which underwent cyclization by adding to the $\mathrm{C} 1$ carbonyl.

In order to investigate the mechanistic hypothesis shown above, ailanthone was converted to $\mathbf{1 0 1 8}$, which is a protected analog of reactive intermediate 1017. Treatment of 1018 with refluxing pyridine resulted in the isomerization of the exo-methylene to give 1019. Conversion of the TBS ether to the corresponding carbonyl gave 1020. This molecule differs from reactive intermediate $\mathbf{1 0 1 7}$ only in the configuration at $C 9$ and the presence of two acetate protecting groups. Refluxing of this molecule in pyridine led to the formation of acetylated shinjulactone $\mathrm{C}$ (1021). The performance of this intermediate in the reaction cascade gives strong support for the mechanistic hypothesis.

Scheme 118. Biomimetic Conversion of Alianthone to Shinjulactone C (Takahashi, 1983)
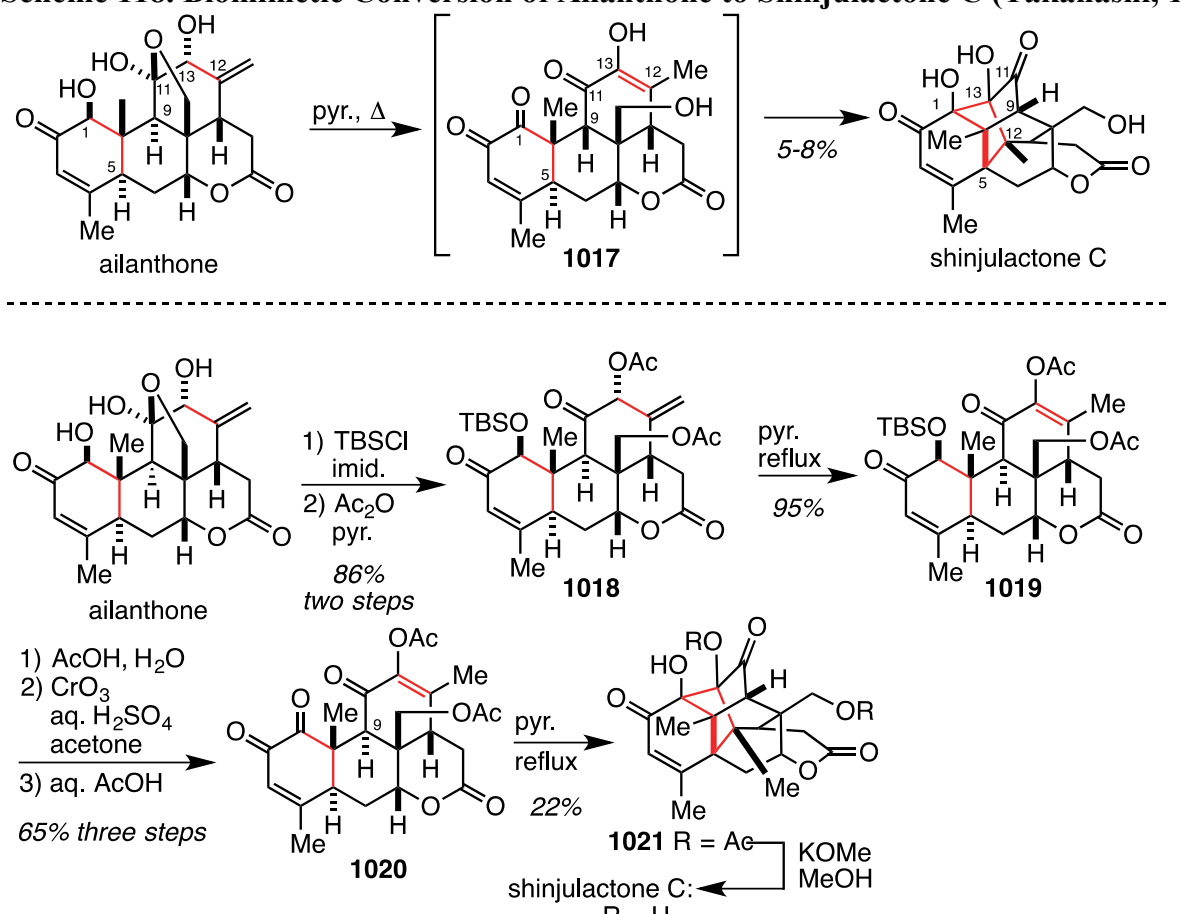

In the first total synthesis of the quassinoid $\left( \pm\right.$ )-shinjulactone $C$, Grieco ${ }^{186}$ utilizes the technology developed by Takahashi (see Scheme 118 above) to assemble a decasubstituted cyclopentane (Scheme 119). The route begins with Wieland-Miescher ketone 853, which was stereoselectively reduced with hydride to give alcohol 1022. This material was advanced over nine steps to cyclization precursor 1023. A Diels-Alder cycloaddition with substituted diene $\mathbf{1 0 2 4}$ using conditions developed by Grieco ${ }^{187}$ produced tricyclic intermediate 1025, which contains all five carbons of the eventual fully functionalized cyclopentane. This material was functionalized over four steps to give tetracyclic compound 1026. A stereoselective hydroboration-oxidation reaction of the tertiary alkene was followed by alcohol oxidation to give ketone 1027. This material was advanced over five steps to ketone 1028. Treatment of $\mathbf{1 0 2 8}$ with DIBAL reduced both carbonyl groups, and subsequent treatment with acidic methanol led to dehydration and acetal formation delivering 1029. Oxidaton of the secondary alcohol provided ketone 1030. Dihydroxylation and protection of the diol as the acetonide afforded 1031. Oxidation of the ketone over three steps led to $\alpha$-methoxy ketone 1032. This material was advanced over seven steps to intermediate 1033. Swern oxidation of the secondary alcohol gives a ketone. Treatment with acetic anhydride in DMAP forms the enol acetate (1034) while simultaneously acetylating the primary alcohol derived from the opening of the hemiketal. Cleavage of the methyl ether with boron tribromide was followed by Jones' oxidation to give cyclization precursor 1020. Following the conditions developed by Takahashi, when $\mathbf{1 0 2 0}$ was heated in pyridine $( \pm$ )-shinjulactone $\mathrm{C}$ diacetate (1021) was produced in moderate yield. Ester hydrolysis gave the natural product.

${ }^{186}$ Collins, J. L.; Grieco, P. A.; Gross, R. S. J. Org. Chem. 1990, 55, 5816-5818.

${ }^{187}$ Grieco, P. A.; Garner, P.; He, Z. Tetrahedron Lett. 1983, 24, 1897-1900. 
Scheme 119. Total Synthesis of ( \pm -Shinjulactone C (Grieco, 1990)

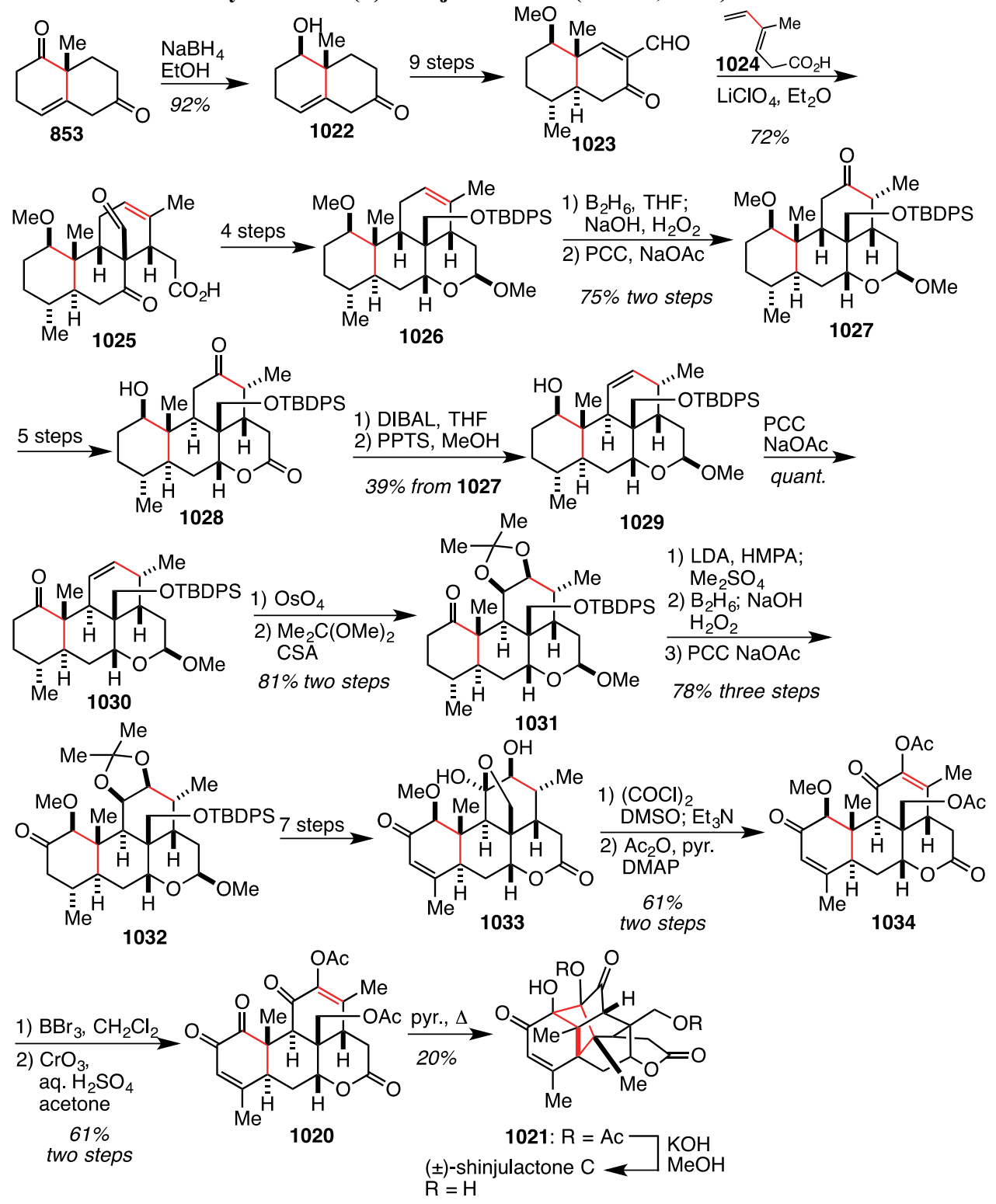

In the Crimmins ${ }^{188}$ synthesis of $( \pm)$-ginkgolide $B$, the five membered ring is assembled in an annulation involving a conjugate addition (Scheme 120). The route begins with furanyl aldehyde 1037, which can be advanced to 1038 over three steps. Addition of ethynylmagnesium bromide delivered syn alcohol $\mathbf{1 0 3 9}$ as the major product in a 1.2:1 diastereomer ratio. The undesired anti diastereomer could be converted to the desired $\mathbf{1 0 3 9}$ via a Mitsunobu inversion. The alcohol was silylated and the alkyne was carboxylated to deliver 1040. Treatment of acetylenic ester 1040 with the zinc-copper homoenolate 1041 delivered the cyclopentenone 1042.

Mechanistically, this transformation proceeds via a conjugate addition followed by a Dieckmann condensation. An intramolecular photocycloaddition resulted in formation of two contiguous quaternary carbon stereocenters on cyclopentanone 1043. Desilylation, and activation of the alcohol as the sulfonate gave intermediate $\mathbf{1 0 4 5 .}$ Lactone formation under acidic conditions gave pentacyclic intermediate 1046. The ketone was oxidized to the corresponding enone with standard conditions to give 1047. Epoxidation of the enol ether with DMDO in the

188 (a) Crimmins, M. T.; Pace, J. M.; Nantermet, P. G.; Kim-Meade, A. S.; Thomas, J. B.; Watterson, S. H.; Wagman, A. S. J. Am. Chem. Soc. 1999, 121, 10249-10250. (b) Crimmins, M. T.; Pace, J. M.; Nantermet, P. G.; Kim-Meade, A. S.; Thomas, J. B.; Watterson, S. H.; Wagman, A. S. J. Am. Chem. Soc. 2000, 122, 8453-8463. 
presence of aqueous acid resulted in spontaneous hydrolyis of the epoxide to a hemiacetal followed by fragmentation of the cyclobutane. Treatment with acidic methanol and trimethylorthoformate led to acetal formation giving 1048. Barton deoxygenation ${ }^{90}$ removed the secondary hydroxyl group to give 1049. Modified conditions of a Davis procedure successfully led to $\alpha$-hydroxylation of the $\beta$-dicarbonyl. Acylation with propionic anhydride delivered 1050. Enolate addition to the cyclopentenone gave the undesired $\mathbf{1 0 5 1}$ as the major product. Epimerization of the methyl stereocenter with $\mathrm{NaOMe} / \mathrm{MeOH}$ generated a 1:1 diastereomeric mixture with desired product 1052. The undesired isomer could then be isolated and resubjected to epimerization conditions to obtain more of the desired material. Lactone cleavage was performed with camphorsulfonic acid and methanol to give 1053. Heating the ketal with PPTS resulted in elimination of methanol and yielded enol ether 1054. Epoxidation of the cyclopentene with concomitant intramolecular lactonization gave fully functionalized cyclopentane $\mathbf{1 0 5 5}$ with the appropriate substitution and stereochemistry found in ginkgolide B. Two additional standard transformations were used to complete the synthesis of the natural product.

Scheme 120. Total Synthesis of ( \pm )-Ginkgolide B (Crimmins, 1999)

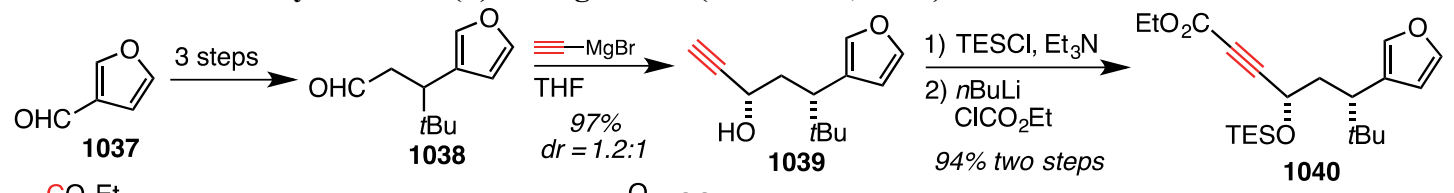

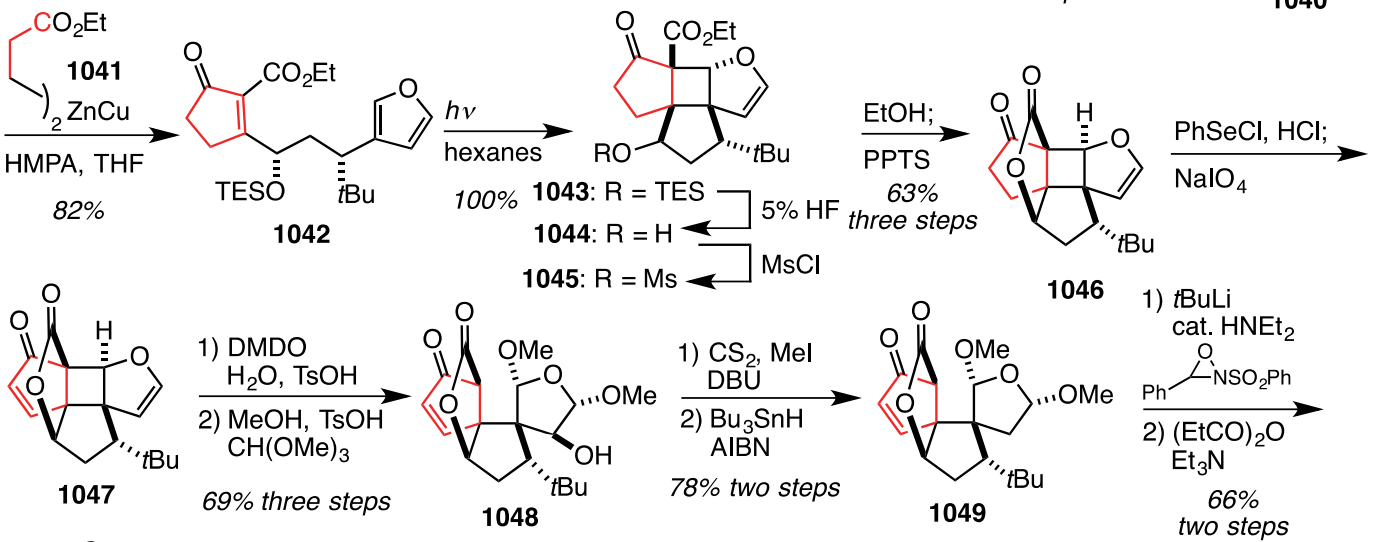

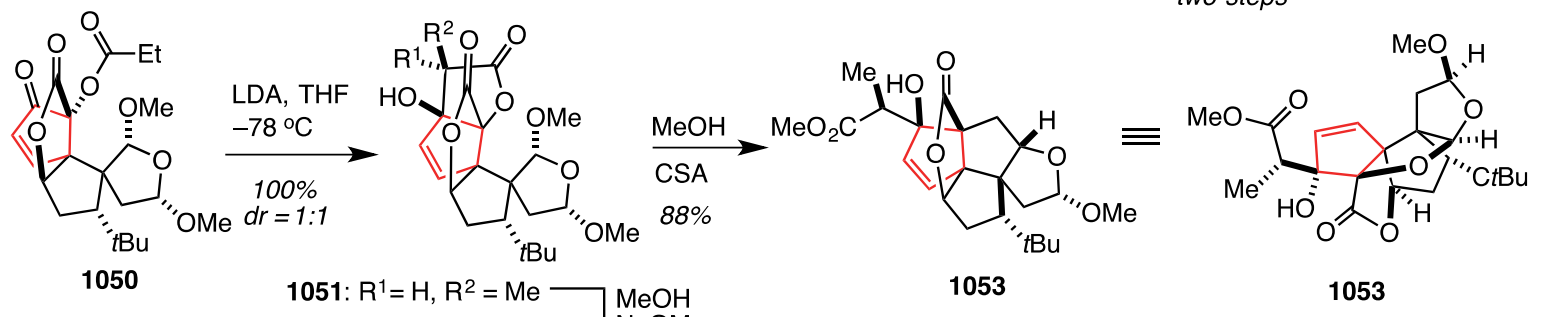
1052: $R^{1}=\mathrm{Me}, \mathrm{R}^{2}=\mathrm{H} \longleftarrow \mathrm{NaOMe}$

$100 \% ; d r=1: 1$

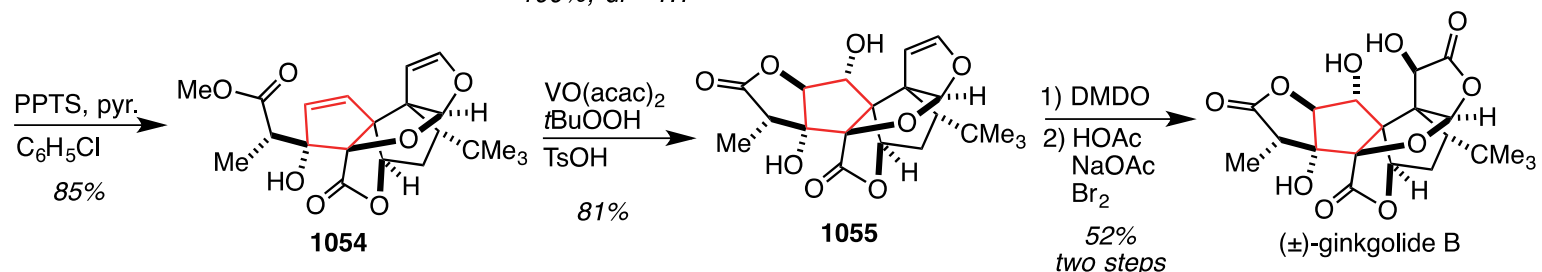

In the synthesis of $( \pm)$-longiborneol by Ihara ${ }^{189}$, a double Michael addition is used to assemble a fully functionalized cyclopentane (Scheme 121). The route begins with dicyclopentadiene derivative $\mathbf{1 0 5 6}$ which was alkylated with iodide 1057 to give 1058. A retro-Diels-Alder reaction releases cyclopentadiene to give cyclopentenone 1059. Addition of methyllithium gave a tertiary alcohol and oxidative transposition with PCC gave enone 1060. Silyl deprotection, alcohol oxidation and olefination gave cyclization precursor 1061. The double Michael reaction proceeds with LHMDS, which constructs a fully functionalized cyclopentane as part of

${ }^{189}$ Takasu, K.; Mizutani, S.; Noguchi, M.; Makita, K.; Ihara, M. J. Org. Chem. 2000, 65, 4112-4119. 
the core molecular architecture of the sesquiterpene natural products (1062); however, the natural products require conversion of the carbomethoxy substituent into a hydroxyl group. Basic hydrolysis of the ester gave 1063. After much experimentation, radical decarboxylation conditions were found to deliver 1064 in good yield. This advanced intermediate contains the appropriate functionality and stereochemistry for ( \pm )-culmorin, and ketone reduction completed the natural product. Intermediate 1063 was taken forward to $( \pm)$-longiborneol by exhaustive ketone reduction to give $\mathbf{1 0 6 5}$ and an oxidative decarboxylation as discussed above.
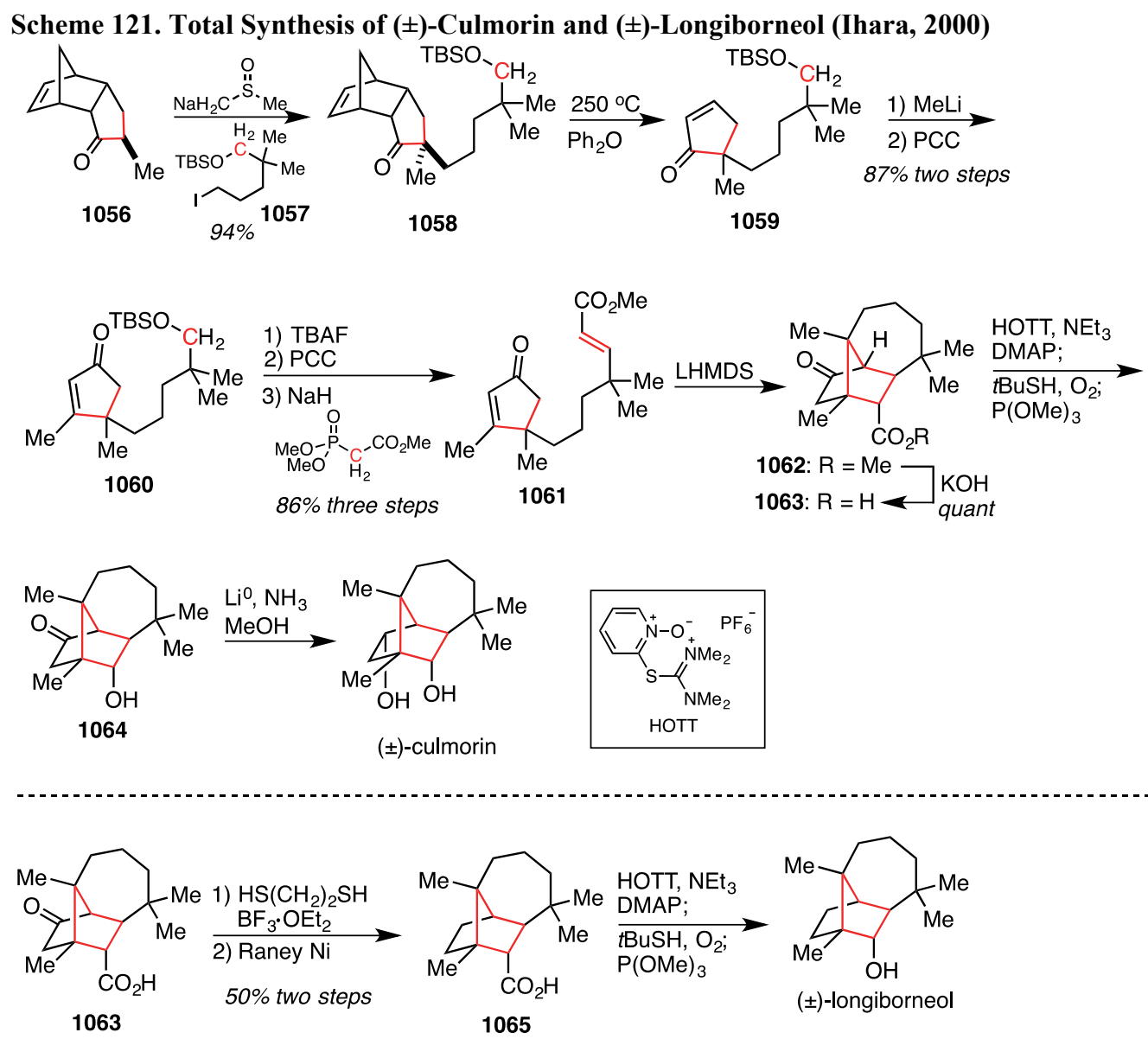

The synthesis of $(+)$-rugulosin by Nicolaou ${ }^{190}$ features two distinct cascade processes based on conjugate addition chemistry that construct two fused fully functionalized cyclopentanes (Scheme 122). The synthesis begins with cyclohexenone $\mathbf{1 0 6 8}$, which was synthesized from the known diacetate $\mathbf{1 0 6 6}$ in four standard steps including an enzymatic desymmetrization. Nitrile $\mathbf{1 0 7 0}^{191}$ was stirred with LHMDS followed by addition of cyclohexenone $\mathbf{1 0 6 8}$ to give anthradihydroquinone 1071. When this material was treated with manganese dioxide (1.5 wt equiv) in $\mathrm{CH}_{2} \mathrm{Cl}_{2}$ intermediate $\mathbf{1 0 7 3}$ was formed. This reactivity has been studied by both Nicolaou and Snider ${ }^{192}$ and mechanistically it could be viewed as sequential conjugate addition reactions or as a hetero-DielsAlder reaction. A tenfold dilution of intermediate 1073 with $\mathrm{CH}_{2} \mathrm{Cl}_{2}$ and treatment with additional managanese dioxide formed 1075. Finally, warming the solution in the presence of triethylamine delivered 1077. The cascade reaction (termed cytoskyrin cascade) involves two sequential Michael reactions to deliver the cage-like structure

${ }^{190}$ Nicolaou, K. C.; Lim, Y. H.; Papageorgiou, C. D.; Piper, J. L. Angew. Chem. Int. Ed. 2005, 44, 7917-7921.

${ }^{191}$ Nitrile 1070 was obtained from benzoic acid derivative 1069 over five steps.

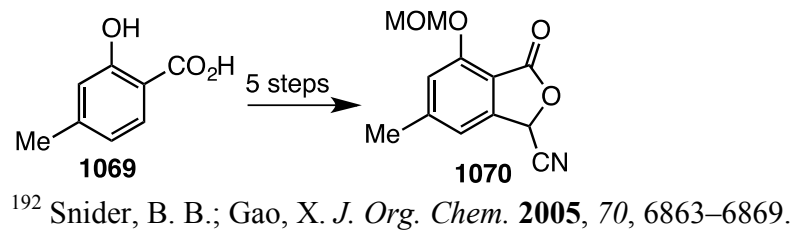


of rugulosin $(\mathbf{1 0 7 4} \rightarrow \mathbf{1 0 7 7})$. Experimentally, it was found that the entire transformation of 1071 to 1077 could occur in a single flask without isolation of intermediates. This single elegant biomimetic transformation builds two fused fully functionalized cyclopentanes with complete control of stereochemical configuration. Acidic removal of the MOM protecting groups completed the synthesis of $(+)$-rugulosin.

Scheme 122. Total Synthesis of (+)-Rugulosin (Nicolaou, 2005)

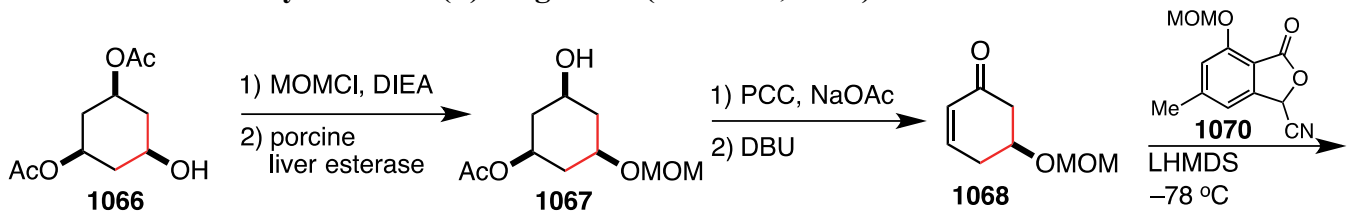

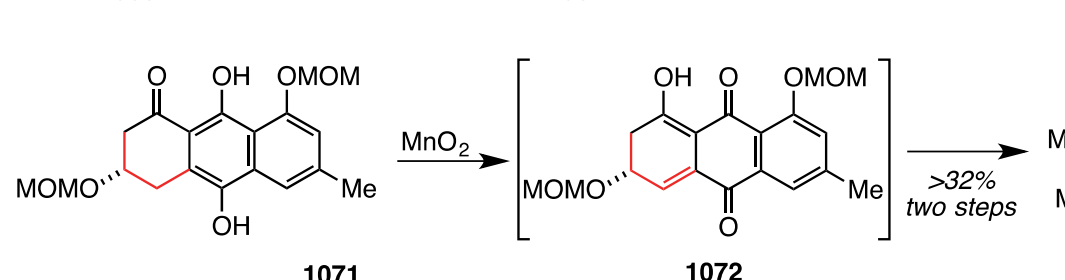<smiles>COCC(=O)OCO</smiles>

1072

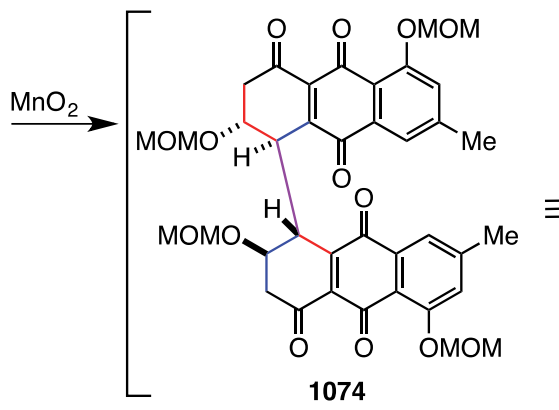

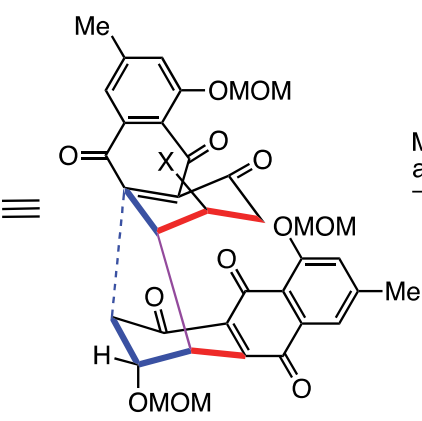

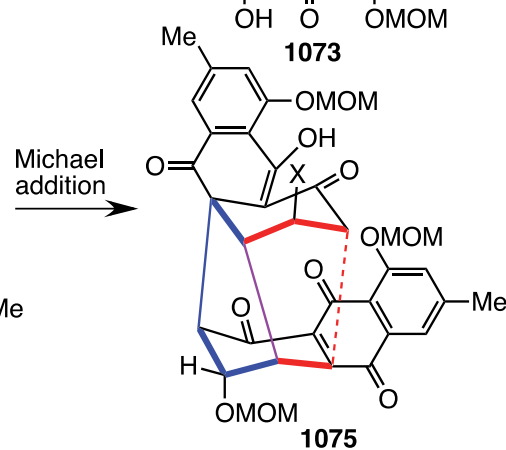

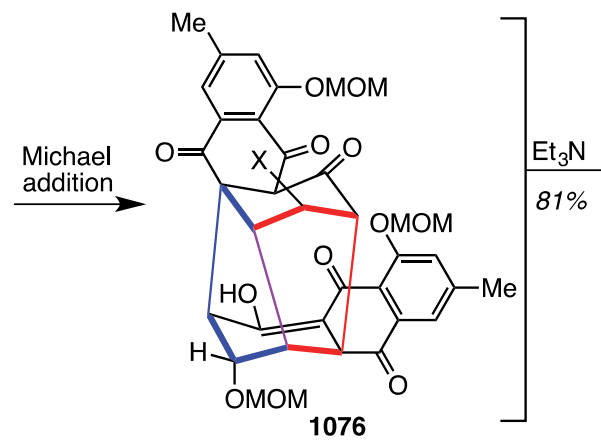
1074; $\mathrm{X}=\mathrm{OMOM}$ 1075

In the asymmetric synthesis of $(+)$-gelsemine by $\operatorname{Qin}^{193}$, the cyclopentane ring is constructed by a Michael addition of a nitrile enolate and an oxindole (Scheme 123). The synthesis begins by conversion of $(R, R)-\mathbf{1 0 7 8}$ into the alkyne $\mathbf{1 0 8 0}$ by reduction, tosylation, and addition of the lithiated acetylide (1079). This material was advanced to cis-olefin 1081 over eight steps. Condensation of the acid and hydroxyl groups delivered oxepinone 1082. The first of two intramolecular Michael additions gave a mixture of inseparable diastereomers in a $66 \%$ yield favoring the desired 1083. Reductive cleavage of the lactone was followed by acetal formation and oxidation of the primary alcohol to give aldehyde 1084. Condensation with $N$-methoxyindole 1085 and subsequent dehydration of the resulting alcohol with thionyl chloride gave a mixture of alkenes $\mathbf{1 0 8 6}$ and 996 in a 1.5:1 ratio. This mixture underwent the second Michael addition, which constructed the fully functionalized cyclopentane yielding $\mathbf{1 0 8 8}$ and $\mathbf{1 0 8 9}$ as a pair of C7 epimers. This transformation successfully synthesized the C20 quaternary stereocenter on the fully functionalized cyclopentane, but led to the undesired $(S)$-configuration at C6. To invert the configuration at C6, the oxindole was deprotonated, and the resulting enolate was treated

${ }^{193}$ Zhou, X.; Xiao, T.; Iwama, Y.; Qin, Y. Angew. Chem. Int. Ed. 2012, 51, 4909-4912. 
with $\mathrm{PhSeCl}$. Oxidative elimination gave an alkene, which was hydrogenated over the Lindlar catalyst to produce two diastereomers favoring the desired $\mathrm{C} 7$ epimer (1090) in a 2:1 ratio. This advanced intermediate contains a fully functionalized cyclopentane, and four additional steps were used to complete the synthesis of $(+)$ gelsemine.

Scheme 123. Total Synthesis of ( \pm )-Gelsemine (Qin, 2012)

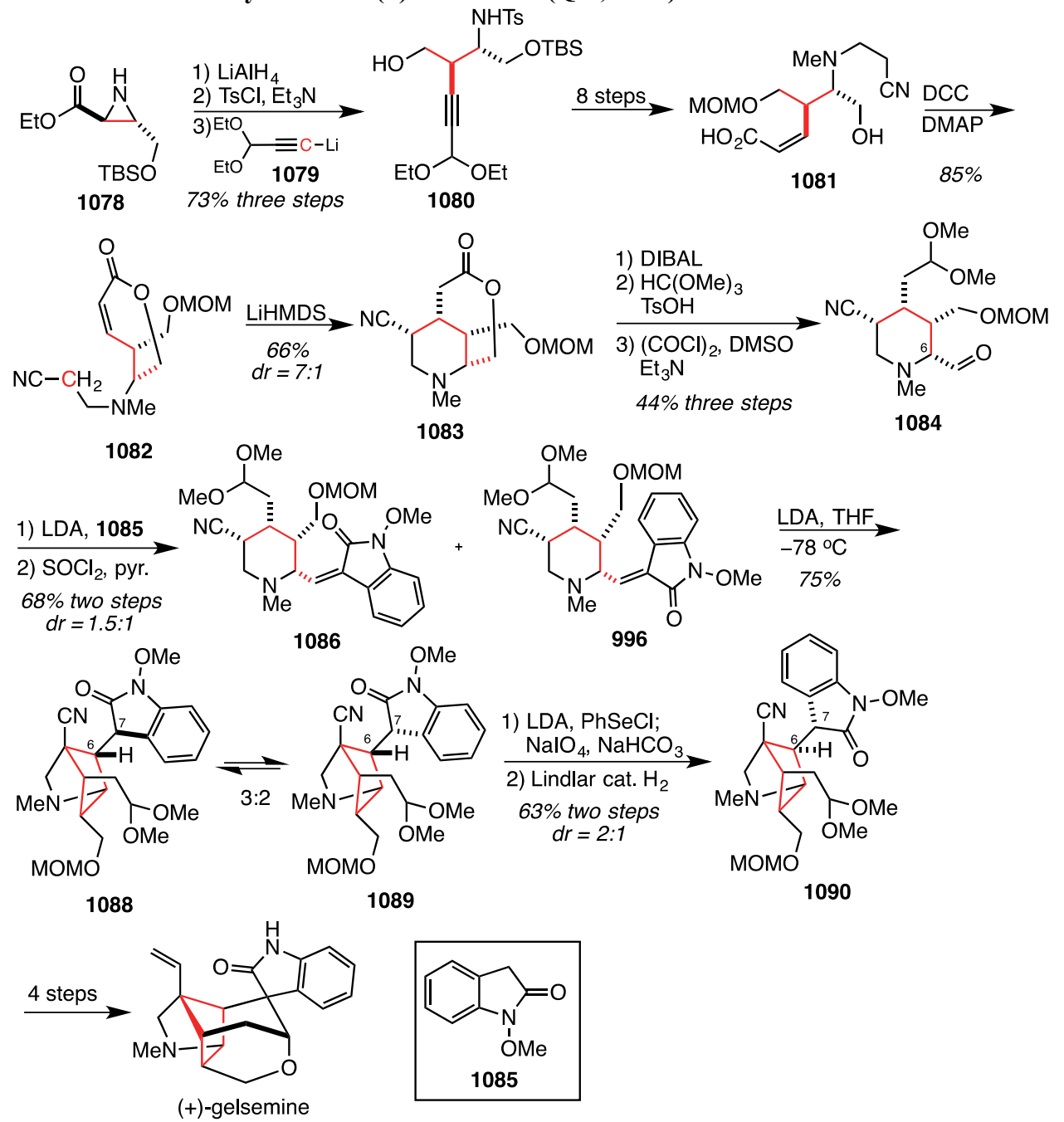

In a formal synthesis of $( \pm)$-gelsemine, Aubé ${ }^{194}$ performed a double conjugate addition to construct a bicyclo [3.2.1] octanone containing a fully functionalized cyclopentane (Scheme 124). In the first step of the synthesis, the dianion of ethyl 3-nitropropionate (1061) was generated with LDA. This was reacted with $p$-benzoquinone dimethyl ketal (963) to afford bicycle 964 as an inconsequential mixture of diastereomers. Treatment of the diastereomeric mixture 964 with DBU led to a single unsaturated ester 1062. Introduction of the spirooxindole was achieved over six steps to give 1063. Ketal removal and Horner-Wadsworth-Emmons reaction with tertbutyl diethylphosphonoacetate (1064) delivered 1065 predominantly as the desired $E$-isomer $(E / Z=3: 1)$. Conjugate addition of methylamine on the less hindered face, alloc-protection, reduction with lithium borohydride, and acetylation furnished 1066. This intermediate has previously been taken to $( \pm)$-gelsemine by Fukuyama. $^{97}$

Scheme 124. Formal Synthesis of $( \pm)$-Gelsemine (Aubé, 2007)

${ }^{194}$ Grecian, S.; Aubé, J. Org. Lett. 2007, 9, 3153-3156. 

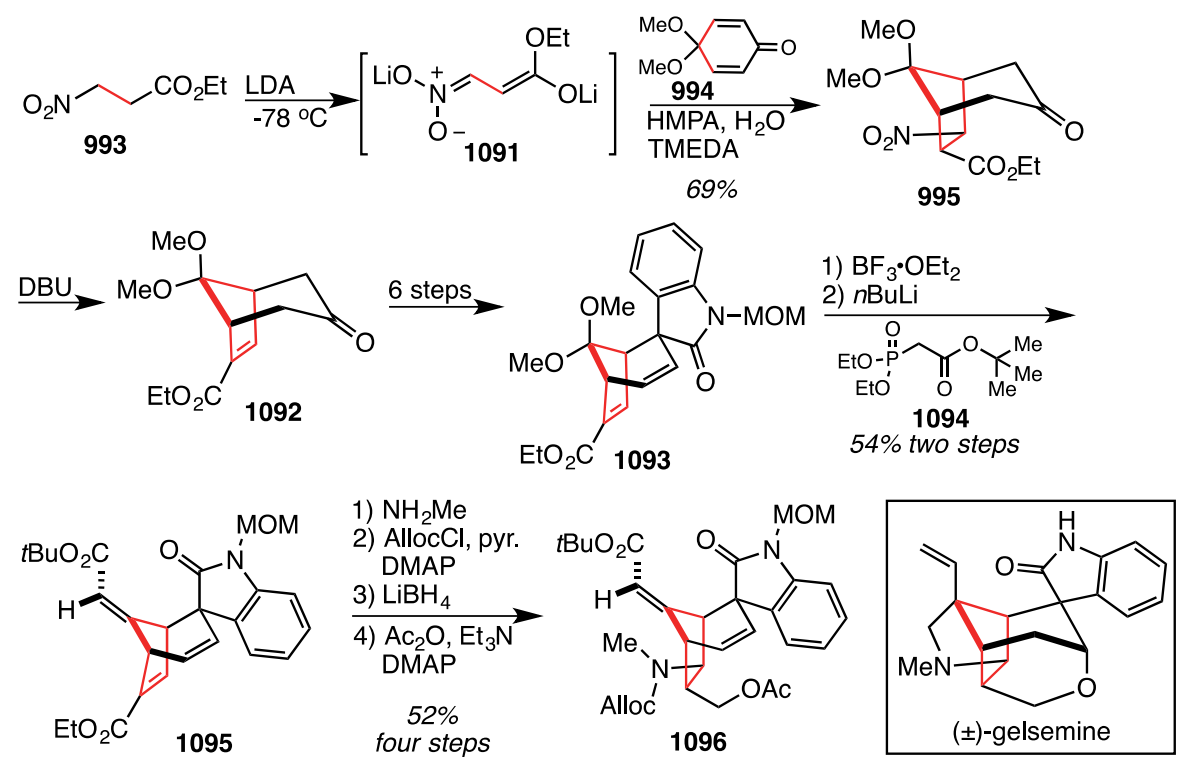

Synthetic efforts towards the hetidine and hetisine diterpenoid natural product frameworks (e.g. kobusine), were reported by Sarpong ${ }^{195}$ (Scheme 125). These skeletons have dense polycyclic structures where the cyclopentane displays four carbon stereocenters, two of which are quaternary. The synthesis begins with alkylation of commercially available $\beta$-ketoester 1097 with known iodoalkyne 1098. Saponification and decarboxylation delivered indanone 1099. Hydride reduction of the ketone and alcohol elimination with PPTS gave indene 1100. Cycloisomerization with gallium(III) iodide provided benzannulated cycloheptadiene 1101. Selective reduction of the disubstituted alkene was performed with diimide. Treatment with ceric ammonium nitrate oxidized the benzylic position to give an enone, which underwent stereoselective hydrogenation furnishing 1102. Allylation gave a mixture of $O$ - and $C$-allylation products; heating the mixture resulted in a Claisen rearrangement simplifying the mixture to $\mathbf{1 1 0 3}$. Simultaneous reduction of the ketone and nitrile was followed by Bocprotection of the resulting amine to give 1104. Treatment of the hydroxyl 1104 with thionyl chloride resulted in chlorination and spontaneous substitution by the tethered carbamate to give 1105. Demethylation with sodium ethanethiolate yielded 1106. Oxidative dearomatization performed with [bis(trifluoroacetoxy)iodo]benzene gave cyclohexadienone 1107. Finally, oxidative cleavage yielded a ketoaldehyde which cyclized via a conjugate addition upon stirring in silica gel to furnish 1108. This advanced intermediate contains many of the structural features of hetidine and hetisine-type alkaloids including a fully functionalized cyclopentane with the appropriate stereochemical configuration.

Scheme 125. Synthetic Studies Towards the Hetidine and Hetisine Skeletons (Sarpong, 2013)

${ }^{195}$ Hamlin, A. M.; Cortez, F. de J.; Lapointe, D.; Sarpong, R. Angew. Chem. Int. Ed. 2013, 52, 4854-4857. 

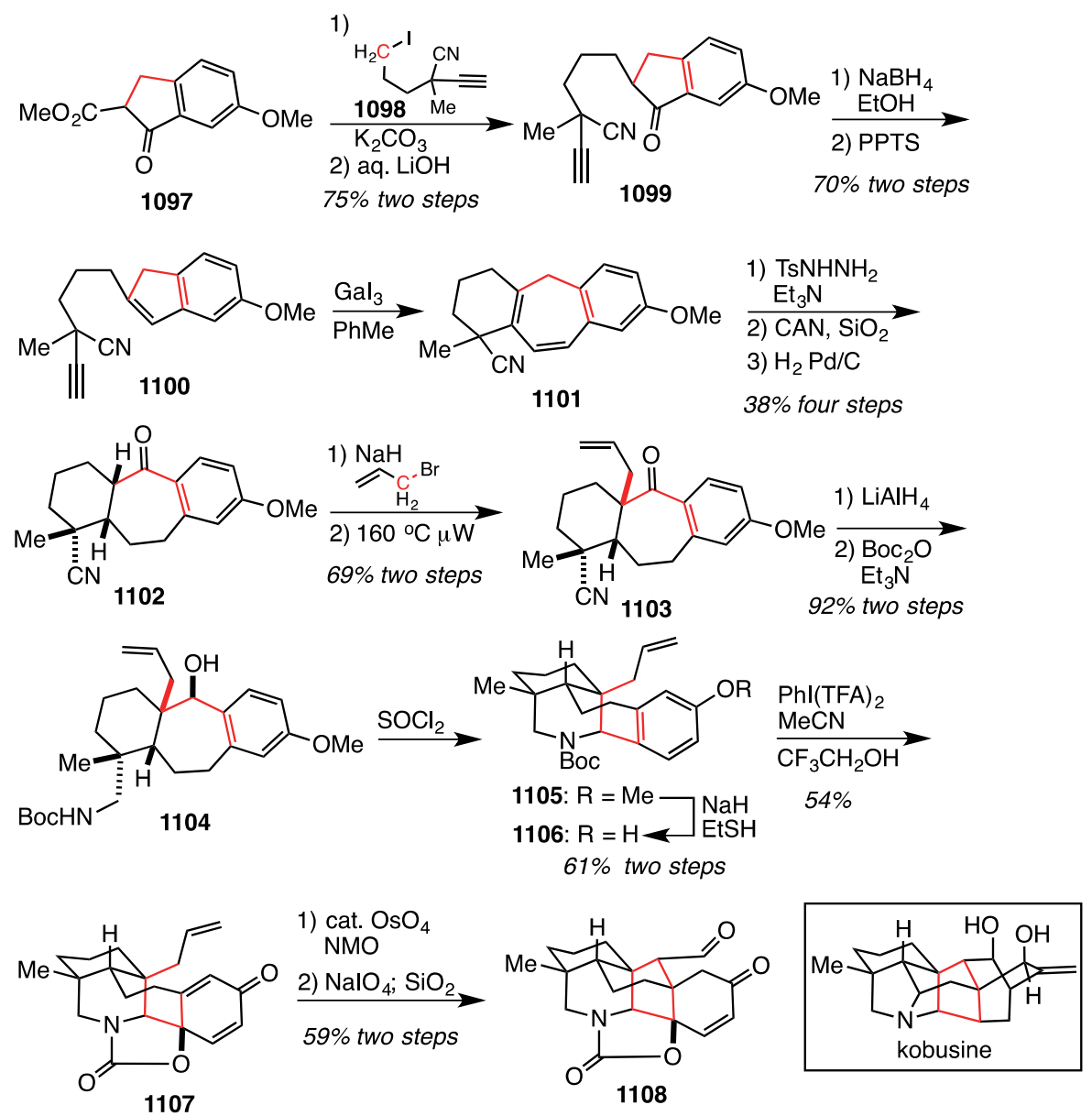

\section{Friedel-Crafts and Related Reactions of Nucleophilic Alkenes}

The intramolecular Friedel-Crafts acylation is a reliable process for the formation of five-membered carbocycles as part of fused polycyclic architectures. The Friedel-Crafts acylation has been used in two distinct strategies for the synthesis of gibberellin natural products; both approaches used the reaction to build the core dodecahydrofluorane substructure. In the first approach (Scheme 126, eq. 1), Lewis acid-promoted cyclization of 1109 led to the formation of the tetrahydrofluorenone 1110. In the second strategy, Brønsted acid mediated cyclization of $\mathbf{1 1 1 1}$ gave tetrahydrofluorene $\mathbf{1 1 1 2}$.

Scheme 126.<smiles>CC1(C)CC=CC(c2ccc(O[N+](=O)[O-])cc2)[C@H]1C(=O)O</smiles>

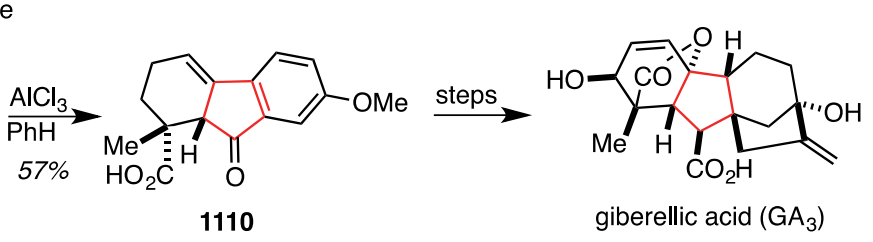<smiles>COC(=O)C1=C(OC)CC=C(OC)C1(Cc1cccc(OC)c1C(=O)OC)OC</smiles>

1111<smiles>COc1ccc2c(c1OC)CC1=C(CC(=O)CC1)C2</smiles>

1112 
The strategic use of the Friedel-Crafts reaction benefits from the wide range of substituted benzenoid starting materials that may be employed in a regioselective bond-forming reaction. As seen in the above examples, the dodecahydrofluorene core of the gibberellins is expeditiously constructed. After the Friedel-Crafts cyclization, the six carbons of the benzene undergo functionalization to reveal the substituted cyclohexane present in the natural product. The products of the Friedel-Crafts cyclization (1110 and 1112) displayed functional handles for further manipulations, and both strategies provided intermediates that could be transformed to the gibberellins. One potential limitation of such Friedel-Crafts reactions in the synthesis of fully functionalized cyclopentanes is the requirement that the product five-membered ring contain (at least) two $\mathrm{sp}^{2}$-hybridized carbon atoms. These carbons must undergo additional transformations en route to the fully saturated ( $\mathrm{sp}^{3}$-hybridized) carbocycle.

Two approaches to the natural product cyclosativene have used a cyclization of a substituted norbornene to build the fully functionalized cyclopentane. Activation of the suitably substituted starting material 1113 leads to an electrophilic intermediate 1114 (Scheme 127). The electrophilic (i.e. carbocationic) carbon is trapped by the nearby alkene with concomitant introduction of a nucleophile. The nucleophile may be tethered to the substrate, or it may be an external reagent. The structure of these cyclization products displays the same tricyclo[2.2.1.0 $0^{2,6}$ hexane core as cyclosativene, and as a result the reactions are a good fit for the topology of this interesting target.

\section{Scheme 127.}

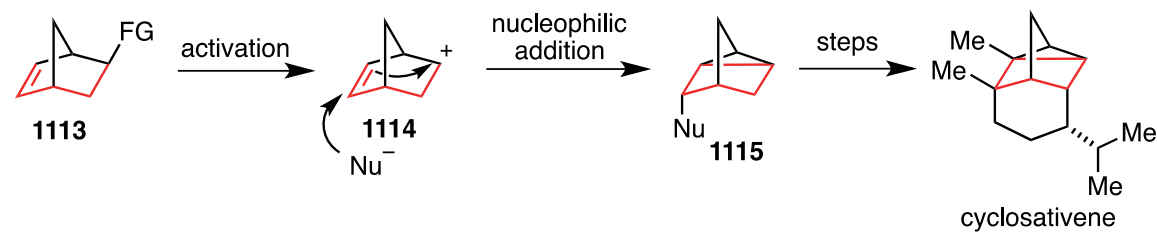

In the progress towards the synthesis of $( \pm)$-gibberelic acid $\left(\mathrm{GA}_{3}\right)$, Nakanishi ${ }^{196}$ constructs a cyclopentane using an intramolecular Friedel-Crafts acylation (Scheme 128). The synthesis commences with a Diels-Alder reaction between diene 1116 and acrylate 1117 to give adduct 1118 as a mixture of endo/exo diastereomers. Hydrolysis of the esters and nitrile was accompanied by a decarboxylation to yield dicarboxylic acid 1119. Heating in acetic anhydride delivered the cyclic anhydride 1109. The intramolecular Friedel-Crafts cyclization occurred upon treatment with $\mathrm{AlCl}_{3}$ in benzene to give cyclopentenone 1110. Epoxidation with peracid and subsequent opening by the tethered carboxylic acid resulted in a lactone 1121. This hydrofluorene derivative was the most advanced intermediate obtained by Nakanishi.

Completion of the synthesis from intermediate $\mathbf{1 1 2 1}$ was accomplished by Yamada $^{197}$ approximately twenty years later. The secondary alcohol was protected as the TMS ether (1122). A Corey-Chaykovsky epoxidation was followed by treatment with boron trifluoroetherate to give an aldehyde on the less hindered face of 1123. Seven additional steps were required to advance 1123 to intermediate $\mathbf{1 1 2 4}$. Cyclohexenone $\mathbf{1 1 2 5}$ was obtained through a dissolving metal reduction of the phenyl ring, acidic hydrolysis of the enol ether and double bond isomerization. A photochemical [2+2] cycloaddition with allene gave tetracyclic adduct $\mathbf{1 1 2 6}$ with a syn ring fusion. This material was advanced over five steps to intermediate 1127. Fluoride removal of the SEM ether, activation as the sulfonate and elimination with DBU gave alkene 1128, which could be advanced over twelve steps to 1129. Iodolactonization of $\mathbf{1 1 2 9}$ installs the oxygen-containing stereocenter on the cyclopentane ring. Removal of the MOM protecting group and elimination of the iodide with DBU delivers $\mathrm{GA}_{3}$.

Scheme 128. Total Synthesis of ( \pm )-Gibberellic Acid (Nakanishi, 1969; Yamada, 1989)

\footnotetext{
${ }^{196}$ Hori, T.; Nakanishi, K. J. Chem. Soc. D, Chem. Commun. 1969, 10, 528-529.
}

${ }^{197}$ Nagaoka, H.; Shimano, M.; Yamada, Y. Tetrahedron Lett. 1989, 30, 971-974. 


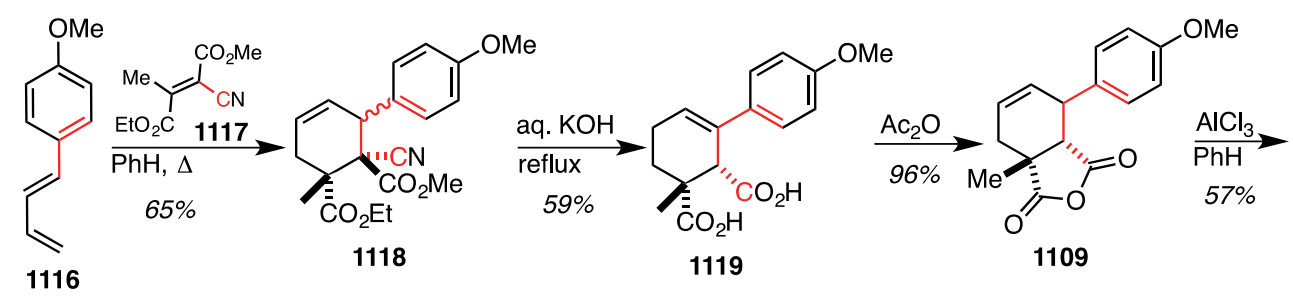

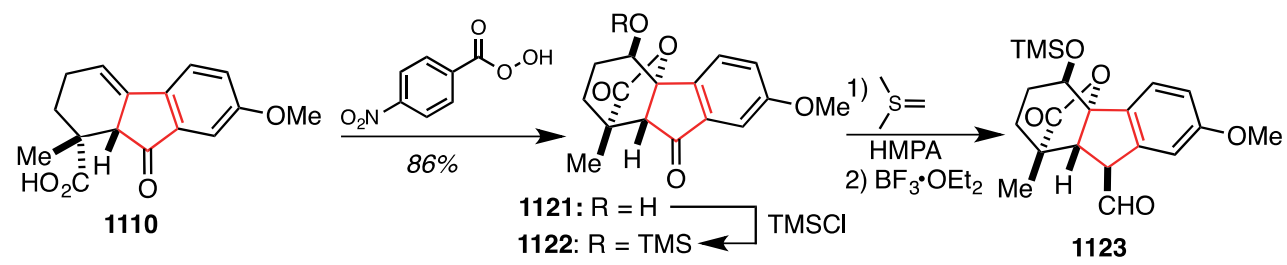<smiles>COC[C@@H](OC)[C@H]1[C@H](COC)c2ccc(OC)cc2[C@@H]1COC</smiles>
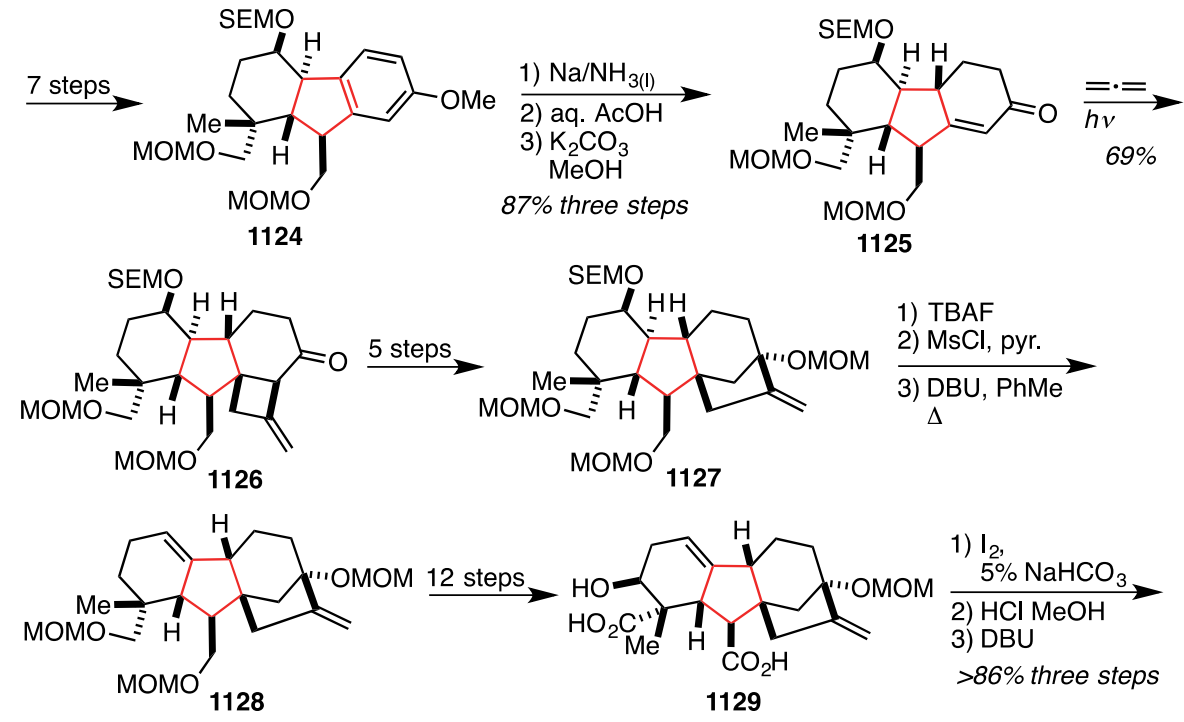

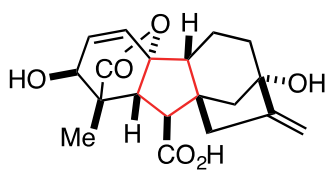

$( \pm)-\mathrm{GA}_{3}$

In a second generation synthesis of $( \pm)$-gibberellic acid by Mander ${ }^{198}$, the key five-membered ring is constructed using an intramolecular Friedel-Crafts acylation (Scheme 129). The synthesis commences with benzyl iodide derivative 1130. Alkylation with enediolate 1131 delivers the hydrofluorene precursor 1111. The Friedel-Crafts reaction was induced with polyphosphoric acid, and a simultaneous decarboxylation furnishes cyclopentadiene 1112. This material was advanced over the next three steps to diazo ketone 1132. Acid-catalyzed cyclization delivered tetracyclic ketone 1133. Standard functional group transformations over the next three steps delivered 1134. Carboxylation of the benzylic position was accomplished by first deprotonation followed by stirring in a carbon dioxide suspension in ether. The configuration of the carboxy bearing benzylic stereocenter was undesired for the synthesis of gibberellic acid, and would require later epimerization. Hydrogenation of the cyclohexene gives the $c i s$-fused ring system in $\mathbf{1 1 3 5}$. This material was advanced four steps to cyclohexadiene 1136. The methyl ester was converted to the corresponding carboxylic acid under nucleophilic conditions, and bromolactonization gave cyclized product 1137. This unstable intermediate was immediately subjected to chromium-based reduction conditions, which gave 1138. Epimerization of the benzylic carboxylate was performed in DBU to correct the stereochemistry for the natural product. A two-step transesterification delivered compound 1139 which had previously been taken to gibberellic acid by Mander. ${ }^{109}$

${ }^{198}$ Hook, J. M.; Mander, L. N.; Urech, R. J. Org. Chem. 1984, 49, 3250-3260. For related studies, see: Hook, J. M.; Mander, L. N.; Urech, R. J. Am. Chem. Soc. 1980, 102, 6628-6629. 
Scheme 129. Total Synthesis of ( \pm )-Gibberellic Acid (Mander, 1975)<smiles>COC(=O)c1cc(COC(=O)c2c(CI)cccc2OC)ccc1OC</smiles><smiles>COC(=O)c1c(CC2(OC)C=C(OC)CC=C2OC)cccc1OC</smiles><smiles>COc1ccc2c(c1C(C)=O)CC1=C(CCC(=O)C1)C2</smiles>

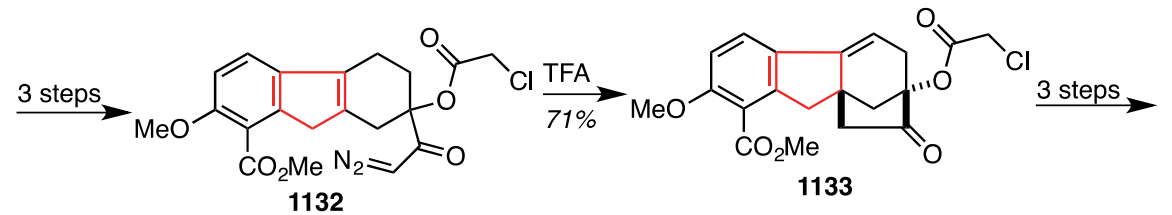

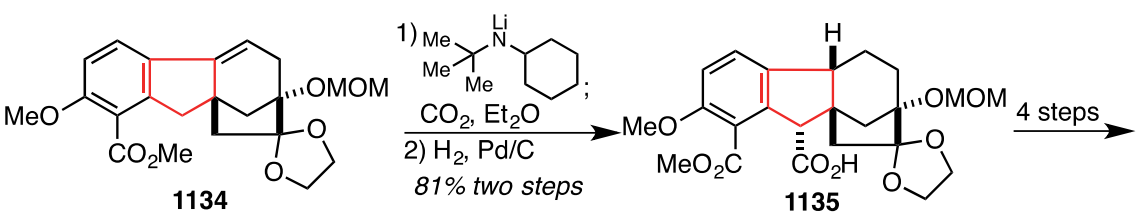

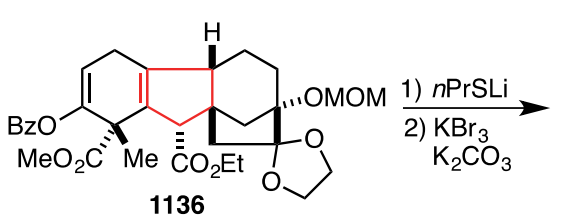

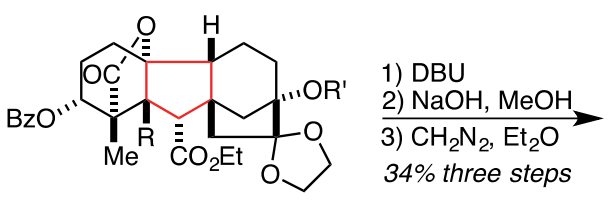
1137: $\mathrm{R}=\mathrm{Br}, \mathrm{R}^{\prime}=\mathrm{MOM} \longrightarrow \mathrm{Cr}(\mathrm{OAc})_{2}$ 1138: $\mathrm{R}, \mathrm{R}^{\prime}=\mathrm{H}$

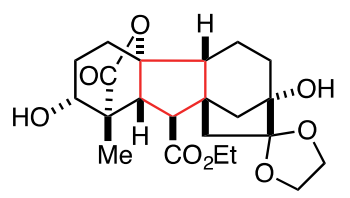
1139

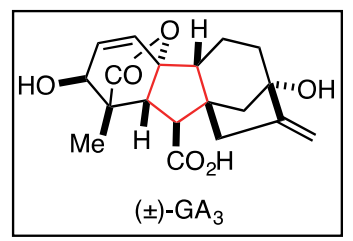

1112 


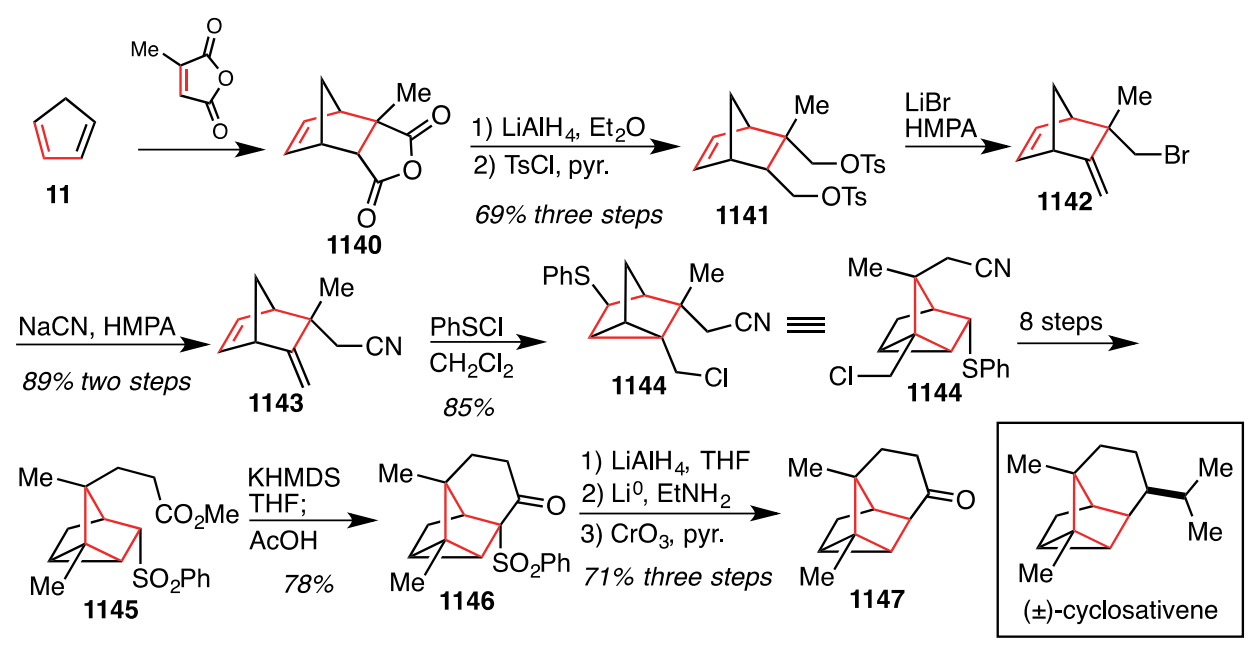

Synthesis of $( \pm)$-cyclosativene was completed by Baldwin ${ }^{201}$ wherein a fully functionalized cyclopentane was constructed with an intramolecular cyclization of a homoallylic carbocation (Scheme 131). The norbornene scaffold was assembled with a Diels-Alder cycloaddition of propynal and 2,3-dimethylcyclopentadiene (1149; generated in situ from 1148) to give 1150. Conjugate addition of allyl alcohol to the unpurified aldehyde delivered ether 1151. Aldehyde reduction and bromination of the resulting alcohol was followed by displacement with lithium acetylide to give 1152. Ether cleavage and subsequent activation of the resulting alcohol as the tosylate gave cyclization precursor 1153. Solvolysis of $\mathbf{1 1 5 3}$ led to a carbocation, which was trapped by the alkene giving the key five-membered ring; the resulting carbocation was intercepted by the tethered alkyne leading to intermediate 1154. Hydrolytic workup gave ketone 1155, which contains the fully functionalized cyclopentane of cyclosativene. The natural product was completed in an additional three steps.

Scheme 131. Total Synthesis of $( \pm)$-Cyclosativene (Baldwin, 1980)
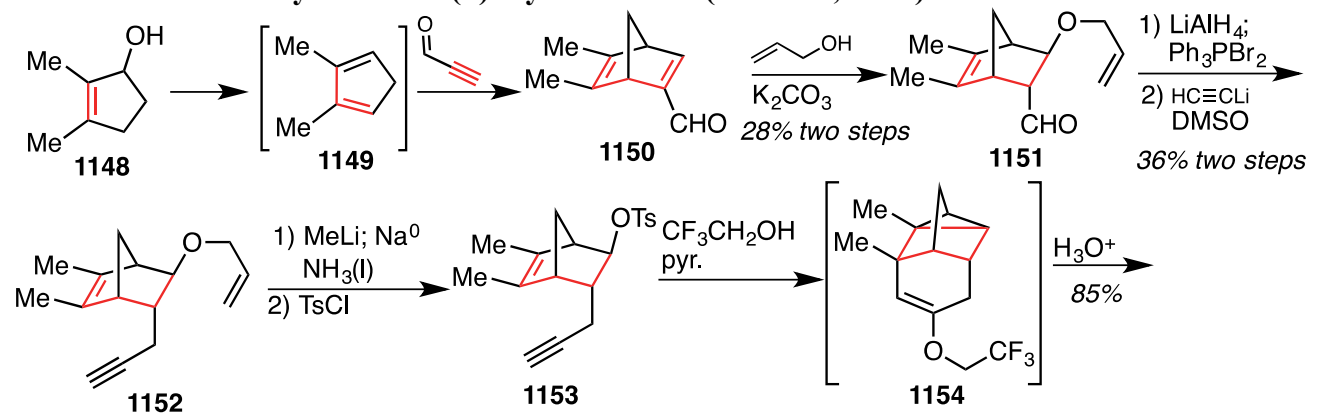

1152

1153

1154

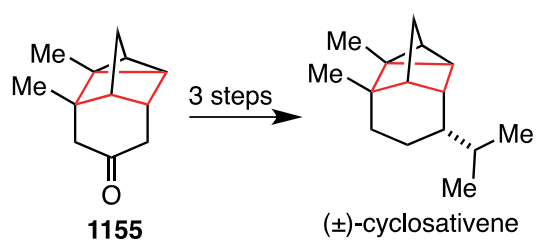

In the synthesis of $( \pm)$-nominine by Muratake and Natsume ${ }^{202}$, a Lewis acid-catalyzed Prins reaction ${ }^{203}$ is used to form a fully functionalized cyclopentane (Scheme 132). The route commences with a palladium-catalyzed intramolecular $\alpha$-arylation of $\mathbf{1 1 5 6}$ to give $\mathbf{1 1 5 7}$ as a mixture of diastereomers favoring the undesired cis isomer (4.2:1). Acetal formation under standard conditions gave 1158. Under the conditions of the acetal formation, an epimerization occurred which decreased the cis:trans ratio to 2:1. The desired trans isomer was isolated and the cis isomer was recycled by re-establishing the equilibrium. Birch reduction of the arene gives predominantly the

${ }^{201}$ Baldwin, S. W.; Tomesch, J. C. Tetrahedron Lett. 1975, 16, 1055-1058.

${ }^{202}$ (a) Muratake, H.; Natsume, M. Tetrahedron Lett. 2002, 43, 2913-2917. (b) Muratake, H.; Natsume, M. Angew. Chem. Int. Ed. 2004, 43, 4646-4649.

${ }^{203}$ The authors describe this transformation as an acetal-ene reaction; however, we describe it as a Prins cyclization. 
undesired $\beta, \gamma$-enone 1159, but this could be isomerized with $\mathrm{NaOMe}$ to the desired $\alpha, \beta$-enone 1160. Luche reduction of 1160 gives allyl alcohol 1161 as the sole isomer. Eschenmoser-Claisen rearrangement of the allylic alcohol was followed by amide reduction and protection to give $\mathbf{1 1 6 2}$. Treatment of $\mathbf{1 1 6 2}$ with Lewis acid initiates opening of the acetal to give an oxocarbenium ion, which is intercepted by the nearby alkene to form a fully functionalized cyclopentane (1163). This material was advanced over 17 steps to intermediate 1164. Removal of the $\mathrm{Cbz}$ protecting group was followed by chlorination of the alcohol and subsequent cyclization to furnish $O$-acetylnominine. Acetate cleavage with base delivered the natural product.

\section{Scheme 132. Total Synthesis of $( \pm)$-Nominine (Muratake and Natsume, 2004)}
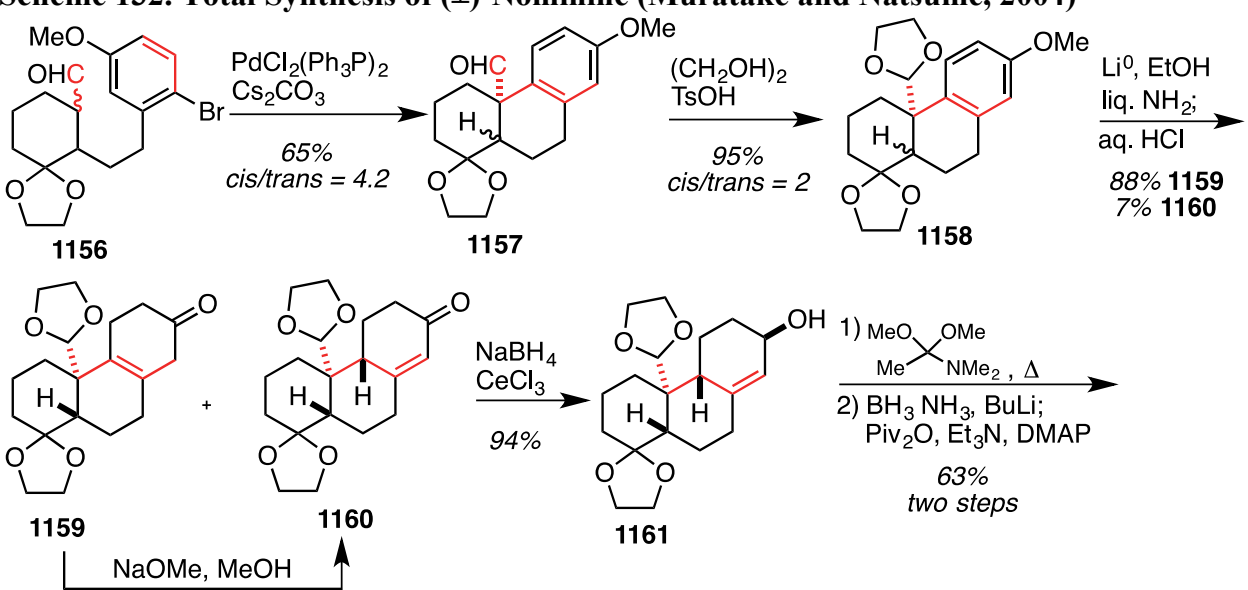

$55 \%$
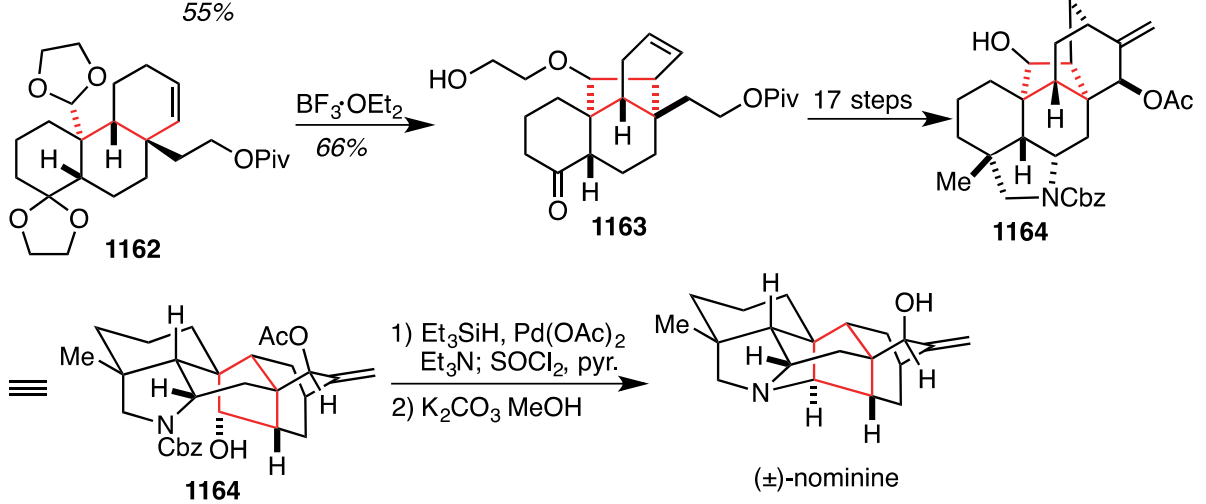

$( \pm)$-nominine

\section{Radical Reactions}

Radical cyclizations represent versatile $\mathrm{C}-\mathrm{C}$ bond formations that can lead to cyclopentane formation with concomitant formation of stereogenic carbon atoms, including quaternary carbon stereocenters. Such reactions are tolerant of steric crowding, and they are commonly used to form stereodiad strucutral motifs as part of larger arrays of contiguous stereocenters.

Typically, a molecule with a 1,5-relationship between $\mathrm{sp}^{2}$-hybridized carbons will undergo an intramolecular pinacol-type coupling to give a substituted cyclopentane (Scheme 133). For example, a 1,5-ketoaldehyde (1165) reacts with $\mathrm{SmI}_{2}$ to give ketyl radical 1166. The ketyl radical undergoes 5-exo-trig cyclization, followed by single electron reduction and protonation to give the diol 1167. Yields are good and the diastereomeric ratio can be quite high.

\section{Scheme 133.}




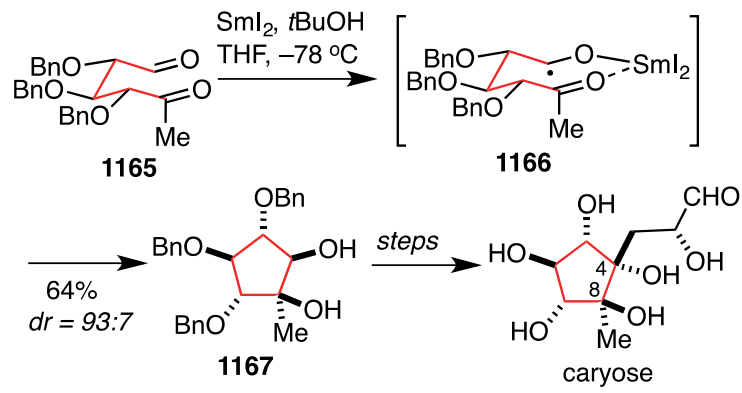

In general for such radical cyclizations, the stereochemical outcome at the forming stereocenters is rationalized by considering the most stable chair-like transition state that places the resident substituents in an equatorial position. Reductive cyclization gives the syn-1,2-diol. The preference for the formation of the syn diol in these substituted cases, and in other unsubstituted dialdehydes (e.g. adipaldehyde), has been explained by a chelate with samarium. ${ }^{204}$ The adjacent alkoxy substituent forces the emerging diol to be anti with respect to the alkoxide. ${ }^{205}$ However, not all cases follow this trend and other factors may complicate this stereochemical model (see discussions of individual syntheses below).

The starting material is not limited to ketoaldehdyes, and a variety of functional group patterns participate in this type of reaction, provided the two $\mathrm{sp}^{2}$-hybridized carbons exist in a 1,5-reationship. For example, 1,5-ketoesters, 1,5-ketonitriles, 1,5-ketooxime ethers, and $\delta, \varepsilon$-unsaturated aldehydes all perform well in this type of reaction.

Such flexibility in starting material allows for strategic placement of a variety of functionality (alcohols, amines, ketones, alkenes, etc.) in the product for subsequent transformations.

Particularly powerful examples of radical cyclizations in the context of this review form $\sigma$-bonds between a fully substituted stereocenter and a heteroatom-bearing stereocenter (e.g. C4-C8 in caryose, Scheme 133). The diastereoselectivity is often high, and this method has been used in elegant syntheses of tertiary alcohol stereocenters.

A second theme in this section involves homolysis of a sigma bond to form a carbon-centered radical that undergoes 5-exo-trig cyclization of a tethered alkene (Scheme 134, eq. 1). A suitable starting material 1168 undergoes bond homolysis to give radical 1169. Cyclization of radical 1169 over a tethered alkene gives a new radical (1170) that abstracts a hydrogen atom to give the product $\mathbf{1 1 7 1}$. The initial $\sigma$-bond homolysis varies widely, and in the context of this review $\mathrm{C}-\mathrm{S}, \mathrm{C}-\mathrm{Hg}, \mathrm{C}-\mathrm{O}$, and $\mathrm{C}-\mathrm{H}$ bonds have been cleaved to provide reactive radical intermediates. This strategy is exemplified by a key cyclization in the Hart synthesis of $( \pm$ )-gelsemine (eq. 2). Homolysis of $N, S$-acetal 1172 gave an $\alpha$-amino radical that cyclized to give intermediate 1173.

Scheme 134.
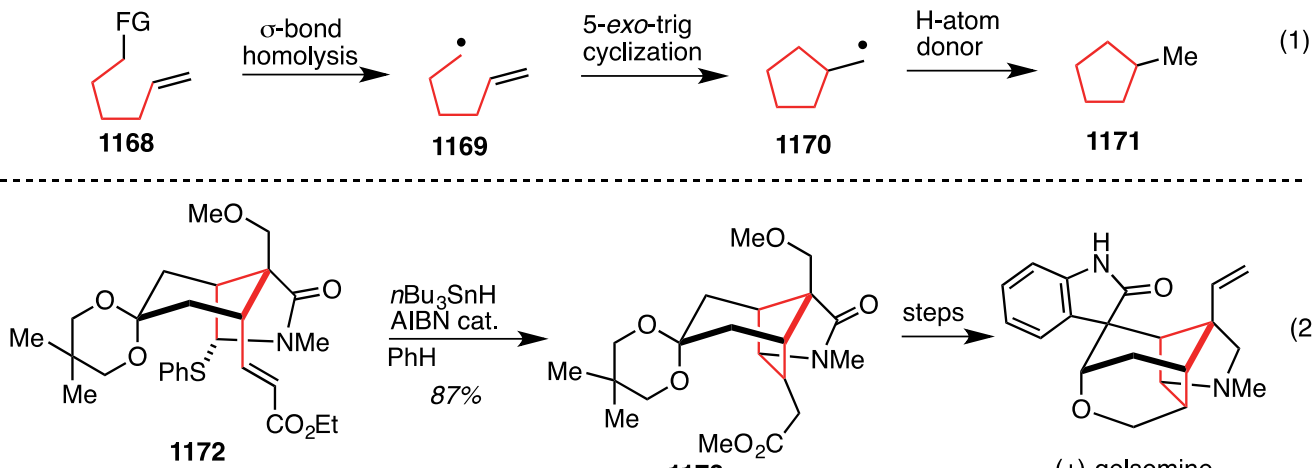

$( \pm)$-gelsemine

Radical reactions are well-suited to cascade processes that create multiple bonds. This review contains three unique reactions that deviate from the typical themes discussed above and create multiple new rings in a single

${ }^{204}$ Chiara, J. L.; Cabri, W.; Hanessian, S. Tetrahedron Lett. 1991, 32, 1125-1128.

${ }^{205}$ For an excellent review of the pinacol coupling, see: Chatterjee, A.; Joshi, N. N. Tetrahedron 2006, 62, 1213712158. 
step (Scheme 135). Reisman reported a reductive cyclization of substrate 1174 to give the molecular architecture of maoecrystal V (eq. 1). Second, Overman reported an elegant radical addition-cyclization-fragmentation cascade reaction between 1176 and radical acceptor 1177 (eq. 2). Finally, Little reported a fragmentationcyclization of 1180 to give triquinane 1181. Intermediate 1181 was advanced to complete a formal synthesis of $\operatorname{coriolin}^{129}$ (eq. 3).

\section{Scheme 135.}
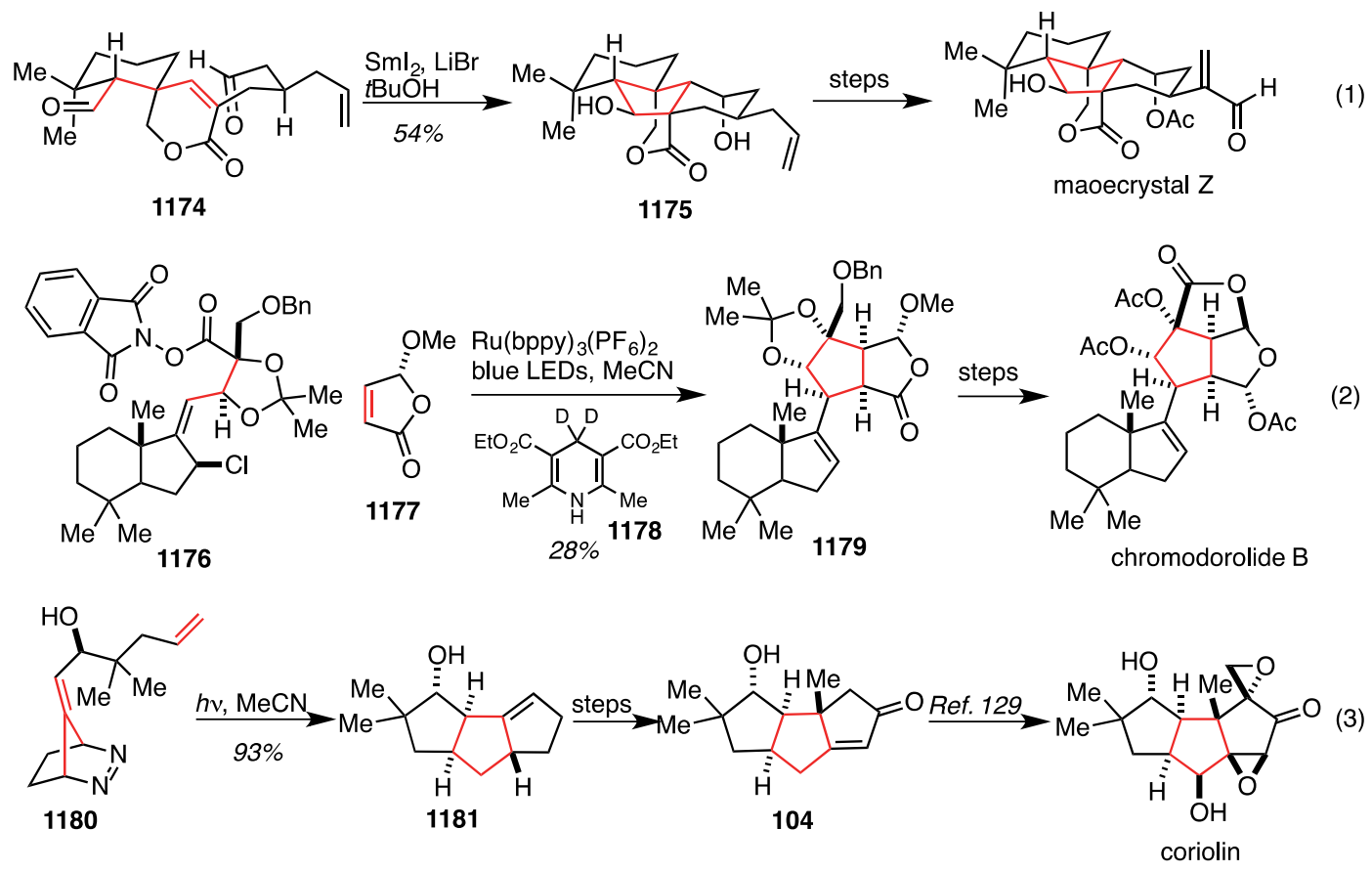

\subsection{Pinacol-type Reactions of Carbon-Heteroatom Double Bonds}

In the synthesis of $( \pm)$-rocaglamide by Taylor ${ }^{206}$ the fully functionalized cyclopentane was constructed with a radical-mediated pinacol coupling (Scheme 136). The route commences with alkylation of benzofuranone 1183 with iodide $\mathbf{1 1 8 4}$ under standard conditions to give $\mathbf{1 1 8 5}$ as a 1:1 mixture of diastereomers. Hydrolysis of the dithiane produced aldehyde 1186. Upon treatment with samarium iodide, the pinacol coupling occurred delivering 1187 as a 1:1 mixture of diastereomers (epimeric at C3). Coordination of samarium between the oxygens of the ketoaldehyde during the formation of the ketyl radical is thought to result in the cis-relationship in diol 1187. However, the target rocaglamide bears an anti-diol relationship, so an inversion of the secondary alcohol would ultimately be required. A Swern oxidation delivered ketone 1188. Conversion of the ketone to the $\beta$-ketoester 1189 was accomplished over three steps following the conditions of Kraus ${ }^{207}$ discussed above. Ester 1189 was converted to the corresponding amide and a stereoselective ketone reduction was directed by the vicinal hydroxyl group to complete $( \pm)$-rocaglamide.

Scheme 136. Total Synthesis of $( \pm)$-Rocaglamide (Taylor, 1991)

${ }^{206}$ Davey, A. E.; Schaeffer, M. J.; Taylor, R. J. K. J. Chem. Soc., Chem. Commun. 1991, 1137-1139. 


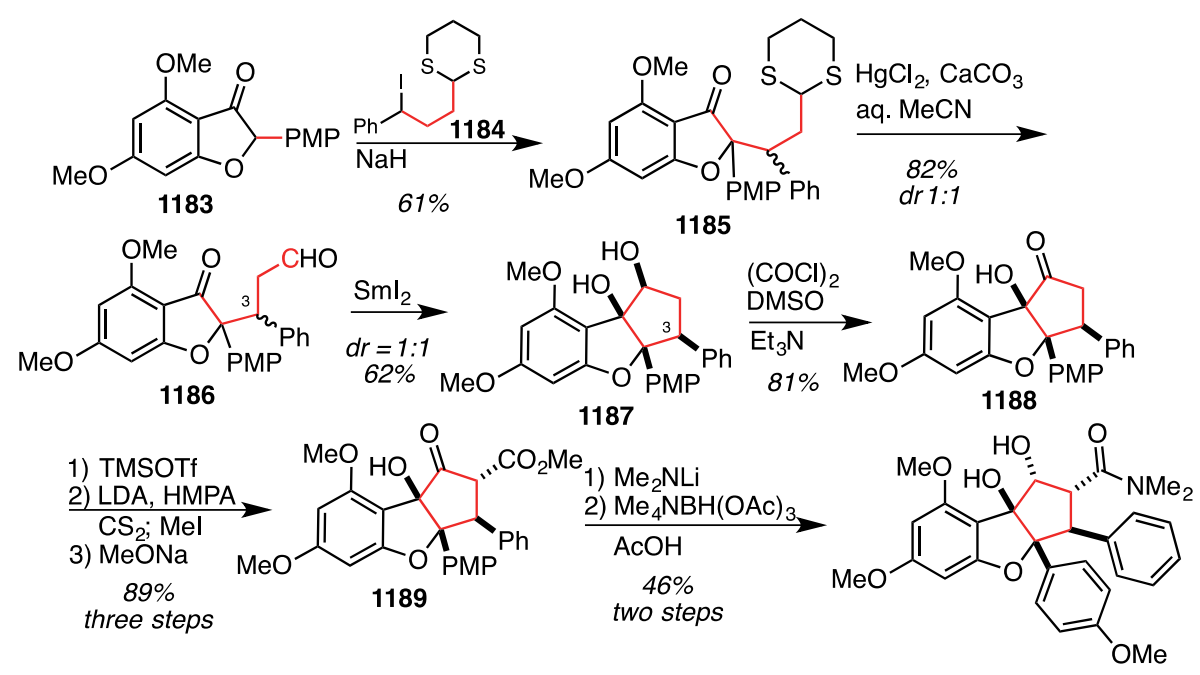

(士)-rocaglamide

In the Qin ${ }^{77}$ synthesis of $( \pm)$-rocaglamide, a radical cyclization was employed for constructing the fully functionalized cyclopentane (Scheme 137). The synthesis began with a Michael addition of benzofuranone 1190 with $\alpha$-methoxycarbonylcinnamate (1191) to furnish the desired intermediate 1192 in modest yield and modest dr. The radical cyclization with samarium metal and 1,2-diiodoethane delivered cyclopentanone 1193, which is in equilibrium with its enol tautomeric form (1194). The radical cyclization gave a single diastereomer of $\mathbf{1 1 9 4}$ resulting from the preferential formation of the cis-fused bicycle. Amidation of the mixture of $\mathbf{1 1 9 3}$ and $\mathbf{1 1 9 4}$ was performed with lithium dimethylamide, and the product $\mathbf{1 1 9 5}$ was formed as a single diastereomer in which the enol tautomer was not observed. It was shown that this diastereomer is the thermodynamic product of the reaction. Finally, ketone reduction proceeds with internal delivery of hydride directed by the vicinal hydroxyl group to give the desired diasteromer completing the synthesis of $( \pm)$-rocaglamide.

\section{Scheme 137. Total Synthesis of $( \pm)$-Rocaglamide (Qin, 2008)}
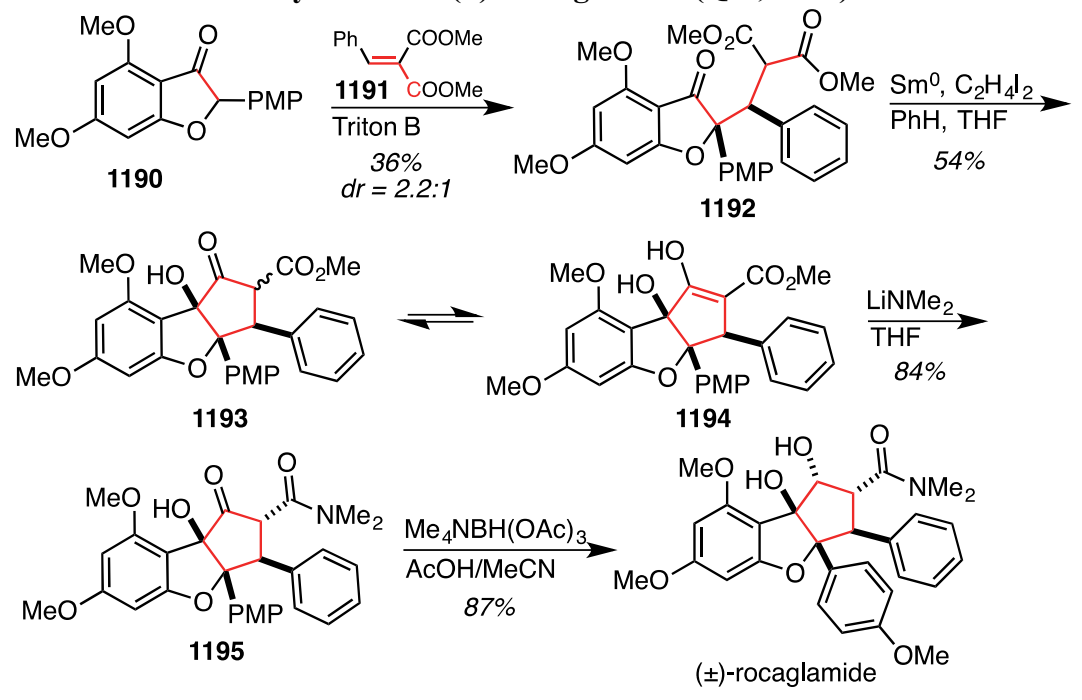

In the synthetic efforts towards rocaglamide, $\mathrm{Kraus}^{207}$ constructs a functionalized cyclopentanone using a samarium-mediated cyclization of a ketonitrile (Scheme 138). Conjugate addition of ketone 1196 to acrylonitrile delivered $\mathbf{1 1 9 7}$ as a racemic mixture. Treatment with samarium iodide induces a radical cyclization exclusively furnishing the cis ring fusion in ketone 1198. This intermediate was converted to the corresponding acyl cyclopentenone 1199 over three steps. Conjugate addition of phenyl cuprate was expected to occur from the convex face to deliver a syn relationship of the two aromatic substituents. However, addition of the

${ }^{207}$ Kraus, G. A.; Sy, J. O. J. Org. Chem. 1989, 54, 77-83. 
organometallic occurred away from the $p$-methoxyphenyl substituent to deliver 1200. Amide formation and ketone reduction with sodium borohydride delivered a fully functionalized cyclopentane 1201. Unfortunately, it was discovered that this intermediate did not display the desired relative stereochemistry of rocaglamide when compared with an authentic sample of the natural product.

Scheme 138. Synthetic Studies Towards Rocaglamide (Kraus, 1989)

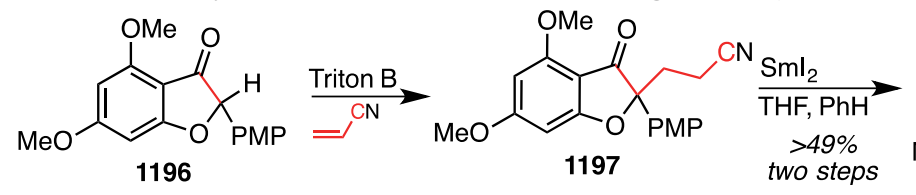<smiles>COc1cc(OC)c2c(c1)OC1([18OH])CCC(=O)C21O</smiles>

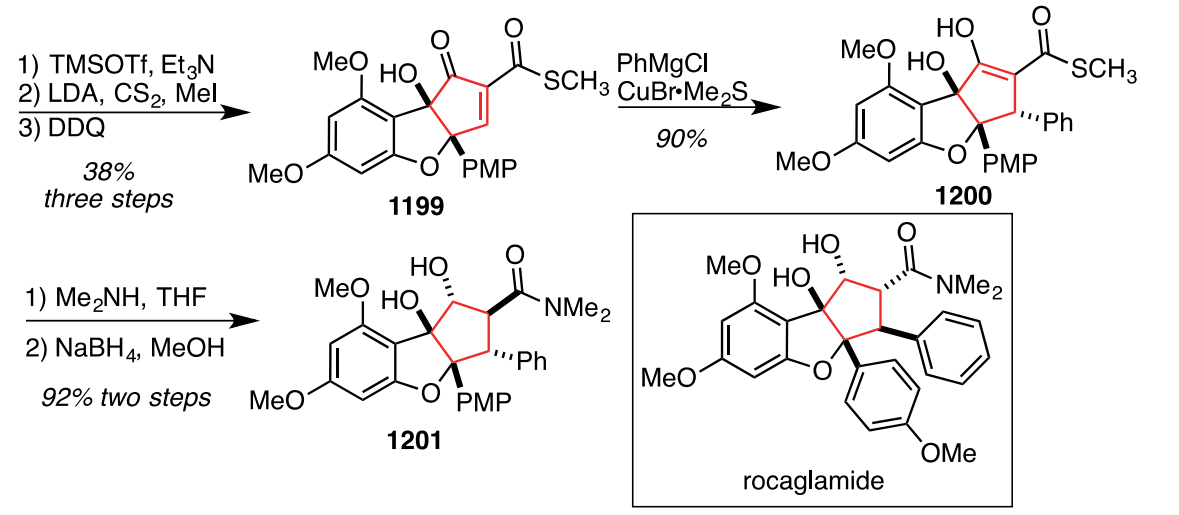

The synthesis of the unusual monosaccharide $(+)$-caryose was completed by Iadonisi, ${ }^{208}$ and it used a radical cyclization of a ketoaldehyde to construct the fully functionalized cyclopentane (Scheme 139). The route begins with 1202, a compound synthesized from D-xylose in five steps. Swern oxidation yields the ketoaldehyde cyclization precursor 1203. Exposure to samarium iodide promotes the expected cyclization to give $\mathbf{1 2 0 4}$ as the major product in a 23:1 diastereomer ratio. As expected, the syn-diol diastereomer is formed (trans with respect to the vicinal benzyloxy substituents) likely as a result of a samarium chelate during the cyclization. ${ }^{204}$ At this stage, a fully functionalized cyclopentane has been synthesized, but the completion of caryose requires further functionalization. The secondary alcohol was oxidized giving ketone 1205. Addition of allyltrimethylsilane and $\mathrm{TiCl}_{4}$ gave homoallylic alcohol 1206 with good selectivity. The authors hypothesize that the stereoselectivity of the addition can be attributed to complexation of the reagents to the adjacent hydroxyl and internal syn-delivery of the allyl group. The fully functionalized cyclopentane now displays all the appropriate stereocenters for the natural product $(+)$-caryose. The natural product was completed in an additional four steps.

Scheme 139. Total Synthesis of (+)-Caryose (Iadonisi, 1997)

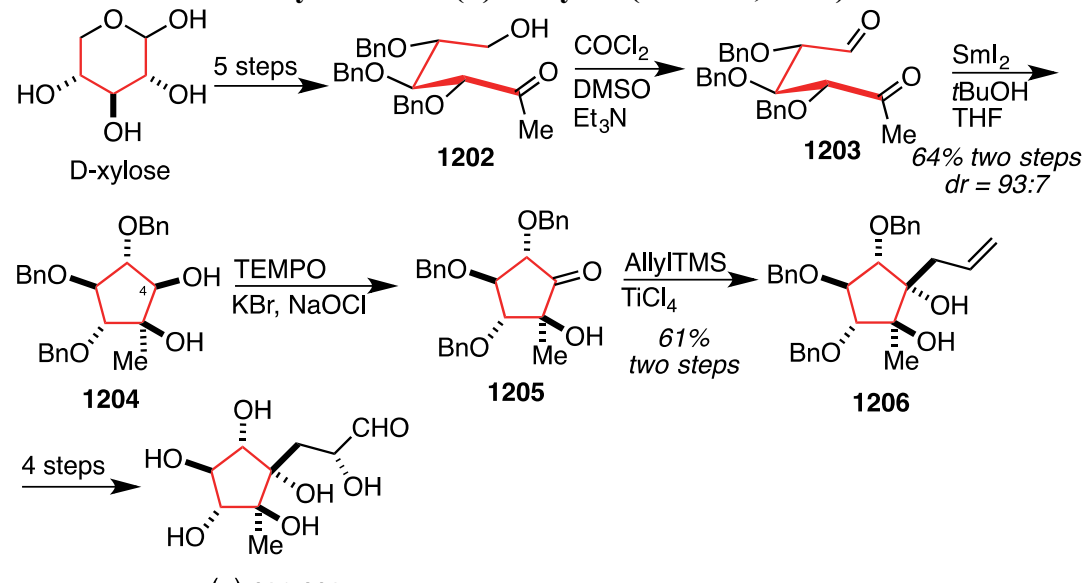

$(+)$-caryose

\footnotetext{
${ }^{208}$ Adinolfi, M.; Barone, G.; Iadonisi, A.; Mangoni, L.; Manna, R. Tetrahedron 1997, 53, 11767-11780.
} 
In the synthesis of $(+)$-trehazolin by Giese $\mathrm{e}^{209}$, a pinacol-type coupling using samarium iodide was used to synthesize the fully functionalized cyclopentane (Scheme 140). Intermediate 1207 was prepared in five steps from D-glucose using standard synthetic transformations. Ring opening of the pyranose with methoxyamine hydrochloride delivers an oxime, and oxidation gives 1208. Radical cyclization of keto-oxime 1208 occurs stereoselectively to form the cis-amino alcohol 1211 as a single diastereomer. In a departure from the stereochemical model invoked in the samarium-chelated pinacol coupling of dicarbonyls, experiments by Giese with 1208 and congeners suggested that interactions between tether substitutents can favor either syn or anti products. The cyclic acetal protecting group in $\mathbf{1 2 0 8}$ was designed to favor conformation 1209, which has a syn co-planar relationship of the ketone and oxime double bonds. Reduction of $\mathbf{1 2 0 9}$ gives radical anion 1210, which leads to formation of the C5,C1 syn amino alcohol 1211. Cyclization product 1211 had the desired configuration of the tertiary alcohol stereocenter, but the amine-bearing stereocenter required stereochemical inversion.

Interestingly, some substrates gave $\mathrm{C} 5, \mathrm{C} 1$ trans-configured amino alcohol products; however, no substrates were found that gave the appropriate trans,trans-diastereomer with respect to $\mathrm{C} 5, \mathrm{C} 1$ and $\mathrm{C} 2$. Although not commented upon by the authors, all cyclizations led to formation of the trans relationship between the emerging amine-bearing stereocenter at $\mathrm{C} 1$ and the resident alkoxy substituent at $\mathrm{C} 2{ }^{210}$ The inversion of the $\mathrm{C} 1$ stereocenter began with ester protection of the tertiary alcohol and oxidation of the hydroxylamine ether to the oxime ether 1212. The acetate was removed, and stereoselective reduction of the oxime ether delivered 1213. Selectivity of the hydride addition may be explained by complexation of the $\mathrm{LiAlH}_{4}$ by the adjacent hydroxyl group promoting delivery from the convex face of the bicycle. The fully functionalized cyclopentane now bears all of the stereocenters contained in $(+)$-trehazolin, and the natural product was completed in an additional four steps.

Scheme 140. Total Synthesis of (+)-Trehazolin (Giese, 1998)
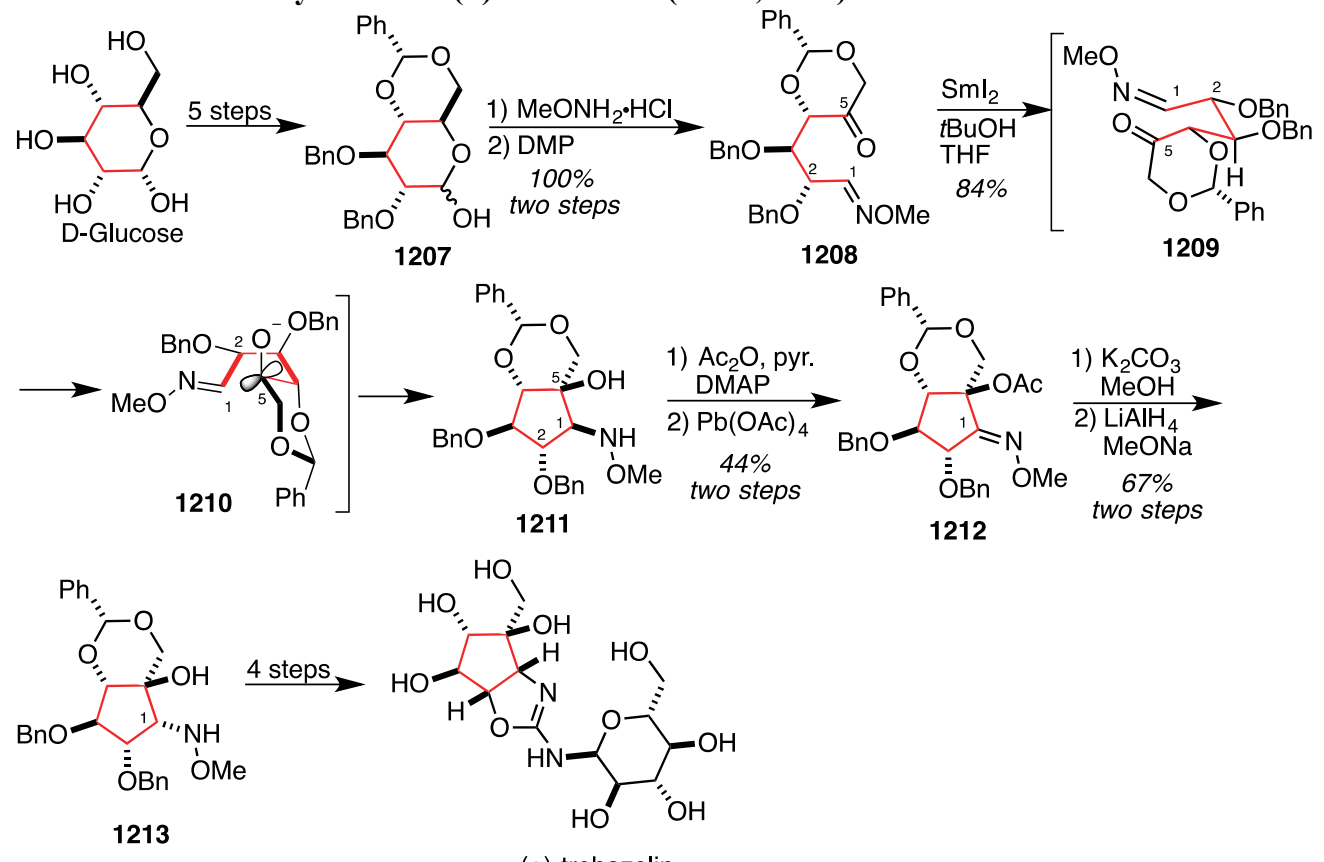

(+)-trehazolin

In the synthesis of $(+)$-trehalamine by Chiara $^{211}$, a similar stereoselective ketone-oxime ether reductive cyclization constructs a fully functionalized cyclopentane (Scheme 141). The synthesis begins with 1214, which was prepared from D-mannose in seven steps. Conversion to the radical cyclization precursor 1215 occurs over two steps. Radical cyclization is promoted by treatment with samarium iodide. Substrate $\mathbf{1 2 1 5}$ contains a cyclic acetal protecting group, similar to the cyclization substrate $\mathbf{1 2 0 8}$ used by Giese. However, a C5,C1 trans-amino alcohol diastereomer was observed in 1216. Also similar to the Giese synthesis, the amino alcohol formed displays a trans relationship between the emerging amine bearing stereocenter at $\mathrm{C} 1$ and the resident substitutent

${ }^{209}$ Boiron, A.; Zillig, P.; Faber, D.; Giese, B. J. Org. Chem. 1998, 63, 5877-5882.

${ }^{210}$ This trans relationship may arise from the preferential pseudo-equatorial positioning of the oxime double bond and the $\mathrm{C} 2$ substituent.

${ }^{211}$ Storch de Gracia, I.; Bobo, S.; Martin-Ortega, M. D.; Chiara, J. L. Org. Lett. 1999, 1, 1705-1708. 
at $\mathrm{C} 2$. The stereochemical result was attributed to minimization of allylic 1,3 strain in the cyclization transition state. $^{212}$ As a result of the stereochemistry present in the D-mannose starting material, intermediate 1216 requires stereochemical inversion of the alcohol bearing stereocenter at $\mathrm{C} 2$. The acetate protecting group was removed with ammonia and methanol to give 1217. Triflation of the secondary alcohol induces an intramolecular substitution to form the oxazoline 1218. An additional two steps were required to complete the synthesis of $(+)$ trehalamine.

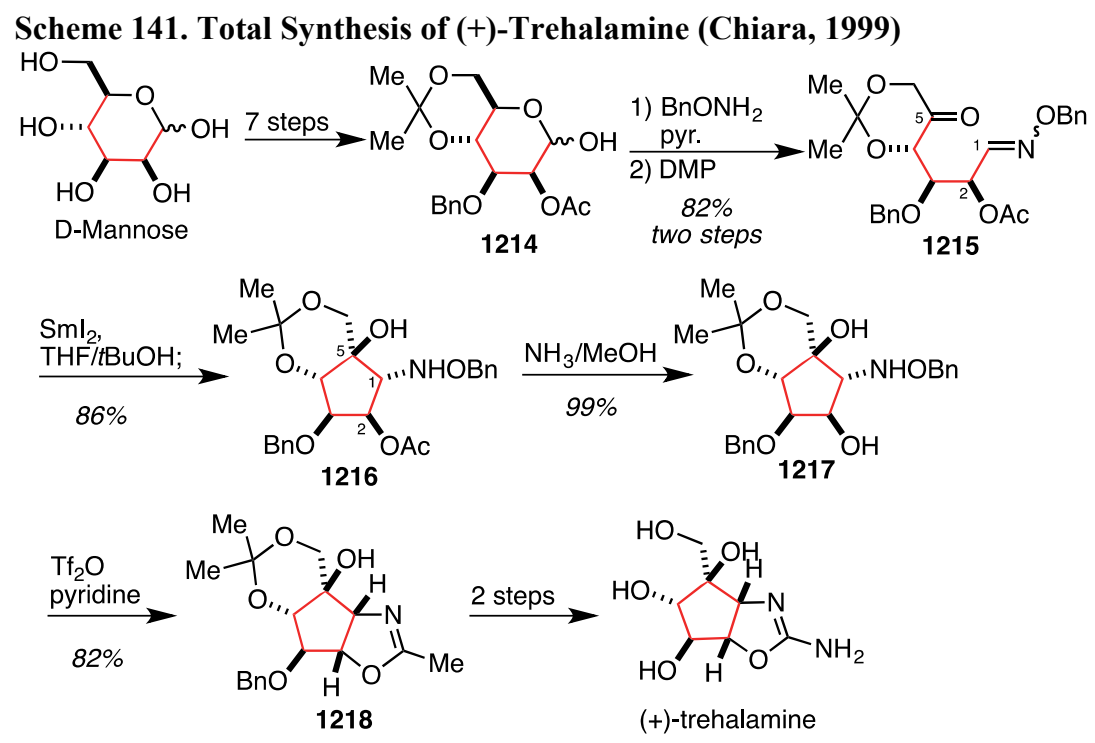

In the synthetic studies toward hexacyclinic acid, Clarke ${ }^{213}$ uses a reductive radical cyclization to build a fully functionalized cyclopentane (Scheme 142). The synthesis begins with an intramolecular Diels-Alder reaction of diene-yne 1219 to yield bicyclic lactone 1220. Conjugate addition of a vinyl cuprate occurs on the diastereoface opposite the benzyloxy methyl substitutent. After an aqueous workup, cis-fused bicycle $\mathbf{1 2 2 1}$ is obtained. Lactone reduction and thioacetal formation gave intermediate 1222. Oxidation of the primary alcohol was followed by addition of vinylmagnesium bromide to give 1223. The desired diastereomer was obtained in a 30:1 ratio and can be rationalized by Felkin-Anh analysis. The alcohol was protected as the silyl ether and the dithiolane protecting group was removed to give aldehyde 1224. With three stereocenters in place, the key reductive cyclization was investigated. The 5-exo-trig cyclization proceeded in a solution of HMPA and water to give fully functionalized cyclopentane 1225. It was hypothesized that the two newly formed stereocenters in this all trans diastereomer is a result of repulsion of the anionic oxygen and carbon atoms in the transition state. Advanced intermediate $\mathbf{1 2 2 5}$ contains a fully functionalized cyclopentane with substituents and stereochemistry found in the natural product.

Scheme 142. Synthetic Studies Towards Hexacyclinic Acid (Clarke, 2009)

\footnotetext{
${ }^{212}$ Alternatively, Giese's stereochemical model applied to substrate $\mathbf{1 2 1 5}$ may give a reactive conformation that predicts the observed stereochemistry.

${ }^{213}$ Clarke, P. A.; Cridland, A. P.; Rolla, G. A.; Iqbal, M.; Bainbridge, N. P.; Whitwood, A. C.; Wilson, C. J. Org. Chem. 2009, 74, 7812-7821.
} 


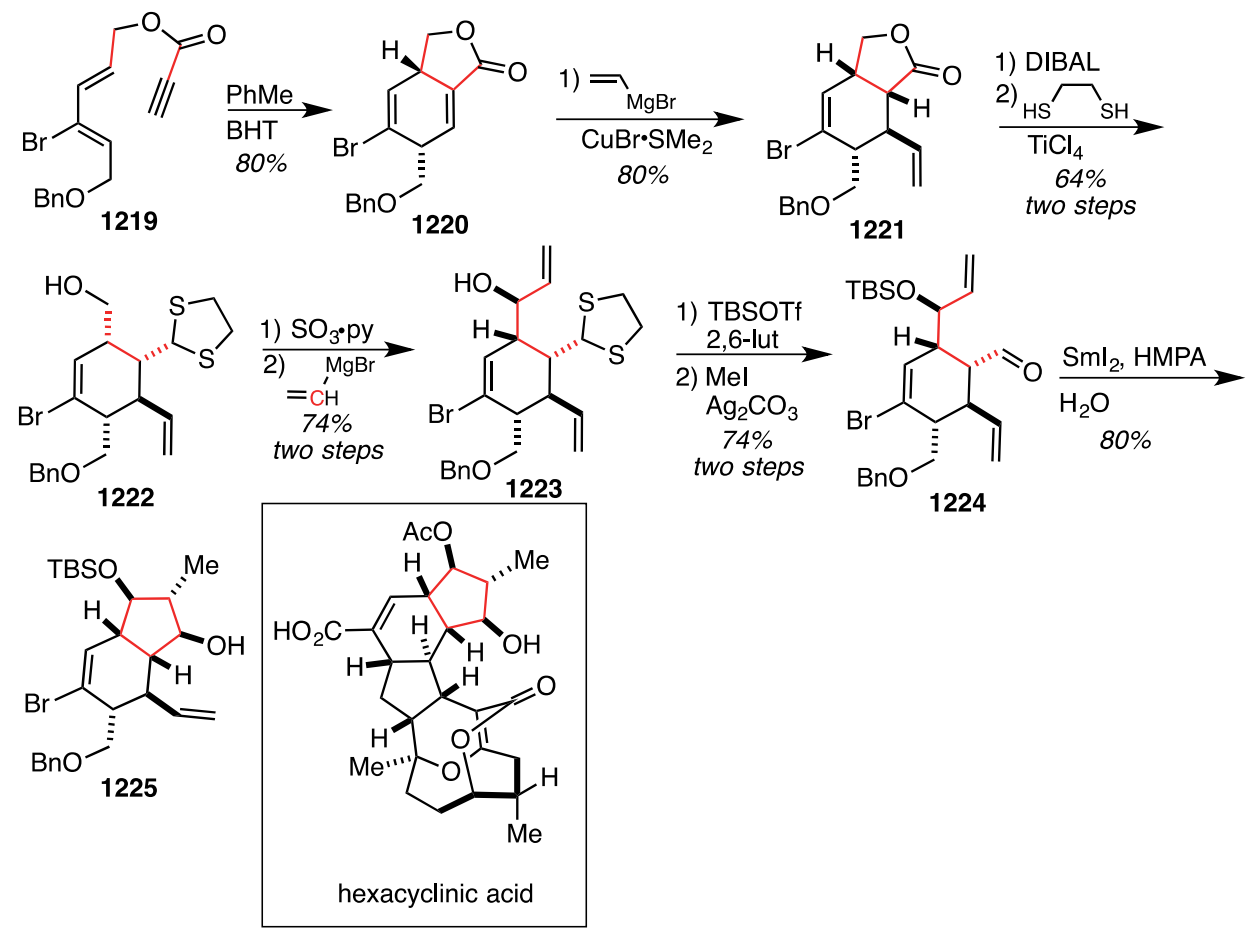

\section{2 $\sigma$-Bond Homolysis-5-exo-trig Cyclizations}

In the Simpkins ${ }^{214}$ synthesis of (-)-allosamizoline, the aglycone of allosamidin, a carbon-centered radical adds to an oxime ether constructing a fully functionalized cyclopentane (Scheme 143). Akin to other aminocyclitol syntheses, stereochemical information in the carbohydrate glucosamine is transferred to the cyclopentane scaffold. Radical precursor $\mathbf{1 2 2 6}$ is obtained from D-glucosamine in five standard transformations. Treatment of 1226 with the tributyltin radical induces $\mathrm{C}-\mathrm{O}$ bond homolysis to give an alkyl radical, which adds to the oxime ether. The major product $\mathbf{1 2 2 7}$ was isolated as an inconsequential epimeric mixture at $\mathrm{C} 1$. The major $\mathrm{C} 5$ diastereomer arises from a preferred chair-like transition state with equatorial tether substituents. ${ }^{215}$ The benzyloxyamino group was oxidized to an oxime ether (1228). Oxidative cleavage with reductive workup delivers alcohol $\mathbf{1 2 2 9}$ in modest yield based on the recovered oxime. Treatment of $\mathbf{1 2 2 9}$ with thionyl chloride leads to alcohol activation and cyclization of the tethered carbamate to give cis-fused bicycle 1230. This advanced intermediate contains the appropriate substitution and stereochemistry for (-)-allosamizoline, and the natural product was completed using an additional two transformations.

\section{Scheme 143. Total Synthesis of (-)-Allosamizoline (Simpkins, 1992)}

\footnotetext{
${ }^{214}$ Simpkins, N. S.; Stokes, S.; Whittle, A. J. J. Chem. Soc., Perkin Trans. 1 1992, 2471-2477.

215 (a) Bartlett, P. A.; McLaren, K. L.; Ting, P. C. J. Am. Chem. Soc. 1988, 110, 1633-1634. (b) Rajanbabu, T. V.; J. Am. Chem. Soc. 1987, 109, 609-611.
} 

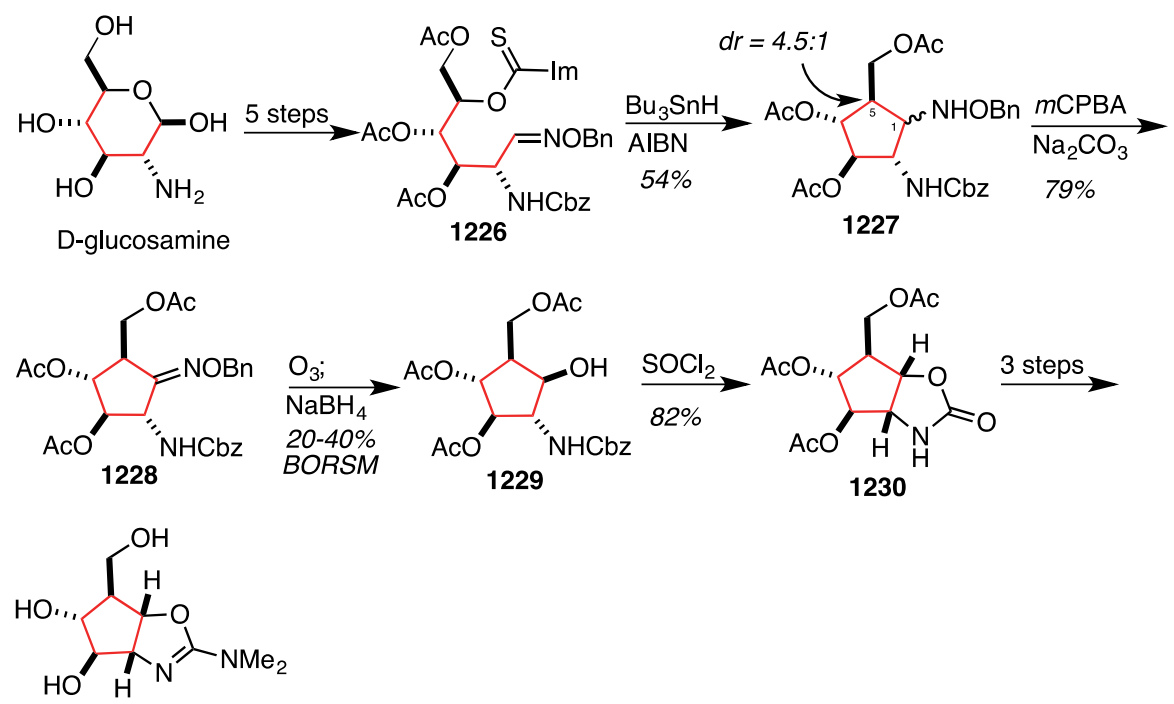

$(-)$-allosamizoline

The synthesis of (-)-coriolin by Weinges ${ }^{216}$ uses an oxymercuration-radical cyclization sequence of $R-(-)$ carvone to construct functionalized cyclopentane 1232 (Scheme 144). The alkyl mercury species, generated after oxymercuration, is reduced to a mercury hydride species that undergoes $\mathrm{C}-\mathrm{Hg} \sigma$-bond homolysis and a 5-exotrig radical cyclization. Treatment with peracid induces an oxidative sequence leading to acetate 1236. The reaction is thought to proceed via Baeyer-Villiger oxidation (to 1233), trans-esterification (to 1234), alcohol oxidation (to 1235) and a subsequent Baeyer-Villiger reaction yielding 1236. Acetate hydrolysis, Mitsunobu bromination, and elimination under basic conditions delivers a mixture of disubstituted alkenes with cyclopentene 1237 isolated as the major regioisomer (5:1). Epoxidation occurs preferentially from the convex face of the ring system to give $\mathbf{1 2 3 8}$ as the major diastereomer. The epoxide was opened with phenylselenide, which was followed by elimination to give an allylic alcohol. The alcohol was then protected as the PMB-ether to furnish 1239. Intermediate 1239 is a related compound to a coriolin precursor published by Curran ${ }^{217}$, but 1239 is non-racemic and it has the eventual secondary hydroxyl of coriolin installed and masked as a PMB-ether. However, many of the following transformations resemble those used in the Curran $( \pm)$-coriolin synthesis. Specifically, organocuprate-mediated allylic substitution occurred following Curran's precedent to give 1241. This intermediate was elaborated to enyne 1242 over six steps. Radical-based cyclization with samarium iodide, again following Curran's route, constructs the triquinane core of (-)-coriolin (1243). Protecting group manipulation over three steps gives enone 1244. A Saegusa oxidation, and silyl deprotection gives common coriolin intermediate 130. Nucleophilic epoxidation gave coriolin as a 2:1 mixture with its spiro-oxirane epimer.

Scheme 144. Total Synthesis of $( \pm)$-Coriolin (Weinges, 1993)

\footnotetext{
${ }^{216}$ (a) Weinges, K.; Braun, R.; Huber-Patz, U.; Irngartinger, H. Liebigs Ann. Chem. 1993, 1133-1140. (b) Weinges, K.; Reichert, H. Synlett 1991, 11, 785-786.

${ }^{217}$ Fevig, T. L.; Elliot, R. L.; Curran, D. P. J. Am. Chem. Soc. 1988, 110, 5064-5067.
} 

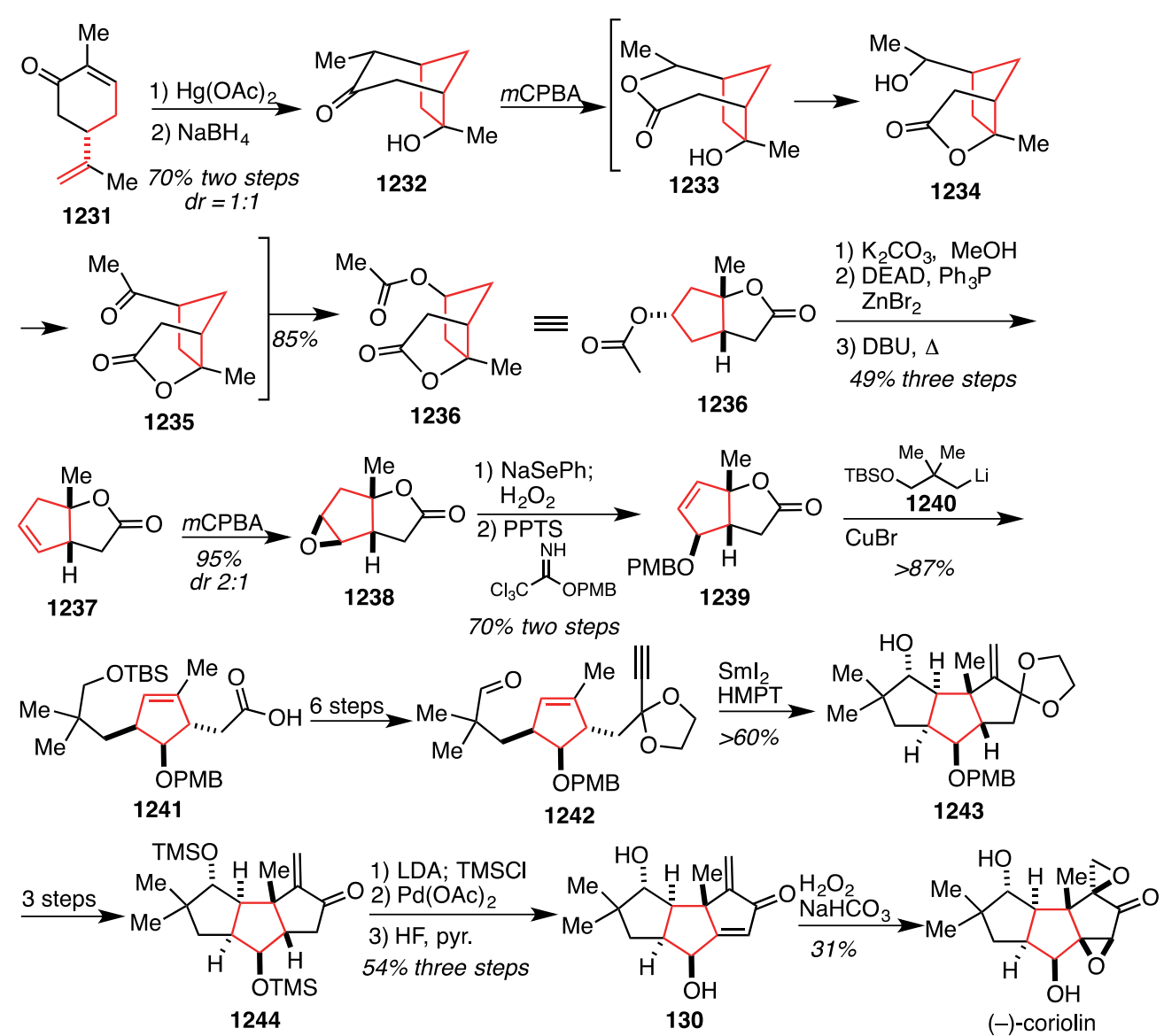

In the synthesis of $( \pm)$-gelsemine by Hart ${ }^{218}$, a free radical is used to construct the tricyclic core and a fully functionalized cyclopentane (Scheme 145). All of the carbon atoms for the cyclopentane were installed with a Diels-Alder cycloaddition of diene 1245 and $N$-methylmaleimide yielding 1246. This material underwent functional group manipulation over three steps to deliver alkene 1247. Reduction of the imide with sodium borohyride occurs from the convex face of the ring system to give a carbinol lactam, which was alkylated with ethyl iodode to yield a mixture of isomeric ethoxy lactams favoring the desired $\mathbf{1 2 4 8}$ in a 7:1 ratio. The lithium enolate generated from 1248 was alkylated on the convex face using MOMCl to deliver 1249. This material was advanced over three steps to thioether 1172. Treatment with standard radical conditions led to carbon-sulfur bond homolysis and cyclization with the tethered $\alpha, \beta$-unsaturated ester to give the tricyclic core of gelsemine (1173). This key transformation leads to a fully functionalized cyclopentane, but requires inversion of configuration at C15. Intermediate 1173 was converted over eight steps to aldehyde $\mathbf{1 2 5 0}$. Treatment with aqueous acid removed the acetate group and spontaneous lactol formation delivered $\mathbf{1 2 5 1}$ with the appropriate substitution and stereochemistry for gelsemine. This advanced intermediate was converted to ( \pm )-gelsemine over an additional five steps.

Scheme 145. Total Synthesis of $( \pm)$-Gelsemine (Hart, 1994)

${ }^{218}$ Atarashi, S.; Choi, J. -K.; Ha, D. -C.; Hart, D. J.; Kuzmich, D.; Lee, C. -S.; Ramesh, S.; Wu, S. C. J. Am. Chem. Soc. 1997, 119, 6226-6241. 


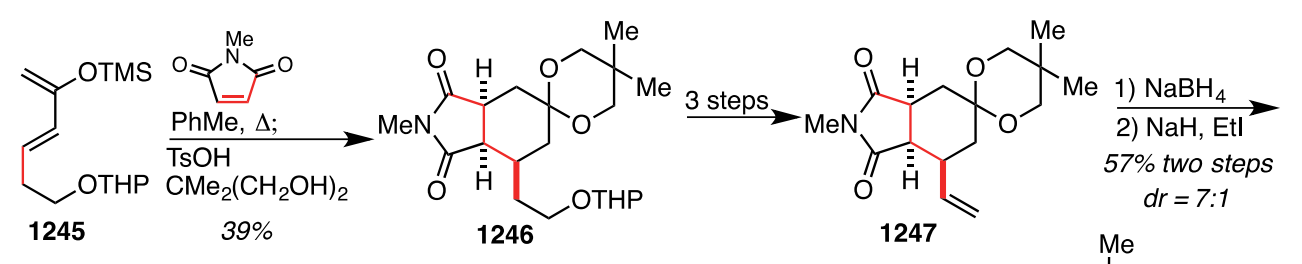

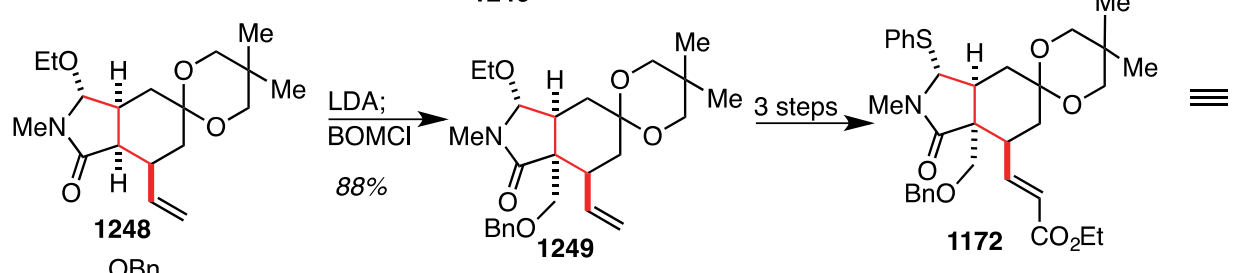

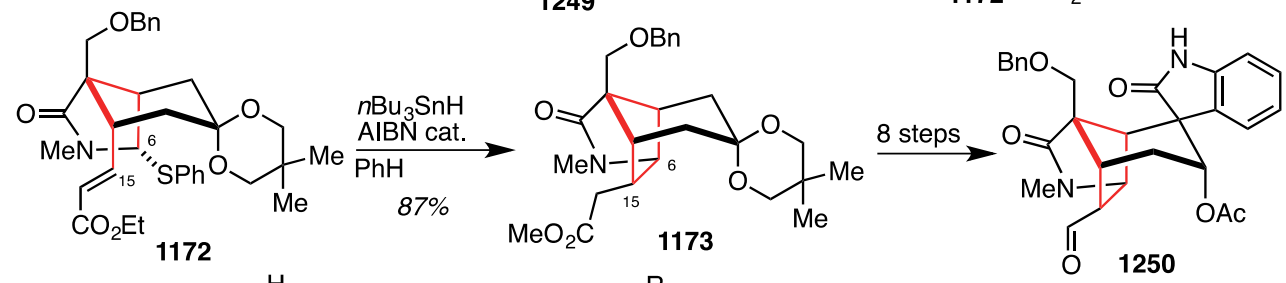

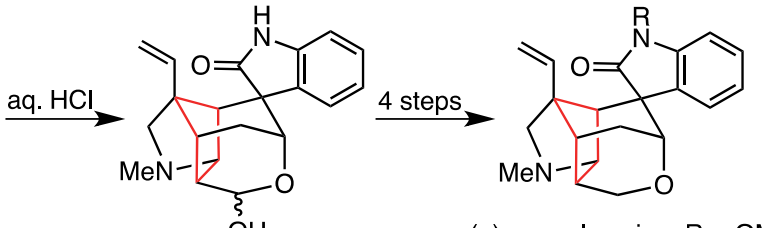

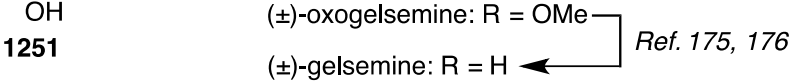

The core of hexacyclinic acid was stereoselectively synthesized by Landais ${ }^{219}$ using a 5-exo-trig radical cyclization of a 1,6-diene (Scheme 146). The synthetic route commences with an aldol reaction between racemic ester 1252 and aldehyde 1253. The major stereoisomer (1254) results from a combination of direction from the silyl substituent and Zimmerman-Traxler considerations. ${ }^{220}$ A palladium-catalyzed sulfonylation delivered the radical cyclization substrate 1255 as a mixture of alkene isomers (E/Z 95:5). The radical cyclization was performed photochemically in the presence of catalytic $p-\mathrm{TolSO}_{2} \mathrm{SePh}$ furnishing fully functionalized cyclopentane 1257. The stereochemical outcome is rationalized by the authors with a chair-like transition state 1256, which places all large substituents pseudo-equatorial. Intermediate 1257 contains a fully functionalized cyclopentane with substitution and stereochemistry corresponding to many of the structural features of hexacyclinic acid. It was advanced four steps to 1258, which displays the ABC ring system of the natural product.

Scheme 146. Synthetic Studies Towards Hexacyclinic Acid (Landais, 2005)

${ }^{219}$ James, P.; Felpin, F. -X.; Landais, Y.; Schenk, K. J. Org. Chem. 2005, 70, 7985-7995.

${ }^{220}$ Fleming, I.; Hill, J. H. M.; Parker, D.; Waterson, D. J. Chem. Soc., Chem. Commun. 1985, 6, 318-320. 


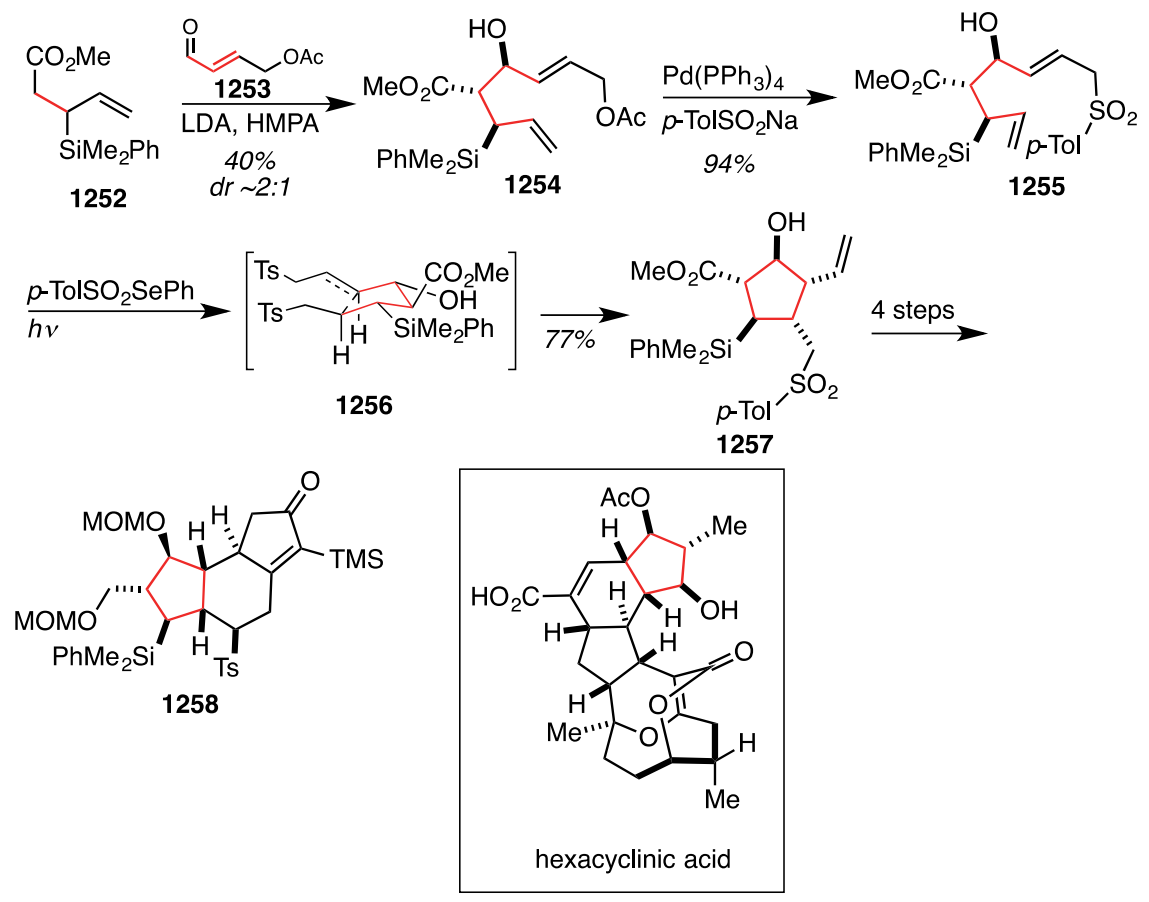

In the synthesis of the reported structures of $( \pm)$-vannusals A and B by Nicolaou, ${ }^{221,222}$ a transannular radical cyclization forms a cyclopentanone substituted at each of the four $\mathrm{sp}^{3}$-hybridized carbons (Scheme 147). Beginning with enone 1259, conjugate addition of the vinyl cuprate derived from 1260 delivers 1261 as an inconsequential mixture of geometric alkene isomers that were advanced through the synthesis. The silylated alcohol was converted to acetal $\mathbf{1 2 6 2}$ over the three steps shown. Intramolecular spirocyclization via a Mukaiyama-type aldol reaction occurred by exposure to excess TMSI and HMDS giving 1263. The cyclization proceeded stereoselectively with the electrophile approaching opposite the vinyl group. Acylation of the ketone was performed using standard conditions to deliver keto-ester $\mathbf{1 2 6 4}$ as a mixture of diastereomers. Treatment of 1264 with conditions developed by Snider ${ }^{223}$ induced the ring closure to form cyclopentanone 1266 via the radical pathway shown. The product was obtained as a single diastereomer unaffected by either the ester stereochemistry or the alkene geometry. At this stage, three of the stereocenters have the desired configuration for vannusal B. A stereoselective ketone reduction with DIBAL delivers alcohol 1267. Presumably, the hydride approaches on the less hindered face away from the ethylene bridge. Functional group transformations delivered intermediate 1268 over four steps. The aldehyde was converted to allyl vinyl ether 1269. The desired $E$-geometry of the enol ether was expected based on steric considerations. Microwave heating induced a Claisen rearrangement which established the quaternary stereocenter. Reduction of the aldehyde gave advanced intermediate 1270. At this stage a fully functionalized cyclopentane with all the required stereochemistry and substitutents for vannusal B has been created. An additional 19 steps were required to complete the reported structure of the natural product.

Scheme 147. Total Synthesis of ( \pm )-Vannusals A and B (Nicolaou, 2010)

221 (a) Nicolaou, N. C.; Jennings, M. P.; Dagneau, P. Chem. Commun. 2002, 21, 2480-2481. (b) Nicolaou, K. C.; Ortiz, A.; Zhang, H.; Dagneau, P.; Lanver, A.; Jennings, M. P. Arseniyadis, S.; Faraoni, R.; Lizos, D. J. Am. Chem. Soc. 2010, 132, 7138-7152.

${ }^{222}$ A related enantioselective approach to the synthesis of the reported structure of vannusals A and B was also published. See: Nicolaou, K. C.; Tang, W.; Dagneau, P.; Faraoni, R. Angew. Chem. Int. Ed. 2005, 44, 3874-3879.

${ }^{223}$ Snider, B. B. Chem. Rev. 1996, 96, 339-364. 

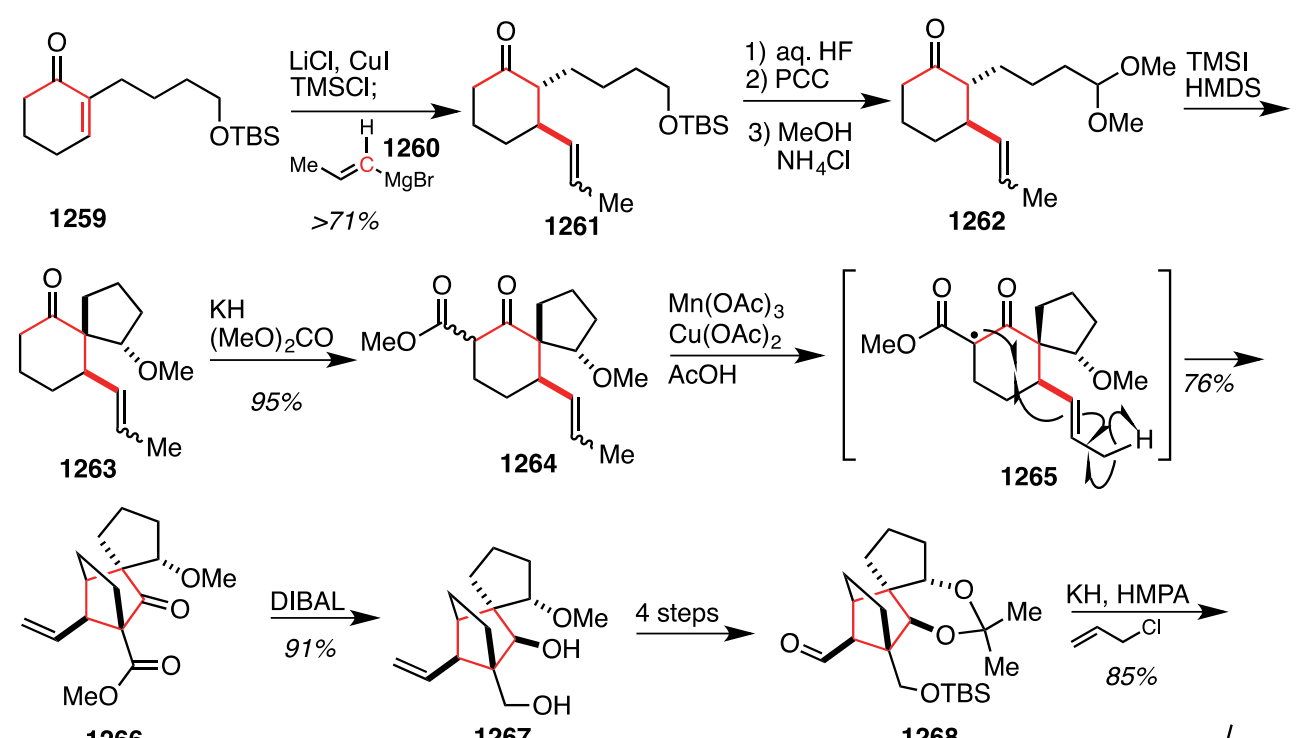

1266

1267

1268

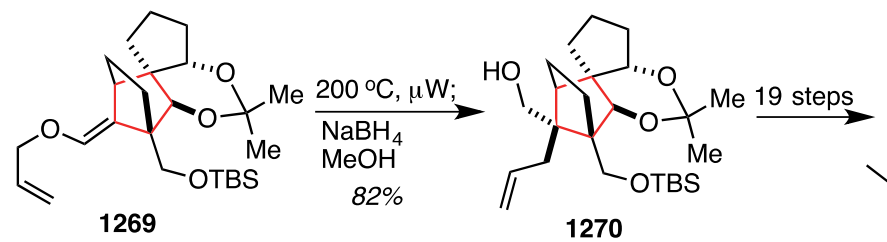

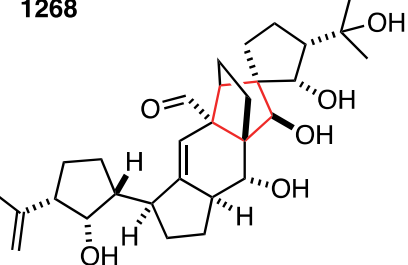

( \pm -vannusal B

(originally assigned)

In the first synthesis of (-)-maoecrystal Z, Reisman ${ }^{224}$ uses a samarium-mediated reductive cascade cyclization reaction to construct a fully functionalized cyclopentane (Scheme 148). The synthesis begins with silation of (-)$\gamma$-cyclogeraniol (1271) followed by epoxidation to give 1272 as the major product. A reductive epoxide coupling with acrylate ester 1273 following Gansäuer's method ${ }^{225}$ delivered spirolactone $\mathbf{1 2 7 4}$ as a single diastereomer. Alkylation with iodide 1275 (prepared from $S, S$-pseudoephedrine) delivered 1276 as an inconsequential mixture of epimers, which was further transformed to enoate 1277 by selenation/selenoxide elimination. Removal of the silyl ethers was followed by oxidation of the diol to dialdehyde $\mathbf{1 1 7 4}$. When radical substrate $\mathbf{1 1 7 4}$ was subjected to samarium iodide and $\mathrm{LiBr}$, the fully functionalized cyclopentane 1175 was obtained. The stereochemical configuration of the alcohols in the product is believed to be the result of a reactive conformation that minimizes steric interactions of the aldehyde carbonyls and the cyclohexane ring. Advanced intermediate 1175 contains a fully functionalized cyclopentane that was converted to the natural product in four additional steps.

\subsection{Radical Cyclizations that Form Multiple Rings}

Scheme 148. Total Synthesis of (-)-Maoecrystal Z (Reisman, 2011)

\footnotetext{
${ }^{224}$ Cha, J. Y.; Yeoman, J. T. S.; Reisman, S. E. J. Am. Chem. Soc. 2011, 133, 14964-14967.

${ }^{225}$ Gansäuer, A.; Pierobon, M.; Bluhm, H. Angew. Chem. Int. Ed. 1998, 37, 101-103.
} 

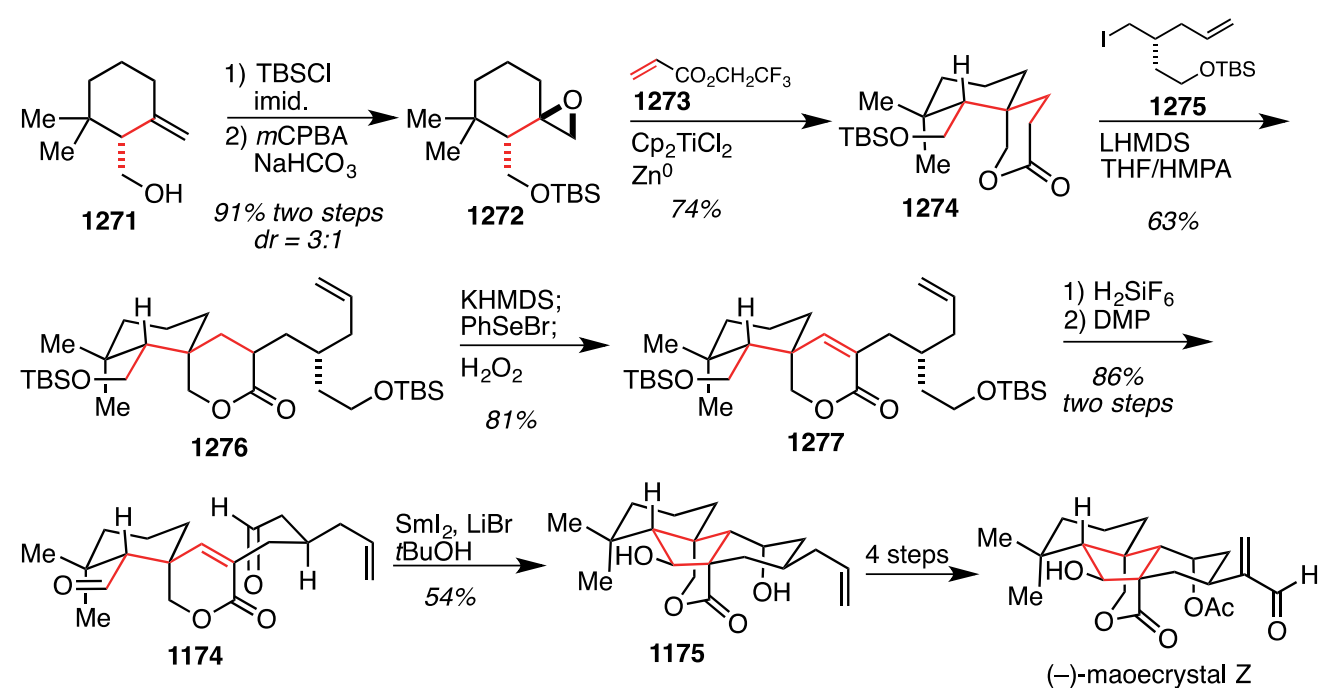

In the first total synthesis of (-)-chromodorolide B, Overman ${ }^{226}$ builds a fully functionalized cyclopentane using a novel radical addition/cyclization/fragmentation cascade (Scheme 149). The cascade brings together a butenolide and trans hydrindane fragment while constructing three stereocenters. The route begins by elaborating known enedione $\mathbf{1 2 7 8}$ over nine steps to vinyl iodide 1279. The hydrindane fragment was coupled with aldehyde $\mathbf{1 2 8 0}^{227}$ using a modified Nozaki-Hiyama-Kishi reaction in the presence of ligand $\mathbf{1 2 8 1}$. The allylic alcohol 1282 was isolated as a single alcohol epimer. Hydrolysis of the methyl ester was followed by esterification with $\mathrm{N}$ hydroxyphthalimide. Suprafacial allylic chlorination with thionyl chloride proceeded regio- and stereoselectively delivering chloride 1176. ${ }^{228}$ The key radical cyclization was performed with blue LEDs in the presence of a ruthenium bipyridyl complex. After much experimentation, optimized conditions were realized with $d 2$-Hantzch ester (1178) to give desired compound 1179 in a 1:1.3 ratio with the C8 epimer in a combined $65 \%$ yield $(28 \%$ of desired 1179). Use of the dideuterio Hantzch ester was to decrease the rate of hydrogen atom transfer to intermediates 1284. The reaction proceeds via the radical cascade mechanism shown. Although the diastereoselectivity and chemical yield was somewhat modest, an impressive increase in molecular complexity transpires over the course of this reaction, and nearly the entire oxygenated moiety of the natural product has been constructed. Moreover, the fully functionalized cyclopentane is delivered in a single step from the coupling of relatively simple functional groups. The benzyl ether was functionalized to carboxylic acid $\mathbf{1 2 8 5}$ over five steps. Treatment with $4 \mathrm{M} \mathrm{HCl}$ resulted in acetonide deprotection and lactonization to form lactol 1286. Global acetyl protection delivered (-)-chromodorolide B.

Scheme 149. Total Synthesis of (-)-Chromodorolide (Overman, 2016)

\footnotetext{
${ }^{226}$ Tao, D. J.; Slutskyy, Y.; Overman, L. E. J. Am. Chem. Soc. 2016, 138, 2186-2189.

${ }^{227}$ Aldehyde 1252 was prepared in three steps from dimethyl 2,3-O-isopropylidene L-tartrate. See: Reference 226

${ }^{228}$ Ireland, R. E.; Wrigley, T. I.; Young, W. G. J. Am. Chem. Soc. 1958, 80, 4604-4606.
} 

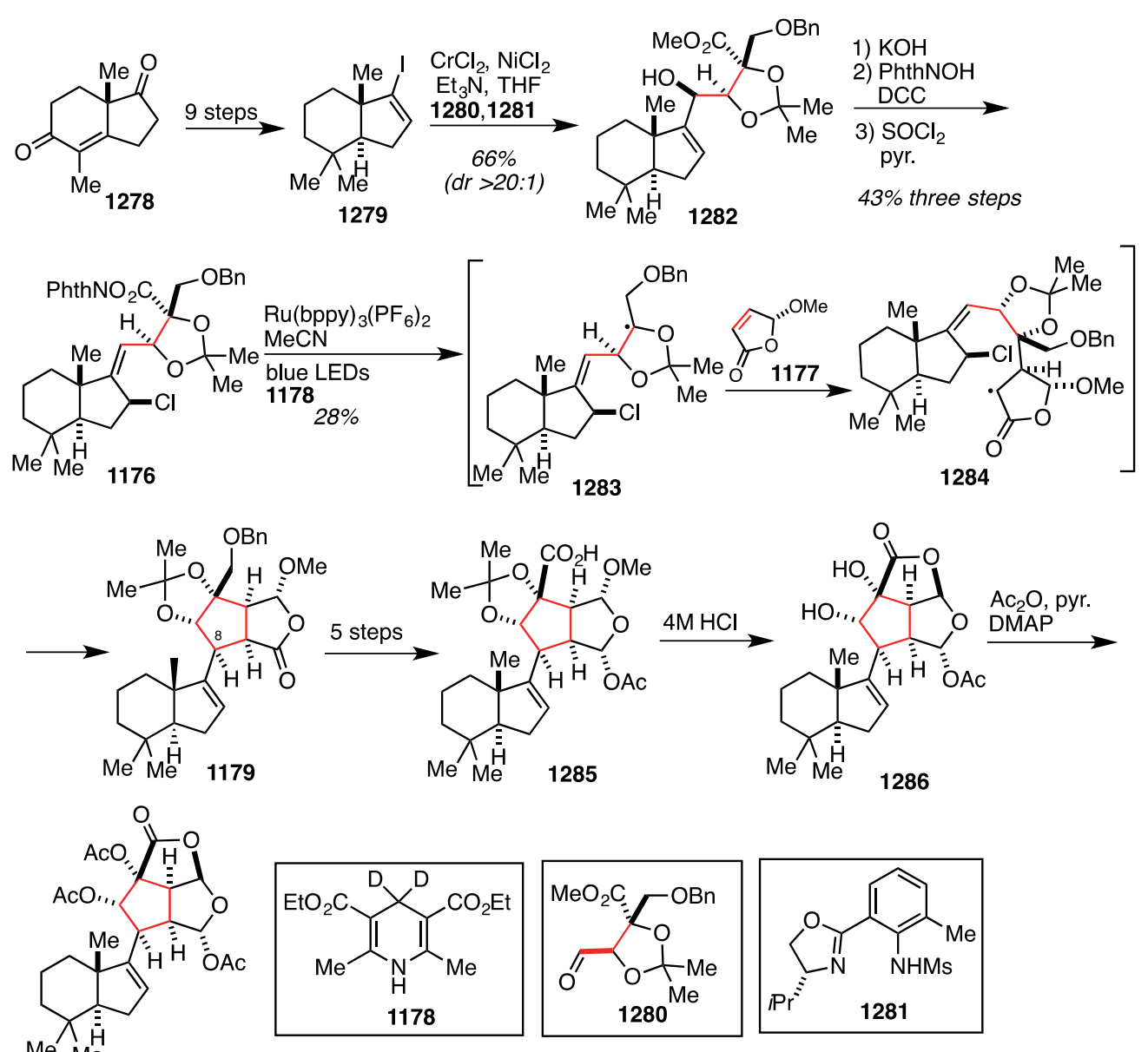

(-)-chromodorolide B

In the formal synthesis of $( \pm)$-coriolin by Little ${ }^{132}$ an intramolecular reaction of an alkene with a 1,3-diyl (i.e. alkyl 1,3-diradical) was used to assemble the cyclopentane (Scheme 150). The synthesis begins with benzyl protection of the commercially available furanone 1287. The lactone was reduced and methylated under acidic conditions to give acetal 1288. The benzyl group was removed and a Swern oxidation gave aldehyde 1289. Condensation of $\mathbf{1 2 8 9}$ with cyclopentadiene (11) delivered fulvene 1290. The bicyclic skeleton of diazene 1292 was formed using a Diels-Alder reaction with dimethylazodicarboxylate (1291). This was advanced to diazene 1293 over three steps. A Wittig reaction with triphenylphosphonium methylide opens the lactol to give cyclization precursor 1180. Photodeazetation of diazene 1180 formed the triquinane 1181. Mechanistically, photolysis leads to 1,3-diyl 1294, which undergoes intramolecular cyclization with the tethered alkene via a favored chair-like transition state (1295) with an equatorial alcohol substituent and suprafacial addition to the diyl. Epoxidation was accomplished with peracid to deliver 1296 in good yield and a 4:1 diastereomer ratio. Interestingly, the minor diastereomer is the product of epoxidation to give a trans-diquinane substructure. This minor diastereomer was confirmed by X-ray crystallographic studies. There are many examples in this review which demonstrate that conversion of similarly unsaturated diquinanes to cis-bicyclo[3.3.0]octanes proceeds reliably with complete diastereocontrol. This is the only example where the trans-diastereomer was observed even as a minor component. Even more interesting, deletion of the alcohol functional group in 1181 was anticipated to prevent Henbest delivery of oxygen to the $\alpha$-face of the alkene; however, the deoxygenated substrate 1299 delivered $\mathbf{1 3 0 0}$ with the trans-bicyclo[3.3.0] octane substructure as the major product. The reason for this diastereoselectivity is unknown. Epoxide 1296 underwent elimination with LDA, selective protection of the secondary alcohol as the benzoate ester and chromium oxidation with transposition of oxygen to deliver enone 1297. Conjugate addition using a higher order cuprate successfully installed the angular methyl group, leading to a cis-fused diquinane substructure (1298). Saegusa oxidation and ester hydrolysis let to intermediate 104, which intersects an intermediate prepared by $\operatorname{Trost}^{129}$ in a synthesis of $( \pm)$-coriolin.

Scheme 150. Formal Synthesis of ( \pm )-Coriolin (Little, 1987) 

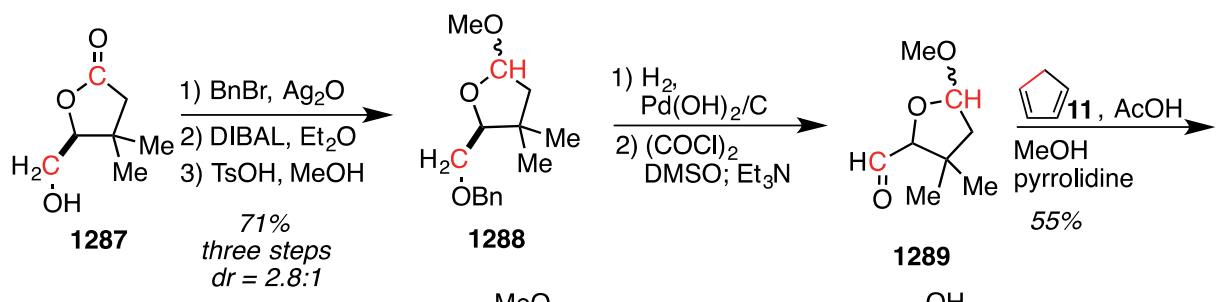<smiles>CO[C@H]1CC(C)(C)C(C=C2C=CC=C2)O1</smiles>

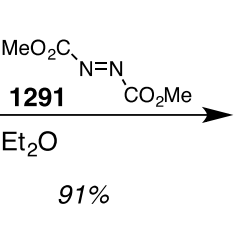

1290
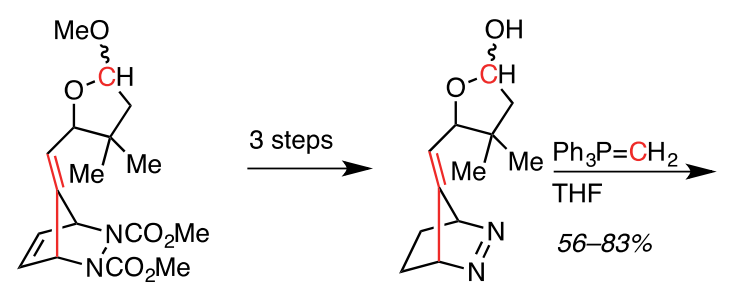

1292
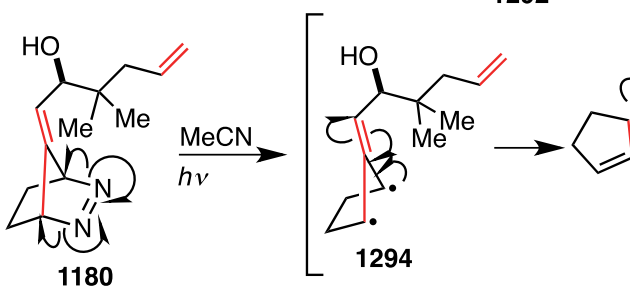

1293
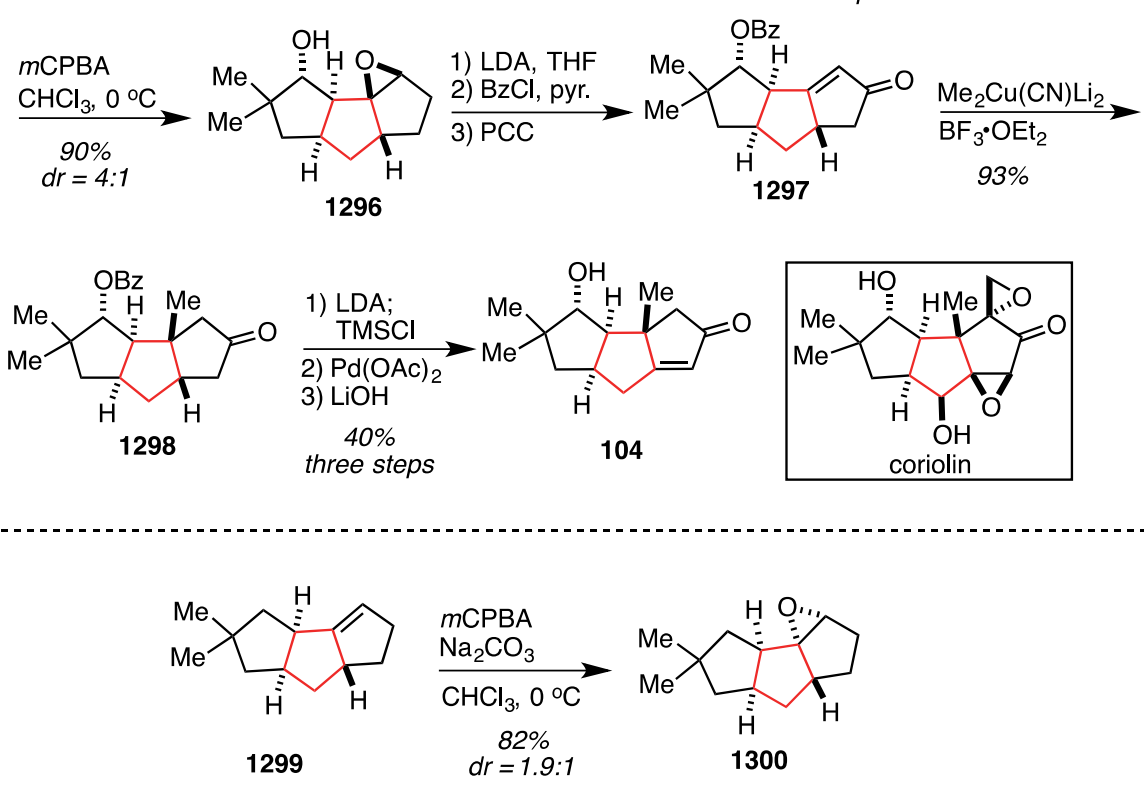

\section{Functionalization of Five-Membered Carbocyclic Starting Materials}

A common strategy for constructing fully functionalized cyclopentanes involves the functionalization of simple five-membered carbocyclic starting materials. With dozens of simple building blocks available, and a variety of transformations in the chemist's toolbox, it is somewhat surprising that many syntheses in this section are represented by a few well-defined patterns.

Cyclopentadiene is an inexpensive starting material common to many syntheses of natural products with fully functionalized cyclopentane architectures. Alkylation of cyclopentadiene gives substituted cyclopentadienes with functionality at all five carbons. A common strategy is to alkylate with an alkoxymethylene group $(\mathbf{1 1} \rightarrow \mathbf{1 3 0 1})$ (shown in Scheme 151); however, dialkylations are also known. ${ }^{244}$ These cyclopentadienes undergo facile DielsAlder cycloaddition on the diene face opposite the substituent. The most common dienophile for this sequence is singlet oxygen; however, nitroso and alkene dienophiles have also participated to give nitrogen- and carbonsubstituted cyclopentenes, respectively. Subsequent fragmentation of the $\mathrm{O}-\mathrm{O}$ sigma bond reveals an all trans 
1,2,3-trisubsituted cyclopentene (1302). Cyclopentene 1302 may undergo 1,2-alkene difunctionalization such as dihydroxylation to give 1303, epoxidation, or bromohydrin formation to reveal the fully functionalized cyclopentane. Depending on the choice of conditions, this 1,2-alkene difunctionalization may occur on the less hindered face, or it may be directed by the substrate to give oxygenation syn to the diol.

\section{Scheme 151.}

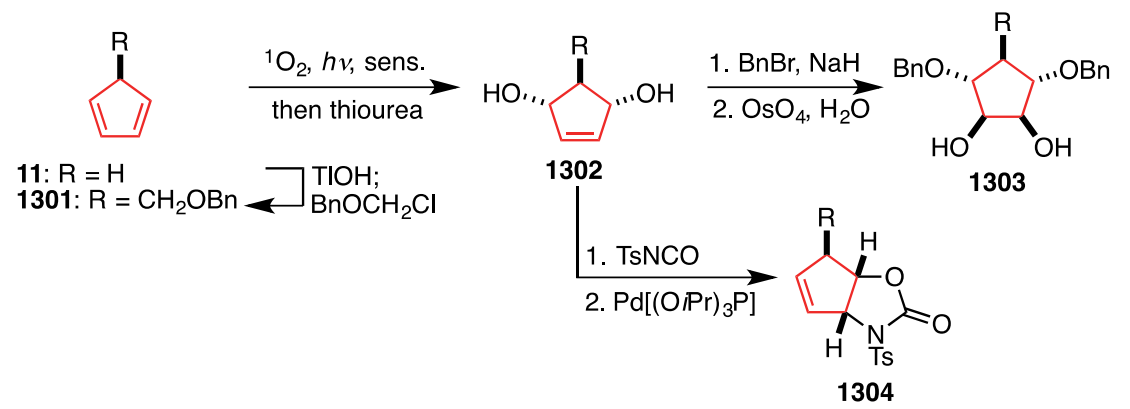

Another common pattern is for $\mathbf{1 3 0 2}$ to first undergo intramolecular $\mathrm{S}_{\mathrm{N}} 2$ '-type cyclization, often via a carbamate intermediate (1304). ${ }^{233}$ A subsequent 1,2-difunctionalization of the transposed alkene leads to substitution at all positions of the cyclopentane. In this strategy, both the Diels-Alder reaction and 1,2-alkene functionalization perform reliably with heteroatom reagents (e.g. ${ }^{1} \mathrm{O}_{2}$ and $\mathrm{OsO}_{4}$, respectively); as a result, many of the natural products (trehazolin, mannostatin A, allosamizoline) made with this synthestic strategy display multiple heteroatom substitutents on the fully functionalized cyclopentane.

Acyl acetal 1305 is a readily available intermediate derived from D-mannose. Addition of phosphonate 1306 under basic conditions gives 1308 (Scheme 152). ${ }^{229}$ Intermediate carbanion 1307 cyclizes via intramolecular Horner-Wadsworth-Emmons olefination to give enone 1308. The enone can be reduced under Luche conditions to form allylic alcohol 1309. Hydride delivery occurs on the less hindered ketone diastereoface to give $\mathbf{1 3 0 9}$ as a single diastereomer. Intermediate $\mathbf{1 3 0 9}$ has been used in multiple syntheses of targets with a fully functionalized cyclopentane.

Scheme 152.

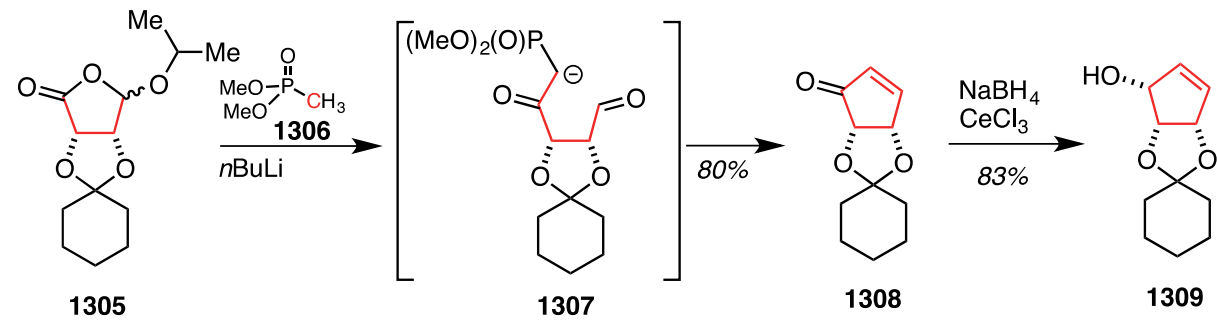

Cyclooctadienes may undergo transannular ring contraction to give cis-bicyclo[3.3.0]octane architectures. The two common starting materials prepared in this strategy are shown in Scheme 153. 1,3-Cyclooctadiene (1310, eq. 1) is oxidized to the corresponding epoxide (1311). Lithiation of the more acidic allylic position is followed by transannular ring closure to give 1312. ${ }^{230}$ Alternatively, 1,5-cyclooctadiene (1313) undergoes Pd-catalyzed cyclization in the presence of acetate to give $C_{2}$-symmetric starting material 1317 (eq. 2). ${ }^{231}$ This intermediate can be processed in a variety of ways, including an enantioselective enzymatic desymmetrization (see below).

\section{Scheme 153.}

\footnotetext{
${ }^{229}$ Borcherding, D. R.; Scholtz, S. A.; Borchardt, R. T. J. Org. Chem. 1987, 52, 5457-5461.

${ }^{230}$ Crandall, J. K.; Chang, L. -H. J. Org. Chem. 1967, 32, 532-536.

${ }^{231}$ Henry, P. M.; Davies, M.; Ferguson, G.; Phillips, S.; Restivo, R. J. Chem. Soc., Chem. Commun. 1974, $112-113$.
} 

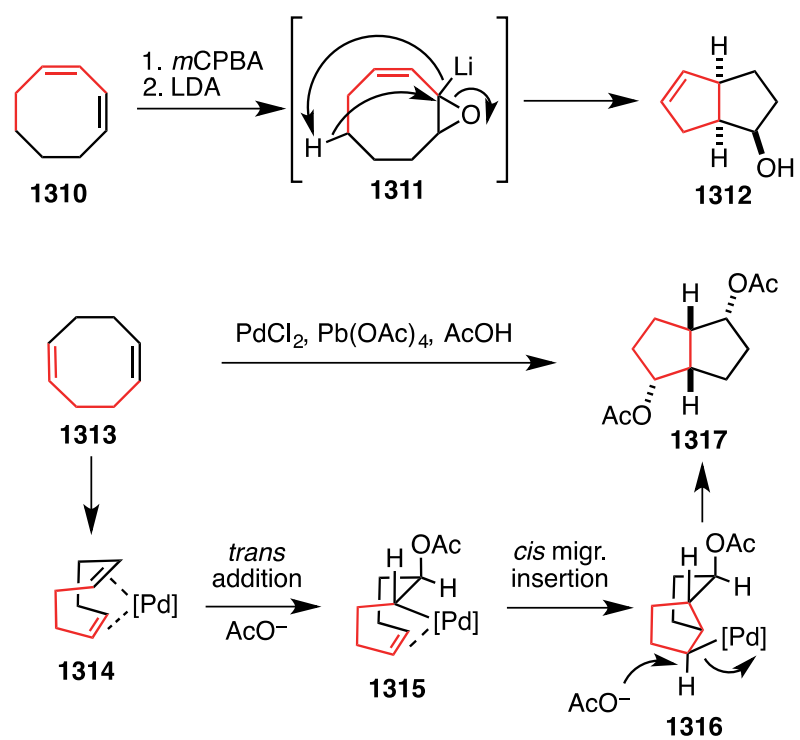

Cyclopentanones have also been used as building-block starting materials for fully functionalized cyclopentane natural products. Four separate syntheses of gymnomitrol begin with cyclopentanone 1321 (Scheme 154). This intermediate is prepared from dimethyl-3-ketoglutarate (1318). ${ }^{232}$ Acid promoted condensation with 2,3butandione (1319) gives 1320. Deoxygenation gives 1321. Ketone 1321 undergoes subsequent transformations that result in a dialkyated ketone intermediate of general structure 1322. This material is advanced to gymnomitrol. Other than ketone 1321, cyclopentane 1323 was used in an anguidine synthesis, and 1324 was used in a synthesis of coriolin.

Scheme 154.
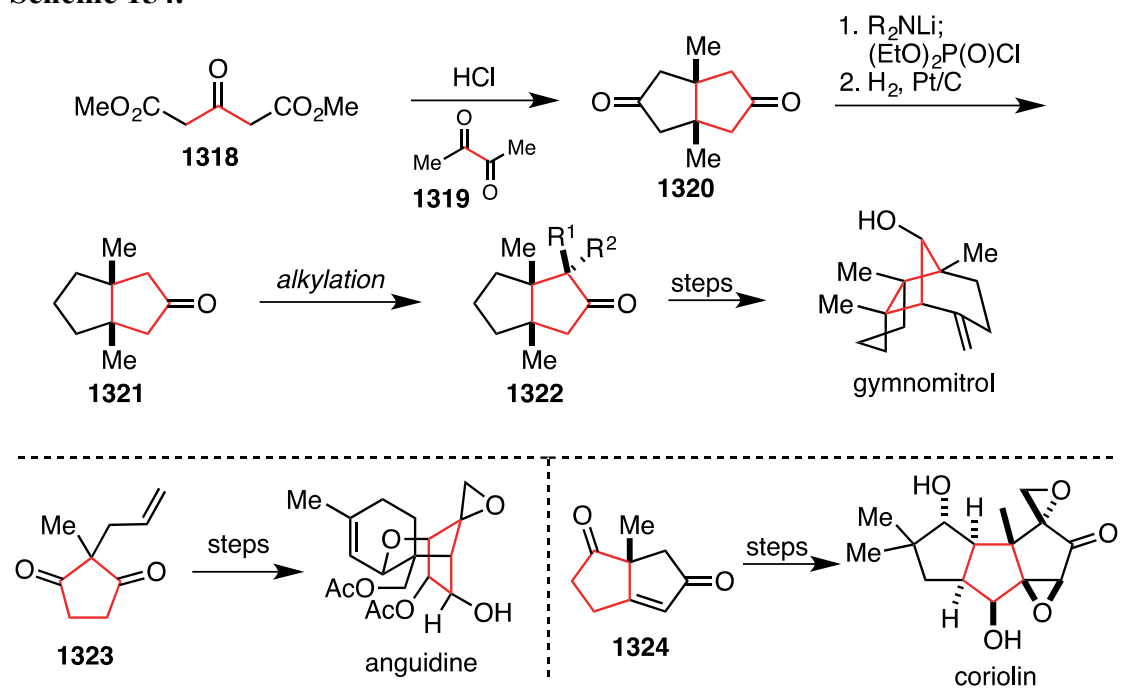

Contrasting with the strategic patterns discussed above for cyclopentadienes and cyclopentanones, the strategic use of cyclopentenone starting materials represents the most varied tactic to approach to fully functionalized cyclopentanes (Figure 3). Many substituted cyclopentenone starting materials have been used, and the enone functional group allows for a wide variety of standard transformations including enolate alkylations, additions to the carbonyl, Baylis-Hillman additions, conjugate additions, and Stork-Danheiser-type sequences.

\section{Figure 3.}

${ }^{232}$ Weiss, U.; Edwards, J. M. Tetrahedron Lett. 1968, 9, 4855-4887. 


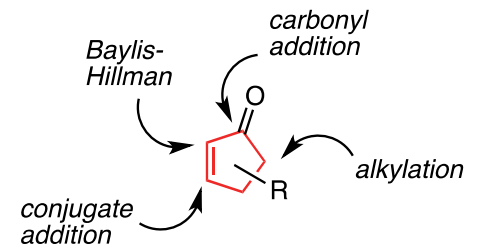

\subsection{Cyclopentadiene Functionalizations}

The Trost ${ }^{233,234}$ synthesis of $( \pm)$-allosamizoline was achieved alongside a general desymmetrization strategy for aminocyclopentitols (Scheme 155). This method of desymmetrizing meso alkenediols involves a palladiumcatalyzed ionization/cyclization reaction to prepare chiral oxazolidinones. The synthesis of allosamizoline begins with meso diol 1325 (derived from cyclopentatdiene). Premixing diol 1325 with $p$-toluenesulfonylisocyanate followed by exposure to a $\mathrm{Pd}(0)$ catalyst promoted conversion to oxazolidin-2-one $\mathbf{1 3 2 6}$ as a single product. An additional three steps were used to convert tosyl oxazolidinone 1326 to amino oxazoline 1328. Alkene 1328 was particularly unreactive, but after much experimentation epoxidation was found to be most successful with peroxytrifluoroacetic acid giving the epoxide on the convex face of the bicycle. Acidic hydrolysis gave a transdiol, which was the final required substituent on the fully functionalized cyclopentane. Hydrogenolysis of the benzyl group completed the synthesis of $( \pm)$-allosamizoline.

\section{Scheme 155. Total Synthesis of ( \pm )-Allosamizoline (Trost, 1990)}

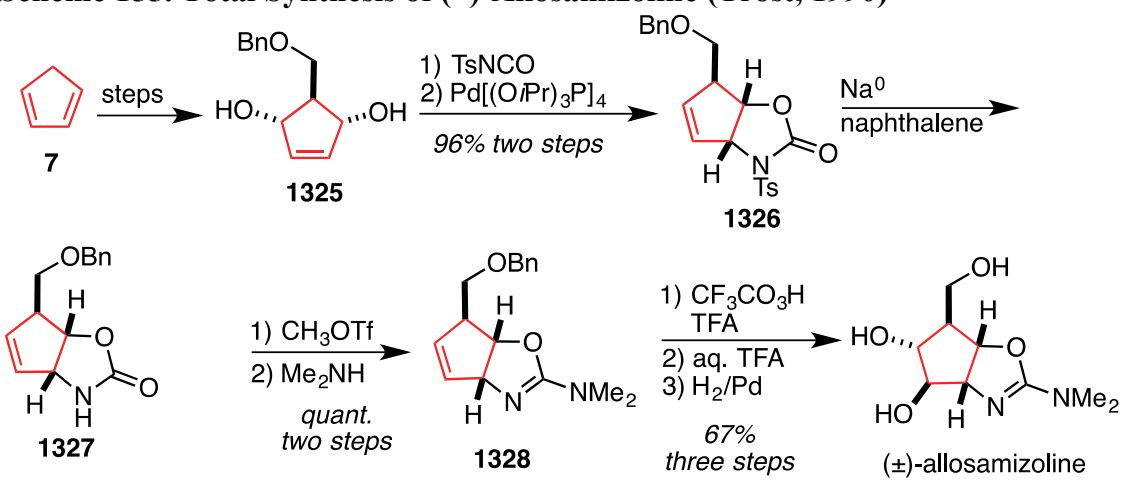

The Danishefsky ${ }^{235}$ synthesis of (-)-allosamidin (Scheme 156) began with meso-diacetate 1329 (derived from cyclopentadiene). An enzymatic desymmetrization ${ }^{236}$ with electric eel acetylcholinesterase delivers chiral alcohol 1330 with excellent enantioselectivity. Standard functional group manipulations were used to convert 1330 to carbamate 1331. Cyclization in the presence of triethylamine and trifluoroacetic anhydride gave oxazolidinone 1327. This material was also an intermediate in the Trost allosamizoline synthesis, and following the precedent of $\operatorname{Trost}^{233}$ underwent aminooxazoline formation (1328), epoxidation and hydrolysis to give diol 1332. An additional four steps were used to convert this intermediate to (-)-allosamidin complete with its disaccharide moiety.

Scheme 156. Total Synthesis of (-)-Allosamidin (Danishefsky, 1991)

\footnotetext{
${ }^{233}$ Trost, B. M.; Van Vranken, D. L. J. Am. Chem. Soc. 1990, 112, 1261-1263.

${ }^{234}$ Trost, B. M.; Van Vranken, D. L. J. Am. Chem. Soc. 1993, 115, 444-458.

${ }^{235}$ Griffith, D. A.; Danishefsky, S. J. J. Am. Chem. Soc. 1991, 113, 5863-5864.

${ }^{236}$ Deardorf, D. R.; Matthews, A. J.; McMeekin, D. S.; Craney, C. L. Tetrahedron Lett. 1986, 27, 1255-1256.
} 

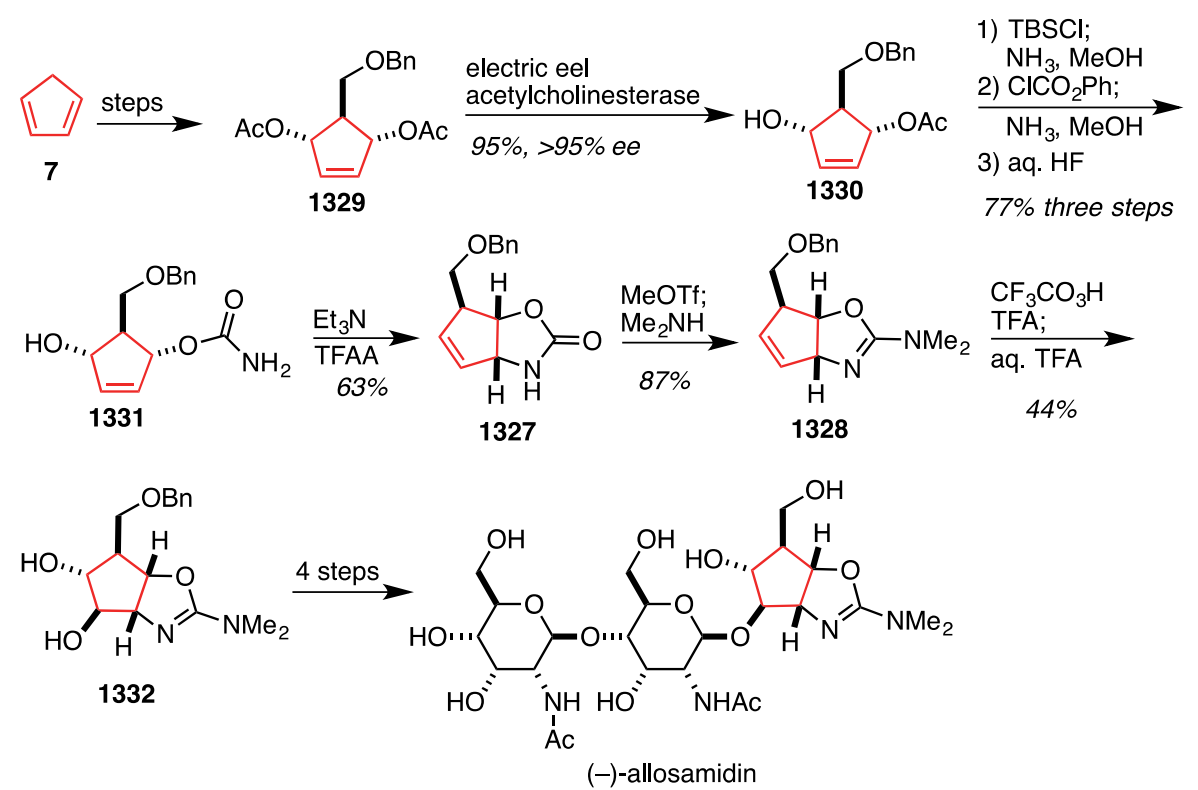

In the synthesis of $( \pm)$-allosamizoline, Ganem ${ }^{237}$ also uses a desymmetrization of a substituted cyclopentene (Scheme 157). The synthesis begins with exposure of 1333 (derived from cyclopentadiene) to NBS in wet DMSO to deliver a single bromohydrin stereoisomer 1334. Mechanistically, bromonium ion formation occurs on the face syn to the hydroxyl groups. ${ }^{238}$ Backside opening of the bromonium ion positions the incoming hydroxyl on the face anti to the resident hydroxyl groups of the substrate. Ring closure was performed with methanolic sodium carbonate to give epoxide 1335. This two-step epoxidation sequence leads to formation of the diastereomer with the epoxide positioned anti to the diol; note that peracid oxidation would lead to the syn diastereomer. Nucleophilic epoxide opening with sodium azide and subsequent reduction delivered aminotriol and fully functionalized cyclopentane 1336. An additional three steps were used to convert this material to $( \pm)$ allosamizoline.

Scheme 157. Total Synthesis of ( \pm )-Allosamizoline (Ganem, 1994)

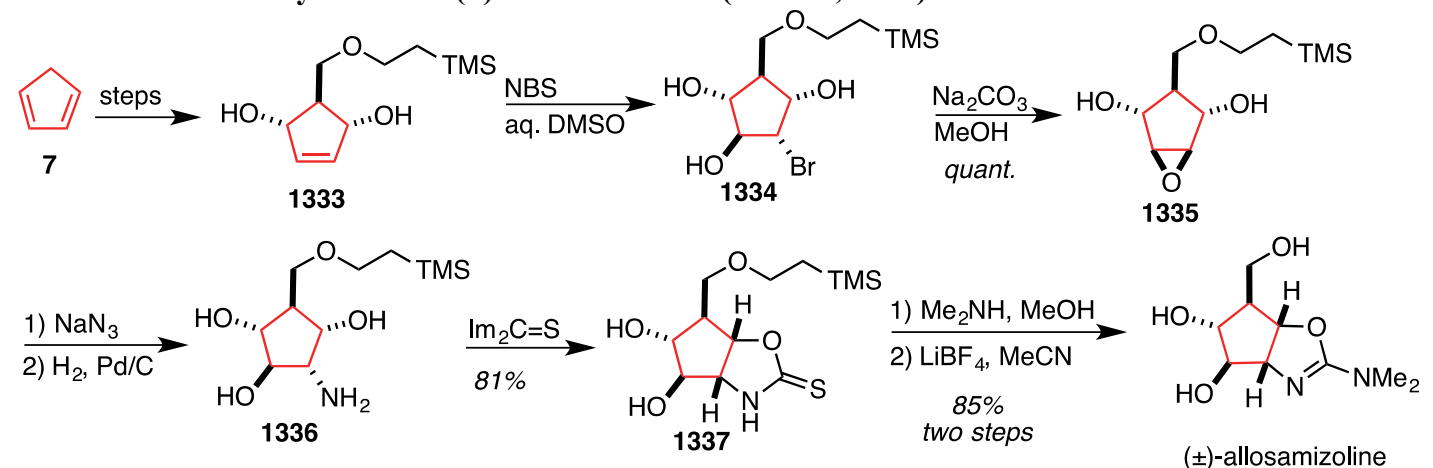

In the synthesis of (-)-allosamidin, Imperiali ${ }^{239}$ uses the enzymatic desymmetrization developed by Danishefsky $^{235}$ to obtain synthetic intermediate 1338 (Scheme 158). An addition reaction of dimethylcyanamide delivers aminoimidate 1339. This material was cyclized via a $\mathrm{Hg}(\mathrm{II})$ mediated ring closure which successfully transfers the stereochemistry to the newly formed center in the desired oxazoline 1341. Oxidative demercuration

${ }^{237}$ Goering, B. K.; Ganem, B. Tetrahedron Lett. 1994, 35, 6997-7000.

238 (a) Bannard, R. A. B.; Casselman, A. A.; Hawkins, L. R. Can. J. Chem. 1965, 43, 2398-2407. (b) Langstaff, E. J.; Hamanaka, E.; Neville, G. A.; Moir, R. Y. Can. J. Chem. 1967, 45, 1906-1920. (c) Bellucci, G.; Berti, G. Ingrosso, G.; Mastrorilli, E. Tetrahedron Lett. 1973, 14, 3911-3914.

${ }^{239}$ Shrader, W. D.; Imperiali, B. Tetrahedron Lett. 1996, 37, 599-602. 
with radical oxygen conditions leads to hydroxyl formation on the convex face of the bicycle to furnish a fully functionalized cyclopentane. ${ }^{240}$ The target (-)-allosamidin was completed in an additional four steps.

Scheme 158. Total Synthesis of (-)-Allosamidin (Imperiali, 1996)
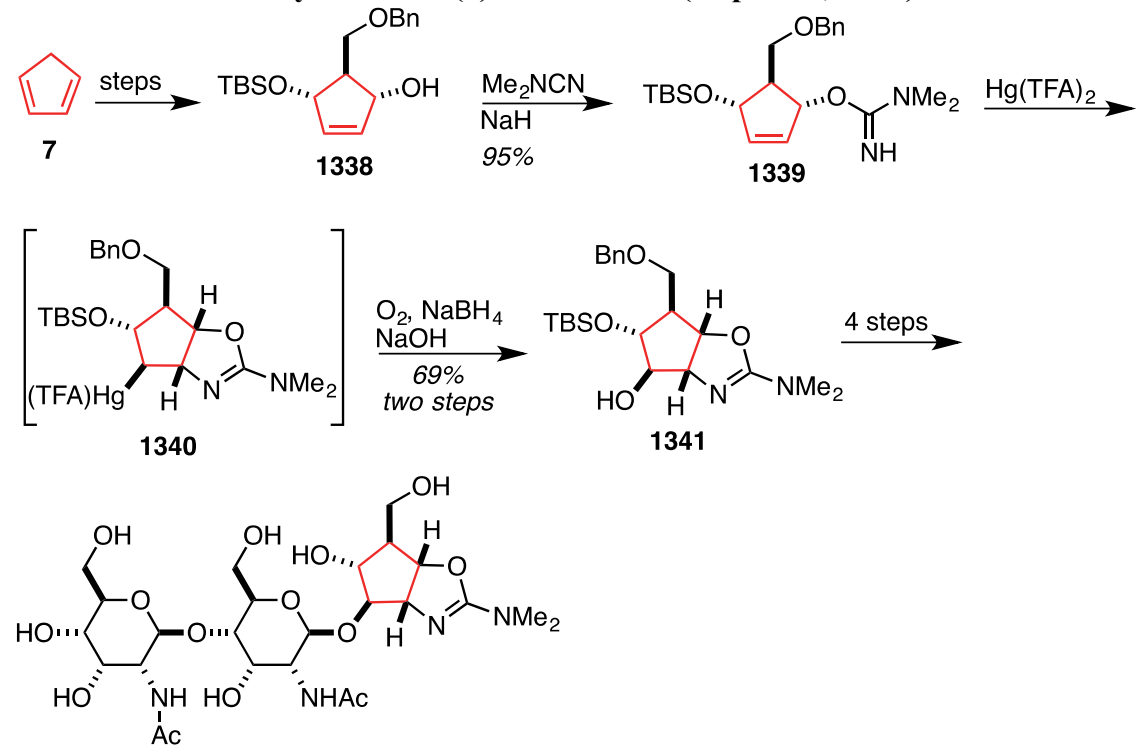

$(-)$-allosamidin

In the synthesis of (-)-allosamizoline, Iwata ${ }^{241}$ desymmetrizes meso-diol 1342 with a chiral auxiliary containing stereogenic sulfoxides (Scheme 159). The synthesis commences with benzyl protection of 1325 (derived from cyclopentadiene 11) followed by dihydroxylation on the less hindered alkene face to deliver $\mathbf{1 3 4 2}$ with good dr. Conversion of meso diol 1342 to chiral acetal 1344 was accomplished in two steps using chiral sulfoxide 1343 and Lewis acid conditons. Treatment with KHMDS and benzyl bromide induces elimination followed by benzylation to give $\mathbf{1 3 4 5}$ with high diastereoselectivity. Acidic hydrolysis of the chiral auxiliary yields 1346. Activation of the alcohol as a sulfonate, displacement with azide, and reduction produces fully functionalized aminocyclopentatol 1347 with the required stereochemistry for the target. This material was advanced to 1348 over four steps which is an intermediate previously taken to (-)-allosamizoline by Tatsuta. ${ }^{23}$

Scheme 159. Total Synthesis of (-)-Allosamizoline (Iwata, 1998)

\footnotetext{
${ }^{240}$ Note that the oxidative demercurization process is likely not stereospecific. See: Hill, C. L.; Whitesides, G. M. J. Am. Chem. Soc. 1974, 96, 870-876.

${ }^{241}$ Maezaki, N.; Sakamoto, A.; Tanaka, T.; Iwata, C. Tetrahedron: Asymmetry 1998, 9, 179-182.
} 


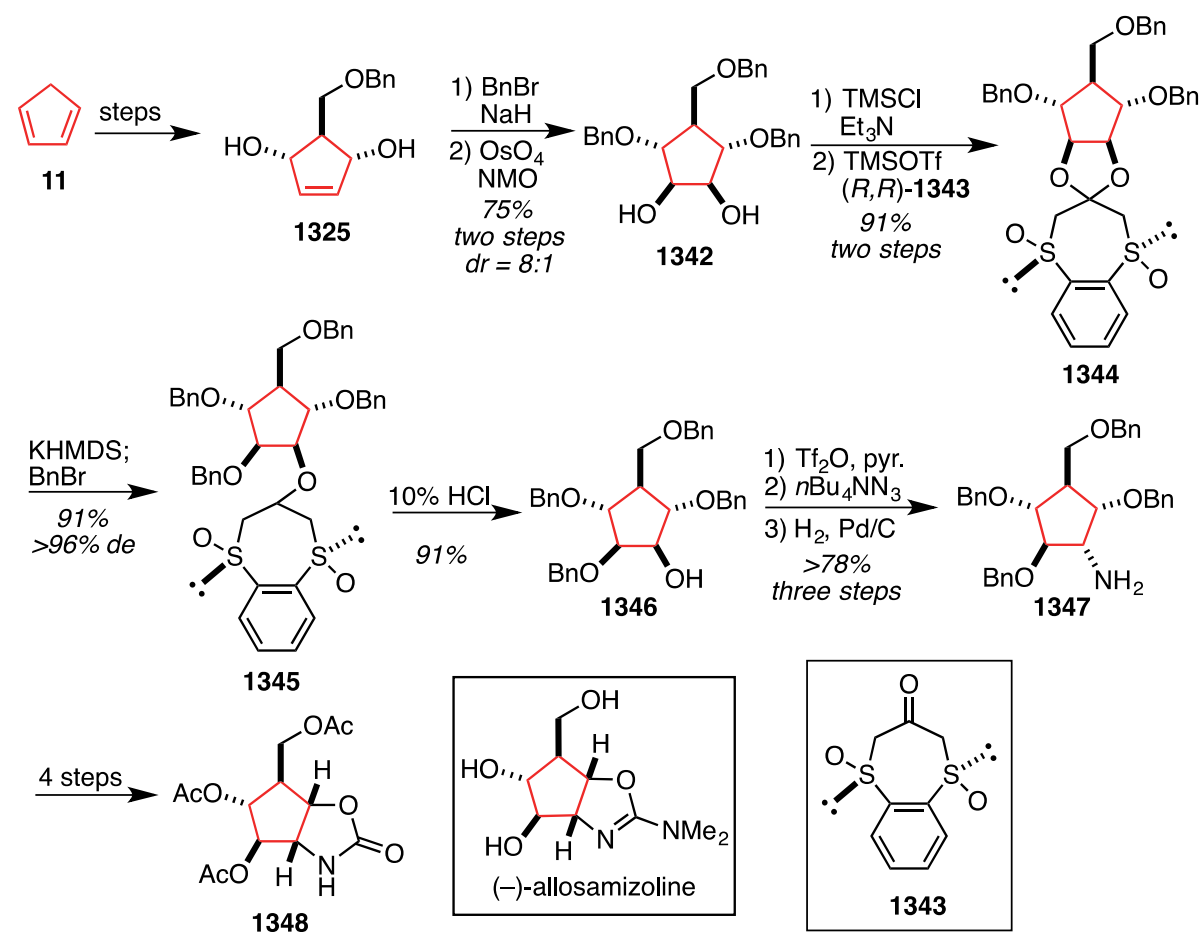

In the synthesis of $(+)$-mannostatin $\mathrm{A}, \mathrm{Ganem}^{242}$ constructs the fully functionalized cyclopentane by desymmetrizing 1-(methylthio)cyclopentadiene (1349, Scheme 160). The synthesis begins with a hetero-DielsAlder reaction of 1319 and the chiral acyl-nitroso compound derived from oxidation of 1350. This step delivers 1351 with three stereocenters, each appropriately functionalized for mannostatin A. Reduction of the $\mathrm{N}-\mathrm{O}$ bond with aluminum amalgam and subsequent acetyl protection delivers 1352. Dihydroxylation of 1352 proceeded with complete facial selectivity to give a fully functionalized cyclopentane. Such dihydroxylations have been studied $^{243}$, and the major diastereomer is believed to be the result of a directing effect on osmium from the neighboring heteroatoms. Acetylation of the diol furnishes 1353. Hydrolysis of the amide under acidic conditions provided (+)-mannostatin A as the hydrochloride salt.

\section{Scheme 160. Total Synthesis of (+)-Mannostatin A (Ganem, 1991)}

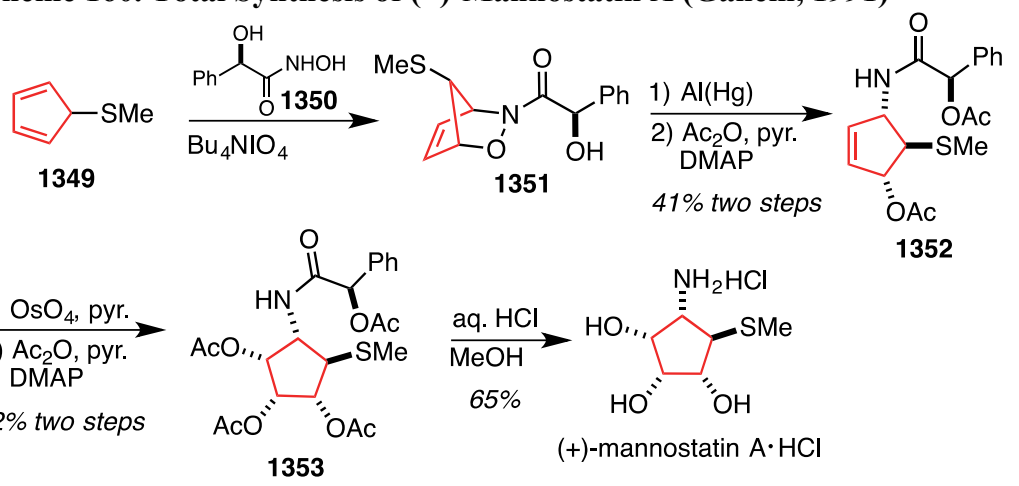

In the Trost synthesis of $( \pm)$-mannostatin $\mathrm{A}^{234}$, the desymmetrization strategy developed for $( \pm)$-allosamizoline ${ }^{233}$ was applied (Scheme 161). Beginning with meso diol 1354 (derived from cyclopentadiene) and toluenesulfonyl isocyanate, a palladium-catalyzed ionization/cyclization reaction was performed to deliver oxazolidinone 1355. Allylic oxidation with selenium dioxide introduced the required hydroxyl group found in the mannostatin A structure. The crude mixture was oxidized with Dess-Martin periodinane to an enone. Luche reduction of this enone delivers the desired $\alpha$-alcohol 1356 in a 7:1 mixture with the undesired stereoisomer. Opening of the

${ }^{242}$ King, B. S.; Ganem, B. J. Am. Chem. Soc. 1991, 113, 5089-5090.

${ }^{243}$ Trost, B. M.; Kuo, G. -H.; Benneche, T. J. Am. Chem. Soc. 1988, 110, 621-622. 
carbamate with base delivers amino diol 1357. The diol was protected as the acetonide, and substrate directed epoxidation with peroxytrifluoroacetic acid furnished 1358. Treatment with lithium methanethiolate opened the epoxide to 1359, which completes the fully functionalized cyclopentane. The regioselectivity in the epoxide opening was explained by stereoelectronic considerations. The acetonide protecting group leads to a more rigidified bicyclo[3.3.0] octane structure, and Furst-Plattner-type considerations favor pseduoaxial attack of the thiolate nucleophile with the observed regioselectivity. Removal of the sulfonate and acetonide protecting groups completes the synthesis of $( \pm)$-mannostatin A.

Scheme 161. Total Synthesis of ( \pm )-Mannostatin A (Trost, 1993)

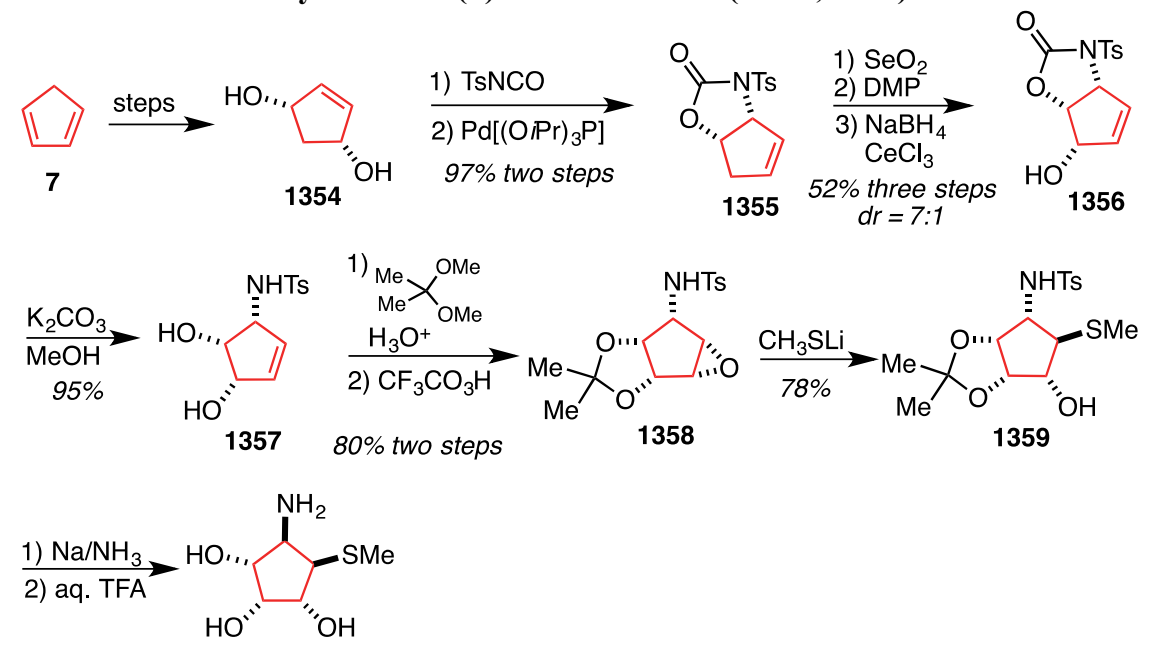

( \pm -mannostatin A

In the synthesis of $(+)$-trehazolin, Carreira ${ }^{244}$ begins with lithium cyclopentadienide (163), which undergoes double addition with $(R)$-epichlorohydrin (164) to deliver 1360 as a pure enantiomer (Scheme 162). Treatment with sodium hydride and $\mathrm{Cl}_{3} \mathrm{CCN}$ delivered trichloroacetimidate $\mathbf{1 3 6 2}$. Iodocyclization presumably gave the allylic iodide $\mathbf{1 3 6 4}$ which underwent $\mathrm{S}_{\mathrm{N}} 2$ ' addition with water to yield the observed allylic alcohol product 1365 . Sillylation of the secondary alcohol and nucleophilic opening of the imidate furnishes cyclopropylcarbinyl bromide 1366. Epoxidation from the less hindered top face with dimethyldioxirane delivers 1367. Epoxide opening with the tethered trichloroacetamide occurs under Lewis acid conditions to give 1368. Radical fragmentation of the cyclopropane to give alkene $\mathbf{1 3 6 9}$ proceeded with partial reduction of the trichloromethyl group. This material was advanced four steps to phenyl ketone 1370. A Norrish Type II cleavage of the aryl ketone gave alkene 1371. The unpurified material was dihydroxylated with $\mathrm{OsO}_{4}$ furnishing 1372 which displays the desired functionality and stereochemistry for the target. This material was advanced to $(+)$-trehazolin over three steps.

Scheme 162. Total Synthesis of (+)-Trehazolin (Carreira, 1995)

${ }^{244}$ Ledford, B. E.; Carreira, E. M. J. Am. Chem. Soc. 1995, 117, 11811-11812. 

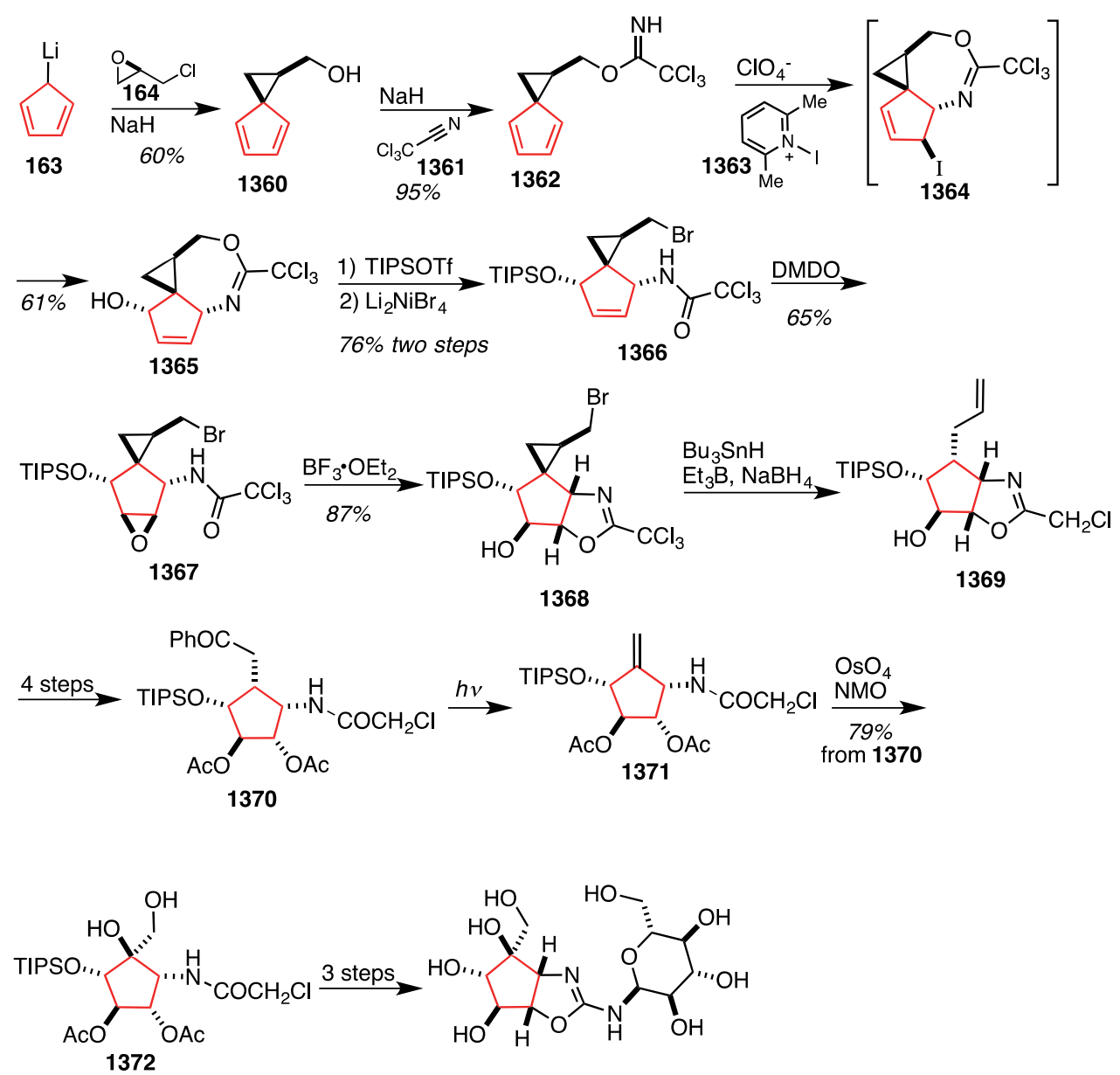

(+)-trehazolin

The Ganem ${ }^{245}$ synthesis of $(+)$-trehazolin capitalizes on the desymmetrization approach developed for their mannostatin synthesis discussed above (Scheme 163). Specifically, the Diels-Alder reaction of cyclopentadiene 1301 with the chiral nitroso species derived from 1373 gave the bicyclic product 1374 . Reduction of the $\mathrm{N}-\mathrm{O}$ bond was performed with sodium amalgam, and the product (1375) was separated from its diastereomer. Cyclopentene 1375 was epoxidized to give 1376. As before, epoxidation results in the syn diastereomer as a result of peracid coordination to the substrate. Hydrolysis of the epoxide was slow, but could be promoted using 2:1 $\mathrm{H}_{2} \mathrm{O}$ /TFA to furnish a diol albeit in a 1:1.6 ratio in favor of the undesired diastereomer. The diol was converted to the corresponding acetate 1377. At this stage, a fully functionalized cyclopentane has been constructed, but the tertiary alcohol has yet to be installed. Intermediate $\mathbf{1 3 7 7}$ was converted to exocyclic alkene 1378 using Grieco's method. ${ }^{42}$ Stereoselective dihydroxylation avoiding steric interactions with the adjacent substituents furnished diol 1379. This advanced intermediate has been synthesized by Shiozaki ${ }^{27}$ in a synthesis of trehazolin.

Scheme 163. Total Synthesis of (+)-Trehazolin (Ganem, 1998)

${ }^{245}$ Li, J.; Lang, F.; Ganem, B. J. Org. Chem. 1998, 63, 3403-3410. 


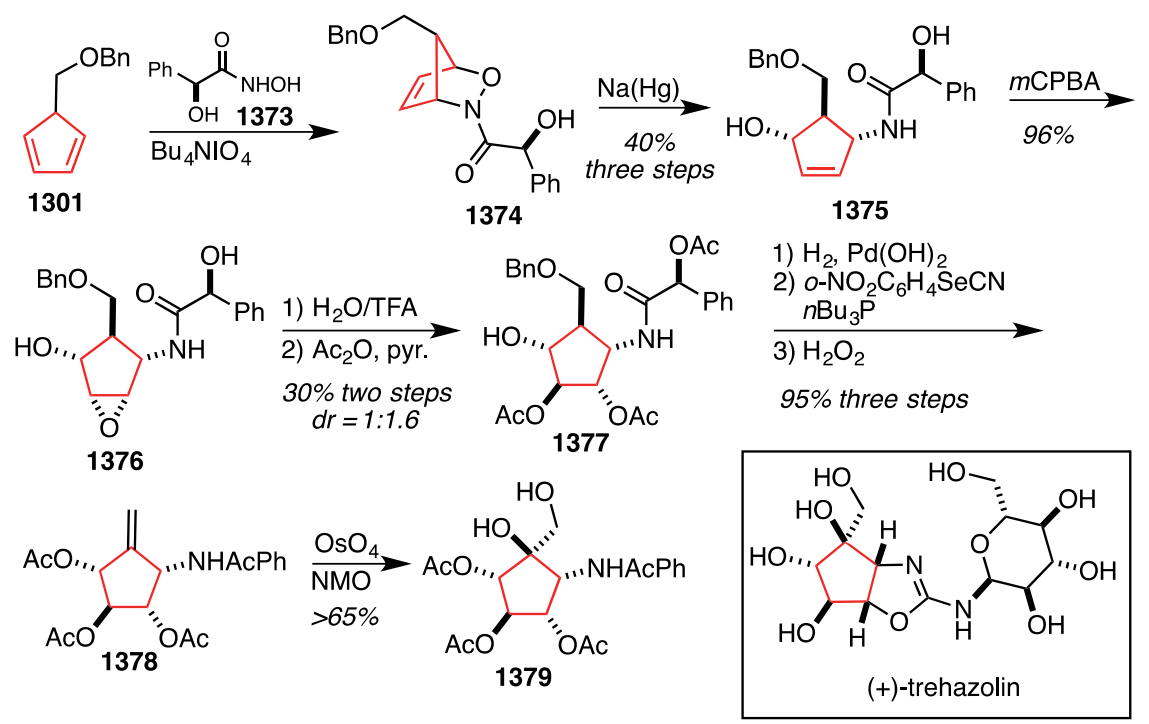

The Carreira ${ }^{246}$ synthesis of the pallambins uses two distinct processes to create each of the fully functionalized carbocycles, and this synthesis could fall into either section 11 or $\mathbf{4}$ of this review (Scheme 164). The first cyclopentane begins as fulvene and the second cyclopentane is created in a rhodium catalyzed $\mathrm{C}-\mathrm{H}$ insertion reaction. We choose to discuss the synthesis here because more than half of the fused 5,5 fully functionalized system is installed with the fulvene. The route begins with a Diels-Alder reaction of methyl acrylate with fulvene (1381), the latter of which was generated in situ by a reduction and Hoffman elimination of the bench-stable dimethylaminofulvene (1380). Cyclopropanation with conditions developed by Denmark ${ }^{247}$ delivered a tricyclic intermediate. The exocyclic olefin could be stereoselectively hydrogenated with Wilkinson's catalyst to give $\mathbf{1 3 8 3}$ as the only product. Installation of the ketone in $\mathbf{1 3 8 4}$ was accomplished by a three-step sequence involving $\alpha$-hydroxylation, ester reduction, and oxidative cleavage of a diol. Methylation of ketone $\mathbf{1 3 8 4}$ was followed by a Mukaiyama aldol reaction with acetaldehyde and Dess-Martin oxidation of the resulting $\beta$-hydroxy ketone to form 1,3-diketone 1385. Formation of the diazoketone 1387 was performed with conditions developed by Danheiser. ${ }^{248}$ Unpurified $\mathbf{1 3 8 7}$ was subjected directly to rhodium conditions, which resulted in $\mathrm{C}-\mathrm{H}$ insertion to deliver desired tetracycle 1388. This $\mathrm{C}-\mathrm{H}$ insertion reaction sets the final substitution on one fully functionalized cyclopentane and constructs the carbocyclic structure of the second key five-membered ring. The structure of pallambins $\mathrm{A}$ and $\mathrm{B}$ required that the $\mathrm{C} 8$ carbonyl served as a functional handle for the installation of the remaining substitution on the five-membered ring. This is a particularly demanding requirement due to the steric hindrance present in the substrate and the presence of two distinct ketone carbonyls. In the event, enolization of the acidic ketone was followed by formation of an enol triflate and subsequent Negishi cross-coupling to give 1389. Dipolar cycloaddition with bromonitrile oxide approached from the convex face resulting in the desired stereochemistry. This advanced intermediate (1390) contains both fully functionalized cyclopentanes with the appropriate stereochemistry for the synthesis of $( \pm)$-pallambins A and B. An additional six steps was required to complete the natural products.

Scheme 164. Total Synthesis of ( \pm )-Pallambins A and B (Carreira, 2015)

\footnotetext{
${ }^{246}$ Ebner, C.; Carreira, E. M. Angew. Chem. Int. Ed. 2015, 54, 11227-11230.

${ }^{247}$ Denmark, S. E.; Edwards, J. P.; J. Org. Chem. 1991, 56, 6974-6981.

${ }^{248}$ Danheiser, R. L.; Miller, R. F.; Brisbois, R. G.; Park, S. Z. J. Org. Chem. 1990, 55, 1959-1964.
} 
<smiles>CN(C)C=C1C=CC=C1</smiles>

1380

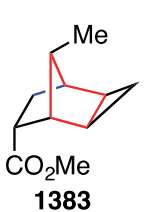

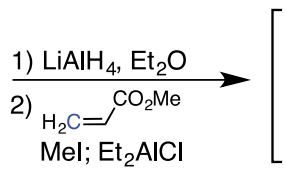

$\mathrm{Mel} ; \mathrm{Et}_{2} \mathrm{AlCl}$

1) $\mathrm{LDA},-78^{\circ} \mathrm{C}$ $\mathrm{P}(\mathrm{OEt})_{3}$, DMPU $\mathrm{O}_{2}$ bubbling 2) $\mathrm{LiAlH}_{4}, \mathrm{Et}_{2} \mathrm{O}$ 3) $\mathrm{NaIO}_{2}$

$82 \%$ three steps

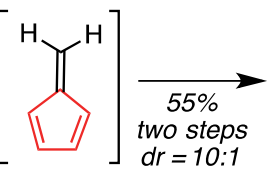

1381

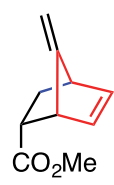

1) $\mathrm{ZnEt}_{2}$

$\mathrm{CICH}_{2}$

2) $\left[\mathrm{RhCl}\left(\mathrm{PPh}_{3}\right)_{3}\right]$

$\mathrm{H}_{2}$

$82 \%$ two steps

1382

1) $L D A-78^{\circ} \mathrm{C}$;

$\mathrm{Mel} ; \mathrm{NEt}_{3}$

3) $\mathrm{CH}_{3} \mathrm{CHO}$

$\mathrm{BF}_{3} \cdot \mathrm{OEt}_{2}$

4) $\mathrm{DMP}, t-\mathrm{BuOH}$

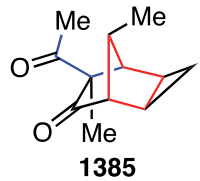

$62 \%$ four steps

1) LHMDS;
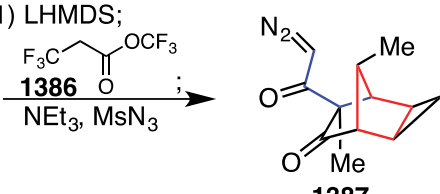

$\underset{76 \% \text { two steps }}{\left[\mathrm{Rh}_{2}(\mathrm{OAc})_{4}\right] \text { cat. }} \underset{\mathrm{CH}_{2} \mathrm{Cl}_{2}}{\mathrm{O}}$

1387
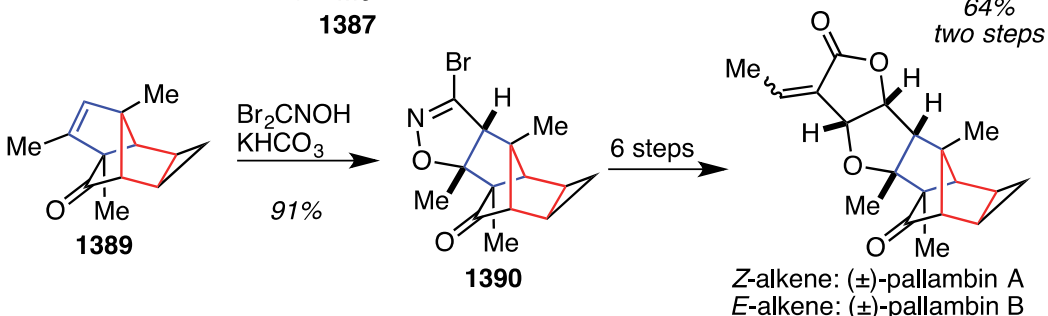

Schoop ${ }^{249}$ reported synthetic studies toward rocaglamide wherein the key five-membered carbocycle was forged using an unusual alkylation-olefination ${ }^{250}$ sequence (Scheme 165). Alkylation of phosphorane 1391 with bromoketone 1392 and subsequent deprotonation and intramolecular Wittig olefination delivers functionalized cyclopentadiene 1392. Enol ether hydrolysis under acidic conditions furnishes cyclopentenone 1394. Hydride reduction of the ketone to $\mathbf{1 3 9 5}$ occurs stereoselectively. This two-step method results in three stereocenters appropriately set for rocaglamide. Oxidative cyclization with ceric ammonium nitrate and 1,3-cyclohexanedione provided dihydrofuran 1396. The cyclization results in a fully functionalized cyclopentane, but the product does not possess the tertiary alcohol found in rocaglamide. Oxidative aromatization of this intermediate in the presence of methanol gave 1397. A two-step oxidation/reduction sequence was used to invert the alcohol stereocenter to give advanced intermediate 1399. This rocaglamide intermediate contains many of the structural features found in the natural product; however, it lacks the tertiary alcohol and methyl ether.

Scheme 165. Synthetic Studies Towards Rocaglamide (Schoop, 2000)

${ }^{249}$ Schoop, A.; Greiving, H.; Göhrt, A. Tetrahedron Lett. 2000, 41, 1913-1916.

${ }^{250}$ Hatanaka, M.; Himeda, Y.; Imashiro, R.; Tanaka, Y.; Ueda, I. J. Org. Chem. 1994, 59, 111-119. 

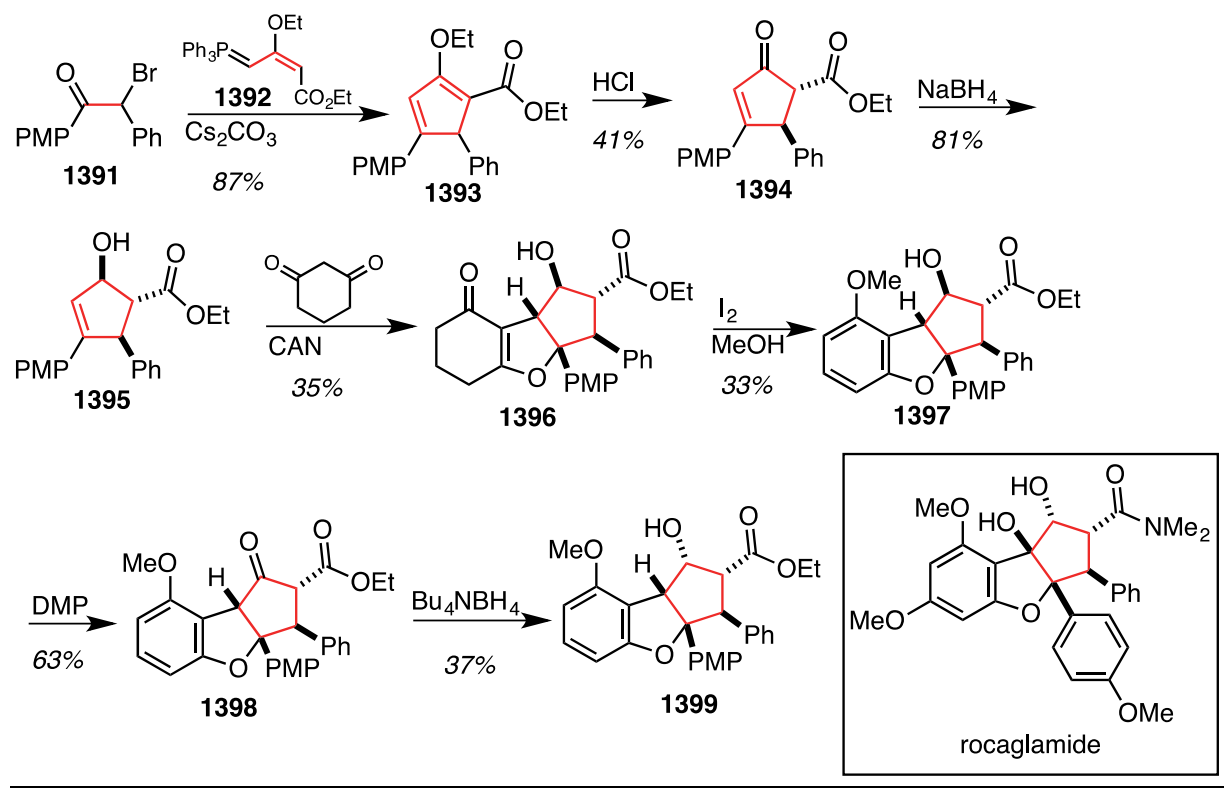

\subsection{Cyclopentene Functionalizations}

The Ikegami ${ }^{38}$ synthesis of $( \pm)$-coriolin begins with a substituted cyclopentene derived from 1,3-cyclooctadiene (1312) (Scheme 166). Benzylation of the alcohol and bromination with $N$-bromosuccinimide resulted in the regio- and stereoselective formation of a bromohydrin. Hydrogenation of the benzyl ether gave 1400. Chemoselective oxidation was possible due to the steric hindrance of the bromohydrin. Silylation of the unreacted hydroxyl furnished bromoketone 1401. Elimination of bromide with DBU yields enone 1402. Conjugate addition from the convex face and subsequent enolate protonation delivered the cis-fused ring system of 1403. This material was advanced over four steps to intermediate 1404. Silyl deprotection and oxidation of the resulting alcohol gave ketone $\mathbf{1 4 0 5}$. Formation of the thermodynamic enolate and addition to allyl bromide on the convex face gave 1406, which sets the quaternary stereogenic carbon in coriolin. A Wacker-Tsuji oxidation with $\mathrm{PdCl}_{2}$ and $\mathrm{CuCl}$ delivered a methyl ketone. The 1,4-dicarbonyl underwent an aldol condensation upon treatment with potassium tert-butoxide furnishing 1407 and the tricyclic core of coriolin. The $\alpha$-methylene functionality was installed by enolate addition to methyl iodide followed by selenation and oxidation with peroxide. Acidic removal of the pyranyl ether delivers 142. Deconjugation of the enone with tert-butoxide affords the $\beta, \gamma-$ unsaturated carbonyl. Epoxidation with peracid occurred with facial selectivity favoring the cis fusion of the five membered rings in 1408. Elimination with DBU provided the $\gamma$-hydroxy enone 130, which intersects an intermediate previously taken to coriolin by Danishefsky ${ }^{34}$ and Tatsuta. ${ }^{35}$

Scheme 166. Total Synthesis of $( \pm)$-Coriolin (Ikegami, 1981) 

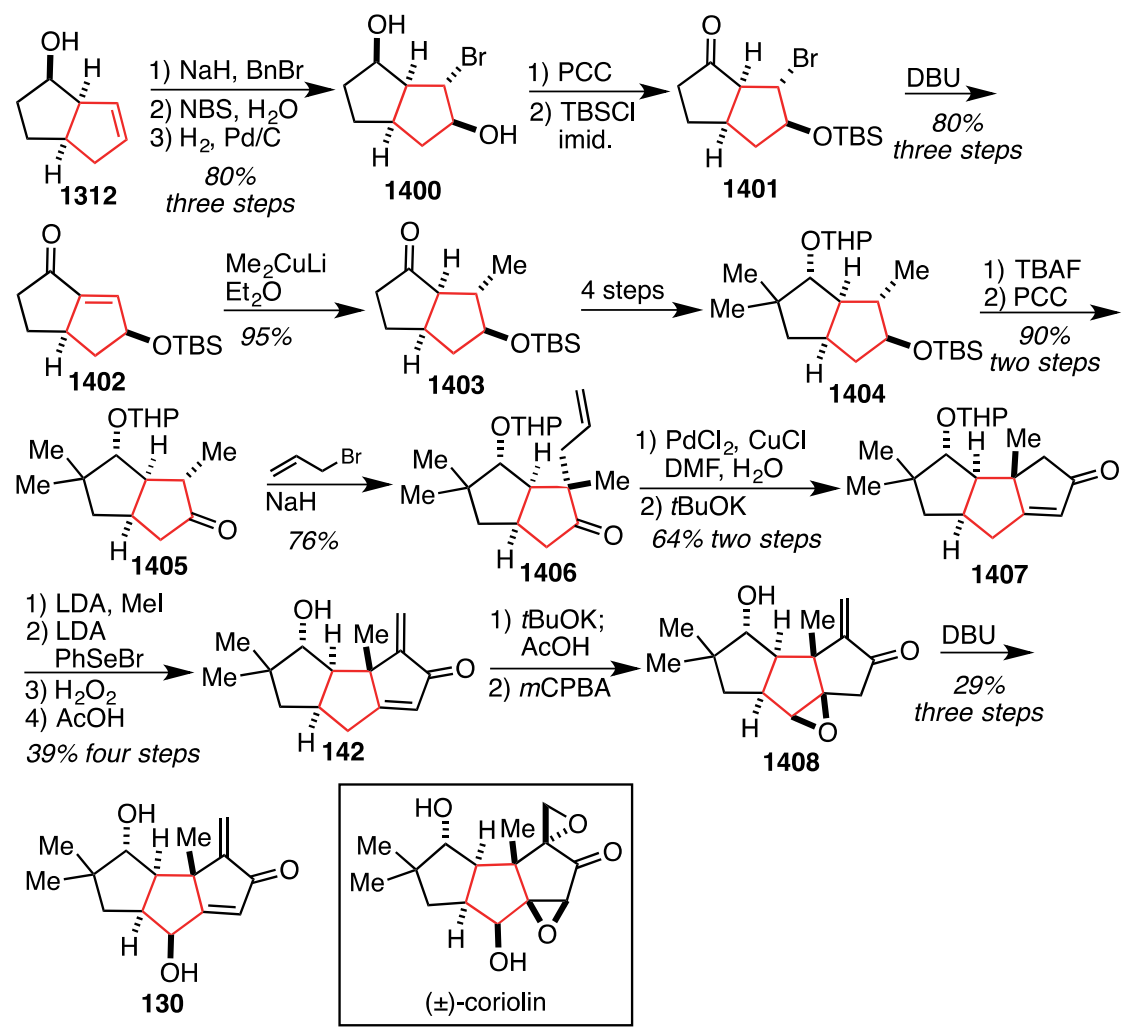

In the synthesis of (+)-mannostatin A by Knapp ${ }^{251}$, the route begins with known cyclopentene $\mathbf{1 3 0 9}$ (Scheme 167), which is obtained from D-ribonolactone as discussed in the beginning of this section. This material could be advanced over two steps to imidothioate 1409. Iodocyclization gave oxazolidinone 1410. Removal of the PMB protecting group and substitution with methyl thiolate gave 1411, which contains a fully functionalized cyclopentane. Substitution reaction proceeded with retention of configuration, likely as a result of anchimeric assistance from the vicinal nitrogen atom. Hydrolysis of the oxazolidinone and ketal groups over two steps completed the synthesis of (+)-mannostatin A.

\section{Scheme 167. Total Synthesis of (+)-Mannostatin A (Knapp, 1991)}
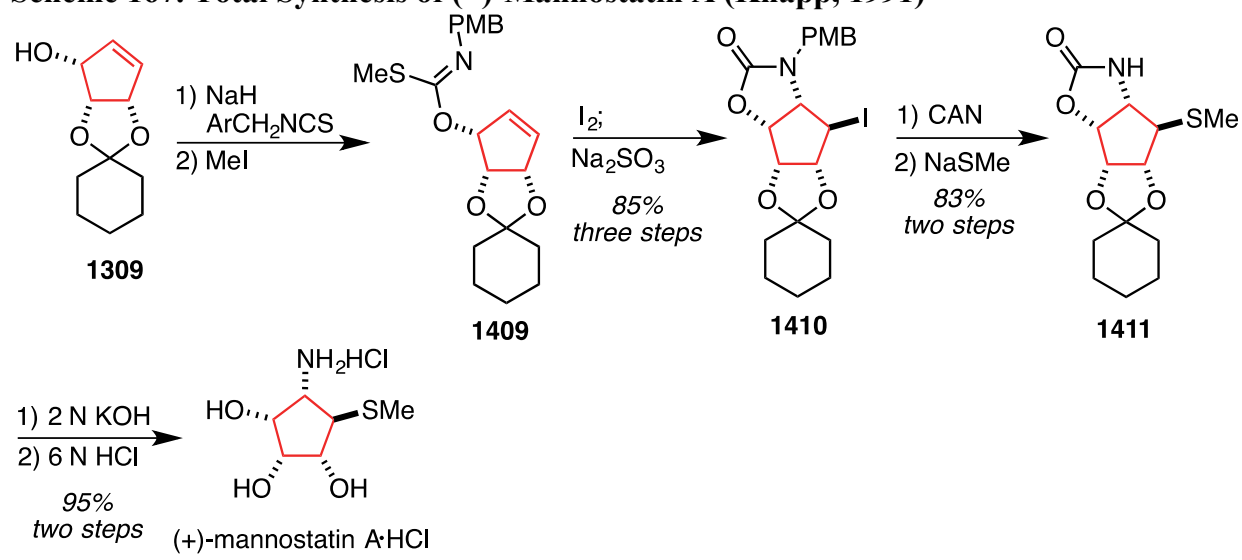

${ }^{251}$ Knapp, S.; Dhar, T. G. M. J. Org. Chem. 1991, 56, 4096-4097. 
Fuchs $^{252}$ synthesized (+)-mannostatin A using the same cyclopentene intermediate (1309) as Knapp ${ }^{251}$ (Scheme 168). The allylic alcohol $\mathbf{1 3 0 9}$ was converted to a trichloroacetimidate. After substantial experimentation, it was found that addition of DIEA and methanesulfenyl triflate in portions led to the isolation of $N$-sulfenylimidate 1412. Resubjection of 1412 to similar conditions led to cyclization delivering 1413. While cyclized product 1413 could be achieved from the acetimidate in a single step, superior yields were obtained if performed as a two-step transformation. Acidic methanolysis of $\mathbf{1 4 1 3}$ delivered (+)-mannostatin A as the hydrochloride salt.

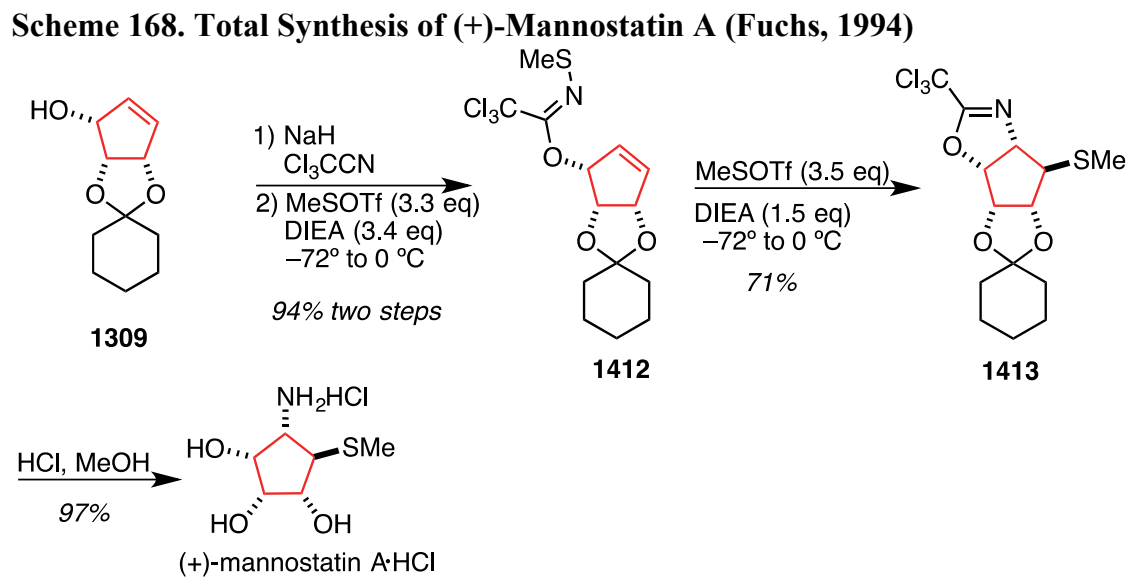

The Mehta ${ }^{253}$ synthesis of (+)-kelsoene begins with chiral diacetate 1317 (Scheme 169), which is available from 1,5-cyclooctadiene (1313) as discussed in the intro. Acetate hydrolysis and oxidation of the corresponding diol gave dione 1414. Wittig olefination desymmetrizes the diketone and hydrogenation occurs from the convex face of the diquinane to deliver 1415. Enolization under kinetic conditions and silylation gave the corresponding enol ether, which underwent Saegusa oxidation to give enone 1416. Subjection of 1416 to methyllithium gave the 1,2addition product, and treatment with PCC led to oxidation with allylic transposition (1417). A [2+2]photocycloaddition with trans-1,2-dicholoroethylene from the convex face of the diquinane furnishes tricyclic intermediate 1418. Three additional steps featuring a reductive dechlorination were used to form intermediate 1419. Homologation of the ketone by olefination following Kluge's method ${ }^{62}$ (to 1420) and hydrolysis gave aldehyde 1421. The favored diastereomer positions the aldehyde on the less hindered face of the polycyclic framework. Four additional steps were required to complete the synthesis of $(+)$-kelsoene.

Scheme 169. Total Synthesis of $(+)$-Kelsoene (Mehta, 2001)

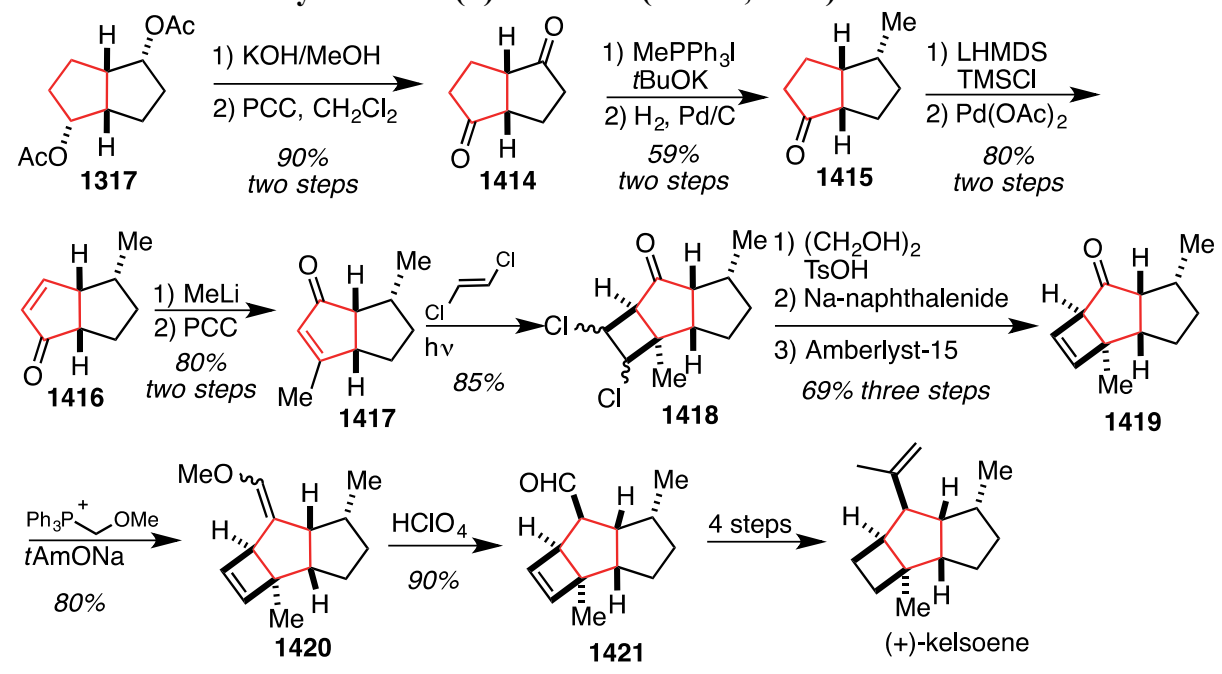

${ }^{252}$ Li. C.; Fuchs, P. L. Tetrahedron Lett. 1994, 35, 5121-5124.

253 (a) Mehta, G.; Srinivas, K. Tetrahedron Lett. 2001, 42, 2855-2857. (b) Mehta, G.; Srinivas, K. Synlett. 1999, 5, $555-556$. 
A synthesis of (-)-kelsoene was completed by Schulz ${ }^{254}$ beginning with Mehta's intermediate 1419 (Scheme 170) ${ }^{255}$ A Corey-Chaykovsky epoxidation delivered 1422. Hydrogenation the cyclobutene proceeded with concomitant epoxide pinacol rearrangement to give aldehyde $\mathbf{1 4 2 3}$ as the major stereoisomer. Addition of methyllithium and oxidation of the resulting alcohol gave methyl ketone 1424. Treatment with base epimerized the ketone stereocenter delivering a fully functionalized cyclopentane containing the desired stereochemistry for the natural product. Olefination with the Petasis reagent completed (-)-kelsoene.

\section{Scheme 170. Total Synthesis of (-)-Kelsoene (Schulz, 2001)}

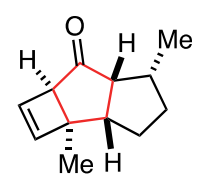

1419

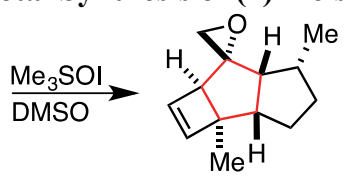

1422

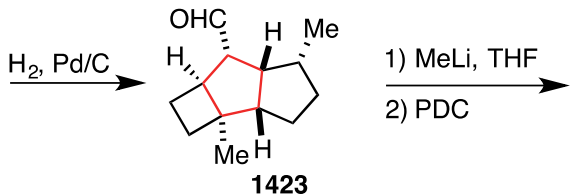

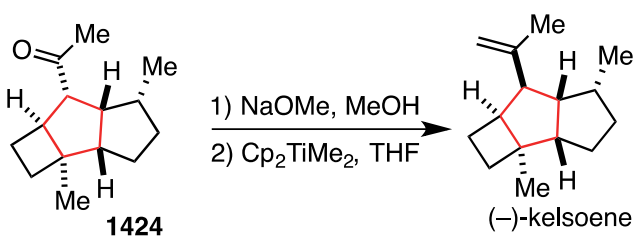

The Danishefsky ${ }^{256}$ synthesis of $( \pm)$-gelsemine begins with epoxidation of the bicyclo[2.2.1]heptadiene 1425 to give 1426 (Scheme 171). Alumina-promoted rearrangement delivers the aldehyde 1427. Through a divinylcyclopropane-Cope rearrangement, the carbocyclic core of gelsemine (1430) was established. Three of the cyclopentane stereocenters have been set at this point in the synthesis. Hydroboration-oxidation of the cyclopentene proceeded with complete chemoselectivity and excellent regioselectively to deliver $\mathbf{1 4 3 1}$. The chemoselective preference arises from the greater strain in the cyclopentene double bond. The regiochemical preference arises from homo-allylic stabilization of partial carbocation character at $\mathrm{C} 16$ that developed during an asynchronous hydroboration. Swern oxidation of the resulting alcohol delivers a ketone. Formation of the silyl enol ether and treatment with Eschenmoser's salt gives $\beta$-amino ketone 1432, and a subsequent Hofmann elimination delivers enone 1433. Luche reduction from the less hindered face gives an allylic alcohol, and a second hydroboration-oxidation sequence generates diol 1434. Selective activation of the primary alcohol was followed by oxetane formation (1435). Ether cleavage and Swern oxidation gave cyclopentanone 1436. A Horner-Wadsworth-Emmons olefination of the ketone led to a mixture of separable alkene isomers (3:2 favoring the $Z$-alkene). Both alkene isomers were independently reduced with DIBAL to give 1437. A Johnson-Claisen rearrangement was performed to install the quaternary stereocenter of intermediate 1438. Formation of the acyl azide and subsequent Curtius rearrangement (Shiori modification ${ }^{257}$ ) gave rise to carbamate 1439. The oxetane was activated with boron trifluoride etherate and addition by the carbamate nitrogen delivered 1440. This advanced intermediate contains the fully functionalized cyclopentane found in ( \pm )-gelsemine. An additional 17 steps were used to complete the synthesis of the natural product.

Scheme 171. Total Synthesis of $( \pm)$-Gelsemine (Danishefsky, 2002)

\footnotetext{
${ }^{254}$ Fietz-Razavian, S.; Schulz, S.; Dix, I.; Jones, P. G. Chem. Commun. 2001, 2154-2155.

${ }^{255}$ Concomitantly with the synthetic studies towards (-)-kelsoene, Schulz also published degradation studies that determined the absolute configuration of the natural product.

${ }^{256}$ Ng. F. W.; Lin, H.; Danishefsky, S. D. J. Am. Chem. Soc. 2002, 124, 9812-9824.

${ }^{257}$ Shiori, T.; Ninomiya, K.; Yamada, S. J. Am. Chem. Soc. 1972, 94, 6203-6205.
} 

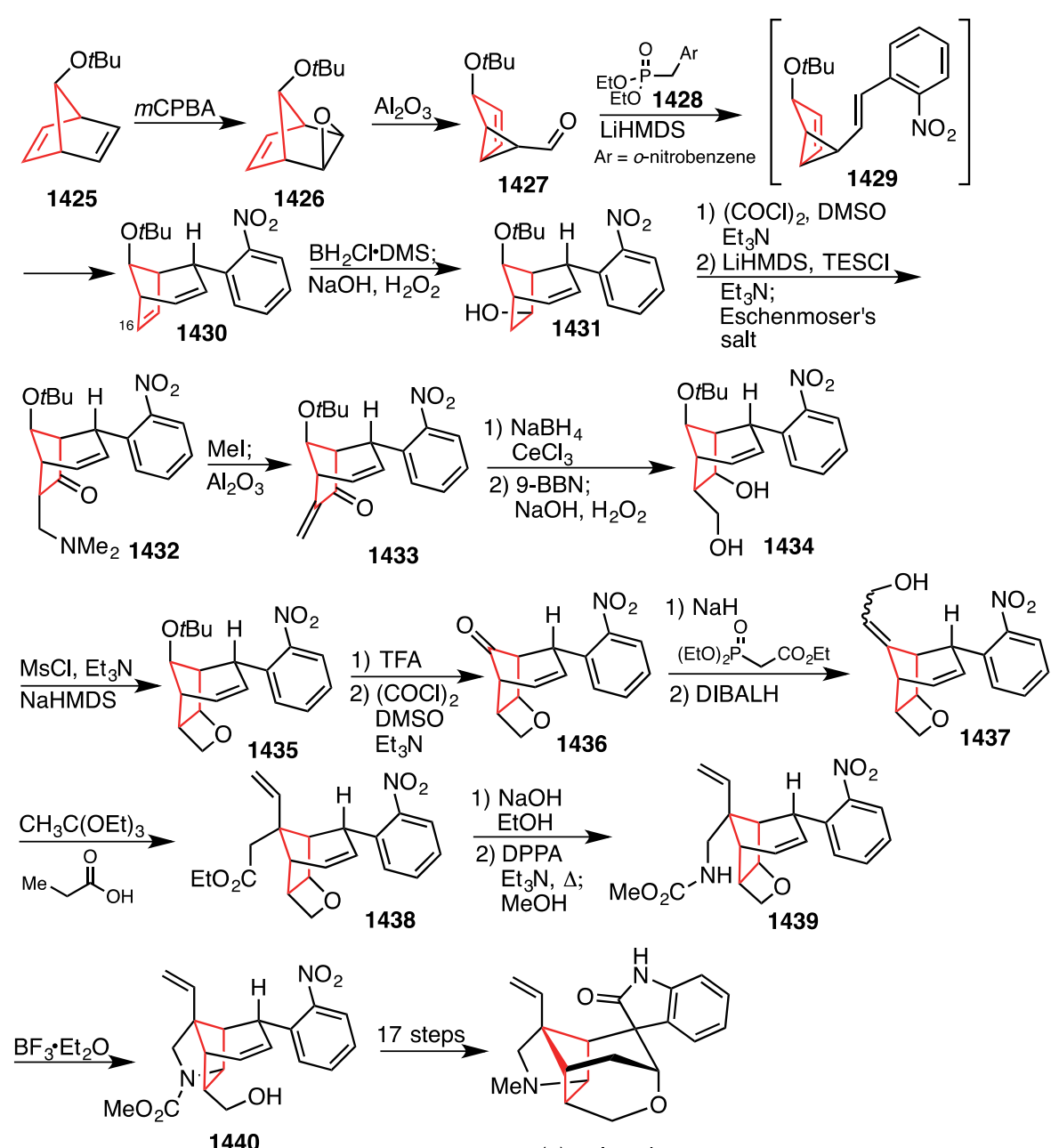

$( \pm)$-gelsemine

In the synthesis of (-)-sulcatine $\mathrm{G}$ (Scheme 172), Mehta ${ }^{258}$ utilizes a similar strategy developed for their kelsoene synthesis discussed above. Desymmetrization of the ring system 1441 was accomplished over five steps to give cyclopentanol 1442. Alcohol oxidation yielded ketone 1443. Acylation with dimethyl carbonate, selenation, and oxidation provides enone 1444. The required methyl group for sulcatine $\mathrm{G}$ was introduced by cuprate addition, and the resulting diketone was re-oxidized to form 1445. Cyclobutane 14146 was obtained by a [2+2]photocycloaddition with trans-1,2-dichloroethylene. Addition occurred exclusively from the less hindered convex face of the diquinane ring system to deliver tricyclic intermediate 14146. Treatment of the diastereomeric chloride mixture with DIBAL resulted in ketone reduction with low diastereoselectivity $(d r=55: 45)$. The complicated mixture of diastereomers was subjected to reductive dechlorination and hydrogenation to produce a mixture of alcohol epimers 14147. Selective silylation of the primary alcohol was followed by acetate formation, at which time the desired diastereomer (1448) could be purified. Advanced intermediate 1448 contains the fully functionalized cyclopentane with the desired stereochemistry for (-)-sulcatine G. An additional eight steps was required to complete the synthesis of the natural product.

Scheme 172. Total Synthesis of (-)-Sulcatine G (Mehta, 2002)

${ }^{258}$ Mehta, G.; Sreenivas, K. Tetrahedron Letters 2002, 43, 3319-3321. 

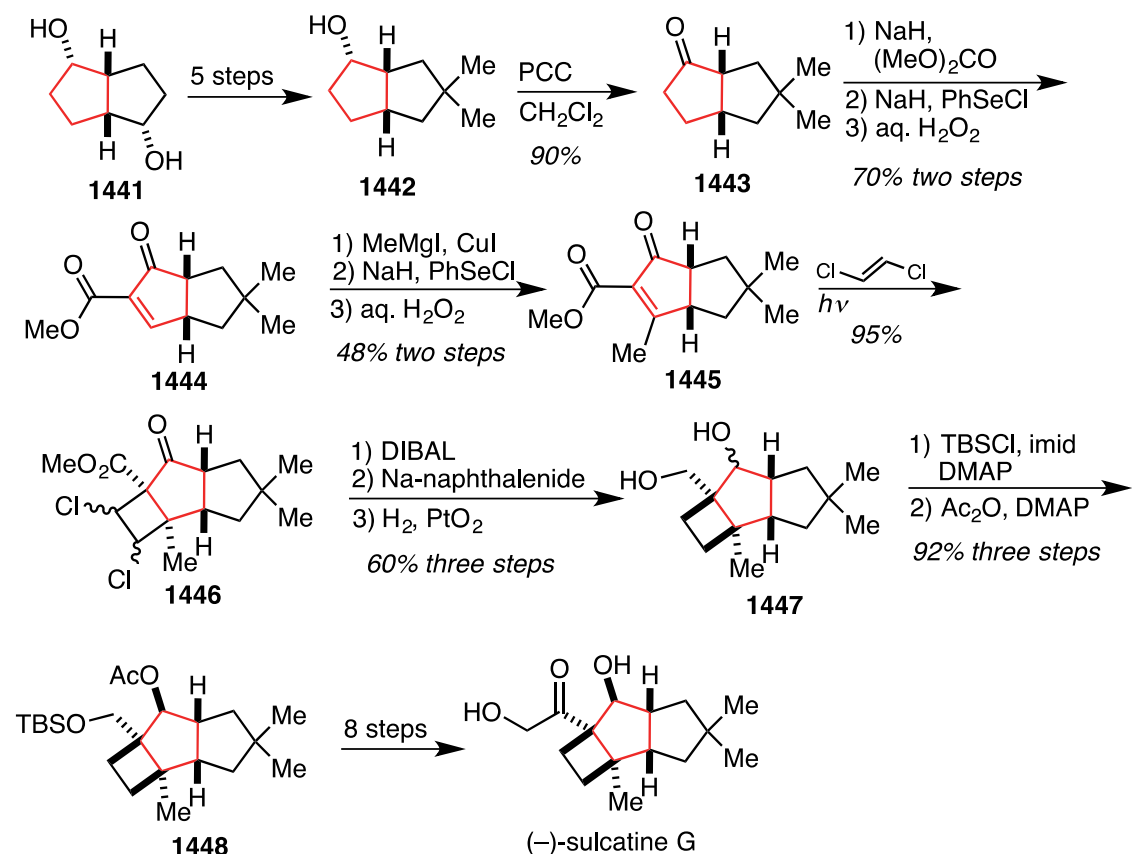

$(-)$-sulcatine $G$

The synthesis of epoxyqueuosine by Carell ${ }^{259,260}$ begins with cyclopentene intermediate 1309 (Scheme 173). A Mitsunobu reaction with hydrazoic acid followed by a Staudinger reduction delivers allyl amine 1449. Protection of the nitrogen as the trichloroacetimide was followed by acetonide cleavage and silylation with TBSCl to give 1450. Epoxidation occurs on the diastereoface opposite the silyl ethers to furnish fully functionalized cyclopentane 1451. This intermediate could be advanced to epoxyqueuosine over an additional three steps.

Scheme 173. Total Synthesis of Epoxyqueuosine (Carell, 2013)<smiles>O[C@@H]1C=C[C@@H]2OC3(CCCCC3)O[C@@H]21</smiles>

1309

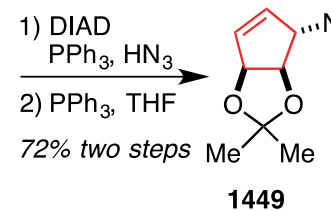

1449

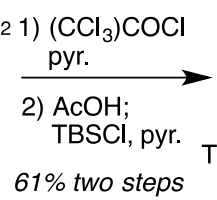

$61 \%$ two steps

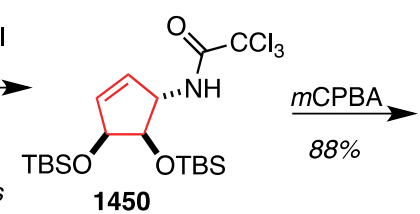

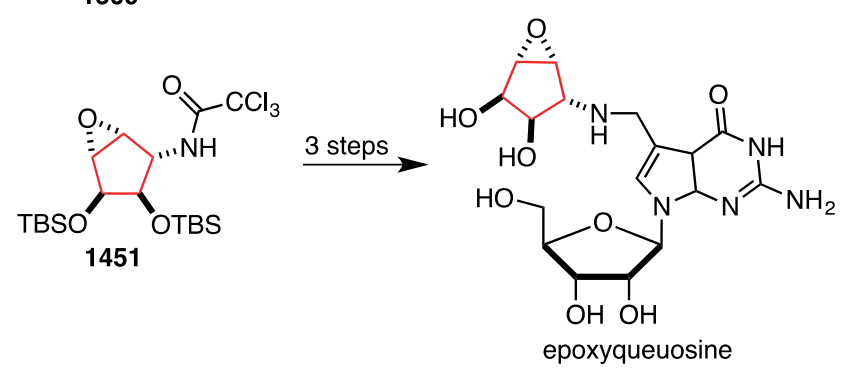

\subsection{Cyclopentanone Functionalizations}

The sesquiterpene $\left( \pm\right.$ )-gymnomitrol was synthesized by Paquette ${ }^{261}$ beginning with ketone 1321 (Scheme 174). Methylenation was accomplished by heating with formalin and $N$-methylanilinium trifluoroacetate to give 1452. Copper-catalyzed conjugate addition of the vinyl Grignard species 1453 was followed by methylation on the convex face of the bicycle to obtain 1454. Epoxidation gave silyl-oxirane 1455, which was hydrolyzed to

259 Thoma, I.; Carell, T. Eur. J. Org. Chem. 2013, 4483-4485.

${ }^{260}$ Epoxyqueuosine has not been isolated from natural sources; however, it is the anticipated biosynthetic precursor to the known natural product queuosine.

${ }^{261}$ Paquette, L. A.; Han, Y. -K. J. Am. Chem. Soc. 1981, 103, 1831-1835. Han, Y. -K.; Paquette, L. A. J. Org. Chem. 1979, 44, 3731-3733. 
aldehyde 1456. Aldol addition of the keto-aldehyde gave the tricyclic scaffold of gymnomitrol. The secondary alcohol stereocenter was then oxidized to diketone 1457. Methylation of the less hindered ketone was followed by dehydration with $\mathrm{POCl}_{3}$ to give an exocyclic alkene. Finally, stereoselective reduction of the ketone delivered a fully functionalized cyclopentane and completed the synthesis of ( \pm )-gymnomitrol.

Scheme 174. Total Synthesis of ( \pm )-Gymnomitrol (Paquette, 1979)

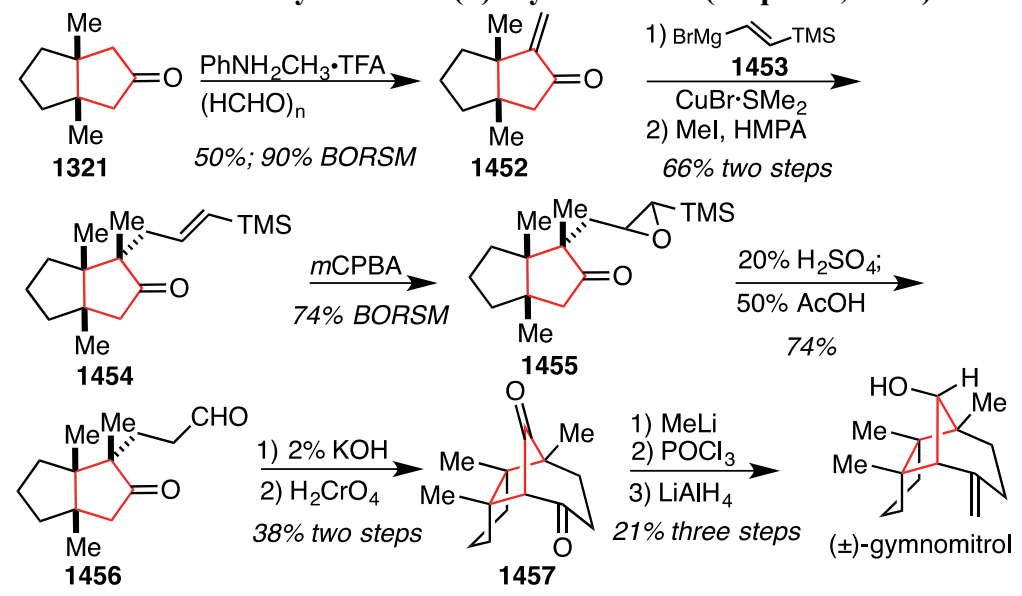

In the synthesis of $( \pm)$-gymnomitrol by Coates ${ }^{121}$, a condensation of cyclopentanone $\mathbf{1 3 2 1}$ with ethyl formate delivered intermediate 1458 (Scheme 175). Reaction with hydroxylamine in sodium methoxide led to formation of the $\alpha$-cyano ketone $\mathbf{1 4 5 9}$ as an epimeric mixture. Upon heating with acrolein diethyl acetal (1460), alkylation occurred from the convex face of the bicycle yielding the ethoxyallyl ketone 1461. Refluxing with ethylene glycol and acid afforded 1,3-dioxolane 1462. Reductive decyanation was accomplished with lithium in liquid ammonia, and the resulting enolate ion was trapped as its silyl ether. Regeneration of the lithium enolate with methyllithium was followed by stereoselective methylation again on the convex face to give $\mathbf{1 4 6 3}$, which contains the three vicinal quaternary carbon stereocenters of gymnomitrol. Acid catalyzed removal of the acetal gave an aldehyde, which was resistant to a planned aldol cyclization. Chromium oxidation gave the corresponding carboxylic acid 1464. Lactonization occurred upon treatment with acetic anhydride to deliver 1465. Reduction of the ester carbonyl with DIBAL gave an aluminum hemiacetal, which fragmented to an aluminum enolate and a tethered aldehyde. Aldol cyclization successfully occurred under these conditions, presumably due to the enhanced reactivity of the enolate (compared with the unsuccessful aldol reaction of $\mathbf{1 4 6 3}$ under acidic conditions) Oxidation of the aldol product delivered bridged diketone 1466. A regioselective methylation, dehydration and carbonyl reduction completed the synthesis of $( \pm)$-gymnomitrol.

Scheme 175. Total Synthesis of ( \pm )-Gymnomitrol (Coates, 1982) 


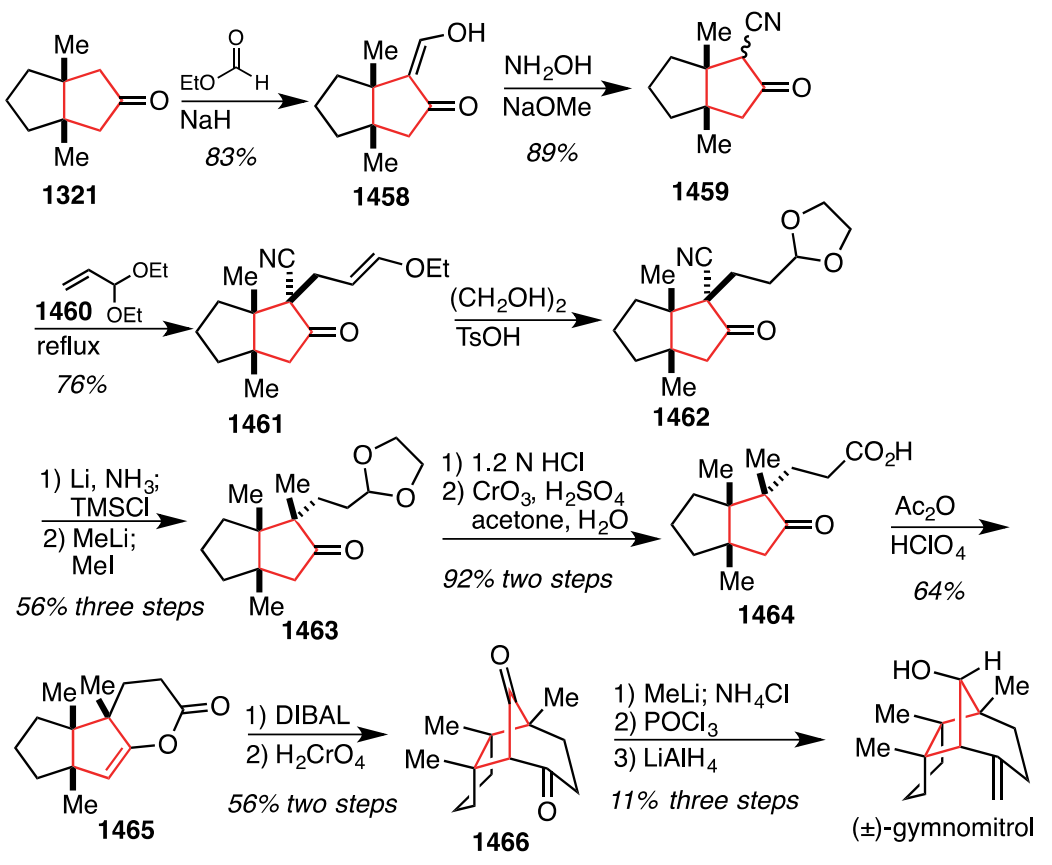

Synthetic efforts towards gymnomitrol and related compounds was performed by Imanishi (Scheme 176). ${ }^{262}$ Beginning with cyclopentanone starting material 1321, the lithium enolate was formed and added to diphenyldisulfide. Reformation of the lithium enolate and $O$-allylation delivers allyl vinyl ether 1467. Heating in toluene gave rise to a Claisen rearrangement, which occurred on the convex face to afford 1468 as a single product. Desfulfurization was accomplished with dissolving metal conditions to give the corresponding enolate. Methyl iodide approaches the generated enolate on the less hindered convex face to give a single diastereomer 1469. Ozonlysis gives an aldehyde that was protected as the acetal (1470). Reduction of the ketone, mesylate formation and elimination gave cyclopentene 1471. A four-step sequence converted the protected aldehyde to a diazoketone (1472). Upon exposure to copper(II) acetylacetonate, cyclopropanation occurred yielding 1473. Cleavage of the cylopropane ring with sulfuric acid in methanol gave fully functionalized cyclopentane 1474 bearing the desired stereochemistry found in gymnomitrol.

Scheme 176. Synthetic Studies Towards Gymnomitrol (Imanishi, 1993)

\footnotetext{
${ }^{262}$ Imanishi, T.; Yamashita, M.; Hirokawa, Y.; Tanaka, T.; Miyashita, K.; Iwata, C. Chem. Pharm. Bull. 1993, 41,
} 1695-1697. 


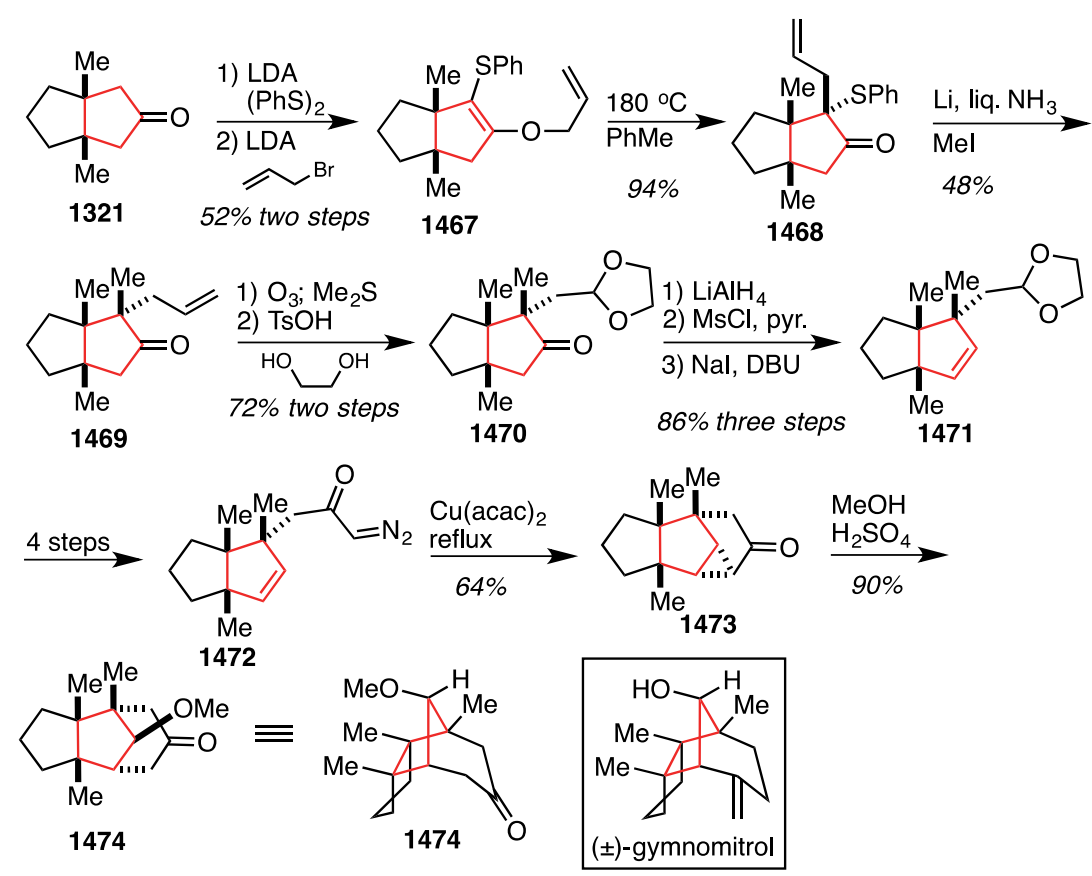

The synthesis of $( \pm)$-gymnomitrol by Snider ${ }^{263}$ begins with ketone 1321 (Scheme 177). Alkylation of the bicyclic ketone with LHMDS and 1-iodo-2-butyne (1475) delivers a mixture of monoalkylated epimers along with some dialkylated material. Treatment of the mixture with standard alkylation conditions gave 1476 in good yield over the two steps. Exposure to potassium 3-aminopropanamide (1477) induces an alkyne-zipper isomerization, and silylation of the acetylide gives 1478. The key step in the synthesis is an oxidative free-radical cyclization to establish the tricyclic architecture of gymnomitrol. The oxidative cyclization of 1478 with $\mathrm{Mn}(\mathrm{OAc})_{3}$ gives 1479 as a mixture of $(E)$ - and $(Z)$-vinyl silanes. Desilylation of the mixture was effected by treatment with hot acetic acid to give exocyclic alkene 1480. Completion of $( \pm)$-gymnomitrol is accomplished by ketone reduction with sodium borohydride delivering the fully functionalized cyclopentane.

\section{Scheme 177. Total Synthesis of ( \pm )-Gymnomitrol (Snider, 1997)}
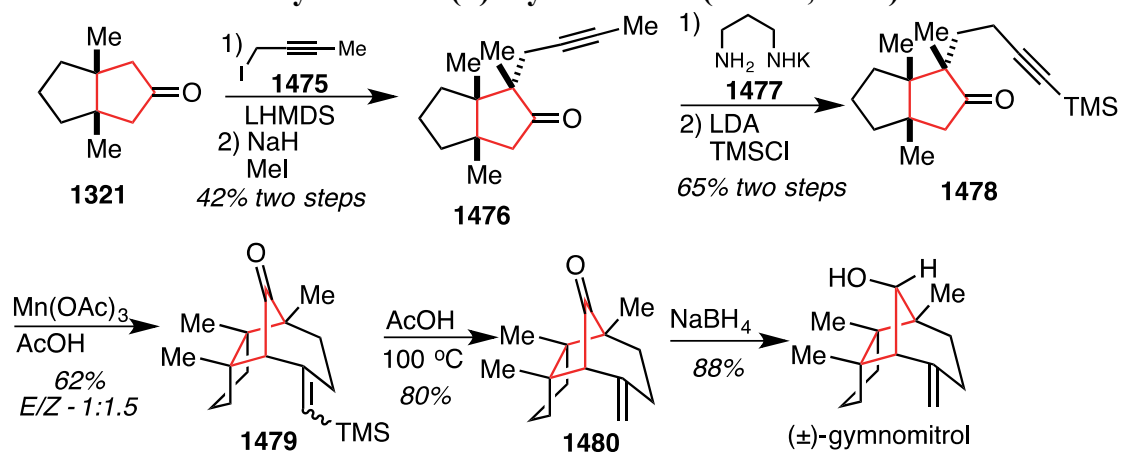

In the synthesis of anguidine by Brooks ${ }^{264}$, a 1,3-cyclopentanedione (1323) is desymmetrized by enantioselective carbonyl reduction with Baker's yeast to give chiral ketol 1481 (Scheme 178). ${ }^{265}$ Hydroxyl inversion occurred over two steps to give 1482. This material was converted to ester 1483 over three steps. Bromination occurred following the method of Marquet ${ }^{266}$, and subsequent elimination gave cyclopentene 1484. The cyclopentene was dihydroxylated to give the desired diastereomer in a 5:1 ratio (1485). Presumably the stereoselectivity results

${ }^{263}$ O’Neil, S. V.; Quickley, C. A.; Snider, B. B. J. Org. Chem. 1997, 62, 1970-1975.

264 (a) Brooks, D. W.; Grothaus, P. G.; Palmer, J. T. Tetrahedron Lett. 1982, 23, 4187-4190. (b) Brooks, D. W.; Grothaus, P. G.; Mazdiyasni, H. J. Am. Chem. Soc. 1983, 105, 4472-4473.

${ }^{265}$ Brooks, D. W.; Grothaus, P. G.; Irwin, W. L. J. Org. Chem. 1982, 47, $2820-2821$.

${ }^{266}$ Marquet, A.; Duolaitsky, M.; Kogan, H. B.; Mamlok, L.; Ouanees, C.; Jacques, J. Bull. Soc. Chim. Fr. 1962, 90. 
from the steric size of the adjacent silyl ether. Treatment of this intermediate with base induced lactonization; the regioselectivity can be attributed to reaction of the less hindered hydroxyl group. Intermediate 1486 was advanced 12 steps to intermediate 1487. A Wititig olefination and removal of the silyl group yielded 1488 . Finally, epoxidation with peracid delivered the fully functionalized cyclopentane (1489) of anguidine. Completion of the synthesis required an additional two transformations.

\section{Scheme 178. Total Synthesis of Anguidine (Brooks, 1983)}

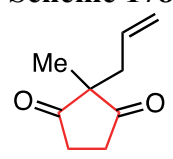

1323

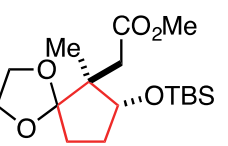

1483

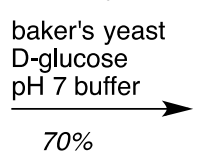

$70 \%$

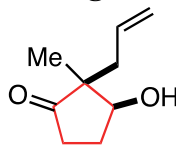

1481
1) $\mathrm{TsCl}$, pyr. 2) $\mathrm{KNO}_{2}$, DMF

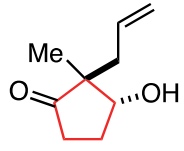

3 steps

1482
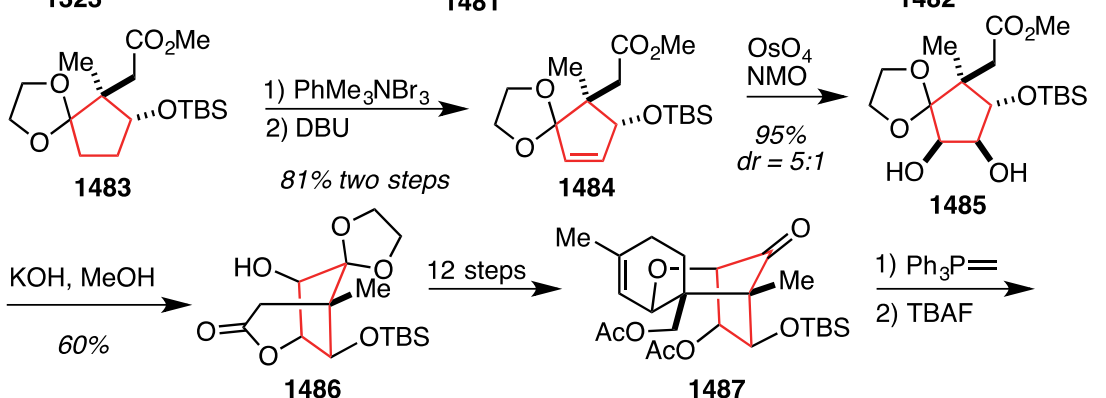

$\mathrm{Me}$

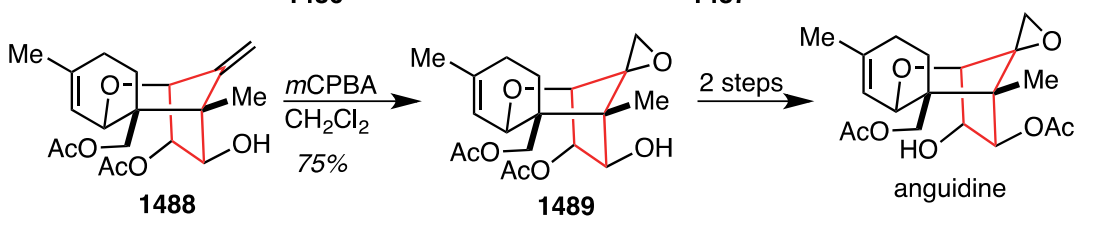

In a synthesis of $( \pm)$-coriolin, Trost begins the synthesis with enedione starting material 1490, which has become an important building block in chemical synthesis ${ }^{267}$ (Scheme 179). The enone functionality was masked as a thioether by conjugate addition of methanethiol. A chemoselective ketalization protected the less hindered ketone. Finally, monosulfenylation using dimethyl disulfide occurred to give 1491. Alkylation with the substituted allyl iodide 1492 gives 1493. Peracid oxidation of the sulfides to the corresponding sulfones yielded 1494. Treatment with fluoride promoted allyl silane addition to the ketone giving triquinane $\mathbf{1 4 9 5}$. This material was advanced three steps to intermediate 1496, which was epoxidized with peracid to give 1497 . The stereoselectivity of the epoxidation can be explained by the preferential formation of the cis diquinane substructure. Acetal hydrolysis and elimination of the sulfones gave dienone 1498. Reductive opening of the epoxide gives a mixture of isomeric products, and subsequent treatment with DBU delivers a single dienone alcohol 1499. Conjugate reduction with lithium in liquid ammonia gave an 80:20 mixture of $\beta, \gamma-$ and $\alpha, \beta-$ unsaturated ketones. Epoxidation of this crude mixture was followed by isomerization with DBU to give the allylic alcohol 129. This material was advanced four steps to intermediate $\mathbf{1 3 0}$, which is an intermediate previously taken to the natural product by Danishefsky. ${ }^{34}$

\section{Scheme 179. Formal Synthesis of $( \pm)$-Coriolin (Trost, 1981)}

${ }^{267}$ Trost, B. M.; Curran, D. P. J. Am. Chem. Soc. 1980, 102, 5699-5700. 


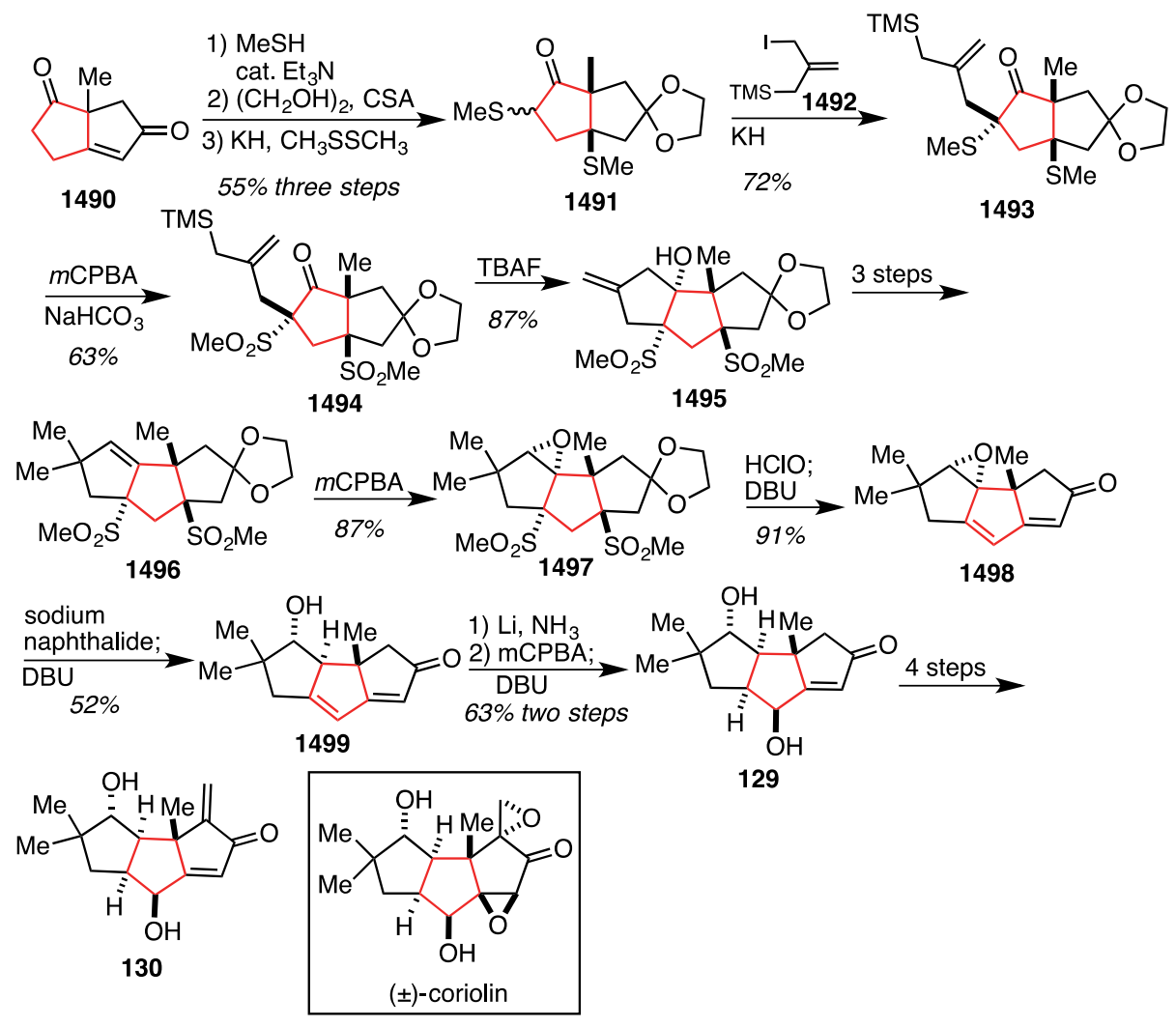

The formal synthesis of $( \pm)$-coriolin by Curran ${ }^{217}$ begins with 2-methylcyclopentenone (1500) (Scheme 180). This material was advanced using an Ireland-Claisen rearrangement, selenolactonization and elimination to give bicyclic intermediate 1505. Treatment of $\mathbf{1 5 0 5}$ with the cuprate derived from $\mathbf{1 4 7 3}$ induced substitution with opening of the bicycle furnishing 1507. Functional group manipulations over five steps occurred to give 1508. An elegant cascade radical cyclization was promoted by treatment with samarium iodide to give the desired triquinane ring system with cis ring fusions. Hydrolysis of the ketal furnishes 1509. Conversion to the crossconjugated dienone 142 was accomplished by formation of the silyl enol ether and oxidation with DDQ. This advanced intermediate was previously prepared and taken to coriolin by Danishefsky ${ }^{34}$ and Tatsuta. ${ }^{35}$

\section{Scheme 180. Formal Synthesis of $( \pm)$-Coriolin (Curran, 1988)}



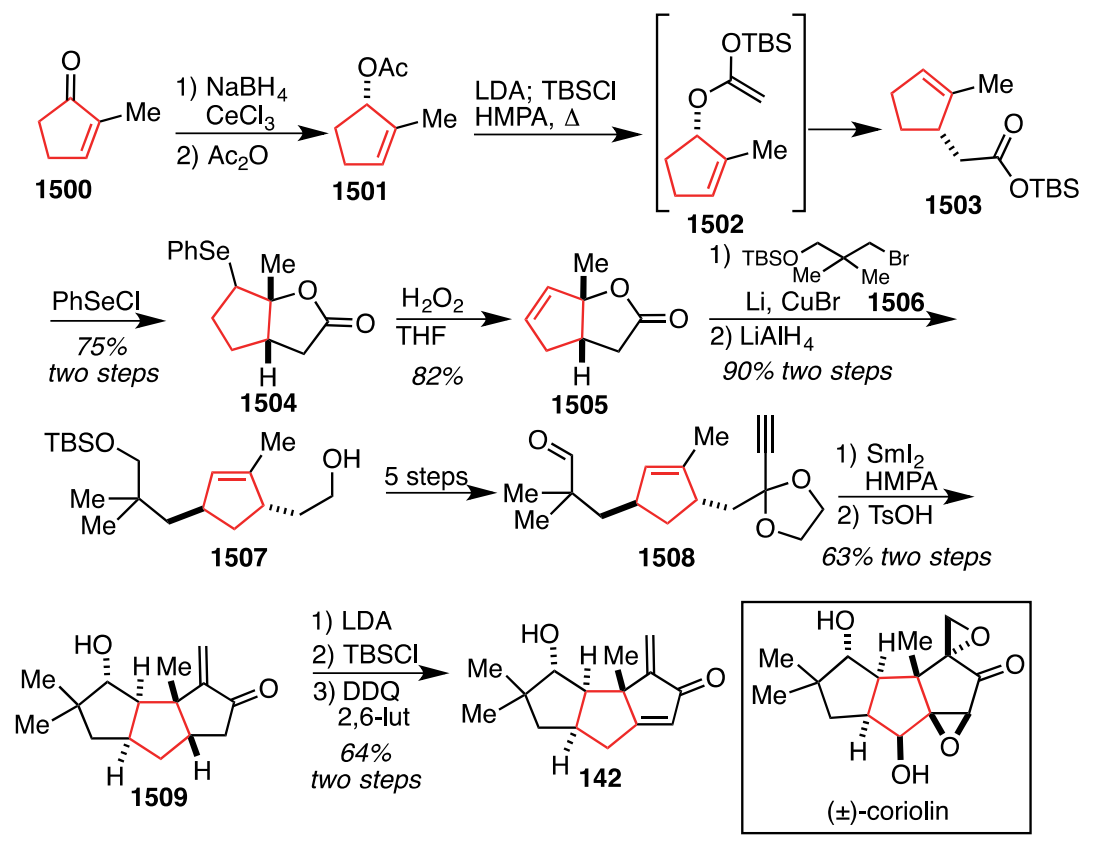

\subsection{Cyclopentenone Functionalizations}

In the synthesis of $( \pm)$-coriolin, Koreeda ${ }^{268}$ uses a one-step aldol-alkylation to construct a bicyclo[3.3.0]octenone system (Scheme 181). The dialkylation reaction begins with a substituted cyclopentenone 1510, which was treated with base to generate dianionic intermediate (1511). This intermediate undergoes double addition to 3 iodo-2,2-dimethylpropanal (1512), and the aldol functional group is protected as its MOM ether to give bicyclic product 1513. The regioselectivity of the alkylation is the result of a more reactive anion at $\mathrm{C} 5$; reaction of the C5 anion leaves a fully conjugated enolate intermediate, which undergoes subsequent alkylation. Alternatively, initial alkylation at $\mathrm{C} 4$ would leave a less stable cross-conjugated anion. Addition of methyllithium to enone 1513 and elimination upon acidic workup delivered enone 1514. A conjugate addition of the cuprate derived from 1515 occurred from the convex face of the bicycle. Acid-catalyzed acetal removal and subsequent aldol cyclization delivered triquinane scaffold 1516. Alcohol protection and elimination delivers enone 1517. A stereoselective and regioselective carbonyl reduction was performed under Luche conditions. Interestingly, the diastereomer formed in the Luche reduction arises from hydride addition to the concave face of the bicycle. After evaluating other hydride reducing reagents and substrates, it was postulated that the steric size of the benzoate group is responsible for the unexpected diastereoselectivity. A subsequent acetylation gave 1518. A Salmond ${ }^{269}$ oxidation installs the carbonyl yielding cyclopentenone 1519. Reductve removal of the acetate and benzoyl deprotection furnishes intermediate 104, which has been taken forward to $( \pm)$-coriolin by Trost. ${ }^{129}$

Scheme 181. Total Synthesis of $( \pm)$-Coriolin (Koreeda, 1983)

${ }^{268}$ Koreeda, M.; Mislankar, S. G. J. Am. Chem. Soc. 1983, 105, 7203-7205.

${ }^{269}$ Salmond, W. G.; Barta, M. A.; Havens, J. L. J. Org. Chem. 1978, 43, 2057-2059. 

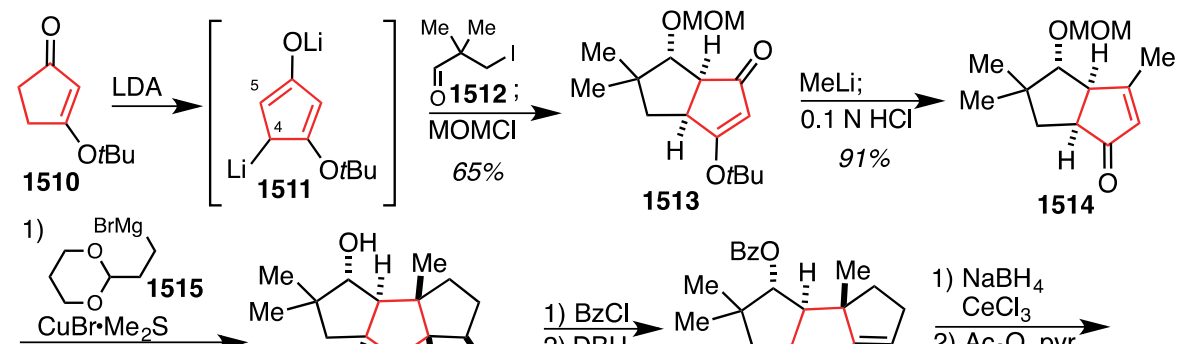

2) aq. $\mathrm{HCl}$

$79 \%$ two steps

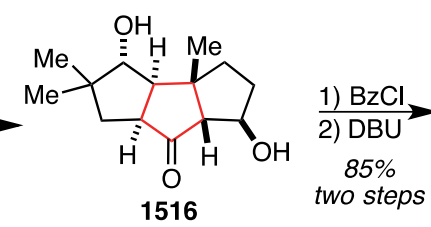

1513

1514
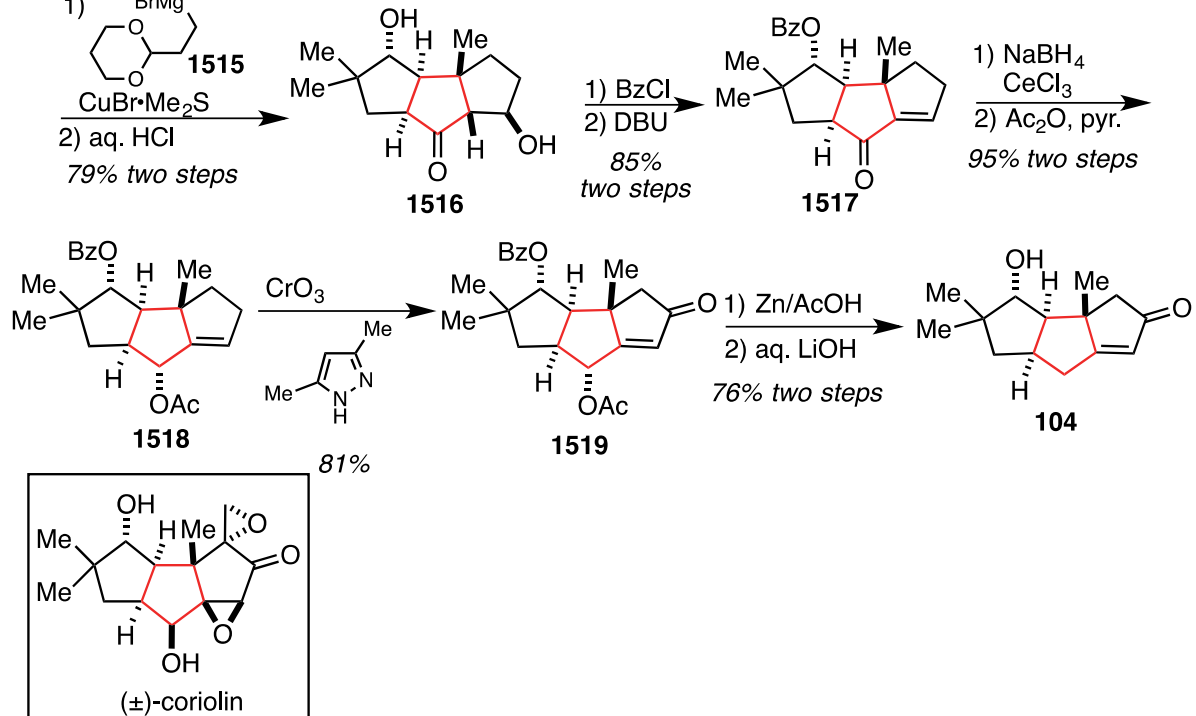

The synthesis of $( \pm)$-kelsoene by Piers ${ }^{270}$ begins with cyclopentenone (1520). Conjugate addition of the lower order heterocuprate 1521 delivers 1522 (Scheme 182). Intramolecular alkylation delivered the cis-fused bicyclo[3.3.0] octane substructure of kelsoene. Hydrogenation using Crabtree's catalyst ${ }^{271}$ occurred from the less hindered face of the bicycle to give $\mathbf{1 5 2 3}$ with good diastereoselectivity. Selenium based oxidation of ketone 1523 gave cyclopentenone 1524. Addition of methyllithium formed a tertiary alcohol that could be oxidized with PCC on alumina to give the transposed enone 1525. A [2+2] photocycloaddition with ethylene proceeded stereoselectively from the less hindered face of the bicycle furnishing $\mathbf{1 5 2 6}$ and completing the tricyclic core of kelsoene. After many unsuccessful attempts at olefination of the ketone, the Lombardo ${ }^{272}$ reagent was effective at delivering alkene 1527. Hydroboration-oxidation delivered fully functionalized cyclopentene 1528. This material was advanced to the methyl ketone $\mathbf{1 5 2 9}$ over three transformations. Treatment with acid induced ketone epimerization, which established the desired stereochemistry for the target. Finally, olefination with the Lombardo reagent completed the synthesis of $( \pm)$-kelsoene.

Scheme 182. Total Synthesis of $( \pm)$-Kelsoene (Piers, 2001)

\footnotetext{
${ }^{270}$ Piers, E.; Orellana, A. Synthesis 2001, 14, 2138-2142.

${ }^{271}$ Suggs, J. W.; Cox, S. D.; Crabtree, R. H.; Quirk, J. M. Tetrahedron Lett. 1981, 22, 303-306.

${ }^{272}$ Lombardo, L.; Tetrahedron. Lett. 1982, 23, 4293-4296.
} 


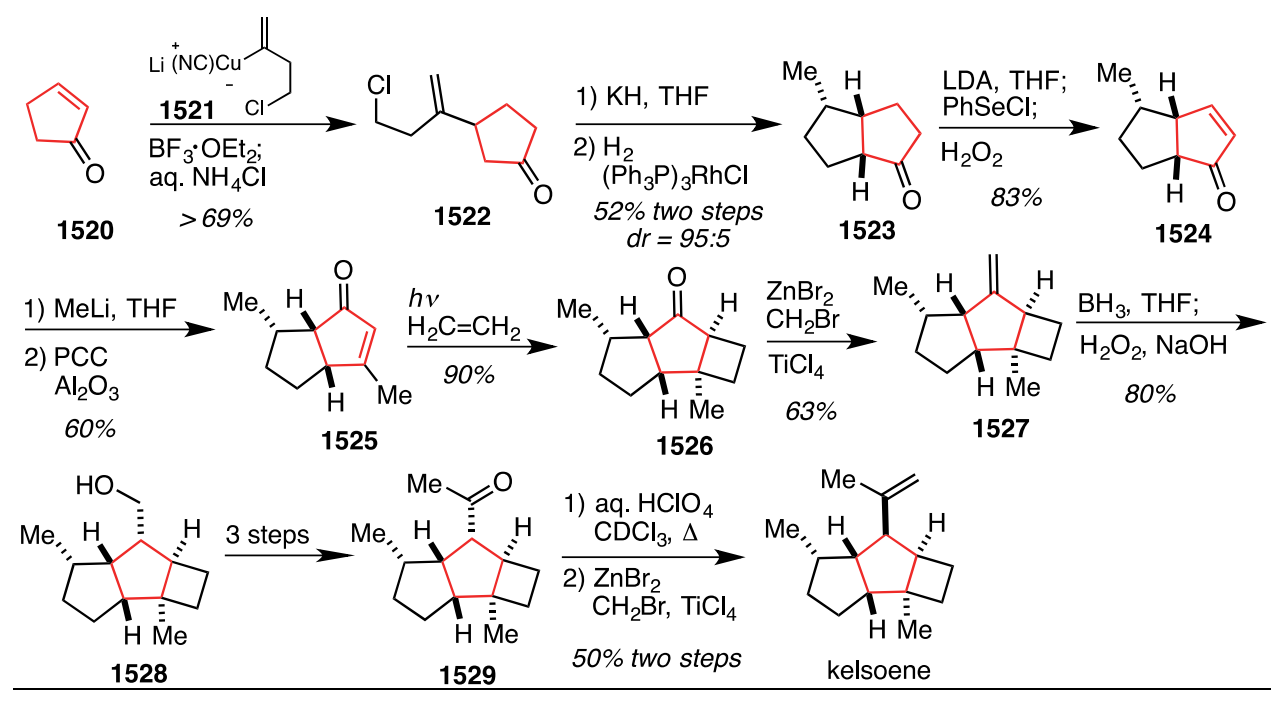

In the synthesis of $\left( \pm\right.$-merrilactone A, Mehta ${ }^{273}$ begins with a substituted cyclopentene-1,3-dione (Scheme 183). Desymmetrization of $\mathbf{1 5 3 0}$ was performed over three steps requiring double hydroxymethylation, protection of the diol as the acetonide and Luche reduction to give 1531. A 1,2-addition of an allylcerium reagent was followed by hydroxyl oxidation with manganese dioxide to give hydroxyenone 1532. A two-step transketalization sequence delivered the desired acetonide 1533 in a 1:1 ratio with $\mathbf{1 5 3 2}$, which could be recycled. This material was advanced four steps to diene 1534. Ring closing metathesis ${ }^{274}$ delivered tricyclic intermediate 1535. A [2+2] cycloaddition with trans-dichloroethylene was successful in promoting the formation of vicinal quaternary stereocenters as a complex mixture of diastereomers. The cycloaddition proceeds with modest facial selectivity $(d r=2: 1)$ with respect to the enone because the acetonide blocks the $\alpha$-diastereoface. The desired major isomer $\mathbf{1 5 3 6}$ was isolated as an inconsequential mixture of chloride diastereomers. Reductive dehalogenation was followed by ketone reduction with DIBAL, which proceeds with complete diastereoselectivity to give 1537. It was speculated that the diastereoselective ketone reduction is the result of either the steric bias of the polycyclic architecture or coordination of the hydride reagent to the acetonide oxygens. The resulting fully functionalized cyclopentane contains all the desired stereochemistry for $( \pm)$ merrilactone $\mathrm{A}$, and the natural product was completed over an additional ten steps.

Scheme 183. Total Synthesis of ( \pm )-Merrilactone A (Mehta, 2006)
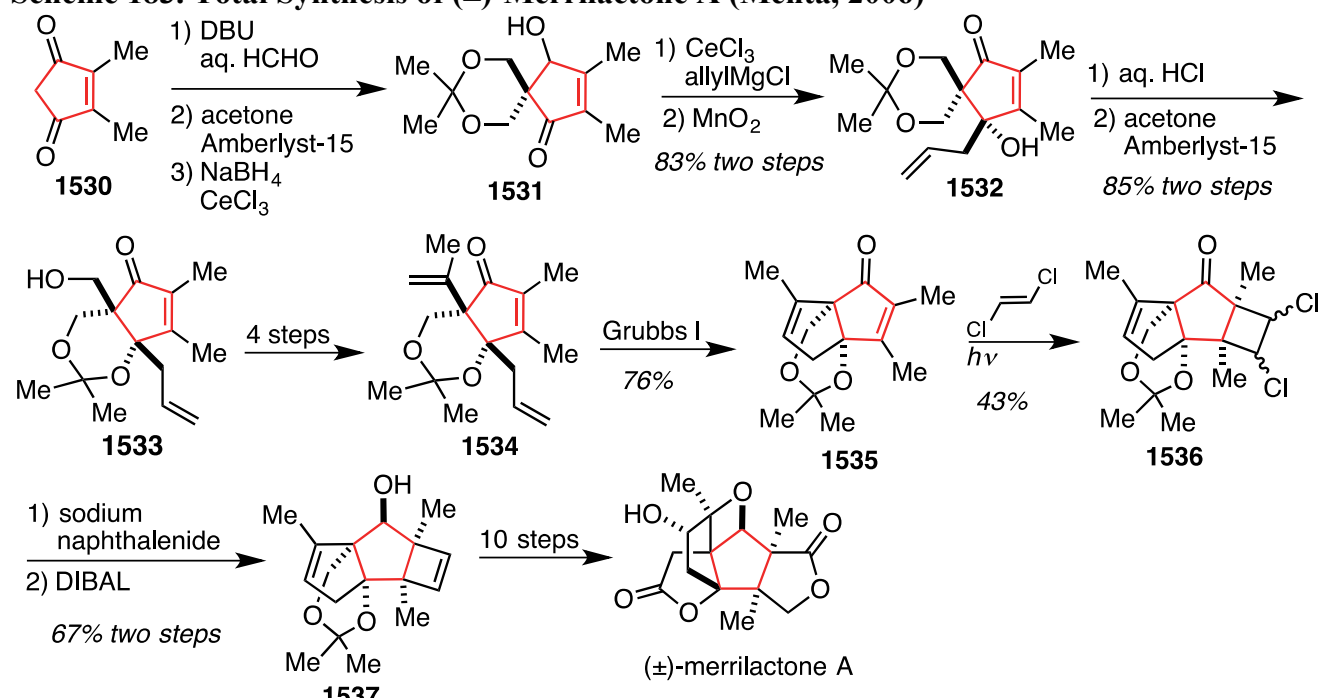

${ }^{273}$ Mehta, G.; Singh, S. R. Tetrahedron Lett. 2005, 46, 2079-2082.

${ }^{274}$ Grubbs, R. H.; Chang, H. Tetrahedron 1998, 54, 4413-4450. 
In a formal synthesis of $( \pm)$-merrilactone A, Greaney ${ }^{275}$ begins with functionalized cyclopentenone 1538 (Scheme 184). Silylation of the alcohol was followed by a 1,2-addition of the organolithium derived from 1539 furnishing 1540. Fluoride deprotection and oxidation gave a cyclopentenone. The tertiary alcohol was then protected as a silyl ether to give 1541. A two-step sequence involving Eschenmoser's reagent yielded 1542. The key conjugate addition-aldol process was promoted by $\mathrm{Et}_{2} \mathrm{AlCN}$ and $\mathrm{TiCl}_{4}$, and 1543 is obtained as the major diastereomer $(d r=7: 1)$. Mechanistically, addition of cyanide gives a cyclic $E$-configured ketone enolate. The aldol reaction presumably proceeds through a closed titanium-chelated transition state leading to the favored anti-aldol diastereomer. Desilylation and treatment with boron trifluoride furnished the oxatriquinane core 1544. Using a similar approach to Mehta ${ }^{273}$, photocycloaddition with dichloroethylene proceeded to give a mixture of diastereomers favoring addition on the $\beta$-diastereoface of the enone (1545). Dechlorination with zinc dust gave cyclobutene 1546. A stereoselective ketone reduction delivered fully substituted cyclopentane $\mathbf{1 5 4 7}$, which is an intermediate previously prepared in the total syntheses of $( \pm)$-merrilactone A by Mehta and Singh. ${ }^{273}$

\section{Scheme 184. Formal Synthesis of ( \pm )-Merrilactone A (Greaney, 2012)}
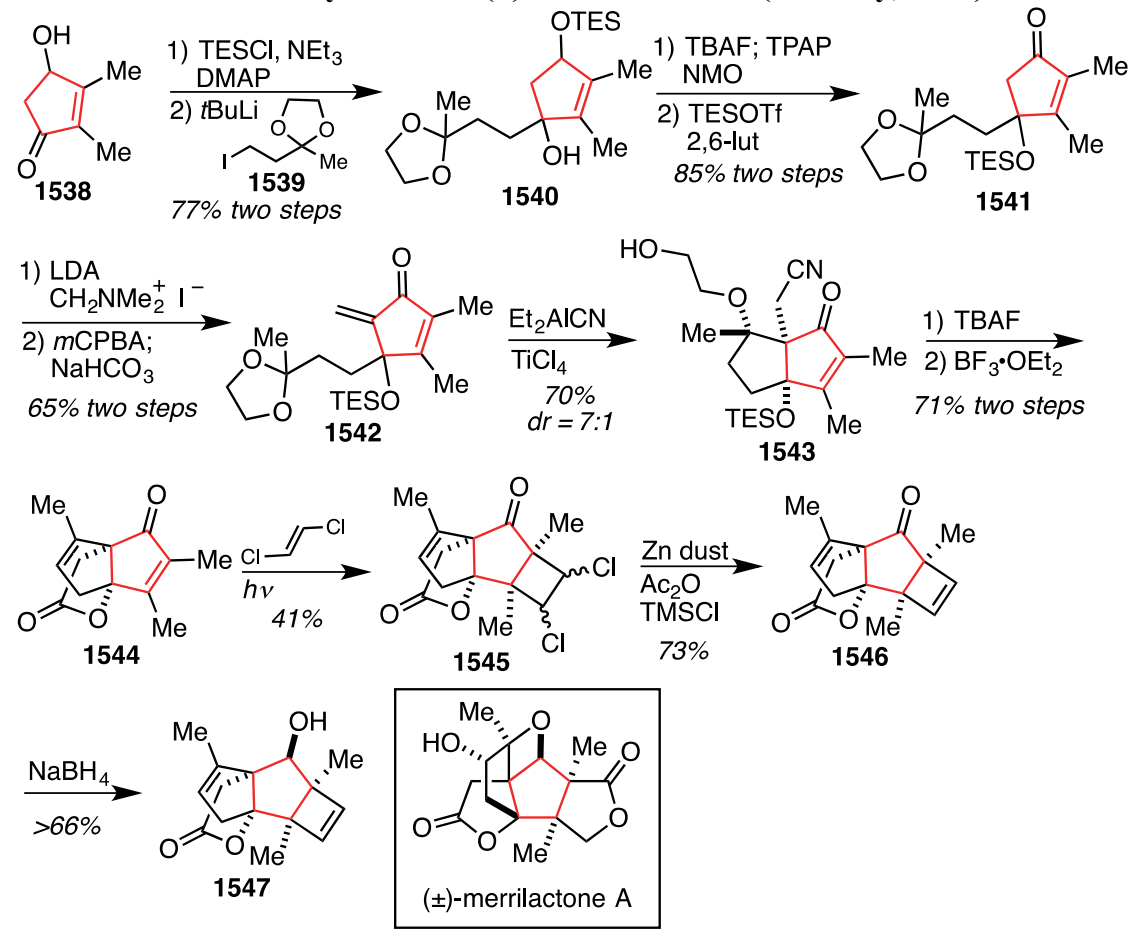

The synthesis of $( \pm)$-palau'amine accomplished by Namba, Nishizawa and Tanino ${ }^{276,277}$ begins with cyclopentenone (1520, Scheme 185). A Morita-Baylis-Hilman reaction with TBS-protected glycolaldehyde (1548) delivers 1549. Acetylation, Luche reduction, and TBS protection delivers a 2:1 mixture of diastereomers (1550). This diastereomeric mixture was carried several steps through the synthesis. This mixture was subjected to an Ireland-Claisen rearrangement to give 1552. The geometry of the trisubstituted alkene is the result of a favored chair-like transition state with equatorial positioning of the silyloxymethyl group. The carboxylic acid was converted to the acyl tosylhydrazide, and the silyl ethers were cleaved under acidic conditions furnishing diol 1553. Cyclization of $\mathbf{1 5 5 3}$ was catalyzed by $\mathrm{Hg}(\mathrm{OTf})_{2}$ to give $\mathbf{1 5 5 4}$ exclusively as the cis-fused bicycle; however, the product remained an inconsequential mixture of alcohol diastereomers. Alcohol oxidation simplified the diastereomer mixture giving good yields of ketone 1555. Saegusa-Ito oxidation provided 1556. A second Morita-Baylis-Hilman reaction with formaldehyde delivered alcohol 1557. Conjugate addition of nitromethane under basic conditions occurred from the convex face of the bicycle to deliver 1558. The unpurified

${ }^{275}$ Nazef, N.; Davies, R. D. M.; Greaney, M. F. Org. Lett. 2012, 14, 3720-3723.

${ }^{276}$ (a) Namba, K.; Kaihara, Y.; Yamamoto, H.; Imagawa, H.; Tanino, K.; Williams, R. M.; Nishizawa, M. Chem. Eur. J. 2009, 15, 6560-6563. (b) Namba, K.; Takeuchi, K.; Kaihara, Y.; Oda, M.; Nakayama, A.; Nakayama, A.; Yoshida, M.; Tanino, K. Nat. Commun. 2015, 6, 8731.

${ }^{277}$ This synthesis was disclosed in two distinct manuscripts each depicting racemic intermediates in opposite enantiomeric series. Herein, we depict all the racemic intermediates from both papers in the same enantiomeric series. 
material was subjected to hydride reduction to yield a fully functionalized cyclopentane 1559. Interestingly, the hydride addition occurred from the concave face of the bicycle; no explanation was offered for this

diastereoselectivity, but it may result from a greater steric demand of the vinyl and nitro methyl groups compared with the cyclic acyl hydrazide. This material was advanced ten steps to intermediate 1560. The central ring architecture of palau'amine was then constructed in a cascade reaction initiated by treatment of 1560 with LHMDS. Deprotonation induces $\beta$-elimination of a trifluoromethyl amide, which simultaneously cleaves the N$\mathrm{N}$ bond and generates an imine (1562). Intramolecular nucleophilic addition to the activated imine delivers the trans-bicyclo[3.3.0] octane ring system. The cascade continues with the addition of the tethered pyrrole to the methyl ester to form diketopiperizine 1564. This intermediate was advanced over 12 steps to alcohol 1565. The activation of the secondary alcohol as the chloride proceeded with retention of stereochemistry and induced a cyclization with the guandine nitrogen to form an intermediate aziridine (1566). Subsequent backside attack of chloride resulted in 1567, which has the required stereogenic chloride configuration and the fully functionalized cyclopentane found in ( \pm )-palau'amine. The natural product was completed in an additional seven steps.

Scheme 185. Total Synthesis of ( \pm )-Palau'amine (Namba, Nishizawa, Tanino, 2015) 

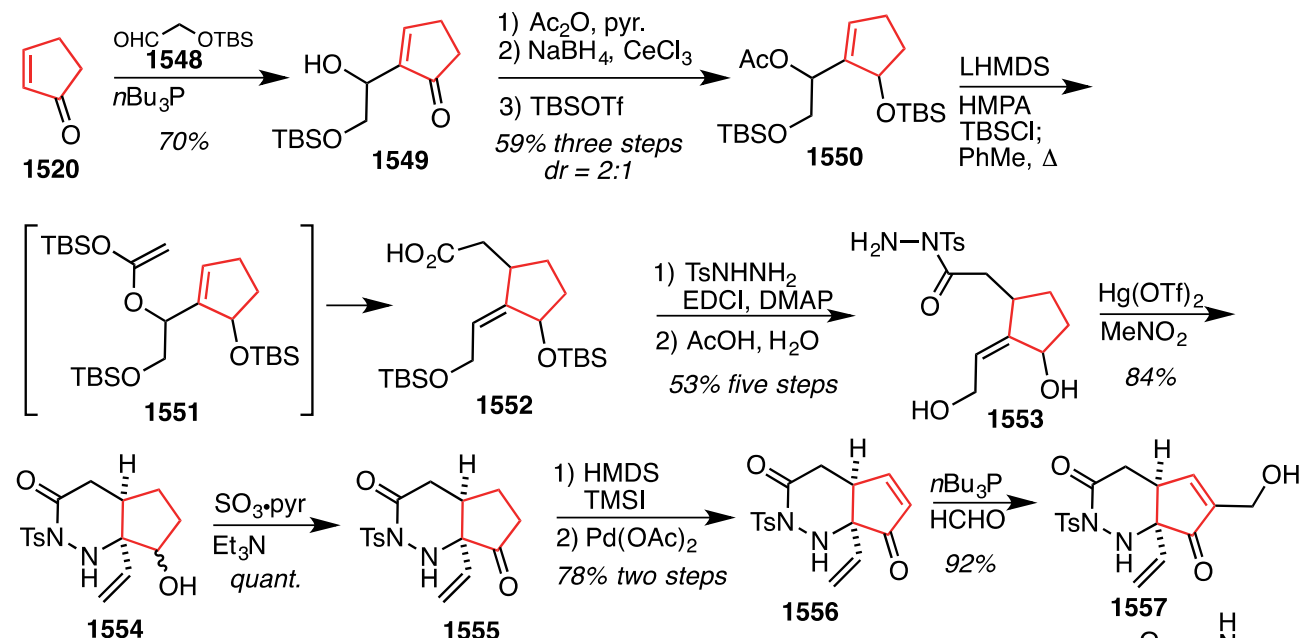

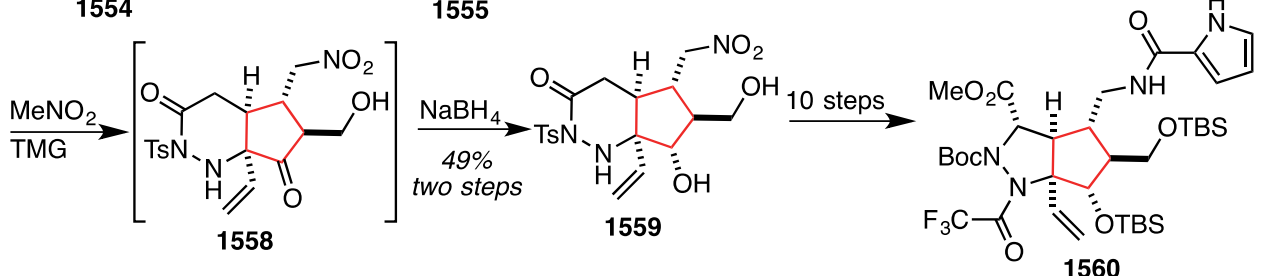

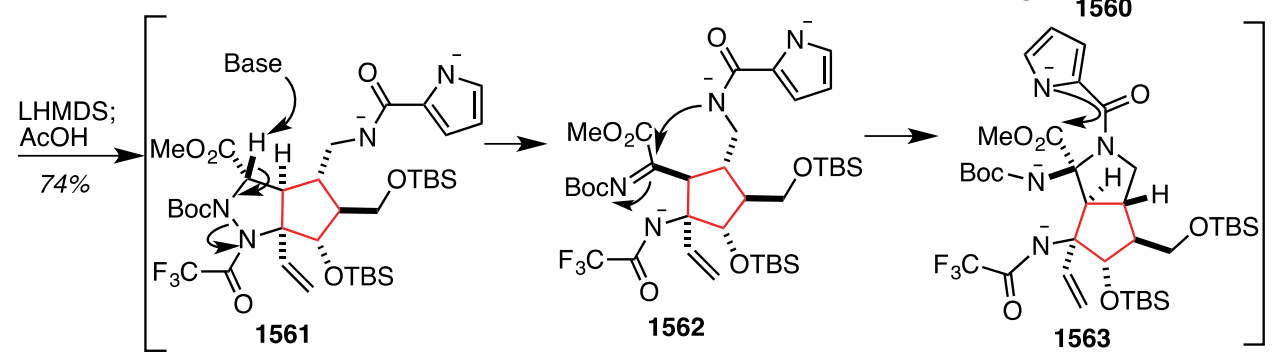

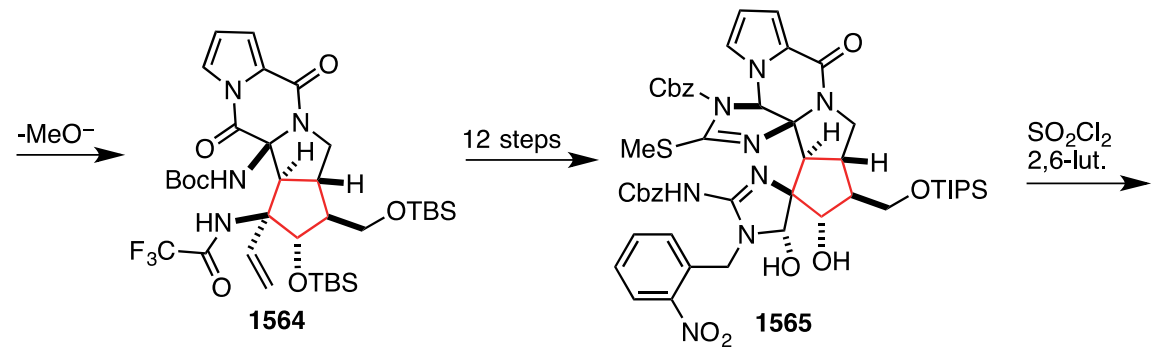

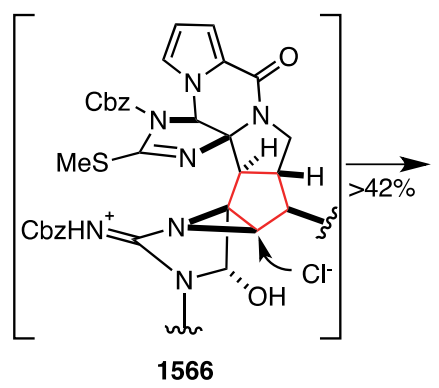

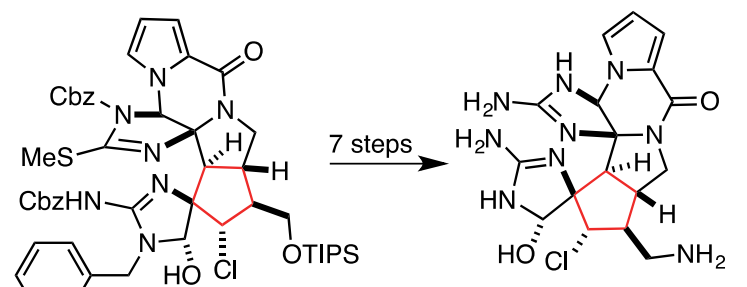

$( \pm)$-palau'amine·3TFA

In the Bruce $^{278}$ synthesis of a $( \pm)$-rocaglamide analog, the opening synthetic sequence capitalizes on the rich reactivity of the enone functional group (Scheme 186). Specifically, conjugate addition of phenyl cuprate to enone 1569 gives an anti-diarylcyclopentanone 1570 after an aqueous workup. Thermodynamic enolate formation and alkylation with chloride $\mathbf{1 5 7 1}$ delivered trisubstituded cyclopentanone $\mathbf{1 5 7 2}$ with control of

${ }^{278}$ Bruce, I.; Cooke, N. G.; Diorazio, L. J.; Hall, R. G.; Irving, E. Tetrahedron Lett. 1999, 40, 4279-4282. 
diastereoselectivity. Intramolecular electrophilic aromatic substitution was followed by an acid promoted dehydration to give the fused tricyclic intermediate 1573. Dihydroxylation of the trisubstituted alkene in 1573 was problematic, but a $28 \%$ yield of $\mathbf{1 5 7 4}$ was obtained using optimized conditions. Steric crowding from the nearby aryl groups hinders the convex face of the ring system making formation and/or cleavage of the osmate ester low yielding. Oxidation of the secondary alcohol, $\alpha$-carboxylation with the Stiles reagent ${ }^{166}$, and methyl ester hydrolysis delivers $\beta$-ketoacid 1575. Formation of the ketoamide was performed with dimethylamine hydrochloride, and the product existed exclusively in the keto form with the amide substituent anti to the vicinal phenyl group. Finally, hydride reduction of the ketone gave target compound $\mathbf{1 5 7 6}$ with modest diastereoselectivity. This final product contains a fully functionalized cyclopentane, and it is an analog of $( \pm)$ rocaglamide where the furanyl oxygen has been replaced with a methylene group.

\section{Scheme 186. Synthetic Studies Towards ( \pm )-Rocaglamide (Bruce, 1999)}

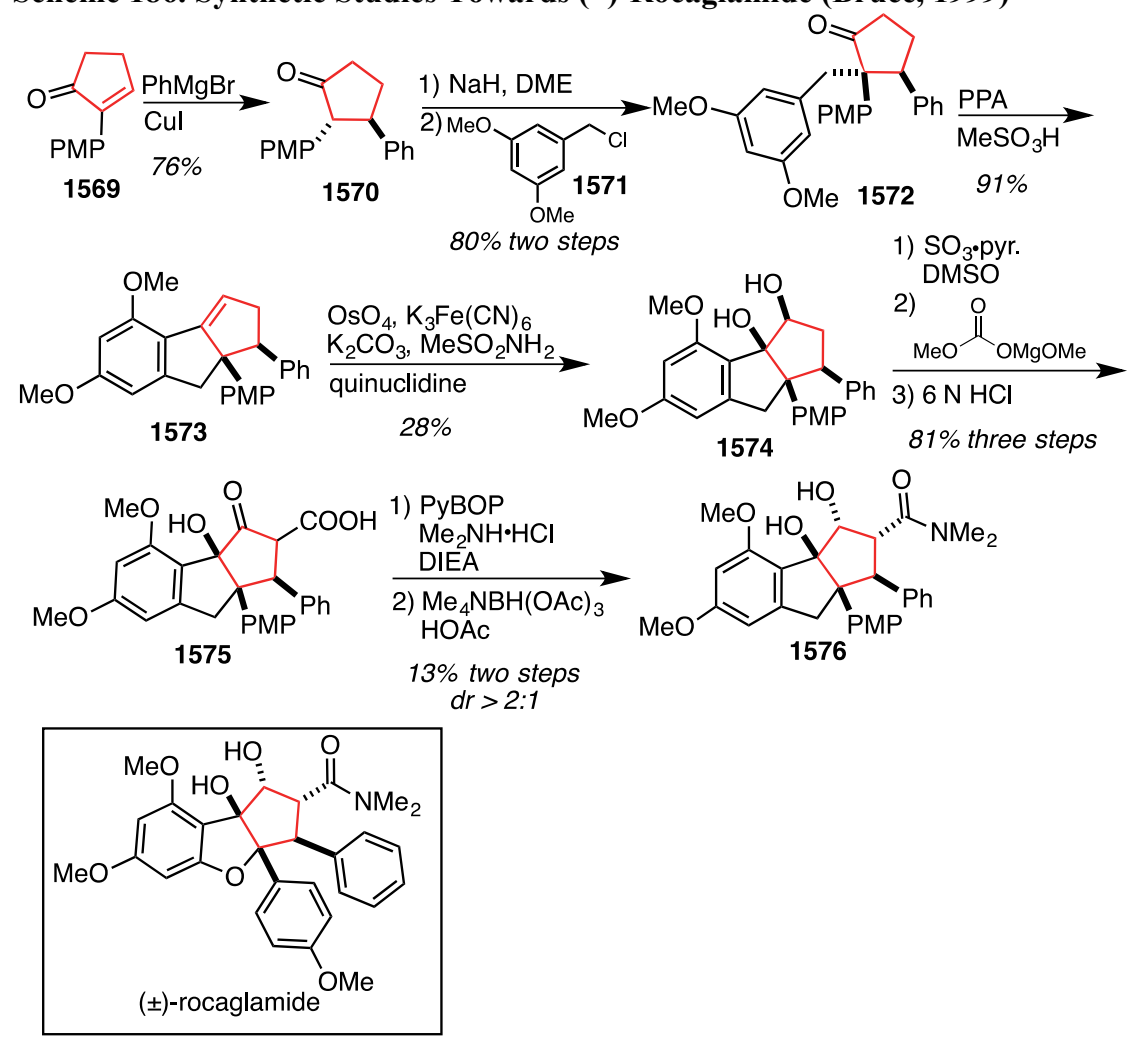

In the Nemoto ${ }^{279}$ synthesis of (-)-allosamizoline, cyclopentenone $\mathbf{1 5 2 0}$ undergoes an asymmetric aziridination using chiral diamine catalyst 1577 (Scheme 187). The key aziridination is believed to proceed through assembly 1578, wherein the primary amine first forms an iminium cation intermediate and the secondary amine aids in delivery of the sulfonyloxy carbamate to the $R e$-face of the ring yielding 1579 . Selenation and ketone reduction each occurred stereoselectively from the convex face of $\mathbf{1 5 7 9}$ to deliver $\mathbf{1 5 8 0}$. Treatment with aqueous acetic acid resulted in regioselective opening of the aziridine ring to furnish $\mathbf{1 5 8 1}$. Oxidative elimination of the selenide delivered cyclopentene 1582. Acidic removal of the Boc protecting group and treatment with triphosgene produces cyclic carbamate 1583. This material was advanced three steps to allyl ether 1584. A suprafacial 2,3Wittig rearrangement resulted in homo-allylic alcohol 1585. Stereoselective epoxidation is thought to arise due to hydroxyl direction to deliver fully functionalized cyclopentane 1586. Acidic opening of the epoxide and acetate protection of the resulting triol was followed by oxidative removal of the PMB ether delivering 1230. Intermediate was previously prepared and taken to allosamizoline by Simpkins ${ }^{214}$ and Tatsuta ${ }^{23}$.

Scheme 187. Formal Synthesis of (-)-Allosamizoline (Nemoto, 2016)

${ }^{279}$ Nemoto, T.; Muramoto, R.; Ruengsatra, T.; Hamada, Y. Tetrahedron 2016, 72, 1991-1997. 

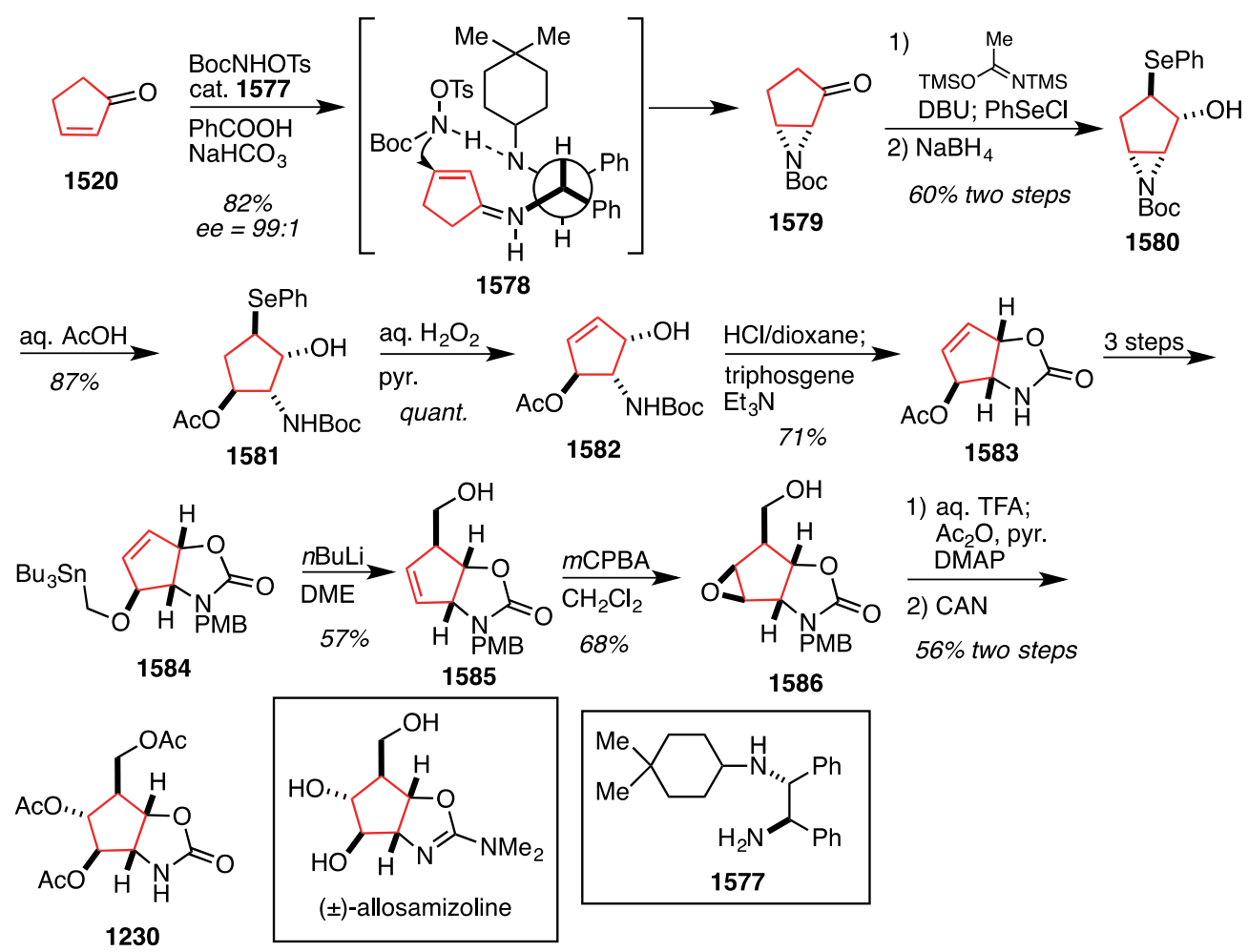

\section{Conclusion}

This review has presented the syntheses of natural products containing fully-functionalized cyclopentanes. Substituted cyclopentanes are a common and central structural feature in many important synthetic target molecules, and this molecular feature has inspired and challenged synthetic chemists for decades. Strategies for constructing fully-functionalized cyclopentanes were organized into ten well-defined patterns. All major reaction types including pericyclic, ionic, and radical reactions have been used to prepare such molecular architectures. Cascade processes are often particularly powerful strategies used to create cyclic motifs with control of multiple stereogenic carbon atoms. Moreover, those cascade sequences that are biomimetic or bioinspired have been particularly useful in creating the most highly substituted cyclopentanes.

\section{Acknowledgment}

We gratefully acknowledge the National Science Foundation for support of our research group under Grant Number 1465287. 